\title{
INTER-UNIVERSAL TEICHMÜLLER THEORY I: CONSTRUCTION OF HODGE THEATERS
}

\author{
SHINICHI MochIZUKI
}

May 2020

\begin{abstract}
The present paper is the first in a series of four papers, the goal of which is to establish an arithmetic version of Teichmüller theory for number fields equipped with an elliptic curve - which we refer to as "inter-universal Teichmüller theory" - by applying the theory of semi-graphs of anabelioids, Frobenioids, the étale theta function, and log-shells developed in earlier papers by the author. We begin by fixing what we call "initial $\Theta$-data", which consists of an elliptic curve $E_{F}$ over a number field $F$, and a prime number $l \geq 5$, as well as some other technical data satisfying certain technical properties. This data determines various hyperbolic orbicurves that are related via finite étale coverings to the once-punctured elliptic curve $X_{F}$ determined by $E_{F}$. These finite étale coverings admit various symmetry properties arising from the additive and multiplicative structures on the ring $\mathbb{F}_{l}=\mathbb{Z} / l \mathbb{Z}$ acting on the $l$-torsion points of the elliptic curve. We then construct " $\Theta \pm$ ell $N F$-Hodge theaters" associated to the given $\Theta$-data. These $\Theta^{ \pm \text {ell }} \mathrm{NF}$-Hodge theaters may be thought of as miniature models of conventional scheme theory in which the two underlying combinatorial dimensions of a number field - which may be thought of as corresponding to the additive and multiplicative structures of a ring or, alternatively, to the group of units and value group of a local field associated to the number field — are, in some sense, "dismantled" or "disentangled" from one another. All $\Theta^{ \pm \text {ell } N F-H o d g e ~ t h e a t e r s ~}$ are isomorphic to one another, but may also be related to one another by means of a " $\Theta$-link", which relates certain Frobenioid-theoretic portions of one $\Theta^{ \pm e l l}$ NF-Hodge theater to another in a fashion that is not compatible with the respective conventional ring/scheme theory structures. In particular, it is a highly nontrivial problem to relate the ring structures on either side of the $\Theta$-link to one another. This will be achieved, up to certain "relatively mild indeterminacies", in future papers in the series by applying the absolute anabelian geometry developed in earlier papers by the author. The resulting description of an "alien ring structure" [associated, say, to the domain of the $\Theta$-link] in terms of a given ring structure [associated, say, to the codomain of the $\Theta$-link] will be applied in the final paper of the series to obtain results in diophantine geometry. Finally, we discuss certain technical results concerning profinite conjugates of decomposition and inertia groups in the tempered fundamental group of a $p$-adic hyperbolic curve that will be of use in the development of the theory of the present series of papers, but are also of independent interest.
\end{abstract}

\section{Contents:}

Introduction

$\S 0$. Notations and Conventions

$\S 1$. Complements on Coverings of Punctured Elliptic Curves 
$\S 2$. Complements on Tempered Coverings

$\S 3$. Chains of $\Theta$-Hodge Theaters

$\S 4$. Multiplicative Combinatorial Teichmüller Theory

$\S 5$. $\Theta N F-H o d g e$ Theaters

$\S 6$. Additive Combinatorial Teichmüller Theory

\section{Introduction}

$\S$ I1. Summary of Main Results

$\S$ I2. Gluing Together Models of Conventional Scheme Theory

$\S I 3$. Basepoints and Inter-universality

$\S$ I4. Relation to Complex and $p$-adic Teichmüller Theory

$\S$ I5. Other Galois-theoretic Approaches to Diophantine Geometry

Acknowledgements

\section{$\S$ I1. Summary of Main Results}

The present paper is the first in a series of four papers, the goal of which is to establish an arithmetic version of Teichmüller theory for number fields equipped with an elliptic curve, by applying the theory of semi-graphs of anabelioids, Frobenioids, the étale theta function, and log-shells developed in [SemiAnbd], [FrdI], [FrdII], [EtTh], and [AbsTopIII] [cf., especially, [EtTh] and [AbsTopIII]]. Unlike many mathematical papers, which are devoted to verifying properties of mathematical objects that are either well-known or easily constructed from wellknown mathematical objects, in the present series of papers, most of our efforts will be devoted to constructing new mathematical objects. It is only in the final portion of the third paper in the series, i.e., [IUTchIII], that we turn to the task of proving properties of interest concerning the mathematical objects constructed. In the fourth paper of the series, i.e., [IUTchIV], we show that these properties may be combined with certain elementary computations to obtain diophantine results concerning elliptic curves over number fields.

We refer to $\S 0$ below for more on the notations and conventions applied in the present series of papers. The starting point of our constructions is a collection of initial $\Theta$-data [cf. Definition 3.1]. Roughly speaking, this data consists, essentially, of

- an elliptic curve $E_{F}$ over a number field $F$,

- an algebraic closure $\bar{F}$ of $F$,

- a prime number $l \geq 5$,

- a collection of valuations $\mathbb{V}$ of a certain subfield $K \subseteq \bar{F}$, and

- a collection of valuations $\overline{\mathbb{V}}$ mod of a certain subfield $F_{\bmod } \subseteq F$

that satisfy certain technical conditions - we refer to Definition 3.1 for more details. Here, we write $F_{\text {mod }} \subseteq F$ for the field of moduli of $E_{F}, K \subseteq \bar{F}$ for the extension field of $F$ determined by the l-torsion points of $E_{F}, X_{F} \subseteq E_{F}$ for the once-punctured elliptic curve obtained by removing the origin from $E_{F}$, and $X_{F} \rightarrow C_{F}$ for the hyperbolic orbicurve obtained by forming the stack-theoretic quotient of $X_{F}$ by the 
natural action of $\{ \pm 1\}$. Then $F$ is assumed to be Galois over $F_{\bmod }, \operatorname{Gal}(K / F)$ is assumed to be isomorphic to a subgroup of $G L_{2}\left(\mathbb{F}_{l}\right)$ that contains $S L_{2}\left(\mathbb{F}_{l}\right), E_{F}$ is assumed to have stable reduction at all of the nonarchimedean valuations of $F$, $C_{K} \stackrel{\text { def }}{=} C_{F} \times_{F} K$ is assumed to be a $K$-core [cf. [CanLift], Remark 2.1.1], $\underline{\mathbb{V}}$ is assumed to be a collection of valuations of $K$ such that the natural inclusion $F_{\text {mod }} \subseteq F \subseteq K$ induces a bijection $\underline{\mathbb{V}} \stackrel{\sim}{\rightarrow} \mathbb{V}_{\text {mod }}$ between $\underline{\mathbb{V}}$ and the set $\mathbb{V}$ mod of all valuations of the number field $F_{\text {mod }}$, and

$$
\mathbb{V}_{\bmod }^{\text {bad }} \subseteq \mathbb{V}_{\bmod }
$$

is assumed to be some nonempty set of nonarchimedean valuations of odd residue characteristic over which $E_{F}$ has bad [i.e., multiplicative] reduction - i.e., roughly speaking, the subset of the set of valuations where $E_{F}$ has bad multiplicative reduction that will be "of interest" to us in the context of the theory of the present series of papers. Then we shall write $\underline{\mathbb{V}}^{\text {bad }} \stackrel{\text { def }}{=} \mathbb{V}_{\text {mod }}^{\text {bad }} \times_{\mathbb{V}_{\text {mod }}} \underline{\mathbb{V}} \subseteq \underline{\mathbb{V}}, \mathbb{V}_{\text {mod }}^{\text {good def }} \stackrel{\mathbb{V}_{\text {mod }} \backslash \mathbb{V} \text { mod }}{\text { bad }}$, $\underline{\mathbb{V}}^{\text {good }} \stackrel{\text { def }}{=} \underline{\mathbb{V}} \backslash \underline{\mathbb{V}}^{\text {bad }}$. Also, we shall apply the superscripts "non" and "arc" to $\underline{\mathbb{V}}, \mathbb{V}$ mod to denote the subsets of nonarchimedean and archimedean valuations, respectively.

This data determines, up to $K$-isomorphism [cf. Remark 3.1.3], a finite étale covering $\underline{C}_{K} \rightarrow C_{K}$ of degree $l$ such that the base-changed covering

$$
\underline{X}_{K} \stackrel{\text { def }}{=} \underline{C}_{K} \times_{C_{F}} X_{F} \rightarrow X_{K} \stackrel{\text { def }}{=} X_{F} \times_{F} K
$$

arises from a rank one quotient $E_{K}[l] \rightarrow Q(\cong \mathbb{Z} / l \mathbb{Z})$ of the module $E_{K}[l]$ of $l$ torsion points of $E_{K}(K)$ [where we write $E_{K} \stackrel{\text { def }}{=} E_{F} \times_{F} K$ ] which, at $\underline{v} \in \underline{\mathbb{V}}^{\text {bad }}$, restricts to the quotient arising from coverings of the dual graph of the special fiber. Moreover, the above data also determines a cusp

of $\underline{C}_{K}$ which, at $\underline{v} \in \underline{\mathbb{V}}^{\text {bad }}$, corresponds to the canonical generator, up to \pm 1 , of $Q$ [i.e., the generator determined by the unique loop of the dual graph of the special fiber]. Furthermore, at $\underline{v} \in \underline{\mathbb{V}}^{\text {bad }}$, one obtains a natural finite étale covering of degree $l$

$$
\underline{\underline{X}}_{\underline{v}} \rightarrow \underline{X}_{\underline{v}} \stackrel{\text { def }}{=} \quad \underline{X}_{K} \times_{K} K_{\underline{v}} \quad\left(\rightarrow \quad \underline{C}_{\underline{v}} \stackrel{\text { def }}{=} \quad \underline{C}_{K} \times_{K} K_{\underline{v}}\right)
$$

by extracting $l$-th roots of the theta function; at $\underline{v} \in \underline{\mathbb{V}}^{\text {good }}$, one obtains a natural finite étale covering of degree $l$

$$
\underline{X}_{\underline{v}} \rightarrow \underline{X}_{\underline{v}} \stackrel{\text { def }}{=} \underline{X}_{K} \times K_{K} K_{\underline{v}} \quad\left(\rightarrow \quad \underline{C}_{\underline{v}} \stackrel{\text { def }}{=} \quad \underline{C}_{K} \times_{K} K_{\underline{v}}\right)
$$

determined by $\underline{\epsilon}$. More details on the structure of the coverings $\underline{C}_{K}, \underline{X}_{K}, \underline{\underline{X}}_{\underline{v}}$ [for $\left.\underline{v} \in \underline{\mathbb{V}}^{\text {bad }}\right], \underline{X}_{\underline{v}}\left[\right.$ for $\left.\underline{v} \in \underline{\mathbb{V}}^{\text {good }}\right]$ may be found in [EtTh], $\S 2$, as well as in $\S 1$ of the present paper.

In this situation, the objects

$$
l^{*} \stackrel{\text { def }}{=}(l-1) / 2 ; \quad l^{ \pm} \stackrel{\text { def }}{=}(l+1) / 2 ; \quad \mathbb{F}_{l}^{*} \stackrel{\text { def }}{=} \mathbb{F}_{l}^{\times} /\{ \pm 1\} ; \quad \mathbb{F}_{l}^{\rtimes \pm} \stackrel{\text { def }}{=} \mathbb{F}_{l} \rtimes\{ \pm 1\}
$$


[cf. the discussion at the beginning of $\S 4$; Definitions 6.1, 6.4] will play an important role in the discussion to follow. The natural action of the stabilizer in $\operatorname{Gal}(K / F)$ of the quotient $E_{K}[l] \rightarrow Q$ on $Q$ determines a natural poly-action of $\mathbb{F}_{l}^{*}$ on $\underline{C}_{K}$, i.e., a natural isomorphism of $\mathbb{F}_{l}^{*}$ with some subquotient of Aut $\left(\underline{C}_{K}\right)$ [cf. Example 4.3, (iv)]. The $\mathbb{F}_{l}^{*}$-symmetry constituted by this poly-action of $\mathbb{F}_{l}^{*}$ may be thought of as being essentially arithmetic in nature, in the sense that the subquotient of Aut $\left(\underline{C}_{K}\right)$ that gives rise to this poly-action of $\mathbb{F}_{l}^{*}$ is induced, via the natural map $\operatorname{Aut}\left(\underline{C}_{K}\right) \rightarrow \operatorname{Aut}(K)$, by a subquotient of $\operatorname{Gal}(K / F) \subseteq \operatorname{Aut}(K)$. In a similar vein, the natural action of the automorphisms of the scheme $\underline{X}_{K}$ on the cusps of $\underline{X}_{K}$ determines a natural poly-action of $\mathbb{F}_{l}^{\rtimes \pm}$ on $\underline{X}_{K}$, i.e., a natural isomorphism of $\mathbb{F}_{l}^{\rtimes \pm}$ with some subquotient of $\operatorname{Aut}\left(\underline{X}_{K}\right)$ [cf. Definition 6.1, (v)]. The $\mathbb{F}_{l}^{\rtimes \pm}$-symmetry constituted by this poly-action of $\mathbb{F}_{l}^{\rtimes \pm}$ may be thought of as being essentially geometric in nature, in the sense that the subgroup $\operatorname{Aut}_{K}\left(\underline{X}_{K}\right) \subseteq \operatorname{Aut}\left(\underline{X}_{K}\right)$ [i.e., of $K$-linear automorphisms] maps isomorphically onto the subquotient of $\operatorname{Aut}\left(\underline{X}_{K}\right)$ that gives rise to this poly-action of $\mathbb{F}_{l}^{\rtimes \pm}$. On the other hand, the global $\mathbb{F}_{l}^{*}$. symmetry of $\underline{C}_{K}$ only extends to a " $\{1\}$-symmetry" [i.e., in essence, fails to extend!] of the local coverings $\underline{\underline{X}}_{\underline{v}}\left[\right.$ for $\left.\underline{v} \in \underline{\mathbb{V}}^{\text {bad }}\right]$ and $\underline{X}_{\underline{v}}$ [for $\left.\underline{v} \in \underline{\mathbb{V}}^{\text {good }}\right]$, while the global $\mathbb{F}_{l}^{\rtimes \pm}$-symmetry of $\underline{X}_{K}$ only extends to a " $\{ \pm 1\}$-symmetry" [i.e., in essence, fails to extend!] of the local coverings $\underline{\underline{X}}_{\underline{v}}\left[\right.$ for $\left.\underline{v} \in \underline{\mathbb{V}}^{\text {bad }}\right]$ and $\underline{X}_{\underline{v}}\left[\right.$ for $\left.\underline{v} \in \underline{\mathbb{V}}^{\text {good }}\right]-$ cf. Fig. I1.1 below.

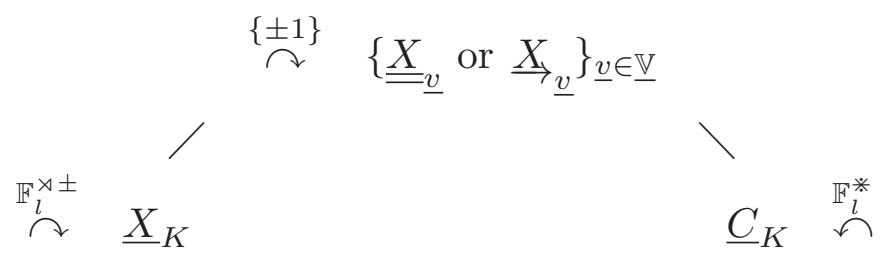

Fig. I1.1: Symmetries of coverings of $X_{F}$

We shall write $\Pi_{\underline{v}}$ for the tempered fundamental group of $\underline{\underline{X}}_{\underline{v}}$, when $\underline{v} \in \underline{\mathbb{V}}^{\text {bad }}$ [cf. Definition $3.1,(\mathrm{e})]$; we shall write $\Pi_{\underline{v}}$ for the étale fundamental group of $\underline{X}_{\underline{v}}$, when $\underline{v} \in \underline{\mathbb{V}}^{\text {good }}\left[\right.$ cf. Definition 3.1, (f)]. Also, for $\underline{v} \in \underline{\mathbb{V}}^{\text {non }}$, we shall write $\Pi_{\underline{v}} \rightarrow G_{\underline{v}}$ for the quotient determined by the absolute Galois group of the base field $K_{\underline{v}}$. Often, in the present series of papers, we shall consider various types of collections of data — which we shall refer to as "prime-strips" — indexed by $\underline{v} \in \underline{\mathbb{V}}(\stackrel{\sim}{\rightarrow} \mathbb{V}$ mod $)$ that are isomorphic to certain data that arise naturally from $\underline{\underline{X}}_{\underline{v}}\left[\right.$ when $\left.\underline{v} \in \underline{\mathbb{V}}^{\text {bad }}\right]$ or $\underline{X}_{\underline{v}}$ [when $\left.\underline{v} \in \underline{\mathbb{V}}^{\text {good }}\right]$. The main types of prime-strips that will be considered in the present series of papers are summarized in Fig. I1.2 below.

Perhaps the most basic kind of prime-strip is a $\mathcal{D}$-prime-strip. When $\underline{v} \in$ $\underline{V}^{\text {non }}$, the portion of a $\mathcal{D}$-prime-strip labeled by $\underline{v}$ is given by a category equivalent to [the full subcategory determined by the connected objects of] the category of tempered coverings of $\underline{\underline{X}}_{\underline{v}}$ [when $\left.\underline{v} \in \underline{\mathbb{V}}^{\text {bad }}\right]$ or finite étale coverings of $\underline{X}_{\underline{v}}$ [when $\left.\underline{v} \in \underline{\mathbb{V}}^{\text {good }}\right]$. When $\underline{v} \in \underline{\mathbb{V}}^{\text {arc }}$, an analogous definition may be obtained by applying the theory of Aut-holomorphic orbispaces developed in [AbsTopIII], $§ 2$. One variant of the notion of a $\mathcal{D}$-prime-strip is the notion of a $\mathcal{D}^{\vdash}$-prime-strip. When $\underline{v} \in \underline{\mathbb{V}}^{\text {non }}$, the portion of a $\mathcal{D}^{\vdash}$-prime-strip labeled by $\underline{v}$ is given by a category equivalent to [the full subcategory determined by the connected objects of] the Galois category 
associated to $G_{v}$; when $\underline{v} \in \underline{\mathbb{V}}^{\text {arc }}$, an analogous definition may be given. In some sense, $\mathcal{D}$-prime-strips may be thought of as abstractions of the "local arithmetic holomorphic structure" of [copies of] $F_{\text {mod }}$ [which we regard as equipped with the once-punctured elliptic curve $\left.X_{F}\right]$ - cf. the discussion of [AbsTopIII], §I3. On the other hand, $\mathcal{D}^{\vdash}$-prime-strips may be thought of as "mono-analyticizations" [i.e., roughly speaking, the arithmetic version of the underlying real analytic structure associated to a holomorphic structure] of $\mathcal{D}$-prime-strips - cf. the discussion of [AbsTopIII], §I3. Throughout the present series of papers, we shall use the notation

to denote mono-analytic structures.

Next, we recall the notion of a Frobenioid over a base category [cf. [FrdI] for more details]. Roughly speaking, a Frobenioid [typically denoted " $\mathcal{F}$ "] may be thought of as a category-theoretic abstraction of the notion of a category of line bundles or monoids of divisors over a base category [typically denoted " $\mathcal{D}$ "] of topological localizations [i.e., in the spirit of a "topos"] such as a Galois category. In addition to $\mathcal{D}$ - and $\mathcal{D}^{\vdash}$-prime-strips, we shall also consider various types of prime-strips that arise from considering various natural Frobenioids - i.e., more concretely, various natural monoids equipped with a Galois action - at $\underline{v} \in \underline{\mathbb{V}}$. Perhaps the most basic type of prime-strip arising from such a natural monoid is an $\mathcal{F}$-prime-strip. Suppose, for simplicity, that $\underline{v} \in \underline{\mathbb{V}}^{\text {bad }}$. Then $\underline{v}$ and $\bar{F}$ determine, up to conjugacy, an algebraic closure $\bar{F}_{\underline{v}}$ of $K_{\underline{v}}$. Write

- $\mathcal{O}_{\bar{F}_{\underline{v}}}$ for the ring of integers of $\bar{F}_{\underline{v}}$;

- $\mathcal{O} \bar{F}_{\underline{v}} \subseteq \mathcal{O}_{\bar{F}_{\underline{v}}}$ for the multiplicative monoid of nonzero integers;

- $\mathcal{O}_{\bar{F}_{\underline{v}}}^{\times} \subseteq \mathcal{O}_{\bar{F}_{\underline{v}}}$ for the multiplicative monoid of units;

- $\mathcal{O}_{\bar{F}_{\underline{v}}}^{\mu} \subseteq \mathcal{O}_{\bar{F}_{\underline{v}}}$ for the multiplicative monoid of roots of unity;

- $\mathcal{O}_{\bar{F}_{\underline{v}}}^{\boldsymbol{\mu}_{2 l}} \subseteq \mathcal{O}_{\bar{F}_{\underline{v}}}$ for the multiplicative monoid of $2 l$-th roots of unity;

- $\underline{\underline{q}}_{\underline{v}} \in \mathcal{O}_{\bar{F}_{\underline{v}}}$ for a $2 l$-th root of the $q$-parameter of $E_{F}$ at $\underline{v}$.

Thus, $\mathcal{O}_{\overline{F_{\underline{v}}}}, \mathcal{O}_{\bar{F}_{\underline{v}}}^{\triangleright}, \mathcal{O}_{\bar{F}_{\underline{v}}}^{\times}, \mathcal{O}_{\bar{F}_{\underline{v}}}^{\mu}$, and $\mathcal{O}_{\bar{F}_{\underline{v}}}^{\mu_{2 l}}$ are equipped with natural $G_{\underline{\underline{v}}}$ actions. The

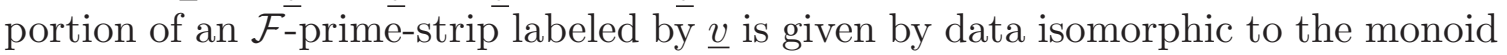
$\mathcal{O} \bar{F}_{\underline{\underline{v}}}$, equipped with its natural $\Pi_{\underline{v}}\left(\rightarrow G_{\underline{v}}\right)$-action [cf. Fig. I1.2]. There are various mono-analytic versions of the notion of an $\mathcal{F}$-prime-strip; perhaps the most basic is the notion of an $\mathcal{F}^{\vdash}$-prime-strip. The portion of an $\mathcal{F}^{\vdash}$-prime-strip labeled by $\underline{v}$ is given by data isomorphic to the monoid $\mathcal{O}_{\bar{F}_{\underline{v}}}^{\times} \times \underline{\underline{q}}_{\underline{v}}^{\mathbb{N}}$, equipped with its natural $G_{\underline{v}^{-}}$action [cf. Fig. I1.2]. Often we shall regard these various mono-analytic versions of an $\mathcal{F}$-prime-strip as being equipped with an additional global realified Frobenioid, which, at a concrete level, corresponds, essentially, to considering various arithmetic degrees $\in \mathbb{R}$ at $\underline{v} \in \underline{\mathbb{V}}\left(\stackrel{\sim}{\rightarrow} \mathbb{V}_{\text {mod }}\right)$ that are related to one another by means of the product formula. Throughout the present series of papers, we shall use the notation 
to denote such prime-strips.

\begin{tabular}{|c|c|c|}
\hline Type of prime-strip & Model at $v \in \mathbb{V}^{\mathrm{bad}}$ & $\underline{\text { Reference }}$ \\
\hline $\mathcal{D}$ & $\Pi_{\underline{v}}$ & $\mathrm{I}, 4.1,(\mathrm{i})$ \\
\hline $\mathcal{D}^{\vdash}$ & $G_{\underline{v}}$ & I, 4.1, (iii) \\
\hline $\mathcal{F}$ & $\Pi_{\underline{v}} \curvearrowright \mathcal{O} \bar{F}_{\underline{\underline{v}}}^{\triangleright}$ & $\mathrm{I}, 5.2,(\mathrm{i})$ \\
\hline $\mathcal{F}^{\vdash}$ & $G_{\underline{v}} \curvearrowright \mathcal{O}_{\bar{F}_{\underline{v}}}^{\times} \times \underline{q}_{\underline{\underline{v}}}^{\mathbb{N}}$ & I, 5.2, (ii) \\
\hline $\mathcal{F}^{\vdash x}$ & $G_{\underline{v}} \curvearrowright \mathcal{O}_{\bar{F}_{\underline{v}}}^{\times}$ & II, 4.9, (vii) \\
\hline $\mathcal{F}^{\vdash \times \mu}$ & $G_{\underline{v}} \curvearrowright \mathcal{O}_{\bar{F}_{\underline{v}}}^{\times \mu} \stackrel{\text { def }}{=} \mathcal{O}_{\bar{F}_{\underline{v}}}^{\times} / \mathcal{O}_{\bar{F}_{\underline{v}}}^{\mu}$ & II, 4.9, (vii) \\
\hline $\mathcal{F}^{\vdash} \triangleright \times \mu$ & $G_{\underline{v}} \curvearrowright \mathcal{O}_{\bar{F}_{\underline{v}}}^{\times \mu} \times \underline{q}_{\underline{v}}^{\mathbb{N}}$ & II, 4.9, (vii) \\
\hline $\mathcal{F}^{\vdash}$ & $G_{\underline{v}} \curvearrowright \underline{q}_{\underline{v}}^{\mathbb{N}}$ & III, 2.4, (ii) \\
\hline $\mathcal{F}^{\vdash \perp}$ & $G_{\underline{v}} \curvearrowright \quad \mathcal{O}_{\bar{F}_{\underline{v}}}^{\mu_{2 l}} \times \underline{q}_{\underline{v}}^{\mathbb{N}}$ & III, 2.4 , (ii) \\
\hline
\end{tabular}

$\mathcal{F}^{\Vdash \cdots}=\mathcal{F}^{\vdash \ldots}+\left\{\right.$ global realified Frobenioid associated to $\left.F_{\text {mod }}\right\}$

Fig. I1.2: Types of prime-strips

In some sense, the main goal of the present paper may be thought of as the construction of $\Theta^{ \pm \text {ell }}$ NF-Hodge theaters [cf. Definition 6.13, (i)]

$$
{ }^{\dagger} \mathcal{H} \mathcal{T}^{\Theta^{ \pm e l l} \mathrm{NF}}
$$

- which may be thought of as "miniature models of conventional scheme theory" - given, roughly speaking, by systems of Frobenioids. To any such 
$\Theta^{ \pm \text {ell }} \mathrm{NF}$-Hodge theater ${ }^{\dagger} \mathcal{H} \mathcal{T}^{\Theta^{ \pm \text {ell }} \mathrm{NF}}$, one may associate a $\mathcal{D}-\Theta^{ \pm \text {ell }} \mathbf{N F}$-Hodge theater [cf. Definition 6.13, (ii)]

$$
{ }^{\dagger} \mathcal{H} \mathcal{T}^{\mathcal{D}-\Theta^{ \pm \mathrm{ell}} \mathrm{NF}}
$$

— i.e., the associated system of base categories.

One may think of a $\Theta^{ \pm \text {ell }} \mathrm{NF}$-Hodge theater ${ }^{\dagger} \mathcal{H} \mathcal{T}^{\Theta^{ \pm e l l} \mathrm{NF}}$ as the result of gluing together a $\Theta^{ \pm \text {ell }}$-Hodge theater ${ }^{\dagger} \mathcal{H} \mathcal{T}^{\Theta^{ \pm e l l}}$ to a $\Theta \mathrm{NF}-$ Hodge theater ${ }^{\dagger} \mathcal{H} \mathcal{T}^{\Theta N F}$ [cf. Remark 6.12.2, (ii)]. In a similar vein, one may think of a $\mathcal{D}-\Theta^{ \pm \text {ell }} \mathrm{NF}-$ Hodge theater ${ }^{\dagger} \mathcal{H} \mathcal{T}^{\mathcal{D}-\Theta^{ \pm \text {ell }} \mathrm{NF}}$ as the result of gluing together a $\mathcal{D}$ - $\Theta^{ \pm \text {ell }}$-Hodge theater ${ }^{\dagger} \mathcal{H} \mathcal{T}^{\mathcal{D}-\Theta^{ \pm \text {ell }}}$ to a $\mathcal{D}$ - $\Theta N F$-Hodge theater ${ }^{\dagger} \mathcal{H} \mathcal{T}^{\mathcal{D} \text { - } \Theta N F}$. A $\mathcal{D}$ - $\Theta^{ \pm \text {ell }}$-Hodge theater ${ }^{\dagger} \mathcal{H} \mathcal{T}^{\mathcal{D} \text { - } \Theta^{ \pm \text {ell }}}$ may be thought of as a bookkeeping device that allows one to keep track of the action of the $\mathbb{F}_{l}^{\rtimes \pm}$-symmetry on the labels

$$
\left(-l^{*}<\ldots<-1<0<1<\ldots<l^{*}\right)
$$

- which we think of as elements $\in \mathbb{F}_{l}$ - in the context of the [orbi]curves $\underline{X}_{K}$, $\underline{\underline{X}}_{\underline{v}}\left[\right.$ for $\left.\underline{v} \in \underline{\mathbb{V}}^{\text {bad }}\right]$, and $\underline{X}_{\underline{v}}\left[\right.$ for $\left.\underline{v} \in \underline{\mathbb{V}}^{\text {good }}\right]$. The $\mathbb{F}_{l}^{\rtimes \pm}$-symmetry is represented in a $\mathcal{D}-\Theta^{ \pm \text {ell }}$-Hodge theater ${ }^{\dagger} \mathcal{H} \mathcal{T}^{\mathcal{D}-\Theta^{ \pm \text {ell }}}$ by a category equivalent to [the full subcategory determined by the connected objects of] the Galois category of finite étale coverings of $\underline{X}_{K}$. On the other hand, each of the labels referred to above is represented in a $\mathcal{D}$ - $\Theta^{ \pm \text {ell }}$-Hodge theater ${ }^{\dagger} \mathcal{H} \mathcal{T}^{\mathcal{D}-\Theta^{ \pm \text {ell }}}$ by a $\mathcal{D}$-prime-strip. In a similar vein, a $\mathcal{D}$ - $\Theta N F$-Hodge theater ${ }^{\dagger} \mathcal{H} \mathcal{T}^{\mathcal{D}-\Theta N F}$ may be thought of as a bookkeeping device that allows one to keep track of the action of the $\mathbb{F}_{l}^{*}$-symmetry on the labels

$$
\left(1<\ldots<l^{*}\right)
$$

- which we think of as elements $\in \mathbb{F}_{l}^{*}$ - in the context of the orbicurves $\underline{C}_{K}$, $\underline{\underline{X}}_{\underline{v}}$ [for $\left.\underline{v} \in \underline{\mathbb{V}}^{\text {bad }}\right]$, and $\underline{X}_{\underline{v}}$ [for $\left.\underline{v} \in \underline{\mathbb{V}}^{\text {good }}\right]$. The $\mathbb{F}_{l}^{*}$-symmetry is represented in a $\mathcal{D}$ - $\Theta N F$-Hodge theater ${ }^{\dagger} \mathcal{H} \overline{\mathcal{T}}^{\mathcal{D}-\Theta N F}$ by a category equivalent to [the full subcategory determined by the connected objects of] the Galois category of finite étale coverings of $\underline{C}_{K}$. On the other hand, each of the labels referred to above is represented in a $\mathcal{D}$ $\Theta N F-H o d g e$ theater ${ }^{\dagger} \mathcal{H} \mathcal{T}^{\mathcal{D}-\Theta N F}$ by a $\mathcal{D}$-prime-strip. The combinatorial structure of $\mathcal{D}-\Theta N F-$ and $\mathcal{D}-\Theta^{ \pm \text {ell }}$-Hodge theaters summarized above [cf. also Fig. I1.3 below] is one of the main topics of the present paper and is discussed in detail in $\S 4$ and $\S 6$. The left-hand portion of Fig. I1.3 corresponds to the $\mathcal{D}$ - $\Theta^{ \pm \text {ell }}$-Hodge theater; the right-hand portion of Fig. I1.3 corresponds to the $\mathcal{D}$ - $\Theta N F-H o d g e$ theater; these left-hand and right-hand portions are glued together by identifying $\mathcal{D}$-prime-strips in such a way that the labels $0 \neq \pm t \in \mathbb{F}_{l}$ on the left are identified with the corresponding label $j \in \mathbb{F}_{i}^{*}$ on the right [cf. Proposition 6.7; Remark 6.12.2; Fig. $6.5]$.

In this context, we remark that many of the constructions of [AbsTopIII] were intended as prototypes for constructions of the present series of papers. For instance, the global theory of [AbsTopIII], §5, was intended as a sort of simplified prototype for the $\Theta^{ \pm \text {ell }} N F$-Hodge theaters of the present paper, i.e., except with the various label bookkeeping devices deleted. The various panalocal objects of [AbsTopIII], §5, were intended as prototypes for the various types of prime-strips that 
appear in the present series of papers. Perhaps most importantly, the theory of the log-Frobenius functor and log-shells developed in [AbsTopIII], §3, §4, §5, was intended as a prototype for the theory of the log-link that is developed in [IUTchIII]. In particular, although most of the main ideas and techniques of [AbsTopIII], $\S 3, \S 4, \S 5$, will play an important role in the present series of papers, many of the constructions performed in [AbsTopIII], $\S 3, \S 4, \S 5$, will not be applied in a direct, literal sense in the present series of papers.

The $\mathbb{F}_{l}^{\rtimes \pm}$-symmetry has the advantange that, being geometric in nature, it allows one to permute various copies of " $G_{\underline{v}}$ " [where $\left.\underline{v} \in \underline{\mathbb{V}}^{\text {non }}\right]$ associated to distinct labels $\in \mathbb{F}_{l}$ without inducing conjugacy indeterminacies. This phenomenon, which we shall refer to as conjugate synchronization, will play a key role in the Kummer theory surrounding the Hodge-Arakelov-theoretic evaluation of the theta function at $\boldsymbol{l}$-torsion points that is developed in [IUTchII] - cf. the discussion of Remark 6.12.6; [IUTchII], Remark 3.5.2, (ii), (iii); [IUTchII], Remark 4.5.3, (i). By contrast, the $\mathbb{F}_{l}^{*}$-symmetry is more suited to situations in which one must descend from $K$ to $F_{\text {mod }}$. In the present series of papers, the most important such situation involves the Kummer theory surrounding the reconstruction of the number field $F_{\text {mod }}$ from the étale fundamental group of $\underline{C}_{K}$ - cf. the discussion of Remark 6.12.6; [IUTchII], Remark 4.7.6. This reconstruction will be discussed in Example 5.1 of the present paper. Here, we note that such situations necessarily induce global Galois permutations of the various copies of " $G_{\underline{v}}$ " [where $\left.\underline{v} \in \underline{\mathbb{V}}^{\text {non }}\right]$ associated to distinct labels $\in \mathbb{F}_{l}^{*}$ that are only well-defined up to conjugacy indeterminacies. In particular, the $\mathbb{F}_{l}^{*}$-symmetry is ill-suited to situations, such as those that appear in the theory of Hodge-Arakelov-theoretic evaluation that is developed in [IUTchII], that require one to establish conjugate synchronization.

$$
\begin{aligned}
& \stackrel{\{ \pm 1\}}{\curvearrowright}\left(\begin{array}{r}
-l^{*} \\
<\ldots<-1<0 \\
<1<\ldots<l^{*}
\end{array}\right) \Rightarrow\left[\begin{array}{r}
1<\ldots \\
<l^{*}
\end{array}\right] \Leftarrow\left(\begin{array}{r}
1<\ldots \\
<l^{*}
\end{array}\right) \\
& \Downarrow \\
& \begin{array}{l}
\underset{\mathbb{F}_{l}^{\rtimes \pm}}{\curvearrowright} \\
\uparrow \\
\stackrel{\downarrow}{\curvearrowright}
\end{array} \\
& \pm \leftarrow \pm \\
& \uparrow \stackrel{\mathbb{F}_{i}^{*}}{\curvearrowright} \downarrow \\
& * \leftarrow *
\end{aligned}
$$

Fig. I1.3: The combinatorial structure of a $\mathcal{D}-\Theta^{ \pm e l l}$ NF-Hodge theater [cf. Figs. 4.4, 4.7, 6.1, 6.3, 6.5 for more details]

Ultimately, when, in [IUTchIV], we consider diophantine applications of the theory developed in the present series of papers, we will take the prime number $l$ to be "large", i.e., roughly of the order of the square root of the height of the elliptic curve $E_{F}$ [cf. [IUTchIV], Corollary 2.2, (ii), (C1)]. When $l$ is regarded as large, the arithmetic of the finite field $\mathbb{F}_{l}$ "tends to approximate" the arithmetic of the ring of rational integers $\mathbb{Z}$. That is to say, the decomposition that occurs in a $\Theta^{ \pm \text {ell }}$ NF-Hodge theater into the "additive" [i.e., $\mathbb{F}_{l}^{\rtimes \pm}$ ] and "multiplicative" [i.e., $\mathbb{F}_{l}^{*}$-] symmetries of the ring $\mathbb{F}_{l}$ may be regarded as a sort of rough, approximate approach to the issue of "disentangling" the multiplicative and additive structures, i.e., "dismantling" the "two underlying combinatorial dimensions" [cf. 
the discussion of [AbsTopIII], §I3], of the ring $\mathbb{Z}$ - cf. the discussion of Remarks 6.12.3, 6.12.6.

Alternatively, this decomposition into additive and multiplicative symmetries in the theory of $\Theta^{ \pm \text {ell }} \mathrm{NF}-$ Hodge theaters may be compared to groups of additive and multiplicative symmetries of the upper half-plane [cf. Fig. I1.4 below]. Here, the "cuspidal" geometry expressed by the additive symmetries of the upper half-plane admits a natural "associated coordinate", namely, the classical $\boldsymbol{q}$-parameter, which is reminiscent of the way in which the $\mathbb{F}_{l}^{\rtimes \pm}$-symmetry is well-adapted to the Kummer theory surrounding the Hodge-Arakelov-theoretic evaluation of the theta function at $\boldsymbol{l}$-torsion points [cf. the above discussion]. By contrast, the "toral", or "nodal" [cf. the classical theory of the structure of Hecke correspondences modulo $p$ ], geometry expressed by the multiplicative symmetries of the upper half-plane admits a natural "associated coordinate", namely, the classical biholomorphic isomorphism of the upper half-plane with the unit disc, which is reminiscent of the way in which the $\mathbb{F}_{l}^{*}$-symmetry is well-adapted to the Kummer theory surrounding the number field $F_{\bmod }$ [cf. the above discussion]. For more details, we refer to the discussion of Remark 6.12.3, (iii).

From the point of view of the scheme-theoretic Hodge-Arakelov theory developed in [HASurI], [HASurII], the theory of the combinatorial structure of a $\Theta^{ \pm \text {ell }} \mathrm{NF}$ Hodge theater - and, indeed, the theory of the present series of papers! - may be regarded as a sort of

solution to the problem of constructing "global multiplicative subspaces" and "global canonical generators" [cf. the quotient "Q" and

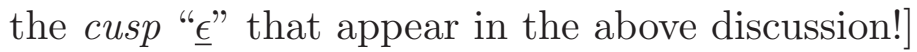

- the nonexistence of which in a "naive, scheme-theoretic sense" constitutes the main obstruction to applying the theory of [HASurI], [HASurII] to diophantine geometry [cf. the discussion of Remark 4.3.1]. Indeed, prime-strips may be thought of as "local analytic sections" of the natural morphism $\operatorname{Spec}(K) \rightarrow$ $\operatorname{Spec}\left(F_{\bmod }\right)$. Thus, it is precisely by working with such "local analytic sections" i.e., more concretely, by working with the collection of valuations $\underline{\mathbb{V}}$, as opposed to the set of all valuations of $K$ - that one can, in some sense, "simulate" the notions of a "global multiplicative subspace" or a "global canonical generator". On the other hand, such "simulated global objects" may only be achieved at the cost of

"dismantling", or performing "surgery" on, the global prime structure of the number fields involved [cf. the discussion of Remark 4.3.1]

- a quite drastic operation, which has the effect of precipitating numerous technical difficulties, whose resolution, via the theory of semi-graphs of anabelioids, Frobenioids, the étale theta function, and log-shells developed in [SemiAnbd], [FrdI], [FrdII], [EtTh], and [AbsTopIII], constitutes the bulk of the theory of the present series of papers! From the point of view of "performing surgery on the global prime structure of a number field", the labels $\in \mathbb{F}_{l}^{*}$ that appear in the "arithmetic" $\mathbb{F}_{l}^{*}$-symmetry may be thought of as a sort of "miniature finite approximation" of this global prime structure, in the spirit of the idea of "Hodge theory at finite resolution" discussed in [HASurI], §1.3.4. On the other hand, the labels $\in \mathbb{F}_{l}$ that appear in the "geometric" $\mathbb{F}_{l}^{\rtimes \pm}$-symmetry may be thought of as a sort 
of "miniature finite approximation" of the natural tempered $\mathbb{Z}$-coverings [i.e., tempered coverings with Galois group $\mathbb{Z}$ ] of the Tate curves determined by $E_{F}$ at $\underline{v} \in \underline{\mathbb{V}}^{\text {bad }}$, again in the spirit of the idea of "Hodge theory at finite resolution" discussed in [HASurI], §1.3.4.

\begin{tabular}{|c|c|c|}
\hline & $\frac{\text { Classical }}{\text { upper half-plane }}$ & 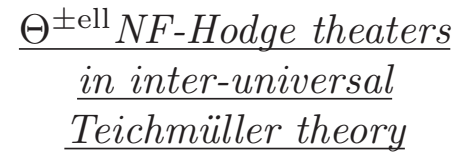 \\
\hline $\begin{array}{l}\text { Additive } \\
\text { symmetry }\end{array}$ & $\begin{array}{l}z \mapsto \quad z+a, \\
z \mapsto-\bar{z}+a \quad(a \in \mathbb{R})\end{array}$ & $\begin{array}{c}\mathbb{F}_{l}^{\rtimes \pm}- \\
\text { symmetry }\end{array}$ \\
\hline $\begin{array}{l}\text { "Functions" assoc'd } \\
\text { to add. symm. }\end{array}$ & $q \stackrel{\text { def }}{=} e^{2 \pi i z}$ & $\begin{array}{l}\text { theta fn. evaluated at } \\
\boldsymbol{l} \text {-tors. }[\text { cf. I, } 6.12 .6, \text { (ii)] }\end{array}$ \\
\hline $\begin{array}{l}\text { Basepoint assoc'd } \\
\text { to add. symm. }\end{array}$ & $\begin{array}{l}\text { single cusp } \\
\text { at infinity }\end{array}$ & {$\left[\right.$ cf. I, $\frac{\mathbb{V}^{ \pm}}{6.1,(\mathrm{v})]}$} \\
\hline $\begin{array}{l}\text { Combinatorial } \\
\text { prototype assoc'd } \\
\text { to add. symm. }\end{array}$ & cusp & cusp \\
\hline $\begin{array}{l}\text { Multiplicative } \\
\text { symmetry }\end{array}$ & $\begin{array}{l}z \mapsto \frac{z \cdot \cos (t)-\sin (t)}{z \cdot \sin (t)+\cos (t)} \\
z \mapsto \frac{\bar{z} \cdot \cos (t)+\sin (t)}{\bar{z} \cdot \sin (t)-\cos (t)} \quad(t \in \mathbb{R})\end{array}$ & $\begin{array}{c}\mathbb{F}_{l}^{*}- \\
\text { symmetry }\end{array}$ \\
\hline $\begin{array}{l}\text { "Functions" } \\
\text { assoc'd to } \\
\text { mult. symm. }\end{array}$ & $w \stackrel{\text { def }}{=} \frac{z-i}{z+i}$ & $\begin{array}{l}\text { elements of the } \\
\text { number field } F_{\bmod } \\
\text { [cf. I, 6.12.6, (iii)] }\end{array}$ \\
\hline $\begin{array}{l}\text { Basepoints assoc'd } \\
\text { to mult. symm. }\end{array}$ & $\begin{array}{c}\left(\begin{array}{cc}\cos (t) & -\sin (t) \\
\sin (t) & \cos (t)\end{array}\right),\left(\begin{array}{cc}\cos (t) & \sin (t) \\
\sin (t) & -\cos (t)\end{array}\right) \\
\curvearrowright\{\text { entire boundary of } \mathfrak{H}\}\end{array}$ & $\begin{array}{c}\mathbb{F}_{i}^{*} \curvearrowright \underline{\mathbb{V}}^{\text {Bor }}=\mathbb{F}_{l}^{*} \cdot \underline{V}^{ \pm \text {un }} \\
{[\text { cf. I, 4.3, (i)] }}\end{array}$ \\
\hline $\begin{array}{l}\text { Combinatorial } \\
\text { prototype assoc'd } \\
\text { to mult. symm. }\end{array}$ & $\begin{array}{l}\text { nodes of } \bmod p \\
\text { Hecke correspondence } \\
{[\text { cf. II, } 4.11 .4,(\text { iii }),(\mathrm{c})]}\end{array}$ & $\begin{array}{c}\text { nodes of } \bmod p \\
\text { Hecke correspondence } \\
{[\text { cf. II, } 4.11 .4,(\mathrm{iii}),(\mathrm{c})]}\end{array}$ \\
\hline
\end{tabular}

Fig. I1.4: Comparison of $\mathbb{F}_{l}^{\rtimes \pm}-, \mathbb{F}_{l}^{*}$-symmetries with the geometry of the upper half-plane 
As discussed above in our explanation of the models at $\underline{v} \in \underline{\mathbb{V}}^{\text {bad }}$ for $\mathcal{F}^{\vdash}$-primestrips, by considering the $2 l$-th roots of the $\boldsymbol{q}$-parameters of the elliptic curve $E_{F}$ at $\underline{v} \in \underline{\mathbb{V}}^{\text {bad }}$, and, roughly speaking, extending to $\underline{v} \in \underline{\mathbb{V}}^{\text {good }}$ in such a way as to satisfy the product formula, one may construct a natural $\mathcal{F}^{\Vdash}$-prime-strip "F $\mathfrak{F}_{\text {mod }}$ " [cf. Example 3.5, (ii); Definition 5.2, (iv)]. This construction admits an abstract, algorithmic formulation that allows one to apply it to the underlying " $\Theta$-Hodge theater" of an arbitrary $\Theta^{ \pm \text {ell }} N F$-Hodge theater ${ }^{\dagger} \mathcal{H} \mathcal{T}^{\Theta^{ \pm \mathrm{ell}} \mathrm{NF}}$ so as to obtain an $\mathcal{F}^{\Vdash}$ prime-strip

$$
\dagger \mathfrak{F}_{\text {mod }}^{\Vdash-}
$$

[cf. Definitions 3.6, (c); 5.2, (iv)]. On the other hand, by formally replacing the $2 l$-th roots of the $q$-parameters that appear in this construction by the reciprocal of the l-th root of the Frobenioid-theoretic theta function, which we shall denote " $\underline{\underline{\Theta}}_{\underline{v}} "\left[\right.$ for $\left.\underline{v} \in \underline{\mathbb{V}}^{\text {bad }}\right]$, studied in [EtTh] [cf. also Example 3.2, (ii), of the present paper], one obtains an abstract, algorithmic formulation for the construction of an $\mathcal{F}^{\Vdash}$-prime-strip

$$
\dagger \mathfrak{F}_{\text {tht }}^{\vdash-}
$$

[cf. Definitions 3.6, (c); 5.2, (iv)] from [the underlying $\Theta$-Hodge theater of] the $\Theta^{ \pm \text {ell }} \mathrm{NF}$-Hodge theater ${ }^{\dagger} \mathcal{H} \mathcal{T}^{\Theta^{ \pm \text {ell }} \mathrm{NF}}$.

Now let ${ }^{\ddagger} \mathcal{H} \mathcal{T}^{\Theta^{ \pm \text {ell }} \mathrm{NF}}$ be another $\Theta^{ \pm \text {ell }} \mathrm{NF}$-Hodge theater [relative to the given initial $\Theta$-data]. Then we shall refer to the "full poly-isomorphism" of [i.e., the collection of all isomorphisms between] $\mathcal{F}^{\Vdash}$-prime-strips

$$
{ }^{\dagger} \mathfrak{F}_{\text {tht }}^{\Vdash} \stackrel{\sim}{\rightarrow} \ddagger \mathfrak{F}_{\text {mod }}^{\Vdash}
$$

as the $\Theta$-link from [the underlying $\Theta$-Hodge theater of] ${ }^{\dagger} \mathcal{H} \mathcal{T}^{\Theta^{ \pm e l l}} \mathrm{NF}$ to [the underlying $\Theta$-Hodge theater of] $\ddagger \mathcal{H} \mathcal{T}^{\Theta^{ \pm e l l} \mathrm{NF}}$ [cf. Corollary 3.7, (i); Definition 5.2, (iv)]. One fundamental property of the $\Theta$-link is the property that it induces a collection of isomorphisms [in fact, the full poly-isomorphism] between the $\mathcal{F}^{\vdash \times}$-prime-strips

$$
{ }^{\dagger} \mathfrak{F}_{\text {mod }}^{\vdash \times} \stackrel{\sim}{\rightarrow} \underset{\ddagger}{\ddagger} \mathfrak{F}_{\text {mod }}^{\vdash \times}
$$

associated to ${ }^{\dagger} \mathfrak{F}_{\text {mod }}^{\vdash}$ and $\ddagger \mathfrak{F}_{\text {mod }}^{\vdash}$ [cf. Corollary 3.7, (ii), (iii); [IUTchII], Definition 4.9, (vii)].

Now let $\left\{{ }^{n} \mathcal{H} \mathcal{T}^{\Theta^{ \pm e l l} \mathrm{NF}}\right\}_{n \in \mathbb{Z}}$ be a collection of distinct $\Theta^{ \pm \text {ell }} N F$-Hodge theaters [relative to the given initial $\Theta$-data] indexed by the integers. Thus, by applying the constructions just discussed, we obtain an infinite chain

$\ldots \quad \stackrel{\Theta}{\longrightarrow}{ }^{(n-1)} \mathcal{H} \mathcal{T}^{\Theta^{ \pm \mathrm{ell}} \mathrm{NF}} \stackrel{\Theta}{\longrightarrow}{ }^{n} \mathcal{H} \mathcal{T}^{\Theta^{ \pm \mathrm{ell}} \mathrm{NF}} \stackrel{\Theta}{\longrightarrow} \quad{ }^{(n+1)} \mathcal{H} \mathcal{T}^{\Theta^{ \pm \mathrm{ell}} \mathrm{NF}} \stackrel{\Theta}{\longrightarrow} \quad \ldots$

of $\Theta$-linked $\Theta^{ \pm \text {ell }} \mathbf{N F - H o d g e}$ theaters [cf. Corollary 3.8], which will be referred to as the Frobenius-picture [associated to the $\Theta$-link]. One fundamental property of this Frobenius-picture is the property that it fails to admit permutation automorphisms that switch adjacent indices $n, n+1$, but leave the remaining indices $\in \mathbb{Z}$ fixed [cf. Corollary 3.8]. Roughly speaking, the $\Theta$-link ${ }^{n} \mathcal{H} \mathcal{T}^{\Theta^{ \pm e l l} \mathrm{NF}} \stackrel{\Theta}{\longrightarrow}{ }^{(n+1)} \mathcal{H} \mathcal{T}^{\Theta^{ \pm e l l} \mathrm{NF}}$ may be thought of as a formal correspondence

$$
{ }^{n} \underline{\underline{\Theta}}_{\underline{v}} \quad \mapsto \quad{ }^{(n+1)} \underline{\underline{q}}_{\underline{v}}
$$


[cf. Remark 3.8.1, (i)], which is depicted in Fig. I1.5 below.

In fact, the $\Theta$-link discussed in the present paper is only a simplified version of the " $\Theta$-link" that will ultimately play a central role in the present series of papers. The construction of the version of the $\Theta$-link that we shall ultimately be interested in is quite technically involved and, indeed, occupies the greater part of the theory to be developed in [IUTchII], [IUTchIII]. On the other hand, the simplified version discussed in the present paper is of interest in that it allows one to give a relatively straightforward introduction to many of the important qualitative properties of the $\Theta$-link — such as the Frobenius-picture discussed above and the étale-picture to be discussed below - that will continue to be of central importance in the case of the versions of the $\Theta$-link that will be developed in [IUTchII], [IUTchIII].

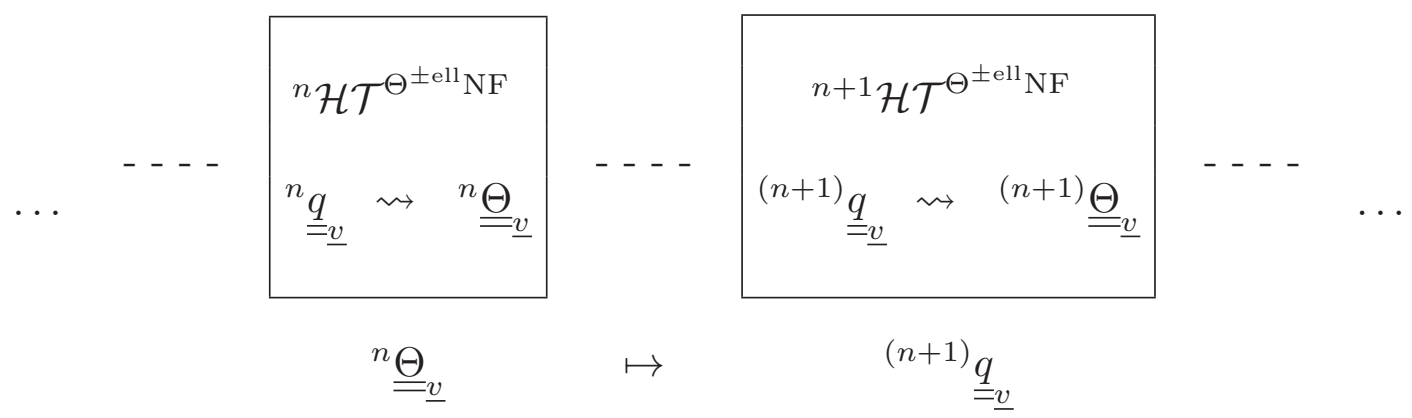

Fig. I1.5: Frobenius-picture associated to the $\Theta$-link

Now let us return to our discussion of the Frobenius-picture associated to the $\Theta$ link. The $\mathcal{D}^{\vdash}$-prime-strip associated to the $\mathcal{F}^{\vdash \times}$-prime-strip ${ }^{\dagger} \mathfrak{F}_{\text {mod }}^{\vdash \times}$ may, in fact, be naturally identified with the $\mathcal{D}^{\vdash}$-prime-strip ${ }^{\dagger} \mathfrak{D}_{>}^{\vdash}$ associated to a certain $\mathcal{F}$-primestrip $^{\dagger} \mathfrak{F}_{>}$[cf. the discussion preceding Example 5.4] that arises from the $\Theta$-Hodge theater underlying the $\Theta^{ \pm \text {ell }} \mathrm{NF}$-Hodge theater ${ }^{\dagger} \mathcal{H} \mathcal{T}^{\Theta^{ \pm e l l} \mathrm{NF}}$. The $\mathcal{D}$-prime-strip $\dagger \mathfrak{D}_{>}$associated to the $\mathcal{F}$-prime-strip ${ }^{\dagger} \mathfrak{F}_{>}$is precisely the $\mathcal{D}$-prime-strip depicted as "[1 $<\ldots<l *]$ " in Fig. I1.3. Thus, the Frobenius-picture discussed above induces an infinite chain of full poly-isomorphisms

$$
\ldots \quad \stackrel{\sim}{\rightarrow} \quad(n-1) \mathfrak{D}_{>}^{\vdash} \stackrel{\sim}{\rightarrow}{ }^{n} \mathfrak{D}_{>}^{\vdash} \quad \stackrel{\sim}{\rightarrow} \quad(n+1) \mathfrak{D}_{>}^{\vdash} \quad \stackrel{\sim}{\rightarrow} \ldots
$$

of $\mathcal{D}^{\vdash}$-prime-strips. That is to say, when regarded up to isomorphism, the $\mathcal{D}^{\vdash}$ prime-strip "(-) $\mathfrak{D}_{>}^{\vdash}$ " may be regarded as an invariant - i.e., a "mono-analytic core" - of the various $\Theta^{ \pm \text {ell }}$ NF-Hodge theaters that occur in the Frobenius-picture [cf. Corollaries 4.12, (ii); 6.10, (ii)]. Unlike the case with the Frobenius-picture, the relationships of the various $\mathcal{D}-\Theta^{ \pm \text {ell }} \mathrm{NF}$-Hodge theaters ${ }^{n} \mathcal{H} \mathcal{T}^{\mathcal{D}-\Theta^{ \pm \text {ell }} \mathrm{NF}}$ to this mono-analytic core - relationships that are depicted by spokes in Fig. I1.6 below - are compatible with arbitrary permutation symmetries among the spokes [i.e., among the labels $n \in \mathbb{Z}$ of the $\mathcal{D}$ - $\Theta^{ \pm \text {ell }}$ NF-Hodge theaters] — cf. Corollaries 4.12, (iii); 6.10, (iii), (iv). The diagram depicted in Fig. I1.6 below will be referred to as the étale-picture.

Thus, the étale-picture may, in some sense, be regarded as a collection of canonical splittings of the Frobenius-picture. The existence of such splittings suggests that 
by applying various results from absolute anabelian geometry to the various tempered and étale fundamental groups that constitute each $\mathcal{D}$ $\Theta^{ \pm \text {ell }} \mathrm{NF}-\mathrm{Hodge}$ theater in the étale-picture, one may obtain algorithmic descriptions of - i.e., roughly speaking, one may take a "glimpse" inside - the conventional scheme theory of one $\Theta^{ \pm \text {ell } N F-H o d g e ~ t h e-~}$ ater ${ }^{m} \mathcal{H} \mathcal{T}^{\Theta^{ \pm e l l} \mathrm{NF}}$ in terms of the conventional scheme theory associated to another $\Theta^{ \pm \text {ell }} \mathrm{NF}$-Hodge theater ${ }^{n} \mathcal{H} \mathcal{T}^{\Theta^{ \pm e l l}} \mathrm{NF}$ [i.e., where $n \neq m$ ].

Indeed, this point of view constitutes one of the main themes of the theory developed in the present series of papers and will be of particular importance in our treatment in [IUTchIII] of the main results of the theory.

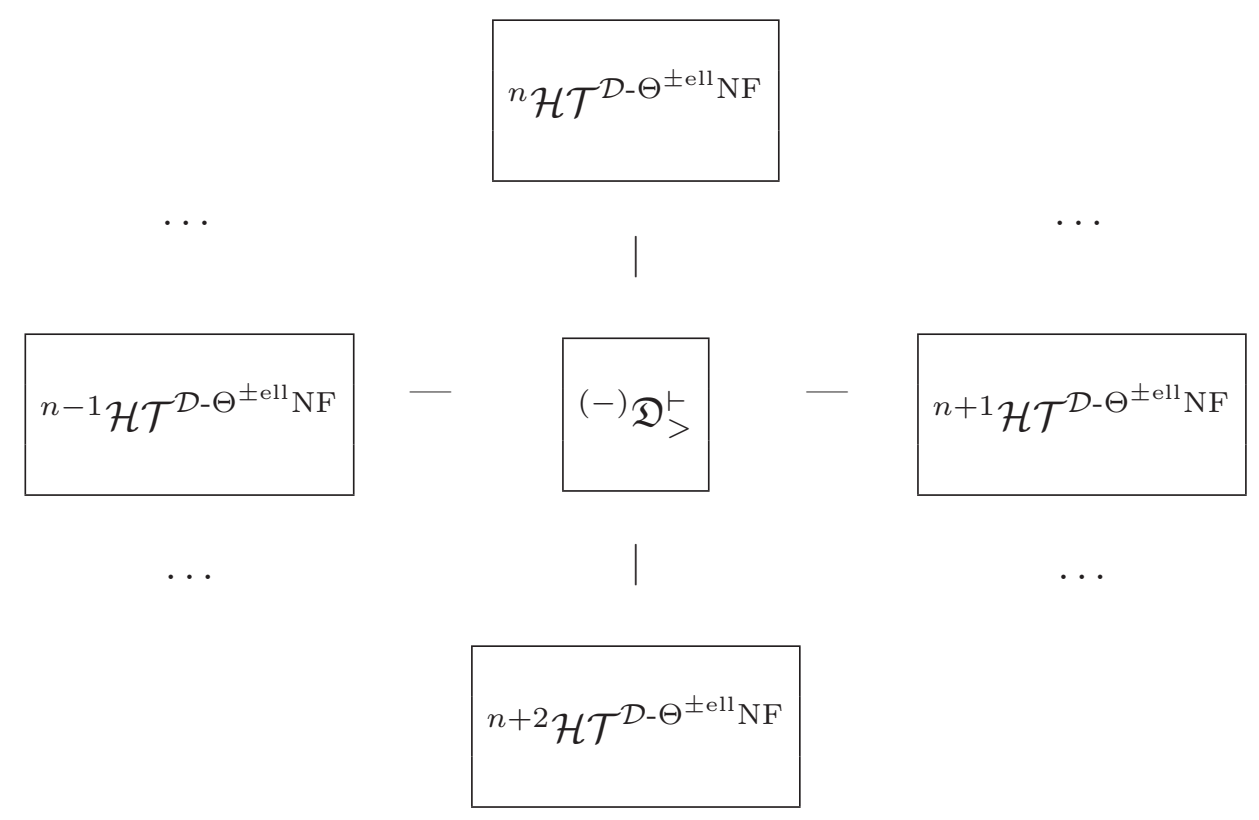

Fig. I1.6: Étale-picture of $\mathcal{D}-\Theta^{ \pm e l l} \mathrm{NF}-$ Hodge theaters

Before proceeding, we recall the "heuristic" notions of Frobenius-like - i.e., "order-conscious" - and étale-like - i.e., "indifferent to order" — mathematical structures discussed in [FrdI], §I4. These notions will play a key role in the theory developed in the present series of papers. In particular, the terms "Frobeniuspicture" and "étale-picture" introduced above are motivated by these notions.

The main result of the present paper may be summarized as follows.

Theorem A. $\quad\left(\mathbb{F}_{l}^{\rtimes \pm}-/ \mathbb{F}_{l}^{*}\right.$-Symmetries, $\Theta$-Links, and Frobenius-/Étale-Pictures Associated to $\Theta^{ \pm \text {ell }} \mathbf{N F - H o d g e ~ T h e a t e r s ) ~ F i x ~ a ~ c o l l e c t i o n ~ o f ~ i n i t i a l ~} \Theta$ data [cf. Definition 3.1], which determines, in particular, data $\left(E_{F}, \bar{F}, l, \underline{\mathbb{V}}\right)$ as in the above discussion. Then one may construct a $\Theta^{ \pm e l l} \mathbf{N F - H o d g e ~ t h e a t e r ~ [ c f . ~}$ Definition 6.13, (i)]

$$
\dagger \mathcal{H} \mathcal{T}^{\Theta^{ \pm \mathrm{ell}} \mathrm{NF}}
$$

- in essence, a system of Frobenioids - associated to this initial $\Theta$-data, as well as an associated $\mathcal{D}-\Theta^{ \pm \text {ell }}$ NF-Hodge theater ${ }^{\dagger} \mathcal{H} \mathcal{T}^{\mathcal{D}-\Theta^{ \pm \mathrm{ell}} \mathrm{NF}}$ [cf. Definition 6.13, (ii)] 
- in essence, the system of base categories associated to the system of Frobenioids $\dagger \mathcal{H} \mathcal{T}^{\Theta^{ \pm \mathrm{ell}} \mathrm{NF}}$.

(i) $\left(\mathbb{F}_{l}^{\rtimes \pm}\right.$ - and $\mathbb{F}_{l}^{*}$-Symmetries) The $\Theta^{ \pm \text {ell }} N F$-Hodge theater ${ }^{\dagger} \mathcal{H} \mathcal{T}^{\Theta^{ \pm \mathrm{ell}} \mathrm{NF}}$ may be obtained as the result of gluing together a $\Theta^{ \pm \text {ell }}$-Hodge theater ${ }^{\dagger} \mathcal{H} \mathcal{T}^{\Theta^{ \pm \mathrm{ell}}}$ to a $\Theta \mathrm{NF}$-Hodge theater ${ }^{\dagger} \mathcal{H} \mathcal{T}^{\Theta \mathrm{NF}}$ [cf. Remark 6.12.2, (ii)]; a similar statement holds for the $\mathcal{D}-\Theta^{ \pm \text {ell }} N F$-Hodge theater ${ }^{\dagger} \mathcal{H} \mathcal{T}^{\mathcal{D}-\Theta^{ \pm e l l}} \mathrm{NF}$. The global portion of a $\mathcal{D}-\Theta^{ \pm e l l}$ Hodge theater ${ }^{\dagger} \mathcal{H} \mathcal{T}^{\mathcal{D}-\Theta^{ \pm e l 1}}$ consists of a category equivalent to the full subcategory determined by the connected objects of] the Galois category of finite étale coverings of the [orbi]curve $\underline{X}_{K}$. This global portion is equipped with an $\mathbb{F}_{l}^{\rtimes \pm}$-symmetry, i.e., a poly-action by $\mathbb{F}_{l}^{\rtimes \pm}$ on the labels

$$
\left(-l^{*}<\ldots<-1<0<1<\ldots<l^{*}\right)
$$

- which we think of as elements $\in \mathbb{F}_{l}$ - each of which is represented in the $\mathcal{D}$ $\Theta^{ \pm \text {ell }}$-Hodge theater ${ }^{\dagger} \mathcal{H} \mathcal{T}^{\mathcal{D}-\Theta^{ \pm e l l}}$ by a $\mathcal{D}$-prime-strip [cf. Fig. I1.3]. The global portion of a $\mathcal{D}$ - $\Theta \mathrm{NF}-$ Hodge theater ${ }^{\dagger} \mathcal{H} \mathcal{T}^{\mathcal{D}-\Theta N F}$ consists of a category equivalent to [the full subcategory determined by the connected objects of] the Galois category of finite étale coverings of the orbicurve $\underline{C}_{K}$. This global portion is equipped with an $\mathbb{F}_{l}^{*}$-symmetry, i.e., a poly-action by $\mathbb{F}_{l}^{*}$ on the labels

$$
\left(1<\ldots<l^{*}\right)
$$

- which we think of as elements $\in \mathbb{F}_{l}^{*}$ - each of which is represented in the

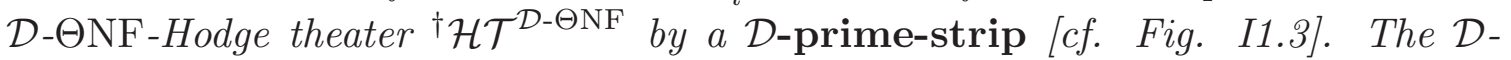
$\Theta^{ \pm \text {ell }}$-Hodge theater ${ }^{\dagger} \mathcal{H} \mathcal{T}^{\mathcal{D}-\Theta^{ \pm e l l}}$ is glued to the $\mathcal{D}$ - $\Theta \mathrm{NF}$-Hodge theater ${ }^{\dagger} \mathcal{H} \mathcal{T}^{\mathcal{D}-\Theta \mathrm{NF}}$ by identifying $\mathcal{D}$-prime-strips in such a way that the labels $0 \neq \pm t \in \mathbb{F}_{l}$ that arise in the $\mathbb{F}_{l}^{\rtimes \pm}$-symmetry are identified with the corresponding label $j \in \mathbb{F}_{l}^{*}$ that arises in the $\mathbb{F}_{l}^{*}$-symmetry [cf. Proposition 6.7; Remark 6.12.2; Fig. 6.5].

(ii) ( $\Theta$-links) By considering the $2 l$-th roots of the $\boldsymbol{q}$-parameters " $\underline{\underline{q}} \underline{\underline{v}}$ " of the elliptic curve $E_{F}$ at $\underline{v} \in \underline{\mathbb{V}}^{\text {bad }}$ and extending to other $\underline{v} \in \underline{\mathbb{V}}$ in such a way as to satisfy the product formula, one may construct a natural $\mathcal{F}^{\Vdash}$-prime-strip ${ }^{\dagger} \mathfrak{F}_{\text {mod }}^{\mid-}$associated to the $\Theta^{ \pm \text {ell }} N F$-Hodge theater ${ }^{\dagger} \mathcal{H} \mathcal{T}^{\Theta^{ \pm e l l} \mathrm{NF}}$ [cf. Definitions 3.6, (c); 5.2, (iv)]. In a similar vein, by considering the reciprocal of the $l$-th root of the Frobenioid-theoretic theta function " $\underline{\underline{\Theta}}_{\underline{v}}$ " associated to the elliptic curve $E_{F}$ at $\underline{v} \in \underline{\mathbb{V}}^{\text {bad }}$ and extending to other $\underline{v} \in \underline{\mathbb{V}}$ in such a way as to satisfy the product formula, one may construct a natural $\mathcal{F}^{\Vdash}$-prime-strip ${ }^{\dagger} \mathfrak{F}_{\text {tht }}^{t}$ associated to the $\Theta^{ \pm \text {ell }} N F$-Hodge theater ${ }^{\dagger} \mathcal{H} \mathcal{T}^{\Theta^{ \pm \mathrm{ell}} \mathrm{NF}}$ [cf. Definitions 3.6, (c); 5.2, (iv)]. Now let $\ddagger \mathcal{H} \mathcal{T}^{\Theta^{ \pm e l l} \mathrm{NF}}$ be another $\Theta^{ \pm \text {ell }} N F$-Hodge theater [relative to the given initial $\Theta$ data]. Then we shall refer to the "full poly-isomorphism" of [i.e., the collection of all isomorphisms between] $\mathcal{F}^{\Vdash}$-prime-strips

$$
\dagger \mathfrak{F}_{\text {tht }}^{\vdash} \stackrel{\sim}{\rightarrow} \quad{ }^{\ddagger} \mathfrak{F}_{\text {mod }}^{\Vdash}
$$

as the $\Theta$-link from [the underlying $\Theta$-Hodge theater of] ${ }^{\dagger} \mathcal{H} \mathcal{T}^{\Theta^{ \pm e l l} \mathrm{NF}}$ to [the underlying $\Theta$-Hodge theater of] ${ }^{\ddagger} \mathcal{H} \mathcal{T}^{\Theta^{ \pm \mathrm{ell}} \mathrm{NF}}$ [cf. Corollary 3.7, (i); Definition 5.2, (iv)]. The $\Theta$-link induces the full poly-isomorphism between the $\mathcal{F}^{\vdash \times}$-prime-strips

$$
{ }^{\dagger} \mathfrak{F}_{\text {mod }}^{\vdash \times} \stackrel{\sim}{\rightarrow} \quad \ddagger \mathfrak{F}_{\text {mod }}^{\vdash \times}
$$


associated to ${ }^{\dagger} \mathfrak{F}_{\text {mod }}^{\Vdash}$ and ${ }^{\ddagger} \mathfrak{F}_{\text {mod }}^{\Vdash}$ [cf. Corollary 3.7, (ii), (iii); [IUTchII], Definition 4.9, (vii)].

(iii) (Frobenius-/Étale-Pictures) Let $\left\{{ }^{n} \mathcal{H} \mathcal{T}^{\Theta^{ \pm e l l} \mathrm{NF}}\right\}_{n \in \mathbb{Z}}$ be a collection of distinct $\Theta^{ \pm \text {ell }} \mathbf{N F - H o d g e ~ t h e a t e r s ~ [ r e l a t i v e ~ t o ~ t h e ~ g i v e n ~ i n i t i a l ~} \Theta$-data] indexed by the integers. Then the infinite chain

$$
\ldots \quad \stackrel{\Theta}{\longrightarrow}{ }^{(n-1)} \mathcal{H} \mathcal{T}^{\Theta^{ \pm e l l} \mathrm{NF}} \stackrel{\Theta}{\longrightarrow}{ }^{n} \mathcal{H} \mathcal{T}^{\Theta^{ \pm e l l} \mathrm{NF}} \stackrel{\Theta}{\longrightarrow} \quad{ }^{(n+1)} \mathcal{H} \mathcal{T}^{\Theta^{ \pm e l l} \mathrm{NF}} \stackrel{\Theta}{\longrightarrow} \quad \ldots
$$

of $\Theta$-linked $\Theta^{ \pm \text {ell }}$ NF-Hodge theaters will be referred to as the Frobeniuspicture [associated to the $\Theta$-link] — cf. Fig. I1.5; Corollary 3.8. The Frobeniuspicture fails to admit permutation automorphisms that switch adjacent indices $n, n+1$, but leave the remaining indices $\in \mathbb{Z}$ fixed. The Frobenius-picture induces an infinite chain of full poly-isomorphisms

$$
\ldots \quad \stackrel{\sim}{\rightarrow} \quad(n-1) \mathfrak{D}_{>}^{\vdash} \quad \stackrel{\sim}{\rightarrow} \quad n \mathfrak{D}_{>}^{\vdash} \quad \stackrel{\sim}{\rightarrow} \quad(n+1) \mathfrak{D}_{>}^{\vdash} \quad \stackrel{\sim}{\rightarrow} \quad \ldots
$$

between the various $\mathcal{D}^{\vdash}$-prime-strips ${ }^{n} \mathfrak{D}_{>}^{\vdash}$, i.e., in essence, the $\mathcal{D}^{\vdash}$-prime-strips associated to the $\mathcal{F}^{\vdash \times}$-prime-strips ${ }^{n} \mathfrak{F}_{\text {mod }}^{\vdash \times}$. The relationships of the various $\mathcal{D}$ $\Theta^{ \pm \text {ell }} N F$-Hodge theaters ${ }^{n} \mathcal{H} \mathcal{T}^{\mathcal{D}-\Theta^{ \pm e l l} \mathrm{NF}}$ to the "mono-analytic core" constituted by the $\mathcal{D}^{\vdash}$-prime-strip "(-) $\mathfrak{D}_{>}^{\vdash}$ " regarded up to isomorphism - relationships that are depicted by spokes in Fig. I1.6 - are compatible with arbitrary permutation symmetries among the spokes, i.e., among the labels $n \in \mathbb{Z}$ of the $\mathcal{D}-\Theta^{ \pm \text {ell }} N F$ Hodge theaters [cf. Corollaries 4.12, (ii), 6.10, (i)]. The diagram depicted in Fig. I1.6 will be referred to as the étale-picture.

In addition to the main result discussed above, we also prove a certain technical result concerning tempered fundamental groups - cf. Theorem B below that will be of use in our development of the theory of Hodge-Arakelov-theoretic evaluation in [IUTchII]. This result is essentially a routine application of the theory of maximal compact subgroups of tempered fundamental groups developed in [SemiAnbd] [cf., especially, [SemiAnbd], Theorems 3.7, 5.4, as well as Remark 2.5.3, (ii), of the present paper]. Here, we recall that this theory of [SemiAnbd] may be thought of as a sort of "Combinatorial Section Conjecture" [cf. Remark 2.5.1 of the present paper; [IUTchII], Remark 1.12.4] — a point of view that is of particular interest in light of the historical remarks made in $\S I 5$ below. Moreover, Theorem $\mathrm{B}$ is of interest independently of the theory of the present series of papers in that it yields, for instance, a new proof of the normal terminality of the tempered fundamental group in its profinite completion, a result originally obtained in [André], Lemma 3.2.1, by means of other techniques [cf. Remark 2.4.1]. This new proof is of interest in that, unlike the techniques of [André], which are only available in the profinite case, this new proof [cf. Proposition 2.4, (iii)] holds in the case of pro- $\widehat{\Sigma}$-completions, for more general $\widehat{\Sigma}$ [i.e., not just the case of $\widehat{\Sigma}=\mathfrak{P r i m}$ ra] .

Theorem B. (Profinite Conjugates of Tempered Decomposition and Inertia Groups) Let $k$ be $a$ mixed-characteristic [nonarchimedean] local field, $X$ a hyperbolic curve over $k$. Write

$$
\Pi_{X}^{\mathrm{tp}}
$$


for the tempered fundamental group $\pi_{1}^{\mathrm{tp}}(X)$ [relative to a suitable basepoint] of X [cf. [André], §4; [SemiAnbd], Example 3.10]; $\widehat{\Pi}_{X}$ for the étale fundamental group [relative to a suitable basepoint] of $X$. Thus, we have a natural inclusion

$$
\Pi_{X}^{\mathrm{tp}} \hookrightarrow \widehat{\Pi}_{X}
$$

which allows one to identify $\widehat{\Pi}_{X}$ with the profinite completion of $\Pi_{X}^{\mathrm{tp}}$. Then every decomposition group in $\widehat{\Pi}_{X}$ (respectively, inertia group in $\widehat{\Pi}_{X}$ ) associated to a closed point or cusp of $X$ (respectively, to a cusp of $X$ ) is contained in $\Pi_{X}^{\mathrm{tp}}$ if and only if it is a decomposition group in $\Pi_{X}^{\mathrm{tp}}$ (respectively, inertia group in $\Pi_{X}^{\mathrm{tp}}$ ) associated to a closed point or cusp of X (respectively, to a cusp of X). Moreover, a $\widehat{\Pi}_{X}$-conjugate of $\Pi_{X}^{\mathrm{tp}}$ contains a decomposition group in $\Pi_{X}^{\mathrm{tp}}$ (respectively, inertia group in $\Pi_{X}^{\mathrm{tp}}$ ) associated to a closed point or cusp of $X$ (respectively, to a cusp of $X)$ if and only if it is equal to $\Pi_{X}^{\mathrm{tp}}$.

Theorem B is [essentially] given as Corollary 2.5 [cf. also Remark 2.5.2] in $\S 2$. Here, we note that although, in the statement of Corollary 2.5, the hyperbolic curve $X$ is assumed to admit stable reduction over the ring of integers $\mathcal{O}_{k}$ of $k$, one verifies immediately [by applying Proposition 2.4, (iii)] that this assumption is, in fact, unnecessary.

Finally, we remark that one important reason for the need to apply Theorem B in the context of the theory of $\Theta^{ \pm \text {ell }} \mathrm{NF}$-Hodge theaters summarized in Theorem A is the following. The $\mathbb{F}_{l}^{\rtimes \pm}$-symmetry, which will play a crucial role in the theory of the present series of papers [cf., especially, [IUTchII], [IUTchIII]], depends, in an essential way, on the synchronization of the \pm -indeterminacies that occur locally at each $\underline{v} \in \mathbb{V}$ [cf. Fig. I1.1]. Such a synchronization may only be obtained by making use of the global portion of the $\Theta^{ \pm \text {ell }}$-Hodge theater under consideration. On the other hand, in order to avail oneself of such global \pm -synchronizations [cf. Remark 6.12.4, (iii)], it is necessary to regard the various labels of the $\mathbb{F}_{l}^{\rtimes \pm}$ symmetry

$$
\left(-l^{*}<\ldots<-1<0<1<\ldots<l^{*}\right)
$$

as conjugacy classes of inertia groups of the [necessarily] profinite geometric étale fundamental group of $\underline{X}_{K}$. That is to say, in order to relate such global profinite conjugacy classes to the corresponding tempered conjugacy classes [i.e., conjugacy classes with respect to the geometric tempered fundamental group] of inertia groups at $\underline{v} \in \underline{\mathbb{V}}^{\text {bad }}$ [i.e., where the crucial Hodge-Arakelov-theoretic evaluation is to be performed!], it is necessary to apply Theorem B — cf. the discussion of Remark 4.5.1; [IUTchII], Remark 2.5.2, for more details.

\section{$\S$ I2. Gluing Together Models of Conventional Scheme Theory}

As discussed in $\S I 1$, the system of Frobenioids constituted by a $\Theta^{ \pm \text {ell }}$ NF-Hodge theater is intended to be a sort of miniature model of conventional scheme theory. One then glues multiple $\Theta^{ \pm \text {ell }} \mathrm{NF}$-Hodge theaters $\left\{{ }^{n} \mathcal{H} \mathcal{T}^{\Theta^{ \pm e l l}} \mathrm{NF}\right\}_{n \in \mathbb{Z}}$ together 
by means of the full poly-isomorphisms between the "subsystems of Frobenioids" constituted by certain $\mathcal{F}^{\Vdash}$-prime-strips

$$
{ }^{\dagger} \mathfrak{F}_{\text {tht }}^{\vdash} \stackrel{\sim}{\rightarrow} \quad \ddagger \mathfrak{F}_{\text {mod }}^{\Vdash}
$$

to form the Frobenius-picture. One fundamental observation in this context is the following:

these gluing isomorphisms - i.e., in essence, the correspondences

$$
\underline{\underline{\Theta}}_{\underline{v}} \quad \mapsto \quad{ }^{(n+1)} \underline{\underline{q}}_{\underline{v}}
$$

- and hence the geometry of the resulting Frobenius-picture lie outside the framework of conventional scheme theory in the sense that they do not arise from ring homomorphisms!

In particular, although each particular model ${ }^{n} \mathcal{H} \mathcal{T}^{\Theta^{ \pm e l l}} \mathrm{NF}$ of conventional scheme theory is constructed within the framework of conventional scheme theory, the relationship between the distinct [albeit abstractly isomorphic, as $\Theta^{ \pm \text {ell }} \mathrm{NF}-\mathrm{Hodge}$ theaters!] conventional scheme theories represented by, for instance, neighboring $\Theta^{ \pm \text {ell }} \mathrm{NF}$-Hodge theaters ${ }^{n} \mathcal{H} \mathcal{T}^{\Theta^{ \pm e l l} \mathrm{NF}},{ }^{n+1} \mathcal{H} \mathcal{T}^{\Theta^{ \pm e l l} \mathrm{NF}}$ cannot be expressed schemetheoretically. In this context, it is also important to note that such gluing operations are possible precisely because of the relatively simple structure - for instance, by comparison to the structure of a ring! - of the Frobenius-like structures constituted by the Frobenioids that appear in the various $\mathcal{F}^{\Vdash}$-prime-strips involved, i.e., in essence, collections of monoids isomorphic to $\mathbb{N}$ or $\mathbb{R}_{\geq 0}$ [cf. Fig. I1.2].

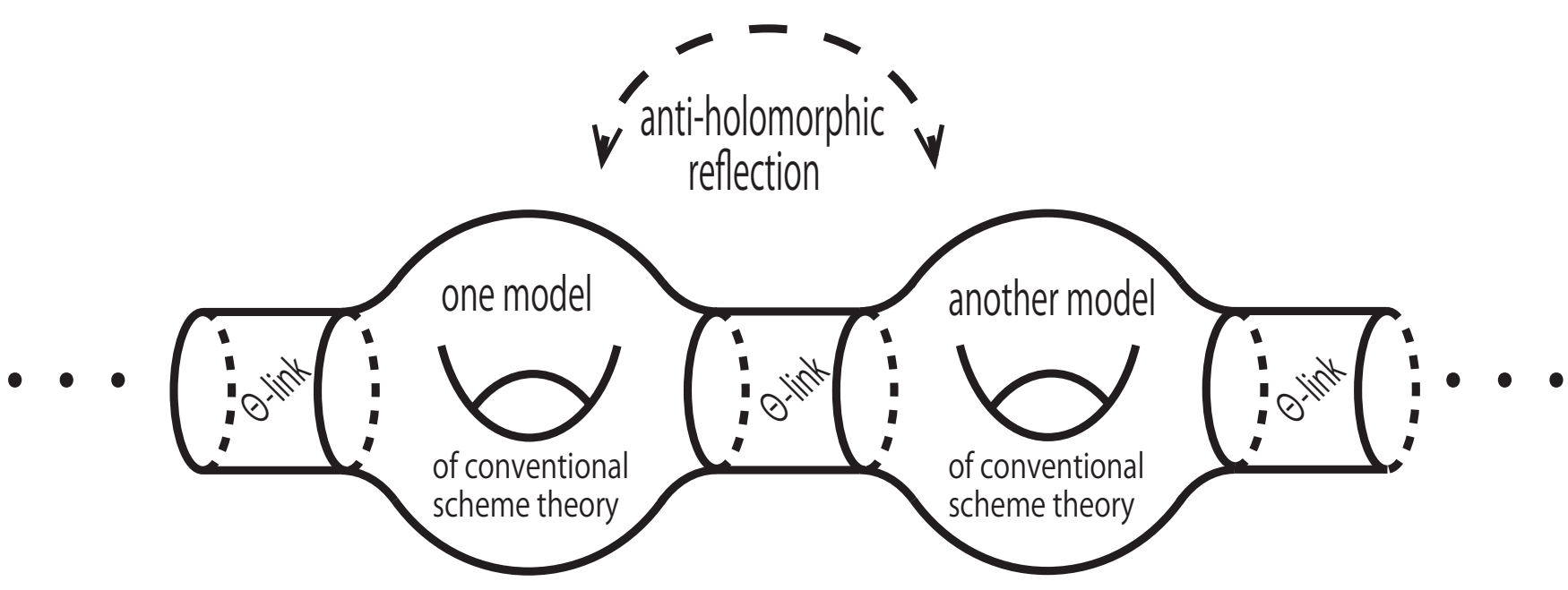

Fig. I2.1: Depiction of Frobenius- and étale-pictures of $\Theta^{ \pm \text {ell }}$ NF-Hodge theaters via glued topological surfaces 
If one thinks of the geometry of "conventional scheme theory" as being analogous to the geometry of "Euclidean space", then the geometry represented by the Frobenius-picture corresponds to a "topological manifold", i.e., which is obtained by gluing together various portions of Euclidean space, but which is not homeomorphic to Euclidean space. This point of view is illustrated in Fig. I2.1 above, where the various $\Theta^{ \pm e l l}$ NF-Hodge theaters in the Frobenius-picture are depicted as [twodimensional! - cf. the discussion of $\S \mathrm{I} 1]$ twice-punctured topological surfaces of genus one, glued together along tubular neighborhoods of cycles, which correspond to the [one-dimensional! — cf. the discussion of $\S \mathrm{I1}$ ] mono-analytic data that appears in the isomorphism that constitutes the $\Theta$-link. The permutation symmetries in the étale-picture [cf. the discussion of $\S I 1]$ are depicted in Fig. I2.1 as the anti-holomorphic reflection [cf. the discussion of multiradiality in [IUTchII], Introduction!] around a gluing cycle between topological surfaces.

Another elementary example that illustrates the spirit of the gluing operations discussed in the present series of papers is the following. For $i=0,1$, let $\mathbb{R}_{i}$ be a copy of the real line; $I_{i} \subseteq \mathbb{R}_{i}$ the closed unit interval [i.e., corresponding to $[0,1] \subseteq \mathbb{R}]$. Write $C_{0} \subseteq I_{0}$ for the Cantor set and

$$
\phi: C_{0} \stackrel{\sim}{\rightarrow} I_{1}
$$

for the bijection arising from the Cantor function. Then if one thinks of $\mathbb{R}_{0}$ and $\mathbb{R}_{1}$ as being glued to one another by means of $\phi$, then it is a highly nontrivial problem

to describe structures naturally associated to the "alien" ring structure of $\mathbb{R}_{0}$ — such as, for instance, the subset of algebraic numbers $\in \mathbb{R}_{0}$ in terms that only require the use of the ring structure of $\mathbb{R}_{1}$.

A slightly less elementary example that illustrates the spirit of the gluing operations discussed in the present series of papers is the following. This example is technically much closer to the theory of the present series of papers than the examples involving topological surfaces and Cantor sets given above. For simplicity, let us write

$$
G \curvearrowright \mathcal{O}^{\times}, \quad G \curvearrowright \mathcal{O}^{\triangleright}
$$

for the pairs " $G_{\underline{v}} \curvearrowright \mathcal{O}_{\bar{F}_{\underline{v}}}^{\times}$", " $G_{\underline{v}} \curvearrowright \mathcal{O} \bar{F}_{\underline{v}}^{\triangleright}$ " [cf. the notation of the discussion surrounding Fig. I1.2]. Recall from [AbsTopIII], Proposition 3.2, (iv), that the operation

$$
\left(G \curvearrowright \mathcal{O}^{\triangleright}\right) \mapsto G
$$

of "forgetting $\mathcal{O}^{\triangleright}$ " determines a bijection from the group of automorphisms of the pair $G \curvearrowright \mathcal{O}^{\triangleright}$ - i.e., thought of as an abstract ind-topological monoid equipped with a continuous action by an abstract topological group - to the group of automorphisms of the topological group $G$. By contrast, we recall from [AbsTopIII], Proposition 3.3, (ii), that the operation

$$
\left(G \curvearrowright \mathcal{O}^{\times}\right) \mapsto G
$$

of "forgetting $\mathcal{O}^{\times}$" only determines a surjection from the group of automorphisms of the pair $G \curvearrowright \mathcal{O}^{\times}$- i.e., thought of as an abstract ind-topological monoid 
equipped with a continuous action by an abstract topological group - to the group of automorphisms of the topological group $G$; that is to say, the kernel of this surjection is given by the natural action of $\widehat{\mathbb{Z}}^{\times}$on $\mathcal{O}^{\times}$. In particular, if one works with two copies $G_{i} \curvearrowright \mathcal{O}_{i}^{\triangleright}$, where $i=0,1$, of $G \curvearrowright \mathcal{O}^{\triangleright}$, which one thinks of as being glued to one another by means of an indeterminate isomorphism

$$
\left(G_{0} \curvearrowright \mathcal{O}_{0}^{\times}\right) \stackrel{\sim}{\rightarrow}\left(G_{1} \curvearrowright \mathcal{O}_{1}^{\times}\right)
$$

[i.e., where one thinks of each $\left(G_{i} \curvearrowright \mathcal{O}_{i}^{\times}\right)$, for $i=0,1$, as an abstract ind-topological monoid equipped with a continuous action by an abstract topological group], then, in general, it is a highly nontrivial problem

to describe structures naturally associated to $\left(G_{0} \curvearrowright \mathcal{O}_{0}^{\triangleright}\right)$ in terms that only require the use of $\left(G_{1} \curvearrowright \mathcal{O}_{1}^{\triangleright}\right)$.

One such structure which is of interest in the context of the present series of papers [cf., especially, the theory of [IUTchII], $§ 1]$ is the natural cyclotomic rigidity isomorphism between the group of torsion elements of $\mathcal{O}_{0}^{\triangleright}$ and an analogous group of torsion elements naturally associated to $G_{0}$ - i.e., a structure that is manifestly not preserved by the natural action of $\widehat{\mathbb{Z}}^{\times}$on $\mathcal{O}_{0}^{\times}$!

In the context of the above discussion of Fig. I2.1, it is of interest to note the important role played by Kummer theory in the present series of papers [cf. the Introductions to [IUTchII], [IUTchIII]]. From the point of view of Fig. I2.1, this role corresponds to the precise specification of the gluing cycle within each twicepunctured genus one surface in the illustration. Of course, such a precise specification depends on the twice-punctured genus one surface under consideration, i.e., the same gluing cycle is subject to quite different "precise specifications", relative to the twice-punctured genus one surface on the left and the twice-punctured genus one surface on the right. This state of affairs corresponds to the quite different Kummer theories to which the monoids/Frobenioids that appear in the $\Theta$-link are subject, relative to the $\Theta^{ \pm \text {ell }} \mathrm{NF}$-Hodge theater in the domain of the $\Theta$-link and the $\Theta^{ \pm \text {ell }} \mathrm{NF}$-Hodge theater in the codomain of the $\Theta$-link. At first glance, it might appear that the use of Kummer theory, i.e., of the correspondence determined by constructing Kummer classes, to achieve this precise specification of the relevant monoids/Frobenioids within each $\Theta^{ \pm \text {ell }} \mathrm{NF-Hodge} \mathrm{theater} \mathrm{is} \mathrm{somewhat} \mathrm{arbitrary,}$ i.e., that one could perhaps use other correspondences [i.e., correspondences not determined by Kummer classes] to achieve such a precise specification. In fact, however, the rigidity of the relevant local and global monoids equipped with Galois actions [cf. Corollary 5.3, (i), (ii), (iv)] implies that, if one imposes the natural condition of Galois-compatibility, then

the correspondence furnished by Kummer theory is the only acceptable choice for constructing the required "precise specification of the relevant monoids/Frobenioids within each $\Theta^{ \pm \text {ell }}$ NF-Hodge theater"

— cf. also the discussion of [IUTchII], Remark 3.6.2, (ii).

The construction of the Frobenius-picture described in $§ I 1$ is given in the present paper. More elaborate versions of this Frobenius-picture will be discussed in [IUTchII], [IUTchIII]. Once one constructs the Frobenius-picture, one natural 
and fundamental problem, which will, in fact, be one of the main themes of the present series of papers, is the problem of

describing an alien "arithmetic holomorphic structure" [i.e., an alien "conventional scheme theory"] corresponding to some ${ }^{m} \mathcal{H}^{\Theta^{ \pm \text {ell }} \mathrm{NF}}$ in terms of a "known arithmetic holomorphic structure" corresponding to ${ }^{n} \mathcal{H} \mathcal{T}^{\Theta}{ }^{ \pm \text {ell }} \mathrm{NF}$ [where $n \neq m$ ]

- a problem, which, as discussed in $\S \mathrm{I} 1$, will be approached, in the final portion of [IUTchIII], by applying various results from absolute anabelian geometry [i.e., more explicitly, the theory of [SemiAnbd], [EtTh], and [AbsTopIII]] to the various tempered and étale fundamental groups that appear in the étale-picture.

The relevance to this problem of the extensive theory of "reconstruction of ring/scheme structures" provided by absolute anabelian geometry is evident from the statement of the problem. On the other hand, in this context, it is of interest to note that, unlike conventional anabelian geometry, which typically centers on the goal of reconstructing a "known scheme-theoretic object", in the present series of papers, we wish to apply techniques and results from anabelian geometry in order to analyze the structure of an unknown, essentially non-scheme-theoretic object, namely, the Frobenius-picture, as described above. Put another way, relative to the point of view that "Galois groups are arithmetic tangent bundles" [cf. the theory of the arithmetic Kodaira-Spencer morphism in [HASurI]], one may think of conventional anabelian geometry as corresponding to the computation of the automorphisms of a scheme as

$$
H^{0} \text { (arithmetic tangent bundle) }
$$

and of the application of absolute anabelian geometry to the analysis of the Frobeniuspicture, i.e., to the solution of the problem discussed above, as corresponding to the computation of

$$
H^{1} \text { (arithmetic tangent bundle) }
$$

- i.e., the computation of "deformations of the arithmetic holomorphic structure" of a number field equipped with an elliptic curve.

In the context of the above discussion, we remark that the word "Hodge" in the term "Hodge theater" was intended as a reference to the use of the word "Hodge" in such classical terminology as "variation of Hodge structure" [cf. also the discussion of Hodge filtrations in [AbsTopIII], §I5], for instance, in discussions of Torelli maps [the most fundamental special case of which arises from the tautological family of one-dimensional complex tori parametrized by the upper half-plane!], where a "Hodge structure" corresponds precisely to the specification of a particular holomorphic structure in a situation in which one considers variations of the holomorphic structure on a fixed underlying real analytic structure. That is to say, later, in [IUTchIII], we shall see that the position occupied by a "Hodge theater" within a much larger framework that will be referred to as the "log-theta-lattice" [cf. the discussion of $\S \mathrm{I} 4$ below] corresponds precisely to the specification of a particular arithmetic holomorphic structure in a situation in which such arithmetic holomorphic structures are subject to deformation. 


\section{$\S$ I3. Basepoints and Inter-universality}

As discussed in $\S \mathrm{I} 2$, the present series of papers is concerned with considering "deformations of the arithmetic holomorphic structure" of a number field - i.e., so to speak, with performing "surgery on the number field". At a more concrete level, this means that one must consider situations in which two distinct "theaters" for conventional ring/scheme theory - i.e., two distinct $\Theta^{ \pm \mathrm{ell}} \mathrm{NF}-\mathrm{Hodge}$ theaters - are related to one another by means of a "correspondence", or "filter", that fails to be compatible with the respective ring structures. In the discussion so far of the portion of the theory developed in the present paper, the main example of such a "filter" is given by the $\Theta$-link. As mentioned earlier, more elaborate versions of the $\Theta$-link will be discussed in [IUTchII], [IUTchIII]. The other main example of such a non-ring/scheme-theoretic "filter" in the present series of papers is the log-link, which we shall discuss in [IUTchIII] [cf. also the theory of [AbsTopIII]].

One important aspect of such non-ring/scheme-theoretic filters is the property that they are incompatible with various constructions that depend on the ring structure of the theaters that constitute the domain and codomain of such a filter. From the point of view of the present series of papers, perhaps the most important example of such a construction is given by the various étale fundamental groups - e.g., Galois groups - that appear in these theaters. Indeed, these groups are defined, essentially, as automorphism groups of some separably closed field, i.e., the field that arises in the definition of the fiber functor associated to the basepoint determined by a geometric point that is used to define the étale fundamental group - cf. the discussion of [IUTchII], Remark 3.6.3, (i); [IUTchIII], Remark 1.2.4, (i); [AbsTopIII], Remark 3.7.7, (i). In particular, unlike the case with ring homomorphisms or morphisms of schemes with respect to which the étale fundamental group satisfies well-known functoriality properties, in the case of nonring/scheme-theoretic filters, the only "type of mathematical object" that makes sense simultaneously in both the domain and codomain theaters of the filter is the notion of a topological group. In particular, the only data that can be considered in relating étale fundamental groups on either side of a filter is the étale-like structure constituted by the underlying abstract topological group associated to such an étale fundamental group, i.e., devoid of any auxiliary data arising from the construction of the group "as an étale fundamental group associated to a basepoint determined by a geometric point of a scheme". It is this fundamental aspect of the theory of the present series of papers - i.e.,

of relating the distinct set-theoretic universes associated to the distinct fiber functors/basepoints on either side of such a non-ring/scheme-theoretic filter

- that we refer to as inter-universal. This inter-universal aspect of the theory manifestly leads to the issue of considering

the extent to which one can understand various ring/scheme structures by considering only the underlying abstract topological group of some étale fundamental group arising from such a ring/scheme structure

- i.e., in other words, of considering the absolute anabelian geometry [cf. the Introductions to [AbsTopI], [AbsTopII], [AbsTopIII]] of the rings/schemes under consideration. 
At this point, the careful reader will note that the above discussion of the inter-universal aspects of the theory of the present series of papers depends, in an essential way, on the issue of distinguishing different "types of mathematical objects" and hence, in particular, on the notion of a "type of mathematical object". This notion may be formalized via the language of "species", which we develop in the final portion of [IUTchIV].

Another important "inter-universal" phenomenon in the present series of papers - i.e., phenomenon which, like the absolute anabelian aspects discussed above, arises from a "deep sensitivity to particular choices of basepoints" - is the phenomenon of conjugate synchronization, i.e., of synchronization between conjugacy indeterminacies of distinct copies of various local Galois groups, which, as was mentioned in $\S I 1$, will play an important role in the theory of [IUTchII], [IUTchIII]. The various rigidity properties of the étale theta function established in [EtTh] constitute yet another inter-universal phenomenon that will play an important role in theory of [IUTchII], [IUTchIII].

\section{§I4. Relation to Complex and $p$-adic Teichmüller Theory}

In order to understand the sense in which the theory of the present series of papers may be thought of as a sort of "Teichmüller theory" of number fields equipped with an elliptic curve, it is useful to recall certain basic, well-known facts concerning the classical complex Teichmüller theory of Riemann surfaces of finite type [cf., e.g., [Lehto], Chapter V, §8]. Although such a Riemann surface is one-dimensional from a complex, holomorphic point of view, this single complex dimension may be thought of consisting of two underlying real analytic dimensions. Relative to a suitable canonical holomorphic coordinate $z=x+i y$ on the Riemann surface, the Teichmüller deformation may be written in the form

$$
z \mapsto \zeta=\xi+i \eta=K x+i y
$$

- where $1<K<\infty$ is the dilation factor associated to the deformation. That is to say, the Teichmüller deformation consists of dilating one of the two underlying real analytic dimensions, while keeping the other dimension fixed. Moreover, the theory of such Teichmüller deformations may be summarized as consisting of

the explicit description of a varying holomorphic structure within a fixed real analytic "container"

- i.e., the underlying real analytic surface associated to the given Riemann surface.

On the other hand, as discussed in [AbsTopIII], $\S \mathrm{I} 3$, one may think of the ring structure of a number field $F$ as a single "arithmetic holomorphic dimension", which, in fact, consists of two underlying "combinatorial dimensions", corresponding to

- its additive structure " $\boxplus$ " and its multiplicative structure " $\square$ ".

When, for simplicity, the number field $F$ is totally imaginary, one may think of these two combinatorial dimensions as corresponding to the 
- two cohomological dimensions of the absolute Galois group $G_{F}$ of $F$.

A similar statement holds in the case of the absolute Galois group $G_{k}$ of a nonarchimedean local field $k$. In the case of complex archimedean fields $k$ [i.e., topological fields isomorphic to the field of complex numbers equipped with its usual topology], the two combinatorial dimensions of $k$ may also be thought of as corresponding to the

- two underlying topological/real dimensions of $k$.

Alternatively, in both the nonarchimedean and archimedean cases, one may think of the two underlying combinatorial dimensions of $k$ as corresponding to the

- group of units $\mathcal{O}_{k}^{\times}$and value group $k^{\times} / \mathcal{O}_{k}^{\times}$of $k$.

Indeed, in the nonarchimedean case, local class field theory implies that this last point of view is consistent with the interpretation of the two underlying combinatorial dimensions via cohomological dimension; in the archimedean case, the consistency of this last point of view with the interpretation of the two underlying combinatorial dimensions via topological/real dimension is immediate from the definitions.

This last interpretation in terms of groups of units and value groups is of particular relevance in the context of the theory of the present series of papers. That is to say, one may think of the $\Theta$-link

$$
\begin{aligned}
\dagger \mathfrak{F}_{\text {tht }}^{\vdash} & \stackrel{\sim}{\rightarrow} \quad{ }^{\ddagger} \mathfrak{F}_{\text {mod }}^{\Vdash} \\
\left\{{ }^{\dagger} \underline{\underline{\Theta}}_{\underline{v}}\right. & \left.\mapsto \quad{ }^{\ddagger} \underline{\underline{q}}_{\underline{v}}\right\}_{\underline{v} \in \underline{\mathbb{V}}^{\text {bad }}}
\end{aligned}
$$

— which, as discussed in $§ I 1$, induces a full poly-isomorphism

$$
\begin{aligned}
& \dagger \mathfrak{F}_{\text {mod }}^{\vdash \times} \stackrel{\sim}{\rightarrow} \ddagger \mathfrak{F}_{\text {mod }}^{-\times} \\
& \left\{\mathcal{O}_{\bar{F}_{\underline{v}}}^{\times} \stackrel{\sim}{\rightarrow} \mathcal{O}_{\bar{F}_{\underline{v}}}^{\times}\right\}_{\underline{v} \in \underline{\mathbb{V}} \text { bad }}
\end{aligned}
$$

- as a sort of "Teichmüller deformation relative to a $\Theta$-dilation", i.e., a deformation of the ring structure of the number field equipped with an elliptic curve constituted by the given initial $\Theta$-data in which one dilates the underlying combinatorial dimension corresponding to the local value groups relative to a " $\Theta$ factor", while one leaves fixed, up to isomorphism, the underlying combinatorial dimension corresponding to the local groups of units [cf. Remark 3.9.3]. This point of view is reminiscent of the discussion in $\S \mathrm{I} 1$ of "disentangling/dismantling" of various structures associated to a number field.

In [IUTchIII], we shall consider two-dimensional diagrams of $\Theta^{ \pm e l l} \mathrm{NF}-H o d g e$ theaters which we shall refer to as log-theta-lattices. The two dimensions of such diagrams correspond precisely to the two underlying combinatorial dimensions of a ring. Of these two dimensions, the "theta dimension" consists of the Frobeniuspicture associated to [more elaborate versions of] the $\Theta$-link. Many of the important properties that involve this "theta dimension" are consequences of the theory of [FrdI], [FrdII], [EtTh]. On the other hand, the "log dimension" consists of iterated copies of the log-link, i.e., diagrams of the sort that are studied in [AbsTopIII]. 
That is to say, whereas the "theta dimension" corresponds to "deformations of the arithmetic holomorphic structure" of the given number field equipped with an elliptic curve, this "log dimension" corresponds to "rotations of the two underlying combinatorial dimensions" of a ring that leave the arithmetic holomorphic structure fixed - cf. the discussion of the "juggling of $\boxplus, \otimes$ induced by log" in [AbsTopIII], §I3. The ultimate conclusion of the theory of [IUTchIII] is that

the "a priori unbounded deformations" of the arithmetic holomorphic structure given by the $\Theta$-link in fact admit canonical bounds, which may be thought of as a sort of reflection of the "hyperbolicity" of the given number field equipped with an elliptic curve

- cf. [IUTchIII], Corollary 3.12. Such canonical bounds may be thought of as analogues for a number field of canonical bounds that arise from differentiating Frobenius liftings in the context of $p$-adic hyperbolic curves - cf. the discussion in the final portion of [AbsTopIII], §I5. Moreover, such canonical bounds are obtained in [IUTchIII] as a consequence of

\section{the explicit description of a varying arithmetic holomorphic struc-} ture within a fixed mono-analytic "container"

- cf. the discussion of $\S I 2$ ! - furnished by [IUTchIII], Theorem 3.11 [cf. also the discussion of [IUTchIII], Remarks 3.12.2, 3.12.3, 3.12.4], i.e., a situation that is entirely formally analogous to the summary of complex Teichmüller theory given above.

The significance of the log-theta-lattice is best understood in the context of the analogy between the inter-universal Teichmüller theory developed in the present series of papers and the $\boldsymbol{p}$-adic Teichmüller theory of [ $p$ Ord], $[p$ Teich]. Here, we recall for the convenience of the reader that the $p$-adic Teichmüller theory of $[p$ Ord], $[p$ Teich] may be summarized, [very!] roughly speaking, as a sort of generalization, to the case of "quite general" $p$-adic hyperbolic curves, of the classical $p$-adic theory surrounding the canonical representation

$$
\pi_{1}\left(\left(\mathbb{P}^{1} \backslash\{0,1, \infty\}\right)_{\mathbb{Q}_{p}}\right) \quad \rightarrow \quad \pi_{1}\left(\left(\mathcal{M}_{\mathrm{ell}}\right)_{\mathbb{Q}_{p}}\right) \quad \rightarrow \quad P G L_{2}\left(\mathbb{Z}_{p}\right)
$$

— where the " $\pi_{1}(-)$ 's" denote the étale fundamental group, relative to a suitable basepoint; $\left(\mathcal{M}_{\mathrm{ell}}\right)_{\mathbb{Q}_{p}}$ denotes the moduli stack of elliptic curves over $\mathbb{Q}_{p}$; the first arrow denotes the morphism induced by the elliptic curve over the projective line minus three points determined by the classical Legendre form of the Weierstrass equation; the second arrow is the representation determined by the $p$-power torsion points of the tautological elliptic curve over $\left(\mathcal{M}_{\text {ell }}\right)_{\mathbb{Q}_{p}}$. In particular, the reader who is familiar with the theory of the classical representation of the above display, but not with the theory of $[p$ Ord], $[p$ Teich $]$, may nevertheless appreciate, to a substantial degree, the analogy between the inter-universal Teichmüller theory developed in the present series of papers and the $p$-adic Teichmüller theory of $[p \mathrm{Ord}],[p$ Teich] by

thinking in terms of the

well-known classical properties of this classical representation.

In some sense, the gap between the "quite general" $p$-adic hyperbolic curves that appear in $p$-adic Teichmüller theory and the classical case of $\left(\mathbb{P}^{1} \backslash\{0,1, \infty\}\right)_{\mathbb{Q}_{p}}$ may 
be thought of, roughly speaking, as corresponding, relative to the analogy with the theory of the present series of papers, to the gap between arbitrary number fields and the rational number field $\mathbb{Q}$. This point of view is especially interesting in the context of the discussion of $§ I 5$ below.

The analogy between the inter-universal Teichmüller theory developed in the present series of papers and the $\boldsymbol{p}$-adic Teichmüller theory of [ $p$ Ord], $[p$ Teich] is described to a substantial degree in the discussion of [AbsTopIII], §I5, i.e., where the "future Teichmüller-like extension of the mono-anabelian theory" may be understood as referring precisely to the inter-universal Teichmüller theory developed in the present series of papers. The starting point of this analogy is the correspondence between a number field equipped with a [once-punctured] elliptic curve [in the present series of papers] and a hyperbolic curve over a positive characteristic perfect field equipped with a nilpotent ordinary indigenous bundle [in p-adic Teichmüller theory] — cf. Fig. I4.1 below. That is to say, in this analogy, the number field which may be regarded as being equipped with a finite collection of "exceptional" valuations, namely, in the notation of $\S \mathrm{I} 1$, the valuations lying over $\mathbb{V}_{\bmod }^{\mathrm{bad}}$ - corresponds to the hyperbolic curve over a positive characteristic perfect field - which may be thought of as a one-dimensional function field over a positive characteristic perfect field, equipped with a finite collection of "exceptional" valuations, namely, the valuations corresponding to the cusps of the curve.

On the other hand, the [once-punctured] elliptic curve in the present series of papers corresponds to the nilpotent ordinary indigenous bundle in p-adic Teichmüller theory. Here, we recall that an indigenous bundle may be thought of as a sort of "virtual analogue" of the first cohomology group of the tautological elliptic curve over the moduli stack of elliptic curves. Indeed, the canonical indigenous bundle over the moduli stack of elliptic curves arises precisely as the first de Rham cohomology module of this tautological elliptic curve. Put another way, from the point of view of fundamental groups, an indigenous bundle may be thought of as a sort of "virtual analogue" of the abelianized fundamental group of the tautological elliptic curve over the moduli stack of elliptic curves. By contrast, in the present series of papers, it is of crucial importance to use the entire nonabelian profinite étale fundamental group - i.e., not just its abelizanization! — of the given once-punctured elliptic curve over a number field. Indeed, only by working with the entire profinite étale fundamental group can one avail oneself of the crucial absolute anabelian theory developed in [EtTh], [AbsTopIII] [cf. the discussion of $§ I 3]$. This state of affairs prompts the following question:

To what extent can one extend the indigenous bundles that appear in classical complex and $p$-adic Teichmüller theory to objects that serve as "virtual analogues" of the entire nonabelian fundamental group of the tautological once-punctured elliptic curve over the moduli stack of [oncepunctured] elliptic curves?

Although this question lies beyond the scope of the present series of papers, it is 
the hope of the author that this question may be addressed in a future paper.

\begin{tabular}{|c|c|}
\hline Inter-universal Teichmüller theory & p-adic Teichmüller theory \\
\hline $\begin{array}{c}\text { number field } \\
F\end{array}$ & $\begin{array}{l}\text { hyperbolic curve } C \text { over a } \\
\text { positive characteristic perfect field }\end{array}$ \\
\hline $\begin{array}{c}\text { [once-punctured] } \\
\text { elliptic curve } \\
X \text { over } F\end{array}$ & $\begin{array}{c}\text { nilpotent ordinary } \\
\text { indigenous bundle } \\
P \text { over } C\end{array}$ \\
\hline $\begin{array}{l}\Theta \text {-link arrows of the } \\
\text { log-theta-lattice }\end{array}$ & $\begin{array}{l}\text { mixed characteristic extension } \\
\text { structure of a ring of Witt vectors }\end{array}$ \\
\hline $\begin{array}{l}\text { log-link arrows of the } \\
\text { log-theta-lattice }\end{array}$ & $\begin{array}{l}\text { the Frobenius morphism } \\
\text { in positive characteristic }\end{array}$ \\
\hline $\begin{array}{c}\text { the entire } \\
\text { log-theta-lattice }\end{array}$ & $\begin{array}{c}\text { the resulting canonical lifting } \\
+ \text { canonical Frobenius action; } \\
\text { canonical Frobenius lifting } \\
\text { over the ordinary locus }\end{array}$ \\
\hline $\begin{array}{l}\text { relatively straightforward } \\
\text { original construction of } \\
\text { log-theta-lattice }\end{array}$ & $\begin{array}{l}\text { relatively straightforward } \\
\text { original construction of } \\
\text { canonical liftings }\end{array}$ \\
\hline $\begin{array}{l}\text { highly nontrivial } \\
\text { description of alien arithmetic } \\
\text { holomorphic structure } \\
\text { via absolute anabelian geometry }\end{array}$ & $\begin{array}{l}\text { highly nontrivial } \\
\text { absolute anabelian } \\
\text { reconstruction of } \\
\text { canonical liftings }\end{array}$ \\
\hline
\end{tabular}

Fig. I4.1: Correspondence between inter-universal Teichmüller theory and p-adic Teichmüller theory

Now let us return to our discussion of the log-theta-lattice, which, as discussed above, consists of two types of arrows, namely, $\Theta$-link arrows and log-link arrows. As discussed in [IUTchIII], Remark 1.4.1, (iii) — cf. also Fig. I4.1 above, as well as Remark 3.9.3, (i), of the present paper - the $\Theta$-link arrows correspond to the "transition from $p^{n} \mathbb{Z} / p^{n+1} \mathbb{Z}$ to $p^{n-1} \mathbb{Z} / p^{n} \mathbb{Z}$ ", i.e., the mixed characteristic extension structure of a ring of Witt vectors, while the log-link arrows, i.e., 
the portion of theory that is developed in detail in [AbsTopIII], and which will be incorporated into the theory of the present series of papers in [IUTchIII], correspond to the Frobenius morphism in positive characteristic. As we shall see in [IUTchIII], these two types of arrows fail to commute [cf. [IUTchIII], Remark 1.4.1, (i)]. This noncommutativity, or "intertwining", of the $\Theta$-link and log-link arrows of the log-theta-lattice may be thought of as the analogue, in the context of the theory of the present series of papers, of the well-known "intertwining between the mixed characteristic extension structure of a ring of Witt vectors and the Frobenius morphism in positive characteristic" that appears in the classical $p$-adic theory. In particular, taken as a whole, the log-theta-lattice in the theory of the present series of papers may be thought of as an analogue, for number fields equipped with a [once-punctured] elliptic curve, of the canonical lifting, equipped with a canonical Frobenius action - hence also the canonical Frobenius lifting over the ordinary locus of the curve - associated to a positive characteristic hyperbolic curve equipped with a nilpotent ordinary indigenous bundle in $p$-adic Teichmüller theory [cf. Fig. I4.1 above; the discussion of [IUTchIII], Remarks 3.12.3, 3.12.4].

Finally, we observe that it is of particular interest in the context of the present discussion that a theory is developed in [CanLift], §3, that yields an absolute anabelian reconstruction for the canonical liftings of p-adic Teichmüller theory. That is to say, whereas the original construction of such canonical liftings given in $[p$ Ord], $\S 3$, is relatively straightforward, the anabelian reconstruction given in [CanLift], $\S 3$, of, for instance, the canonical lifting modulo $p^{2}$ of the logarithmic special fiber consists of a highly nontrivial anabelian argument. This state of affairs is strongly reminiscent of the stark contrast between the relatively straightforward construction of the log-theta-lattice given in the present series of papers and the description of an "alien arithmetic holomorphic structure" given in [IUTchIII], Theorem 3.11 [cf. the discussion in the earlier portion of the present §I4], which is achieved by applying highly nontrivial results in absolute anabelian geometry cf. Fig. I4.1 above. In this context, we observe that the absolute anabelian theory of [AbsTopIII], §1, which plays a central role in the theory surrounding [IUTchIII], Theorem 3.11, corresponds, in the theory of [CanLift], $\S 3$, to the absolute anabelian reconstruction of the logarithmic special fiber given in [AbsAnab], $§ 2$ [i.e., in essence, the theory of absolute anabelian geometry over finite fields developed in [Tama1]; cf. also [Cusp], §2]. Moreover, just as the absolute anabelian theory of [AbsTopIII], §1, follows essentially by combining a version of "Uchida's Lemma" [cf. [AbsTopIII], Proposition 1.3] with the theory of Belyi cuspidalization - i.e.,

\section{[AbsTopIII], $\S 1=$ Uchida Lem. + Belyi cuspidalization}

— the absolute anabelian geometry over finite fields of [Tama1], [Cusp], follows essentially by combining a version of "Uchida's Lemma" with an application [to the counting of rational points] of the Lefschetz trace formula for [powers of] the Frobenius morphism on a curve over a finite field - i.e.,

\section{$[$ Tama1], [Cusp] = Uchida Lem. + Lefschetz trace formula for Frob.}

- cf. the discussion of [AbsTopIII], §I5. That is to say, it is perhaps worthy of note that in the analogy between the inter-universal Teichmüller theory developed in the present series of papers and the $p$-adic Teichmüller theory of [ $p$ Ord], [ $p$ Teich], [CanLift], the application of the theory of Belyi cuspidalization over number fields 
and mixed characteristic local fields may be thought of as corresponding to the Lefschetz trace formula for [powers of] the Frobenius morphism on a curve over a finite field, i.e.,

\section{Belyi cuspidalization $\longleftrightarrow \quad$ Lefschetz trace formula for Frobenius}

[Here, we note in passing that this correspondence may be related to the correspondence discussed in [AbsTopIII], §I5, between Belyi cuspidalization and the Verschiebung on positive characteristic indigenous bundles by considering the geometry of Hecke correspondences modulo $p$, i.e., in essence, graphs of the Frobenius morphism in characteristic $p$ !] It is the hope of the author that these analogies and correspondences might serve to stimulate further developments in the theory.

\section{$\S I 5$. Other Galois-theoretic Approaches to Diophantine Geometry}

The notion of anabelian geometry dates back to a famous "letter to Faltings" [cf. [Groth]], written by Grothendieck in response to Faltings' work on the Mordell Conjecture [cf. [Falt]]. Anabelian geometry was apparently originally conceived by Grothendieck as a new approach to obtaining results in diophantine geometry such as the Mordell Conjecture. At the time of writing, the author is not aware of any expositions by Grothendieck that expose this approach in detail. Nevertheless, it appears that the thrust of this approach revolves around applying the Section Conjecture for hyperbolic curves over number fields to obtain a contradiction by applying this Section Conjecture to the "limit section" of the Galois sections associated to any infinite sequence of rational points of a proper hyperbolic curve over a number field [cf. [MNT], $\S 4.1(\mathrm{~B})$, for more details]. On the other hand, to the knowledge of the author, at least at the time of writing, it does not appear that any rigorous argument has been obtained either by Grothendieck or by other mathematicians for deriving a new proof of the Mordell Conjecture from the as yet unproven] Section Conjecture for hyperbolic curves over number fields. Nevertheless, one result that has been obtained is a new proof by M. Kim [cf. [Kim]] of Siegel's theorem concerning $\mathbb{Q}$-rational points of the projective line minus three points - a proof which proceeds by obtaining certain bounds on the cardinality of the set of Galois sections, without applying the Section Conjecture or any other results from anabelian geometry.

In light of the historical background just discussed, the theory exposed in the present series of papers — which yields, in particular, a method for applying results in absolute anabelian geometry to obtain diophantine results such as those given in [IUTchIV] - occupies a somewhat curious position, relative to the historical development of the mathematical ideas involved. That is to say, at a purely formal level, the implication

$$
\text { anabelian geometry } \Longrightarrow \text { diophantine results }
$$

at first glance looks something like a "confirmation" of Grothendieck's original intuition. On the other hand, closer inspection reveals that the approach of the theory of the present series of papers - that is to say, the precise content of the relationship between anabelian geometry and diophantine geometry established in 
the present series of papers - differs quite fundamentally from the sort of approach that was apparently envisioned by Grothendieck.

Perhaps the most characteristic aspect of this difference lies in the central role played by anabelian geometry over $\boldsymbol{p}$-adic fields in the present series of papers. That is to say, unlike the case with number fields, one central feature of anabelian geometry over $p$-adic fields is the fundamental gap between relative and absolute results [cf., e.g., [AbsTopI], Introduction]. This fundamental gap is closely related to the notion of an "arithmetic Teichmüller theory for number fields" [cf. the discussion of $\S$ I4 of the present paper; [AbsTopIII], $\S \mathrm{I} 3$, §I5] - i.e., a theory of deformations not for the "arithmetic holomorphic structure" of a hyperbolic curve over a number field, but rather for the "arithmetic holomorphic structure" of the number field itself! To the knowledge of the author, there does not exist any mention of such ideas [i.e., relative vs. absolute $p$-adic anabelian geometry; the notion of an arithmetic Teichmüller theory for number fields] in the works of Grothendieck.

As discussed in $\S \mathrm{I} 4$, one fundamental theme of the theory of the present series of papers is the issue of the

explicit description of the relationship between the additive structure and the multiplicative structure of a ring/number field/local field.

Relative to the above discussion of the relationship between anabelian geometry and diophantine geometry, it is of interest to note that this issue of understanding/describing the relationship between addition and multiplication is, on the one hand, a central theme in the proofs of various results in anabelian geometry [cf., e.g., [Tama1], [pGC], [AbsTopIII]] and, on the other hand, a central aspect of the diophantine results obtained in [IUTchIV].

From a historical point of view, it is also of interest to note that results from absolute anabelian geometry are applied in the present series of papers in the context of the canonical splittings of the Frobenius-picture that arise by considering the étale-picture [cf. the discussion in $\S \mathrm{I} 1$ preceding Theorem A]. This state of affairs is reminiscent - relative to the point of view that the Grothendieck Conjecture constitutes a sort of "anabelian version" of the Tate Conjecture for abelian varieties [cf. the discussion of [MNT], $\S 1.2]$ — of the role played by the Tate Conjecture for abelian varieties in obtaining the diophantine results of [Falt], namely, by means of the various semi-simplicity properties of the Tate module that arise as formal consequences of the Tate Conjecture. That is to say, such semi-simplicity properties may also be thought of as "canonical splittings" that arise from Galois-theoretic considerations [cf. the discussion of "canonical splittings" in the final portion of [CombCusp], Introduction].

Certain aspects of the relationship between the inter-universal Teichmüller theory of the present series of papers and other Galois-theoretic approaches to diophantine geometry are best understood in the context of the analogy, discussed in $\S$ I4, between inter-universal Teichmüller theory and $\boldsymbol{p}$-adic Teichmüller theory. One way to think of the starting point of $p$-adic Teichmüller is as an attempt to construct a $p$-adic analogue of the theory of the action of $S L_{2}(\mathbb{Z})$ on the upper half-plane, i.e., of the natural embedding

$$
\rho_{\mathbb{R}}: S L_{2}(\mathbb{Z}) \quad \hookrightarrow \quad S L_{2}(\mathbb{R})
$$


of $S L_{2}(\mathbb{Z})$ as a discrete subgroup. This leads naturally to consideration of the representation

$$
\rho_{\widehat{\mathbb{Z}}}=\prod_{p} \rho_{\mathbb{Z}_{p}}: \quad S L_{2}(\mathbb{Z})^{\wedge} \rightarrow S L_{2}(\widehat{\mathbb{Z}})=\prod_{p \in \mathfrak{P r i m e s}} S L_{2}\left(\mathbb{Z}_{p}\right)
$$

— where we write $S L_{2}(\mathbb{Z})^{\wedge}$ for the profinite completion of $S L_{2}(\mathbb{Z})$. If one thinks of $S L_{2}(\mathbb{Z})^{\wedge}$ as the geometric étale fundamental group of the moduli stack of elliptic curves over a field of characteristic zero, then the $p$-adic Teichmüller theory of $\left[p\right.$ Ord], $[p$ Teich $]$ does indeed constitute a generalization of $\rho_{\mathbb{Z}_{p}}$ to more general $p$ adic hyperbolic curves.

From a representation-theoretic point of view, the next natural direction in which to further develop the theory of $[p \mathrm{Ord}],[p$ Teich $]$ consists of attempting to generalize the theory of representations into $S L_{2}\left(\mathbb{Z}_{p}\right)$ obtained in $[p$ Ord], $[p$ Teich $]$ to a theory concerning representations into $S L_{n}\left(\mathbb{Z}_{p}\right)$ for arbitrary $n \geq 2$. This is precisely the motivation that lies, for instance, behind the work of Joshi and Pauly [cf. [JP]].

On the other hand, unlike the original motivating representation $\rho_{\mathbb{R}}$, the representation $\rho_{\widehat{\mathbb{Z}}}$ is far from injective, i.e., put another way, the so-called Congruence Subgroup Property fails to hold in the case of $S L_{2}$. This failure of injectivity means that working with

$\rho_{\widehat{\mathbb{Z}}}$ only allows one to access a relatively limited portion of $S L_{2}(\mathbb{Z})^{\wedge}$.

From this point of view, a more natural direction in which to further develop the theory of $[p$ Ord $],[p$ Teich $]$ is to consider the "anabelian version"

$$
\rho_{\Delta}: \quad S L_{2}(\mathbb{Z})^{\wedge} \rightarrow \operatorname{Out}\left(\Delta_{1,1}\right)
$$

of $\rho_{\widehat{\mathbb{Z}}}$ - i.e., the natural outer representation on the geometric étale fundamental group $\Delta_{1,1}$ of the tautological family of once-punctured elliptic curves over the moduli stack of elliptic curves over a field of characteristic zero. Indeed, unlike the case with $\rho_{\widehat{\mathbb{Z}}}$, one knows [cf. [Asada]] that $\rho_{\Delta}$ is injective. Thus, the "arithmetic Teichmüller theory for number fields equipped with a [once-punctured] elliptic curve" constituted by the inter-universal Teichmüller theory developed in the present series of papers may [cf. the discussion of $\S \mathbf{I} 4$ !] be regarded as a realization of this sort of "anabelian" approach to further developing the $p$-adic Teichmüller theory of $[p$ Ord $],[p$ Teich $]$.

In the context of these two distinct possible directions for the further development of the $p$-adic Teichmüller theory of $[p \mathrm{Ord}],[p$ Teich $]$, it is of interest to recall the following elementary fact:

If $G$ is a free pro-p group of rank $\geq 2$, then a [continuous] representation

$$
\rho_{G}: G \rightarrow G L_{n}\left(\mathbb{Q}_{p}\right)
$$

can never be injective!

Indeed, assume that $\rho_{G}$ is injective and write $\ldots \subseteq H_{j} \subseteq \ldots \subseteq \operatorname{Im}\left(\rho_{G}\right) \subseteq G L_{n}\left(\mathbb{Q}_{p}\right)$ for an exhaustive sequence of open normal subgroups of the image of $\rho_{G}$. Then since 
the $H_{j}$ are closed subgroups of $G L_{n}\left(\mathbb{Q}_{p}\right)$, hence $p$-adic Lie groups, it follows that the $\mathbb{Q}_{p}$-dimension $\operatorname{dim}\left(H_{j}^{\mathrm{ab}} \otimes \mathbb{Q}_{p}\right)$ of the tensor product with $\mathbb{Q}_{p}$ of the abelianization of $H_{j}$ may be computed at the level of Lie algebras, hence is bounded by the $\mathbb{Q}_{p^{-}}$ dimension of the $p$-adic Lie group $G L_{n}\left(\mathbb{Q}_{p}\right)$, i.e., we have $\operatorname{dim}\left(H_{j}^{\mathrm{ab}} \otimes \mathbb{Q}_{p}\right) \leq n^{2}$, in contradiction to the well-known fact since $G \cong \operatorname{Im}\left(\rho_{G}\right)$ is free pro-p of rank $\geq 2$, it holds that $\operatorname{dim}\left(H_{j}^{\mathrm{ab}} \otimes \mathbb{Q}_{p}\right) \rightarrow \infty$ as $j \rightarrow \infty$. Note, moreover, that

this sort of argument, i.e., concerning the asymptotic behavior of the abelianizations - or, more generally, of the Lie algebras associated to the pro-algebraic groups determined by associated Tannakian categories of representations - of open subgroups, is characteristic of the sort of proofs that typically occur in anabelian geometry [cf., e.g., the proofs of [Tama1], $[p \mathrm{GC}]$, [CombGC], as well as [Cusp], §3!].

That is to say, the above argument to the effect that $\rho_{G}$ can never be injective is a typical instance of the more general phenomenon that

so long as one restricts oneself to representation theory into $G L_{n}\left(\mathbb{Q}_{p}\right)$ [or even more general groups that arise as groups of $\mathbb{Q}_{p}$-valued points of pro-algebraic groups], one can never access the sort of asymptotic phenomena that form the "technical core" [cf., e.g., the proofs of [Tama1], $[p \mathrm{GC}]$, [CombGC], as well as [Cusp], §3!] of various important results in anabelian geometry.

Put another way, the two "directions" discussed above - i.e., representationtheoretic and anabelian - appear to be essentially mutually alien to one another.

In this context, it is of interest to observe that the diophantine results derived in [IUTchIV] from the inter-universal Teichmüller theory developed in the present series of papers concern essentially asymptotic behavior, i.e., they do not concern properties of "a specific rational point over a specific number field", but rather properties of the asymptotic behavior of "varying rational points over varying number fields". One important aspect of this asymptotic nature of the diophantine results derived in [IUTchIV] is that there are no distinguished number fields that occur in the theory, i.e., the theory - being essentially asymptotic in nature! - is "invariant" with respect to the operation of passing to finite extensions of the number field involved [which, from the point of view of the absolute Galois group $G_{\mathbb{Q}}$ of $\mathbb{Q}$, corresponds precisely to the operation of passing to smaller and smaller open subgroups, as in the above discussion!]. This contrasts sharply with the "representation-theoretic approach to diophantine geometry" constituted by such works as [Wiles], where specific rational points over the specific number field $\mathbb{Q}$ - or, for instance, in generalizations of [Wiles] involving Shimura varieties, over specific number fields characteristically associated to the Shimura varieties involved — play a central role.

\section{Acknowledgements:}

The research discussed in the present paper profited enormously from the generous support that the author received from the Research Institute for Mathematical 
Sciences, a Joint Usage/Research Center located in Kyoto University. At a personal level, I would like to thank Fumiharu Kato, Akio Tamagawa, Go Yamashita, Mohamed Saïdi, Yuichiro Hoshi, Ivan Fesenko, Fucheng Tan, Emmanuel Lepage, Arata Minamide, and Wojciech Porowski for many stimulating discussions concerning the material presented in this paper. In particular, I would like to thank Emmanuel Lepage for his stimulating comments [summarized in Remark 2.5.3] on [SemiAnbd]. Also, I feel deeply indebted to Go Yamashita, Mohamed Saïdi, and Yuichiro Hoshi for their meticulous reading of and numerous comments concerning the present paper. In particular, the introduction of the theory of $\kappa$-coric functions was motivated by various stimulating discussions with Yuichiro Hoshi. Finally, I would like to express my deep gratitude to Ivan Fesenko for his quite substantial efforts to disseminate - for instance, in the form of a survey that he wrote - the theory discussed in the present series of papers. 


\section{Section 0: Notations and Conventions}

\section{Monoids and Categories:}

We shall use the notation and terminology concerning monoids and categories of $[\mathrm{FrdI}], \S 0$.

We shall refer to a topological space $P$ equipped with a continuous map

$$
P \times P \supseteq S \rightarrow P
$$

as a topological pseudo-monoid if there exists a topological abelian group $M$ [whose group operation will be written multiplicatively] and an embedding of topological spaces $\iota: P \hookrightarrow M$ such that $S=\{(a, b) \in P \times P \mid \iota(a) \cdot \iota(b) \in \iota(P) \subseteq M\}$, and the map $S \rightarrow P$ is obtained by restricting the group operation $M \times M \rightarrow M$ on $M$ to $P$ by means of $\iota$. Here, if $M$ is equipped with the discrete topology, then we shall refer to the resulting $P$ simply as a pseudo-monoid. In particular, every topological pseudo-monoid determines, in an evident fashion, an underlying pseudomonoid. Let $P$ be a pseudo-monoid. Then we shall say that the pseudo-monoid $P$ is divisible if $M$ and $\iota$ may be taken such that for each positive integer $n$, every element of $M$ admits an $n$-th root in $M$, and, moreover, an element $a \in M$ lies in $\iota(P)$ if and only if $a^{n}$ lies in $\iota(P)$. We shall say that the pseudo-monoid $P$ is cyclotomic if $M$ and $\iota$ may be taken such that the subgroup $\boldsymbol{\mu}_{M} \subseteq M$ of torsion elements of $M$ is isomorphic to the group $\mathbb{Q} / \mathbb{Z}, \boldsymbol{\mu}_{M} \subseteq \iota(P)$, and $\boldsymbol{\mu}_{M} \cdot \iota(P) \subseteq \iota(P)$.

We shall refer to an isomorphic copy of some object as an isomorph of the object.

If $\mathcal{C}$ and $\mathcal{D}$ are categories, then we shall refer to as an isomorphism $\mathcal{C} \rightarrow \mathcal{D}$ any isomorphism class of equivalences of categories $\mathcal{C} \rightarrow \mathcal{D}$. [Note that this terminology differs from the standard terminology of category theory, but will be natural in the context of the theory of the present series of papers.] Thus, from the point of view of "coarsifications of 2-categories of 1-categories" [cf. [FrdI], Appendix, Definition A.1, (ii)], an "isomorphism $\mathcal{C} \rightarrow \mathcal{D}$ " is precisely an "isomorphism in the usual sense" of the [1-]category constituted by the coarsification of the 2-category of all small 1 -categories relative to a suitable universe with respect to which $\mathcal{C}$ and $\mathcal{D}$ are small.

Let $\mathcal{C}$ be a category; $A, B \in \mathrm{Ob}(\mathcal{C})$. Then we define a poly-morphism $A \rightarrow B$ to be a collection of morphisms $A \rightarrow B$ [i.e., a subset of the set of morphisms $A \rightarrow B]$; if all of the morphisms in the collection are isomorphisms, then we shall refer to the poly-morphism as a poly-isomorphism; if $A=B$, then we shall refer to a poly-isomorphism $A \stackrel{\sim}{\rightarrow} B$ as a poly-automorphism. We define the full poly-isomorphism $A \stackrel{\sim}{\rightarrow} B$ to be the poly-morphism given by the collection of all isomorphisms $A \stackrel{\sim}{\rightarrow} B$. The composite of a poly-morphism $\left\{f_{i}: A \rightarrow B\right\}_{i \in I}$ with a poly-morphism $\left\{g_{j}: B \rightarrow C\right\}_{j \in J}$ is defined to be the poly-morphism given by the set [i.e., where "multiplicities" are ignored] $\left\{g_{j} \circ f_{i}: A \rightarrow C\right\}_{(i, j) \in I \times J}$.

Let $\mathcal{C}$ be a category. We define a capsule of objects of $\mathcal{C}$ to be a finite collection $\left\{A_{j}\right\}_{j \in J}$ [i.e., where $J$ is a finite index set] of objects $A_{j}$ of $\mathcal{C}$; if $|J|$ denotes the 
cardinality of $J$, then we shall refer to a capsule with index set $J$ as a $|J|$-capsule; also, we shall write $\pi_{0}\left(\left\{A_{j}\right\}_{j \in J}\right) \stackrel{\text { def }}{=} J$. A morphism of capsules of objects of $\mathcal{C}$

$$
\left\{A_{j}\right\}_{j \in J} \rightarrow\left\{A_{j^{\prime}}^{\prime}\right\}_{j^{\prime} \in J^{\prime}}
$$

is defined to consist of an injection $\iota: J \hookrightarrow J^{\prime}$, together with, for each $j \in J$, a morphism $A_{j} \rightarrow A_{\iota(j)}^{\prime}$ of objects of $\mathcal{C}$. Thus, the capsules of objects of $\mathcal{C}$ form a category Capsule $(\mathcal{C})$. A capsule-full poly-morphism

$$
\left\{A_{j}\right\}_{j \in J} \rightarrow\left\{A_{j^{\prime}}^{\prime}\right\}_{j^{\prime} \in J^{\prime}}
$$

between two objects of Capsule $(\mathcal{C})$ is defined to be the poly-morphism associated to some [fixed] injection $\iota: J \hookrightarrow J^{\prime}$ which consists of the set of morphisms of Capsule $(\mathcal{C})$ given by collections of [arbitrary] isomorphisms $A_{j} \stackrel{\sim}{\rightarrow} A_{\iota(j)}^{\prime}$, for $j \in$ $J$. A capsule-full poly-isomorphism is a capsule-full poly-morphism for which the associated injection between index sets is a bijection.

If $X$ is a connected noetherian algebraic stack which is generically scheme-like [cf. [Cusp], §0], then we shall write

$$
\mathcal{B}(X)
$$

for the category of finite étale coverings of $X$ [and morphisms over $X$ ]; if $A$ is a noetherian [commutative] ring [with unity], then we shall write $\mathcal{B}(A) \stackrel{\text { def }}{=} \mathcal{B}(\operatorname{Spec}(A))$. Thus, [cf. [FrdI], §0] the subcategory of connected objects $\mathcal{B}(X)^{0} \subseteq \mathcal{B}(X)$ may be thought of as the subcategory of connected finite étale coverings of $X$ [and morphisms over $X]$.

Let $\Pi$ be a topological group. Then let us write

$$
\mathcal{B}^{\text {temp }}(\Pi)
$$

for the category whose objects are countable [i.e., of cardinality $\leq$ the cardinality of the set of natural numbers], discrete sets equipped with a continuous $\Pi$-action, and whose morphisms are morphisms of $\Pi$-sets [cf. [SemiAnbd], §3]. If $\Pi$ may be written as an inverse limit of an inverse system of surjections of countable discrete topological groups, then we shall say that $\Pi$ is tempered [cf. [SemiAnbd], Definition 3.1, (i)]. A category $\mathcal{C}$ equivalent to a category of the form $\mathcal{B}^{\text {temp }}(\Pi)$, where $\Pi$ is a tempered topological group, is called a connected temperoid [cf. [SemiAnbd], Definition 3.1, (ii)]. Thus, if $\mathcal{C}$ is a connected temperoid, then $\mathcal{C}$ is naturally equivalent to $\left(\mathcal{C}^{0}\right)^{\top}$ [cf. [FrdI], $\left.\S 0\right]$. Moreover, if $\Pi$ is Galois-countable [cf. Remark 2.5.3, (i), (T1)], then one can reconstruct [cf. Remark 2.5.3, (i), (T5)] the topological group $\Pi$, up to inner automorphism, category-theoretically from $\mathcal{B}^{\text {temp }}(\Pi)$ or $\mathcal{B}^{\text {temp }}(\Pi)^{0}$ [i.e., the subcategory of connected objects of $\left.\mathcal{B}^{\text {temp }}(\Pi)\right]$; in particular, for any Galoiscountable [cf. Remark 2.5.3, (i), (T1)] connected temperoid $\mathcal{C}$, it makes sense to write

$$
\pi_{1}(\mathcal{C}), \quad \pi_{1}\left(\mathcal{C}^{0}\right)
$$

for the topological groups, up to inner automorphism, obtained by applying this reconstruction algorithm [cf. Remark 2.5.3, (i), (T5)]. 
In this context, if $\mathcal{C}_{1}, \mathcal{C}_{2}$ are connected temperoids, then it is natural to define a morphism

$$
\mathcal{C}_{1} \rightarrow \mathcal{C}_{2}
$$

to be an isomorphism class of functors $\mathcal{C}_{2} \rightarrow \mathcal{C}_{1}$ that preserves finite limits and countable colimits. [Note that this differs — but only slightly! — from the definition given in [SemiAnbd], Definition 3.1, (iii). This difference does not, however, have any effect on the applicability of results of [SemiAnbd] in the context of the present series of papers.] In a similar vein, we define a morphism

$$
\mathcal{C}_{1}^{0} \rightarrow \mathcal{C}_{2}^{0}
$$

to be a morphism $\left(\mathcal{C}_{1}^{0}\right)^{\top} \rightarrow\left(\mathcal{C}_{2}^{0}\right)^{\top}$ [where we recall that we have natural equivalences of categories $\mathcal{C}_{i} \stackrel{\sim}{\rightarrow}\left(\mathcal{C}_{i}^{0}\right)^{\top}$ for $\left.i=1,2\right]$. One verifies immediately that an "isomorphism" relative to this terminology is equivalent to an "isomorphism of categories" in the sense defined at the beginning of the present discussion of "Monoids and Categories". Finally, if $\Pi_{1}, \Pi_{2}$ are Galois-countable [cf. Remark 2.5.3, (i), (T1)] tempered topological groups, then we recall that there is a natural bijective correspondence between

(a) the set of continuous outer homomorphisms $\Pi_{1} \rightarrow \Pi_{2}$,

(b) the set of morphisms $\mathcal{B}^{\text {temp }}\left(\Pi_{1}\right) \rightarrow \mathcal{B}^{\text {temp }}\left(\Pi_{2}\right)$, and

(c) the set of morphisms $\mathcal{B}^{\text {temp }}\left(\Pi_{1}\right)^{0} \rightarrow \mathcal{B}^{\text {temp }}\left(\Pi_{2}\right)^{0}$

— cf. Remark 2.5.3, (ii), (E7); [SemiAnbd], Proposition 3.2.

Suppose that for $i=1,2, \mathcal{C}_{i}$ and $\mathcal{C}_{i}^{\prime}$ are categories. Then we shall say that two isomorphism classes of functors $\phi: \mathcal{C}_{1} \rightarrow \mathcal{C}_{2}, \phi^{\prime}: \mathcal{C}_{1}^{\prime} \rightarrow \mathcal{C}_{2}^{\prime}$ are abstractly equivalent if, for $i=1,2$, there exist isomorphisms $\alpha_{i}: \mathcal{C}_{i} \stackrel{\sim}{\rightarrow} \mathcal{C}_{i}^{\prime}$ such that $\phi^{\prime} \circ \alpha_{1}=\alpha_{2} \circ \phi$. We shall also apply this terminology to morphisms between [connected] temperoids, as well as to morphisms between subcategories of connected objects of [connected] temperoids.

\section{Numbers:}

We shall use the abbreviations NF ("number field"), MLF ("mixed-characteristic [nonarchimedean] local field"), CAF ("complex archimedean field"), as defined in $[\mathrm{AbsTopI}], \S 0 ;$ [AbsTopIII], §0. We shall denote the set of prime numbers by Primes.

Let $F$ be a number field [i.e., a finite extension of the field of rational numbers]. Then we shall write

$$
\mathbb{V}(F)=\mathbb{V}(F)^{\operatorname{arc}} \bigcup \mathbb{V}(F)^{\text {non }}
$$

for the set of valuations of $F$, that is to say, the union of the sets of archimedean [i.e., $\mathbb{V}(F)^{\text {arc }}$ ] and nonarchimedean [i.e., $\mathbb{V}(F)^{\text {non }}$ ] valuations of $F$. Here, we note that this terminology "valuation", as it is applied in the present series of papers, corresponds to such terminology as "place" or "absolute value" in the work of other authors. Let $v \in \mathbb{V}(F)$. Then we shall write $F_{v}$ for the completion of $F$ at $v$ and say that an element of $F$ or $F_{v}$ is integral [at $v$ ] if it is of norm $\leq 1$ with respect to the valuation $v$; if, moreover, $L$ is any [possibly infinite] Galois extension of $F$, 
then, by a slight abuse of notation, we shall write $L_{v}$ for the completion of $L$ at some valuation $\in \mathbb{V}(L)$ that lies over $v$. If $v \in \mathbb{V}(F)^{\text {non }}$, then we shall write $p_{v}$ for the residue characteristic of $v$. If $v \in \mathbb{V}(F)^{\text {arc }}$, then we shall write $p_{v} \in F_{v}$ for the unique positive real element of $F_{v}$ whose natural logarithm is equal to 1 [i.e., "e $=2.71828 \ldots$.."]. By passing to appropriate projective or inductive limits, we shall also apply the notation "V $(F)$ ", " $F_{v}$ ", " $p_{v}$ " in situations where " $F$ " is an infinite extension of $\mathbb{Q}$.

\section{Curves:}

We shall use the terms hyperbolic curve, cusp, stable log curve, and smooth $\log$ curve as they are defined in [SemiAnbd], §0. We shall use the term hyperbolic orbicurve as it is defined in [Cusp], §0. 


\section{Section 1: Complements on Coverings of Punctured Elliptic Curves}

In the present $\S 1$, we discuss certain routine complements - which will be of use in the present series of papers - to the theory of coverings of once-punctured elliptic curves, as developed in [EtTh], $§ 2$.

Let $l \geq 5$ be an integer prime to $6 ; X$ a hyperbolic curve of type $(1,1)$ over a field $k$ of characteric zero; $\underline{C}$ a hyperbolic orbicurve of type $(1, l \text {-tors })_{ \pm}[\mathrm{cf}$. [EtTh], Definition 2.1] over $k$, whose $k$-core $C$ [cf. [CanLift], Remark 2.1.1; [EtTh], the discussion at the beginning of $\S 2]$ also forms a $k$-core of $X$. Thus, $\underline{C}$ determines, up to $k$-isomorphism, a hyperbolic orbicurve $\underline{X} \stackrel{\text { def }}{=} \underline{C} \times_{C} X$ of type (1,l-tors) [cf. [EtTh], Definition 2.1] over $k$. Moreover, if we write $G_{k}$ for the absolute Galois group of $k$ [relative to an appropriate choice of basepoint], $\Pi_{(-)}$for the arithmetic fundamental group of a geometrically connected, geometrically normal, generically scheme-like $k$-algebraic stack of finite type " $(-)$ " [i.e., the étale fundamental group $\pi_{1}((-))$ ], and $\Delta_{(-)}$for the geometric fundamental group of "(-)" [i.e., the kernel of the natural surjection $\Pi_{(-)} \rightarrow G_{k}$, then we obtain natural cartesian diagrams

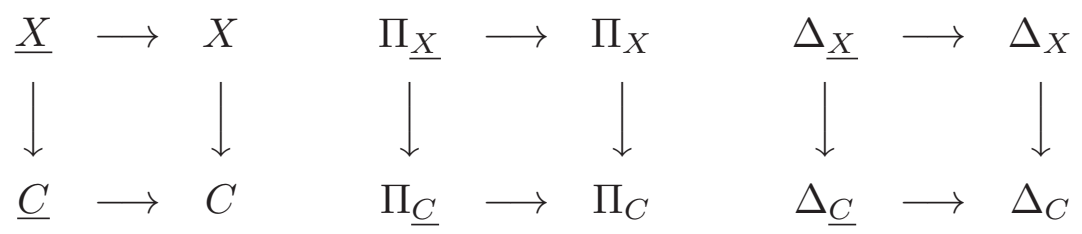

of finite étale coverings of hyperbolic orbicurves and open immersions of profinite groups. Finally, let us make the following assumption:

(*) The natural action of $G_{k}$ on $\Delta_{X}^{\mathrm{ab}} \otimes(\mathbb{Z} / l \mathbb{Z})$ [where the superscript "ab" denotes the abelianization] is trivial.

Next, let $\underline{\epsilon}$ be a nonzero cusp of $\underline{C}$ - i.e., a cusp that arises from a nonzero element of the quotient " $Q$ " that appears in the definition of a "hyperbolic orbicurve of type $(1, l \text {-tors })_{ \pm}$" given in [EtTh], Definition 2.1. Write $\underline{\epsilon}^{0}$ for the unique "zero cusp" [i.e., "non-nonzero cusp"] of $\underline{X} ; \underline{\epsilon}^{\prime}, \underline{\epsilon}^{\prime \prime}$ for the two cusps of $\underline{X}$ that lie over $\underline{\epsilon}$; and

$$
\Delta_{\underline{X}} \rightarrow \Delta_{\underline{X}}^{\mathrm{ab}} \otimes(\mathbb{Z} / l \mathbb{Z}) \rightarrow \Delta_{\underline{\epsilon}}
$$

for the quotient of $\Delta_{X}^{\mathrm{ab}} \otimes(\mathbb{Z} / l \mathbb{Z})$ by the images of the inertia groups of all nonzero cusps $\neq \underline{\epsilon}^{\prime}, \underline{\epsilon}^{\prime \prime}$ of $\underline{X}$. Thus, we obtain a natural exact sequence

$$
0 \quad \longrightarrow \quad I_{\underline{\epsilon}^{\prime}} \times I_{\underline{\epsilon}^{\prime \prime}} \longrightarrow \Delta_{\underline{\epsilon}} \longrightarrow \Delta_{\underline{E}} \otimes(\mathbb{Z} / l \mathbb{Z}) \longrightarrow 0
$$

- where we write $\underline{E}$ for the genus one compactification of $\underline{X}$, and $I_{\underline{\epsilon}^{\prime}}, I_{\underline{\epsilon}^{\prime \prime}}$ for the respective images in $\Delta_{\underline{\epsilon}}$ of the inertia groups of the cusps $\underline{\epsilon}^{\prime}, \underline{\epsilon}^{\prime \prime}$ [so we have noncanonical isomorphisms $\left.I_{\underline{\epsilon}^{\prime}} \cong \mathbb{Z} / l \mathbb{Z} \cong I_{\underline{\epsilon}^{\prime \prime}}\right]$.

Next, let us observe that $G_{k}, \operatorname{Gal}(\underline{X} / \underline{C})(\cong \mathbb{Z} / 2 \mathbb{Z})$ act naturally on the above exact sequence. Write $\iota \in \operatorname{Gal}(\underline{X} / \underline{C})$ for the unique nontrivial element. Then $\iota$ induces an isomorphism $I_{\underline{\epsilon}^{\prime}} \cong I_{\underline{\epsilon}^{\prime \prime}}$; if we use this isomorphism to identify $I_{\underline{\epsilon}^{\prime}}, I_{\underline{\epsilon}^{\prime \prime}}$, then one verifies immediately that $\iota$ acts on the term " $I_{\underline{\epsilon}^{\prime}} \times I_{\underline{\epsilon}^{\prime}}$ " of the above exact sequence by switching the two factors. Moreover, one verifies immediately that $\iota$ 
acts on $\Delta_{\underline{E}} \otimes(\mathbb{Z} / l \mathbb{Z})$ via multiplication by -1 . In particular, since $l$ is $o d d$, it follows that the action by $\iota$ on $\Delta_{\underline{\epsilon}}$ determines a decomposition into eigenspaces

$$
\Delta_{\underline{\epsilon}} \stackrel{\sim}{\rightarrow} \Delta_{\underline{\epsilon}}^{+} \times \Delta_{\underline{\epsilon}}^{-}
$$

— i.e., where $\iota$ acts on $\Delta_{\underline{\epsilon}}^{+}$(respectively, $\Delta_{\underline{\epsilon}}^{-}$) by multiplication by +1 (respectively, $-1)$. Moreover, the natural composite maps

$$
I_{\underline{\epsilon}^{\prime}} \hookrightarrow \Delta_{\underline{\epsilon}} \rightarrow \Delta_{\underline{\epsilon}}^{+} ; \quad I_{\underline{\epsilon}^{\prime \prime}} \hookrightarrow \Delta_{\underline{\epsilon}} \rightarrow \Delta_{\underline{\epsilon}}^{+}
$$

determine isomorphisms $I_{\underline{\epsilon}^{\prime}} \stackrel{\sim}{\rightarrow} \Delta_{\underline{\epsilon}}^{+}, I_{\underline{\epsilon}^{\prime \prime}} \stackrel{\sim}{\rightarrow} \Delta_{\underline{\epsilon}}^{+}$. Since the natural action of $G_{k}$ on $\Delta_{\epsilon}$ clearly commutes with the action of $\iota$, we thus conclude that the quotient $\Delta_{\underline{X}} \rightarrow \Delta_{\underline{\epsilon}} \rightarrow \Delta_{\underline{\epsilon}}^{+}$determines quotients

$$
\Pi_{\underline{X}} \rightarrow J_{\underline{X}} ; \quad \Pi_{\underline{C}} \rightarrow J_{\underline{C}}
$$

- where the surjections $\Pi_{\underline{X}} \rightarrow G_{k}, \Pi_{\underline{C}} \rightarrow G_{k}$ induce natural exact sequences $1 \rightarrow \Delta_{\underline{\epsilon}}^{+} \rightarrow J_{\underline{X}} \rightarrow G_{k} \rightarrow 1,1 \rightarrow \Delta_{\underline{\epsilon}}^{+} \times \overline{G a l}(\underline{X} / \underline{C}) \rightarrow J_{\underline{C}} \rightarrow G_{k} \rightarrow 1$; we have a natural inclusion $J_{\underline{X}} \hookrightarrow J_{\underline{C}}$.

Next, let us consider the cusp " $2 \underline{\epsilon}$ " of $\underline{C}$ - i.e., the cusp whose inverse images in $\underline{X}$ correspond to the points of $\underline{E}$ obtained by multiplying $\underline{\epsilon}^{\prime}, \underline{\epsilon}^{\prime \prime}$ by 2 , relative to the group law of the elliptic curve determined by the pair $\left(\underline{X}, \underline{\epsilon}^{0}\right)$. Since $2 \neq \pm 1(\bmod l)$ [a consequence of our assumption that $l \geq 5$ ], it follows that the decomposition group associated to this cusp " $2 \underline{\epsilon}$ " determines a section

$$
\sigma: G_{k} \rightarrow J_{\underline{C}}
$$

of the natural surjection $J_{\underline{C}} \rightarrow G_{k}$. Here, we note that although, a priori, $\sigma$ is only determined by $2 \underline{\epsilon}$ up to composition with an inner automorphism of $J_{\underline{C}}$ determined by an element of $\Delta_{\underline{\epsilon}}^{+} \times \operatorname{Gal}(\underline{X} / \underline{C})$, in fact, since [in light of the assumption (*)!] the natural [outer] action of $G_{k}$ on $\Delta_{\underline{\epsilon}}^{+} \times \operatorname{Gal}(\underline{X} / \underline{C})$ is trivial, we conclude that $\sigma$ is completely determined by $2 \underline{\epsilon}$, and that the subgroup $\operatorname{Im}(\sigma) \subseteq J_{\underline{C}}$ determined by the image of $\sigma$ is normal in $J_{C}$. Moreover, by considering the decomposition groups associated to the cusps of $\underline{X}$ lying over $2 \underline{\epsilon}$, we conclude that $\operatorname{Im}(\sigma)$ lies inside the subgroup $J_{\underline{X}} \subseteq J_{\underline{C}}$. Thus, the subgroups $\operatorname{Im}(\sigma) \subseteq J_{\underline{X}}, \operatorname{Im}(\sigma) \times \operatorname{Gal}(\underline{X} / \underline{C}) \subseteq J_{\underline{C}}$ determine [the horizontal arrows in] cartesian diagrams

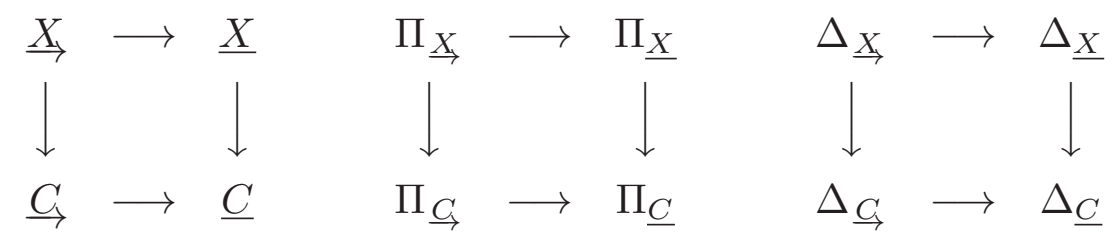

of finite étale cyclic coverings of hyperbolic orbicurves and open immersions [with normal image] of profinite groups; we have $\operatorname{Gal}(\underline{C} / \underline{C}) \cong \mathbb{Z} / l \mathbb{Z}, \operatorname{Gal}(\underline{X} / \underline{C}) \cong \mathbb{Z} / 2 \mathbb{Z}$, and $\operatorname{Gal}(\underline{X} / \underline{C}) \stackrel{\sim}{\rightarrow} \operatorname{Gal}(\underline{X} / \underline{C}) \times \operatorname{Gal}(\underline{C} / \underline{C}) \cong \mathbb{Z} / 2 l \mathbb{Z}$.

Definition 1.1. We shall refer to a hyperbolic orbicurve over $k$ that arises, up to isomorphism, as the hyperbolic orbicurve $\underline{X}$ (respectively, $\underline{C}_{\rightarrow}$ ) constructed above for some choice of $l, \underline{\epsilon}$ as being of type (1, $l$-tors) (respectively, $\left.(1, l \text {-tors })_{ \pm}\right)$. 
Remark 1.1.1. The arrow " $\rightarrow$ " in the notation " $\underline{X}$ ", " $C$ ", "( $1, l$-tors $)$ ", " $(1, l \text {-tors })_{ \pm}$" may be thought of as denoting the "archimedean, ordered labels $1,2, \ldots "$ - i.e., determined by the choice of $\underline{\epsilon} !$ - on the $\{ \pm 1\}$-orbits of elements of the quotient " $Q$ " that appears in the definition of a "hyperbolic orbicurve of type $(1, l \text {-tors })_{ \pm} "$ given in [EtTh], Definition 2.1 .

Remark 1.1.2. We observe that $\underline{X}, \underline{C}$ are completely determined, up to $k$ isomorphism, by the data $(X / k, \underline{C}, \underline{\epsilon})$.

Corollary 1.2. (Characteristic Nature of Coverings) Suppose that $k$ is an NF or an $M L F$. Then there exists a functorial group-theoretic algorithm [cf. [AbsTopIII], Remark 1.9.8, for more on the meaning of this terminology] to reconstruct

$$
\left.\Pi_{\underline{X}}, \Pi_{\underline{G}}, \Pi_{\underline{C}} \text { (respectively, } \Pi_{\underline{C}}\right)
$$

together with the conjugacy classes of the decomposition group $(s)$ determined by the set(s) of cusps $\left\{\underline{\epsilon}^{\prime}, \underline{\epsilon}^{\prime \prime}\right\} ;\{\underline{\epsilon}\}$ (respectively, $\left.\{\underline{\epsilon}\}\right)$ from $\Pi_{\underline{X}}$ (respectively, $\Pi_{\underline{G}}$ ). Here, the asserted functoriality is with respect to isomorphisms of topological groups; we reconstruct $\Pi_{\underline{X}}, \Pi_{\underline{G}}, \Pi_{\underline{C}}$ (respectively, $\Pi_{\underline{C}}$ ) as a subgroup of $\operatorname{Aut}\left(\Pi_{\underline{X}}\right)$ (respectively, $\left.\operatorname{Aut}\left(\Pi_{\underline{C}_{\rightarrow}}\right)\right)$.

Proof. For simplicity, we consider the non-resp'd case; the resp'd case is entirely similar [but slightly easier]. The argument is similar to the arguments applied in [EtTh], Proposition 1.8; [EtTh], Proposition 2.4. First, we recall that $\Pi_{\underline{X}}, \Pi_{\underline{X}}$, and $\Pi_{\underline{C}}$ are slim [cf., e.g., [AbsTopI], Proposition 2.3, (ii)], hence embed naturally into $\operatorname{Aut}\left(\Pi_{\underline{X}}\right)$, and that one may recover the subgroup $\Delta_{\underline{X}} \subseteq \Pi_{\underline{X}}$ via the algorithms of [AbsTopI], Theorem 2.6, (v), (vi). Next, we recall that the algorithms of [AbsTopII], Corollary 3.3, (i), (ii) — which are applicable in light of [AbsTopI], Example 4.8 - allow one to reconstruct $\Pi_{C}$ [together with the natural inclusion $\Pi_{\underline{X}} \hookrightarrow \Pi_{C}$ ], as well as the subgroups $\Delta_{X} \subseteq \Delta_{C} \subseteq \Pi_{C}$. In particular, $l$ may be recovered via the formula $l^{2}=\left[\Delta_{X}: \Delta_{\underline{X}}\right] \cdot\left[\Delta_{\underline{X}}: \Delta_{\underline{X}}\right]=\left[\Delta_{X}: \Delta_{\underline{X}}\right]=\left[\Delta_{C}: \Delta_{\underline{X}}\right] / 2$. Next, let us set $H \stackrel{\text { def }}{=} \operatorname{Ker}\left(\Delta_{X} \rightarrow \Delta_{X}^{\text {ab }} \otimes(\mathbb{Z} / l \mathbb{Z})\right)$. Then $\Pi_{\underline{X}} \subseteq \Pi_{C}$ may be recovered via the [easily verified] equality of subgroups $\Pi_{\underline{X}}=\Pi_{\underline{X}} \cdot H$. The conjugacy classes of the decomposition groups of $\underline{\epsilon}^{0}, \underline{\epsilon}^{\prime}, \underline{\epsilon}^{\prime \prime}$ in $\Pi_{\underline{X}}$ may be recovered as the decomposition groups of cusps [cf. [AbsTopI], Lemma 4.5, as well as Remark 1.2.2, (ii), below] whose image in $\operatorname{Gal}(\underline{X} / \underline{X})=\Pi_{\underline{X}} / \Pi_{\underline{X}}$ is nontrivial. Next, to reconstruct $\Pi_{\underline{C}} \subseteq \Pi_{C}$, it suffices to reconstruct the splitting of the surjection $\operatorname{Gal}(\underline{X} / C)=\Pi_{C} / \Pi_{\underline{X}} \rightarrow$ $\Pi_{C} / \Pi_{X}=\operatorname{Gal}(X / C)$ determined by $\operatorname{Gal}(\underline{X} / \underline{C})=\Pi_{\underline{C}} / \Pi_{X}$; but [since $l$ is prime to 3 !] this splitting may be characterized [group-theoretically!] as the unique splitting that stabilizes the collection of conjugacy classes of subgroups of $\Pi_{\underline{X}}$ determined by the decomposition groups of $\underline{\epsilon}^{0}, \underline{\epsilon}^{\prime}, \underline{\epsilon}^{\prime \prime}$. Now $\Pi_{\underline{G}} \subseteq \Pi_{\underline{C}}$ may be reconstructed by applying the observation that $(\mathbb{Z} / 2 \mathbb{Z} \cong) \operatorname{Gal}(\underline{X} / \underline{C}) \subseteq \operatorname{Gal}(\underline{X} / \underline{C})(\cong \mathbb{Z} / 2 l \mathbb{Z})$ is the unique maximal subgroup of odd index. Finally, the conjugacy classes of the decomposition groups of $\underline{\epsilon}^{\prime}, \underline{\epsilon}^{\prime \prime}$ in $\Pi_{\underline{X}}$ may be recovered as the decomposition groups of cusps [cf. [AbsTopI], Lemma 4.5, as well as Remark 1.2.2, (ii), below] whose image in $\operatorname{Gal}(\underline{X} / \underline{X})=\Pi_{\underline{X}} / \Pi_{\underline{X}}$ is nontrivial, but which are not fixed [up to 
conjugacy] by the outer action of $\operatorname{Gal}(\underline{X} / \underline{C})=\Pi_{\underline{C}} / \Pi_{\underline{X}}$ on $\Pi_{\underline{X}}$. This completes the proof of Corollary 1.2.

Remark 1.2.1. It follows immediately from Corollary 1.2 that

$$
\operatorname{Aut}_{k}(\underline{X})=\operatorname{Gal}(\underline{X} / \underline{C})(\cong \mathbb{Z} / 2 l \mathbb{Z}) ; \quad \operatorname{Aut}_{k}(\underline{C})=\operatorname{Gal}(\underline{C} / \underline{C})(\cong \mathbb{Z} / l \mathbb{Z})
$$

[cf. [EtTh], Remark 2.6.1].

Remark 1.2.2. The group-theoretic algorithm for reconstructing the decomposition groups of cusps given [AbsTopI], Lemma 4.5 - which is based on the argument given in the proof of [AbsAnab], Lemma 1.3.9 - contains some minor, inessential inaccuracies. In light of the importance of this group-theoretic algorithm for the theory of the present series of papers, we thus pause to discuss how these inaccuracies may be amended.

(i) The final portion [beginning with the third sentence] of the second paragraph of the proof of [AbsAnab], Lemma 1.3.9, should be replaced by the following text:

Since $r_{i}$ may be recovered group-theoretically, given any finite étale coverings

$$
Z_{i} \rightarrow V_{i} \rightarrow X_{i}
$$

such that $Z_{i}$ is cyclic [hence Galois], of degree a power of $l$, over $V_{i}$, one may determine group-theoretically whether or not $Z_{i} \rightarrow V_{i}$ is totally ramified [i.e., at some point of $Z_{i}$ ], since this condition is easily verified to be equivalent to the condition that the covering $Z_{i} \rightarrow V_{i}$ admit a factorization $Z_{i} \rightarrow W_{i} \rightarrow V_{i}$, where $W_{i} \rightarrow V_{i}$ is finite étale of degree $l$, and $r_{W_{i}}<l \cdot r_{V_{i}}$. Moreover, this group-theoreticity of the condition that a cyclic covering be totally ramified extends immediately to the case of pro-l cyclic coverings $Z_{i} \rightarrow V_{i}$. Thus, by Lemma 1.3.7, we conclude that the inertia groups of cusps in $\left(\Delta_{X_{i}}\right)^{(l)}$ [i.e., the maximal pro-l quotient of $\Delta_{X_{i}}$ ] may be characterized [group-theoretically!] as the maximal subgroups of $\left(\Delta_{X_{i}}\right)^{(l)}$ that correspond to [profinite] coverings satisfying this condition.

(ii) The final portion [beginning with the third sentence] of the statement of [AbsTopI], Lemma 4.5, (iv), should be replaced by the following text:

Then the decomposition groups of cusps $\subseteq H^{*}$ may be characterized ["group-theoretically"] as the maximal closed subgroups $I \subseteq H^{*}$ isomorphic to $\mathbb{Z}_{l}$ which satisfy the following condition: We have

$$
d_{\chi_{G}^{\text {cyclo }}}\left(\left(I^{l} \cdot J\right)^{\mathrm{ab}} \otimes \mathbb{Q}_{l}\right)+1<l \cdot\left\{d_{\chi_{G}^{\text {cyclo }}}\left((I \cdot J)^{\mathrm{ab}} \otimes \mathbb{Q}_{l}\right)+1\right\}
$$

[i.e., "the covering of curves corresponding to $J \subseteq I \cdot J$ is totally ramified at some cusp"] for every characteristic open subgroup $J \subseteq H^{*}$ such that $J \neq I \cdot J$.

Remark 1.2.3. The minor, inessential inaccuracies in the group-theoretic algorithms of [AbsAnab], Lemma 1.3.9; [AbsTopI], Lemma 4.5, that were discussed 
in Remark 1.2.2 are closely related to certain minor, inessential inaccuracies in the theory of [CombGC]. Thus, it is of interest, in the context of the discussion of Remark 1.2.2, to pause to discuss how these inaccuracies may be amended. These inaccuracies arise in the arguments applied in [CombGC], Definition 1.4, (v), (vi), and [CombGC], Remarks 1.4.2, 1.4.3, and 1.4.4, to prove [CombGC], Theorem 1.6. These arguments are formulated in a somewhat confusing way and should be modified as follows:

(i) First of all, we remark that in [CombGC], as well as in the following discussion, a "Galois" finite étale covering is to be understood as being connected.

(ii) In the second sentence of [CombGC], Definition 1.4, (v), the cuspidal and nodal cases of the notion of a purely totally ramified covering are in fact unnecessary and may be deleted. Also, the terminology introduced in [CombGC], Definition 1.4, (vi), concerning finite étale coverings that descend is unnecessary and may be deleted. text:

(iii) The text of [CombGC], Remark 1.4.2, should be replaced by the following

Let $\mathcal{G}^{\prime} \rightarrow \mathcal{G}$ be a Galois finite étale covering of degree a positive power of $l$, where $\mathcal{G}$ is of pro- $\Sigma$ PSC-type, $\Sigma=\{l\}$. Then one verifies immediately that, if we assume further that the covering $\mathcal{G}^{\prime} \rightarrow \mathcal{G}$ is cyclic, then $\mathcal{G}^{\prime} \rightarrow \mathcal{G}$ is cuspidally totally ramified if and only if the inequality

$$
\underline{r}\left(\mathcal{G}^{\prime \prime}\right)<l \cdot \underline{r}(\mathcal{G})
$$

- where we write $\mathcal{G}^{\prime} \rightarrow \mathcal{G}^{\prime \prime} \rightarrow \mathcal{G}$ for the unique [up to isomorphism] factorization of the finite étale covering $\mathcal{G}^{\prime} \rightarrow \mathcal{G}$ as a composite of finite étale coverings such that $\mathcal{G}^{\prime \prime} \rightarrow \mathcal{G}$ is of degree $l-$ is satisfied. Suppose further that $\mathcal{G}^{\prime} \rightarrow \mathcal{G}$ is a [not necessarily cyclic! $] \Pi_{\mathcal{G}}^{\text {unr }}$-covering $\left[\operatorname{so} \underline{n}\left(\mathcal{G}^{\prime}\right)=\right.$ $\left.\operatorname{deg}\left(\mathcal{G}^{\prime} / \mathcal{G}\right) \cdot \underline{n}(\mathcal{G})\right]$. Then one verifies immediately that $\mathcal{G}^{\prime} \rightarrow \mathcal{G}$ is verticially purely totally ramified if and only if the equality

$$
\underline{i}\left(\mathcal{G}^{\prime}\right)=\operatorname{deg}\left(\mathcal{G}^{\prime} / \mathcal{G}\right) \cdot(\underline{i}(\mathcal{G})-1)+1
$$

is satisfied. Also, we observe that this last inequality is equivalent to the following equality involving the expression " $\underline{i}(\ldots)-\underline{n}(\ldots)$ " [cf. Remark 1.1.3]:

$$
\underline{i}\left(\mathcal{G}^{\prime}\right)-\underline{n}\left(\mathcal{G}^{\prime}\right)=\operatorname{deg}\left(\mathcal{G}^{\prime} / \mathcal{G}\right) \cdot(\underline{i}(\mathcal{G})-\underline{n}(\mathcal{G})-1)+1
$$

(iv) The text of [CombGC], Remark 1.4.3, should be replaced by the following text:

Suppose that $\mathcal{G}$ is of pro- $\Sigma$ PSC-type, $\Sigma=\{l\}$. Then one verifies immediately that the cuspidal edge-like subgroups of $\Pi_{\mathcal{G}}$ may be characterized as the maximal [cf. Proposition 1.2, (i)] closed subgroups $A \subseteq \Pi_{\mathcal{G}}$ isomorphic to $\mathbb{Z}_{l}$ which satisfy the following condition:

for every characteristic open subgroup $\Pi_{\mathcal{G}^{\prime}} \subseteq \Pi_{\mathcal{G}}$, if we write $\mathcal{G}^{\prime} \rightarrow \mathcal{G}^{\prime \prime} \rightarrow \mathcal{G}$ for the finite étale coverings corresponding to 
$\Pi_{\mathcal{G}^{\prime}} \subseteq \Pi_{\mathcal{G}^{\prime \prime}} \stackrel{\text { def }}{=} A \cdot \Pi_{\mathcal{G}^{\prime}} \subseteq \Pi_{\mathcal{G}}$, then the cyclic finite étale covering $\mathcal{G}^{\prime} \rightarrow \mathcal{G}^{\prime \prime}$ is cuspidally totally ramified.

[Indeed, the necessity of this characterization is immediate from the definitions; the sufficiency of this characterization follows by observing that since the set of cusps of a finite étale covering of $\mathcal{G}$ is always finite, the above condition implies that there exists a compatible system of cusps of the various $\mathcal{G}^{\prime}$ that arise, each of which is stabilized by the action of $A$.] On the other hand, in order to characterize the unramified verticial subgroups of $\Pi_{\mathcal{G}}^{\mathrm{unr}}$, it suffices - by considering stabilizers of vertices of underlying semi-graphs of finite étale $\Pi_{\mathcal{G}}^{u n}$-coverings of $\mathcal{G}$ - to give a functorial characterization of the set of vertices of $\mathcal{G}$ [i.e., which may also be applied to finite étale $\Pi_{\mathcal{G}}^{\text {unr }}$-coverings of $\mathcal{G}$ ]. This may be done, for sturdy $\mathcal{G}$, as follows. Write $M_{\mathcal{G}}^{\mathrm{unr}}$ for the abelianization of $\Pi_{\mathcal{G}}^{\mathrm{unr}}$. For each vertex $v$ of the underlying semi-graph $\mathbb{G}$ of $\mathcal{G}$, write $M_{\mathcal{G}}^{\text {unr }}[v] \subseteq M_{\mathcal{G}}^{\text {unr }}$ for the image of the $\Pi_{\mathcal{G}}^{u n r}$-conjugacy class of unramified verticial subgroups of $\Pi_{\mathcal{G}}^{\text {unr }}$ associated to $v$. Then one verifies immediately, by constructing suitable abelian $\Pi_{\mathcal{G}}^{u n r}$-coverings of $\mathcal{G}$ via suitable gluing operations [i.e., as in the proof of Proposition 1.2], that the inclusions $M_{\mathcal{G}}^{\text {unr }}[v] \subseteq M_{\mathcal{G}}^{\text {unr }}$ determine a split injection

$$
\bigoplus_{v} M_{\mathcal{G}}^{\mathrm{unr}}[v] \hookrightarrow M_{\mathcal{G}}^{\mathrm{unr}}
$$

[where $v$ ranges over the vertices of $\mathbb{G}$ ], whose image we denote by $M_{\mathcal{G}}^{\text {unr-vert }} \subseteq$ $M_{\mathcal{G}}^{u n r}$. Now we consider elementary abelian quotients

$$
\phi: M_{\mathcal{G}}^{\mathrm{unr}} \rightarrow Q
$$

- i.e., where $Q$ is an elementary abelian group. We identify such quotients whenever their kernels coincide and order such quotients by means of the relation of "domination" [i.e., inclusion of kernels]. Then one verifies immediately that such a quotient $\phi: M_{\mathcal{G}}^{\text {unr }} \rightarrow Q$ corresponds to a verticially purely totally ramified covering of $\mathcal{G}$ if and only if there exists a vertex $v$ of $\mathbb{G}$ such that $\phi\left(M_{\mathcal{G}}^{\text {unr }}[v]\right)=Q, \phi\left(M_{\mathcal{G}}^{\text {unr }}\left[v^{\prime}\right]\right)=0$ for all vertices $v^{\prime} \neq v$ of $\mathbb{G}$. In particular, one concludes immediately that

the elementary abelian quotients $\phi: M_{\mathcal{G}}^{\text {unr }} \rightarrow Q$ whose restriction to $M_{\mathcal{G}}^{\text {unr-vert }}$ surjects onto $Q$ and has the same kernel as the quotient

$$
M_{\mathcal{G}}^{\text {unr-vert }} \rightarrow M_{\mathcal{G}}^{\text {unr }}[v] \rightarrow M_{\mathcal{G}}^{\text {unr }}[v] \otimes \mathbb{F}_{l}
$$

— where the first " $\rightarrow$ " is the natural projection; the second " $\rightarrow$ " is given by reduction modulo $l$ - may be characterized as the maximal quotients [i.e., relative to the relation of domination] among those elementary abelian quotients of $M_{\mathcal{G}}^{\text {unr }}$ that correspond to verticially purely totally ramified coverings of $\mathcal{G}$.

Thus, since $\mathcal{G}$ is sturdy, the set of vertices of $\mathcal{G}$ may be characterized as the set of [nontrivial!] quotients $M_{\mathcal{G}}^{\text {unr-vert }} \rightarrow M_{\mathcal{G}}^{\text {unr }}[v] \otimes \mathbb{F}_{l}$. 
(v) The text of [CombGC], Remark 1.4.4, should be replaced by the following text:

Suppose that $\mathcal{G}$ is of pro- $\Sigma$ PSC-type, where $\Sigma=\{l\}$, and that $\mathcal{G}$ is noncuspidal. Then, in the spirit of the cuspidal portion of Remark 1.4.3, we observe the following: One verifies immediately that the nodal edge-like subgroups of $\Pi_{\mathcal{G}}$ may be characterized as the maximal [cf. Proposition 1.2, (i)] closed subgroups $A \subseteq \Pi_{\mathcal{G}}$ isomorphic to $\mathbb{Z}_{l}$ which satisfy the following condition:

for every characteristic open subgroup $\Pi_{\mathcal{G}^{\prime}} \subseteq \Pi_{\mathcal{G}}$, if we write $\mathcal{G}^{\prime} \rightarrow \mathcal{G}^{\prime \prime} \rightarrow \mathcal{G}$ for the finite étale coverings corresponding to $\Pi_{\mathcal{G}^{\prime}} \subseteq \Pi_{\mathcal{G}^{\prime \prime}} \stackrel{\text { def }}{=} A \cdot \Pi_{\mathcal{G}^{\prime}} \subseteq \Pi_{\mathcal{G}}$, then the cyclic finite étale covering $\mathcal{G}^{\prime} \rightarrow \mathcal{G}^{\prime \prime}$ is nodally totally ramified.

Here, we note further that [one verifies immediately that] the finite étale covering $\mathcal{G}^{\prime} \rightarrow \mathcal{G}^{\prime \prime}$ is nodally totally ramified if and only if it is module-wise nodal.

(vi) The text of the second paragraph of the proof of [CombGC], Theorem 1.6, should be replaced by the following text [which may be thought as being appended to the end of the first paragraph of the proof of [CombGC], Theorem 1.6]:

Then the fact that $\alpha$ is group-theoretically cuspidal follows formally from the characterization of cuspidal edge-like subgroups given in Remark 1.4.3 and the characterization of cuspidally totally ramified cyclic finite étale coverings given in Remark 1.4.2.

(vii) The text of the final paragraph of the proof of [CombGC], Theorem 1.6, should be replaced by the following text [which may be thought of as a sort of "easy version" of the argument given in the proof of the implication "(iii) $\Longrightarrow$ (i)" of [CbTpII], Proposition 1.5]:

Finally, we consider assertion (iii). Sufficiency is immediate. On the other hand, necessity follows formally from the characterization of unramified verticial subgroups given in Remark 1.4 .3 and the characterization of verticially purely totally ramified finite étale coverings given in Remark 1.4.2. 


\section{Section 2: Complements on Tempered Coverings}

In the present $\S 2$, we discuss certain routine complements - which will be of use in the present series of papers - to the theory of tempered coverings of graphs of anabelioids, as developed in [SemiAnbd], $§ 3$ [cf. also the closely related theory of [CombGC]].

Let $\Sigma, \widehat{\Sigma}$ be nonempty sets of prime numbers such that $\Sigma \subseteq \widehat{\Sigma}$;

a semi-graph of anabelioids of pro- $\Sigma$ PSC-type [cf. [CombGC], Definition 1.1, (i)], whose underlying semi-graph we denote by $\mathbb{G}$. Write $\Pi_{\mathcal{G}}^{\mathrm{tp}}$ for the tempered fundamental group of $\mathcal{G}$ [cf. the discussion preceding [SemiAnbd], Proposition 3.6, as well as Remark 2.5.3, (i), (T6), of the present paper] and $\widehat{\Pi}_{\mathcal{G}}$ for the pro- $\widehat{\Sigma}$ [i.e., maximal pro- $\widehat{\Sigma}$ quotient of the profinite] fundamental group of $\mathcal{G}$ [cf. the discussion preceding [SemiAnbd], Definition 2.2] — both taken with respect to appropriate choices of basepoints. Thus, since discrete free groups of finite rank inject into their pro- $l$ completions for any prime number $l$ [cf., e.g., [RZ], Proposition 3.3.15], it follows that we have a natural injection [cf. [SemiAnbd], Proposition 3.6, (iii), as well as Remark 2.5.3, (ii), (E7), of the present paper, when $\widehat{\Sigma}=\mathfrak{P r i m e s}$; the proof in the case of arbitrary $\widehat{\Sigma}$ is entirely similar]

$$
\Pi_{\mathcal{G}}^{\mathrm{tp}} \hookrightarrow \widehat{\Pi}_{\mathcal{G}}
$$

that we shall use to regard $\Pi_{\mathcal{G}}^{\text {tp }}$ as a subgroup of $\widehat{\Pi}_{\mathcal{G}}$ and $\widehat{\Pi}_{\mathcal{G}}$ as the pro- $\widehat{\Sigma}$ completion of $\Pi_{\mathcal{G}}^{\text {tp }}$.

Next, let

\section{$\mathcal{H}$}

be the semi-graph of anabelioids associated to a connected sub-semi-graph $\mathbb{H} \subseteq$ $\mathbb{G}$. One verifies immediately that the restriction of $\mathcal{H}$ to the maximal subgraph [cf. the discussion at the beginning of [SemiAnbd], §1] of $\mathbb{H}$ coincides with the restriction to the maximal subgraph of the underlying semi-graph of some semigraph of anabelioids of pro- $\Sigma$ PSC-type. That is to say, roughly speaking, up to the possible omission of some of the cuspidal edges, $\mathcal{H}$ "is" a semi-graph of anabelioids of pro- $\Sigma$ PSC-type. In particular, since the omission of cuspidal edges clearly does not affect either the tempered or pro- $\widehat{\Sigma}$ fundamental groups, we shall apply the notation introduced above for " $\mathcal{G}$ " to $\mathcal{H}$. We thus obtain a natural commutative diagram

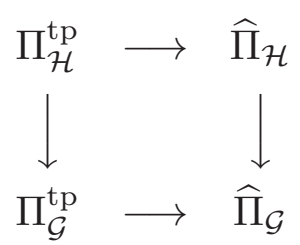

of [outer] inclusions [cf. [SemiAnbd], Proposition 2.5, (i), when $\widehat{\Sigma}=$ Primes; in light of the well-known structure of fundamental groups of hyperbolic Riemann surfaces of finite type, a similar proof may be given in the case of arbitrary $\widehat{\Sigma}$, i.e., 
by considering successive composites of finite étale Galois coverings that restrict to trivial coverings over the closed edges and finite étale abelian [Galois] coverings obtained by gluing together suitable abelian coverings] of topological groups, which we shall use to regard all of the groups in the diagram as subgroups of $\widehat{\Pi}_{\mathcal{G}}$. In particular, one may think of $\Pi_{\mathcal{H}}^{\text {tp }}$ (respectively, $\widehat{\Pi}_{\mathcal{H}}$ ) as the decomposition subgroup in $\Pi_{\mathcal{G}}^{\mathrm{tp}}$ (respectively, $\widehat{\Pi}_{\mathcal{G}}$ ) [which is well-defined up to $\Pi_{\mathcal{G}}^{\text {tp }}$ (respectively, $\widehat{\Pi}_{\mathcal{G}^{-}}$)conjugacy] associated to the sub-semi-graph $\mathcal{H}$.

The following result is the central technical result underlying the theory of the present $\S 2$.

Proposition 2.1. (Profinite Conjugates of Nontrivial Compact Subgroups) In the notation of the above discussion, let $\Lambda \subseteq \Pi_{\mathcal{G}}^{\text {tp }}$ be a nontrivial compact subgroup, $\gamma \in \widehat{\Pi}_{\mathcal{G}}$ an element such that $\gamma \cdot \Lambda \cdot \gamma^{-1} \subseteq \Pi_{\mathcal{G}}^{\mathrm{tp}}$ [or, equivalently, $\left.\Lambda \subseteq \gamma^{-1} \cdot \Pi_{\mathcal{G}}^{\mathrm{tp}} \cdot \gamma\right]$. Then $\gamma \in \Pi_{\mathcal{G}}^{\mathrm{tp}}$.

Proof. Write $\widehat{\Gamma}$ for the "pro- $\widehat{\Sigma}$ semi-graph" associated to the universal pro- $\widehat{\Sigma}$ étale covering of $\mathcal{G}$ [i.e., the covering corresponding to the subgroup $\{1\} \subseteq \widehat{\Pi}_{\mathcal{G}}$; $\Gamma^{\text {tp }}$ for the "pro-semi-graph" associated to the universal tempered covering of $\mathcal{G}$ [i.e., the covering corresponding to the subgroup $\left.\{1\} \subseteq \Pi_{\mathcal{G}}^{\text {tp }}\right]$. Thus, we have a natural dense map $\Gamma^{\mathrm{tp}} \rightarrow \widehat{\Gamma}$. Let us refer to a ["pro-"] vertex of $\widehat{\Gamma}$ that occurs as the image of a ["pro-"]vertex of $\Gamma^{\mathrm{tp}}$ as tempered. Since $\Lambda, \gamma \cdot \Lambda \cdot \gamma^{-1}$ are compact subgroups of $\Pi_{\mathcal{G}}^{\mathrm{tp}}$, it follows from [SemiAnbd], Theorem 3.7, (iii) [cf. also [SemiAnbd], Example 3.10, as well as Remark 2.5.3, (ii), (E7), of the present paper], that there exist verticial subgroups $\Lambda^{\prime}, \Lambda^{\prime \prime} \subseteq \Pi_{\mathcal{G}}^{\text {tp }}$ such that $\Lambda \subseteq \Lambda^{\prime}, \gamma \cdot \Lambda \cdot \gamma^{-1} \subseteq \Lambda^{\prime \prime}$. Thus, $\Lambda^{\prime}$, $\Lambda^{\prime \prime}$ correspond to tempered vertices $v^{\prime}$, $v^{\prime \prime}$ of $\widehat{\Gamma} ;\{1\} \neq \gamma \cdot \Lambda \cdot \gamma^{-1} \subseteq \gamma \cdot \Lambda^{\prime} \cdot \gamma^{-1}$, so $\left(\gamma \cdot \Lambda^{\prime} \cdot \gamma^{-1}\right) \cap \Lambda^{\prime \prime} \neq\{1\}$. Since $\Lambda^{\prime \prime}, \gamma \cdot \Lambda^{\prime} \cdot \gamma^{-1}$ are both verticial subgroups of $\widehat{\Pi}_{\mathcal{G}}$, it thus follows either from [AbsTopII], Proposition 1.3, (iv), or from [NodNon], Proposition 3.9, (i), that the corresponding vertices $v^{\prime \prime},\left(v^{\prime}\right)^{\gamma}$ of $\widehat{\Gamma}$ are either equal or adjacent. In particular, since $v^{\prime \prime}$ is tempered, we thus conclude that $\left(v^{\prime}\right)^{\gamma}$ is tempered. Thus, $v^{\prime},\left(v^{\prime}\right)^{\gamma}$ are tempered, so $\gamma \in \Pi_{\mathcal{G}}^{\text {tp }}$, as desired.

Next, relative to the notation " $C$ ", " $N$ " and related terminology concerning commensurators and normalizers discussed, for instance, in [SemiAnbd], §0; [CombGC], §0, we have the following result.

Proposition 2.2. (Commensurators of Decomposition Subgroups Associated to Sub-semi-graphs) In the notation of the above discussion, $\widehat{\Pi}_{\mathcal{H}}$ (respectively, $\Pi_{\mathcal{H}}^{\mathrm{tp}}$ ) is commensurably terminal in $\widehat{\Pi}_{\mathcal{G}}$ (respectively, $\widehat{\Pi}_{\mathcal{G}}$ [hence, also in $\left.\left.\Pi_{\mathcal{G}}^{\mathrm{tp}}\right]\right)$. In particular, $\Pi_{\mathcal{G}}^{\mathrm{tp}}$ is commensurably terminal in $\widehat{\Pi}_{\mathcal{G}}$.

Proof. First, let us observe that by allowing, in Proposition 2.1, $\Lambda$ to range over the open subgroups of any verticial [hence, in particular, nontrivial compact!] subgroup of $\Pi_{\mathcal{G}}^{\text {tp }}$, we conclude from Proposition 2.1 that

$$
\Pi_{\mathcal{G}}^{\mathrm{tp}} \text { is commensurably terminal in } \widehat{\Pi}_{\mathcal{G}}
$$


- cf. Remark 2.2.2 below. In particular, by applying this fact to $\mathcal{H}$ [cf. the discussion preceding Proposition 2.1], we conclude that $\Pi_{\mathcal{H}}^{\text {tp }}$ is commensurably terminal in $\widehat{\Pi}_{\mathcal{H}}$. Next, let us observe that it is immediate from the definitions that

$$
\Pi_{\mathcal{H}}^{\mathrm{tp}} \subseteq C_{\Pi_{\mathcal{G}}^{\mathrm{tp}}}\left(\Pi_{\mathcal{H}}^{\mathrm{tp}}\right) \subseteq C_{\widehat{\Pi}_{\mathcal{G}}}\left(\Pi_{\mathcal{H}}^{\mathrm{tp}}\right) \subseteq C_{\widehat{\Pi}_{\mathcal{G}}}\left(\widehat{\Pi}_{\mathcal{H}}\right)
$$

[where we think of $\widehat{\Pi}_{\mathcal{H}}, \widehat{\Pi}_{\mathcal{G}}$, respectively, as the pro- $\widehat{\Sigma}$ completions of $\Pi_{\mathcal{H}}^{\mathrm{tp}}, \Pi_{\mathcal{G}}^{\mathrm{tp}}$ ]. On the other hand, by the evident pro- $\widehat{\Sigma}$ analogue of [SemiAnbd], Corollary 2.7, (i) [cf. also the argument involving gluing of abelian coverings in the discussion preceding Proposition 2.1], we have $C_{\widehat{\Pi}_{\mathcal{G}}}\left(\widehat{\Pi}_{\mathcal{H}}\right)=\widehat{\Pi}_{\mathcal{H}}$. Thus, by the commensurable terminality of $\Pi_{\mathcal{H}}^{\mathrm{tp}}$ in $\widehat{\Pi}_{\mathcal{H}}$, we conclude that

$$
\Pi_{\mathcal{H}}^{\mathrm{tp}} \subseteq C_{\widehat{\Pi}_{\mathcal{G}}}\left(\Pi_{\mathcal{H}}^{\mathrm{tp}}\right) \subseteq C_{\widehat{\Pi}_{\mathcal{H}}}\left(\Pi_{\mathcal{H}}^{\mathrm{tp}}\right)=\Pi_{\mathcal{H}}^{\mathrm{tp}}
$$

- as desired.

Remark 2.2.1. It follows immediately from the theory of [SemiAnbd] [cf., e.g., [SemiAnbd], Corollary 2.7, (i)] that, in fact, Propositions 2.1 and 2.2 can be proven for much more general semi-graphs of anabelioids $\mathcal{G}$ than the sort of $\mathcal{G}$ that appears in the above discussion. We leave the routine details of such generalizations to the interested reader.

Remark 2.2.2. Recall that when $\widehat{\Sigma}=\mathfrak{P r i m e s}$, the fact that

$$
\Pi_{\mathcal{G}}^{\text {tp }} \text { is normally terminal in } \widehat{\Pi}_{\mathcal{G}}
$$

may also be derived from the fact that any nonabelian finitely generated free group is normally terminal [cf. [André], Lemma 3.2.1; [SemiAnbd], Lemma 6.1, (i)] in its profinite completion. In particular, the proof of the commensurable terminality of $\Pi_{\mathcal{G}}^{\mathrm{tp}}$ in $\widehat{\Pi}_{\mathcal{G}}$ that is given in the proof of Proposition 2.2 may be thought of as a new proof of this normal terminality that does not require one to invoke [André], Lemma 3.2.1, which is essentially an immediate consequence of the rather difficult conjugacy separability result given in [Stb1], Theorem 1. This relation of Proposition 2.1 to the theory of [Stb1] is interesting in light of the discrete analogue given in Theorem 2.6 below of [the "tempered version of Theorem 2.6" constituted by] Proposition 2.4 [which is essentially a formal consequence of Proposition 2.1].

Now let $k$ be an $M L F, \bar{k}$ an algebraic closure of $k, G_{k} \stackrel{\text { def }}{=} \operatorname{Gal}(\bar{k} / k), X$ a hyperbolic curve over $k$ that admits stable reduction over the ring of integers $\mathcal{O}_{k}$ of $k$. Write

$$
\Pi_{X}^{\mathrm{tp}}, \quad \Delta_{X}^{\mathrm{tp}}
$$

for the respective " $\widehat{\Sigma}$-tempered" quotients of the tempered fundamental groups $\pi_{1}^{\mathrm{tp}}(X)$, $\pi_{1}^{\mathrm{tp}}\left(X_{\bar{k}}\right)$ [relative to suitable basepoints] of $X, X_{\bar{k}} \stackrel{\text { def }}{=} X \times_{k} \bar{k}$ [cf. [André], §4; [SemiAnbd], Example 3.10]. That is to say, $\pi_{1}^{\mathrm{tp}}\left(X_{\bar{k}}\right) \rightarrow \Delta_{X}^{\mathrm{tp}}$ is the quotient determined by the intersection of the kernels of all continuous surjections of $\pi_{1}^{\mathrm{tp}}\left(X_{\bar{k}}\right)$ onto extensions of a finite group of order a product [possibly with multiplicities] of primes 
$\in \widehat{\Sigma}$ by a discrete free group of finite rank; $\pi_{1}^{\mathrm{tp}}(X) \rightarrow \Pi_{X}^{\mathrm{tp}}$ is the quotient of $\pi_{1}^{\mathrm{tp}}(X)$ determined by the kernel of the quotient of $\pi_{1}^{\mathrm{tp}}\left(X_{\bar{k}}\right) \rightarrow \Delta_{X}^{\mathrm{tp}}$. Write $\widehat{\Delta}_{X}$ for the pro- $\widehat{\Sigma}$ [i.e., maximal pro- $\widehat{\Sigma}$ quotient of the profinite] fundamental group of $X_{\bar{k}}$; $\widehat{\Pi}_{X}$ for the quotient of the profinite fundamental group of $X$ by the subgroup of the profinite fundamental group of $X_{\bar{k}}$ that determines the quotient $\widehat{\Delta}_{X}$. Thus, since discrete free groups of finite rank inject into their pro- $l$ completions for any prime number $l$ [cf., e.g., [RZ], Proposition 3.3.15], we have natural inclusions

$$
\Pi_{X}^{\mathrm{tp}} \hookrightarrow \widehat{\Pi}_{X}, \quad \Delta_{X}^{\mathrm{tp}} \hookrightarrow \widehat{\Delta}_{X}
$$

[cf., e.g., [SemiAnbd], Proposition 3.6, (iii), as well as Remark 2.5.3, (ii), (E7), of the present paper, when $\widehat{\Sigma}=$ Primes] ; $\widehat{\Delta}_{X}$ may be identified with the pro- $\widehat{\Sigma}$ completion of $\Delta_{X}^{\mathrm{tp}} ; \widehat{\Pi}_{X}$ is generated by the images of $\Pi_{X}^{\mathrm{tp}}$ and $\widehat{\Delta}_{X}$.

Now suppose that the residue characteristic $p$ of $k$ is not contained in $\Sigma$; that the semi-graph of anabelioids $\mathcal{G}$ of the above discussion is the pro- $\Sigma$ semi-graph of anabelioids associated to the geometric special fiber of the stable model $\mathcal{X}$ of $X$ over $\mathcal{O}_{k}$ [cf., e.g., [SemiAnbd], Example 3.10]; and that the sub-semi-graph $\mathbb{H} \subseteq \mathbb{G}$ is stabilized by the natural action of $G_{k}$ on $\mathbb{G}$. Thus, we have natural surjections

$$
\Delta_{X}^{\mathrm{tp}} \rightarrow \Pi_{\mathcal{G}}^{\mathrm{tp}} ; \quad \widehat{\Delta}_{X} \rightarrow \widehat{\Pi}_{\mathcal{G}}
$$

of topological groups.

Corollary 2.3. (Subgroups of Tempered Fundamental Groups Associated to Sub-semi-graphs) In the notation of the above discussion:

(i) The closed subgroups

$$
\Delta_{X, \mathbb{H}}^{\mathrm{tp}} \stackrel{\text { def }}{=} \Delta_{X}^{\mathrm{tp}} \times_{\Pi_{\mathcal{G}}^{\mathrm{tp}}} \Pi_{\mathcal{H}}^{\mathrm{tp}} \subseteq \Delta_{X}^{\mathrm{tp}} ; \quad \widehat{\Delta}_{X, \mathbb{H}} \stackrel{\text { def }}{=} \widehat{\Delta}_{X} \times_{\widehat{\Pi}_{\mathcal{G}}} \widehat{\Pi}_{\mathcal{H}} \subseteq \widehat{\Delta}_{X}
$$

are commensurably terminal. In particular, the natural outer actions of $G_{k}$ on $\Delta_{X}^{\mathrm{tp}}, \widehat{\Delta}_{X}$ determine natural outer actions of $G_{k}$ on $\Delta_{X, \mathbb{H}}^{\mathrm{tp}}, \widehat{\Delta}_{X, \mathbb{H}}$.

(ii) The closure of $\Delta_{X, \mathbb{H}}^{\mathrm{tp}} \subseteq \Delta_{X}^{\mathrm{tp}} \subseteq \widehat{\Delta}_{X}$ in $\widehat{\Delta}_{X}$ is equal to $\widehat{\Delta}_{X, \mathbb{H}}$.

(iii) Suppose that [at least] one of the following conditions holds: (a) $\widehat{\Sigma}$ contains a prime number $l \notin \Sigma \bigcup\{p\} ;$ (b) $\widehat{\Sigma}=\mathfrak{P r i m e s}$. Then $\widehat{\Delta}_{X, \mathbb{H}}$ is slim. In particular, the natural outer actions of $G_{k}$ on $\Delta_{X, \mathbb{H}}^{\mathrm{tp}}, \widehat{\Delta}_{X, \mathbb{H}}[c f$. (i)] determine natural exact sequences of center-free topological groups [cf. (ii); the slimness of $\widehat{\Delta}_{X, \mathbb{H}}$; [AbsAnab], Theorem 1.1.1, (ii)]

$$
\begin{aligned}
1 & \rightarrow \Delta_{X, \mathbb{H}}^{\mathrm{tp}} \rightarrow \Pi_{X, \mathbb{H}}^{\mathrm{tp}} \rightarrow G_{k} \rightarrow 1 \\
1 & \rightarrow \widehat{\Delta}_{X, \mathbb{H}} \rightarrow \widehat{\Pi}_{X, \mathbb{H}} \rightarrow G_{k} \rightarrow 1
\end{aligned}
$$

- where $\Pi_{X, \mathbb{H}}^{\mathrm{tp}}, \widehat{\Pi}_{X, \mathbb{H}}$ are defined so as to render the sequences exact.

(iv) Suppose that the hypothesis of (iii) holds. Then the images of the natural inclusions $\Pi_{X, \mathbb{H}}^{\mathrm{tp}} \hookrightarrow \Pi_{X}^{\mathrm{tp}}, \widehat{\Pi}_{X, \mathbb{H}} \hookrightarrow \widehat{\Pi}_{X}$ are commensurably terminal. 
(v) We have: $\widehat{\Delta}_{X, \mathbb{H}} \cap \Delta_{X}^{\mathrm{tp}}=\Delta_{X, \mathbb{H}}^{\mathrm{tp}} \subseteq \widehat{\Delta}_{X}$.

(vi) Let

$$
I_{x} \subseteq \Delta_{X}^{\text {tp }}\left(\text { respectively, } I_{x} \subseteq \widehat{\Delta}_{X}\right)
$$

be an inertia group associated to a cusp $x$ of $X$. Write $\xi$ for the cusp of the stable model $\mathcal{X}$ corresponding to $x$. Then the following conditions are equivalent:

(a) $I_{x}$ lies in a $\Delta_{X^{-}}^{\mathrm{tp}}$ (respectively, $\widehat{\Delta}_{X^{-}}$) conjugate of $\Delta_{X, \mathbb{H}}^{\mathrm{tp}}$ (respectively, $\left.\widehat{\Delta}_{X, \mathbb{H}}\right)$;

(b) $\xi$ meets an irreducible component of the special fiber of $\mathcal{X}$ that is contained in $\mathbb{H}$.

Proof. Assertion (i) follows immediately from Proposition 2.2. Assertion (ii) follows immediately from the definitions of the various tempered fundamental groups involved, together with the following elementary observation: If $G \rightarrow F$ is a surjection of finitely generated free discrete groups, which induces a surjection $\widehat{G} \rightarrow \widehat{F}$ between the respective pro- $\widehat{\Sigma}$ completions [so, since discrete free groups of finite rank inject into their pro- $l$ completions for any prime number $l$ [cf., e.g., [RZ], Proposition 3.3.15], we think of $G$ and $F$ as subgroups of $\widehat{G}$ and $\widehat{F}$, respectively], then $H \stackrel{\text { def }}{=} \operatorname{Ker}(G \rightarrow F)$ is dense in $\widehat{H} \stackrel{\text { def }}{=} \operatorname{Ker}(\widehat{G} \rightarrow \widehat{F})$, relative to the pro- $\widehat{\Sigma}$ topology of $\widehat{G}$. Indeed, let $\iota: F \hookrightarrow G$ be a section of the given surjection $G \rightarrow F$ [which exists since $F$ is free]. Then if $\left\{g_{i}\right\}_{i \in \mathbb{N}}$ is a sequence of elements of $G$ that converges, in the pro- $\widehat{\Sigma}$ topology of $\widehat{G}$, to a given element $h \in \widehat{H}$, and maps to a sequence of elements $\left\{f_{i}\right\}_{i \in \mathbb{N}}$ of $F$ [which necessarily converges, in the pro- $\widehat{\Sigma}$ topology of $\widehat{F}$, to the identity element $1 \in \widehat{F}]$, then one verifies immediately that $\left\{g_{i} \cdot \iota\left(f_{i}\right)^{-1}\right\}_{i \in \mathbb{N}}$ is a sequence of elements of $H$ that converges, in the pro- $\widehat{\Sigma}$ topology of $\widehat{G}$, to $h$. This completes the proof of the observation and hence of assertion (ii).

Next, we consider assertion (iii). In the following, we give, in effect, two distinct proofs of the slimness of $\widehat{\Delta}_{X, \mathbb{H}}$ : one is elementary, but requires one to assume that condition (a) holds; the other depends on the highly nontrivial theory of [Tama2] and requires one to assume that condition (b) holds. If condition (a) holds, then let us set $\Sigma^{*} \stackrel{\text { def }}{=} \Sigma \bigcup\{l\}$. If condition (b) holds, but condition (a) does not hold [so $\widehat{\Sigma}=\mathfrak{P r i m e s}=\Sigma \bigcup\{p\}]$, then let us set $\Sigma^{*} \stackrel{\text { def }}{=} \Sigma$. Thus, in either case, $p \notin \Sigma^{*}$, and $\Sigma \subseteq \Sigma^{*} \subseteq \widehat{\Sigma}$.

Let $J \subseteq \widehat{\Delta}_{X}$ be a normal open subgroup. Write $J_{\mathbb{H}} \stackrel{\text { def }}{=} J \cap \widehat{\Delta}_{X, \mathbb{H}} ; J \rightarrow J^{*}$ for the maximal pro- $\Sigma^{*}$ quotient; $J_{\mathbb{H}}^{*} \subseteq J^{*}$ for the image of $J_{\mathbb{H}}$ in $J^{*}$. Now suppose that $\alpha \in \widehat{\Delta}_{X, \mathbb{H}}$ commutes with $J_{\mathbb{H}}$. Let $v$ be a vertex of the dual graph of the geometric special fiber of a stable model $\mathcal{X}_{J}$ of the covering $X_{J}$ of $X_{\bar{k}}$ determined by $J$. Write $J_{v} \subseteq J$ for the decomposition group [well-defined up to conjugation in $J$ ] associated to $v ; J_{v}^{*} \subseteq J^{*}$ for the image of $J_{v}$ in $J^{*}$. Then let us observe that

( $\dagger$ ) there exists an open subgroup $J_{0} \subseteq \widehat{\Delta}_{X}$ which is independent of $J, v$, and $\alpha$ such that if $J \subseteq J_{0}$, then for arbitrary $v$ [and $\alpha$ ] as above, it holds that $J_{v}^{*} \cap J_{\mathbb{H}}^{*}\left(\subseteq J^{*}\right)$ is infinite and nonabelian.

Indeed, suppose that condition (a) holds. Now it follows immediately from the definitions that the image of the homomorphism $J_{v} \subseteq J \subseteq \widehat{\Delta}_{X} \rightarrow \widehat{\Pi}_{\mathcal{G}}$ is pro- $\Sigma$; in 
particular, since $l \notin \Sigma$, and $\operatorname{Ker}\left(J_{v} \subseteq J \subseteq \widehat{\Delta}_{X} \rightarrow \widehat{\Pi}_{\mathcal{G}}\right) \subseteq J_{v} \bigcap J_{\mathbb{H}}$, it follows that $J_{v} \cap J_{\mathbb{H}}$, hence also $J_{v}^{*} \cap J_{\mathbb{H}}^{*}$, surjects onto the maximal pro-l quotient of $J_{v}$, which is isomorphic to the pro- $l$ completion of the fundamental group of a hyperbolic Riemann surface, hence [as is well-known] is infinite and nonabelian [so we may take $J_{0} \stackrel{\text { def }}{=} \widehat{\Delta}_{X}$ ]. Now suppose that condition (b) holds, but condition (a) does not hold. Then it follows immediately from [Tama2], Theorem 0.2, (v), that, for an appropriate choice of $J_{0}$, if $J \subseteq J_{0}$, then every $v$ corresponds to an irreducible component that either maps to a point in $\mathcal{X}$ or contains a node that maps to a smooth point of $\mathcal{X}$. In particular, it follows that for every choice of $v$, there exists at least one pro- $\Sigma$, torsion-free, pro-cyclic subgroup $F \subseteq J_{v}$ that lies in $\operatorname{Ker}\left(J_{v} \subseteq\right.$ $\left.J \subseteq \widehat{\Delta}_{X} \rightarrow \widehat{\Pi}_{\mathcal{G}}\right) \subseteq J_{v} \cap J_{\mathbb{H}}$ and, moreover, maps injectively into $J^{*}$. Thus, we obtain an injection $F \hookrightarrow J_{v}^{*} \cap J_{\mathbb{H}}^{*}$; a similar statement holds when $F$ is replaced by any $J_{v}$-conjugate of $F$. Moreover, it follows from the well-known structure of the pro- $\Sigma$ completion of the fundamental group of a hyperbolic Riemann surface [such as $J_{v}^{*}$ ] that the image of the $J_{v}$-conjugates of such a group $F$ topologically generate a closed subgroup of $J_{v}^{*} \bigcap J_{\mathbb{H}}^{*}$ which is infinite and nonabelian. This completes the proof of $(\dagger)$.

Next, let us observe that it follows by applying either [AbsTopII], Proposition 1.3 , (iv), or [NodNon], Proposition 3.9, (i), to the various $\widehat{\Delta}_{X}$-conjugates in $J^{*}$ of $J_{v}^{*} \cap J_{\mathbb{H}}^{*}$ as in ( $\left.\dagger\right)$ that the fact that $\alpha$ commutes with $J_{v}^{*} \cap J_{\mathbb{H}}^{*}$ implies that $\alpha$ fixes $v$. If condition (a) holds, then the fact that conjugation by $\alpha$ on the maximal pro-l quotient of $J_{v}$ [which, as we saw above, is a quotient of $J_{v}^{*} \cap J_{\mathbb{H}}^{*}$ ] is trivial implies [cf. the argument concerning the inertia group " $I_{v} \subseteq D_{v}$ " in the latter portion of the proof of [SemiAnbd], Corollary 3.11] that $\alpha$ not only fixes $v$, but also acts trivially on the irreducible component of the special fiber of $\mathcal{X}_{J}$ determined by $v$; since $v$ as in $(\dagger)$ is arbitrary, we thus conclude that $\alpha$ acts on the abelianization $\left(J^{*}\right)^{\text {ab }}$ of $J^{*}$ as a unipotent automorphism of finite order, hence that $\alpha$ acts trivially on $\left(J^{*}\right)^{\text {ab }}$; since $J$ as in $(\dagger)$ is arbitrary, we thus conclude [cf., e.g., the proof of [Config], Proposition 1.4] that $\alpha$ is the identity element, as desired. Now suppose that condition (b) holds, but condition (a) does not hold. Then since $J$ and $v$ as in $(\dagger)$ are arbitrary, we thus conclude again from [Tama2], Theorem 0.2, (v), that $\alpha$ fixes not only $v$, but also every closed point on the irreducible component of the special fiber of $\mathcal{X}_{J}$ determined by $v$, hence that $\alpha$ acts trivially on this irreducible component. Again since $J$ and $v$ as in $(\dagger)$ are arbitrary, we thus conclude that $\alpha$ is the identity element, as desired. This completes the proof of assertion (iii). In light of the exact sequences of assertion (iii), assertion (iv) follows immediately from assertion (i). Assertion (vi) follows immediately from a similar argument to the argument applied in the proof of [CombGC], Proposition 1.5, (i), by passing to pro- $\Sigma$ completions.

Finally, it follows immediately from the definitions of the various tempered fundamental groups involved that to verify assertion (v), it suffices to verify the following analogue of assertion (v) for a nonabelian finitely generated free discrete group $G$ : for any finitely generated subgroup $F \subseteq G$, if we use the notation " $\wedge$ " to denote the pro- $\widehat{\Sigma}$ completion, then $\widehat{F} \cap G=F$. But to verify this assertion concerning $G$, it follows immediately from [SemiAnbd], Corollary 1.6, (ii), that we may assume without loss of generality that the inclusion $F \subseteq G$ admits a splitting $G \rightarrow F$ [i.e., such that the composite $F \hookrightarrow G \rightarrow F$ is the identity on $F$ ], in which 
case the desired equality " $\widehat{F} \cap G=F$ " follows immediately. This completes the proof of assertion (v), and hence of Corollary 2.3.

Next, we observe the following arithmetic analogue of Proposition 2.1.

\section{Proposition 2.4. (Profinite Conjugates of Nontrivial Arithmetic Com- pact Subgroups) In the notation of the above discussion:}

(i) Let $\Lambda \subseteq \Delta_{X}^{\text {tp }}$ be a nontrivial pro- $\Sigma$ compact subgroup, $\gamma \in \widehat{\Pi}_{X}$ an element such that $\gamma \cdot \Lambda \cdot \gamma^{-1} \subseteq \Delta_{X}^{\mathrm{tp}}$ [or, equivalently, $\left.\Lambda \subseteq \gamma^{-1} \cdot \Delta_{X}^{\mathrm{tp}} \cdot \gamma\right]$. Then $\gamma \in \Pi_{X}^{\mathrm{tp}}$

(ii) Suppose that $\widehat{\Sigma}=$ Primes. Let $\Lambda \subseteq \Pi_{X}^{\mathrm{tp}}$ be a [nontrivial] compact subgroup whose image in $G_{k}$ is open, $\gamma \in \widehat{\Pi}_{X}$ an element such that $\gamma \cdot \Lambda \cdot \gamma^{-1} \subseteq$ $\Pi_{X}^{\mathrm{tp}}\left[\right.$ or, equivalently, $\left.\Lambda \subseteq \gamma^{-1} \cdot \Pi_{X}^{\mathrm{tp}} \cdot \gamma\right]$. Then $\gamma \in \Pi_{X}^{\mathrm{tp}}$.

(iii) $\Delta_{X}^{\mathrm{tp}}$ (respectively, $\Pi_{X}^{\mathrm{tp}}$ ) is commensurably terminal in $\widehat{\Delta}_{X}$ (respectively, $\left.\widehat{\Pi}_{X}\right)$.

Proof. First, we consider assertion (i). We begin by observing that since as is well-known - cf., e.g., [Config], Remark 1.2.2] $\widehat{\Delta}_{X}$ is strongly torsion-free, it follows that there exists a finite index characteristic open subgroup $J \subseteq \Delta_{X}^{\text {tp }}$ such that, if we write $\mathcal{G}_{J}$ for the pro- $\Sigma$ semi-graph of anabelioids associated to the special fiber of the stable model [i.e., over the ring of integers $\mathcal{O}_{\bar{k}}$ of $\bar{k}$ ] of the finite étale covering of $X \times_{k} \bar{k}$ determined by $J$, then $J \bigcap \Lambda$ has nontrivial image in the pro- $\Sigma$ completion of the abelianization of $J$, hence in $\Pi_{\mathcal{G}_{J}}^{\mathrm{tp}}$ [since, as is well-known, our assumption that $p \notin \Sigma$ implies that the surjection $J \rightarrow \Pi_{\mathcal{G}_{J}}^{\text {tp }}$ induces an isomorphism between the pro- $\Sigma$ completions of the respective abelianizations]. Since the quotient $\Pi_{X}^{\mathrm{tp}}$ surjects onto $G_{k}$, and $J$ is open of finite index in $\Delta_{X}^{\text {tp }}$, we may assume without loss of generality that $\gamma$ lies in the closure $\widehat{J}$ of $J$ in $\widehat{\Pi}_{X}$. Since $J \bigcap \Lambda$ has nontrivial image in $\Pi_{\mathcal{G}_{J}}^{\mathrm{tp}}$, it thus follows from Proposition 2.1 [applied to $\mathcal{G}_{J}$ ] that the image of $\gamma$ via the natural surjection on pro- $\widehat{\Sigma}$ completions $\widehat{J} \rightarrow \widehat{\Pi}_{\mathcal{G}_{J}}$ lies in $\Pi_{\mathcal{G}_{J}}^{\mathrm{tp}}$. Since, by allowing $J$ to vary, $\Pi_{X}^{\text {tp }}$ (respectively, $\widehat{\Pi}_{X}$ ) may be written as an inverse limit of the topological groups $\Pi_{X}^{\mathrm{tp}} / \operatorname{Ker}\left(J \rightarrow \Pi_{\mathcal{G}_{J}}^{\mathrm{tp}}\right)\left(\right.$ respectively, $\left.\widehat{\Pi}_{X} / \operatorname{Ker}\left(\widehat{J} \rightarrow \widehat{\Pi}_{\mathcal{G}_{J}}\right)\right)$, we thus conclude that [the original] $\gamma$ lies in $\Pi_{X}^{\mathrm{tp}}$, as desired.

Next, we consider assertion (ii). First, let us observe that it follows from a similar argument to the argument applied to prove Proposition 2.1 - where, instead of applying [SemiAnbd], Theorem 3.7, (iii), we apply its arithmetic analogue, namely, [SemiAnbd], Theorem 5.4, (ii); [SemiAnbd], Example 5.6 [cf. also Remark 2.5.3, (ii), (E5), (E7), of the present paper] — that the image of $\gamma$ in $\widehat{\Pi}_{X} / \operatorname{Ker}\left(\widehat{\Delta}_{X} \rightarrow \widehat{\Pi}_{\mathcal{G}^{*}}\right)$ lies in $\Pi_{X}^{\mathrm{tp}} / \operatorname{Ker}\left(\Delta_{X}^{\mathrm{tp}} \rightarrow \Pi_{\mathcal{G}^{*}}^{\mathrm{tp}}\right)$, where [by invoking the hypothesis that $\widehat{\Sigma}=\mathfrak{P r i m e s}$ ] we take $\mathcal{G}^{*}$ to be a semi-graph of anabelioids as in [SemiAnbd], Example 5.6, i.e., the semi-graph of anabelioids whose finite étale coverings correspond to arbitrary admissible coverings of the geometric special fiber of the stable model $\mathcal{X}$. Here, we note that when one applies either [AbsTopII], Proposition 1.3, (iv), or [NodNon], 
Proposition 3.9, (i) - after, say, restricting the outer action of $G_{k}$ on $\Pi_{\mathcal{G}^{*}}^{\mathrm{tp}}$ to a closed pro- $\Sigma$ subgroup of the inertia group $I_{k}$ of $G_{k}$ that maps isomorphically onto the maximal pro- $\Sigma$ quotient of $I_{k}$ - to the vertices " $v^{\prime \prime}$ ", " $\left(v^{\prime}\right)^{\gamma}$ ", one may only conclude that these two vertices either coincide, are adjacent, or admit a common adjacent vertex; but this is still sufficient to conclude the temperedness of " $\left(v^{\prime}\right)^{\gamma}$ " from that of " $v$ " ". Now [just as in the proof of assertion (i)] by applying [the evident analogue of] this observation to the quotients $\Pi_{X}^{\mathrm{tp}} \rightarrow \Pi_{X}^{\mathrm{tp}} / \operatorname{Ker}\left(J \rightarrow \Pi_{\mathcal{G}^{*}}^{\mathrm{tp}}\right)$ - where $J \subseteq \Delta_{X}^{\text {tp }}$ is a finite index characteristic open subgroup, and $\mathcal{G}_{J}^{*}$ is the semi-graph of anabelioids whose finite étale coverings correspond to arbitrary admissible coverings of the special fiber of the stable model over $\mathcal{O}_{\bar{k}}$ of the finite étale covering of $X \times_{k} \bar{k}$ determined by $J$ - we conclude that $\gamma \in \Pi_{X}^{\mathrm{tp}}$, as desired.

Finally, we consider assertion (iii). Just as in the proof of Proposition 2.2, the commensurable terminality of $\Delta_{X}^{\text {tp }}$ in $\widehat{\Delta}_{X}$ follows immediately from assertion (i), by allowing, in assertion (i), $\Lambda$ to range over the open subgroups of a pro- $\Sigma$ Sylow subgroup of a decomposition group $\subseteq \Delta_{X}^{\text {tp }}$ associated to an irreducible component of the special fiber of $\mathcal{X}$. The commensurable terminality of $\Pi_{X}^{\mathrm{tp}}$ in $\widehat{\Pi}_{X}$ then follows immediately from the commensurable terminality of $\Delta_{X}^{\text {tp }}$ in $\widehat{\Delta}_{X}$.

Remark 2.4.1. Thus, when $\widehat{\Sigma}=\mathfrak{P r i m e s}$, the proof given above of Proposition 2.4, (iii), yields a new proof of [André], Corollary 6.2.2 [cf. also [SemiAnbd], Lemma 6.1, (ii), (iii)] which is independent of [André], Lemma 3.2.1, hence also of [Stb1], Theorem 1 [cf. the discussion of Remark 2.2.2].

Corollary 2.5. (Profinite Conjugates of Tempered Decomposition and Inertia Groups) In the notation of the above discussion, suppose further that $\widehat{\Sigma}=$ Primes. Then every decomposition group in $\widehat{\Pi}_{X}$ (respectively, inertia group in $\widehat{\Pi}_{X}$ ) associated to a closed point or cusp of $X$ (respectively, to a cusp of $X)$ is contained in $\Pi_{X}^{\mathrm{tp}}$ if and only if it is a decomposition group in $\Pi_{X}^{\mathrm{tp}}$ (respectively, inertia group in $\Pi_{X}^{\mathrm{tp}}$ ) associated to a closed point or cusp of $X$ (respectively, to a cusp of X). Moreover, a $\widehat{\Pi}_{X}$-conjugate of $\Pi_{X}^{\mathrm{tp}}$ contains a decomposition group in $\Pi_{X}^{\mathrm{tp}}$ (respectively, inertia group in $\Pi_{X}^{\mathrm{tp}}$ ) associated to a closed point or cusp of $X$ (respectively, to a cusp of $X$ ) if and only if it is equal to $\Pi_{X}^{\mathrm{tp}}$.

Proof. Let $D_{x} \subseteq \Pi_{X}^{\mathrm{tp}}$ be the decomposition group in $\Pi_{X}^{\mathrm{tp}}$ associated to a closed point or cusp $x$ of $X ; I_{x} \stackrel{\text { def }}{=} D_{x} \cap \Delta_{X}^{\text {tp }}$. Then the decomposition groups of $\widehat{\Pi}_{X}$ associated to $x$ are precisely the $\widehat{\Pi}_{X}$-conjugates of $D_{x}$; the decomposition groups of $\Pi_{X}^{\mathrm{tp}}$ associated to $x$ are precisely the $\Pi_{X}^{\mathrm{tp}}$-conjugates of $D_{x}$. Since $D_{x}$ is compact and surjects onto an open subgroup of $G_{k}$, it thus follows from Proposition 2.4, (ii), that a $\widehat{\Pi}_{X}$-conjugate of $D_{x}$ is contained in $\Pi_{X}^{\mathrm{tp}}$ if and only if it is, in fact, a $\Pi_{X}^{\mathrm{tp}}$-conjugate of $D_{x}$, and that a $\widehat{\Pi}_{X}$-conjugate of $\Pi_{X}^{\mathrm{tp}}$ contains $D_{x}$ if and only if it is, in fact, equal to $\Pi_{X}^{\text {tp }}$. In a similar vein, when $x$ is a cusp of $X$ [so $I_{x} \cong \widehat{\mathbb{Z}}$ ], it follows - i.e., by applying Proposition 2.4, (i), to the unique maximal pro- $\Sigma$ subgroup of $I_{x}$ - that a $\widehat{\Pi}_{X}$-conjugate of $I_{x}$ is contained in $\Pi_{X}^{\text {tp }}$ if and only if it is, in fact, a $\Pi_{X}^{\mathrm{tp}}$-conjugate of $I_{x}$, and that a $\widehat{\Pi}_{X}$-conjugate of $\Pi_{X}^{\mathrm{tp}}$ contains $I_{x}$ if and only if it is, in fact, equal to $\Pi_{X}^{\mathrm{tp}}$. This completes the proof of Corollary 2.5. 
Remark 2.5.1. The content of Corollary 2.5 may be regarded as a sort of [very weak!] version of the "Section Conjecture" of anabelian geometry - i.e., as the assertion that certain sections of the tempered fundamental group [namely, those that arise from geometric sections of the profinite fundamental group] are geometric as sections of the tempered fundamental group. This point of view is reminiscent of the point of view of [SemiAnbd], Remark 6.9.1. Perhaps one way of summarizing this circle of ideas is to state that one may think of

(i) the classification of maximal compact subgroups of tempered fundamental groups given in [SemiAnbd], Theorem 3.7, (iv); [SemiAnbd], Theorem 5.4, (ii) [cf. also Remark 2.5.3, (ii), (E5), (E7), of the present paper], or, for that matter,

(ii) the more elementary fact that "any finite group acting on a tree [without inversion] fixes at least one vertex" [cf. [SemiAnbd], Lemma 1.8, (ii)] from which these results of [SemiAnbd] are derived

as a sort of combinatorial version of the Section Conjecture.

Remark 2.5.2. Ultimately, when we apply Corollary 2.5 in [IUTchII], it will only be necessary to apply the portion of Corollary 2.5 that concerns inertia groups of cusps, i.e., the portion whose proof only requires the use of Proposition 2.4, (i), which is essentially an immediate consequence of Proposition 2.1. That is to say, the theory developed in [IUTchII] [and indeed throughout the present series of papers] will never require the application of Proposition 2.4, (ii), i.e., whose proof depends on a slightly more complicated version of the proof of Proposition 2.1.

Remark 2.5.3. In light of the importance of the theory of [SemiAnbd] in the present $\S 2$, we pause to discuss certain minor oversights on the part of the author in the exposition of [SemiAnbd].

(i) Certain pathologies occur in the theory of tempered fundamental groups if one does not impose suitable countability hypotheses. In order to discuss these countability hypotheses, it will be convenient to introduce some terminology as follows:

(T1) We shall say that a tempered group is Galois-countable if its topology admits a countable basis. We shall say that a connected temperoid is Galois-countable if it arises from a Galois-countable tempered group. We shall say that a temperoid is Galois-countable if it arises from a collection of Galois-countable connected temperoids. We shall say that a connected quasi-temperoid is Galois-countable if it arises from a Galoiscountable connected temperoid. We shall say that a quasi-temperoid is Galois-countable if it arises from a collection of Galois-countable connected quasi-temperoids.

(T2) We shall say that a semi-graph of anabelioids $\mathcal{G}$ is Galois-countable if it is countable, and, moreover, admits a countable collection of finite étale coverings $\left\{\mathcal{G}_{i} \rightarrow \mathcal{G}\right\}_{i \in I}$ such that for any finite étale covering $\mathcal{H} \rightarrow \mathcal{G}$, there exists an $i \in I$ such that the base-changed covering $\mathcal{H} \times{ }_{\mathcal{G}} \mathcal{G}_{i} \rightarrow \mathcal{G}_{i}$ splits over the constituent anabelioid associated to each component of [the underlying semi-graph of] $\mathcal{G}_{i}$. 
(T3) We shall say that a semi-graph of anabelioids $\mathcal{G}$ is strictly coherent if it is coherent [cf. [SemiAnbd], Definition 2.3, (iii)], and, moreover, each of the profinite groups associated to components $c$ of [the underlying semigraph of] $\mathcal{G}$ [cf. the final sentence of [SemiAnbd], Definition 2.3, (iii)] is topologically generated by $N$ generators, for some positive integer $N$ that is independent of $c$. In particular, it follows that if $\mathcal{G}$ is finite and coherent, then it is strictly coherent.

(T4) One verifies immediately that every strictly coherent, countable semigraph of anabelioids is Galois-countable.

(T5) One verifies immediately that if, in [SemiAnbd], Remark 3.2.1, one assumes in addition that the temperoid $\mathcal{X}$ is Galois-countable, then it follows that its associated tempered fundamental group $\pi_{1}^{\text {temp }}(\mathcal{X})$ is well-defined and Galois-countable.

(T6) One verifies immediately that if, in the discussion of the paragraph preceding [SemiAnbd], Proposition 3.6, one assumes in addition that the semi-graph of anabelioids $\mathcal{G}$ is Galois-countable, then it follows that its associated tempered fundamental group $\pi_{1}^{\text {temp }}(\mathcal{G})$ and temperoid $\mathcal{B}^{\text {temp }}(\mathcal{G})$ are well-defined and Galois-countable.

Here, we note that, in (T5) and (T6), the Galois-countability assumption is necessary in order to ensure that the index sets of "universal covering pro-objects" implicit in the definition of the tempered fundamental group may to be taken to be countable. This countability of the index sets involved implies that the various objects that constitute such a universal covering pro-object admit a compatible system of basepoints, i.e., that the obstruction to the existence of such a compatible system - which may be thought of as an element of a sort of "nonabelian $\mathbb{R}^{1}$ lim" - vanishes. In order to define the tempered fundamental group in an intrinsically meaningful fashion, it is necessary to know the existence of such a compatible system of basepoints.

(ii) The effects of the omission of Galois-countability hypotheses in [SemiAnbd], $\S 3$ [cf. the discussion of (i)], on the remainder of [SemiAnbd], as well as on subsequent papers of the author, may be summarized as follows:

(E1) First of all, we observe that all topological subquotients of absolute Galois groups of fields of countable cardinality are Galois-countable.

(E2) Also, we observe that if $k$ is a field whose absolute Galois group is Galoiscountable, and $U$ is a nonempty open subscheme of a connected proper $k$-scheme $X$ that arises as the underlying scheme of a log scheme that is $\log$ smooth over $k$ [where we regard $\operatorname{Spec}(k)$ as equipped with the trivial $\log$ structure], and whose interior is equal to $U$, then the tamely ramified arithmetic fundamental group of $U$ [i.e., that arises by considering finite étale coverings of $U$ with tame ramification over the divisors that lie in the complement of $U$ in $X$ ] is itself Galois-countable [cf., e.g., [AbsTopI], Proposition 2.2].

(E3) Next, we observe, with regard to [SemiAnbd], Examples 2.10, 3.10, and 5.6, that the tempered groups and temperoids that appear in these Examples are Galois-countable [cf. (E1), (E2)], while the semi-graphs of 
anabelioids that appear in these Examples are strictly coherent [cf. item (T3) of (i)], hence [cf. item (T4) of (i)] Galois-countable. In particular, there is no effect on the theory of objects discussed in these Examples.

(E4) It follows immediately from (E3) that there is no effect on [SemiAnbd], $\S 6$.

(E5) It follows immediately from items (T3), (T4) of (i), together with the assumptions of finiteness and coherence in the discussion of the paragraph immediately preceding [SemiAnbd], Definition 4.2, the assumption of coherence in [SemiAnbd], Definition 5.1, (i), and the assumption of [SemiAnbd], Definition 5.1, (i), (d), that there is no effect on [SemiAnbd], $\S 4, \S 5$. [Here, we note that since the notion of a tempered covering of a semi-graph of anabelioids is only defined in the case where the semi-graph of anabelioids is countable, it is implicit in [SemiAnbd], Proposition 5.2, and [SemiAnbd], Definition 5.3, that the semi-graphs of anabelioids under consideration are countable.]

(E6) There is no effect on [SemiAnbd], $11, \S 2$, or the Appendix of [SemiAnbd], since tempered fundamental groups are never discussed in these portions of [SemiAnbd].

(E7) In the Definitions/Propositions/Theorems/Corollaries of [SemiAnbd] that are numbered 3.2, 3.3, 3.4, 3.5, 3.6, 3.7, 3.8, 3.9, one must assume that all tempered groups, temperoids, and semi-graphs of anabelioids that appear are Galois-countable. On the other hand, it follows immediately from (E1), (E2), and (E3) that there is no effect on the remaining portions of [SemiAnbd], $\S 3$.

(E8) In [QuCnf] and [FrdII], one must assume that all tempered groups and [quasi-]temperoids that appear are Galois-countable.

(E9) There is no effect on any papers of the author other than [SemiAnbd] and the papers discussed in (E8).

(iii) The assertion stated in the second display of [SemiAnbd], Remark 2.4.2, is false as stated. [The automorphisms of the semi-graphs of anabelioids in [SemiAnbd], Example 2.10, that arise from "Dehn twists" constitute a well-known counterexample to this assertion.] This assertion should be replaced by the following slightly modified version of this assertion:

The isomorphism classes of the $\phi_{v}$ completely determine the isomorphism class of each of the $\phi_{e}$, as well as each isomorphism $\phi_{b}$, up to composition with an automorphism of the composite 1-morphism of anabelioids $\mathcal{G}_{e} \rightarrow \mathcal{H}_{f} \rightarrow \mathcal{H}_{w}$ that arises from an automorphism of the 1-morphism of anabelioids $\mathcal{G}_{e} \rightarrow \mathcal{H}_{f}$.

Also, in the discussion following this assertion [as well as the various places where this discussion is applied, i.e., [SemiAnbd], Remark 3.5.2; the second paragraph of [SemiAnbd], §4; [SemiAnbd], Definition 5.1, (iv)], it is necessary to assume further that the semi-graphs of anabelioids that appear satisfy the condition that every edge abuts to at least one vertex. 
(iv) The phrase "is Galois" at the end of the first sentence of the proof of [SemiAnbd], Proposition 3.2, should read "is a countable coproduct of Galois objects".

(v) In the first sentence of [SemiAnbd], Definition 3.5, (ii), the phrase "Suppose that" should read "Suppose that each connected component of"; the phrase "splits the restriction of" should read "splits the restriction of this connected component of".

(vi) In order to carry out the argument stated in the proof of [SemiAnbd], Proposition 5.2, (i), it is necessary to strengthen the conditions (c) and (d) of [SemiAnbd], Definition 5.1, (i), as follows. This strengthening of the conditions (c) and (d) of [SemiAnbd], Definition 5.1, (i), has no effect either on the remainder of [SemiAnbd] or on subsequent papers of the author. Suppose that $\mathcal{G}$ is as in [SemiAnbd], Definition 5.1, (i). Then we begin by making the following observation:

(O1) Suppose that $\mathcal{G}$ is finite. Then $\mathcal{G}$ admits a cofinal, countable collection of connected finite étale Galois coverings $\left\{\mathcal{G}^{i} \rightarrow \mathcal{G}\right\}_{i \in I}$, each of which is characteristic [i.e., any pull-back of the covering via an element of $\operatorname{Aut}(\mathcal{G})$ is isomorphic to the original covering]. [For instance, one verifies immediately, by applying the finiteness and coherence of $\mathcal{G}$, that such a collection of coverings may be obtained by considering, for $n$ a positive integer, the composite of all connected finite étale Galois coverings of degree $\leq n$.] We may assume, without loss of generality, that this collection of coverings arises from a projective system, which we denote by $\widetilde{\mathcal{G}}$. Thus, we obtain a natural exact sequence

$$
1 \longrightarrow \operatorname{Gal}(\widetilde{\mathcal{G}} / \mathcal{G}) \longrightarrow \operatorname{Aut}(\widetilde{\mathcal{G}} / \mathcal{G}) \longrightarrow \operatorname{Aut}(\mathcal{G}) \longrightarrow 1
$$

— where we write "Aut $(\widetilde{\mathcal{G}} / \mathcal{G})$ " for the group of pairs of compatible automorphisms of $\widetilde{\mathcal{G}}$ and $\mathcal{G}$.

This observation (O1) has the following immediate consequence:

(O2) Suppose that we are in the situation of (O1). Consider, for $i \in I$, the finite index normal subgroup

$$
\operatorname{Aut}^{i}(\widetilde{\mathcal{G}} / \mathcal{G}) \subseteq \operatorname{Aut}(\widetilde{\mathcal{G}} / \mathcal{G})
$$

of elements of $\operatorname{Aut}(\widetilde{\mathcal{G}} / \mathcal{G})$ that induce the identity automorphism on the underlying semi-graph $\mathbb{G}^{i}$ of $\mathcal{G}^{i}$, as well as on $\operatorname{Gal}\left(\mathcal{G}^{i} / \mathcal{G}\right)$. Then one verifies immediately [from the definition of a semi-graph of anabelioids; cf. also [SemiAnbd], Proposition 2.5, (i)] that the intersection of the $\operatorname{Aut}^{i}(\widetilde{\mathcal{G}} / \mathcal{G})$, for $i \in I$, is $=\{1\}$. Thus, the $\operatorname{Aut}^{i}(\widetilde{\mathcal{G}} / \mathcal{G})$, for $i \in I$, determine a natural profinite topology on $\operatorname{Aut}(\widetilde{\mathcal{G}} / \mathcal{G})$ and hence also on the quotient $\operatorname{Aut}(\mathcal{G})$, which is easily seen to be compatible with the profinite topology on $\operatorname{Gal}(\widetilde{\mathcal{G}} / \mathcal{G})$ and, moreover, independent of the choice of $\widetilde{\mathcal{G}}$.

The new version of the condition (c) of [SemiAnbd], Definition 5.1, (i), that we wish to consider is the following:

( $\left.\mathrm{c}^{\text {new }}\right)$ The action of $H$ on $\mathbb{G}$ is trivial; the resulting homomorphism $H \rightarrow$ $\operatorname{Aut}(\mathcal{G}[c])$, where $c$ ranges over the components [i.e., vertices and edges] 
of $\mathbb{G}$, is continuous [i.e., relative to the natural profinite group topology defined in $(\mathrm{O} 2)$ on $\operatorname{Aut}(\mathcal{G}[c])]$.

It is immediate that $\left(\mathrm{c}^{\text {new }}\right)$ implies $(\mathrm{c})$. Moreover, we observe in passing that:

(O3) In fact, since $H$ is topologically finitely generated [cf. [SemiAnbd], Definition 5.1, (i), (a)], it holds [cf. [NS], Theorem 1.1] that every finite index subgroup of $H$ is open in $H$. Thus, the conditions (c) and ( $\left.\mathrm{c}^{\text {new }}\right)$ in fact hold automatically.

The new version of the condition (d) of [SemiAnbd], Definition 5.1, (i), that we wish to consider is the following:

$\left(\mathrm{d}^{\text {new }}\right)$ There is a finite set $C^{*}$ of components [i.e., vertices and edges] of $\mathbb{G}$ such that for every component $c$ of $\mathbb{G}$, there exists a $c^{*} \in C^{*}$ and an isomorphism of semi-graphs of anabelioids $\mathcal{G}[c] \stackrel{\sim}{\rightarrow} \mathcal{G}\left[c^{*}\right]$ that is compatible with the action of $H$ on both sides.

It is immediate that $\left(\mathrm{d}^{\text {new }}\right)$ implies $(\mathrm{d})$. The reason that, in the context of the proof of [SemiAnbd], Proposition 5.2, (i), it is necessary to consider the stronger conditions $\left(\mathrm{c}^{\text {new }}\right)$ and $\left(\mathrm{d}^{\text {new }}\right)$ is as follows. It suffices to show that, given a connected finite étale covering $\mathcal{G}^{\prime} \rightarrow \mathcal{G}$, after possibly replacing $H$ by an open subgroup of $H$, the action of $H$ on $\mathcal{G}$ lifts to an action on $\mathcal{G}^{\prime}$ that satisfies the conditions of [SemiAnbd], Definition 5.1, (i). Such a lifting of the action of $H$ on $\mathcal{G}$ to an action on the portion of $\mathcal{G}^{\prime}$ that lies over the vertices of $\mathbb{G}$ follows in a straightforward manner from the original conditions (a), (b), (c), and (d). On the other hand, in order to conclude that such a lifting is [after possibly replacing $H$ by an open subgroup of $H$ ] compatible with the gluing conditions arising from the structure of $\mathcal{G}^{\prime}$ over the edges of $\mathbb{G}$, it is necessary to assume further that the "component-wise versions ( $\mathrm{c}^{\text {new }}$ ), ( $\mathrm{d}^{\text {new }}$ )" of the original "vertex-wise conditions $(c),(d)$ " hold. This issue is closely related to the issue discussed in (iii) above.

Finally, we observe that Proposition 2.4, Corollary 2.5 admit the following discrete analogues, which may be regarded as generalizations of [André], Lemma 3.2.1 [cf. Theorem 2.6 below in the case where $H=F=G$ is free]; [EtTh], Lemma 2.17, (i).

Theorem 2.6. (Profinite Conjugates of Discrete Subgroups) Let $F$ be a group that contains a subgroup of finite index $G \subseteq F$ such that $G$ is either a free discrete group of finite rank or an orientable surface group [i.e., a fundamental group of a compact orientable topological surface of genus $\geq 2$ ]; $H \subseteq F$ an infinite subgroup. Since $F$ is residually finite [cf., e.g., [Config], Proposition 7.1, (ii)], we shall write $H, G \subseteq F \subseteq \widehat{F}$, where $\widehat{F}$ denotes the profinite completion of $F$. Let $\gamma \in \widehat{F}$ be an element such that

$$
\left.\gamma \cdot H \cdot \gamma^{-1} \subseteq F \quad \text { [or, equivalently, } H \subseteq \gamma^{-1} \cdot F \cdot \gamma\right] .
$$

Write $H_{G} \stackrel{\text { def }}{=} H \bigcap G$. Then $\gamma \in F \cdot N_{\widehat{F}}\left(H_{G}\right)$, i.e., $\gamma \cdot H_{G} \cdot \gamma^{-1}=\delta \cdot H_{G} \cdot \delta^{-1}$, for some $\delta \in F$. If, moreover, $H_{G}$ is nonabelian, then $\gamma \in F$.

Proof. Let us first consider the case where $H_{G}$ is abelian. In this case, it follows from Lemma 2.7, (iv), below, that $H_{G}$ is cyclic. Thus, by applying Lemma 2.7, 
(ii), it follows that by replacing $G$ by an appropriate finite index subgroup of $G$, we may assume that the natural composite homomorphism $H_{G} \hookrightarrow G \rightarrow G^{\text {ab }}$ is a split injection. In particular, by Lemma $2.7,(\mathrm{v})$, we conclude that $N_{\widehat{G}}\left(H_{G}\right)=\widehat{H}_{G}$, where we write $\widehat{H}_{G}$ for the closure of $H_{G}$ in the profinite completion $\widehat{G}$ of $G$. Next, let us observe that by multiplying $\gamma$ on the left by an appropriate element of $F$, we may assume that $\gamma \in \widehat{G}$. Thus, we have $\gamma \cdot H_{G} \cdot \gamma^{-1} \subseteq F \cap \widehat{G}=G$. Next, let us recall that $G$ is conjugacy separable. Indeed, this is precisely the content of [Stb1], Theorem 1, when $G$ is free; [Stb2], Theorem 3.3, when $G$ is an orientable surface group. Since $G$ is conjugacy separable, it follows that $\gamma \cdot H_{G} \cdot \gamma^{-1}=\epsilon \cdot H_{G} \cdot \epsilon^{-1}$ for some $\epsilon \in G$, so $\gamma \in G \cdot N_{\widehat{G}}\left(H_{G}\right)=G \cdot \widehat{H}_{G} \subseteq F \cdot N_{\widehat{F}}\left(H_{G}\right)$, as desired. This completes the proof of Theorem 2.6 when $H_{G}$ is abelian.

Thus, let us assume for the remainder of the proof of Theorem 2.6 that $H_{G}$ is nonabelian. Then, by applying Lemma 2.7, (iii), it follows that, after replacing $G$ by an appropriate finite index subgroup of $G$, we may assume that there exist elements $x, y \in H_{G}$ that generate a free abelian subgroup of rank two $M \subseteq G^{\text {ab }}$ such that the injection $M \hookrightarrow G^{\text {ab }}$ splits. Write $H_{x}, H_{y} \subseteq H_{G}$ for the subgroups generated, respectively, by $x$ and $y ; \widehat{H}_{x}, \widehat{H}_{y} \subseteq \widehat{G}$ for the respective closures of $H_{x}, H_{y}$. Then by Lemma 2.7 , (v), we conclude that $N_{\widehat{G}}\left(H_{x}\right)=\widehat{H}_{x}, N_{\widehat{G}}\left(H_{y}\right)=\widehat{H}_{y}$. Next, let us observe that by multiplying $\gamma$ on the left by an appropriate element of $F$, we may assume that $\gamma \in \widehat{G}$. Thus, we have $\gamma \cdot H_{G} \cdot \gamma^{-1} \subseteq F \cap \widehat{G}=G$. In particular, by applying the portion of Theorem 2.6 that has already been proven to the subgroups $H_{x}, H_{y} \subseteq G$, we conclude that $\gamma \in G \cdot N_{\widehat{G}}\left(H_{x}\right)=G \cdot \widehat{H}_{x}, \gamma \in G \cdot N_{\widehat{G}}\left(H_{y}\right)=G \cdot \widehat{H}_{y}$. Thus, by projecting to $\widehat{G}^{\mathrm{ab}}$, and applying the fact that $M$ is of rank two, we conclude that $\gamma \in G$, as desired. This completes the proof of Theorem 2.6.

Remark 2.6.1. Note that in the situation of Theorem 2.6, if $H_{G}$ is abelian, then - unlike the tempered case discussed in Proposition 2.4! - it is not necessarily the case that $F=\gamma^{-1} \cdot F \cdot \gamma$.

Lemma 2.7. (Well-known Properties of Free Groups and Orientable Surface Groups) Let $G$ be a group as in Theorem 2.6. Write $\widehat{G}$ for the profinite completion of $G$. Then:

(i) Any subgroup of $G$ generated by two elements of $G$ is free.

(ii) Let $x \in G$ be an element $\neq 1$. Then there exists a finite index subgroup $G_{1} \subseteq G$ such that $x \in G_{1}$, and $x$ has nontrivial image in the abelianization $G_{1}^{\text {ab }}$ of $G_{1}$.

(iii) Let $x, y \in G$ be noncommuting elements of $G$. Then there exists a finite index subgroup $G_{1} \subseteq G$ and a positive integer $n$ such that $x^{n}, y^{n} \in G_{1}$, and the images of $x^{n}$ and $y^{n}$ in the abelianization $G_{1}^{\mathrm{ab}}$ of $G_{1}$ generate a free abelian subgroup of rank two.

(iv) Any abelian subgroup of $G$ is cyclic.

(v) Let $\widehat{T} \subseteq \widehat{G}$ be a closed subgroup such that there exists a continuous surjection of topological groups $\widehat{G} \rightarrow \widehat{\mathbb{Z}}$ that induces an isomorphism $\widehat{T} \stackrel{\sim}{\rightarrow} \widehat{\mathbb{Z}}$. Then $\widehat{T}$ is normally terminal in $\widehat{G}$. 
(vi) Suppose that $G$ is nonabelian. Write $\widehat{N} \subseteq \widehat{G}$ for the kernel of the natural surjection $\widehat{G} \rightarrow \widehat{G}^{\text {ab }}$ to the abelianization $\widehat{G}^{\text {ab }}$ of $\widehat{G}$. Then the centralizer $Z_{\widehat{G}}(\widehat{N})$ of $\widehat{N}$ in $\widehat{G}$ is trivial.

(vii) In the notation of (vi), let $\alpha$ be an automorphism of the profinite group $\widehat{G}$ that preserves and restricts to the identity on the subgroup $\widehat{N}$. Then $\alpha$ is the identity automorphism of $\widehat{G}$.

Proof. First, we consider assertion (i). If $G$ is free, then assertion (i) follows from the well-known fact that any subgroup of a free group is free. If $G$ is an orientable surface group, then assertion (i) follows immediately — i.e., by considering the noncompact covering of a compact surface that corresponds to an infinite index subgroup of $G$ of the sort discussed in assertion (i) - from a classical result concerning the fundamental group of a noncompact surface due to Johansson [cf. [Stl], p. 142; the discussion preceding [FRS], Theorem A1]. This completes the proof of assertion (i). Next, we consider assertion (ii). Since $G$ is residually finite [cf., e.g., [Config], Proposition 7.1, (ii)], it follows that there exists a finite index normal subgroup $G_{0} \subseteq G$ such that $x \notin G_{0}$. Thus, it suffices to take $G_{1}$ to be the subgroup of $G$ generated by $G_{0}$ and $x$. This completes the proof of assertion (ii).

Next, we consider assertion (iii). By applying assertion (i) to the subgroup $J$ of $G$ generated by $x$ and $y$, it follows from the fact that $x$ and $y$ are noncommuting elements of $G$ that $J$ is a free group of rank 2 , hence that $x^{a} \cdot y^{b} \neq 1$, for all $(a, b) \in \mathbb{Z} \times \mathbb{Z}$ such that $(a, b) \neq(0,0)$. Next, let us recall the well-known fact that the abelianization of any finite index subgroup of $G$ is torsion-free. Thus, by applying assertion (ii) to $x$ and $y$, we conclude that there exists a finite index subgroup $G_{0} \subseteq G$ and a positive integer $m$ such that $x^{m}, y^{m} \in G_{0}$, and $x^{m}$ and $y^{m}$ have nontrivial image in the abelianization $G_{0}^{\mathrm{ab}}$ of $G_{0}$. Now suppose that $x^{m a} \cdot y^{m b}$ lies in the kernel of the natural surjection $G_{0} \rightarrow G_{0}^{\mathrm{ab}}$ for some $(a, b) \in \mathbb{Z} \times \mathbb{Z}$ such that $(a, b) \neq(0,0)$. Since $G$ is residually finite, and [as we observed above] $x^{m a} \cdot y^{m b} \neq 1$, it follows, by applying assertion (ii) to $G_{0}$, that there exists a finite index subgroup $G_{1} \subseteq G_{0}$ and a positive integer $n$ that is divisible by $m$ such that $x^{n}, y^{n}, x^{m a} \cdot y^{m b} \in G_{1}$, and the image of $x^{m a} \cdot y^{m b}$ in $G_{1}^{\mathrm{ab}}$ is nontrivial. Since $G_{1}^{\mathrm{ab}}$ is torsion-free, it thus follows that the image of $x^{n a} \cdot y^{n b}$ in $G_{1}^{\text {ab }}$ is nontrivial. On the other hand, by considering the natural homomorphism $G_{1}^{\mathrm{ab}} \rightarrow G_{0}^{\mathrm{ab}}$, we thus conclude that the images of $x^{n}$ and $y^{n}$ in $G_{1}^{\text {ab }}$ generate a free abelian subgroup of rank two, as desired. This completes the proof of assertion (iii).

Next, we consider assertion (iv). By assertion (i), it follows that any abelian subgroup of $G$ generated by two elements is free, hence cyclic. In particular, we conclude that any abelian subgroup $J$ of $G$ is equal to the union of the groups that appear in some chain $G_{1} \subseteq G_{2} \subseteq \ldots \subseteq G$ of cyclic subgroups of $G$. On the other hand, by applying assertion (ii) to some generator of $G_{1}$, it follows that there exists a finite index subgroup $G_{0}$ and a positive integer $n$ such that $G_{j}^{n} \subseteq G_{0}$ for all $j=1,2, \ldots$, and, moreover, $G_{1}^{n}$ has nontrivial image in $G_{0}^{\mathrm{ab}}$. Thus, by considering the image in [the finitely generated abelian group] $G_{0}^{\text {ab }}$ of the chain of cyclic subgroups $G_{1}^{n} \subseteq G_{2}^{n} \subseteq \ldots$, we conclude that this chain, hence also the original chain $G_{1} \subseteq G_{2} \subseteq \ldots$, must terminate. Thus, $J$ is cyclic, as desired. This completes the proof of assertion (iv). 
Next, we consider assertion (v). By considering the surjection $\widehat{G} \rightarrow \widehat{\mathbb{Z}}$, we conclude immediately that the normalizer $N_{\widehat{G}}(\widehat{T})$ of $\widehat{T}$ in $\widehat{G}$ is equal to the centralizer $Z_{\widehat{G}}(\widehat{T})$ of $\widehat{T}$ in $\widehat{G}$. If $Z_{\widehat{G}}(\widehat{T}) \neq \widehat{T}$, then it follows immediately that, for some prime number $l$, there exists a closed [abelian] subgroup $\widehat{T}_{1} \subseteq Z_{\widehat{G}}(\widehat{T})$ containing the pro- $l$ portion of $\widehat{T}$ such that there exists a continuous surjection $\mathbb{Z}_{l} \times \mathbb{Z}_{l} \rightarrow \widehat{T}_{1}$ whose kernel lies in $l \cdot\left(\mathbb{Z}_{l} \times \mathbb{Z}_{l}\right)$. In particular, one computes easily that the $l$-cohomological dimension of $\widehat{T}_{1}$ is $\geq 2$. On the other hand, since $\widehat{T}_{1}$ is of infinite index in $\widehat{G}$, it follows immediately that there exists an open subgroup $\widehat{G}_{1} \subseteq \widehat{G}$ of $\widehat{G}$ such that $\widehat{T}_{1} \subseteq \widehat{G}_{1}$, and, moreover, there exists a continuous surjection $\phi: \widehat{G}_{1} \rightarrow \mathbb{Z}_{l}$ whose kernel $\operatorname{Ker}(\phi)$ contains $\widehat{T}_{1}$. In particular, since the cohomology of $\widehat{T}_{1}$ may be computed as the direct limit of the cohomologies of open subgroups of $\widehat{G}$ containing $\widehat{T}_{1}$, it follows immediately from the existence of $\phi$, together with the well-known structure of the cohomology of open subgroups of $\widehat{G}$, that the $l$-cohomological dimension of $\widehat{T}_{1}$ is 1 , a contradiction. This completes the proof of assertion (v).

Next, we consider assertion (vi). Write $N \subseteq G$ for the kernel of the natural surjection $G \rightarrow G^{\text {ab }}$ to the abelianization $G^{\text {ab }}$ of $G$. It follows immediately from the "tautological universal property" of a free group or an orientable surface group [i.e., regarded as the quotient of a free group by a single relation] that $N$ is not cyclic, hence by assertion (iv), that $N$ is nonabelian. Thus, by assertion (iii), there exist a finite index subgroup $G_{1} \subseteq G$ equipped with a surjection $\beta: G_{1} \rightarrow \mathbb{Z} \times \mathbb{Z}$ and elements $x, y \in N \cap G_{1}$ such that $\beta(x)=(1,0)$ and $\beta(y)=(0,1)$. In particular, it follows from assertion $(\mathrm{v})$ that the closed subgroups $\widehat{T}_{x}, \widehat{T}_{y} \subseteq \widehat{G}$ topologically generated by $x$ and $y$, respectively, are normally terminal in the profinite completion $\widehat{G}_{1} \subseteq \widehat{G}$ of $G_{1}$. But this implies formally that $Z_{\widehat{G}}(\widehat{N}) \bigcap \widehat{G}_{1} \subseteq Z_{\widehat{G}_{1}}\left(\widehat{T}_{x}\right) \bigcap Z_{\widehat{G}_{1}}\left(\widehat{T}_{y}\right) \subseteq \widehat{T}_{x} \bigcap \widehat{T}_{y}=\{1\}$ [where the last equality follows from the existence of the surjection $\widehat{G}_{1} \rightarrow \widehat{\mathbb{Z}} \times \widehat{\mathbb{Z}}$ induced by $\beta$ ]. Since [as is well-known] the abelianizations of all open subgroups of $\widehat{G}$ are torsion-free, we thus conclude that $Z_{\widehat{G}}(\widehat{N})=\{1\}$, as desired. This completes the proof of assertion (vi). Finally, we consider assertion (vii). If $x \in \widehat{G}, y \in \widehat{N}$ [so $\left.x \cdot y \cdot x^{-1} \in \widehat{N}\right]$, then $x \cdot y \cdot x^{-1}=\alpha\left(x \cdot y \cdot x^{-1}\right)=\alpha(x) \cdot \alpha(y) \cdot \alpha(x)^{-1}=\alpha(x) \cdot y \cdot \alpha(x)^{-1}$. We thus conclude from assertion (vi) that $\alpha(x) \cdot x^{-1} \in Z_{\widehat{G}}(\widehat{N})=\{1\}$, i.e., that $\alpha(x)=x$. This completes the proof of assertion (vii).

Corollary 2.8. (Subgroups of Topological Fundamental Groups of Complex Hyperbolic Curves) Let $Z$ be a hyperbolic curve over $\mathbb{C}$. Write $\Pi_{Z}$ for the usual topological fundamental group of $Z ; \widehat{\Pi}_{Z}$ for the profinite completion of $\Pi_{Z}$. Let $H \subseteq \Pi_{Z}$ be an infinite subgroup [such as a cuspidal inertia group!]; $\gamma \in \widehat{\Pi}_{Z}$ an element such that

$$
\left.\gamma \cdot H \cdot \gamma^{-1} \subseteq \Pi_{Z} \quad \text { [or, equivalently, } H \subseteq \gamma^{-1} \cdot \Pi_{Z} \cdot \gamma\right] .
$$

Then $\gamma \in \Pi_{Z} \cdot N_{\widehat{\Pi}_{Z}}(H)$, i.e., $\gamma \cdot H \cdot \gamma^{-1}=\delta \cdot H \cdot \delta^{-1}$, for some $\delta \in \Pi_{Z}$. If, moreover, $H$ is nonabelian, then $\gamma \in \Pi_{Z}$.

Remark 2.8.1. Corollary 2.8 is an immediate consequence of Theorem 2.6. In fact, in the present series of papers, we shall only apply Corollary 2.8 in the case 
where $Z$ is non-proper, and $H$ is a cuspidal inertia group. In this case, the proof of Theorem 2.6 may be simplified somewhat, but we chose to include the general version given here, for the sake of completeness. 


\section{Section 3: Chains of $\Theta$-Hodge Theaters}

In the present $\S 3$, we construct chains of " $\Theta$-Hodge theaters". Each " $\Theta$-Hodge theater" is to be thought of as a sort of miniature model of the conventional scheme-theoretic arithmetic geometry that surrounds the theta function. This miniature model is formulated via the theory of Frobenioids [cf. [FrdI]; [FrdII]; [EtTh], §3, §4, §5]. On the other hand, the link [cf. Corollary 3.7, (i)] between adjacent members of such chains is purely Frobenioid-theoretic, i.e., it lies outside the framework of ring theory/scheme theory. It is these chains of $\Theta$-Hodge theaters that form the starting point of the theory of the present series of papers.

Definition 3.1. We shall refer to as initial $\Theta$-data any collection of data

$$
\left(\bar{F} / F, X_{F}, l, \underline{C}_{K}, \underline{\mathbb{V}}, \mathbb{V}_{\bmod }^{\mathrm{bad}}, \underline{\epsilon}\right)
$$

that satisfies the following conditions:

(a) $F$ is a number field such that $\sqrt{-1} \in F ; \bar{F}$ is an algebraic closure of $F$. Write $G_{F} \stackrel{\text { def }}{=} \operatorname{Gal}(\bar{F} / F)$.

(b) $X_{F}$ is a once-punctured elliptic curve [i.e., a hyperbolic curve of type $(1,1)$ ] over $F$ that admits stable reduction over all $v \in \mathbb{V}(F)^{\text {non }}$. Write $E_{F}$ for the elliptic curve over $F$ determined by $X_{F}$ [so $\left.X_{F} \subseteq E_{F}\right]$;

$$
X_{F} \rightarrow C_{F}
$$

for the hyperbolic orbicurve [cf. $§ 0]$ over $F$ obtained by forming the stacktheoretic quotient of $X_{F}$ by the unique $F$-involution [i.e., automorphism of order two] " -1 " of $X_{F} ; F_{\bmod } \subseteq F$ for the field of moduli [cf., e.g., [AbsTopIII], Definition 5.1, (ii)] of $X_{F} ; F_{\text {sol }} \subseteq \bar{F}$ for the maximal solvable extension of $F_{\text {mod }}$ in $\bar{F} ; \mathbb{V}_{\text {mod }} \stackrel{\text { def }}{=} \mathbb{V}\left(F_{\text {mod }}\right)$. Then

$$
\mathbb{V}_{\bmod }^{\text {bad }} \subseteq \mathbb{V}_{\text {mod }}
$$

is a nonempty set of nonarchimedean valuations of $F_{\bmod }$ of odd residue characteristic such that $X_{F}$ has bad [i.e., multiplicative] reduction at the

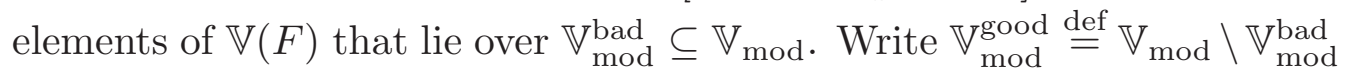
[where we note that $X_{F}$ may in fact have bad reduction at some of the elements of $\mathbb{V}(F)$ that lie over $\mathbb{V}_{\bmod }^{\text {good }} \subseteq \mathbb{V}_{\text {mod }} ! ; ; \mathbb{V}(F) \stackrel{\text { def }}{=} \mathbb{V}_{\bmod }^{\square} \times_{\mathbb{V}_{\text {mod }}} \mathbb{V}(F)$ for $\square \in\{$ bad, good $\}$;

$$
\begin{gathered}
\Pi_{X_{F}} \stackrel{\text { def }}{=} \pi_{1}\left(X_{F}\right) \subseteq \Pi_{C_{F}} \stackrel{\text { def }}{=} \pi_{1}\left(C_{F}\right) \\
\Delta_{X} \stackrel{\text { def }}{=} \pi_{1}\left(X_{F} \times_{F} \bar{F}\right) \subseteq \Delta_{C} \stackrel{\text { def }}{=} \pi_{1}\left(C_{F} \times_{F} \bar{F}\right)
\end{gathered}
$$

for the étale fundamental groups [relative to appropriate choices of basepoints] of $X_{F}, C_{F}, X_{F} \times_{F} \bar{F}, C_{F} \times_{F} \bar{F}$. [Thus, we have natural exact sequences $1 \rightarrow \Delta_{(-)} \rightarrow \Pi_{(-)_{F}} \rightarrow G_{F} \rightarrow 1$ for "(-)" taken to be either " $X$ " or " $C$ ".] Here, we suppose further that the field extension $F / F_{\bmod }$ 
is Galois of degree prime to $l$, and that the $2 \cdot 3$-torsion points of $E_{F}$ are rational over $F$.

(c) $l$ is a prime number $\geq 5$ such that the image of the outer homomorphism

$$
G_{F} \rightarrow G L_{2}\left(\mathbb{F}_{l}\right)
$$

determined by the l-torsion points of $E_{F}$ contains the subgroup $S L_{2}\left(\mathbb{F}_{l}\right) \subseteq$ $G L_{2}\left(\mathbb{F}_{l}\right)$; write $K \subseteq \bar{F}$ for the finite Galois extension of $F$ determined by the kernel of this homomorphism. Also, we suppose that $l$ is prime to the [residue characteristics of the] elements of $\mathbb{V}_{\text {mod }}^{\text {mad }}$, as well as to the orders of the q-parameters of $E_{F}$ [i.e., in the terminology of [GenEll], Definition 3.3 , the "local heights" of $\left.E_{F}\right]$ at the primes of $\mathbb{V}(F)$ bad.

(d) $\underline{C}_{K}$ is a hyperbolic orbicurve of type $(1, l \text {-tors })_{ \pm}$[cf. [EtTh], Definition 2.1] over $K$, with $K$-core [cf. [CanLift], Remark 2.1.1; [EtTh], the discussion at the beginning of $\S 2$ ] given by $C_{K} \stackrel{\text { def }}{=} C_{F} \times_{F} K$. [Thus, by (c), it follows that $\underline{C}_{K}$ is completely determined, up to isomorphism over $F$, by $C_{F}$.] In particular, $\underline{C}_{K}$ determines, up to $K$-isomorphism, a hyperbolic orbicurve $\underline{X}_{K}$ of type (1,l-tors) [cf. [EtTh], Definition 2.1] over $K$, together with natural cartesian diagrams

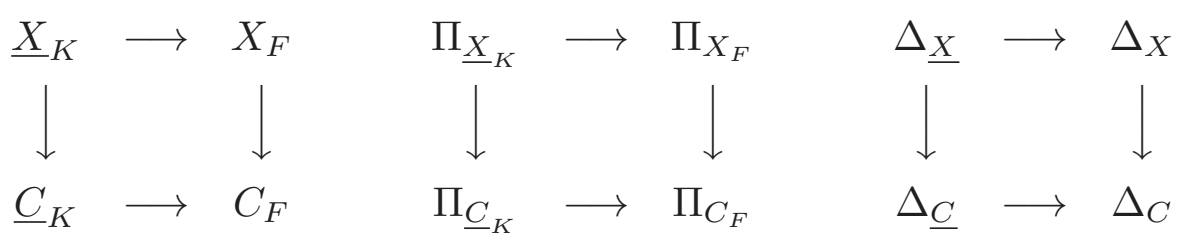

of finite étale coverings of hyperbolic orbicurves and corresponding open immersions of profinite groups. Finally, we recall from [EtTh], Proposition 2.2 , that $\Delta_{\underline{C}}$ admits uniquely determined open subgroups $\Delta_{\underline{\underline{X}}} \subseteq \Delta_{\underline{\underline{C}}} \subseteq$ $\Delta_{\underline{C}}$, which may be thought of as corresponding to finite étale coverings of $\underline{C_{\bar{F}}} \stackrel{\text { def }}{=} \underline{C} \times_{F} \bar{F}$ by hyperbolic orbicurves $\underline{\underline{X}}_{\bar{F}}, \underline{\underline{C}}_{\bar{F}}$ of type $\left(1, l\right.$-tors $\left.{ }^{\Theta}\right)$, $\left(1, l \text {-tors }{ }^{\Theta}\right)_{ \pm}$, respectively [cf. [EtTh], Definition 2.3].

(e) $\mathbb{V} \subseteq \mathbb{V}(K)$ is a subset that induces a natural bijection

$$
\underline{\mathbb{V}} \stackrel{\sim}{\rightarrow} \mathbb{V}_{\bmod }
$$

- i.e., a section of the natural surjection $\mathbb{V}(K) \rightarrow \mathbb{V}_{\text {mod }}$. Write $\underline{\mathbb{V}}^{\text {non }} \stackrel{\text { def }}{=}$ $\underline{\mathbb{V}} \cap \mathbb{V}(K)^{\text {non }}, \underline{\mathbb{V}}^{\text {arc }} \stackrel{\text { def }}{=} \underline{\mathbb{V}} \cap \mathbb{V}(K)^{\text {arc }}, \underline{\mathbb{V}}^{\text {good }} \stackrel{\text { def }}{=} \underline{\mathbb{V}} \cap \mathbb{V}(K)^{\text {good }}, \underline{\mathbb{V}}^{\text {bad }} \stackrel{\text { def }}{=}$ $\underline{\mathbb{V}} \cap \mathbb{V}(K)^{\text {bad }}$. For each $\underline{v} \in \mathbb{V}(K)$, we shall use the subscript $\underline{v}$ to denote the result of base-changing hyperbolic orbicurves over $F$ or $K$ to $K_{\underline{v}}$. Thus, for each $\underline{v} \in \mathbb{V}(K)$ lying under a $\bar{v} \in \mathbb{V}(\bar{F})$, we have natural cartesian diagrams

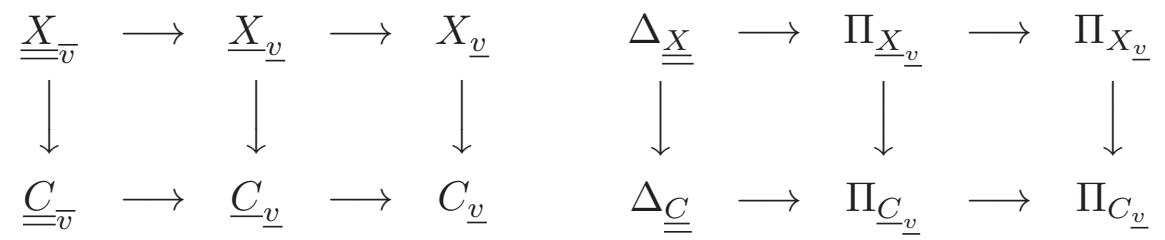

of profinite étale coverings of hyperbolic orbicurves and corresponding injections of profinite groups [i.e., étale fundamental groups]. Here, the 
subscript $\bar{v}$ denotes base-change with respect to $\bar{F} \hookrightarrow \bar{F}_{\bar{v}}$; the various profinite groups " $\Pi_{(-)}$" admit natural outer surjections onto the decomposition group $G_{\underline{v}} \subseteq G_{K} \stackrel{\text { def }}{=} \operatorname{Gal}(\bar{F} / K)$ determined, up to $G_{K}$-conjugacy, by $\underline{v}$. If $\underline{v} \in \underline{\mathbb{V}}^{\text {bad }}$, then we assume further that the hyperbolic orbicurve $\underline{C}_{\underline{v}}$ is of type $(1, \mathbb{Z} / l \mathbb{Z})_{ \pm}[\mathrm{cf}$. [EtTh], Definition 2.5, (i)]. [Here, we note that it follows from the portion of (b) concerning 2-torsion points that the base field $K_{\underline{v}}$ satisfies the assumption " $K=\ddot{K}$ " of [EtTh], Definition 2.5, (i).] Finally, we observe that when $\underline{v} \in \underline{\mathbb{V}}^{\text {bad }}$, it follows from the theory of [EtTh], $\S 2$ - i.e., roughly speaking, "by extracting an l-th root of the theta function" - that $\underline{\underline{X}}_{\bar{v}}, \underline{\underline{C}}_{\bar{v}}$ admit natural models

$$
\underline{\underline{X}} \underline{\underline{v}}, \quad \underline{\underline{C}}
$$

over $K_{\underline{v}}$, which are hyperbolic orbicurves of type $\left(1,(\mathbb{Z} / l \mathbb{Z})^{\Theta}\right),\left(1,(\mathbb{Z} / l \mathbb{Z})^{\Theta}\right)_{ \pm}$, respectively [cf. [EtTh], Definition 2.5, (i)]; these models determine open subgroups $\Pi_{\underline{\underline{X}}_{v}} \subseteq \Pi_{\underline{\underline{C}}_{v}} \subseteq \Pi_{\underline{C}_{\underline{v}}}$. If $\underline{v} \in \underline{\mathbb{V}}^{\text {bad }}$, then, relative to the notation of Remark 3.1.1 below, we shall write $\Pi_{\underline{v}} \stackrel{\text { def }}{=} \Pi_{\underline{\underline{X}}}^{\text {tp }}$.

(f) $\underline{\epsilon}$ is a cusp of the hyperbolic orbicurve $\underline{C}_{K}[\mathrm{cf}$. (d)] that arises from a nonzero element of the quotient " $Q$ " that appears in the definition of a "hyperbolic orbicurve of type $(1, l \text {-tors })_{ \pm}$" given in [EtTh], Definition 2.1. If $\underline{v} \in \underline{\mathbb{V}}$, then let us write $\underline{\epsilon}_{\underline{v}}$ for the cusp of $\underline{C}_{\underline{v}}$ determined by $\underline{\epsilon}$. If $\underline{v} \in \underline{\mathbb{V}}^{\text {bad }}$, then we assume that $\underline{\epsilon}_{\underline{v}}$ is the cusp that arises from the canonical generator [up to sign] " \pm 1 " of the quotient " $\widehat{\mathbb{Z} " ~ t h a t ~ a p p e a r s ~}$ in the definition of a "hyperbolic orbicurve of type $(1, \mathbb{Z} / l \mathbb{Z})_{ \pm}$" given in [EtTh], Definition 2.5, (i). Thus, the data $\left(X_{K} \stackrel{\text { def }}{=} X_{F} \times_{F} K, \underline{C}_{K}, \underline{\epsilon}\right)$ determines hyperbolic orbicurves

$$
\stackrel{X}{\rightarrow}_{K}, \quad \underline{C}_{K}
$$

of type $(1, l$-tors $),(1, l \text {-tors })_{ \pm}$, respectively [cf. Definition 1.1, Remark 1.1.2], as well as open subgroups $\Pi_{\underline{X}_{K}} \subseteq \Pi_{\underline{G}_{K}} \subseteq \Pi_{C_{F}}, \Delta_{\underline{X}} \subseteq \Delta_{\underline{G}} \subseteq \Delta_{C}$, and, for $\underline{v} \in \underline{\mathbb{V}}^{\text {good }}, \Pi_{\underline{X}_{\underline{v}}} \subseteq \Pi_{\underline{C}_{\underline{v}}} \subseteq \Pi_{C_{\underline{v}}}$. If $\underline{v} \in \underline{\mathbb{V}}^{\text {good }}$, then we shall write $\Pi_{\underline{v}} \stackrel{\text { def }}{=} \Pi_{\underline{X}_{\underline{v}}}$.

Remark 3.1.1. Relative to the notation of Definition 3.1, (e), suppose that $\underline{v} \in \underline{\mathbb{V}}^{\text {non }}$. Then in addition to the various profinite groups $\Pi_{(-)_{v}}, \Delta_{(-)}$, one also has corresponding tempered fundamental groups

$$
\Pi_{(-)_{\underline{v}}}^{\mathrm{tp}} ; \quad \Delta_{(-)_{\underline{v}}}^{\mathrm{tp}}
$$

[cf. [André], §4; [SemiAnbd], Example 3.10], whose profinite completions may be identified with $\Pi_{(-)_{\underline{v}}}, \Delta_{(-)}$. Here, we note that unlike " $\Delta_{(-)}$", the topological group $\Delta_{(-)_{\underline{v}}}^{\mathrm{tp}}$ depends, a priori, on $\underline{v}$. 


\section{Remark 3.1.2.}

(i) Observe that the open subgroup $\Pi_{\underline{X}_{K}} \subseteq \Pi_{\underline{C}_{K}}$ may be constructed grouptheoretically from the topological group $\Pi_{\underline{C}_{K}}$. Indeed, it follows immediately from the construction of the coverings " $\underline{X}$ ", " $\underline{C}$ " in the discussion at the beginning of [EtTh], $\S 2$ [cf. also [AbsAnab], Lemma 1.1.4, (i)], that the closed subgroup $\Delta_{\underline{X}} \subseteq \Pi_{\underline{C}_{K}}$ may be characterized by a rather simple explicit algorithm. Since the decomposition groups of $\Pi_{\underline{C}_{K}}$ at the nonzero cusps - i.e., the cusps whose inertia groups are contained in $\Delta_{X}$ [cf. the discussion at the beginning of $\S 1$ - are also group-theoretic [cf., e.g., [AbsTopI], Lemma 4.5, as well as Remark 1.2.2, (ii), of the present paper], the above observation follows immediately from the easily verified fact that the image of any of these decomposition groups associated to nonzero cusps coincides with the image of $\Pi_{\underline{X}_{K}}$ in $\Pi_{\underline{C}_{K}} / \Delta_{\underline{X}}$.

(ii) In light of the observation of (i), it makes sense to adopt the following convention:

Instead of applying the group-theoretic reconstruction algorithm of [AbsTopIII], Theorem 1.9 [cf. also the discussion of [AbsTopIII], Remark 2.8.3], directly to $\Pi_{\underline{C}_{K}}$ [or topological groups isomorphic to $\Pi_{\underline{C}_{K}}$, we shall apply this reconstruction algorithm to the open subgroup $\underline{\Pi}_{\underline{X}_{K}} \subseteq$ $\Pi_{\underline{C}_{K}}$ to reconstruct the function field of $\underline{X}_{K}$, equipped with its natural $\operatorname{Gal}\left(\underline{X}_{K} / \underline{C}_{K}\right) \cong \Pi_{\underline{C}_{K}} / \Pi_{\underline{X}_{K}}$-action.

In this context, we shall refer to this approach of applying [AbsTopIII], Theorem 1.9, as the $\Theta$-approach to [AbsTopIII], Theorem 1.9. Note that, for $\underline{v} \in \underline{\mathbb{V}}^{\text {good }} \cap \underline{\mathbb{V}}^{\text {non }}$ (respectively, $\underline{v} \in \underline{\mathbb{V}}^{\text {bad }}$ ), one may also adopt a " $\Theta$-approach" to applying [AbsTopIII], Theorem 1.9 , to $\Pi_{\underline{C}_{\underline{v}}}$ or [by applying Corollary 1.2] $\Pi_{\underline{X}_{\underline{v}}}, \Pi_{\underline{C}_{\underline{v}}}$ (respectively, to $\Pi_{\underline{C}_{\underline{v}}}^{\mathrm{tp}}$ or [by applying [EtTh], Proposition 2.4] $\Pi_{\underline{\underline{X}}_{\underline{v}}}^{\mathrm{tp}}$ ). In the present series of papers, we shall always think of [AbsTopIII], Theorem 1.9 [as well as the other results of [AbsTopIII] that arise as consequences of [AbsTopIII], Theorem 1.9] as being applied to [isomorphs of] $\Pi_{\underline{C}_{K}}$ or, for $\underline{v} \in \underline{\mathbb{V}}^{\text {good }} \cap \underline{\mathbb{V}}^{\text {non }}$ (respectively, $\underline{v} \in \underline{\mathbb{V}}^{\text {bad }}$ ),

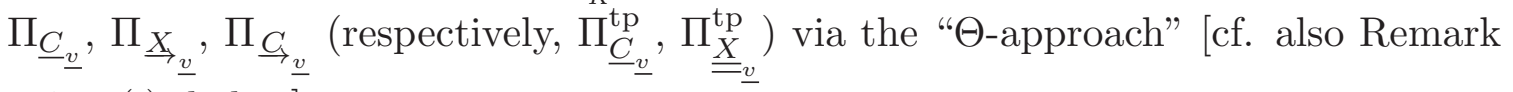
3.4.3, (i), below].

(iii) Recall from the discussion at the beginning of [EtTh], §2, the tautological extension

$$
1 \rightarrow \Delta_{\Theta} \rightarrow \Delta_{X}^{\Theta} \rightarrow \Delta_{X}^{\mathrm{ell}} \rightarrow 1
$$

— where $\Delta_{\Theta} \stackrel{\text { def }}{=}\left[\Delta_{X}, \Delta_{X}\right] /\left[\Delta_{X},\left[\Delta_{X}, \Delta_{X}\right]\right] ; \Delta_{X}^{\Theta} \stackrel{\text { def }}{=} \Delta_{X} /\left[\Delta_{X},\left[\Delta_{X}, \Delta_{X}\right]\right] ; \Delta_{X}^{\text {ell }} \stackrel{\text { def }}{=}$ $\Delta_{X}^{\mathrm{ab}}$. The extension class $\in H^{2}\left(\Delta_{X}^{\mathrm{ell}}, \Delta_{\Theta}\right)$ of this extension determines a tautological isomorphism

$$
M_{X} \stackrel{\sim}{\rightarrow} \Delta_{\Theta}
$$

- where we recall from [AbsTopIII], Theorem 1.9, (b), that the module " $M_{X}$ " of [AbsTopIII], Theorem 1.9, (b) [cf. also [AbsTopIII], Proposition 1.4, (ii); [AbsTopIII], Remark 1.10.1, (ii)], may be naturally identified with $\operatorname{Hom}\left(H^{2}\left(\Delta_{X}^{\text {ell }}, \widehat{\mathbb{Z}}\right), \widehat{\mathbb{Z}}\right)$. In particular, we obtain a tautological isomorphism

$$
M_{\underline{X}} \stackrel{\sim}{\rightarrow}\left(l \cdot \Delta_{\Theta}\right)
$$


[i.e., since $\left.\left[\Delta_{X}: \Delta_{X}\right]=l\right]$. In particular, we observe that if we write $\Pi_{C_{F_{\bmod }}}$ for the étale fundamental group of the orbicurve $C_{F_{\text {mod }}}$ discussed in Remark 3.1.7, (i), below, then $M_{\underline{X}} \stackrel{\sim}{\rightarrow}\left(l \cdot \Delta_{\Theta}\right)$ may be regarded as a characteristic subquotient of $\Pi_{C_{F_{\text {mod }}}}$, hence admits a natural conjugation action by $\Pi_{C_{F_{\bmod }}}$. From the point of view of the theory of the present series of papers, the significance of the " $\Theta$ approach" lies precisely in the existence of this tautological isomorphism $M_{\underline{X}} \stackrel{\sim}{\rightarrow}(l$. $\left.\Delta_{\Theta}\right)$, which will be applied in [IUTchII] at $\underline{v} \in \underline{\mathbb{V}}^{\text {bad }}$. That is to say, the $\Theta$-approach involves applying the reconstruction algorithm of [AbsTopIII], Theorem 1.9, via the cyclotome $M_{\underline{X}}$, which may be identified, via the above tautological isomorphism, with the cyclotome $\left(l \cdot \Delta_{\Theta}\right)$, which plays a central role in the theory of [EtTh] cf., especially, the discussion of "cyclotomic rigidity" in [EtTh], Corollary 2.19, (i).

(iv) If one thinks of the prime number $l$ as being "large", then the role played by the covering $\underline{X}$ in the above discussion of the " $\Theta$-approach" is reminiscent of the role played by the universal covering of a complex elliptic curve by the complex plane in the holomorphic reconstruction theory of [AbsTopIII], $§ 2$ [cf., e.g., [AbsTopIII], Propositions 2.5, 2.6].

Remark 3.1.3. Since $\mathbb{V}_{\text {mod }}^{\text {bad }} \neq \emptyset[$ cf. Definition 3.1, (b)], it follows immediately from Definition 3.1, (d), (e), (f), that the data $\left(\bar{F} / F, X_{F}, l, \underline{C}_{K}, \underline{\mathbb{V}}, \mathbb{V}_{\bmod }^{\text {bad }}, \underline{\epsilon}\right)$ is, in fact, completely determined by the data $\left(\bar{F} / F, X_{F}, \underline{C}_{K}, \underline{\mathbb{V}}, \mathbb{V}_{\bmod }^{\text {bad }}\right)$, and that $\underline{C}_{K}$ is completely determined up to $K$-isomorphism by the data $\left(\bar{F} / F, X_{F}, l, \underline{\mathbb{V}}\right)$. Finally, we remark that for given data $\left(X_{F}, l, \mathbb{V}_{\bmod }^{\text {bad }}\right)$, distinct choices of " $\mathbb{V}$ " will not affect the theory in any significant way.

Remark 3.1.4. It follows immediately from the definitions that at each $\underline{v} \in \underline{\mathbb{V}}^{\text {bad }}$ [which is necessarily prime to $l$ - cf. Definition 3.1, (c)] (respectively, each $\underline{v} \in$ $\underline{\mathbb{V}}^{\text {good }} \cap \underline{\mathbb{V}}^{\text {non }}$ which is prime to $l$; each $\left.\underline{v} \in \underline{\mathbb{V}}^{\text {good }} \cap \underline{\mathbb{V}}^{\text {non }}\right), \underline{\underline{X}}_{\underline{v}}$ (respectively, $\underline{X}_{\underline{v}}$; $\left.\underline{X}_{\underline{v}}\right)$ admits a stable model over the ring of integers of $K_{\underline{v}}$.

Remark 3.1.5. Note that since the 3-torsion points of $E_{F}$ are rational over $F$, and $F$ is Galois over $F_{\text {mod }}$ [cf. Definition 3.1, (b)], it follows [cf., e.g., [IUTchIV], Proposition 1.8, (iv)] that $K$ is Galois over $F_{\bmod }$. In addition to working with the field $F_{\text {mod }}$ and various extensions of $F_{\text {mod }}$ contained in $\bar{F}$, we shall also have occasion to work with the algebraic stack

$$
S_{\text {mod }} \stackrel{\text { def }}{=} \operatorname{Spec}\left(\mathcal{O}_{K}\right) / / \operatorname{Gal}\left(K / F_{\text {mod }}\right)
$$

obtained by forming the stack-theoretic quotient [i.e., "//"] of the spectrum of the ring of integers $\mathcal{O}_{K}$ of $K$ by the Galois group $\operatorname{Gal}\left(K / F_{\text {mod }}\right)$. Thus, any finite extension $L \subseteq \bar{F}$ of $F_{\text {mod }}$ in $\bar{F}$ determines, by forming the integral closure of $S_{\bmod }$ in $L$, an algebraic stack $S_{\text {mod } L}$ over $S_{\text {mod }}$. In particular, by considering arithmetic line bundles over such $S_{\text {mod }, L}$, one may associate to any finite quotient $\operatorname{Gal}\left(\bar{F} / F_{\bmod }\right) \rightarrow Q$ a Frobenioid via [the easily verified "stack-theoretic version" of] the construction of [FrdI], Example 6.3. One verifies immediately that an appropriate analogue of [FrdI], Theorem 6.4, holds for such stack-theoretic versions of the Frobenioids constructed in $[\mathrm{FrdI}]$, Example 6.3. Also, we observe that upon passing to either the 
perfection or the realification, such stack-theoretic versions become naturally isomorphic to the non-stack-theoretic versions [i.e., of [FrdI], Example 6.3, as stated].

Remark 3.1.6. In light of the important role played by the various orbicurves constructed in [EtTh], $\S 2$, in the present series of papers, we take the opportunity to correct an unfortunate - albeit in fact irrelevant! - error in [EtTh]. In the discussion preceding [EtTh], Definition 2.1, one must in fact assume that the integer $l$ is odd in order for the quotient $\bar{\Delta}_{X}$ to be well-defined. Since, ultimately, in [EtTh] [cf. the discussion following [EtTh], Remark 5.7.1], as well as in the present series of papers, this is the only case that is of interest, this oversight does not affect either the present series of papers or the bulk of the remainder of [EtTh]. Indeed, the only places in [EtTh] where the case of even $l$ is used are [EtTh], Remark 2.2.1, and the application of [EtTh], Remark 2.2.1, in the proof of [EtTh], Proposition

2.12 , for the orbicurves " $\dot{\underline{C}}$ ". Thus, [EtTh], Remark 2.2.1, must be deleted; in [EtTh], Proposition 2.12, one must in fact exclude the case where the orbicurve under consideration is " $\underline{\underline{C}}$ ". On the other hand, this theory involving [EtTh], Proposition 2.12 [cf., especially, [EtTh], Corollaries 2.18, 2.19] is only applied after the discussion following [EtTh], Remark 5.7.1, i.e., which only treats the curves " $\underline{\underline{X}}$ ". That is to say, ultimately, in [EtTh], as well as in the present series of papers, one is only interested in the curves " $\underline{\underline{X}}$ ", whose treatment only requires the case of odd $l$.

\section{Remark 3.1.7.}

(i) Observe that it follows immediately from the definition of $F_{\bmod }$ and the $K$ coricity of $C_{K}$ [cf. Definition 3.1, (b), (d)] that $C_{F}$ admits a unique [up to unique isomorphism] model

$$
C_{F_{\text {mod }}}
$$

over $F_{\text {mod }}$. If $v \in \mathbb{V}_{\text {mod }}$, then we shall write $C_{v}$ for the result of base-changing this model to $\left(F_{\bmod }\right)_{v}$. When applying the group-theoretic reconstruction algorithm of [AbsTopIII], Theorem 1.9 [cf. Remark 3.1.2, (ii)], it will frequently be useful to consider certain special types of rational functions on $C_{F_{\mathrm{mod}}}$ and $C_{v}$, as follows. Let $L$ be a field which is equal either to $F_{\bmod }$ or to $\left(F_{\bmod }\right)_{v}$ for some $v \in \mathbb{V}_{\text {mod }}$. Write $C_{L}$ for the model just discussed of $C_{F}$ over $L$. Thus, one verifies immediately that the coarse space $\left|C_{L}\right|$ associated to the algebraic stack $C_{L}$ is isomorphic to the affine line over $L$. Now suppose that we are given an algebraic closure $\bar{L}_{C}$ of the function field $L_{C}$ of $C_{L}$. Write $\bar{L}$ for the algebraic closure of $L$ determined by $\bar{L}_{C}$. We shall refer to a closed point of the proper smooth curve determined by some finite subextension $\subseteq \bar{L}_{C}$ of $L_{C}$ as a critical point if it maps to a closed point of the [proper smooth] compactification $\left|C_{L}\right|^{\text {cpt }}$ of $\left|C_{L}\right|$ that arises from one of the 2-torsion points of $E_{F}$; we shall refer to a critical point which does not map to the closed point of $\left|C_{L}\right|^{\text {cpt }}$ that arises from the unique cusp of $C_{L}$ as strictly critical. Thus, as one might imagine from the central importance of 2-torsion points in the elementary theory of elliptic curves, the strictly critical points of $\left|C_{L}\right|^{\mathrm{cpt}}$ may be thought of as the "most fundamental/canonical non-cuspidal points" of $\left|C_{L}\right|^{\text {cpt }}$. We shall refer to a rational function $f \in L_{C}$ on $C_{L}$ as $\kappa$-coric - where we think of the $\kappa$ as standing for "Kummer" — if 
- whenever $f \notin L$, it holds that, over $\bar{L}, f$ has precisely one pole [of unrestricted order], but at least two distinct zeroes;

- the divisor of zeroes and poles of $f$ is defined over a number field and avoids the critical points;

- $f$ restricts to a root of unity at every strictly critical point of $\left|C_{L}\right|^{\mathrm{cpt}}$.

Thus, the first displayed condition, taken together with the latter portion of the second displayed condition, may be understood as the condition that there exist a unique non-critical L-rational point of $\left|C_{L}\right|^{\mathrm{cpt}}$ with respect to which [i.e., if one takes this $L$-rational point to be the "point at infinity"] $f$ may be thought of as a polynomial on the affine line over $L$ with non-critical zeroes. In particular, it follows from the first displayed condition that, whenever $f \notin L$, it is never the case that both $f$ and $f^{-1}$ are $\kappa$-coric. By contrast, the third displayed condition may be understood as the condition that restriction to the strictly critical points determines a sort of canonical splitting up to roots of unity [which will play an important role in the present series of papers - cf., e.g., the discussion of Example 5.1, (v); Definition 5.2, (vi), (viii); Remark 5.2.3, below] of the set of nonzero constant [i.e., $L$-] multiples of $\kappa$-coric functions into a direct product, up to roots of unity, of the set of $\kappa$-coric functions and the set of nonzero elements of $L$. In particular, it follows from the third displayed condition that if $c \in L$ and $f \in L_{C}$ are such that both $f$ and $c \cdot f$ are $\kappa$-coric, then $c$ is a root of unity.

(ii) We maintain the notation of (i). Let $L^{\square}$ be an intermediate field between $L$ and $\bar{L}$ that is solvably closed [cf. [GlSol], Definition 1, (i)], i.e., has no nontrivial abelian extensions. Observe that, since $\left|C_{L}\right|^{\text {cpt }}$ has precisely 4 critical points, it follows immediately from the elementary theory of polynomial functions on the affine line over $L$ [i.e., the complement in $\left|C_{L}\right|^{\mathrm{cpt}}$ of some $L$-rational point $\left|C_{L}\right|^{\mathrm{cpt}}$ ] that there exists a $\kappa$-coric $f_{\text {sol }} \in L_{C}$ [i.e., a rational function on the affine line over $L]$ of degree 4 . In particular, it follows immediately from the elementary theory of polynomial functions on the affine line [i.e., $\left.\left|C_{L}\right|\right]$ over $L$ [together with "Hensel's lemma" - cf., e.g., the method of proof of [AbsTopII], Lemma 2.1] (respectively, from the existence of $f_{\text {sol }}$ [together with the well-known fact that the symmetric group on 4 letters is solvable]) that

every element of $L$ (respectively, $L^{\square}$ ) appears as a value of some $\kappa$-coric rational function on $C_{L}$ at some $L$ - (respectively, $L^{\square}$ ) valued point of $C_{L}$ that is not critical.

If $L=F_{\text {mod }}$, then write $\mathcal{U}_{\bar{L}}$ for the group $\bar{L}^{\times}$of nonzero elements of $\bar{L}$; if $L=$ $\left(F_{\text {mod }}\right)_{v}$ for some $v \in \mathbb{V}_{\text {mod }}$, then write $\mathcal{U}_{\bar{L}}$ for the group of units [i.e., relative to the unique valuation on $\bar{L}$ that extends $v$ ] of $\bar{L}$. We shall say that an element $f \in \bar{L}_{C}$ is $\infty \kappa$-coric if there exists a positive integer $n$ such that $f^{n}$ is a $\kappa$-coric element of $L_{C}$; we shall say that an element $f \in \bar{L}_{C}$ is $\infty \kappa \times$-coric if there exists an element $c \in \mathcal{U}_{\bar{L}}$ such that $c \cdot f \in \bar{L}_{C}$ is $\infty \kappa$-coric. Thus, an element $f \in L_{C}$ is $\kappa$-coric if and only if it is $\infty \kappa$-coric. Also, one verifies immediately that

an $\infty \kappa \times$-coric element $f \in \bar{L}_{C}$ is ${ }_{\infty} \kappa$-coric if and only if it restricts to a root of unity at some [or, equivalently, every] strictly critical point of the proper smooth curve determined by some finite subextension $\subseteq \bar{L}_{C}$ of the function field $L_{C}$ that contains $f$. 
Finally, one verifies immediately that the operation of multiplication determines a structure of pseudo-monoid [cf. §0] on the sets of $\kappa^{-}, \infty^{-} \kappa^{-}$, and $\infty \kappa \times$-coric rational functions; moreover, in the case of $\infty \kappa$ - and $\infty \kappa \times$-coric rational functions, the resulting pseudo-monoid is divisible and cyclotomic. These pseudo-monoids will be of use in discussions concerning the Kummer theory of rational functions on $C_{L}$ [cf. Example 5.1, (i), (v); Definition 5.2, (v), (vi), (vii), (viii), below].

(iii) We maintain the notation of (i) and (ii) and assume further that $L=F_{\bmod }$, $\bar{L}=\bar{F}$. We shall say that an element $f \in \bar{L}_{C}$ is $\kappa$-solvable if it is an $F_{\text {sol }}^{\times}$-multiple [cf. Definition 3.1, (b)] of a $\infty \kappa$-coric element of $\bar{L}_{C}$. Thus, one verifies immediately that an element $f \in \bar{L}_{C}$ is $\kappa$-solvable if and only if there exists a positive integer $n$ such that $f^{n}$ is a $\infty \kappa \times$-coric element of $F_{\text {sol }} \cdot L_{C}$. Write $F\left(\boldsymbol{\mu}_{l}\right) \subseteq K$ for the subextension of $K$ generated by the $l$-th roots of unity; $L_{C}(\kappa$-sol $) \subseteq \bar{L}_{C}$ for the subfield of $\bar{L}_{C}$ generated by the $\kappa$-solvable elements of $\bar{L}_{C} ; L_{C}\left(\underline{C}_{K}\right) \subseteq \bar{L}_{C}$ for the subfield of $\bar{L}_{C}$ generated over $L_{C}$ by the images of the $F\left(\boldsymbol{\mu}_{l}\right) \cdot L_{C}$-linear embeddings into $\bar{L}_{C}$ of the function field of $\underline{C}_{K}$. Thus, the fact that the extension $F / F_{\text {mod }}$ is Galois of degree prime to $l$ [cf. Definition 3.1, (b)] implies that

the subgroup $\operatorname{Gal}\left(K / F\left(\boldsymbol{\mu}_{l}\right)\right) \subseteq \operatorname{Gal}\left(K / F_{\text {mod }}\right)$ is normal and may be characterized as the unique subgroup of $\operatorname{Gal}\left(K / F_{\text {mod }}\right)$ that is [abstractly] isomorphic to $S L_{2}\left(\mathbb{F}_{l}\right)$

[cf. Remark 3.1.5; [GenEll], Lemma 3.1, (i)]. Moreover, we observe that it follows immediately from the well-known fact that the finite group $S L_{2}\left(\mathbb{F}_{l}\right)$ is perfect [cf. Definition 3.1, (c); [GenEll], Lemma 3.1, (ii)], together with the definition of the term " $\infty \kappa \times$-coric" [cf., especially, the fact that the zeroes and poles avoid the critical points!], that

the subfields $L_{C}\left(\underline{C}_{K}\right) \subseteq \bar{L}_{C} \supseteq F\left(\boldsymbol{\mu}_{l}\right) \cdot L_{C}(\kappa$-sol $)$ are linearly disjoint over $F\left(\boldsymbol{\mu}_{l}\right) \cdot L_{C}$.

In particular, it follows that there is a natural isomorphism

$$
\operatorname{Gal}\left(L_{C}\left(\underline{C}_{K}\right) / F\left(\boldsymbol{\mu}_{l}\right) \cdot L_{C}\right) \stackrel{\sim}{\rightarrow} \operatorname{Gal}\left(L_{C}\left(\underline{C}_{K}\right) \cdot L_{C}(\kappa-\text { sol }) / F\left(\boldsymbol{\mu}_{l}\right) \cdot L_{C}(\kappa-\text { sol })\right)
$$

- i.e., one may regard $\operatorname{Gal}\left(L_{C}\left(\underline{C}_{K}\right) / F\left(\boldsymbol{\mu}_{l}\right) \cdot L_{C}\right)$ as being equipped with an action on $L_{C}\left(\underline{C}_{K}\right) \cdot L_{C}(\kappa$-sol $)$ that restricts to the trivial action on $F\left(\boldsymbol{\mu}_{l}\right) \cdot L_{C}(\kappa$-sol).

(iv) We maintain the notation of (iii). In the following, we shall write "Out(-)" for the group of outer automorphisms of the topological group in parentheses. Consider the tautological exact sequence of Galois groups

$$
1 \rightarrow \operatorname{Gal}\left(\bar{L}_{C} / L_{C}(\kappa \text {-sol })\right) \rightarrow \operatorname{Gal}\left(\bar{L}_{C} / L_{C}\right) \rightarrow \operatorname{Gal}\left(L_{C}(\kappa \text {-sol }) / L_{C}\right) \rightarrow 1
$$

[cf. the discussion of (iii)]. Let us refer to a subgroup of $\operatorname{Gal}\left(\bar{L}_{C} / L_{C}(\kappa\right.$-sol $\left.)\right)$ as a $\kappa$-sol-open subgroup if it is the intersection with $\operatorname{Gal}\left(\bar{L}_{C} / L_{C}(\kappa\right.$-sol $\left.)\right)$ of a normal open subgroup of $\operatorname{Gal}\left(\bar{L}_{C} / L_{C}\right)$. Thus, the subgroups

$$
\begin{aligned}
\operatorname{Aut}^{\kappa-\operatorname{sol}}\left(\operatorname{Gal}\left(\bar{L}_{C} / L_{C}(\kappa \text {-sol })\right)\right) & \subseteq \operatorname{Aut}\left(\operatorname{Gal}\left(\bar{L}_{C} / L_{C}(\kappa \text {-sol })\right)\right) \\
\operatorname{Out}^{\kappa-\operatorname{sol}}\left(\operatorname{Gal}\left(\bar{L}_{C} / L_{C}(\kappa \text {-sol })\right)\right) & \subseteq \operatorname{Out}\left(\operatorname{Gal}\left(\bar{L}_{C} / L_{C}(\kappa \text {-sol })\right)\right)
\end{aligned}
$$


of automorphisms/outer automorphisms of the topological group $\operatorname{Gal}\left(\bar{L}_{C} / L_{C}(\kappa\right.$-sol $\left.)\right)$ that preserve each $\kappa$-sol-open subgroup - i.e., of " $\kappa$-sol-automorphisms $/ \kappa$-sol-outer automorphisms" — admit natural compatible homomorphisms

$$
\begin{aligned}
\operatorname{Aut}^{\kappa-\text { sol }}\left(\operatorname{Gal}\left(\bar{L}_{C} / L_{C}(\kappa \text {-sol })\right)\right) & \rightarrow \operatorname{Aut}(Q) \\
\operatorname{Out}^{\kappa-\operatorname{sol}}\left(\operatorname{Gal}\left(\bar{L}_{C} / L_{C}(\kappa \text {-sol })\right)\right) & \rightarrow \operatorname{Out}(Q)
\end{aligned}
$$

for each quotient $\operatorname{Gal}\left(\bar{L}_{C} / L_{C}(\kappa\right.$-sol $\left.)\right) \rightarrow Q$ by a $\kappa$-sol-open subgroup. The kernels of these natural homomorphisms [for varying " $Q$ "] determine natural profinite topologies on $\mathrm{Aut}^{\kappa \text {-sol }}\left(\operatorname{Gal}\left(\bar{L}_{C} / L_{C}(\kappa\right.\right.$-sol $\left.\left.)\right)\right)$, Out ${ }^{\kappa-\text { sol }}\left(\operatorname{Gal}\left(\bar{L}_{C} / L_{C}(\kappa\right.\right.$-sol $\left.\left.)\right)\right)$, with respect to which each arrow of the commutative diagram of homomorphisms

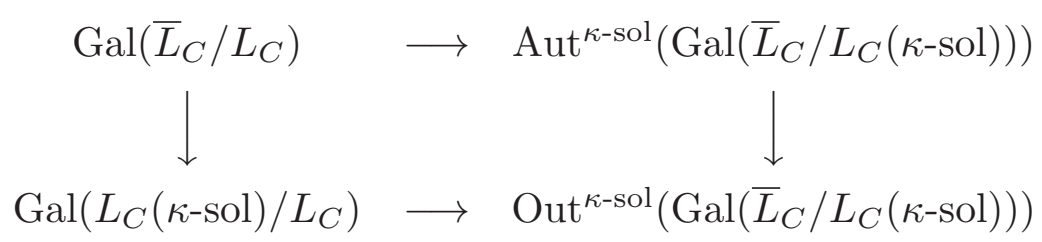

that arises, via conjugation, from the exact sequence considered above is continuous. Finally, we observe that

$\operatorname{Gal}\left(\bar{L}_{C} / L_{C}(\kappa\right.$-sol $\left.)\right)$ is center-free; in particular, the above commutative diagram of homomorphisms of topological groups is cartesian.

Indeed, let us first observe that it follows immediately from the definitions that $\operatorname{Gal}\left(\bar{F} \cdot L_{C}(\kappa-\mathrm{sol}) / \bar{F} \cdot L_{C}\right)$ is abelian. Thus, it follows formally, by applying Lemma 2.7, (vi), (vii), to the geometric fundamental groups of the various genus zero affine hyperbolic curves whose function field is equal to $L_{C}$, that the conjugacy action by any element $\alpha$ in the center of $\operatorname{Gal}\left(\bar{L}_{C} / L_{C}(\kappa\right.$-sol $\left.)\right)$ on such a [center-free] geometric fundamental group is trivial and hence, by [the special case that was already known to Belyi of] the Galois injectivity result discussed in [NodNon], Theorem C, that $\alpha$ is the identity element of $\operatorname{Gal}\left(\bar{L}_{C} / L_{C}(\kappa\right.$-sol $\left.)\right)$, as desired.

Given initial $\Theta$-data as in Definition 3.1, the theory of Frobenioids given in [FrdI], [FrdII], [EtTh] allows one to construct various associated Frobenioids, as follows.

Example 3.2. $\quad$ Frobenioids at Bad Nonarchimedean Primes. Let $\underline{v} \in$ $\underline{\mathbb{V}}^{\text {bad }}=\mathbb{V} \cap \mathbb{V}(K)^{\text {bad }}$. The theory of the "Frobenioid-theoretic theta function" discussed in [EtTh], $\S 5$, may be thought of as a sort of formal, category-theoretic way to formulate various elementary classical facts [which are reviewed in [EtTh], §1] concerning the theory of the line bundles and divisors related to the classical theta function on a Tate curve over an MLF. We give a brief review of this theory of [EtTh], §5, as follows:

(i) By the theory of [EtTh], the hyperbolic curve $\underline{\underline{X}}_{\underline{v}}$ determines a tempered Frobenioid 
[i.e., the Frobenioid denoted "C $\mathrm{C}$ " in the discussion at the beginning of [EtTh], $\S 5$; cf. also the discussion of Remark 3.2.4 below] over a base category

$$
\mathcal{D}_{\underline{v}}
$$

[i.e., the category denoted " $\mathcal{D}$ " in the discussion at the beginning of [EtTh], §5]. We recall from the theory of [EtTh] that $\mathcal{D}_{\underline{v}}$ may be thought of as the category of connected tempered coverings - i.e., " $\mathcal{B}^{\text {temp }}\left(\underline{\underline{X}}_{\underline{v}}\right)^{0}$ " in the notation of [EtTh], Example 3.9 - of the hyperbolic curve $\underline{\underline{X}}_{\underline{v}}$. In the following, we shall write

$$
\mathcal{D}_{\underline{v}}^{\vdash} \stackrel{\text { def }}{=} \mathcal{B}\left(K_{\underline{v}}\right)^{0}
$$

[cf. the notational conventions concerning categories discussed in §0]. Also, we observe that $\mathcal{D}_{\underline{v}}^{\vdash}$ may be naturally regarded [by pulling back finite étale coverings via the structure morphism $\left.\underline{\underline{X}}_{\underline{v}} \rightarrow \operatorname{Spec}\left(K_{\underline{v}}\right)\right]$ as a full subcategory

$$
\mathcal{D}_{\underline{v}}^{\vdash} \subseteq \mathcal{D}_{\underline{v}}
$$

of $\mathcal{D}_{\underline{v}}$, and that we have a natural functor $\mathcal{D}_{\underline{v}} \rightarrow \mathcal{D}_{\underline{v}}^{\vdash}$, which is left-adjoint to the natural inclusion functor $\mathcal{D}_{\underline{v}}^{\vdash} \hookrightarrow \mathcal{D}_{\underline{v}}$ [cf. [FrdII], Example 1.3, (ii)]. If (-) is an object of $\mathcal{D}_{\underline{v}}$, then we shall denote by " $\mathbb{T}_{(-)}$" the Frobenius-trivial object [a notion which is category-theoretic - cf. [FrdI], Definition 1.2, (iv); [FrdI], Corollary 4.11, (iv); [EtTh], Proposition 5.1] of $\underline{\underline{\mathcal{F}}}_{\underline{v}}$ [which is completely determined up to isomorphism] that lies over "(-)".

(ii) Next, let us recall [cf. [EtTh], Proposition 5.1; [FrdI], Corollary 4.10] that the birationalization

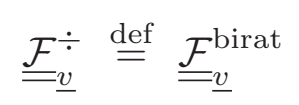

may be reconstructed category-theoretically from $\underline{\underline{\mathcal{F}}}_{\underline{v}}$ [cf. Remark 3.2 .1 below]. Write

$$
\underline{\underline{Y}}_{\underline{v}} \rightarrow \underline{\underline{X}}_{\underline{v}}
$$

for the tempered covering determined by the object " $\ddot{\underline{Y}}^{\log }$ " in the discussion at the beginning of [EtTh], §5. Thus, we may think of $\underline{\underline{Y}}_{\underline{v}}$ as an object of $\mathcal{D}_{\underline{v}}$ [cf. the

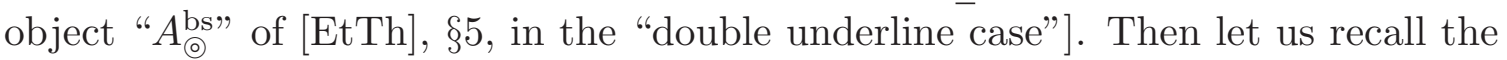
"Frobenioid-theoretic l-th root of the theta function", which is normalized so as to attain the value 1 at the point " $\sqrt{-1}$ " [cf. [EtTh], Theorem 5.7]; we shall denote the reciprocal of [i.e., "1 over"] this theta function by

$$
\underline{\underline{\Theta}}_{\underline{v}} \in \mathcal{O}^{\times}\left(\mathbb{T}_{\underline{\underline{\underline{Y}}}_{\underline{v}}^{\dot{\vdots}}}^{\dot{\div}}\right)
$$

— where we use the superscript " $\div$ " to denote the image in $\underline{\underline{\mathcal{F}}} \underline{\dot{v}}$ of an object of $\underline{\underline{\mathcal{F}}} \underline{\underline{v}}$. Here, we recall that $\underline{\underline{\Theta}}_{\underline{v}}$ is completely determined up to multiplication by a $2 l$-th $\underline{\bar{r}}^{\underline{v}} \underline{\underline{v}}$ of unity [i.e., an element of $\boldsymbol{\mu}_{2 l}\left(\mathbb{T}_{\underline{\underline{\dot{\hat{Y}}}}_{\underline{v}}}^{\dot{\dot{v}}}\right)$ ] and the action of the group of automorphisms 
$l \cdot \underline{\mathbb{Z}} \subseteq \operatorname{Aut}\left(\mathbb{T}_{\underline{\underline{\underline{Y}}}}\right)$ [i.e., we write $\underline{\mathbb{Z}}$ for the group denoted " $\underline{\mathbb{Z}}$ " in [EtTh], Theorem 5.7; cf. also the discussion preceding [EtTh], Definition 1.9]. Moreover, we recall from the theory of [EtTh], $\S 5$ [cf. the discussion at the beginning of [EtTh], 55 ; [EtTh], Theorem 5.7] that

$\mathbb{T}_{\underline{\underline{\underline{Y}}}} \quad[$ regarded up to isomorphism] and

$\underline{\underline{\Theta}}_{\underline{v}} \quad$ [regarded up to the $\boldsymbol{\mu}_{2 l}\left(\mathbb{T}_{\underline{\underline{\dot{Y}}}_{\underline{v}}^{\dot{\dot{S}}}}^{\dot{\underline{Z}}}\right), l \cdot \underline{\mathbb{Z}}$ indeterminacies discussed above] may be reconstructed category-theoretically from $\underline{\underline{\mathcal{F}}}_{v}$ [cf. Remark 3.2.1 below].

(iii) Next, we recall from [EtTh], Corollary 3.8, (ii) [cf. also [EtTh], Proposition 5.1], that the $p_{\underline{v}}$-adic Frobenioid constituted by the "base-field-theoretic hull" [cf. [EtTh], Remark 3.6.2]

$$
\mathcal{C}_{\underline{v}} \subseteq \underline{\underline{\mathcal{F}}}_{\underline{v}}
$$

[i.e., we write $\mathcal{C}_{v}$ for the subcategory "C ${ }^{\text {bs-fld" }}$ of [EtTh], Definition 3.6, (iv)] may be reconstructed category-theoretically from $\underline{\underline{\mathcal{F}}}_{v}$ [cf. Remark 3.2.1 below].

(iv) Write $q_{\underline{v}}$ for the $q$-parameter of the elliptic curve $E_{\underline{v}}$ over $K_{\underline{v}}$. Thus, we may think of $q_{\underline{v}}$ as an element $q_{\underline{v}} \in \mathcal{O}^{\triangleright}\left(\mathbb{T}_{\underline{\underline{X}}_{v}}\right)\left(\cong \mathcal{O}_{K_{\underline{v}}}^{\triangleright}\right)$. Note that it follows from our assumption concerning 2-torsion [cf. Definition 3.1, (b)], together with the definition of " $K$ " [cf. Definition 3.1, (c)], that $q_{\underline{v}}$ admits a $2 l$-th root in $\mathcal{O}^{\triangleright}\left(\mathbb{T}_{\underline{\underline{X}}_{\underline{v}}}\right)\left(\cong \mathcal{O}_{K_{\underline{v}}}^{\triangleright}\right)$. Then one computes immediately from the final formula of [EtTh], Proposition 1.4, (ii), that the value of $\underline{\underline{\Theta}}_{\underline{v}}$ at $\sqrt{-q_{\underline{v}}}$ is equal to

$$
\underline{\underline{q}} \stackrel{\underline{v}}{\stackrel{\text { def }}{=}} q_{\underline{v}}^{1 / 2 l} \in \mathcal{O}^{\triangleright}\left(\mathbb{T}_{\underline{\underline{X}}_{\underline{v}}}\right)
$$

— where the notation " $q_{\underline{\underline{v}}}^{1 / 2 l}$ " [hence also $\left.\underline{\underline{q}}_{\underline{v}}\right]$ is completely determined up to a $\boldsymbol{\mu}_{2 l}\left(\mathbb{T}_{\underline{\underline{X}}}\right)$-multiple. Write $\Phi_{\mathcal{C}_{\underline{v}}}$ for the divisor monoid [cf. [FrdI], Definition 1.1, (iv)] of the $p_{\underline{v}}$-adic Frobenioid $\mathcal{C}_{\underline{v}}$. Then the image of $\underline{\underline{q}} \underline{\underline{v}}$ determines a constant section [i.e., a sub-monoid on $\mathcal{D}_{\underline{v}}$ isomorphic to $\mathbb{N}$ ] " $\log _{\Phi}(\underline{\underline{q}} \underline{\underline{v}})$ " of $\Phi_{\mathcal{C}_{\underline{v}}}$. Moreover, the resulting submonoid [cf. Remark 3.2.2 below]

$$
\left.\left.\Phi_{\mathcal{C}_{\underline{v}}^{\vdash}} \stackrel{\text { def }}{=} \mathbb{N} \cdot \log _{\Phi}(\underline{\underline{q}})\right|_{\mathcal{D}_{\underline{v}}^{\vdash}} \subseteq \Phi_{\mathcal{C}_{\underline{v}}}\right|_{\mathcal{D}_{\underline{v}}^{\vdash}}
$$

determines a $p_{\underline{v}}$-adic Frobenioid with base category given by $\mathcal{D}_{\underline{v}}^{\vdash}[\mathrm{cf}$. [FrdII], Example $1.1,(\mathrm{ii})]$

$$
\mathcal{C}_{\underline{v}}^{\vdash}\left(\subseteq \mathcal{C}_{\underline{v}} \subseteq \underline{\underline{\mathcal{F}}}_{\underline{v}} \rightarrow \underline{\underline{\mathcal{F}}}_{\underline{\dot{v}}}^{\dot{*}}\right)
$$

- which may be thought of as a subcategory of $\mathcal{C}_{\underline{v}}$. Also, we observe that [since the $q$-parameter $\underline{\underline{q}} \in K_{\underline{v}}$, it follows that] $\underline{\underline{q}}_{\underline{v}}$ determines a $\boldsymbol{\mu}_{2 l}(-)$-orbit of characteristic splittings [cf. [FrdI], Definition 2.3] 
on $\mathcal{C}_{\underline{v}}^{\vdash}$.

(v) Next, let us recall that the base field of $\underline{\underline{Y}}_{\underline{v}}$ is equal to $K_{\underline{v}}$ [cf. the discussion of Definition 3.1, (e)]. Write

$$
\mathcal{D}_{\underline{v}}^{\Theta} \subseteq\left(\mathcal{D}_{\underline{v}}\right)_{\underline{\underline{Y}}_{\underline{v}}}
$$

for the full subcategory of the category $\left(\mathcal{D}_{\underline{\underline{v}}}\right)_{\underline{\underline{Y}}_{\underline{v}}}[$ cf. the notational conventions concerning categories discussed in $\S 0$ ] determined by the products in $\mathcal{D}_{\underline{v}}$ of $\underline{\underline{Y}}_{\underline{v}}$ with objects of $\mathcal{D}_{\underline{v}}^{\vdash}$. Thus, one verifies immediately that "forming the product with $\underline{\underline{\underline{Y}}}$ " determines a natural equivalence of categories $\mathcal{D}_{\underline{v}}^{\vdash} \stackrel{\sim}{\rightarrow} \mathcal{D}_{\underline{v}}^{\Theta}$. Moreover, for $A^{\Theta} \in \overline{\mathrm{Ob}}\left(\mathcal{D}_{\underline{v}}^{\Theta}\right)$, the assignment

$$
A^{\Theta} \mapsto \mathcal{O}^{\times}\left(\mathbb{T}_{A^{\Theta}}\right) \cdot\left(\left.\underline{\underline{\Theta}}_{\underline{v}}^{\mathbb{N}}\right|_{\mathbb{T}_{A}}\right) \subseteq \mathcal{O}^{\times}\left(\mathbb{T}_{\dot{A}^{\Theta}}^{\dot{ }}\right)
$$

determines a monoid $\mathcal{O}_{\mathcal{C}_{\underline{v}}^{\Theta}}^{\triangleright}(-)$ on $\mathcal{D}_{\underline{v}}^{\Theta}$ [in the sense of [FrdI], Definition 1.1, (ii)]; write $\mathcal{O}_{\mathcal{C}_{\underline{v}}^{\ominus}}^{\times}(-) \subseteq \mathcal{O}_{\mathcal{C}_{\underline{v}}^{\ominus}}^{\triangleright}(-)$ for the submonoid determined by the invertible elements. Next, let us observe that, relative to the natural equivalence of categories $\mathcal{D}_{\underline{v}}^{\vdash} \stackrel{\sim}{\rightarrow} \mathcal{D}_{\underline{v}}^{\Theta}$ — which we think of as mapping $\operatorname{Ob}\left(\mathcal{D}_{\underline{v}}^{+}\right) \ni A \mapsto A^{\Theta} \stackrel{\text { def }}{=} \underline{\underline{Y}}_{\underline{v}} \times A \in \mathrm{Ob}\left(\mathcal{D}_{\underline{v}}^{\Theta}\right)$ - we have natural isomorphisms

$$
\mathcal{O}_{\mathcal{C}_{\underline{v}}^{\vdash}}^{\triangleright}(-) \stackrel{\sim}{\rightarrow} \mathcal{O}_{\mathcal{C}_{\underline{v}}^{\ominus}}^{\triangleright}(-) ; \quad \mathcal{O}_{\mathcal{C}_{\underline{v}}^{\vdash}}^{\times}(-) \stackrel{\sim}{\rightarrow} \mathcal{O}_{\mathcal{C}_{\underline{v}}^{\Theta}}^{\times}(-)
$$

[where $\mathcal{O}_{\mathcal{C}_{\underline{v}}^{\vdash}}^{\triangleright}(-), \mathcal{O}_{\mathcal{C}_{\underline{v}}^{\vdash}}^{\times}(-)$are the monoids associated to the Frobenioid $\mathcal{C}_{\underline{v}}^{\vdash}$ as in [FrdI], Proposition 2.2] which are compatible with the assignment

$$
\underline{\underline{q}}_{\underline{v}}\left|\mathbb{T}_{A} \mapsto \underline{\underline{\Theta}}_{\underline{v}}\right| \mathbb{T}_{A \Theta}
$$

and the natural isomorphism [i.e., induced by the natural projection $A^{\Theta}=\underline{\underline{Y}}_{\underline{v}} \times$ $A \rightarrow A] \mathcal{O}^{\times}\left(\mathbb{T}_{A}\right) \stackrel{\sim}{\rightarrow} \mathcal{O}^{\times}\left(\mathbb{T}_{A^{\Theta}}\right)$. In particular, we conclude that the monoid $\left.\mathcal{O}_{\mathcal{C}_{\underline{v}}^{\Theta}}^{\triangleright} \bar{v}^{-}\right)$ determines - in a fashion consistent with the notation of [FrdI], Proposition 2.2! - a $p_{\underline{v}}$-adic Frobenioid with base category given by $\mathcal{D}_{\underline{v}}^{\Theta}$ [cf. [FrdII], Example 1.1, (ii)]

$$
\mathcal{C}_{\underline{v}}^{\Theta} \quad\left(\subseteq \underline{\underline{\mathcal{F}}_{\underline{v}}^{\dot{*}}}\right)
$$

- which may be thought of as a subcategory of $\underline{\underline{\mathcal{F}^{*}}} \underline{\dot{v}}$, and which is equipped with a $\boldsymbol{\mu}_{2 l}(-)$-orbit of characteristic splittings [cf. [FrdI], Definition 2.3]

$$
\tau_{\underline{v}}^{\Theta}
$$

determined by $\underline{\underline{\Theta}}_{\underline{v}}$. Moreover, we have a natural equivalence of categories

$$
\mathcal{C}_{\underline{v}}^{\vdash} \stackrel{\sim}{\rightarrow} \mathcal{C}_{\underline{v}}^{\Theta}
$$


that maps $\tau_{\underline{v}}^{\vdash}$ to $\tau_{\underline{v}}^{\Theta}$. This fact may be stated more succinctly by writing

$$
\mathcal{F}_{\underline{v}}^{\vdash} \stackrel{\sim}{\rightarrow} \mathcal{F}_{\underline{v}}^{\Theta}
$$

— where we write $\mathcal{F}_{\underline{v}}^{\vdash} \stackrel{\text { def }}{=}\left(\mathcal{C}_{\underline{v}}^{\vdash}, \tau_{\underline{v}}^{\vdash}\right) ; \mathcal{F}_{\underline{v}}^{\Theta} \stackrel{\text { def }}{=}\left(\mathcal{C}_{\underline{v}}^{\Theta}, \tau_{\underline{v}}^{\Theta}\right)$. In the following, we shall refer to a pair such as $\mathcal{F}_{v}^{\vdash}$ or $\mathcal{F}_{v}^{\Theta}$ consisting of a Frobenioid equipped with a collection of characteristic splittings as a split Frobenioid.

(vi) Here, it is useful to recall [cf. Remark 3.2.1 below] that:

(a) the subcategory $\mathcal{D}_{\underline{v}}^{\vdash} \subseteq \mathcal{D}_{\underline{v}}$ may be reconstructed category-theoretically from $\mathcal{D}_{\underline{v}}[\mathrm{cf}$. [AbsAnāb], Lemma 1.3.8];

(b) the category $\mathcal{D}_{v}^{\Theta}$ may be reconstructed category-theoretically from $\mathcal{D}_{\underline{v}}[\mathrm{cf}$. (a); the discussion at the beginning of [EtTh], §5];

(c) the category $\mathcal{D}_{\underline{v}}^{\vdash}$ (respectively, $\mathcal{D}_{\underline{v}}^{\Theta}$ ) may be reconstructed category-theoretically from $\mathcal{C}_{\underline{v}}^{\vdash}$ (respectively, $\mathcal{C}_{\underline{v}}^{\Theta}$ ) [cf. [FrdI], Theorem 3.4, (v); [FrdII], Theorem 1.2, (i); [FrdII], Example 1.3, (i); [AbsAnab], Theorem 1.1.1, (ii)];

(d) the category $\mathcal{D}_{\underline{v}}$ may be reconstructed category-theoretically either from $\underline{\underline{\mathcal{F}}}_{\underline{v}}\left[\mathrm{cf} . \quad[\mathrm{EtTh}]\right.$, Theorem 4.4; [EtTh], Proposition 5.1] or from $\mathcal{C}_{\underline{v}}[\mathrm{cf}$. [FrdI], Theorem 3.4, (v); [FrdII], Theorem 1.2, (i); [FrdII], Example 1.3, (i); [SemiAnbd], Example 3.10; [SemiAnbd], Remark 3.4.1].

Next, let us observe that by (b), (d), together with the discussion of (ii) concerning the category-theoreticity of $\underline{\underline{\Theta}}_{v}$, it follows [cf. Remark 3.2.1 below] that

(e) one may reconstruct the split Frobenioid $\mathcal{F}_{v}^{\Theta}$ [up to the $l \cdot \underline{\mathbb{Z}}$ indeterminacy in $\underline{\underline{\Theta}}_{\underline{v}}$ discussed in (ii); cf. also Remark 3.2.3 below] category-theoretically from $\underline{\underline{\mathcal{F}}}_{\underline{v}}$ [cf. [FrdI], Theorem 3.4, (i), (v); [EtTh], Proposition 5.1].

Next, let us recall that the values of $\underline{\underline{\Theta}}_{v}$ may be computed by restricting the corresponding Kummer class, i.e., the "étale theta function" [cf. [EtTh], Proposition 1.4, (iii); the proof of [EtTh], Theorem 1.10, (ii); the proof of [EtTh], Theorem 5.7], which may be reconstructed category-theoretically from $\mathcal{D}_{\underline{v}}[\mathrm{cf}$. [EtTh], Corollary 2.8, (i)]. Thus, by applying the isomorphisms of cyclotomes of [AbsTopIII], Corollary 1.10, (c); [AbsTopIII], Remark 3.2.1 [cf. also [AbsTopIII], Remark 3.1.1], to these Kummer classes, one concludes from (a), (d) that

(f) one may reconstruct the split Frobenioid $\mathcal{F}_{v}^{\vdash}$ category-theoretically from $\mathcal{C}_{\underline{v}}$, hence also [cf. (iii)] from $\underline{\underline{\mathcal{F}}}_{\underline{v}}$ [cf. Remark 3.2.1 below].

\section{Remark 3.2.1.}

(i) In [FrdI], [FrdII], and [EtTh] [cf. [EtTh], Remark 5.1.1], the phrase "reconstructed category-theoretically" is interpreted as meaning "preserved by equivalences of categories". From the point of view of the theory of [AbsTopIII] - i.e., the discussion of "mono-anabelian" versus "bi-anabelian" geometry [cf. [AbsTopIII], §I2, 
(Q2)] — this sort of definition is "bi-anabelian" in nature. In fact, it is not difficult to verify that the techniques of [FrdI], [FrdII], and [EtTh] all result in explicit reconstruction algorithms, whose input data consists solely of the category structure of the given category, of a "mono-anabelian" nature that do not require the use of some fixed reference model that arises from scheme theory [cf. the discussion of [AbsTopIII], §I4]. For more on the foundational aspects of such "mono-anabelian reconstruction algorithms", we refer to the discussion of [IUTchIV], Example 3.5.

(ii) One reason that we do not develop in detail here a "mono-anabelian approach to the geometry of categories" along the lines of [AbsTopIII] is that, unlike the case with the mono-anabelian theory of [AbsTopIII], which plays a quite essential role in the theory of the present series of papers, much of the category-theoretic reconstruction theory of $[\mathrm{FrdI}],[\mathrm{FrdII}]$, and [EtTh] is not of essential importance in the development of the theory of the present series of papers. That is to say, for instance, instead of quoting results to the effect that the base categories or divisor monoids of various Frobenioids may be reconstructed category-theoretically, one could instead simply work with the data consisting of "the category constituted by the Frobenioid equipped with its pre-Frobenioid structure" [cf. [FrdI], Definition 1.1, (iv)]. Nevertheless, we chose to apply the theory of [FrdI], [FrdII], and [EtTh] partly because it simplifies the exposition [i.e., reduces the number of auxiliary structures that one must carry around], but more importantly because it renders explicit precisely which structures arising from scheme-theory are "categorically intrinsic" and which merely amount to "arbitrary, non-intrinsic choices" which, when formulated intrinsically, correspond to various "indeterminacies". This explicitness is of particular importance with respect to phenomena related to the unitlinear Frobenius functor [cf. [FrdI], Proposition 2.5] and the Frobenioid-theoretic indeterminacies studied in [EtTh], §5.

Remark 3.2.2. Although the submonoid $\Phi_{\mathcal{C}_{\underline{v}}^{\vdash}}$ is not "absolutely primitive" in the sense of [FrdII], Example 1.1, (ii), it is "very close to being absolutely primitive", in the sense that [as is easily verified] there exists a positive integer $N$ such that $N \cdot \Phi_{\mathcal{C}_{\underline{v}}^{\vdash}}$ is absolutely primitive. This proximity to absolute primitiveness may also be seen in the existence of the characteristic splittings $\tau_{\underline{v}}^{\vdash}$.

\section{Remark 3.2.3.}

(i) Let $\alpha \in \operatorname{Aut}_{\mathcal{D}_{\underline{v}}}\left(\underline{\underline{Y}}_{\underline{v}}\right)$. Then observe that $\alpha$ determines, in a natural way, an automorphism $\alpha_{\mathcal{D}}$ of the functor $\mathcal{D}_{v}^{\vdash} \rightarrow \mathcal{D}_{\underline{v}}$ obtained by composing the equivalence of categories $\mathcal{D}_{\underline{v}}^{\vdash} \stackrel{\sim}{\rightarrow} \mathcal{D}_{\underline{v}}^{\Theta}$ [i.e., which maps $\left.\mathrm{Ob}\left(\mathcal{D}_{\underline{v}}^{\vdash}\right) \ni A \mapsto A^{\Theta} \in \mathrm{Ob}\left(\mathcal{D}_{\underline{v}}^{\Theta}\right)\right]$ discussed in Example 3.2, (v), with the natural functor $\mathcal{D}_{\underline{\underline{v}}}^{\Theta} \subseteq\left(\mathcal{D}_{\underline{\underline{v}}}\right)_{\underline{\underline{\underline{Y}}}} \rightarrow \mathcal{D}_{\underline{v}}$. Moreover, $\alpha_{\mathcal{D}}$ induces, in a natural way, an isomorphism $\alpha_{\mathcal{O}}$ of the monoid $\mathcal{O}_{\mathcal{C}_{\underline{v}}^{\ominus}}^{\triangleright}(-)$ on $\mathcal{D}_{\underline{v}}^{\Theta}$ associated to $\underline{\underline{\Theta}}_{\underline{v}}$ in Example 3.2, (v), onto the corresponding monoid on $\mathcal{D}_{\underline{v}}^{\Theta}$ associated to the $\alpha$-conjugate $\underline{\underline{\Theta}}_{\underline{v}}^{\alpha}$ of $\underline{\underline{\Theta}}_{\underline{v}}$. Thus, it follows immediately from the discussion of Example 3.2, (v), that

$\alpha_{\mathcal{O} \triangleright}$ - hence also $\alpha$ - induces an isomorphism of the split Frobenioid 
$\mathcal{F}_{\underline{v}}^{\Theta}$ associated to $\underline{\underline{\Theta}}_{\underline{v}}$ onto the split Frobenioid $\mathcal{F}_{\underline{\underline{v}}}^{\Theta^{\alpha}}$ associated to $\underline{\underline{\Theta}}_{\underline{v}}^{\alpha}$ which lies over the identity functor on $\mathcal{D}_{\underline{v}}^{\Theta}$.

In particular, the expression " $\mathcal{F}_{\underline{v}}^{\Theta}$, regarded up to the $l \cdot \underline{\mathbb{Z}}$ indeterminacy in $\underline{\underline{\Theta}}_{\underline{v}}$ discussed in Example 3.2, (ii)" may be understood as referring to the various split Frobenioids " $\mathcal{F}_{\underline{v}}^{\Theta^{\alpha}}$ ", as $\alpha$ ranges over the elements of $\operatorname{Aut}_{\mathcal{D}_{\underline{v}}}\left(\underline{\underline{\underline{Y}}}_{\underline{v}}\right)$, relative to the identifications given by these isomorphisms of split Frobenioids induced by the various elements of $\operatorname{Aut}_{\mathcal{D}_{\underline{v}}}\left(\underline{\underline{Y}}_{\underline{v}}\right)$.

(ii) Suppose that $A \in \operatorname{Ob}\left(\mathcal{D}_{\underline{v}}\right)$ lies in the image of the natural functor $\mathcal{D}_{v}^{\Theta} \subseteq$ $\left(\mathcal{D}_{\underline{\underline{v}}}\right)_{\underline{\underline{\underline{Y}}}} \rightarrow \mathcal{D}_{\underline{v}}$, and that $\psi: B \rightarrow \mathbb{T}_{A}$ is a linear morphism in the Frobenioid $\underline{\underline{\mathcal{F}}}_{\underline{\underline{v}}}$. Then $\bar{\psi}$ induces an injective homomorphism

$$
\mathcal{O}^{\times}\left(\mathbb{T}_{\dot{A}}^{\dot{\dot{A}}}\right) \hookrightarrow \mathcal{O}^{\times}\left(B^{\dot{\div}}\right)
$$

[cf. [FrdI], Proposition 1.11, (iv)]. In particular, one may pull-back sections of the monoid $\mathcal{O}_{\mathcal{C}_{\underline{v}}^{\Theta}}^{\triangleright}(-)$ on $\mathcal{D}_{\underline{v}}^{\Theta}$ of Example 3.2, (v), to B. Such pull-backs are useful, for instance, when one considers the roots of $\underline{\underline{\Theta}}_{\underline{v}}$, as in the theory of [EtTh], $\S 5$.

Remark 3.2.4. Before proceeding, we pause to discuss certain minor oversights on the part of the author in the discussion of the theory of tempered Frobenioids in [EtTh], $33, \S 4$. Let $\mathfrak{Z}_{\infty}^{\log }$ be as in the discussion at the beginning of [EtTh], 33 . Here, we recall that $\mathfrak{Z}_{\infty}^{\log }$ is obtained as the "universal combinatorial covering" of the formal log scheme associated to a stable log curve with split special fiber over the ring of integers of a finite extension of an MLF of residue characteristic $p$ [cf. loc. cit. for more details]; we write $Z^{\log }$ for the generic fiber of the stable log curve under consideration.

(i) First, let us consider the following conditions on a nonzero meromorphic function $f$ on $\mathfrak{Z}_{\infty}^{\log \text { : }}$

(a) For every $N \in \mathbb{N}_{\geq 1}$, it holds that $f$ admits an $N$-th root over some tempered covering of $Z^{\log }$.

(b) For every $N \in \mathbb{N}_{\geq 1}$ which is prime to $p$, it holds that $f$ admits an $N$-th root over some tempered covering of $Z^{\mathrm{log}}$.

(c) The divisor of zeroes and poles of $f$ is a log-divisor.

It is immediate that (a) implies (b). Moreover, one verifies immediately, by considering the ramification divisors of the tempered coverings that arise from extracting roots of $f$, that (b) implies (c). When $N$ is prime to $p$, if $f$ satisfies (c), then it follows immediately from the theory of admissible coverings [cf., e.g., [PrfGC], $\S 2, \S 8]$ that there exists a finite $\log$ étale covering $Y^{\log } \rightarrow Z^{\log }$ whose pull-back $Y_{\infty}^{\log } \rightarrow Z_{\infty}^{\log }$ to the generic fiber $Z_{\infty}^{\log }$ of $\mathfrak{Z}_{\infty}^{\log }$ is sufficient

(R1) to annihilate all ramification over the cusps or special fiber of $\mathfrak{Z}_{\infty}^{\log }$ that might arise from extracting an $N$-th root of $f$, as well as 
(R2) to split all extensions of the function fields of irreducible components of the special fiber of $\mathfrak{Z}_{\infty}^{\log }$ that might arise from extracting an $N$-th root of $f$.

That is to say, in this situation, it follows that $f$ admits an $N$-th root over the tempered covering of $Z^{\log }$ given by the "universal combinatorial covering" of $Y^{\log }$. In particular, it follows that (c) implies (b). Thus, in summary, we have:

$$
\text { (a) } \Longrightarrow(\mathrm{b}) \Longleftrightarrow(\mathrm{c}) \text {. }
$$

On the other hand, unfortunately, it is not clear to the author at the time of writing whether or not (c) [or (b)] implies (a).

(ii) Observe that it follows from the theory of [EtTh], 11 [cf., especially, [EtTh], Proposition 1.3] that the theta function that forms the main topic of interest of [EtTh] satisfies condition (a) of (i).

(iii) In [EtTh], Definition 3.1, (ii), a meromorphic function $f$ as in (i) is defined to be "log-meromorphic" if it satisfies condition (c) of (i). On the other hand, in the proof of [EtTh], Proposition 4.2, (iii), it is necessary to use property (a) of (i) i.e., despite the fact that, as remarked in (i), it is not clear whether or not property (c) implies property (a). The author apologizes for any confusion caused by this oversight on his part.

(iv) The problem pointed out in (iii) may be remedied - at least from the point of view of the theory of [EtTh] — via either of the following two approaches:

(A) One may modify [EtTh], Definition 3.1, (ii), by taking the definition of a "log-meromorphic" function to be a function that satisfies condition (a) [i.e., as opposed to condition (c)] of (i). [In light of the content of this modified definition, perhaps a better term for this class of meromorphic functions would be "temperedmeromorphic".] Then the remainder of the text of [EtTh] goes through without change.

(B) One may modify [EtTh], Definition 4.1, (i), by assuming that the meromorphic function " $f \in \mathcal{O}^{\times}\left(A^{\text {birat }}\right)$ " of [EtTh], Definition 4.1, (i), satisfies the following "Frobenioid-theoretic version" of condition (a):

(d) For every $N \in \mathbb{N}_{\geq 1}$, there exists a linear morphism $A^{\prime} \rightarrow A$ in $\mathcal{C}$ such that the pull-back of $f$ to $A^{\prime}$ admits an $N$-th root.

[Here, we recall that, as discussed in (ii), the Frobenioid-theoretic theta functions that appear in [EtTh] satisfy (d).] Note that since the rational function monoid of the Frobenioid $\mathcal{C}$, as well as the linear morphisms of $\mathcal{C}$, are category-theoretic [cf. [FrdI], Theorem 3.4, (iii), (v); [FrdI], Corollary 4.10], this condition (d) is categorytheoretic. Thus, if one modifies [EtTh], Definition 4.1, (i), in this way, then the remainder of the text of [EtTh] goes through without change, except that one must replace the reference to the definition of "log-meromorphic" [i.e., [EtTh], Definition 3.1 , (ii)] that occurs in the proof of [EtTh], Proposition 4.2, (iii), by a reference to condition (d) [i.e., in the modified version of [EtTh], Definition 4.1, (i)].

(v) In the discussion of (iv), we note that the approach of (A) results in a slightly different definition of the notion of a "tempered Frobenioid" from the original 
definition given in [EtTh]. Put another way, the approach of (B) has the advantage that it does not result in any modification of the definition of the notion of a "tempered Frobenioid"; that is to say, the approach of (B) only results in a slight reduction in the range of applicability of the theory of [EtTh], $\S 4$, which is essentially irrelevant from the point of view of the present series of papers, since [cf. (ii)] theta functions lie within this reduced range of applicability. On the other hand, the approach of (A) has the advantage that one may consider the Kummer theory of arbitrary rational functions of the tempered Frobenioid without imposing any further hypotheses. Thus, for the sake of simplicity, in the present series of papers, we shall interpret the notion of a "tempered Frobenioid" via the approach of (A).

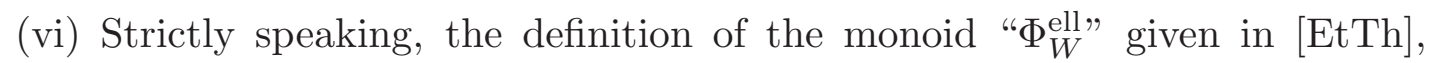
Example 3.9, (iii), leads to certain technical difficulties, which are, in fact, entirely irrelevant to the theory of [EtTh]. These technical difficulties may be averted by making the following slight modifications to the text of [EtTh], Example 3.9, as follows:

(1) In the discussion following the first display of [EtTh], Example 3.9, (i), the phrase " $Y$ log is of genus 1 " should be replaced by the phrase " $Y$ log is of genus 1 and has either precisely one cusp or precisely two cusps whose difference is a 2-torsion element of the underlying elliptic curve".

(2) In the discussion following the first display of [EtTh], Example 3.9, (i), the phrase

the lower arrow of the diagram to be " $\underline{\dot{X}}^{\log } \rightarrow \underline{\dot{C}}^{\log }$ "

should be replaced by the phrase

the lower arrow of the diagram to be " $\dot{X}^{\log } \rightarrow \dot{C}^{\log }$ ".

(3) In the discussion following the first display of [EtTh], Example 3.9, (ii), the phrase "unramified over the cusps of ..." should be replaced by the phrase "unramified over the cusps as well as over the generic points of the irreducible components of the special fibers of the stable models of ...". Also, the phrase "tempered coverings of the underlying ..." should be replaced by the phrase "tempered admissible coverings of the underlying ...".

In a word, the thrust of both the original text and the slight modifications just discussed is that the monoid " $\Phi_{W}^{\text {ell }}$ " is to be defined to be just large enough to include precisely those divisors which are necessary in order to treat the theta functions that appear in [EtTh].

Example 3.3. Frobenioids at Good Nonarchimedean Primes. Let $\underline{v} \in$ $\underline{\mathbb{V}}^{\text {good }} \cap \underline{\mathbb{V}}^{\text {non }}$. Then:

(i) Write

$$
\mathcal{D}_{\underline{v}} \stackrel{\text { def }}{=} \mathcal{B}\left(\underline{X}_{\underline{\underline{v}}}\right)^{0} ; \quad \mathcal{D}_{\underline{v}}^{\vdash} \stackrel{\text { def }}{=} \mathcal{B}\left(K_{\underline{v}}\right)^{0}
$$


[cf. §0]. Thus, $\mathcal{D}_{\underline{v}}^{\vdash}$ may be naturally regarded [by pulling back finite étale coverings via the structure morphism $\left.\underline{X}_{\underline{v}} \rightarrow \operatorname{Spec}\left(K_{\underline{v}}\right)\right]$ as a full subcategory

$$
\mathcal{D}_{\underline{v}}^{\vdash} \subseteq \mathcal{D}_{\underline{v}}
$$

of $\mathcal{D}_{\underline{v}}$, and we have a natural functor $\mathcal{D}_{\underline{v}} \rightarrow \mathcal{D}_{\underline{v}}^{\vdash}$, which is left-adjoint to the natural inclusion functor $\mathcal{D}_{\underline{v}}^{\vdash} \hookrightarrow \mathcal{D}_{\underline{v}}$ [cf. [FrdII], Example 1.3, (ii)]. For $\operatorname{Spec}(L) \in \operatorname{Ob}\left(\mathcal{D}_{\underline{v}}^{\vdash}\right)$ [i.e., $L$ is a finite separable extension of $K_{\underline{v}}$ ], write $\operatorname{ord}\left(\mathcal{O}_{L}^{\triangleright}\right) \stackrel{\text { def }}{=} \mathcal{O}_{L}^{\triangleright} / \mathcal{O}_{L}^{\times}$as in [FrdII], Example 1.1, (i). Thus, the assignment [cf. §0]

$$
\Phi_{\mathcal{C}_{\underline{v}}}: \operatorname{Spec}(L) \mapsto \operatorname{ord}\left(\mathcal{O}_{L}^{\triangleright}\right)^{\mathrm{pf}}
$$

determines a monoid $\Phi_{\mathcal{C}_{\underline{v}}}$ on $\left[\mathcal{D}_{\underline{v}}^{\vdash}\right.$, hence, by pull-back via the natural functor $\mathcal{D}_{\underline{v}} \rightarrow$ $\mathcal{D}_{\underline{v}}^{\vdash}$, on] $\mathcal{D}_{\underline{v}}$; the assignment

$$
\Phi_{\mathcal{C}_{\underline{v}}^{\vdash}}: \operatorname{Spec}(L) \mapsto \operatorname{ord}\left(\mathbb{Z}_{p_{\underline{v}}}^{\triangleright}\right)\left(\subseteq \operatorname{ord}\left(\mathcal{O}_{L}^{\triangleright}\right)^{\mathrm{pf}}\right)
$$

determines an absolutely primitive [cf. [FrdII], Example 1.1, (ii)] submonoid $\Phi_{\mathcal{C}_{\underline{v}}} \subseteq$ $\left.\Phi_{\mathcal{C}_{\underline{v}}}\right|_{\mathcal{D}_{\underline{v}}^{\vdash}}$ on $\mathcal{D}_{\underline{v}}^{\vdash}$; these monoids $\Phi_{\mathcal{C}_{\underline{v}}^{\vdash}}, \Phi_{\mathcal{C}_{\underline{v}}}$ determine $p_{\underline{\underline{v}}}$-adic Frobenioids

$$
\mathcal{C}_{\underline{v}}^{\vdash} \subseteq \mathcal{C}_{\underline{v}}
$$

[cf. [FrdII], Example 1.1, (ii), where we take " $\Lambda$ " to be $\mathbb{Z}]$, whose base categories are given by $\mathcal{D}_{\underline{v}}^{\vdash}, \mathcal{D}_{\underline{v}}$ [in a fashion compatible with the natural inclusion $\mathcal{D}_{\underline{v}}^{\vdash} \subseteq \mathcal{D}_{\underline{v}}$ ], respectively. Also, we shall write

$$
\underline{\underline{\mathcal{F}}}_{\underline{v}} \stackrel{\text { def }}{=} \mathcal{C}_{\underline{v}}
$$

[cf. the notation of Example 3.2, (i)]. Finally, let us observe that the element $p_{\underline{v}} \in \mathbb{Z}_{p_{\underline{v}}}^{\triangleright} \subseteq \mathcal{O}_{K_{\underline{v}}}^{\triangleright}$ determines a characteristic splitting

$$
\tau_{\underline{v}}^{\vdash}
$$

on $\mathcal{C}_{\underline{v}}^{\vdash}$ [cf. [FrdII], Theorem 1.2, (v)]. Write $\mathcal{F}_{\underline{v}}^{\vdash} \stackrel{\text { def }}{=}\left(\mathcal{C}_{\underline{v}}^{\vdash}, \tau_{\underline{v}}^{\vdash}\right)$ for the resulting split Frobenioid.

(ii) Next, let us write $\log \left(p_{\underline{v}}\right)$ for the element $p_{\underline{v}}$ of (i) considered additively and consider the monoid on $\mathcal{D}_{\underline{v}}^{\vdash}$

$$
\mathcal{O}_{\mathcal{C}_{\underline{v}}^{\vdash}}^{\triangleright}(-)=\mathcal{O}_{\mathcal{C}_{\underline{v}}^{\vdash}}^{\times}(-) \times\left(\mathbb{N} \cdot \log \left(p_{\underline{v}}\right)\right)
$$

associated to $\mathcal{C}_{v}^{\vdash}$ [cf. [FrdI], Proposition 2.2]. By replacing " $\log \left(p_{\underline{v}}\right)$ " by the formal symbol " $\log \left(p_{\underline{v}}\right) \cdot \log (\underline{\underline{\Theta}})=\log \left(p_{\underline{v}} \log (\underline{\underline{\Theta}})\right)$ ", we obtain a monoid

$$
\mathcal{O}_{\mathcal{C}_{\underline{v}}^{\ominus}}^{\triangleright}(-) \stackrel{\text { def }}{=} \mathcal{O}_{\mathcal{C}_{\underline{v}}^{\Theta}}^{\times}(-) \times\left(\mathbb{N} \cdot \log \left(p_{\underline{v}}\right) \cdot \log (\underline{\underline{\Theta}})\right)
$$


[i.e., where $\left.\mathcal{O}_{\mathcal{C}_{\underline{v}}^{\ominus}}^{\times}(-) \stackrel{\text { def }}{=} \mathcal{O}_{\mathcal{C}_{\underline{v}}^{\vdash}}^{\times}(-)\right]$, which is naturally isomorphic to $\mathcal{O}_{\mathcal{C}_{\underline{v}}^{\vdash}}^{\triangleright}$ and which arises as the monoid " $\mathcal{O}^{\triangleright}\left(-\frac{\underline{-}}{-}\right)$ " of [FrdI], Proposition 2.2, associated to some $p_{\underline{v}}$-adic Frobenioid $\mathcal{C}_{\underline{v}}^{\Theta}$ with base category $\mathcal{D}_{\underline{\underline{v}}}^{\Theta} \stackrel{\text { def }}{=} \mathcal{D}_{\underline{\underline{v}}}^{\vdash}$ equipped with a characteristic splitting $\tau_{\underline{v}}^{\Theta}$ determined by $\log \left(p_{\underline{v}}\right) \cdot \log (\underline{\underline{\Theta}})$. In particular, we have a natural equivalence

$$
\mathcal{F}_{\underline{v}}^{\vdash} \stackrel{\sim}{\rightarrow} \mathcal{F}_{\underline{v}}^{\Theta}
$$

— where $\mathcal{F}_{\underline{v}}^{\Theta} \stackrel{\text { def }}{=}\left(\mathcal{C}_{\underline{v}}^{\Theta}, \tau_{\underline{v}}^{\Theta}\right)$ — of split Frobenioids.

(iii) Here, it is useful to recall that

(a) the subcategory $\mathcal{D}_{\underline{v}}^{\vdash} \subseteq \mathcal{D}_{\underline{v}}$ may be reconstructed category-theoretically from $\mathcal{D}_{\underline{v}}[\mathrm{cf} .[$ AbsAnab], Lemma 1.3.8];

(b) the category $\mathcal{D}_{\underline{v}}^{+}$(respectively, $\mathcal{D}_{\underline{v}}^{\Theta}$ ) may be reconstructed category-theoretically from $\mathcal{C}_{v}^{\vdash}$ (respectively, $\mathcal{C}_{v}^{\Theta}$ ) [cf. [FrdI], Theorem 3.4, (v); [FrdII], Theorem 1.2, (i); [FrdII], Example 1.3, (i); [AbsAnab], Theorem 1.1.1, (ii)];

(c) the category $\mathcal{D}_{\underline{v}}$ may be reconstructed category-theoretically from $\underline{\underline{\mathcal{F}}}_{v}=\mathcal{C}_{\underline{v}}$ [cf. [FrdI], Theorem 3.4, (v); [FrdII], Theorem 1.2, (i); [FrdII], Example 1.3, (i); [AbsAnab], Lemma 1.3.1].

Note that it follows immediately from the category-theoreticity of the divisor monoid $\Phi_{\mathcal{C}_{\underline{v}}}$ [cf. [FrdI], Corollary 4.11, (iii); [FrdII], Theorem 1.2, (i)], together with (a), (c), and the definition of $\mathcal{C}_{\underline{v}}^{\vdash}$ [cf. also [AbsAnab], Proposition 1.2.1, (v)], that

(d) $\mathcal{C}_{\underline{v}}^{\vdash}$ may be reconstructed category-theoretically from $\underline{\underline{\mathcal{F}}} \underline{\underline{v}}$.

Finally, by applying the algorithmically constructed field structure on the image of the Kummer map of [AbsTopIII], Proposition 3.2, (iii) [cf. Remark 3.1.2; Remark 3.3.2 below], it follows that one may construct the element " $p_{\underline{v}}$ " of $\mathcal{O}_{K_{\underline{v}}}^{\triangleright}$ category-theoretically from $\underline{\underline{\mathcal{F}}}_{\underline{v}}$, hence that the characteristic splitting $\tau_{\underline{v}}^{\vdash}$ may be reconstructed category-theoretically from $\underline{\underline{\mathcal{F}}}_{\underline{v}}$. [Here, we recall that the curve $X_{F}$ is "of strictly Belyi type" — cf. [AbsTopIII], Remark 2.8.3.] In particular,

(e) one may reconstruct the split Frobenioids $\mathcal{F}_{\underline{v}}^{+}, \mathcal{F}_{\underline{v}}^{\Theta}$ category-theoretically from $\underline{\underline{\mathcal{F}}}_{\underline{v}}$.

Remark 3.3.1. A similar remark to Remark 3.2.1 [i.e., concerning the phrase "reconstructed category-theoretically"] applies to the Frobenioids $\mathcal{C}_{\underline{v}}, \mathcal{C}_{\underline{v}}^{\vdash}$ constructed in Example 3.3.

Remark 3.3.2. Note that the $p_{\underline{v}}$-adic Frobenioid $\mathcal{C}_{\underline{v}}$ (respectively, $\mathcal{C}_{\underline{v}}$ ) of Example 3.3 , (i), consists of essentially the same data as an "MLF-Galois TM-pair of strictly Belyi type" (respectively, "MLF-Galois TM-pair of mono-analytic type"), in the sense of [AbsTopIII], Definition 3.1, (ii) [cf. [AbsTopIII], Remark 3.1.1]. A similar 
remark applies to the $p_{\underline{v}}$-adic Frobenioid $\mathcal{C}_{\underline{v}}$ (respectively, $\mathcal{C}_{\underline{v}}^{\vdash}$ ) of Example 3.2 , (iii), (iv) [cf. [AbsTopIII], Remark 3.1.3].

Example 3.4. Frobenioids at Archimedean Primes. Let $\underline{v} \in \underline{\mathbb{V}}^{\text {arc }}$. Then:

(i) Write

$$
\mathbb{X}_{\underline{v}}, \mathbb{C}_{\underline{v}}, \underline{\mathbb{X}}_{\underline{v}}, \underline{\mathbb{C}}_{\underline{v}}, \underline{\mathbb{X}}_{\underline{v}}, \underline{\mathbb{C}}_{\underline{v}}
$$

for the Aut-holomorphic orbispaces [cf. [AbsTopIII], Definition 2.1, (i); [AbsTopIII], Remark 2.1.1] determined, respectively, by the hyperbolic orbicurves $X_{K}, C_{K}, \underline{X}_{K}$, $\underline{C}_{K}, \underline{X}_{K}, \underline{C}_{K}$ at $\underline{v}$. Thus, for $\square \in\left\{\mathbb{X}_{\underline{v}}, \mathbb{C}_{\underline{v}}, \underline{\mathbb{X}}_{\underline{v}}, \underline{\mathbb{C}}_{\underline{v}}, \underline{\mathbb{X}}_{\underline{v}}, \underline{\mathbb{C}}_{\underline{v}}\right\}$, we have a complex archimedean topological field [i.e., a "CAF" — cf. §0]

\section{$\overline{\mathcal{A}}_{\square}$}

[cf. [AbsTopIII], Definition 4.1, (i)] which may be algorithmically constructed from $\square$; write $\mathcal{A}_{\square} \stackrel{\text { def }}{=} \overline{\mathcal{A}}_{\square} \backslash\{0\}$ [cf. Remark 3.4.3, (i), below]. Next, let us write

$$
\mathcal{D}_{\underline{v}} \stackrel{\text { def }}{=} \underline{\mathbb{X}}_{\underline{v}}
$$

and

$$
\mathcal{C}_{\underline{v}}
$$

for the archimedean Frobenioid as in [FrdII], Example 3.3, (ii) [i.e., "C" of loc. cit.], where we take the base category [i.e., " $\mathcal{D}$ " of loc. cit.] to be the one-morphism category determined by $\operatorname{Spec}\left(K_{\underline{v}}\right)$. Thus, the linear morphisms among the pseudoterminal objects of $\mathcal{C}$ determine unique isomorphisms [cf. [FrdI], Definition 1.3, (iii), (c)] among the respective topological monoids " $\mathcal{O}^{\triangleright}(-)$ " — where we recall [cf. [FrdI], Theorem 3.4, (iii); [FrdII], Theorem 3.6, (i), (vii)] that these topological monoids may be reconstructed category-theoretically from $\mathcal{C}$. In particular, it makes sense to write " $\mathcal{O}^{\triangleright}\left(\mathcal{C}_{\underline{v}}\right)$ ", " $\mathcal{O}^{\times}\left(\mathcal{C}_{\underline{v}}\right) \subseteq \mathcal{O}^{\triangleright}\left(\mathcal{C}_{\underline{v}}\right)$ ". Moreover, we observe that, by construction, there is a natural isomorphism

$$
\mathcal{O}^{\triangleright}\left(\mathcal{C}_{\underline{v}}\right) \stackrel{\sim}{\rightarrow} \mathcal{O}_{K_{\underline{v}}}^{\triangleright}
$$

of topological monoids. Thus, one may also think of $\mathcal{C}_{\underline{v}}$ as a "Frobenioid-theoretic representation" of the topological monoid $\mathcal{O}_{K_{\underline{v}}}^{\triangleright}[$ cf. [A Observe that there is a natural topological isomorphism $K_{\underline{v}} \stackrel{\sim}{\rightarrow} \overline{\mathcal{A}}_{\mathcal{D}_{\underline{v}}}$, which may be restricted to $\mathcal{O}_{K_{\underline{v}}}^{\triangleright}$ to obtain an inclusion of topological monoids

$$
\kappa_{\underline{v}}: \mathcal{O}^{\triangleright}\left(\mathcal{C}_{\underline{v}}\right) \hookrightarrow \mathcal{A}_{\mathcal{D}_{\underline{v}}}
$$

— which we shall refer to as the Kummer structure on $\mathcal{C}_{\underline{v}}$ [cf. Remark 3.4.2 below]. Write

$$
\underline{\underline{\mathcal{F}}} \underline{\underline{v}} \stackrel{\text { def }}{=}\left(\mathcal{C}_{\underline{v}}, \mathcal{D}_{\underline{v}}, \kappa_{\underline{v}}\right)
$$

[cf. Example 3.2, (i); Example 3.3, (i)]. 
(ii) Next, recall the category $\mathbb{T M}^{\vdash}$ of "split topological monoids" of [AbsTopIII], Definition 5.6, (i) - i.e., the category whose objects $(C, \vec{C})$ consist of a topological monoid $C$ isomorphic to $\mathcal{O}_{\mathbb{C}}^{\triangleright}$ and a topological submonoid $\vec{C} \subseteq C$ [necessarily isomorphic to $\mathbb{R}_{>0}$ ] such that the natural inclusions $C^{\times} \hookrightarrow C$ [where $C^{\times}$, which is necessarily isomorphic to $\mathbb{S}^{1}$, denotes the topological submonoid of invertible elements], $\vec{C} \hookrightarrow C$ determine an isomorphism $C^{\times} \times \vec{C} \stackrel{\sim}{\rightarrow} C$ of topological monoids, and whose morphisms $\left(C_{1}, \vec{C}_{1}\right) \rightarrow\left(C_{2}, \vec{C}_{2}\right)$ are isomorphisms of topological monoids $C_{1} \stackrel{\sim}{\rightarrow} C_{2}$ that induce isomorphisms $\vec{C}_{1} \stackrel{\sim}{\rightarrow} \vec{C}_{2}$. Note that the CAF's $K_{\underline{v}}, \overline{\mathcal{A}}_{\mathcal{D}_{\underline{v}}}$ determine, in a natural way, objects of $\mathbb{T M}^{\vdash}$. Write

$$
\tau_{\underline{v}}^{\vdash}
$$

for the resulting characteristic splitting of the Frobenioid $\mathcal{C}_{v}^{\vdash} \stackrel{\text { def }}{=} \mathcal{C}_{\underline{v}}$, i.e., so that we may think of the pair $\left(\mathcal{O}^{\triangleright}\left(\mathcal{C}_{\underline{v}}^{\vdash}\right), \tau_{\underline{v}}^{\vdash}\right)$ as the object of $\mathbb{T M}^{\vdash}$ determined by $K_{\underline{v}}$;

$$
\mathcal{D}_{\underline{v}}^{\vdash}
$$

for the object of $\mathbb{M M}^{\vdash}$ determined by $\overline{\mathcal{A}}_{\mathcal{D}_{\underline{v}}}$;

$$
\mathcal{F}_{\underline{v}}^{\vdash} \stackrel{\text { def }}{=}\left(\mathcal{C}_{\underline{v}}^{\vdash}, \mathcal{D}_{\underline{v}}^{\vdash}, \tau_{\underline{v}}^{\vdash}\right)
$$

for the [ordered] triple consisting of $\mathcal{C}_{\underline{v}}^{\vdash}, \mathcal{D}_{\underline{v}}^{\vdash}$, and $\tau_{\underline{v}}^{\vdash}$. Thus, the object $\left(\mathcal{O}^{\triangleright}\left(\mathcal{C}_{\underline{v}}^{\vdash}\right), \tau_{\underline{v}}^{\vdash}\right)$ of $\mathbb{T M}^{\vdash}$ is isomorphic to $\mathcal{D}_{\underline{v}}^{\vdash}$. Moreover, $\mathcal{C}_{\underline{v}}^{\vdash}$ (respectively, $\mathcal{D}_{\underline{v}}^{\vdash} ; \mathcal{F}_{\underline{v}}^{\vdash}$ ) may be algorithmically reconstructed from $\underline{\underline{\underline{\mathcal{F}}}} \underline{\underline{v}}$ (respectively, $\left.\mathcal{D}_{\underline{v}} ; \underline{\underline{\mathcal{F}}}_{\underline{v}}\right)$.

(iii) Next, let us observe that $p_{\underline{v}} \in K_{\underline{v}}$ [cf. $\left.\S 0\right]$ may be thought of as a(n) [nonidentity] element of the noncompact factor $\Phi_{\mathcal{C}_{v}^{\vdash}}$ [i.e., the factor denoted by a " $\rightarrow$ " in the definition of $\mathbb{T M}^{\vdash}$ ] of the object $\left(\mathcal{O}^{\triangleright}\left(\mathcal{C}_{v}^{\vdash}\right), \tau_{v}^{\vdash}\right)$ of $\mathbb{T M}^{\vdash}$. This noncompact factor $\Phi_{\mathcal{C}_{\underline{v}}}$ is isomorphic, as a topological monoid, to $\mathbb{R}_{\geq 0}$; let us write $\Phi_{\mathcal{C}_{\underline{v}}^{\vdash}}$ additively and denote by $\log \left(p_{\underline{v}}\right)$ the element of $\Phi_{\mathcal{C}_{v}}$ determined by $p_{\underline{v}}$. Thus, relative to the natural action [by multiplication!] of $\overline{\mathbb{R}}_{\geq 0}$ on $\Phi_{\mathcal{C}_{v}}$, it follows that $\log \left(p_{\underline{v}}\right)$ is a generator of $\Phi_{\mathcal{C}_{\underline{v}}^{\vdash}}$. In particular, we may form a new topological monoid

$$
\Phi_{\mathcal{C}_{\underline{v}}^{\ominus}} \stackrel{\text { def }}{=} \mathbb{R}_{\geq 0} \cdot \log \left(p_{\underline{v}}\right) \cdot \log (\underline{\underline{\Theta}})
$$

isomorphic to $\mathbb{R}_{\geq 0}$ that is generated by a formal symbol " $\log \left(p_{\underline{\underline{v}}}\right) \cdot \log (\underline{\underline{\Theta}})=\log \left(p_{\underline{\underline{v}}}(\underline{\underline{\Theta}})\right)$ ".

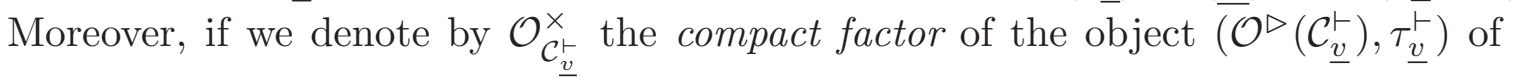
$\mathbb{T M}^{\vdash}$, and set $\mathcal{O}_{\mathcal{C}_{\underline{v}}^{\ominus}}^{\times} \stackrel{\text { def }}{=} \mathcal{O}_{\mathcal{C}_{\underline{v}}^{\vee}}^{\times}$, then we obtain a new split Frobenioid $\left(\mathcal{C}_{\underline{v}}^{\Theta}, \tau_{\underline{v}}^{\Theta}\right)$, isomorphic to $\left(\mathcal{C}_{\underline{v}}^{\vdash}, \tau_{\underline{v}}^{\vdash}\right)$, such that

$$
\mathcal{O}^{\triangleright}\left(\mathcal{C}_{\underline{v}}^{\Theta}\right)=\mathcal{O}_{\mathcal{C}_{\underline{v}}^{\ominus}}^{\times} \times \Phi_{\mathcal{C}_{\underline{v}}^{\Theta}}
$$


- where we note that this equality gives rise to a natural isomorphism of split Frobenioids $\left(\mathcal{C}_{v}^{\vdash}, \tau_{v}^{\vdash}\right) \stackrel{\sim}{\rightarrow}\left(\mathcal{C}_{v}^{\Theta}, \tau_{v}^{\Theta}\right)$, obtained by "forgetting the formal symbol $\log (\underline{\Theta})$ ". In particular, we thus obtain a natural isomorphism

$$
\mathcal{F}_{\underline{v}}^{\vdash} \stackrel{\sim}{\rightarrow} \mathcal{F}_{\underline{v}}^{\Theta}
$$

— where we write $\mathcal{F}_{\underline{v}}^{\Theta} \stackrel{\text { def }}{=}\left(\mathcal{C}_{\underline{v}}^{\Theta}, \mathcal{D}_{\underline{v}}^{\Theta}, \tau_{\underline{v}}^{\Theta}\right)$ for the [ordered] triple consisting of $\mathcal{C}_{\underline{v}}^{\Theta}$, $\mathcal{D}_{\underline{v}}^{\Theta} \stackrel{\text { def }}{=} \mathcal{D}_{\underline{v}}^{+}, \tau_{\underline{v}}^{\Theta}$. Finally, we observe that $\mathcal{F}_{\underline{v}}^{\Theta}$ may be algorithmically reconstructed from $\underline{\underline{\mathcal{F}}} \underline{\underline{v}}$.

Remark 3.4.1. A similar remark to Remark 3.2.1 [i.e., concerning the phrase "reconstructed category-theoretically"] applies to the phrase "algorithmically reconstructed" that was applied in the discussion of Example 3.4.

Remark 3.4.2. One way to think of the Kummer structure

$$
\kappa_{\underline{v}}: \mathcal{O}^{\triangleright}\left(\mathcal{C}_{\underline{v}}\right) \hookrightarrow \mathcal{A}_{\mathcal{D}_{\underline{v}}}
$$

discussed in Example 3.4, (i), is as follows. In the terminology of [AbsTopIII], Definition 2.1, (i), (iv), the structure of CAF on $\overline{\mathcal{A}}_{\mathcal{D}_{\underline{v}}}$ determines, via pull-back by $\kappa_{\underline{v}}$, an Aut-holomorphic structure on the groupification $\mathcal{O}^{\triangleright}\left(\mathcal{C}_{\underline{v}}\right)^{\text {gp }}$ of $\mathcal{O}^{\triangleright}\left(\mathcal{C}_{\underline{v}}\right)$, together with a [tautological!] co-holomorphicization $\mathcal{O}^{\triangleright}\left(\mathcal{C}_{\underline{v}}\right)^{\mathrm{gp}} \rightarrow \mathcal{A}_{\mathcal{D}_{\underline{v}}}$. Conversely, if one starts with this Aut-holomorphic structure on [the groupification of] the topological monoid $\mathcal{O}^{\triangleright}\left(\mathcal{C}_{\underline{v}}\right)$, together with the co-holomorphicization $\mathcal{O}^{\triangleright}\left(\mathcal{C}_{\underline{v}}\right)^{\mathrm{gp}} \rightarrow \mathcal{A}_{\mathcal{D}_{\underline{v}}}$, then one verifies immediately that one may recover the inclusion of topological monoids $\kappa_{v}$. [Indeed, this follows immediately from [AbsTopIII], Corollary 2.3, together with the elementary fact that every holomorphic automorphism of the complex Lie group $\mathbb{C}^{\times}$that preserves the submonoid of elements of norm $\leq 1$ is equal to the identity.] That is to say, in summary,

the Kummer structure $\kappa_{\underline{v}}$ is completely equivalent to the collection of data consisting of the Aut-holomorphic structure [induced by $\kappa_{\underline{v}}$ ] on the groupification $\mathcal{O}^{\triangleright}\left(\mathcal{C}_{\underline{v}}\right)^{\text {gp }}$ of $\mathcal{O}^{\triangleright}\left(\mathcal{C}_{\underline{v}}\right)$, together with the co-holomorphicization [induced by $\left.\kappa_{\underline{v}}\right] \mathcal{O}^{\triangleright}\left(\mathcal{C}_{\underline{v}}\right)^{\mathrm{gp}} \rightarrow \mathcal{A}_{\mathcal{D}_{\underline{v}}}$.

The significance of thinking of Kummer structures in this way lies in the observation that [unlike inclusions of topological monoids!]

the co-holomorphicization induced by $\kappa_{v}$ is compatible with the logarithm operation discussed in [AbsTopIII], Corollary 4.5.

Indeed, this observation may be thought of as a rough summary of a substantial portion of the content of [AbsTopIII], Corollary 4.5. Put another way, thinking of Kummer structures in terms of co-holomorphicizations allows one to separate out the portion of the structures involved that is not compatible with this logarithm operation - i.e., the monoid structures! - from the portion of the structures involved that is compatible with this logarithm operation - i.e., the tautological co-holomorphicization. 


\section{Remark 3.4.3.}

(i) In the notation of Example 3.4, write $\mathcal{A}_{\square}^{\times} \subseteq \overline{\mathcal{A}}_{\square}$ for the topological group of units [i.e., of elements of norm 1] of the CAF $\overline{\mathcal{A}}_{\square}$ [so $\mathcal{A}_{\square}^{\times}$is noncanonically isomorphic to the unit circle $\left.\mathbb{S}^{1}\right] ; \mathcal{A}_{\square}^{\mu} \subseteq \mathcal{A}_{\square}^{\times}$for the subgroup of torsion elements [so $\mathcal{A}_{\square}^{\mu}$ is noncanonically isomorphic to $\left.\mathbb{Q} / \mathbb{Z}\right] ; \underline{\mathbb{E}}_{v}$ for the Aut-holomorphic space [cf. [AbsTopIII], Definition 2.1, (i)] determined by the elliptic curve obtained by compactifying $\underline{X}_{K}$ at $\underline{v}$. Now recall from the construction of " $\overline{\mathcal{A}}_{\square}$ " in [AbsTopIII], Corollary 2.7 [cf. also [AbsTopIII], Definition 4.1, (i)] via the technique of "holomorphic elliptic cuspidalization", that one has a natural isomorphism of CAF's

$$
\overline{\mathcal{A}}_{\underline{\mathbb{X}}_{\underline{v}}}=\overline{\mathcal{A}}_{\mathcal{D}_{\underline{v}}} \stackrel{\sim}{\rightarrow} \overline{\mathcal{A}}_{\underline{\mathbb{X}}_{\underline{v}}}
$$

— which may be used to "identify" $\overline{\mathcal{A}}_{\underline{\mathbb{X}}_{\underline{v}}}=\overline{\mathcal{A}}_{\mathcal{D}_{\underline{v}}}$ with $\overline{\mathcal{A}}_{\underline{\mathbb{X}}_{\underline{v}}}$. Indeed, thinking of " $\overline{\mathcal{A}}_{\underline{\mathbb{X}}_{\underline{v}}}=\overline{\mathcal{A}}_{\mathcal{D}_{\underline{v}}}$ " as " $\overline{\mathcal{A}}_{\underline{\mathbb{X}}_{\underline{v}}}$ " is natural from the point of view of the " $\Theta$-approach" discussed in Remark 3.1.2, (ii). Moreover, by allowing $\mathcal{A}_{\underline{\mathbb{X}}_{v}}^{\times}$to "act" [cf. the algorithm discussed in [AbsTopIII], Corollary 2.7, (e)] on points in a sufficiently small neighborhood of [but not equal to!] a given point " $x$ " of $\underline{\mathbb{E}}_{v}$, one may regard the "circle" $\mathcal{A}_{\underline{\mathbb{X}}_{v}}^{\times}$as a deformation retract of the complement of $x$ in a suitable small neighborhoōd of $x$ in $\underline{\mathbb{E}}_{\underline{v}}$. In particular,

from the point of view of the " $\Theta$-approach" discussed in Remark 3.1.2, (ii), it is natural to think of " $\overline{\mathcal{A}}_{\underline{\mathbb{X}}_{\underline{v}}}=\overline{\mathcal{A}}_{\mathcal{D}_{\underline{v}}}$ " as " $\overline{\mathcal{A}}_{\underline{\mathbb{X}}_{\underline{v}}}$ " and to regard

$$
\operatorname{Hom}\left(\mathbb{Q} / \mathbb{Z}, \mathcal{A}_{\underline{\mathbb{X}}_{\underline{v}}}^{\mu}\right)=\operatorname{Hom}\left(\mathbb{Q} / \mathbb{Z}, \mathcal{A}_{\underline{\mathbb{X}}_{\underline{v}}}^{\times}\right)
$$

[a profinite group which is noncanonically isomorphic to $\widehat{\mathbb{Z}}$ ] as the result of identifying the cuspidal inertia groups of the various points " $x$ " of $\underline{\underline{\mathbb{E}}}$

- cf. discussion of the cuspidal inertia groups " $I_{x}$ " in [AbsTopIII], Proposition 1.4, (i), (ii). Indeed, this interpretation of $\overline{\mathcal{A}}_{\underline{\mathbb{X}}_{v}}=\overline{\mathcal{A}}_{\mathcal{D}_{\underline{v}}}$ via cuspidal inertia groups may be thought of as a sort of archimedean version of the " $\Theta$-approach" discussed in Remark 3.1.2, (ii).

(ii) We observe that just as the theory of elliptic cuspidalization [cf. [AbsTopII], Example 3.2; [AbsTopII], Corollaries 3.3, 3.4] admits a straightforward holomorphic analogue, i.e., the theory of "holomorphic elliptic cuspidalization" [cf. [AbsTopIII], Corollary 2.7] referred to in (i) above, the theory of Belyi cuspidalization [cf. [AbsTopII], Example 3.6; [AbsTopII], Corollaries 3.7, 3.8; [AbsTopIII], Remark 2.8.3] admits a straightforward holomorphic analogue, i.e., a theory of "holomorphic Belyi cuspidalization". We leave the routine details to the reader. Here, we observe that one immediate consequence of such "holomorphic Belyi cuspidalizations" may be stated as follows:

the set of NF-points [i.e., points defined over a number field] of the underlying topological space of the Aut-holomorphic space $\mathcal{D}_{\underline{v}}$ may be reconstructed via a functorial algorithm from the [abstract] Autholomorphic space $\mathcal{D}_{\underline{v}}$. 


\section{Example 3.5. Global Realified Frobenioids.}

(i) Write

$$
\mathcal{C}_{\text {mod }}^{\Vdash}
$$

for the realification [cf. [FrdI], Theorem 6.4, (ii)] of the Frobenioid of [FrdI], Example 6.3 [cf. also Remark 3.1.5 of the present paper], associated to the number field $F_{\text {mod }}$ and the trivial Galois extension [i.e., the Galois extension of degree 1] of $F_{\bmod }$ [so the base category of $\mathcal{C}_{\bmod }^{\Vdash}$ is, in the terminology of [FrdI], equivalent to a onemorphism category]. Thus, the divisor monoid $\Phi_{\mathcal{C}_{\bmod }}^{\Vdash}$ of $\mathcal{C}_{\bmod }^{\Vdash}$ may be thought of as a single abstract monoid, whose set of primes, which we denote $\operatorname{Prime}\left(\mathcal{C}_{\text {mod }}^{\Vdash}\right)$ [cf. [FrdI], §0], is in natural bijective correspondence with $\mathbb{V}_{\bmod }$ [cf. the discussion of [FrdI], Example 6.3]. Moreover, the submonoid $\Phi_{\mathcal{C}_{\text {mod }}^{\Vdash}, v}$ of $\Phi_{\mathcal{C}_{\text {mod }}^{\Vdash}}$ corresponding to $v \in \mathbb{V}_{\text {mod }}$ is naturally isomorphic to $\operatorname{ord}\left(\mathcal{O}_{\left(F_{\text {mod }}\right)_{v}}^{\triangleright}\right)^{\text {pf }} \otimes \mathbb{R}_{\geq 0}\left(\cong \mathbb{R}_{\geq 0}\right)$ [i.e., to $\operatorname{ord}\left(\mathcal{O}_{\left(F_{\text {mod }}\right)_{v}}^{\triangleright}\right)\left(\cong \mathbb{R}_{\geq 0}\right)$ if $\left.v \in \mathbb{V}_{\text {mod }}^{\text {arc }}\right]$. In particular, $p_{v}$ determines an element $\log _{\text {mod }}^{\vdash}\left(p_{v}\right) \in \Phi_{\mathcal{C}_{\text {mod }}^{\Vdash},}$. Write $\underline{v} \in \underline{\mathbb{V}}$ for the element of $\underline{\mathbb{V}}$ that corresponds to $v$. Then observe that regardless of whether $\underline{v}$ belongs to $\underline{\mathbb{V}}^{\text {bad }}, \underline{\mathbb{V}}^{\text {good }} \cap \underline{\mathbb{V}}^{\text {non }}$, or $\underline{\mathbb{V}}^{\text {arc }}$, the realification $\Phi_{\mathcal{C}_{\underline{v}}}^{\text {rlf }}$ of the divisor monoid $\Phi_{\mathcal{C}_{\underline{v}}^{\vdash}}$ of $\mathcal{C}_{\underline{v}}^{\vdash}$ [which, as is easily verified, is a constant monoid over the corresponding base category] may be regarded as a single abstract monoid isomorphic to $\mathbb{R}_{\geq 0}$. Write $\log _{\Phi}\left(p_{\underline{v}}\right) \in \Phi_{\mathcal{C}_{\underline{v}}}^{\text {rlf }}$ for the element defined by $p_{\underline{v}}$ and

$$
\mathcal{C}_{\rho_{\underline{v}}}: \mathcal{C}_{\text {mod }}^{\Vdash} \rightarrow\left(\mathcal{C}_{\underline{v}}^{\vdash}\right)^{\text {rlf }}
$$

for the natural restriction functor [cf. the theory of poly-Frobenioids developed in [FrdII], §5] to the realification of the Frobenioid $\mathcal{C}_{\underline{v}}^{\vdash}$ [cf. [FrdI], Proposition 5.3]. Thus, one verifies immediately that $\mathcal{C}_{\rho_{v}}$ is determined, up to isomorphism, by the isomorphism of topological monoids [which are isomorphic to $\mathbb{R}_{\geq 0}$ ]

$$
\rho_{\underline{v}}: \Phi_{\mathcal{C}_{\text {mod }}^{\Vdash}, v} \stackrel{\sim}{\rightarrow} \Phi_{\mathcal{C}_{\underline{v}}^{\vdash}}^{\mathrm{rlf}}
$$

induced by $\mathcal{C}_{\rho_{\underline{v}}}$ - which, by considering the natural "volume interpretations" of the arithmetic divisors involved, is easily computed to be given by the assignment $\log _{\text {mod }}^{\vdash}\left(p_{v}\right) \mapsto \frac{1}{\left[K_{\underline{v}}:\left(F_{\text {mod }}\right)_{v}\right]} \log _{\Phi}\left(p_{\underline{v}}\right)$.

(ii) In a similar vein, one may construct a " $\Theta$-version" [i.e., as in Examples 3.2 , (v); 3.3, (ii); 3.4, (iii)] of the various data constructed in (i). That is to say, we set

$$
\Phi_{\mathcal{C}_{\text {tht }}} \stackrel{\text { def }}{=} \Phi_{\mathcal{C}_{\text {mod }}^{\Vdash}} \cdot \log (\underline{\underline{\Theta}})
$$

- i.e., an isomorphic copy of $\Phi_{\mathcal{C}_{\bmod }}$ generated by a formal symbol $\log (\underline{\underline{\Theta}})$. This monoid $\Phi_{\mathcal{C}_{\text {tht }}^{\Vdash}}$ thus determines a Frobenioid $\mathcal{C}_{\text {tht }}^{\Vdash}$, equipped with a natural equivalence of categories $\mathcal{C}_{\text {mod }}^{\Vdash} \stackrel{\sim}{\rightarrow} \mathcal{C}_{\text {tht }}^{\Vdash}$ and a natural bijection Prime $\left(\mathcal{C}_{\text {tht }}^{\Vdash}\right) \stackrel{\sim}{\rightarrow} \underset{\mathbb{V} \text { mod. }}{ }$. For $v \in$ $\mathbb{V}_{\text {mod }}$, the element $\log _{\text {mod }}^{\vdash}\left(p_{v}\right)$ of the submonoid $\Phi_{\mathcal{C}_{\text {mod }}^{\Vdash}, v} \subseteq \Phi_{\mathcal{C}_{\text {mod }}^{\Vdash}}$ thus determines an element $\log _{\text {mod }}^{\vdash}\left(p_{v}\right) \cdot \log (\underline{\underline{\Theta}})$ of a submonoid $\Phi_{\mathcal{C}_{\text {tht }}^{\Vdash}, v} \subseteq \Phi_{\mathcal{C}_{\text {tht }}^{\vdash}}$. Write $\underline{v} \in \underline{\mathbb{V}}$ for the element of $\underline{\mathbb{V}}$ that corresponds to $v$. Then the realification $\Phi_{\mathcal{C}_{\underline{v}}^{\ominus}}^{\text {rlf }}$ of the divisor monoid $\Phi_{\mathcal{C}_{\underline{v}}^{\Theta}}$ of $\mathcal{C}_{\underline{v}}^{\Theta}$ [which, as is easily verified, is a constant monoid over the corresponding 
base category] may be regarded as a single abstract monoid isomorphic to $\mathbb{R}_{\geq 0}$. Write

$$
\mathcal{C}_{\rho_{\underline{v}}^{\Theta}}: \mathcal{C}_{\text {tht }}^{\Vdash} \rightarrow\left(\mathcal{C}_{\underline{v}}^{\Theta}\right)^{\text {rlf }}
$$

for the natural restriction functor [cf. (i) above; the theory of poly-Frobenioids developed in [FrdII], \$5] to the realification of the Frobenioid $\mathcal{C}_{\underline{v}}^{\Theta}$ [cf. [FrdI], Proposition 5.3]. Thus, one verifies immediately that $\mathcal{C}_{\rho_{\underline{v}}^{\Theta}}$ is determined, up to isomorphism, by the isomorphism of topological monoids [which are isomorphic to $\mathbb{R}_{\geq 0}$ ]

$$
\rho_{\underline{v}}^{\Theta}: \Phi_{\mathcal{C}_{\mathrm{tht}}^{\Vdash}, v} \stackrel{\sim}{\rightarrow} \Phi_{\mathcal{C}_{\underline{v}}^{\Theta}}^{\mathrm{rlf}}
$$

induced by $\mathcal{C}_{\rho_{\underline{v}}^{\Theta}}$. If $\underline{v} \in \underline{\mathbb{V}}^{\text {good }}$, then write $\log _{\Phi}\left(p_{\underline{v}}\right) \cdot \log (\underline{\underline{\Theta}}) \in \Phi_{\mathcal{C}_{\underline{v}}^{\Theta}}^{\text {rlf }}$ for the element determined by $\log _{\Phi}\left(p_{\underline{v}}\right)$; thus, [cf. (i)] $\rho_{\underline{v}}^{\Theta}$ is given by the assignment $\log _{\bmod }^{\vdash}\left(p_{v}\right)$. $\log (\underline{\underline{\Theta}}) \mapsto \frac{1}{\left[K_{\underline{v}}:\left(F_{\bmod }\right)_{v}\right]} \log _{\Phi}\left(p_{\underline{v}}\right) \cdot \log (\underline{\underline{\Theta}})$. On the other hand, if $\underline{v} \in \underline{\mathbb{V}}^{\text {bad }}$, then let us write

$$
\log _{\Phi}\left(\underline{\underline{\Theta}}_{\underline{v}}\right) \in \Phi_{\mathcal{C}_{\underline{v}}^{\Theta}}^{\mathrm{rlf}}
$$

for the element determined by $\underline{\underline{\Theta}}_{\underline{v}}\left[\right.$ cf. Example 3.2, (v)] and $\log _{\Phi}\left(p_{\underline{v}}\right)$ for the constant section of $\Phi_{\mathcal{C}_{\underline{v}}}$ determined by $p_{\underline{v}}$ [cf. the notation " $\log _{\Phi}(\underline{\underline{q}} \underline{\underline{v}})$ " of Example 3.2 , (iv)]; in particular, it makes sense to write $\log _{\Phi}\left(p_{\underline{v}}\right) / \log _{\Phi}\left(\underline{\underline{q}}_{\underline{v}}\right) \in \mathbb{Q}_{>0} ;$ thus, [cf. (i)] $\rho_{\underline{v}}^{\Theta}$ is given by the assignment

$$
\log _{\text {mod }}^{\vdash}\left(p_{v}\right) \cdot \log (\underline{\underline{\Theta}}) \mapsto \frac{\log _{\Phi}\left(p_{\underline{v}}\right)}{\left[K_{\underline{v}}:\left(F_{\text {mod }}\right)_{v}\right]} \cdot \frac{\log _{\Phi}\left(\underline{\underline{\Theta}}_{\underline{v}}\right)}{\log _{\Phi}\left(\underline{\underline{q}}_{\underline{v}}\right)}
$$

- cf. Remark 3.5.1, (i), below. Note that, for arbitrary $\underline{v} \in \underline{\mathbb{V}}$, the various $\rho_{\underline{v}}$, $\rho_{\underline{v}}^{\Theta}$ are compatible with the natural isomorphisms $\mathcal{C}_{\text {mod }}^{\Vdash} \stackrel{\sim}{\rightarrow} \mathcal{C}_{\text {tht }}^{\Vdash}, \mathcal{C}_{\underline{v}}^{\vdash} \stackrel{\sim}{\rightarrow} \mathcal{C}_{\underline{v}}^{\Theta}$ [cf. $\left.\S 0\right]$. This fact may be expressed as a natural isomorphism between cöllections of data [consisting of a category, a bijection of sets, a collection of data indexed by $\underline{\mathbb{V}}$, and a collection of isomorphisms indexed by $\underline{\mathbb{V}}]$

$$
\mathfrak{F}_{\text {mod }}^{\Vdash} \stackrel{\sim}{\rightarrow} \quad \mathfrak{F}_{\text {tht }}^{\Vdash}
$$

— where we write

$$
\begin{aligned}
& \mathfrak{F}_{\text {mod }}^{\Vdash} \stackrel{\text { def }}{=}\left(\mathcal{C}_{\text {mod }}^{\Vdash}, \operatorname{Prime}\left(\mathcal{C}_{\text {mod }}^{\Vdash}\right) \stackrel{\sim}{\rightarrow} \underline{\mathbb{V}},\left\{\mathcal{F}_{\underline{v}}^{\vdash}\right\}_{\underline{v} \in \underline{\mathbb{V}}},\left\{\rho_{\underline{v}}\right\}_{\underline{v} \in \underline{\mathbb{V}}}\right) \\
& \mathfrak{F}_{\text {tht }}^{\Vdash} \stackrel{\text { def }}{=}\left(\mathcal{C}_{\text {tht }}^{\Vdash}, \operatorname{Prime}\left(\mathcal{C}_{\text {tht }}^{\Vdash}\right) \stackrel{\sim}{\rightarrow} \underline{\mathbb{V}},\left\{\mathcal{F}_{\underline{v}}^{\Theta}\right\}_{\underline{v} \in \underline{\mathbb{V}}},\left\{\rho_{\underline{v}}^{\Theta}\right\}_{\underline{v} \in \underline{\mathbb{V}}}\right)
\end{aligned}
$$

[and we apply the natural bijection $\underline{\mathbb{V}} \stackrel{\sim}{\rightarrow} \mathbb{V}_{\text {mod }}$ ]; cf. Remark 3.5.2 below.

(iii) One may also construct a "D-version" - which, from the point of view of the theory of [AbsTopIII], one may also think of as a "log-shell version" - of the various data constructed in (i), (ii). To this end, we write

$$
\mathcal{D}_{\text {mod }}^{\Vdash}
$$


for a [i.e., another] copy of $\mathcal{C}_{\text {mod }}^{\Vdash}$. Thus, one may associate to $\mathcal{D}_{\text {mod }}^{\Vdash}$ various objects $\Phi_{\mathcal{D}_{\text {mod }}^{\Vdash}}, \operatorname{Prime}\left(\mathcal{D}_{\text {mod }}^{\Vdash}\right) \stackrel{\sim}{\rightarrow} \mathbb{V}_{\text {mod }}, \log _{\text {mod }}^{\mathcal{D}}\left(p_{v}\right) \in \Phi_{\mathcal{D}_{\text {mod }}^{\Vdash}, v} \subseteq \Phi_{\mathcal{D}_{\text {mod }}^{\Vdash}}\left[\right.$ for $\left.v \in \mathbb{V}_{\text {mod }}\right]$ that map to the corresponding objects associated to $\mathcal{C}_{\text {mod }}^{\Vdash}$ under the tautological equivalence of categories $\mathcal{C}_{\text {mod }}^{\Vdash} \stackrel{\sim}{\rightarrow} \mathcal{D}_{\text {mod }}^{\Vdash}$. Write $\underline{v} \in \mathbb{\mathbb { V }}$ for the element of $\underline{\mathbb{V}}$ that corresponds to $v$. Next, suppose that $\underline{v} \in \underline{\mathbb{V}}^{\text {non }}$; then let us recall from [AbsTopIII], Proposition 5.8, (iii), that [since the profinite group associated to $\mathcal{D}_{\underline{v}}^{+}$is the absolute Galois group of an MLF] one may construct algorithmically from $\overline{\mathcal{D}}_{\underline{v}}^{\vdash}$ a topological monoid isomorphic to $\mathbb{R}_{\geq 0}$

$$
\left(\mathbb{R}_{\geq 0}^{\vdash}\right)_{\underline{v}}
$$

[i.e., the topological monoid determined by the nonnegative elements of the ordered topological group " $\mathbb{R}_{\text {non }}(G)$ " of loc. cit.] equipped with a distinguished "Frobenius element" $\in\left(\mathbb{R}_{\geq 0}^{\vdash}\right)_{\underline{v}}$; if $e_{\underline{v}}$ is the absolute ramification index of the MLF $K_{\underline{v}}$, then we shall write $\log _{\Phi} \underset{\mathcal{D}}{\mathcal{D}}\left(p_{\underline{v}}\right) \in\left(\mathbb{R}_{\geq 0}^{\vdash}\right)_{\underline{v}}$ for the result of multiplying this Frobenius element by [the positive real number] $e_{\underline{v}}$. Next, suppose that $\underline{v} \in \underline{\mathbb{V}}^{\text {arc }}$; then let us recall from [AbsTopIII], Proposition 5.8, (vi), that [since, by definition, $\mathcal{D}_{\underline{v}}^{\vdash} \in \mathrm{Ob}\left(\mathbb{T M}^{\vdash}\right)$ ] one may construct algorithmically from $\mathcal{D}_{\underline{v}}^{\vdash}$ a topological monoid isomorphic to $\mathbb{R}_{\geq 0}$

$$
\left(\mathbb{R}_{\geq 0}^{\vdash}\right)_{\underline{v}}
$$

[i.e., the topological monoid determined by the nonnegative elements of the ordered topological group " $\mathbb{R}_{\text {arc }}(G)$ " of loc. cit.] equipped with a distinguished "Frobenius element" $\in\left(\mathbb{R}_{\geq 0}^{\vdash}\right)_{\underline{v}}$; we shall write $\log _{\Phi}^{\mathcal{D}}\left(p_{\underline{v}}\right) \in\left(\mathbb{R}_{\geq 0}^{\vdash}\right)_{\underline{v}}$ for the result of dividing this Frobenius element by [the positive real number] $2 \pi$. In particular, for every $\underline{v} \in \underline{\mathbb{V}}$, we obtain a uniquely determined isomorphism of topological monoids [which are isomorphic to $\mathbb{R}_{\geq 0}$ ]

$$
\rho_{\underline{v}}^{\mathcal{D}}: \Phi_{\mathcal{D}_{\text {mod }}^{\Vdash}, v} \stackrel{\sim}{\rightarrow}\left(\mathbb{R}_{\geq 0}^{\vdash}\right)_{\underline{v}}
$$

by assigning $\log _{\text {mod }}^{\mathcal{D}}\left(p_{v}\right) \mapsto \frac{1}{\left[K_{\underline{v}}:\left(F_{\bmod }\right)_{v}\right]} \log _{\Phi}^{\mathcal{D}}\left(p_{\underline{v}}\right)$. Thus, we obtain data [consisting of a Frobenioid, a bijection of sets, a collection of data indexed by $\underline{\mathbb{V}}$, and a collection of isomorphisms indexed by $\underline{\mathbb{V}}]$

$$
\mathfrak{F}_{\mathcal{D}}^{\Vdash} \stackrel{\text { def }}{=}\left(\mathcal{D}_{\text {mod }}^{\Vdash}, \operatorname{Prime}\left(\mathcal{D}_{\text {mod }}^{\Vdash}\right) \stackrel{\sim}{\rightarrow} \underline{\mathbb{V}},\left\{\mathcal{D}_{\underline{v}}^{\vdash}\right\}_{\underline{v} \in \underline{\mathbb{V}}},\left\{\rho_{\underline{v}}^{\mathcal{D}}\right\}_{\underline{v} \in \underline{\mathbb{V}}}\right)
$$

[where we apply the natural bijection $\underline{\mathbb{V}} \stackrel{\sim}{\rightarrow} \mathbb{V}_{\text {mod }}$ ], which, by [AbsTopIII], Proposition 5.8, (iii), (vi), may be reconstructed algorithmically from the data $\left\{\mathcal{D}_{\underline{v}}^{\vdash}\right\}_{\underline{v}} \in \underline{\mathbb{V}}$.

\section{Remark 3.5.1.}

(i) The formal symbol " $\log (\underline{\underline{\Theta}})$ " may be thought of as the result of identifying the various formal quotients " $\log _{\Phi}\left(\underline{\underline{\Theta}}_{\underline{v}}\right) / \log _{\Phi}\left(\underline{\underline{q}}_{\underline{v}}\right)$ ", as $\underline{v}$ varies over the elements of $\underline{\mathbb{V}}^{\text {bad }}$.

(ii) The global Frobenioids $\mathcal{C}_{\text {mod }}^{\Vdash}, \mathcal{C}_{\text {tht }}^{\Vdash}$ of Example 3.5 may be thought of as "devices for currency exchange" between the various "local currencies" constituted by the divisor monoids at the various $\underline{v} \in \underline{\mathbb{V}}$. 
(iii) One may also formulate the data contained in $\mathfrak{F}_{\text {mod }}^{\Vdash}, \mathfrak{F}_{\text {tht }}^{\Vdash}$ via the language of poly-Frobenioids as developed in [FrdII], $\S 5$, but we shall not pursue this topic in the present series of papers.

Remark 3.5.2. In Example 3.5, as well as in the following discussion, we shall often speak of "isomorphisms of collections of data", relative to the following conventions.

(i) Such isomorphisms are always assumed to satisfy various evident compatibility conditions, relative to the various relationships stipulated between the various constituent data, whose explicit mention we shall omit for the sake of simplicity.

(ii) In situations where the collections of data consist partially of various categories, the portion of the "isomorphism of collections of data" involving corresponding categories is to be understood as an isomorphism class of equivalences of categories [cf. §0].

Definition 3.6. Fix a collection of initial $\Theta$-data $\left(\bar{F} / F, X_{F}, l, \underline{C}_{K}, \underline{\mathbb{V}}, \underset{\mathbb{V}}{\bmod }\right.$ bad $\left.\underline{\epsilon}\right)$ as in Definition 3.1. In the following, we shall use the various notations introduced in Definition 3.1 for various objects associated to this initial $\Theta$-data. Then we define a $\Theta$-Hodge theater [relative to the given initial $\Theta$-data] to be a collection of data

$$
{ }^{\dagger} \mathcal{H} \mathcal{T}^{\Theta}=\left(\left\{\underline{\underline{\mathcal{F}}}_{\underline{v}}^{\dagger}\right\}_{\underline{v} \in \underline{\mathbb{V}}},{ }^{\dagger} \mathfrak{F}_{\text {mod }}^{\vdash}\right)
$$

that satisfies the following conditions:

(a) If $\underline{v} \in \underline{\mathbb{V}}^{\text {non }}$, then ${ }^{\dagger} \underline{\underline{\mathcal{F}}}_{\underline{v}}$ is a category which admits an equivalence of categories ${ }^{\dagger} \underline{\underline{\mathcal{F}}}_{\underline{v}} \stackrel{\sim}{\rightarrow} \underline{\underline{\mathcal{F}}}_{\underline{v}}$ [where $\underline{\underline{\mathcal{F}}}_{\underline{v}}$ is as in Examples 3.2, (i); 3.3, (i)]. In particular, ${ }^{\dagger} \underline{\underline{\mathcal{F}}}_{v}$ admits a natural Frobenioid structure [cf. [FrdI], Corollary 4.11, (iv)], which may be constructed solely from the category-theoretic structure of ${ }^{\dagger} \underline{\underline{\mathcal{F}}}_{\underline{v}}$. Write ${ }^{\dagger} \mathcal{D}_{\underline{v}},{ }^{\dagger} \mathcal{D}_{\underline{\underline{v}}}^{\vdash},{ }^{\dagger} \mathcal{D}_{\underline{v}}^{\Theta},{ }^{\dagger} \mathcal{F}_{\underline{v}}^{\vdash},{ }^{\dagger} \mathcal{F}_{\underline{\underline{v}}}^{\Theta}$ for the objects constructed category-theoretically from ${ }^{\dagger} \underline{\underline{\mathcal{F}}}_{\underline{v}}$ that correspond to the objects without a "†" discussed in Examples 3.2, 3.3 [cf., especially, Examples 3.2, (vi); 3.3, (iii)].

(b) If $\underline{v} \in \underline{\mathbb{V}}^{\text {arc }}$, then ${ }^{\dagger} \underline{\underline{\mathcal{F}}}_{\underline{v}}$ is a collection of data $\left({ }^{\dagger} \mathcal{C}_{\underline{v}},{ }^{\dagger} \mathcal{D}_{\underline{v}},{ }^{\dagger} \kappa_{\underline{v}}\right)$ - where ${ }^{\dagger} \mathcal{C}_{\underline{v}}$ is a category equivalent to the category $\mathcal{C}_{\underline{v}}$ of Example $3.4,(\mathrm{i}) ;{ }^{\dagger} \mathcal{D}_{\underline{v}}$ is an Aut-holomorphic orbispace; and ${ }^{\dagger} \kappa_{\underline{v}}: \mathcal{O}^{\triangleright}\left({ }^{\dagger} \mathcal{C}_{\underline{v}}\right) \hookrightarrow \mathcal{A}_{\dagger \mathcal{D}_{v}}$ is an inclusion of topological monoids, which we shall refer to as the $\overline{\text { Kummer structure }}$ on ${ }^{\dagger} \mathcal{C}_{\underline{v}}$ - such that there exists an isomorphism of collections of data ${ }^{\dagger} \underline{\underline{\mathcal{F}}}_{\underline{v}} \stackrel{\overline{\mathcal{J}}_{\rightarrow}}{\underline{\underline{\mathcal{F}}}_{\underline{v}}}$ [where $\underline{\underline{\mathcal{F}}}_{\underline{v}}$ is as in Example 3.4, (i)]. Write ${ }^{\dagger} \mathcal{D}_{\underline{v}}^{\vdash},{ }^{\dagger} \mathcal{D}_{\underline{v}}^{\Theta},{ }^{\dagger} \mathcal{F}_{\underline{v}}^{\vdash}$, $\dagger \mathcal{F}_{\underline{v}}^{\bar{\Theta}}$ for the objects constructed algorithmically from ${ }^{\dagger} \underline{\underline{\mathcal{F}}}_{\underline{v}}$ that correspond to the objects without a "†" discussed in Example 3.4, (ii), (iii).

(c) ${ }^{\dagger} \mathfrak{F}_{\text {mod }}^{\vdash}$ is a collection of data

$$
\left({ }^{\dagger} \mathcal{C}_{\text {mod }}^{\Vdash}, \operatorname{Prime}\left({ }^{\dagger} \mathcal{C}_{\text {mod }}^{\Vdash}\right) \stackrel{\sim}{\rightarrow} \underline{\mathbb{V}},\left\{{ }^{\dagger} \mathcal{F}_{\underline{v}}^{\vdash}\right\}_{\underline{v} \in \underline{\mathbb{V}}},\left\{{ }^{\dagger} \rho_{\underline{v}}\right\}_{\underline{v} \in \underline{\mathbb{V}}}\right)
$$


— where ${ }^{\dagger} \mathcal{C}_{\text {mod }}^{\Vdash}$ is a category which admits an equivalence of categories ${ }^{\dagger} \mathcal{C}_{\text {mod }}^{\Vdash} \stackrel{\sim}{\rightarrow} \mathcal{C}_{\text {mod }}^{\Vdash}$ [which implies that ${ }^{\dagger} \mathcal{C}_{\text {mod }}^{\Vdash}$ admits a natural category-theoretically constructible Frobenioid structure — cf. [FrdI], Corollary 4.11, (iv); [FrdI], Theorem 6.4, (i)]; Prime $\left({ }^{\dagger} \mathcal{C}_{\text {mod }}^{\Vdash}\right) \stackrel{\sim}{\rightarrow} \mathbb{V}$ is a bijection of sets, where we write Prime $\left({ }^{\dagger} \mathcal{C}_{\text {mod }}^{\Vdash}\right)$ for the set of primes constructed from the category ${ }^{\dagger} \mathcal{C}_{\text {mod }}^{\Vdash}\left[\mathrm{cf} . \quad[\operatorname{FrdI}]\right.$, Theorem 6.4 , (iii)]; ${ }^{\dagger} \mathcal{F}_{v}^{\vdash}$ is as discussed in (a), (b) above; ${ }^{\dagger} \rho_{\underline{v}}: \Phi_{\dagger \mathcal{C}_{\text {mod }}}, v \stackrel{\sim}{\rightarrow} \Phi_{\dagger_{\mathcal{C}_{\underline{v}}}}^{\text {rlf }}$ [where we use notation as in the discussion of Example 3.5, (i)] is an isomorphism of topological monoids. Moreover, we require that there exist an isomorphism of collections of data ${ }^{\dagger} \mathfrak{F}_{\text {mod }}^{\vdash} \stackrel{\sim}{\rightarrow} \mathfrak{F}_{\text {mod }}^{\Vdash}\left[\right.$ where $\mathfrak{F}_{\text {mod }}^{\Vdash}$ is as in Example 3.5, (ii)]. Write ${ }^{\dagger} \mathfrak{F}_{\text {tht }}^{\Vdash},{ }^{\dagger} \mathfrak{F}_{\mathcal{D}}^{\vdash}$ for the objects constructed algorithmically from ${ }^{\dagger} \mathfrak{F}$ mod that correspond to the objects without a "†" discussed in Example 3.5, (ii), (iii).

Remark 3.6.1. When we discuss various collections of $\Theta$-Hodge theaters, labeled by some symbol " $\square$ " in place of a "†", we shall apply the notation of Definition 3.6 with " $\nmid$ " replaced by " $\square$ " to denote the various objects associated to the $\Theta$-Hodge theater labeled by " $\square$ ".

Remark 3.6.2. If $\dagger^{\dagger} \mathcal{H} \mathcal{T}^{\Theta}$ and $\ddagger \mathcal{H} \mathcal{T}^{\Theta}$ are $\Theta$-Hodge theaters, then there is an evident notion of isomorphism of $\Theta$-Hodge theaters ${ }^{\dagger} \mathcal{H} \mathcal{T}^{\Theta} \stackrel{\sim}{\rightarrow} \ddagger \mathcal{H} \mathcal{T}^{\Theta}$ [cf. Remark 3.5.2]. We leave the routine details to the interested reader.

Corollary 3.7. ( $\Theta$-Links Between $\Theta$-Hodge Theaters) Fix a collection of initial $\Theta$-data $\left(\bar{F} / F, X_{F}, l, \underline{C}_{K}, \underline{\mathbb{V}}, \mathbb{V}_{\bmod }^{\text {bad }}, \underline{\epsilon}\right)$ as in Definition 3.1. Let

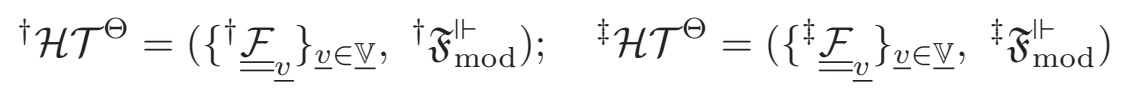

be $\Theta$-Hodge theaters [relative to the given initial $\Theta$-data]. Then:

(i) ( $\Theta$-Link) The full poly-isomorphism [cf. $\S 0]$ between collections of data [cf. Remark 3.5.2]

$$
\dagger \mathfrak{F}_{\text {tht }}^{\vdash} \stackrel{\sim}{\rightarrow} \ddagger \mathfrak{F}_{\text {mod }}^{\Vdash}
$$

is nonempty [cf. Remark 3.7.1 below]. We shall refer to this full poly-isomorphism as the $\Theta$-link

$$
{ }^{\dagger} \mathcal{H} \mathcal{T}^{\Theta} \stackrel{\Theta}{\longrightarrow} \ddagger \mathcal{H} \mathcal{T}^{\Theta}
$$

from ${ }^{\dagger} \mathcal{H} \mathcal{T}^{\Theta}$ to ${ }^{\ddagger} \mathcal{H} \mathcal{T}^{\Theta}$

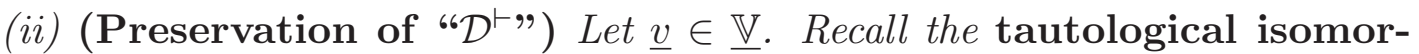
phisms ${ }^{\square} \mathcal{D}_{\underline{v}}^{\vdash} \stackrel{\sim}{\rightarrow} \square \mathcal{D}_{\underline{v}}^{\Theta}$ for $\square=\dagger, \ddagger-i . e$., which arise from the definitions when $\underline{v} \in \underline{\mathbb{V}}^{\text {good }}$ [cf. Examples 3.3, (ii); 3.4, (iii)], and which arise from a natural product functor [cf. Example 3.2, (v)] when $\underline{v} \in \underline{\mathbb{V}}^{\text {bad }}$. Then we obtain a composite [full] poly-isomorphism

$$
{ }^{\dagger} \mathcal{D}_{\underline{v}}^{\vdash} \stackrel{\sim}{\rightarrow}{ }^{\dagger} \mathcal{D}_{\underline{v}}^{\Theta} \stackrel{\sim}{\rightarrow}{ }^{\ddagger} \mathcal{D}_{\underline{v}}^{\vdash}
$$

by composing the tautological isomorphism just mentioned with the poly-isomorphism induced by the $\Theta$-link poly-isomorphism of (i). 
(iii) (Preservation of " $\mathrm{O}^{\times}$") Let $\underline{v} \in \underline{\mathbb{V}}$. Recall the tautological isomorphisms $\mathcal{O}_{\square \mathcal{C}_{\underline{v}}^{\vdash}}^{\times} \stackrel{\sim}{\rightarrow} \mathcal{O}_{\square_{\mathcal{C}_{\underline{v}}^{\ominus}}^{\times}}^{\times}$[where we omit the notation "(-)"] for $\square=\dagger, \ddagger-$ i.e., which arise from the definitions when $\underline{v} \in \underline{\mathbb{V}}^{\text {good }}$ [cf. Examples 3.3, (ii); 3.4, (iii)], and which are induced by the natural product functor [cf. Example 3.2, (v)] when $\underline{v} \in \underline{\mathbb{V}}^{\text {bad }}$. Then, relative to the corresponding composite isomorphism of (ii), we obtain a composite [full] poly-isomorphism

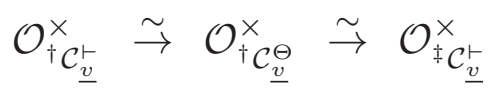

by composing the tautological isomorphism just mentioned with the poly-isomorphism induced by the $\Theta$-link poly-isomorphism of (i).

Proof. The various assertions of Corollary 3.7 follow immediately from the definitions and the discussion of Examples 3.2, 3.3, 3.4, and 3.5.

Remark 3.7.1. One verifies immediately that there exist many distinct isomorphisms ${ }^{\dagger} \mathfrak{F}_{\text {tht }}^{\Vdash} \stackrel{\sim}{\rightarrow} \mathfrak{F}_{\text {mod }}^{\Vdash}$ as in Corollary 3.7, (i), none of which is conferred a "distinguished" status, i.e., in the fashion of the "natural isomorphism $\mathfrak{F}_{\text {mod }}^{\Vdash} \stackrel{\sim}{\rightarrow} \mathfrak{F}_{\text {tht }}^{\Vdash}$ " discussed in Example 3.5, (ii).

The following result follows formally from Corollary 3.7.

Corollary 3.8. (Frobenius-pictures of $\Theta$-Hodge Theaters) Fix a collection of initial $\Theta$-data as in Corollary 3.7. Let $\left\{{ }^{n} \mathcal{H} \mathcal{T}^{\Theta}\right\}_{n \in \mathbb{Z}}$ be a collection of distinct $\Theta$-Hodge theaters indexed by the integers. Then by applying Corollary 3.7, (i), with ${ }^{\dagger} \mathcal{H} \mathcal{T}^{\Theta} \stackrel{\text { def }}{=}{ }^{n} \mathcal{H} \mathcal{T}^{\Theta},{ }^{\ddagger} \mathcal{H} \mathcal{T}^{\Theta} \stackrel{\text { def }}{=}(n+1) \mathcal{H} \mathcal{T}^{\Theta}$, we obtain an infinite chain

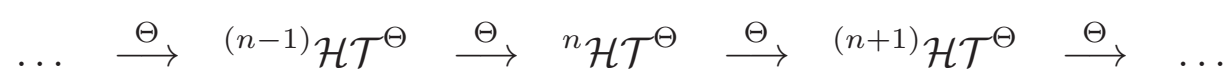

of $\Theta$-linked $\Theta$-Hodge theaters. This infinite chain may be represented symbolically as an oriented graph $\vec{\Gamma}$ [cf. [AbsTopIII], §O]

- i.e., where the arrows correspond to the " $\stackrel{\Theta}{\longrightarrow}$ 's", and the "•' 's" correspond to the ${ }^{n} \mathcal{H} \mathcal{T}^{\Theta} "$. This oriented graph $\vec{\Gamma}$ admits a natural action by $\mathbb{Z}-i . e .$, a translation symmetry - but it does not admit arbitrary permutation symmetries. For instance, $\vec{\Gamma}$ does not admit an automorphism that switches two adjacent vertices, but leaves the remaining vertices fixed. Put another way, from the point of view of the discussion of [FrdI], §I 4, the mathematical structure constituted by this infinite chain is "Frobenius-like", or "order-conscious". It is for this reason that we shall refer to this infinite chain in the following discussion as the Frobeniuspicture. 


\section{Remark 3.8.1.}

(i) Perhaps the central defining aspect of the Frobenius-picture is the fact that the $\Theta$-link maps

$$
{ }^{n} \underline{\underline{\Theta}}_{\underline{v}} \mapsto \quad{ }^{(n+1)} \underline{\underline{q}} \underline{v}
$$

[i.e., where $\underline{v} \in \underline{\mathbb{V}}^{\text {bad }}$ - cf. the discussion of Example 3.2, (v)]. From this point of view, the Frobenius-picture may be depicted as in Fig. 3.1 below — i.e., each box is a $\Theta$-Hodge theater; the " $\rightsquigarrow$ " may be thought of as denoting the scheme theory that lies between " $\underline{\underline{q}} \underline{\underline{v}}$ " and " $\underline{\underline{\Theta}}_{\underline{v}}$ "; the ". . . " denotes the $\Theta$-link.

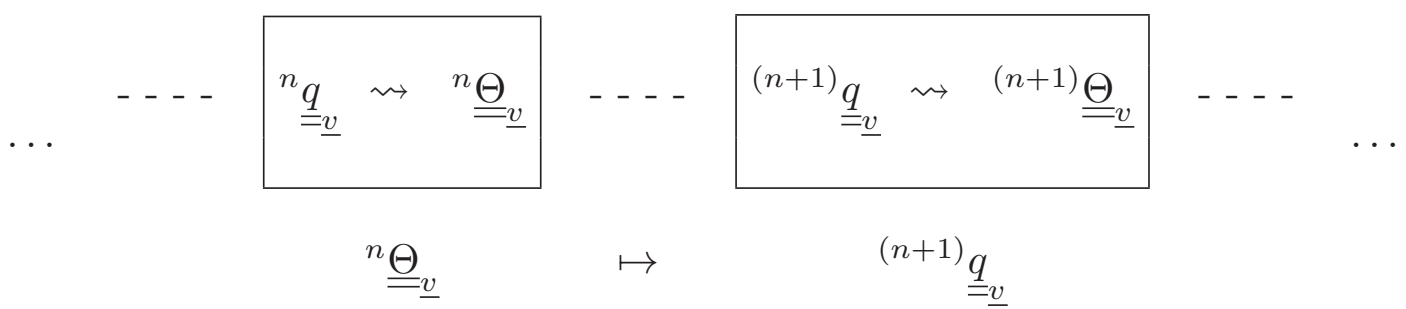

Fig. 3.1: Frobenius-picture of $\Theta$-Hodge theaters

(ii) It is perhaps not surprising [cf. the theory of [FrdI]] that the Frobeniuspicture involves, in an essential way, the divisor monoid portion [i.e., " $\underline{\underline{q}}$ " and " $\underline{\underline{\Theta}}_{v}$ "] of the various Frobenioids that appear in a $\Theta$-Hodge theater. Put another way,

it is as if the "Frobenius-like nature" of the divisor monoid portion of the Frobenioids involved induces the "Frobenius-like nature" of the Frobeniuspicture.

By contrast, observe that for $\underline{v} \in \underline{\mathbb{V}}$, the isomorphisms

$$
\ldots \stackrel{\sim}{\rightarrow}{ }^{n} \mathcal{D}_{\underline{v}}^{\vdash} \stackrel{\sim}{\rightarrow}(n+1) \mathcal{D}_{\underline{v}}^{\vdash} \stackrel{\sim}{\rightarrow} \ldots
$$

of Corollary 3.7, (ii), imply that if one thinks of the various ${ }^{(-)} \mathcal{D}_{\underline{v}}^{\vdash}$ as being only known up to isomorphism, then

one may regard ${ }^{(-)} \mathcal{D}_{v}^{\vdash}$ as a sort of constant invariant of the various $\Theta$-Hodge theaters that constitute the Frobenius-picture

- cf. Remark 3.9.1 below. This observation is the starting point of the theory of the étale-picture [cf. Corollary 3.9, (i), below]. Note that by Corollary 3.7, (iii), we also obtain isomorphisms

$$
\ldots \stackrel{\sim}{\rightarrow} \mathcal{O}_{n}^{\times} \mathcal{C}_{\underline{v}}^{\vdash} \stackrel{\sim}{\rightarrow} \quad \mathcal{O}_{(n+1)}^{\times} \mathcal{C}_{\underline{v}}^{\vdash} \stackrel{\sim}{\rightarrow} \ldots
$$

lying over the isomorphisms involving the "(-) $\mathcal{D}_{\underline{v}}^{+} "$ discussed above.

(iii) In the situation of (ii), suppose that $\underline{v} \in \underline{\mathbb{V}}^{\text {non }}$. Then ${ }^{(-)} \mathcal{D}_{v}^{\vdash}$ is simply the category of connected objects of the Galois category associated to the profinite group $G_{\underline{\underline{v}}}$. That is to say, one may think of ${ }^{(-)} \mathcal{D}_{\underline{v}}^{\vdash}$ as representing " $G_{\underline{v}}$ up to 
isomorphism". Then each ${ }^{n} \mathcal{D}_{v}$ represents an "isomorph of the topological group $\Pi_{v}$, labeled by $n$, which is regarded as an extension of some isomorph of $G_{v}$ that is independent of $n$ ". In particular, the quotients corresponding to $G_{\underline{v}}$ of the copies of $\Pi_{v}$ that arise from ${ }^{n} \mathcal{H} \mathcal{T}^{\Theta}$ for different $n$ are only related to one another via some indeterminate isomorphism. Thus, from the point of view of the theory of [AbsTopIII] [cf. [AbsTopIII], §I3; [AbsTopIII], Remark 5.10.2, (ii)], each $\Pi_{v}$ gives rise to a well-defined ring structure - i.e., a "holomorphic structure" — which is obliterated by the indeterminate isomorphism between the quotient isomorphs of $G_{\underline{v}}$ arising from ${ }^{n} \mathcal{H} \mathcal{T}^{\Theta}$ for distinct $n$.

(iv) In the situation of (ii), suppose that $\underline{v} \in \underline{\mathbb{V}}^{\text {arc }}$. Then ${ }^{(-)} \mathcal{D}_{\underline{v}}^{\vdash}$ is an object of $\mathbb{T M}^{\vdash}$; each ${ }^{n} \mathcal{D}_{\underline{v}}$ represents an "isomorph of the Aut-holomorphic orbispace $\underline{\mathbb{X}}_{\underline{v}}$ ", labeled by $n$, whose associated [complex archimedean] topological field $\overline{\mathcal{A}}_{\underline{\mathbb{X}}_{\underline{v}}}$ gives rise to an isomorph of $\mathcal{D}_{\underline{v}}^{\vdash}$ that is independent of $n$ ". In particular, the various isomorphs of $\mathcal{D}_{\underline{v}}^{\vdash}$ associated to the copies of $\underline{\mathbb{X}}_{\underline{v}}$ that arise from ${ }^{n} \mathcal{H} \mathcal{T}^{\Theta}$ for different $n$ are only related to one another via some indeterminate isomorphism. Thus, from the point of view of the theory of [AbsTopIII] [cf. [AbsTopIII], §I3; [AbsTopIII], Remark 5.10.2, (ii)], each $\underline{\mathbb{X}}_{v}$ gives rise to a well-defined ring structure - i.e., a "holomorphic structure" — which is obliterated by the indeterminate isomorphism between the isomorphs of $\mathcal{D}_{\underline{v}}^{\vdash}$ arising from ${ }^{n} \mathcal{H} \mathcal{T}^{\Theta}$ for distinct $n$.

The discussion of Remark 3.8.1, (iii), (iv), may be summarized as follows.

Corollary 3.9. (Étale-pictures of $\Theta$-Hodge Theaters) In the situation of Corollary 3.8, let $\underline{v} \in \underline{\mathbb{V}}$. Then:

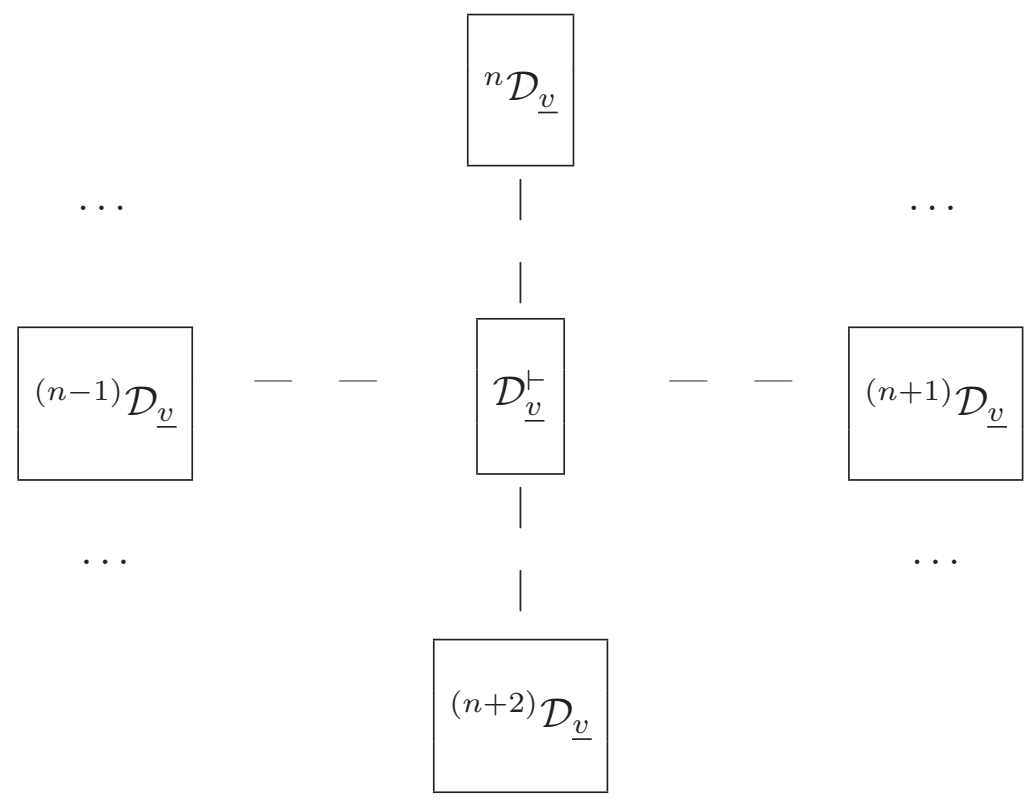

Fig. 3.2: Étale-picture of $\Theta$-Hodge theaters 


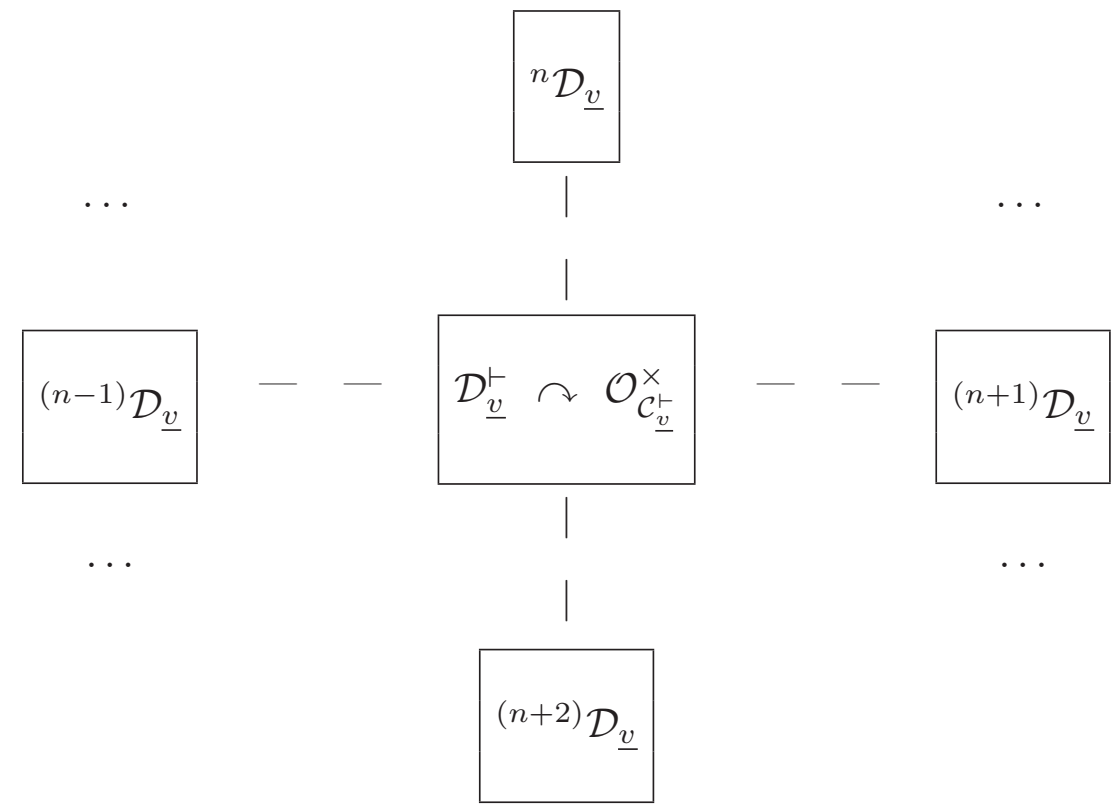

Fig. 3.3: Étale-picture plus units

(i) We have a diagram as in Fig. 3.2 above, which we refer to as the étalepicture. Here, each horizontal and vertical "_ _ " denotes the relationship between ${ }^{(-)} \mathcal{D}_{\underline{v}}$ and $\mathcal{D}_{\underline{v}}^{-}-i . e$. , an extension of topological groups when $\underline{v} \in \underline{\mathbb{V}}^{\text {non }}$, or the underlying object of $\mathbb{T M}^{\vdash}$ arising from the associated topological field when $\underline{v} \in \underline{\mathbb{V}}^{\text {arc }}$ - discussed in Remark 3.8.1, (iii), (iv). The étale-picture [unlike the Frobenius-picture!] admits arbitrary permutation symmetries among the labels $n \in \mathbb{Z}$ corresponding to the various $\Theta$-Hodge theaters. Put another way, the étale-picture may be thought of as a sort of canonical splitting of the Frobeniuspicture.

(ii) In a similar vein, we have a diagram as in Fig. 3.3 above, obtained by replacing the " $\mathcal{D}_{\underline{v}}^{\vdash}$ " in the middle of Fig. 3.2 by " $\mathcal{D}_{\underline{v}}^{\vdash} \curvearrowright \mathcal{O}_{\mathcal{C}_{v}^{\vdash}}^{\times}$". Here, each horizontal and vertical "__ _ " denotes the relationship between ${ }^{(-)} \mathcal{D}_{\underline{v}}$ and $\mathcal{D}_{\underline{v}}^{\vdash}$ discussed in (i); when $\underline{v} \in \underline{\mathbb{V}}^{\text {non }}$, the notation " $\mathcal{D}_{\underline{v}}^{\vdash} \curvearrowright \mathcal{O}_{\mathcal{C}_{\underline{v}}^{\vdash}}^{\times}$" denotes an isomorph of the pair consisting of the category $\mathcal{D}_{\underline{v}}^{\vdash}$ together with the group-like monoid $\mathcal{O}_{\mathcal{C}_{\underline{v}}^{\vdash}}^{\times}$ on $\mathcal{D}_{\underline{v}}^{\vdash}$; when $\underline{v} \in \underline{\mathbb{V}}^{\text {arc }}$, the notation " $\mathcal{D}_{\underline{v}}^{\vdash} \curvearrowright \mathcal{O}_{\mathcal{C}_{\underline{v}}^{\vdash}}^{\times}$" denotes an isomorph of the pair consisting of the object $\mathcal{D}_{\underline{v}}^{\vdash} \in \mathrm{Ob}\left(\mathbb{T M}^{\vdash}\right)$ and the topological group $\mathcal{O}_{\mathcal{C}_{\underline{v}}^{\vdash}}^{\times}$[which is isomorphic - but not canonically! - to the compact factor of $\left.\mathcal{D}_{\underline{v}}^{\vdash}\right]$. Just as in the case of (i), this diagram admits arbitrary permutation symmetries among the labels $n \in \mathbb{Z}$ corresponding to the various $\Theta$-Hodge theaters.

Remark 3.9.1. If one formulates things relative to the language of [AbsTopIII], Definition 3.5, then ${ }^{(-)} \mathcal{D}_{\underline{v}}$ constitutes a core. Relative to the theory of [AbsTopIII], $\S 5$, this core is essentially the mono-analytic core discussed in [AbsTopIII], §I3; [AbsTopIII], Remark 5.10.2, (ii). Indeed, the symbol "-" is intended - both in [AbsTopIII] and in the present series of papers! — as an abbreviation for the term "mono-analytic". 
Remark 3.9.2. Whereas the étale-picture of Corollary 3.9, (i), will remain valid throughout the development of the remainder of the theory of the present series of papers, the local units " $\mathcal{O}_{\mathcal{C}_{v}^{\vdash}}^{\times}$" that appear in Corollary 3.9, (ii), will ultimately cease to be a constant invariant of various enhanced versions of the Frobenius-picture that will arise in the theory of [IUTchIII]. In a word, these enhancements revolve around the incorporation into each Hodge theater of the "rotation of addition [i.e., $\boxplus$ '] and multiplication [i.e., " $\left.\square^{\prime}\right]$ " in the style of the theory of [AbsTopIII].

\section{Remark 3.9.3.}

(i) As discussed in [AbsTopIII], §I3; [AbsTopIII], Remark 5.10.2, (ii), the "mono-analytic core" $\left\{\mathcal{D}_{\underline{v}}^{\vdash}\right\}_{\underline{v} \in \underline{\mathbb{V}}}$ may be thought of as a sort of fixed underlying real-analytic surface associated to a number field on which various holomorphic structures are imposed. Then the Frobenius-picture in its entirety may be thought of as a sort of global arithmetic analogue of the notion of a Teichmüller geodesic in classical complex Teichmüller theory or, alternatively, as a global arithmetic analogue of the canonical liftings of p-adic Teichmüller theory [cf. the discussion of [AbsTopIII], §I5].

(ii) Recall that in classical complex Teichmüller theory, one of the two real dimensions of the surface is dilated as one moves along a Teichmüller geodesic, while the other of the two real dimensions is held fixed. In the case of the Frobeniuspicture of Corollary 3.8, the local units " $\mathrm{O}^{\times}$" correspond to the dimension that is held fixed, while the local value groups are subject to " $\Theta$-dilations" as one moves along the diagram constituted by the Frobenius-picture. Note that in order to construct such a mathematical structure in which the local units and local value groups are treated independently, it is of crucial importance to avail oneself of the various characteristic splittings that appear in the split Frobenioids of Examples 3.2, 3.3, 3.4. Here, we note in passing that, in the case of Example 3.2, this splitting corresponds to the "constant multiple rigidity" of the étale theta function, which forms a central theme of the theory of [EtTh].

(iii) In classical complex Teichmüller theory, the two real dimensions of the surface that are treated independently of one another correspond to the real and imaginary parts of the coordinate obtained by locally integrating the square root of a given square differential. In particular, it is of crucial importance in classical complex Teichmüller theory that these real and imaginary parts not be "subject to confusion with one another". In the case of the square root of a square differential, the only indeterminacy that arises is indeterminacy with respect to multiplication by -1 , an operation that satisfies the crucial property of preserving the real and imaginary parts of a complex number. By contrast, it is interesting to note that

if, for $n \geq 3$, one attempts to construct Teichmüller deformations in the fashion of classical complex Teichmüller theory by means of coordinates obtained by locally integrating the $n$-th root of a given section of the $n$ th tensor power of the sheaf of differentials, then one must contend with an indeterminacy with respect to multiplication by an $n$-th root of unity, an operation that results in an essential confusion between the real and imaginary parts of a complex number. 
(iv) Whereas linear movement along the oriented graph $\vec{\Gamma}$ of Corollary 3.8 corresponds to the linear flow along a Teichmüller geodesic, the "rotation of addition [i.e., "⿴囗十 - which will be incorporated into the theory of the present series of papers in [IUTchIII] [cf. Remark 3.9.2] — corresponds to rotations around a fixed point in the complex geometry arising from Teichmüller theory [cf., e.g., the discussion of [AbsTopIII], §I3; the hyperbolic geometry of the upper half-plane, regarded as the "Teichmüller space" of compact Riemann surfaces of genus 1]. Alternatively, in the analogy with $p$-adic Teichmüller theory, this "rotation of $\boxplus$ and $\nabla$ " corresponds to the Frobenius morphism in positive characteristic - cf. the discussion of [AbsTopIII], §I5.

Remark 3.9.4. At first glance, the assignment "n $\underline{\underline{\Theta}}_{\underline{v}} \mapsto{ }^{(n+1)} \underline{\underline{q}}_{v}$ " [cf. Remark 3.8.1, (i)] may strike the reader as being nothing more than a "conventional evaluation map" [i.e., of the theta function at a torsion point - cf. the discussion of Example 3.2, (iv)]. Although we shall ultimately be interested, in the theory of the present series of papers, in such "Hodge-Arakelov-style evaluation maps" [within a fixed Hodge theater!] of the theta function at torsion points [cf. the theory of [IUTchII]], the $\Theta$-link considered here differs quite fundamentally from such conventional evaluation maps in the following respect:

the value ${ }^{(n+1)} \underline{\underline{q}}_{\underline{v}}$ belongs to a distinct scheme theory - i.e., the scheme theory represented by the distinct $\Theta$-Hodge theater ${ }^{(n+1)} \mathcal{H}^{\Theta}$ from the base ${ }^{n} \underline{\underline{q}}_{\underline{v}}$. Which belongs to the scheme theory represented by the $\Theta$-Hodge theater $\left.{ }^{n} \mathcal{H} \mathcal{T}^{\Theta}\right]$ over which the theta function ${ }^{n} \underline{\underline{\Theta}}_{\underline{v}}$ is constructed.

The distinctness of the ring/scheme theories of distinct $\Theta$-Hodge theaters may be seen, for instance, in the indeterminacy of the isomorphism between the associated isomorphs of $\mathcal{D}_{\underline{v}}^{-}$, an indeterminacy which has the effect of obliterating the ring structure - i.e., the "arithmetic holomorphic structure" — associated to ${ }^{n} \mathcal{D}_{\underline{v}}$ for distinct $n$ [cf. the discussion of Remark 3.8.1, (iii), (iv)]. 


\section{Section 4: Multiplicative Combinatorial Teichmüller Theory}

In the present $\S 4$, we begin to prepare for the construction of the various "enhancements" to the $\Theta$-Hodge theaters of $\S 3$ that will be made in $\S 5$. More precisely, in the present $\S 4$, we discuss the combinatorial aspects of the "D " - i.e., in the terminology of the theory of Frobenioids, the "base category" - portion of the notions to be introduced in $\S 5$ below. In a word, these combinatorial aspects revolve around the "functorial dynamics" imposed upon the various number fields and local fields involved by the "labels"

$$
\in \mathbb{F}_{l}^{*} \stackrel{\text { def }}{=} \mathbb{F}_{l}^{\times} /\{ \pm 1\}
$$

— where we note that the set $\mathbb{F}_{l}^{*}$ is of cardinality $l^{*} \stackrel{\text { def }}{=}(l-1) / 2$ - of the $l$-torsion points at which we intend to conduct, in [IUTchII], the "Hodge-Arakelov-theoretic evaluation" of the étale theta function studied in [EtTh] [cf. Remarks 4.3.1; 4.3.2; 4.5.1, (v); 4.9.1, (i)].

In the following, we fix a collection of initial $\Theta$-data

$$
\left(\bar{F} / F, X_{F}, l, \underline{C}_{K}, \underline{\mathbb{V}}, \mathbb{V}_{\bmod }^{\mathrm{bad}}, \underline{\epsilon}\right)
$$

as in Definition 3.1; also, we shall use the various notations introduced in Definition 3.1 for various objects associated to this initial $\Theta$-data.

\section{Definition 4.1.}

(i) We define a holomorphic base-prime-strip, or $\mathcal{D}$-prime-strip, [relative to the given initial $\Theta$-data] to be a collection of data

$$
{ }^{\dagger} \mathfrak{D}=\left\{{ }^{\dagger} \mathcal{D}_{\underline{v}}\right\}_{\underline{v} \in \mathbb{V}}
$$

that satisfies the following conditions: (a) if $\underline{v} \in \underline{\mathbb{V}}^{\text {non }}$, then ${ }^{\dagger} \mathcal{D}_{\underline{v}}$ is a category which admits an equivalence of categories ${ }^{\dagger} \mathcal{D}_{\underline{v}} \stackrel{\sim}{\rightarrow} \mathcal{D}_{\underline{v}}$ [where $\mathcal{D}_{\underline{v}}$ is as in Examples 3.2, (i); 3.3, (i)]; (b) if $\underline{v} \in \underline{\mathbb{V}}^{\text {arc }}$, then ${ }^{\dagger} \mathcal{D}_{\underline{v}}$ is an Aut-holomorphic orbispace such that there exists an isomorphism of Aut-holomorphic orbispaces ${ }^{\dagger} \mathcal{D}_{\underline{v}} \stackrel{\sim}{\rightarrow} \mathcal{D}_{\underline{v}}$ [where $\mathcal{D}_{\underline{v}}$ is as in Example 3.4, (i)]. Observe that if $\underline{v} \in \underline{\mathbb{V}}^{\text {non }}$, then $\pi_{1}\left({ }^{\dagger} \mathcal{D}_{\underline{v}}\right)$ determines, in a functorial fashion, a topological [in fact, profinite if $\underline{v} \in \underline{\mathbb{V}}^{\text {good }}$ ] group corresponding to " $\underline{C}_{\underline{v}}$ " [cf. Corollary 1.2 if $\underline{v} \in \underline{\mathbb{V}}^{\text {good }}$; [EtTh], Proposition 2.4, if $\underline{v} \in \underline{\mathbb{V}}^{\text {bad }}$ ], which contains $\pi_{1}\left({ }^{\dagger} \mathcal{D}_{\underline{v}}\right)$ as an open subgroup; thus, if we write ${ }^{\dagger} \underline{\mathcal{D}}_{\underline{v}}$ for $\mathcal{B}(-)^{0}$ of this topological group, then we obtain a natural morphism ${ }^{\dagger} \mathcal{D}_{\underline{v}} \rightarrow{ }^{\dagger} \underline{\mathcal{D}}_{\underline{v}}[$ cf. $\S 0]$. In a similar vein, if $\underline{v} \in \underline{\mathbb{V}}^{\text {arc }}$, then since $\underline{X}_{\underline{v}}$ admits a $K_{\underline{v}^{-}}$core, a routine translation into the "language of Aut-holomorphic orbispaces" of the argument given in the proof of Corollary 1.2 [cf. also [AbsTopIII], Corollary 2.4] reveals that ${ }^{\dagger} \mathcal{D}_{\underline{v}}$ determines, in a functorial fashion, an Aut-holomorphic orbispace ${ }^{\dagger} \underline{\mathcal{D}}_{\underline{v}}$ corresponding to " $\underline{C}_{\underline{v}}$ ", together with a natural morphism ${ }^{\dagger} \mathcal{D}_{\underline{v}} \rightarrow{ }^{\dagger} \underline{\mathcal{D}}_{\underline{v}}$ of Aut-holomorphic orbispaces. Thus, in summary, one obtains a collection of data

$$
{ }^{\dagger} \underline{\mathfrak{D}}=\left\{\underline{\mathcal{D}}^{\dagger}\right\}_{\underline{v}} \in \underline{\mathbb{V}}
$$


completely determined by ${ }^{\dagger} \mathfrak{D}$.

(ii) Suppose that we are in the situation of (i). Then observe that by applying the group-theoretic algorithm of [AbsTopI], Lemma 4.5 [cf., especially, [AbsTopI], Lemma 4.5, (v), as well as Remark 1.2.2, (ii), of the present paper], to construct the set of conjugacy classes of cuspidal decomposition groups of the topological group $\pi_{1}\left({ }^{\dagger} \mathcal{D}_{v}\right)$ when $\underline{v} \in \underline{\mathbb{V}}^{\text {non }}$, or by considering $\pi_{0}(-)$ of a cofinal collection of "neighborhoods of infinity" [i.e., complements of compact subsets] of the underlying topological space of ${ }^{\dagger} \mathcal{D}_{\underline{v}}$ when $\underline{v} \in \underline{\mathbb{V}}^{\text {arc }}$, it makes sense to speak of the set of cusps of ${ }^{\dagger} \mathcal{D}_{\underline{v}}$; a similar observation applies to ${ }^{\dagger} \underline{\mathcal{D}}_{\underline{v}}$, for $\underline{v} \in \underline{\mathbb{V}}$. If $\underline{v} \in \underline{\mathbb{V}}$, then we define a label class of cusps of ${ }^{\dagger} \mathcal{D}_{\underline{v}}$ to be the set of cusps of ${ }^{\dagger} \mathcal{D}_{\underline{v}}$ that lie over a single "nonzero cusp" [i.e., a cusp that arises from a nonzero element of the quotient " $Q$ " that appears in the definition of a "hyperbolic orbicurve of type $(1, l \text {-tors })_{ \pm}$" given in [EtTh], Definition 2.1] of ${ }^{\dagger} \underline{\mathcal{D}}_{v}$; write

$$
\operatorname{LabCusp}\left({ }^{\dagger} \mathcal{D}_{\underline{v}}\right)
$$

for the set of label classes of cusps of ${ }^{\dagger} \mathcal{D}_{\underline{v}}$. Thus, for each $\underline{v} \in \underline{\mathbb{V}}, \operatorname{LabCusp}\left({ }^{\dagger} \mathcal{D}_{\underline{v}}\right)$ admits a natural $\mathbb{F}_{l}^{*}$-torsor structure [i.e., which arises from the natural action of $\mathbb{F}_{l}^{\times}$on the quotient " $Q$ " of [EtTh], Definition 2.1]. Moreover, [for any $\underline{v} \in \underline{\mathbb{V}}$ !] one may construct, solely from ${ }^{\dagger} \mathcal{D}_{\underline{v}}$, a canonical element

$$
{ }^{\dagger} \underline{\eta}_{\underline{v}} \in \operatorname{LabCusp}\left({ }^{\dagger} \mathcal{D}_{\underline{v}}\right)
$$

determined by " $\underline{\epsilon}_{v}$ " [cf. the notation of Definition 3.1, (f)]. [Indeed, this follows from [EtTh], Corollary 2.9, for $\underline{v} \in \underline{\mathbb{V}}^{\text {bad }}$, from Corollary 1.2 for $\underline{v} \in \underline{\mathbb{V}}^{\text {good }} \cap \underline{\mathbb{V}}^{\text {non }}$, and from the evident translation into the "language of Aut-holomorphic orbispaces" of Corollary 1.2 for $\underline{v} \in \underline{\mathbb{V}}^{\text {arc }}$.]

(iii) We define a mono-analytic base-prime-strip, or $\mathcal{D}^{\vdash}$-prime-strip, [relative to the given initial $\Theta$-data] to be a collection of data

$$
\dagger \mathfrak{D}^{\vdash}=\left\{{ }^{\dagger} \mathcal{D}_{\underline{v}}^{\vdash}\right\}_{\underline{v}} \in \underline{\mathbb{V}}
$$

that satisfies the following conditions: (a) if $\underline{v} \in \underline{\mathbb{V}}^{\text {non }}$, then ${ }^{\dagger} \mathcal{D}_{v}^{\vdash}$ is a category which admits an equivalence of categories ${ }^{\dagger} \mathcal{D}_{\underline{v}}^{\vdash} \stackrel{\sim}{\rightarrow} \mathcal{D}_{\underline{v}}^{\vdash}$ [where $\mathcal{D}_{\underline{v}}^{\vdash}$ is as in Examples 3.2, (i); 3.3, (i)]; (b) if $\underline{v} \in \underline{\mathbb{V}}^{\text {arc }}$, then ${ }^{\dagger} \mathcal{D}_{\underline{v}}^{\vdash}$ is an object of the category $\mathbb{T M}^{\vdash}$ [so, if $\mathcal{D}_{\underline{v}}^{\vdash}$ is as in Example 3.4, (ii), then there exists an isomorphism ${ }^{\dagger} \mathcal{D}_{\underline{v}}^{\vdash} \stackrel{\sim}{\rightarrow} \mathcal{D}_{\underline{v}}^{\vdash}$ in $\left.\mathbb{T M}^{\vdash}\right]$.

(iv) A morphism of $\mathcal{D}$ - (respectively, $\mathcal{D}^{\vdash}$-) prime-strips is defined to be a collection of morphisms, indexed by $\underline{\mathbb{V}}$, between the various constituent objects of the prime-strips. Following the conventions of $\S 0$, one thus has a notion of capsules of $\mathcal{D}$ - (respectively, $\mathcal{D}^{\vdash}$-) and morphisms of capsules of $\mathcal{D}$ - (respectively, $\mathcal{D}^{\vdash}$-) primestrips. Note that to any $\mathcal{D}$-prime-strip ${ }^{\dagger} \mathfrak{D}$, one may associate, in a natural way, a $\mathcal{D}^{\vdash}$-prime-strip ${ }^{\dagger} \mathfrak{D}^{\vdash}$ — which we shall refer to as the mono-analyticization of $\dagger \mathfrak{D}$ - by considering appropriate subcategories at the nonarchimedean primes [cf. Examples 3.2, (i), (vi); 3.3, (i), (iii)], or by applying the construction of Example 3.4, (ii), at the archimedean primes. 
(v) Write

$$
\mathcal{D}^{\odot} \stackrel{\text { def }}{=} \mathcal{B}\left(\underline{C}_{K}\right)^{0}
$$

[cf. §0]. Then recall from [AbsTopIII], Theorem 1.9 [cf. Remark 3.1.2], that there exists a group-theoretic algorithm for reconstructing, from $\pi_{1}\left(\mathcal{D}^{\odot}\right)$ [cf. $\S 0$ ], the algebraic closure " $F$ " of the base field " $K$ ", hence also the set of valuations "V $(\bar{F})$ " [e.g., as a collection of topologies on $\bar{F}$ - cf., e.g., [AbsTopIII], Corollary 2.8]. Moreover, for $\underline{w} \in \mathbb{V}(K)^{\text {arc }}$, let us recall [cf. Remark 3.1.2; [AbsTopIII], Corollaries 2.8, 2.9] that one may reconstruct group-theoretically, from $\pi_{1}\left(\mathcal{D}^{\odot}\right)$, the Aut-holomorphic orbispace $\underline{\mathbb{C}}_{\underline{w}}$ associated to $\underline{C}_{\underline{w}}$. Let ${ }^{\dagger} \mathcal{D}^{\odot}$ be a category equivalent to $\mathcal{D}^{\odot}$. Then let us write

$$
\overline{\mathbb{V}}\left({ }^{\dagger} \mathcal{D}^{\odot}\right)
$$

for the set of valuations [i.e., " $\mathbb{V}(\bar{F})$ "], equipped with its natural $\pi_{1}\left({ }^{\dagger} \mathcal{D}^{\odot}\right)$-action,

$$
\mathbb{V}\left({ }^{\dagger} \mathcal{D}^{\odot}\right) \stackrel{\text { def }}{=} \overline{\mathbb{V}}\left({ }^{\dagger} \mathcal{D}^{\odot}\right) / \pi_{1}\left({ }^{\dagger} \mathcal{D}^{\odot}\right)
$$

for the quotient of $\overline{\mathbb{V}}\left({ }^{\dagger} \mathcal{D}^{\odot}\right)$ by $\pi_{1}\left({ }^{\dagger} \mathcal{D}^{\odot}\right)$ [i.e., " $\mathbb{V}(K)$ "], and, for $\underline{w} \in \mathbb{V}\left({ }^{\dagger} \mathcal{D}^{\odot}\right)^{\text {arc }}$,

$$
\underline{\mathbb{C}}\left({ }^{\dagger} \mathcal{D}^{\odot}, \underline{w}\right)
$$

[i.e., "드 $\underline{w}_{w}$ " c cf. the discussion of [AbsTopIII], Definition 5.1, (ii)] for the Autholomorphic orbispace obtained by applying these group-theoretic reconstruction algorithms to $\pi_{1}\left({ }^{\dagger} \mathcal{D}^{\odot}\right)$. Now if $\mathbb{U}$ is an arbitrary Aut-holomorphic orbispace, then let us define a morphism

$$
\mathbb{U} \rightarrow{ }^{\dagger} \mathcal{D}^{\odot}
$$

to be a morphism of Aut-holomorphic orbispaces [cf. [AbsTopIII], Definition 2.1, (ii)] $\mathbb{U} \rightarrow \underline{\mathbb{C}}\left({ }^{\dagger} \mathcal{D}^{\odot}, \underline{w}\right)$ for some $\underline{w} \in \mathbb{V}\left({ }^{\dagger} \mathcal{D}^{\odot}\right)^{\text {arc }}$. Thus, it makes sense to speak of the pre-composite (respectively, post-composite) of such a morphism $\mathbb{U} \rightarrow{ }^{\dagger} \mathcal{D}^{\odot}$ with a morphism of Aut-holomorphic orbispaces (respectively, with an isomorphism [cf. $\S 0]^{\dagger} \mathcal{D}^{\odot} \stackrel{\sim}{\rightarrow} \ddagger \mathcal{D}^{\odot}$ [i.e., where ${ }^{\ddagger} \mathcal{D}^{\odot}$ is a category equivalent to $\mathcal{D}^{\odot}$ ]). Finally, just as in

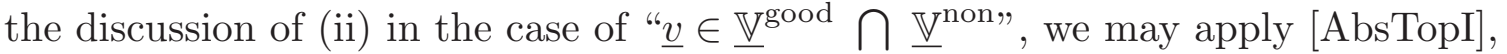
Lemma 4.5 [cf. also Remark 1.2.2, (ii), of the present paper], to conclude that it makes sense to speak of the set of cusps of ${ }^{\dagger} \mathcal{D}^{\odot}$, as well as the set of label classes of cusps

$$
\operatorname{LabCusp}\left({ }^{\dagger} \mathcal{D}^{\odot}\right)
$$

of ${ }^{\dagger} \mathcal{D}^{\odot}$, which admits a natural $\mathbb{F}_{l}^{*}$-torsor structure.

(vi) Let ${ }^{\dagger} \mathcal{D}^{\odot}$ be a category equivalent to $\mathcal{D}^{\odot},{ }^{\dagger} \mathfrak{D}=\left\{{ }^{\dagger} \mathcal{D}_{\underline{\underline{v}}}\right\}_{\underline{v} \in \underline{\mathbb{V}}}$ a $\mathcal{D}$-primestrip. If $\underline{v} \in \mathbb{V}$, then we define a poly-morphism ${ }^{\dagger} \mathcal{D}_{\underline{v}} \rightarrow{ }^{\dagger} \mathcal{D}^{\odot}$ to be a collection of morphisms ${ }^{\dagger} \mathcal{D}_{\underline{v}} \rightarrow{ }^{\dagger} \mathcal{D}^{\odot}\left[\operatorname{cf}\right.$. $\S 0$ when $\underline{v} \in \underline{\mathbb{V}}^{\text {non }} ;(\mathrm{v})$ when $\left.\underline{v} \in \underline{\mathbb{V}}^{\text {arc }}\right]$. We define a poly-morphism

$$
\dagger \mathfrak{D} \rightarrow{ }^{\dagger} \mathcal{D}^{\odot}
$$

to be a collection of poly-morphisms $\left\{{ }^{\dagger} \mathcal{D}_{\underline{v}} \rightarrow{ }^{\dagger} \mathcal{D}^{\odot}\right\}_{\underline{v} \in \underline{\mathbb{V}}}$. Finally, if $\left\{{ }^{e} \mathfrak{D}\right\}_{e \in E}$ is a capsule of $\mathcal{D}$-prime-strips, then we define a poly-morphism

$$
\left.\left\{{ }^{e} \mathfrak{D}\right\}_{e \in E} \rightarrow{ }^{\dagger} \mathcal{D}^{\odot} \text { (respectively, }\left\{{ }^{e} \mathfrak{D}\right\}_{e \in E} \rightarrow{ }^{\dagger} \mathfrak{D}\right)
$$


to be a collection of poly-morphisms $\left\{{ }^{e} \mathfrak{D} \rightarrow^{\dagger} \mathcal{D}^{\odot}\right\}_{e \in E}$ (respectively, $\left.\left\{{ }^{e} \mathfrak{D} \rightarrow{ }^{\dagger} \mathfrak{D}\right\}_{e \in E}\right)$.

The following result follows immediately from the discussion of Definition 4.1, (ii).

Proposition 4.2. (The Set of Label Classes of Cusps of a Base-PrimeStrip) Let ${ }^{\dagger} \mathfrak{D}=\left\{{ }^{\dagger} \mathcal{D}_{\underline{v}}\right\}_{\underline{v} \in \underline{\mathbb{V}}}$ be a $\mathcal{D}$-prime-strip. Then for any $\underline{v}, \underline{w} \in \underline{\mathbb{V}}$, there exist bijections

$$
\operatorname{LabCusp}\left({ }^{\dagger} \mathcal{D}_{\underline{v}}\right) \stackrel{\sim}{\rightarrow} \operatorname{LabCusp}\left({ }^{\dagger} \mathcal{D}_{\underline{w}}\right)
$$

that are uniquely determined by the condition that they be compatible with the assignments ${ }^{\dagger} \underline{\eta}_{\underline{v}} \mapsto{ }^{\dagger} \underline{\eta}_{\underline{w}}$ [cf. Definition 4.1, (ii)], as well as with the $\mathbb{F}_{i}^{*}$ torsor structures on either side. In particular, these bijections are preserved by arbitrary isomorphisms of $\mathcal{D}$-prime-strips. Thus, by identifying the various "LabCusp $\left({ }^{\dagger} \mathcal{D}_{\underline{v}}\right)$ " via these bijections, it makes sense to write $\operatorname{LabCusp}\left({ }^{\dagger} \mathfrak{D}\right)$. Finally, LabCusp $\left({ }^{\dagger} \mathfrak{D}\right)$ is equipped with a canonical element, arising from the ${ }^{\dagger} \underline{\eta}_{\underline{v}}$ $\left[\right.$ for $\underline{v} \in \underline{\mathbb{V}}$ ], as well as a natural $\mathbb{F}_{l}^{*}$-torsor structure; in particular, this canonical element and $\mathbb{F}_{l}^{*}$-torsor structure determine a natural bijection

$$
\operatorname{LabCusp}\left({ }^{\dagger} \mathfrak{D}\right) \stackrel{\sim}{\rightarrow} \mathbb{F}_{l}^{*}
$$

that is preserved by isomorphisms of $\mathcal{D}$-prime-strips.

Remark 4.2.1. Note that if, in Examples 3.3, 3.4 - i.e., at $\underline{v} \in \underline{\mathbb{V}}^{\text {good }}$ one defines " $\mathcal{D}_{\underline{v}}$ " by means of " $\underline{C}_{\underline{v}}$ " instead of " $\underline{X}_{v}$ ", then there does not exist a system of bijections as in Proposition 4.2. Indeed, by the Tchebotarev density theorem [cf., e.g., [Lang], Chapter VIII, $§ 4$, Theorem 10], it follows immediately that there exist $\underline{v} \in \underline{\mathbb{V}}$ such that, for a suitable embedding $\operatorname{Gal}(K / F) \hookrightarrow G L_{2}\left(\mathbb{F}_{l}\right)$, the decomposition subgroup in $\operatorname{Gal}(K / F) \hookrightarrow G L_{2}\left(\mathbb{F}_{l}\right)$ determined [up to conjugation] by $\underline{v}$ is equal to the subgroup of diagonal matrices with determinant 1 . Thus, if ${ }^{\dagger} \mathfrak{D}=\left\{{ }^{\dagger} \mathcal{D}_{\underline{v}}\right\}_{\underline{v} \in \underline{\mathbb{V}}},{ }^{\dagger} \underline{\mathfrak{D}}=\left\{{ }^{\dagger} \underline{\mathcal{D}}_{\underline{v}}\right\}_{\underline{v} \in \underline{\mathbb{V}}}$ are as in Definition 4.1, (i), then for such a $\underline{v}$, the automorphism group of ${ }^{\dagger} \underline{\mathcal{D}}_{\underline{v}}$ acts transitively on the set of label classes of cusps of ${ }^{\dagger} \underline{\mathcal{D}}_{\underline{v}}$, while the automorphism group of ${ }^{\dagger} \underline{\mathcal{D}}_{\underline{w}}$ acts trivially [by [EtTh], Corollary 2.9] on the set of label classes of cusps of ${ }^{\dagger} \underline{\mathcal{D}}_{\underline{w}}$ for any $\underline{w} \in \underline{\mathbb{V}}^{\text {bad }}$.

Example 4.3. Model Base-NF-Bridges. In the following, we construct the "models" for the notion of a "base-NF-bridge" [cf. Definition 4.6, (i), below].

(i) Write

$$
\operatorname{Aut}_{\underline{\epsilon}}\left(\underline{C}_{K}\right) \subseteq \operatorname{Aut}\left(\underline{C}_{K}\right) \cong \operatorname{Out}\left(\Pi_{\underline{C}_{K}}\right) \cong \operatorname{Aut}\left(\mathcal{D}^{\odot}\right)
$$

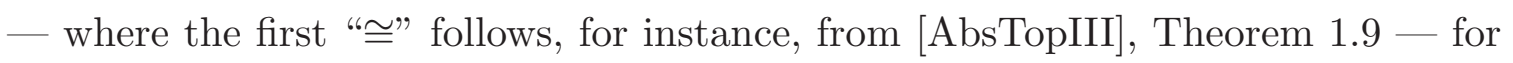
the subgroup of elements which fix the cusp $\underline{\epsilon}$. Now let us recall that the profinite group $\Delta_{X}$ may be reconstructed group-theoretically from $\Pi_{\underline{C}_{K}}$ [cf. [AbsTopII], Corollary 3.3, (i), (ii); [AbsTopII], Remark 3.3.2; [AbsTopI], Example 4.8]. Since 
inner automorphisms of $\Pi_{\underline{C}_{K}}$ clearly act by multiplication by \pm 1 on the $l$-torsion points of $E_{\bar{F}}$ [i.e., on $\left.\Delta_{X}^{\mathrm{ab}} \otimes \mathbb{F}_{l}\right]$, we obtain a natural homomorphism $\operatorname{Out}\left(\Pi_{\underline{C}_{K}}\right) \rightarrow$ $\operatorname{Aut}\left(\Delta_{X}^{\mathrm{ab}} \otimes \mathbb{F}_{l}\right) /\{ \pm 1\}$. Thus, it follows immediately from the discussion of the notation " $K$ ", " $\underline{C}_{K}$ ", and " $\underline{\epsilon}$ " in Definition 3.1, (c), (d), (f) [cf. also Remark 3.1.5; the discussion preceding [EtTh], Definition 2.1; the discussion of [EtTh], Remark 2.6.1], that, relative to an isomorphism $\operatorname{Aut}\left(\Delta_{X}^{\mathrm{ab}} \otimes \mathbb{F}_{l}\right) /\{ \pm 1\} \stackrel{\sim}{\rightarrow} G L_{2}\left(\mathbb{F}_{l}\right) /\{ \pm 1\}$ arising from a suitable choice of basis for $\Delta_{X}^{\mathrm{ab}} \otimes \mathbb{F}_{l}$, if we write $\operatorname{Im}\left(G_{F_{\text {mod }}}\right) \subseteq G L_{2}\left(\mathbb{F}_{l}\right) /\{ \pm 1\}$ for the image of the natural action [i.e., modulo $\{ \pm 1\}]$ of $G_{F_{\text {mod }}} \stackrel{\text { def }}{=} \operatorname{Gal}\left(\bar{F} / F_{\bmod }\right)$ on the l-torsion points of $E_{F}$ [cf. the homomorphism of the display of Definition 3.1, (c); the model " $C_{F_{\text {mod }}}$ " discussed in Remark 3.1.7], then the images of the groups $\operatorname{Aut}_{\underline{\epsilon}}\left(\underline{C}_{K}\right)$, Aut $\left(\underline{C}_{K}\right)$ may be identified with the subgroups consisting of elements of the form

$$
\left\{\left(\begin{array}{cc}
* & * \\
0 & \pm 1
\end{array}\right)\right\} \subseteq\left\{\left(\begin{array}{ll}
* & * \\
0 & *
\end{array}\right)\right\} \subseteq \operatorname{Im}\left(G_{F_{\bmod }}\right)\left(\supseteq \quad S L_{2}\left(\mathbb{F}_{l}\right) /\{ \pm 1\}\right)
$$

- i.e., "semi-unipotent, up to \pm 1 " and "Borel" subgroups - of $\operatorname{Im}\left(G_{F_{\bmod }}\right) \subseteq$ $G L_{2}\left(\mathbb{F}_{l}\right) /\{ \pm 1\}$. Write

$$
\operatorname{Aut}_{\underline{\epsilon}}^{S L}\left(\underline{C}_{K}\right) \subseteq \operatorname{Aut}_{\underline{\epsilon}}\left(\underline{C}_{K}\right), \quad \operatorname{Aut}^{S L}\left(\underline{C}_{K}\right) \subseteq \operatorname{Aut}\left(\underline{C}_{K}\right)
$$

for the respective subgroups of elements that act trivially on the subfield $F\left(\boldsymbol{\mu}_{l}\right) \subseteq K$ [cf. Remark 3.1.7, (iii)] and

$$
\underline{\mathbb{V}}^{ \pm \text {un }} \stackrel{\text { def }}{=} \operatorname{Aut}_{\underline{\epsilon}}\left(\underline{C}_{K}\right) \cdot \underline{\mathbb{V}} \subseteq \underline{\mathbb{V}}^{\text {Bor }} \stackrel{\text { def }}{=} \operatorname{Aut}\left(\underline{C}_{K}\right) \cdot \underline{\mathbb{V}} \subseteq \mathbb{V}(K)
$$

for the resulting subsets of $\mathbb{V}(K)$. Thus, one verifies immediately that the subgroup $\operatorname{Aut}_{\underline{\epsilon}}\left(\underline{C}_{K}\right) \subseteq \operatorname{Aut}\left(\underline{C}_{K}\right)$ is normal, and that we have natural isomorphisms

$$
\operatorname{Aut}^{S L}\left(\underline{C}_{K}\right) / \operatorname{Aut}_{\underline{\epsilon}}^{S L}\left(\underline{C}_{K}\right) \stackrel{\sim}{\rightarrow} \operatorname{Aut}\left(\underline{C}_{K}\right) / \operatorname{Aut}_{\underline{\epsilon}}\left(\underline{C}_{K}\right) \stackrel{\sim}{\rightarrow} \mathbb{F}_{l}^{*}
$$

- so we may think of $\underline{\mathbb{V}}^{\text {Bor }}$ as the $\mathbb{F}_{l}^{*}$-orbit of $\underline{\mathbb{V}}^{ \pm \text {un }}$. Also, we observe that in light of the above discussion] it follows immediately that there exists a group-theoretic algorithm for reconstructing, from $\pi_{1}\left(\mathcal{D}^{\odot}\right)$ [i.e., an isomorph of $\Pi_{\underline{C}_{K}}$ ] the subgroup

$$
\operatorname{Aut}_{\underline{\epsilon}}\left(\mathcal{D}^{\odot}\right) \subseteq \operatorname{Aut}\left(\mathcal{D}^{\odot}\right)
$$

determined by $\operatorname{Aut}_{\underline{\epsilon}}\left(\underline{C}_{K}\right)$.

(ii) Let $\underline{v} \in \underline{\mathbb{V}}^{\text {non }}$. Then the natural restriction functor on finite étale coverings arising from the natural composite morphism $\underline{X}_{\underline{v}_{\underline{v}}} \rightarrow \underline{C}_{\underline{v}} \rightarrow \underline{C}_{K}$ if $\underline{v} \in \underline{\mathbb{V}}^{\text {good }}$ (respectively, $\underline{\underline{X}}_{\underline{v}} \rightarrow \underline{C}_{\underline{v}} \rightarrow \underline{C}_{K}$ if $\underline{v} \in \underline{\mathbb{V}}^{\text {bad }}$ ) determines [cf. Examples 3.2, (i); 3.3 , (i)] a natural morphism $\phi_{\bullet, \underline{v}}^{\mathrm{NF}}: \mathcal{D}_{\underline{v}} \rightarrow \mathcal{D}^{\odot}[$ cf. $\oint 0$ for the definition of the term "morphism"]. Write

$$
\phi_{\underline{v}}^{\mathrm{NF}}: \mathcal{D}_{\underline{v}} \rightarrow \mathcal{D}^{\odot}
$$

for the poly-morphism given by the collection of morphisms $\mathcal{D}_{\underline{v}} \rightarrow \mathcal{D}^{\odot}$ of the form

$$
\beta \circ \phi_{\bullet, \underline{v}}^{\mathrm{NF}} \circ \alpha
$$


- where $\alpha \in \operatorname{Aut}\left(\mathcal{D}_{\underline{v}}\right) \cong \operatorname{Aut}\left(\underline{X}_{\underline{v}}\right)\left(\right.$ respectively, $\left.\alpha \in \operatorname{Aut}\left(\mathcal{D}_{\underline{v}}\right) \cong \operatorname{Aut}\left(\underline{\underline{X}}_{\underline{v}}\right)\right) ; \beta \in$ $\operatorname{Aut}_{\underline{\epsilon}}\left(\mathcal{D}^{\odot}\right) \cong \operatorname{Aut}_{\underline{\epsilon}}\left(\underline{C}_{K}\right)$ [cf., e.g., [A $\operatorname{AbsTopIII]}$, Theorem 1.9].

(iii) Let $\underline{v} \in \underline{\mathbb{V}}^{\text {arc }}$. Thus, [cf. Example 3.4, (i)] we have a tautological morphism $\mathcal{D}_{\underline{v}}=\underline{\mathbb{X}}_{\underline{v}} \rightarrow \underline{\mathbb{C}}_{\underline{v}} \stackrel{\sim}{\rightarrow} \underline{\mathbb{C}}\left(\mathcal{D}^{\odot}, \underline{v}\right)$, hence a morphism $\phi_{\bullet, \underline{v}}^{\mathrm{NF}}: \mathcal{D}_{\underline{v}} \rightarrow \mathcal{D}^{\odot}[$ cf. Definition 4.1, (v)]. Write

$$
\phi_{\underline{v}}^{\mathrm{NF}}: \mathcal{D}_{\underline{v}} \rightarrow \mathcal{D}^{\odot}
$$

for the poly-morphism given by the collection of morphisms $\mathcal{D}_{\underline{v}} \rightarrow \mathcal{D}^{\odot}$ of the form

$$
\beta \circ \phi_{\bullet, \underline{v}}^{\mathrm{NF}} \circ \alpha
$$

- where $\alpha \in \operatorname{Aut}\left(\mathcal{D}_{\underline{v}}\right) \cong \operatorname{Aut}\left(\underline{\mathbb{X}}_{\underline{v}}\right)\left[\operatorname{cf.}[\operatorname{AbsTopIII}]\right.$, Corollary 2.3]; $\beta \in \operatorname{Aut}_{\underline{\underline{\epsilon}}}\left(\mathcal{D}^{\odot}\right) \cong$ $\operatorname{Aut}_{\underline{\epsilon}}\left(\underline{C}_{K}\right)$.

(iv) For each $j \in \mathbb{F}_{l}^{*}$, let

$$
\mathfrak{D}_{j}=\left\{\mathcal{D}_{\underline{\underline{v}}}\right\}_{\underline{v} \in \underline{\mathbb{V}}}
$$

- where we use the notation $\underline{v}_{j}$ to denote the pair $(j, \underline{v})$ - be a copy of the "tautological $\mathcal{D}$-prime-strip" $\left\{\mathcal{D}_{\underline{v}}\right\}_{\underline{v}} \in \underline{\mathbb{V}}$. Let us denote by

$$
\phi_{1}^{\mathrm{NF}}: \mathfrak{D}_{1} \rightarrow \mathcal{D}^{\odot}
$$

[where, by abuse of notation, we write "1" for the element of $\mathbb{F}_{l}^{*}$ determined by 1 ] the poly-morphism determined by the collection $\left\{\phi_{\underline{\underline{v}}_{1}}^{\mathrm{NF}}: \mathcal{D}_{\underline{v}_{1}} \rightarrow \mathcal{D}^{\odot}\right\}_{\underline{\underline{v}} \in \underline{\mathbb{V}}}$ of copies of the poly-morphisms $\phi_{\underline{v}}^{\mathrm{NF}}$ constructed in (ii), (iii). Note that $\phi_{1}^{\mathrm{NF}}$ is stabilized by the action of $\operatorname{Aut}_{\underline{\epsilon}}\left(\underline{C}_{K}\right)$ on $\mathcal{D}^{\odot}$. Thus, it makes sense to consider, for arbitrary $j \in \mathbb{F}_{l}^{*}$, the poly-morphism

$$
\phi_{j}^{\mathrm{NF}}: \mathfrak{D}_{j} \rightarrow \mathcal{D}^{\odot}
$$

obtained [via any isomorphism $\mathfrak{D}_{1} \cong \mathfrak{D}_{j}$ ] by post-composing with the "poly-action" [i.e., action via poly-automorphisms - cf. (i)] of $j \in \mathbb{F}_{l}^{*}$ on $\mathcal{D}^{\odot}$. Let us write

$$
\mathfrak{D}_{*} \stackrel{\text { def }}{=}\left\{\mathfrak{D}_{j}\right\}_{j \in \mathbb{F}_{l}^{*}}
$$

for the capsule of $\mathcal{D}$-prime-strips indexed by $j \in \mathbb{F}_{l}^{*}$ [cf. Definition 4.1, (iv)] and denote by

$$
\phi_{*}^{\mathrm{NF}}: \mathfrak{D}_{*} \rightarrow \mathcal{D}^{\odot}
$$

the poly-morphism given by the collection of poly-morphisms $\left\{\phi_{j}^{\mathrm{NF}}\right\}_{j \in \mathbb{F}_{i}^{*}}$. Thus, $\phi_{*}^{\mathrm{NF}}$ is equivariant with respect to the natural poly-action of $\mathbb{F}_{l}^{*}$ on $\mathcal{D}^{\odot}$ and the natural permutation poly-action of $\mathbb{F}_{l}^{*}$, via capsule-full [cf. $\S 0$ ] poly-automorphisms, on the constituents of the capsule $\mathfrak{D}_{*}^{*}$. In particular, we obtain a natural poly-action of $\mathbb{F}_{l}^{*}$ on the collection of data $\left(\mathfrak{D}_{*}^{*}, \mathcal{D}^{\odot}, \phi_{*}^{\mathrm{NF}}\right)$. 


\section{Remark 4.3.1.}

(i) Suppose, for simplicity, in the following discussion that $F=F_{\text {mod }}$. Note that the morphism of schemes $\operatorname{Spec}(K) \rightarrow \operatorname{Spec}(F)$ [or, equivalently, the homomorphism of rings $F \hookrightarrow K$ ] does not admit a section. This nonexistence of a section is closely related to the nonexistence of a "global multiplicative subspace" of the sort discussed in [HASurII], Remark 3.7. In the context of loc. cit., this nonexistence of a "global multiplicative subspace" may be thought of as a concrete way of representing the principal obstruction to applying the scheme-theoretic HodgeArakelov theory of [HASurI], [HASurII] to diophantine geometry. From this point of view, if one thinks of the ring structure of $F, K$ as a sort of "arithmetic holomorphic structure" [cf. [AbsTopIII], Remark 5.10.2, (ii)], then one may think of the $[\mathcal{D}$-]prime-strips that appear in the discussion of Example 4.3 as defining, via the arrows $\phi_{j}^{\mathrm{NF}}$ of Example 4.3, (iv),

"arithmetic collections of local analytic sections" of $\operatorname{Spec}(K) \rightarrow \operatorname{Spec}(F)$

— cf. Fig. 4.1 below, where each ". $-\cdot \ldots \ldots-\cdot \ldots$. represents a $[\mathcal{D}$ - $]$ prime-strip. In fact, if, for the sake of brevity, we abbreviate the phrase "collection of local analytic" by the term "local-analytic", then each of these sections may be thought of as yielding not only an "arithmetic local-analytic global multiplicative subspace", but also an "arithmetic local-analytic global canonical generator" [i.e., up to multiplication by \pm 1 , of the quotient of the module of $l$-torsion points of the elliptic curve in question by the "arithmetic local-analytic global multiplicative subspace"]. We refer to Remark 4.9.1, (i), below, for more on this point of view.

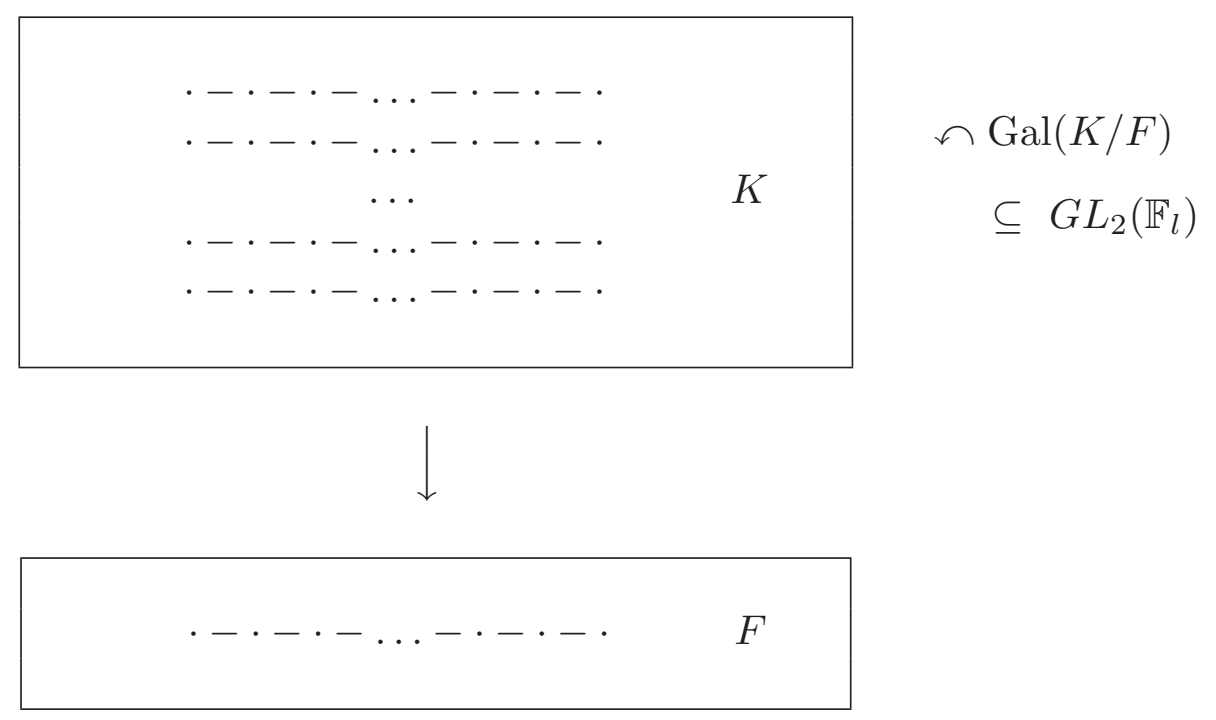

Fig. 4.1: Prime-strips as "sections" of $\operatorname{Spec}(K) \rightarrow \operatorname{Spec}(F)$

(ii) The way in which these "arithmetic local-analytic sections" constituted by the $[\mathcal{D}$-]prime-strips fail to be [globally] "arithmetically holomorphic" may be understood from several closely related points of view. The first point of view was already noted above in (i) - namely:

(a) these sections fail to extend to ring homomorphisms $K \rightarrow F$.

The second point of view involves the classical phenomenon of decomposition of primes in extensions of number fields. The decomposition of primes in extensions 
of number fields may be represented by a tree, as in Fig. 4.2, below. If one thinks of the tree in large parentheses of Fig. 4.2 as representing the decomposition of primes over a prime $v$ of $F$ in extensions of $F$ [such as $K !$ ], then the "arithmetic local-analytic sections" constituted by the $\mathcal{D}$-prime-strips may be thought of as

(b) an isomorphism, or identification, between $v$ [i.e., a prime of $F$ ] and $v^{\prime}$ [i.e., a prime of $K$ ] which [manifestly — cf., e.g., [NSW], Theorem 12.2.5] fails to extend to an isomorphism between the respective prime decomposition trees over $v$ and $v^{\prime}$.

If one thinks of the relation " $\in$ " between sets in axiomatic set theory as determining a "tree", then

the point of view of (b) is reminiscent of the point of view of [IUTchIV], §3, where one is concerned with constructing some sort of artificial solution to the "membership equation $a \in a$ " [cf. the discussion of [IUTchIV], Remark 3.3.1, (i)].

The third point of view consists of the observation that although the "arithmetic local-analytic sections" constituted by the $\mathcal{D}$-prime-strips involve isomorphisms of the various local absolute Galois groups,

(c) these isomorphisms of local absolute Galois groups fail to extend to a section of global absolute Galois groups $G_{F} \rightarrow G_{K}$ [i.e., a section of the natural inclusion $\left.G_{K} \hookrightarrow G_{F}\right]$.

Here, we note that in fact, by the Neukirch-Uchida theorem [cf. [NSW], Chapter XII, §2], one may think of (a) and (c) as essentially equivalent. Moreover, (b) is closely related to this equivalence, in the sense that the proof [cf., e.g., [NSW], Chapter XII, §2] of the Neukirch-Uchida theorem depends in an essential fashion on a careful analysis of the prime decomposition trees of the number fields involved.

$$
\left(\begin{array}{ccc}
\cdots & & \\
V / & \ldots & \ldots \\
v^{\prime} & v^{\prime \prime} & v^{\prime \prime \prime} \\
\backslash & \mid & / \\
& v &
\end{array}\right) \supseteq\left(\begin{array}{c}
\cdots \\
V / \\
v^{\prime}
\end{array}\right)
$$

Fig. 4.2: Prime decomposition trees

(iii) In some sense, understanding more precisely the content of the failure of these "arithmetic local-analytic sections" constituted by the $\mathcal{D}$-prime-strips to be "arithmetically holomorphic" is a central theme of the theory of the present series of papers - a theme which is very much in line with the spirit of classical complex Teichmüller theory.

Remark 4.3.2. The incompatibility of the "arithmetic local-analytic sections" of Remark 4.3.1, (i), with global prime distributions and global absolute Galois groups [cf. the discussion of Remark 4.3.1, (ii)] is precisely the technical obstacle that 
will necessitate the application — in [IUTchIII] — of the absolute p-adic monoanabelian geometry developed in [AbsTopIII], in the form of "panalocalization along the various prime-strips" [cf. [IUTchIII] for more details]. Indeed,

the mono-anabelian theory developed in [AbsTopIII] represents the culmination of earlier research of the author during the years 2000 to 2007 concerning absolute $\boldsymbol{p}$-adic anabelian geometry — research that was motivated precisely by the goal of developing a geometry that would allow one to work with the "arithmetic local-analytic sections" constituted by the prime-strips, so as to overcome the principal technical obstruction to applying the Hodge-Arakelov theory of [HASurI], [HASurII] [cf. Remark 4.3.1, (i)].

Note that the "desired geometry" in question will also be subject to other requirements. For instance, in [IUTchIII] [cf. also [IUTchII], §4], we shall make essential use of the global arithmetic - i.e., the ring structure and absolute Galois groups of number fields. As observed above in Remark 4.3.1, (ii), these global arithmetic structures are not compatible with the "arithmetic local-analytic sections" constituted by the prime-strips. In particular, this state of affairs imposes the further requirement that the "geometry" in question be compatible with globalization, i.e., that it give rise to the global arithmetic of the number fields in question in a fashion that is independent of the various local geometries that appear in the "arithmetic local-analytic sections" constituted by the prime-strips, but nevertheless admits localization operations to these various local geometries [cf. Fig. 4.3; the discussion of [IUTchII], Remark 4.11.2, (iii); [AbsTopIII], Remark 3.7.6, (iii), (v)].

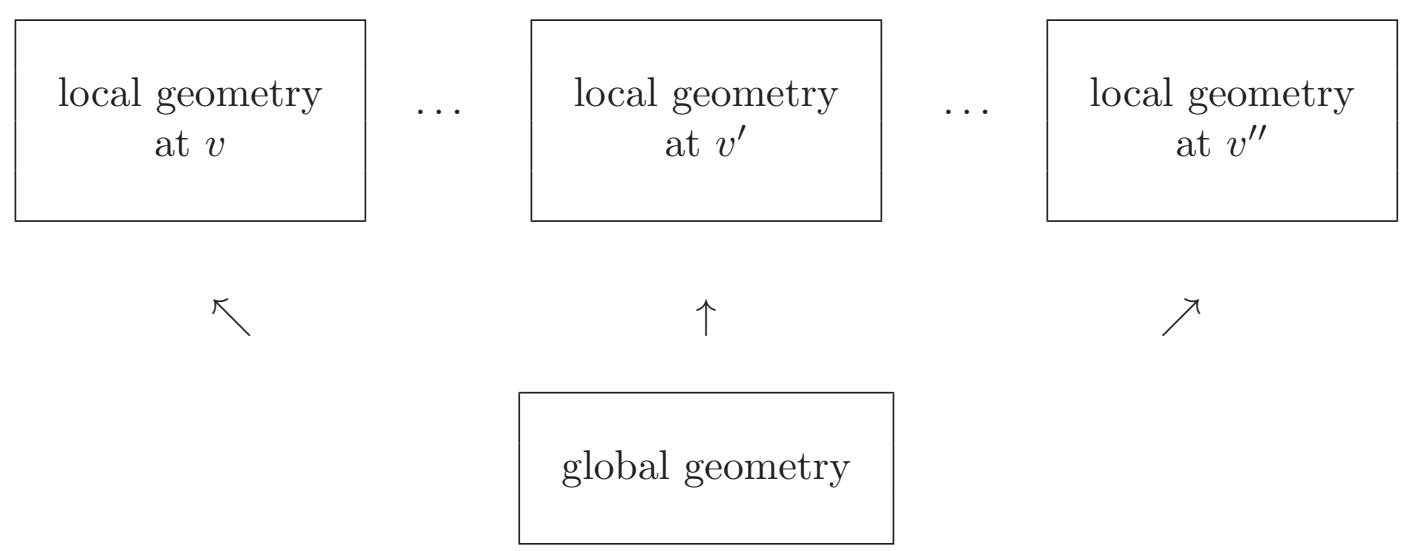

Fig. 4.3: Globalizability

Finally, in order for the "desired geometry" to be applicable to the theory developed in the present series of papers, it is necessary for it to be based on "étale-like structures", so as to give rise to canonical splittings, as in the étale-picture discussed in Corollary 3.9, (i). Thus, in summary, the requirements that we wish to impose on the "desired geometry" are the following:

(a) local independence of global structures,

(b) globalizability, in a fashion that is independent of local structures,

(c) the property of being based on étale-like structures.

Note, in particular, that properties (a), (b) at first glance almost appear to contradict one another. In particular, the simultaneous realization of (a), (b) is highly 
nontrivial. For instance, in the case of a function field of dimension one over a base field, the simultaneous realization of properties (a), (b) appears to require that one restrict oneself essentially to working with structures that descend to the base field! It is thus a highly nontrivial consequence of the theory of [AbsTopIII] that the mono-anabelian geometry of [AbsTopIII] does indeed satisfy all of these requirements (a), (b), (c) [cf. the discussion of [AbsTopIII], §I1].

\section{Remark 4.3.3.}

(i) One important theme of [AbsTopIII] is the analogy between the monoanabelian theory of [AbsTopIII] and the theory of Frobenius-invariant indigenous bundles of the sort that appear in $p$-adic Teichmüller theory [cf. [AbsTopIII], $\S I 5]$. In fact, [although this point of view is not mentioned in [AbsTopIII]] one may "compose" this analogy with the analogy between the $p$-adic and complex theories discussed in $[p$ Ord], Introduction; $[p$ Teich], Introduction, $\S 0$, and consider the analogy between the mono-anabelian theory of [AbsTopIII] and the classical geometry of the upper half-plane $\mathfrak{H}$. In addition to being more elementary than the $p$-adic theory, this analogy with the classical geometry of the upper half-plane $\mathfrak{H}$ also has the virtue that

since it revolves around the canonical Kähler metric - i.e., the Poincaré metric - on the upper half-plane, it renders more transparent the relationship between the theory of the present series of papers and classical Arakelov theory [which also revolves, to a substantial extent, around Kähler metrics at the archimedean primes].

(ii) The essential content of the mono-anabelian theory of [AbsTopIII] may be summarized by the diagram

$$
\Pi \curvearrowright \bar{k}^{\times} \stackrel{\log }{\longrightarrow} \bar{k} \curvearrowleft \Pi
$$

— where $k$ is a finite extension of $\mathbb{Q}_{p} ; \bar{k}$ is an algebraic closure of $k ; \Pi$ is the arithmetic fundamental group of a hyperbolic orbicurve over $k$; $\mathfrak{l o g}$ is the $p$-adic logarithm [cf. [AbsTopIII], §I1]. On the other hand, if $\left(\mathcal{E}, \nabla_{\mathcal{E}}\right)$ denotes the "tautological indigenous bundle" on $\mathfrak{H}$ [i.e., the first de Rham cohomology of the tautological elliptic curve over $\mathfrak{H}]$, then one has a natural Hodge filtration $0 \rightarrow \omega \rightarrow \mathcal{E} \rightarrow \tau \rightarrow 0$ [where $\omega, \tau \stackrel{\text { def }}{=} \omega^{-1}$ are holomorphic line bundles on $\mathfrak{H}$ ], together with a natural complex conjugation operation $\iota_{\mathcal{E}}: \mathcal{E} \rightarrow \mathcal{E}$. The composite

$$
\omega \hookrightarrow \mathcal{E} \stackrel{\iota \mathcal{E}}{\longrightarrow} \mathcal{E} \quad \rightarrow \quad \tau
$$

then determines an Hermitian metric $|-| \omega$ on $\omega$. For any trivializing section $f$ of $\omega$, the $(1,1)$-form

$$
\kappa_{\mathfrak{H}} \stackrel{\text { def }}{=} \frac{1}{2 \pi i} \partial \bar{\partial} \log \left(|f|_{\omega}^{2}\right)
$$

is the canonical Kähler metric [i.e., Poincaré metric] on $\mathfrak{H}$. Then one can already identify various formal similarities between $\kappa_{\mathfrak{H}}$ and the diagram $(*)$ reviewed above: Indeed, at a purely formal [but by no means coincidental!] level, the "log" that 
appears in the definition of $\kappa_{\mathfrak{H}}$ is reminiscent of the "log-Frobenius operation" log. At a less formal level, the "Galois group" $\Pi$ is reminiscent — cf. the point of view that "Galois groups are arithmetic tangent bundles", a point of view that underlies the theory of the arithmetic Kodaira-Spencer morphism discussed in [HASurI]! of $\partial$. If one thinks of complex conjugation as a sort of "archimedean Frobenius" [cf. $[p$ Teich], Introduction, $\S 0]$, then $\bar{\partial}$ is reminiscent of the "Galois group" $\Pi$ operating on the opposite side $\left[\mathrm{cf} . \iota_{\mathcal{E}}\right.$ ] of the log-Frobenius operation $\mathfrak{l o g}$. The Hodge filtration of $\mathcal{E}$ corresponds to the ring structures of the copies of $\bar{k}$ on either side of $\mathfrak{l o g}$ [cf. the discussion of [AbsTopIII], Remark 3.7.2]. Finally, perhaps most importantly from the point of view of the theory of the present series of papers:

the fact that log-shells play the role in the theory of [AbsTopIII] of "canonical rigid integral structures" [cf. [AbsTopIII], §I1] — i.e., "canonical standard units of volume" — is reminiscent of the fact that the Kähler metric $\kappa_{\mathfrak{H}}$ also plays the role of determining a canonical notion of volume on $\mathfrak{H}$.

(iii) From the point of view of the analogy discussed in (ii), property (a) of Remark 4.3.2 may be thought of as corresponding to the local representability via the [positive] $(1,1)$-form $\kappa_{\mathfrak{H}}$ - on, say, a compact quotient $S$ of $\mathfrak{H}$ - of the [positive] global degree of [the result of descending to $S$ ] the line bundle $\omega$; property (b) of Remark 4.3.2 may be thought of as corresponding to the fact that this $(1,1)$-form $\kappa_{\mathfrak{H}}$ that gives rise to a local representation on $S$ of the notion of a positive global degree not only exists locally on $S$, but also admits a canonical global extension to the entire Riemann surface $S$ which may be related to the algebraic theory [i.e., of algebraic rational functions on $S$ ].

(iv) The analogy discussed in (ii) may be summarized as follows:

\begin{tabular}{|c|c|}
\hline mono-anabelian theory & geometry of the upper-half plane $\mathfrak{H}$ \\
\hline the Galois group $\Pi$ & the differential operator $\partial$ \\
\hline $\begin{array}{l}\text { the Galois group } \Pi \\
\text { on the opposite side of } \mathfrak{l o g}\end{array}$ & $\begin{array}{c}\text { the differential operator } \\
\bar{\partial}\end{array}$ \\
\hline $\begin{array}{l}\text { the ring structures of the copies } \\
\text { of } \bar{k} \text { on either side of } \mathfrak{l o g}\end{array}$ & $\begin{array}{l}\text { the Hodge filtration of } \mathcal{E}, \\
\qquad \iota_{\mathcal{E}},|-|_{\mathcal{E}}\end{array}$ \\
\hline $\begin{array}{c}\text { log-shells as } \\
\text { canonical units of volume }\end{array}$ & $\begin{array}{l}\text { the canonical Kähler volume } \\
\kappa_{\mathfrak{H}}\end{array}$ \\
\hline
\end{tabular}

Example 4.4. Model Base- $\Theta$-Bridges. In the following, we construct the "models" for the notion of a "base- $\Theta$-bridge" [cf. Definition 4.6, (ii), below]. We continue to use the notation of Example 4.3.

(i) Let $\underline{v} \in \underline{\mathbb{V}}^{\text {bad }}$. Recall that there is a natural bijection between the set

$$
\left|\mathbb{F}_{l}\right| \stackrel{\text { def }}{=} \mathbb{F}_{l} /\{ \pm 1\}=0 \bigcup \mathbb{F}_{l}^{*}
$$


[i.e., the set of $\{ \pm 1\}$-orbits of $\left.\mathbb{F}_{l}\right]$ and the set of cusps of the hyperbolic orbicurve $\underline{C}_{\underline{v}}$ [cf. [EtTh], Corollary 2.9]. Thus, [by considering fibers over $\underline{C}_{\underline{v}}$ ] we obtain labels $\in\left|\mathbb{F}_{l}\right|$ of various collections of cusps of $\underline{X}_{\underline{v}}, \underline{\underline{X}}_{\underline{v}}$. Write

$$
\mu_{-} \in \underline{X}_{\underline{v}}\left(K_{\underline{v}}\right)
$$

for the unique torsion point of order 2 whose closure in any stable model of $\underline{X}_{\underline{v}}$ over $\mathcal{O}_{K_{\underline{v}}}$ intersects the same irreducible component of the special fiber of the stable model as the [unique] cusp labeled $0 \in\left|\mathbb{F}_{l}\right|$. Now observe that it makes sense to speak of the points $\in \underline{X}_{v}\left(K_{\underline{v}}\right)$ obtained as $\mu_{-}$-translates of the cusps, relative to the group scheme structure of the elliptic curve determined by $\underline{X}_{\underline{v}}$ [i.e., whose origin is given by the cusp labeled $\left.0 \in\left|\mathbb{F}_{l}\right|\right]$. We shall refer to these $\bar{\mu}_{-}$-translates of the cusps with labels $\in\left|\mathbb{F}_{l}\right|$ as the evaluation points of $\underline{X}_{\underline{v}}$. Note that the value of the theta function " $\underline{\underline{\Theta}}_{\underline{v}}$ " of Example 3.2, (ii), at a point lying over an evaluation point arising from a cusp with label $j \in\left|\mathbb{F}_{l}\right|$ is contained in the $\boldsymbol{\mu}_{2 l}$-orbit of

$$
\left\{\underline{\underline{q}} \underline{\underline{v}} \stackrel{j}{=}^{2}\right\} \underset{\underline{j}}{=} \equiv j
$$

[cf. Example 3.2, (iv); [EtTh], Proposition 1.4, (ii)] — where $\underline{\underline{j}}$ ranges over the elements of $\mathbb{Z}$ that map to $j \in\left|\mathbb{F}_{l}\right|$. In particular, it follows immediately from the definition of the covering $\underline{\underline{X}}_{\underline{v}} \rightarrow \underline{X}_{\underline{v}}$ [i.e., by considering l-th roots of the theta function! - cf. [EtTh], Definition 2.5, (i)] that the points of $\underline{\underline{X}}_{\underline{v}}$ that lie over evaluation points of $\underline{X}_{\underline{v}}$ are all defined over $K_{\underline{v}}$. We shall refer to the points $\in \underline{\underline{X}}_{\underline{v}}\left(K_{\underline{v}}\right)$ that lie over the evaluation points of $\underline{X}_{\underline{v}}$ as the evaluation points of $\underline{\underline{X}}_{\underline{v}}$ and to the various sections

$$
G_{\underline{v}} \rightarrow \Pi_{\underline{v}}=\Pi_{\underline{\underline{X}}}^{\mathrm{tp}}
$$

of the natural surjection $\Pi_{\underline{v}} \rightarrow G_{\underline{v}}$ that arise from the evaluation points as the evaluation sections of $\Pi_{\underline{v}}^{-} \rightarrow G_{\underline{v}}^{-}$. Thus, each evaluation section has an associated label $\in\left|\mathbb{F}_{l}\right|$. Note that there is a group-theoretic algorithm for constructing the evaluation sections from [isomorphs of] the topological group $\Pi_{\underline{v}}$. Indeed, this follows immediately from [the proofs of] [EtTh], Corollary 2.9 [concerning the grouptheoreticity of the labels]; [EtTh], Proposition 2.4 [concerning the group-theoreticity of $\Pi_{\underline{C}_{\underline{v}}}, \Pi_{\underline{X}_{\underline{v}}}$; ;SemiAnbd], Corollary 3.11 [concerning the dual semi-graphs of the special fibers of stable models], applied to $\Delta_{\underline{\underline{X}}_{\underline{v}}}^{\mathrm{tp}} \subseteq \Pi_{\underline{\underline{X}}_{\underline{v}}}^{\mathrm{tp}}=\Pi_{\underline{v}}$; [SemiAnbd], Theorem 6.8, (iii) [concerning the group-theoreticity of the decomposition groups of $\mu_{-}$-translates of the cusps].

(ii) We continue to suppose that $\underline{v} \in \underline{\mathbb{V}}^{\text {bad }}$. Let

$$
\mathfrak{D}_{>}=\left\{\mathcal{D}_{>, \underline{w}}\right\}_{\underline{w} \in \underline{\mathbb{V}}}
$$

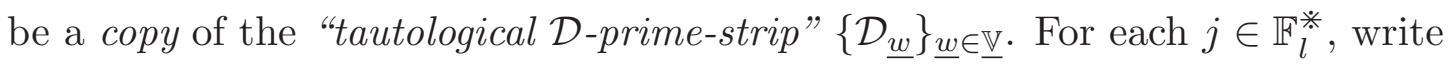

$$
\phi_{\underline{v}_{j}}^{\Theta}: \mathcal{D}_{\underline{v}_{j}} \rightarrow \mathcal{D}_{>, \underline{v}}
$$


for the poly-morphism given by the collection of morphisms [cf. §0] obtained by composing with arbitrary isomorphisms $\mathcal{D}_{\underline{v}_{j}} \stackrel{\sim}{\rightarrow} \mathcal{B}^{\operatorname{temp}}\left(\Pi_{\underline{v}}\right)^{0}, \mathcal{B}^{\operatorname{temp}}\left(\Pi_{\underline{v}}\right)^{0} \stackrel{\sim}{\rightarrow} \mathcal{D}_{>, \underline{v}}$ the various morphisms $\mathcal{B}^{\text {temp }}\left(\Pi_{\underline{v}}\right)^{0} \rightarrow \mathcal{B}^{\text {temp }}\left(\Pi_{\underline{v}}\right)^{0}$ that arise [i.e., via composition with the natural surjection $\Pi_{\underline{v}} \rightarrow G_{\underline{v}}$ ] from the evaluation sections labeled $j$. Now if $\mathcal{C}$ is any isomorph of $\mathcal{B}^{\text {temp }}\left(\Pi_{\underline{v}}\right)^{0}$, then let us write

$$
\pi_{1}^{\text {geo }}(\mathcal{C}) \subseteq \pi_{1}(\mathcal{C})
$$

for the subgroup corresponding to $\Delta_{\underline{\underline{X}}_{v}}^{\mathrm{tp}} \subseteq \Pi_{\underline{\underline{X}}_{v}}^{\mathrm{tp}}=\Pi_{\underline{v}}$, a subgroup which we recall may be reconstructed group-theoretically [cf., e.g., [AbsTopI], Theorem 2.6, (v); [AbsTopI], Proposition 4.10, (i)]. Then we observe that for each constituent morphism $\mathcal{D}_{\underline{v}_{j}} \rightarrow \mathcal{D}_{>, \underline{v}}$ of the poly-morphism $\phi_{\underline{v}_{j}}^{\Theta}$, the induced homomorphism $\pi_{1}\left(\mathcal{D}_{\underline{v}_{j}}\right) \rightarrow \pi_{1}\left(\mathcal{D}_{>, \underline{v}}\right)$ [well-defined, up to composition with an inner automorphism] is compatible with the respective outer actions [of the domain and codomain of this homomorphism] on $\pi_{1}^{\text {geo }}\left(\mathcal{D}_{\underline{v}_{j}}\right), \pi_{1}^{\text {geo }}\left(\mathcal{D}_{>, \underline{v}}\right)$ for some [not necessarily unique, but determined up to finite ambiguity - cf. [SemiAnbd], Theorem 6.4!] outer isomorphism $\pi_{1}^{\text {geo }}\left(\mathcal{D}_{\underline{v}_{j}}\right) \stackrel{\sim}{\rightarrow} \pi_{1}^{\text {geo }}\left(\mathcal{D}_{>, \underline{v}}\right)$. We shall refer to this fact by saying that " $\phi_{\underline{v}_{j}}^{\Theta}$ is compatible with the outer actions on the respective geometric [tempered] fundamental groups".

(iii) Let $\underline{v} \in \underline{\mathbb{V}}^{\text {good }}$. For each $j \in \mathbb{F}_{l}^{*}$, write

$$
\phi_{\underline{v}_{j}}^{\Theta}: \mathcal{D}_{\underline{v}_{j}} \stackrel{\sim}{\rightarrow} \mathcal{D}_{>, \underline{v}}
$$

for the full poly-isomorphism [cf. §0].

(iv) For each $j \in \mathbb{F}_{l}^{*}$, write

$$
\phi_{j}^{\Theta}: \mathfrak{D}_{j} \rightarrow \mathfrak{D}_{>}
$$

for the poly-morphism determined by the collection $\left\{\phi_{\underline{v}_{j}}^{\Theta}: \mathcal{D}_{\underline{v}_{j}} \rightarrow \mathcal{D}_{>, \underline{v}}\right\}_{\underline{v} \in \underline{\mathbb{V}}}$ and

$$
\phi_{*}^{\Theta}: \mathfrak{D}_{*} \rightarrow \mathfrak{D}_{>}
$$

for the poly-morphism $\left\{\phi_{j}^{\Theta}\right\}_{j \in \mathbb{F}_{l}^{*}}$. Thus, whereas the capsule $\mathfrak{D}_{*}$ admits a natural permutation poly-action by $\mathbb{F}_{l}^{*}$, the "labels" — i.e., in effect, elements of $\operatorname{LabCusp}\left(\mathfrak{D}_{>}\right)$[cf. Proposition 4.2] — determined by the various collections of evaluation sections corresponding to a given $j \in \mathbb{F}_{l}^{*}$ are held fixed by arbitrary automorphisms of $\mathfrak{D}_{>}$[cf. Proposition 4.2].

\section{Example 4.5. Transport of Label Classes of Cusps via Model Base-}

Bridges. We continue to use the notation of Examples 4.3, 4.4.

(i) Let $j \in \mathbb{F}_{l}^{*}, \underline{v} \in \underline{\mathbb{V}}$. Recall from Example 4.3, (iv), that the data of the arrow $\phi_{j}^{\mathrm{NF}}: \mathfrak{D}_{j} \rightarrow \mathcal{D}^{\odot}$ at $\underline{v}$ consists of an arrow $\phi_{\underline{v}_{j}}^{\mathrm{NF}}: \mathcal{D}_{\underline{v}_{j}} \rightarrow \mathcal{D}^{\odot}$. If $\underline{v} \in \underline{\mathbb{V}}^{\text {non }}$, then $\phi_{\underline{v}_{j}}^{\mathrm{NF}}$ induces various outer homomorphisms $\pi_{1}\left(\mathcal{D}_{\underline{v}_{j}}\right) \rightarrow \pi_{1}\left(\mathcal{D}^{\odot}\right)$; thus, 
by considering cuspidal inertia groups of $\pi_{1}\left(\mathcal{D}^{\odot}\right)$ whose unique index $l$ subgroup is contained in the image of this homomorphism [cf. Corollary 2.5 when $\underline{v} \in \underline{\mathbb{V}}^{\text {bad }}$; the discussion of Remark 4.5.1 below],

we conclude that these homomorphisms induce a natural isomorphism of $\mathbb{F}_{l}^{*}$-torsors $\operatorname{LabCusp}\left(\mathcal{D}^{\odot}\right) \stackrel{\sim}{\rightarrow} \operatorname{LabCusp}\left(\mathcal{D}_{\underline{v}_{j}}\right)$. In a similar vein, if $\underline{v} \in \underline{\mathbb{V}}^{\text {arc }}$, then it follows from Definition 4.1, (v), that $\phi_{\underline{v}_{j}}^{\mathrm{NF}}$ consists of certain morphisms of Aut-holomorphic orbispaces which induce various outer homomorphisms $\pi_{1}\left(\mathcal{D}_{\underline{v}_{j}}\right) \rightarrow \pi_{1}\left(\mathcal{D}^{\odot}\right)$ from the [discrete] topological fundamental group $\pi_{1}\left(\mathcal{D}_{\underline{v}_{j}}\right)$ to the profinite group $\pi_{1}\left(\mathcal{D}^{\odot}\right)$; thus,

by considering the closures in $\pi_{1}\left(\mathcal{D}^{\odot}\right)$ of the images of cuspidal inertia groups of $\pi_{1}\left(\mathcal{D}_{\underline{v}_{j}}\right)$ [cf. the discussion of Remark 4.5.1 below],

we conclude that these homomorphisms induce a natural isomorphism of $\mathbb{F}_{l}^{*}$-torsors $\operatorname{LabCusp}\left(\mathcal{D}^{\odot}\right) \stackrel{\sim}{\rightarrow} \operatorname{LabCusp}\left(\mathcal{D}_{\underline{v}_{j}}\right)$. Now let us observe that it follows immediately from the definitions that, as one allows $\underline{v}$ to vary, these isomorphisms of $\mathbb{F}_{l}^{*}$-torsors $\operatorname{LabCusp}\left(\mathcal{D}^{\odot}\right) \stackrel{\sim}{\rightarrow} \operatorname{LabCusp}\left(\mathcal{D}_{\underline{v}_{j}}\right)$ are compatible with the natural bijections in the first display of Proposition 4.2, hence determine an isomorphism of $\mathbb{F}_{l}^{*}$-torsors $\operatorname{LabCusp}\left(\mathcal{D}^{\odot}\right) \stackrel{\sim}{\rightarrow} \operatorname{LabCusp}\left(\mathfrak{D}_{j}\right)$. Next, let us note that the data of the arrow $\phi_{j}^{\Theta}: \mathfrak{D}_{j} \rightarrow \mathfrak{D}_{>}$at the various $\underline{v} \in \underline{\mathbb{V}}$ determines an isomorphism of $\mathbb{F}_{l}^{*}$-torsors $\operatorname{LabCusp}\left(\mathfrak{D}_{j}\right) \stackrel{\sim}{\rightarrow} \operatorname{LabCusp}\left(\mathfrak{D}_{>}\right)$[which may be composed with the previous isomorphism of $\mathbb{F}_{l}^{*}$-torsors $\left.\operatorname{LabCusp}\left(\mathcal{D}^{\odot}\right) \stackrel{\sim}{\rightarrow} \operatorname{LabCusp}\left(\mathfrak{D}_{j}\right)\right]$. Indeed, this is immediate from the definitions when $\underline{v} \in \underline{\mathbb{V}}^{\text {good }}$; when $\underline{v} \in \underline{\mathbb{V}}^{\text {bad }}$, it follows immediately from the discussion of Example 4.4, (ii).

(ii) The discussion of (i) may be summarized as follows:

for each $j \in \mathbb{F}_{i}^{*}$, restriction at the various $\underline{v} \in \underline{\mathbb{V}}$ via $\phi_{j}^{\mathrm{NF}}, \phi_{j}^{\Theta}$ determines an isomorphism of $\mathbb{F}_{l}^{*}$-torsors

$$
\phi_{j}^{\mathrm{LC}}: \operatorname{LabCusp}\left(\mathcal{D}^{\odot}\right) \stackrel{\sim}{\rightarrow} \operatorname{LabCusp}\left(\mathfrak{D}_{>}\right)
$$

such that $\phi_{j}^{\mathrm{LC}}$ is obtained from $\phi_{1}^{\mathrm{LC}}$ by composing with the action by $j \in \mathbb{F}_{l}^{*}$.

Write $[\underline{\epsilon}] \in \operatorname{LabCusp}\left(\mathcal{D}^{\odot}\right)$ for the element determined by $\underline{\epsilon}$. Then we observe that

$$
\phi_{j}^{\mathrm{LC}}([\underline{\epsilon}]) \mapsto j ; \quad \phi_{1}^{\mathrm{LC}}(j \cdot[\underline{\epsilon}]) \mapsto j
$$

via the natural bijection LabCusp $\left(\mathfrak{D}_{>}\right) \stackrel{\sim}{\rightarrow} \mathbb{F}_{l}^{*}$ of Proposition 4.2. In particular, the element $[\underline{\epsilon}] \in \operatorname{LabCusp}\left(\mathcal{D}^{\odot}\right)$ may be characterized as the unique element $\eta \in$ $\operatorname{LabCusp}\left(\mathcal{D}^{\odot}\right)$ such that evaluation at $\eta$ yields the assignment $\phi_{j}^{\mathrm{LC}} \mapsto j$.

\section{Remark 4.5.1.}

(i) Let $G$ be a group. If $H \subseteq G$ is a subgroup, $g \in G$, then we shall write $H^{g} \stackrel{\text { def }}{=} g \cdot H \cdot g^{-1}$. Let $J \subseteq H \subseteq G$ be subgroups. Suppose further that each of the 
subgroups $J, H$ of $G$ is only known up to conjugacy in $G$. Put another way, we suppose that we are in a situation in which there are independent $G$-conjugacy indeterminacies in the specification of the subgroups $J$ and $H$. Thus, for instance, there is no natural way to distinguish the given inclusion $\iota: J \hookrightarrow H$ from its $\gamma$ conjugate $\iota^{\gamma}: J^{\gamma} \hookrightarrow H^{\gamma}$, for $\gamma \in G$. Moreover, it may happen to be the case that for some $g \in G$, not only $J$, but also $J^{g} \subseteq H$ [or, equivalently $J \subseteq H^{g^{-1}}$ ]. Here, the subgroups $J, J^{g}$ of $H$ are not necessarily conjugate in $H$; indeed, the abstract pairs of a group and a subgroup given by $(H, J)$ and $\left(H, J^{g}\right)$ need not be isomorphic [i.e., it is not even necessarily the case that there exists an automorphism of $H$ that maps $J$ onto $J^{g}$. In particular, the existence of the independent $G$-conjugacy indeterminacies in the specification of $J$ and $H$ means that one cannot specify the inclusion $\iota: J \hookrightarrow H$ independently of the inclusion $\zeta: J \hookrightarrow H^{g^{-1}}$ [i.e., arising from $\left.J^{g} \subseteq H\right]$. One way to express this state of affairs is as follows. Write " $\stackrel{\text { out }}{\hookrightarrow}$ " for the outer homomorphism determined by an injective homomorphism between groups. Then the collection of factorizations $J \stackrel{\text { out }}{\hookrightarrow} H \stackrel{\text { out }}{\hookrightarrow} G$ of the natural "outer" inclusion $J \stackrel{\text { out }}{\hookrightarrow} G$ through some $G$-conjugate of $H$ - i.e., put another way,

the collection of outer homomorphisms

$$
J \stackrel{\text { out }}{\longrightarrow} H
$$

that are compatible with the "structure morphisms" $J \stackrel{\text { out }}{\hookrightarrow} G$, $H \stackrel{\text { out }}{\hookrightarrow} G$ determined by the natural inclusions

- is well-defined, in a fashion that is compatible with independent $G$-conjugacy indeterminacies in the specification of $J$ and $H$. That is to say, this collection of outer homomorphisms amounts to the collection of inclusions $J^{g_{1}} \hookrightarrow H^{g_{2}}$, for $g_{1}, g_{2} \in G$. By contrast, to specify the inclusion $\iota: J \hookrightarrow H$ [together with, say, its $G$-conjugates $\left\{\iota^{\gamma}\right\}_{\gamma \in G}$ ] independently of the inclusion $\zeta: J \hookrightarrow H^{g^{-1}}$ [and its $G$ conjugates $\left\{\zeta^{\gamma}\right\}_{\gamma \in G}$ ] amounts to the imposition of a partial synchronization i.e., a partial deactivation - of the [a priori!] independent G-conjugacy indeterminacies in the specification of $J$ and $H$. Moreover, such a "partial deactivation" can only be effected at the cost of introducing certain arbitrary choices into the construction under consideration.

(ii) Relative to the factorizations considered in (i), we make the following observation. Given a $G$-conjugate $H^{*}$ of $H$ and a subgroup $I \subseteq H^{*}$, the condition on $I$ that

$$
(* \subseteq) I \text { be a G-conjugate of } J
$$

is a condition that is independent of the datum $H^{*}$, while the condition on $I$ that

$$
\left(*^{\cong}\right) I \text { be a } G \text {-conjugate of } J \text { such that }\left(H^{*}, I\right) \cong(H, J)
$$

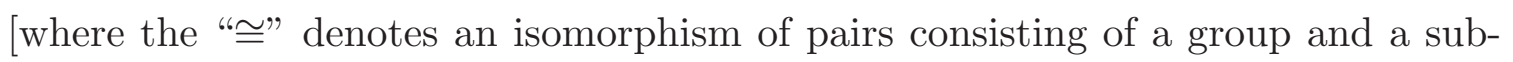
group - cf. the discussion of (i)] is a condition that depends, in an essential fashion, on the datum $H^{*}$. Here, $(* \subseteq)$ is precisely the condition that one must impose when one considers arbitrary factorizations as in $(\mathrm{i})$, while $\left(*^{\simeq}\right)$ is the condition that one must impose when one wishes to restrict one's attention to factorizations whose 
first arrow gives rise to a pair isomorphic to the pair determined by $\iota$. That is to say, the dependence of $\left({ }^{*} \cong\right)$ on the datum $H^{*}$ may be regarded as an explicit formulation of the necessity for the "imposition of a partial synchronization" as discussed in (i), while the corresponding independence, exhibited by $(* \subseteq)$, of the datum $H^{*}$ may be regarded as an explicit formulation of the lack of such a necessity when one considers arbitrary factorizations as in (i). Finally, we note that by reversing the direction of the inclusion " $\subseteq$ ", one may consider a subgroup $I \subseteq G$ that contains a given $G$-conjugate $J^{*}$ of $J$, i.e., $I \supseteq J^{*}$; then analogous observations may be made concerning the condition $(* \supseteq)$ on $I$ that $I$ be a $G$-conjugate of $H$.

(iii) The abstract situation described in (i) occurs in the discussion of Example 4.5, (i), at $\underline{v} \in \underline{\mathbb{V}}^{\text {bad }}$. That is to say, the group "G" (respectively, " $H$ "; " $\left.J "\right)$ of (i) corresponds to the group $\pi_{1}\left(\mathcal{D}^{\odot}\right)$ (respectively, the image of $\pi_{1}\left(\mathcal{D}_{\underline{v}_{j}}\right)$ in $\pi_{1}\left(\mathcal{D}^{\odot}\right)$; the unique index $l$ open subgroup of a cuspidal inertia group of $\left.\pi_{1}\left(\mathcal{D}^{\odot}\right)\right)$ of Example 4.5, (i). Here, we recall that the homomorphism $\pi_{1}\left(\mathcal{D}_{\underline{v}_{j}}\right) \rightarrow \pi_{1}\left(\mathcal{D}^{\odot}\right)$ is only known up to composition with an inner automorphism - i.e., up to $\pi_{1}\left(\mathcal{D}^{\odot}\right)$-conjugacy; a cuspidal inertia group of $\pi_{1}\left(\mathcal{D}^{\odot}\right)$ is also only determined by an element $\in \operatorname{LabCusp}\left(\mathcal{D}^{\odot}\right)$ up to $\pi_{1}\left(\mathcal{D}^{\odot}\right)$-conjugacy. Moreover, it is immediate from the construction of the "model D-NF-bridges" of Example 4.3 [cf. also Definition 4.6, (i), below] that there is no natural way to synchronize these indeterminacies. Indeed, from the point of view of the discussion of Remark 4.3.1, (ii), by considering the actions of the absolute Galois groups of the local and global base fields involved on the cuspidal inertia groups that appear, one sees that such a synchronization would amount, roughly speaking, to a Galois-equivariant splitting [i.e., relative to the global absolute Galois groups that appear] of the "prime decomposition trees" of Remark 4.3.1, (ii) — which is absurd [cf. [IUTchII], Remark 2.5.2, (iii), for a more detailed discussion of this sort of phenomenon]. This phenomenon of the "non-synchronizability" of indeterminacies arising from local and global absolute Galois groups is reminiscent of the discussion of [EtTh], Remark 2.16.2. On the other hand, by Corollary 2.5 , one concludes in the present situation the highly nontrivial fact that

a factorization " $J \hookrightarrow H \hookrightarrow G$ " is uniquely determined by the composite $J \hookrightarrow G$, i.e., by the $G$-conjugate of $J$ that one starts with, without resorting to any a priori "synchronization of indeterminacies".

(iv) A similar situation to the situation of (iii) occurs in the discussion of Example 4.5, (i), at $\underline{v} \in \underline{\mathbb{V}}^{\text {arc }}$. That is to say, in this case, the group "G" (respectively, " $H$ "; " $J ")$ of (i) corresponds to the group $\pi_{1}\left(\mathcal{D}^{\odot}\right)$ (respectively, the image of $\pi_{1}\left(\mathcal{D}_{\underline{v}_{j}}\right)$ in $\pi_{1}\left(\mathcal{D}^{\odot}\right)$; a cuspidal inertia group of $\left.\pi_{1}\left(\mathcal{D}_{\underline{v}_{j}}\right)\right)$ of Example 4.5, (i). In this case, although it does not hold that a factorization " $J \hookrightarrow H \hookrightarrow G$ " is uniquely determined by the composite $J \hookrightarrow G$, i.e., by the $G$-conjugate of $J$ that one starts with [cf. Remark 2.6.1], it does nevertheless hold, by Corollary 2.8, that the $H$-conjugacy class of the image of $J$ via the arrow $J \hookrightarrow H$ that occurs in such a factorization is uniquely determined.

(v) The property observed at $\underline{v} \in \underline{\mathbb{V}}^{\text {arc }}$ in (iv) is somewhat weaker than the rather strong property observed at $\underline{v} \in \underline{\mathbb{V}}^{\text {bad }}$ in (iii). In the present series of papers, however, we shall only be concerned with such subtle factorization properties at $\underline{v} \in \underline{\mathbb{V}}^{\text {bad }}$, where we wish to develop, in [IUTchII], the theory of "Hodge- 
Arakelov-theoretic evaluation" by restricting certain cohomology classes via an arrow " $J \hookrightarrow H$ " appearing in a factorization " $J \hookrightarrow H \hookrightarrow G$ " of the sort discussed in (i). In fact, in the context of the theory of Hodge-Arakelov-theoretic evaluation that will be developed in [IUTchII], a slightly modified version of the phenomenon discussed in (iii) — which involves the "additive" version to be developed in $\S 6$ of the "multiplicative" theory developed in the present $\S 4$ - will be of central importance.

\section{Definition 4.6.}

(i) We define a base-NF-bridge, or $\mathcal{D}$-NF-bridge, [relative to the given initial $\Theta$-data] to be a poly-morphism

$$
\dagger \mathfrak{D}_{J} \stackrel{{ }^{\dagger} \phi_{*}^{\mathrm{NF}}}{\longrightarrow} \dagger^{\dagger} \mathcal{D}^{\odot}
$$

- where ${ }^{\dagger} \mathcal{D}^{\odot}$ is a category equivalent to $\mathcal{D}^{\odot} ;{ }^{\dagger} \mathfrak{D}_{J}=\left\{{ }^{\dagger} \mathfrak{D}_{j}\right\}_{j \in J}$ is a capsule of $\mathcal{D}$ prime-strips, indexed by a finite index set $J$ - such that there exist isomorphisms $\mathcal{D}^{\odot} \stackrel{\sim}{\rightarrow} \dagger \mathcal{D}^{\odot}, \mathfrak{D}_{*} \stackrel{\sim}{\rightarrow} \dagger \mathfrak{D}_{J}$, conjugation by which maps $\phi_{*}^{\mathrm{NF}} \mapsto^{\dagger} \phi_{*}^{\mathrm{NF}}$. We define a $(\mathrm{n})$ [iso]morphism of $\mathcal{D}$-NF-bridges

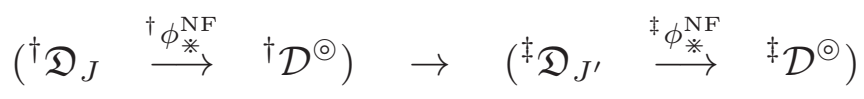

to be a pair of poly-morphisms

$$
\dagger \mathfrak{D}_{J} \stackrel{\sim}{\rightarrow} \ddagger \mathfrak{D}_{J^{\prime}} ; \quad{ }^{\dagger} \mathcal{D}^{\odot} \stackrel{\sim}{\rightarrow}{ }^{\ddagger} \mathcal{D}^{\odot}
$$

— where ${ }^{\dagger} \mathfrak{D}_{J} \stackrel{\sim}{\rightarrow} \ddagger \mathfrak{D}_{J^{\prime}}$ is a capsule-full poly-isomorphism $[\mathrm{cf} . \S 0] ;{ }^{\dagger} \mathcal{D}^{\odot} \rightarrow{ }^{\ddagger} \mathcal{D}^{\odot}$ is a poly-morphism which is an $\operatorname{Aut}_{\underline{\epsilon}}\left({ }^{\dagger} \mathcal{D}^{\odot}\right)$ - [or, equivalently, $\operatorname{Aut}_{\underline{\epsilon}}\left({ }^{\ddagger} \mathcal{D}^{\odot}\right)$-] orbit $[\mathrm{cf}$. the discussion of Example 4.3, (i)] of isomorphisms - which are compatible with ${ }^{\dagger} \phi_{*}^{\mathrm{NF}}$, $\ddagger \phi_{*}^{\mathrm{NF}}$. There is an evident notion of composition of morphisms of $\mathcal{D}$-NF-bridges.

(ii) We define a base- $\Theta$-bridge, or $\mathcal{D}-\Theta$-bridge, [relative to the given initial $\Theta$-data] to be a poly-morphism

$$
\dagger \mathfrak{D}_{J} \stackrel{{ }^{\dagger} \phi_{\text {娄 }}^{\longrightarrow}}{\dagger} \mathfrak{D}_{>}
$$

— where ${ }^{\dagger} \mathfrak{D}_{>}$is a $\mathcal{D}$-prime-strip; ${ }^{\dagger} \mathfrak{D}_{J}=\left\{{ }^{\dagger} \mathfrak{D}_{j}\right\}_{j \in J}$ is a capsule of $\mathcal{D}$-prime-strips, indexed by a finite index set $J$ - such that there exist isomorphisms $\mathfrak{D}_{>} \stackrel{\sim}{\rightarrow}{ }^{\dagger} \mathfrak{D}_{>}$, $\mathfrak{D}_{*} \stackrel{\sim}{\rightarrow} \dagger \mathfrak{D}_{J}$, conjugation by which maps $\phi_{*}^{\Theta} \mapsto^{\dagger} \phi_{*}^{\Theta}$. We define a(n) [iso]morphism of $\mathcal{D}$ - $\Theta$-bridges

$$
\left(\mathfrak{D}_{J} \stackrel{{ }^{\dagger} \phi_{\text {菓 }}^{\longrightarrow}}{\dagger} \mathfrak{D}_{>}\right) \rightarrow\left({ }^{\ddagger} \mathfrak{D}_{J^{\prime}} \stackrel{{ }^{\ddagger} \phi_{\text {* }}^{\ominus}}{\longrightarrow}{ }^{\ddagger} \mathfrak{D}_{>}\right)
$$

to be a pair of poly-morphisms

$$
\dagger \mathfrak{D}_{J} \stackrel{\sim}{\rightarrow} \ddagger \mathfrak{D}_{J^{\prime}} ; \quad \dagger \mathfrak{D}_{>} \stackrel{\sim}{\rightarrow} \ddagger \mathfrak{D}_{>}
$$

— where ${ }^{\dagger} \mathfrak{D}_{J} \stackrel{\sim}{\rightarrow} \ddagger \mathfrak{D}_{J^{\prime}}$ is a capsule-full poly-isomorphism; ${ }^{\dagger} \mathfrak{D}_{>} \stackrel{\sim}{\rightarrow} \ddagger \mathfrak{D}>$ is the full poly-isomorphism — which are compatible with ${ }^{\dagger} \phi_{*}^{\Theta},{ }^{\ddagger} \phi_{*}^{\Theta}$. There is an evident notion of composition of morphisms of $\mathcal{D}$ - $\Theta$-bridges. 


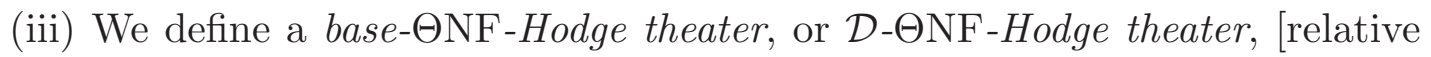
to the given initial $\Theta$-data] to be a collection of data

$$
{ }^{\dagger} \mathcal{H} \mathcal{T}^{\mathcal{D}-\Theta N F}=\left({ }^{\dagger} \mathcal{D}^{\odot} \stackrel{{ }^{\dagger} \phi_{*}^{\mathrm{NF}}}{\longleftarrow} \quad \dagger \mathfrak{D}_{J} \stackrel{{ }^{\dagger} \phi_{\text {* }}^{\ominus}}{\longrightarrow}{ }^{\dagger} \mathfrak{D}_{>}\right)
$$

— where ${ }^{\dagger} \phi_{*}^{\mathrm{NF}}$ is a $\mathcal{D}$-NF-bridge; ${ }^{\dagger} \phi_{*}^{\Theta}$ is a $\mathcal{D}$ - $\Theta$-bridge — such that there exist isomorphisms

$$
\mathcal{D}^{\odot} \stackrel{\sim}{\rightarrow}{ }^{\dagger} \mathcal{D}^{\odot} ; \quad \mathfrak{D}_{*} \stackrel{\sim}{\rightarrow}{ }^{\dagger} \mathfrak{D}_{J} ; \quad \mathfrak{D}_{>} \stackrel{\sim}{\rightarrow}{ }^{\dagger} \mathfrak{D}_{>}
$$

conjugation by which maps $\phi_{*}^{\mathrm{NF}} \mapsto^{\dagger} \phi_{*}^{\mathrm{NF}}, \phi_{*}^{\Theta} \mapsto^{\dagger} \phi_{*}^{\Theta}$. $\mathrm{A}(\mathrm{n})$ [iso]morphism of $\mathcal{D}$ $\Theta N F-H o d g e$ theaters is defined to be a pair of morphisms between the respective associated $\mathcal{D}$-NF- and $\mathcal{D}$ - $\Theta$-bridges that are compatible with one another in the sense that they induce the same bijection between the index sets of the respective capsules of $\mathcal{D}$-prime-strips. There is an evident notion of composition of morphisms of $\mathcal{D}$ - $\Theta N F-H o d g e$ theaters.

\section{Proposition 4.7. (Transport of Label Classes of Cusps via Base-} Bridges) Let

$$
{ }^{\dagger} \mathcal{H} \mathcal{T}^{\mathcal{D}-\Theta \mathrm{NF}}=\left({ }^{\dagger} \mathcal{D}^{\odot} \stackrel{{ }^{\dagger} \phi_{*}^{\mathrm{NF}}}{\longleftarrow}{ }^{\dagger} \mathfrak{D}_{J} \stackrel{{ }^{\dagger} \phi_{*}^{\Theta}}{\longrightarrow}{ }^{\dagger} \mathfrak{D}_{>}\right)
$$

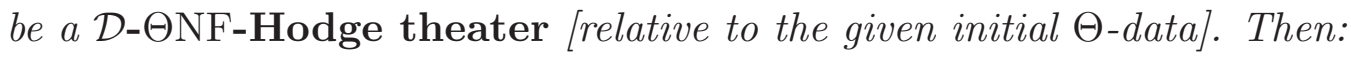

(i) The structure at the various $\underline{v} \in \underline{\mathbb{V}}^{\text {bad }}$ of the $\mathcal{D}$ - $\Theta$-bridge ${ }^{\dagger} \phi_{*}^{\Theta}[$ i.e., involving evaluation sections - cf. Example 4.4, (i), (ii); Definition 4.6, (ii)] determines a bijection

$$
{ }^{\dagger} \chi: \pi_{0}\left({ }^{\dagger} \mathfrak{D}_{J}\right)=J \stackrel{\sim}{\rightarrow} \mathbb{F}_{l}^{*}
$$

- i.e., determines labels $\in \mathbb{F}_{l}^{*}$ for the constituent $\mathcal{D}$-prime-strips of the capsule $\dagger \mathfrak{D}_{J}$.

(ii) For each $j \in J$, restriction at the various $\underline{v} \in \underline{\mathbb{V}}$ [cf. Example 4.5] via the portion of ${ }^{\dagger} \phi_{*}^{\mathrm{NF}},{ }^{\dagger} \phi_{*}^{\Theta}$ indexed by $j$ determines an isomorphism of $\mathbb{F}_{l}^{*}$-torsors

$$
{ }^{\dagger} \phi_{j}^{\mathrm{LC}}: \operatorname{LabCusp}\left({ }^{\dagger} \mathcal{D}^{\odot}\right) \stackrel{\sim}{\rightarrow} \operatorname{LabCusp}\left({ }^{\dagger} \mathfrak{D}_{>}\right)
$$

such that ${ }^{\dagger} \phi_{j}^{\mathrm{LC}}$ is obtained from ${ }^{\dagger} \phi_{1}^{\mathrm{LC}}$ [where, by abuse of notation, we write " $1 \in J$ " for the element of $J$ that maps via ${ }^{\dagger} \chi$ to the image of 1 in $\left.\mathbb{F}_{l}^{*}\right]$ by composing with the action by ${ }^{\dagger} \chi(j) \in \mathbb{F}_{l}^{*}$.

(iii) There exists a unique element

$$
\left[{ }^{\dagger} \underline{\epsilon}\right] \in \operatorname{LabCusp}\left({ }^{\dagger} \mathcal{D}^{\odot}\right)
$$

such that for each $j \in J$, the natural bijection LabCusp $\left({ }^{\dagger} \mathfrak{D}_{>}\right) \stackrel{\sim}{\rightarrow} \mathbb{F}_{l}^{*}$ of the second display of Proposition 4.2 maps ${ }^{\dagger} \phi_{j}^{\mathrm{LC}}\left(\left[{ }^{\dagger} \underline{\epsilon}\right]\right)={ }^{\dagger} \phi_{1}^{\mathrm{LC}}\left({ }^{\dagger} \chi(j) \cdot\left[{ }^{\dagger} \underline{\epsilon}\right]\right) \mapsto{ }^{\dagger} \chi(j)$. In particular, the element $\left[{ }^{\dagger} \underline{\epsilon}\right]$ determines an isomorphism of $\mathbb{F}_{l}^{*}$-torsors

$$
{ }^{\dagger} \zeta_{*}: \operatorname{LabCusp}\left({ }^{\dagger} \mathcal{D}^{\odot}\right) \stackrel{\sim}{\rightarrow} J \quad\left(\stackrel{\sim}{\rightarrow} \mathbb{F}_{l}^{*}\right)
$$


[where the bijection in parentheses is the bijection ${ }^{\dagger} \chi$ of (i)] between "global cusps" [i.e., " " $\chi(j) \cdot\left[{ }^{\dagger} \underline{\epsilon}\right]$ "] and capsule indices [i.e., $\left.j \in J \stackrel{\sim}{\rightarrow} \mathbb{F}_{l}^{*}\right]$. Finally, when considered up to composition with multiplication by an element of $\mathbb{F}_{l}^{*}$, the bijection ${ }^{\dagger} \zeta_{*}$ is independent of the choice of ${ }^{\dagger} \phi_{*}^{\mathrm{NF}}$ within the $\mathbb{F}_{l}^{*}$-orbit of ${ }^{\dagger} \phi_{*}^{\mathrm{NF}}$ relative to the natural poly-action of $\mathbb{F}_{l}^{*}$ on ${ }^{\dagger} \mathcal{D}^{\odot}$ [cf. Example 4.3, (iv); Fig. 4.4 below].

Proof. Assertion (i) follows immediately from the definitions [cf. Example 4.4, (i), (ii), (iv); Definition 4.6], together with the bijection of the second display of Proposition 4.2. Assertions (ii) and (iii) follow immediately from the intrinsic nature of the constructions of Example 4.5.

Remark 4.7.1. The significance of the natural bijection ${ }^{\dagger} \zeta_{*}$ of Proposition 4.7, (iii), lies in the following observation: Suppose that one wishes to work with the global data ${ }^{\dagger} \mathcal{D}^{\odot}$ in a fashion that is independent of the local data [i.e., "prime-strip data" ${ }^{\dagger} \mathfrak{D}_{>},{ }^{\dagger} \mathfrak{D}_{J}[\mathrm{cf}$. Remark 4.3.2, (b)]. Then

by replacing the capsule index set $J$ by the set of global label classes of cusps LabCusp $\left({ }^{\dagger} \mathcal{D}^{\odot}\right)$ via ${ }^{\dagger} \zeta_{*}$, one obtains an object - i.e., LabCusp $\left({ }^{\dagger} \mathcal{D}^{\odot}\right)$ - constructed via [i.e., "native to"] the global data that is immune to the "collapsing" of $J \stackrel{\sim}{\rightarrow} \mathbb{F}_{l}^{*}$ - i.e., of $\mathbb{F}_{l}^{*}$-orbits of $\underline{\mathbb{V}}^{ \pm \text {un }}$ - even at primes $\underline{v} \in \underline{\mathbb{V}}$ of the sort discussed in Remark 4.2.1!

That is to say, this "collapsing" of [i.e., failure of $\mathbb{F}_{l}^{*}$ to act freely on] $\mathbb{F}_{l}^{*}$-orbits of $\underline{\mathbb{V}}^{ \pm \text {un }}$ is a characteristically global consequence of the global prime decomposition trees discussed in Remark 4.3.1, (ii) [cf. the example discussed in Remark 4.2.1]. We refer to Remark 4.9.3, (ii), below for a discussion of a closely related phenomenon.

\section{Remark 4.7.2.}

(i) At the level of labels [cf. the content of Proposition 4.7], the structure of a

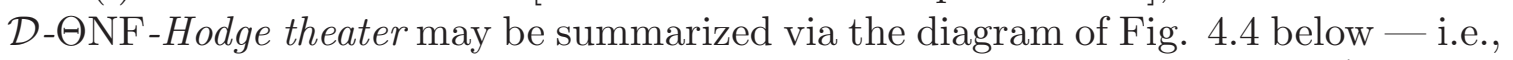
where the expression "[ $\left.1<2<\ldots<\left(l^{*}-1\right)<l^{*}\right]$ " corresponds to ${ }^{\dagger} \mathfrak{D}_{>}$; the expression "( $\left.1 \quad 2 \quad \ldots l l * l^{*}-1 \quad l^{*}\right)$ " corresponds to ${ }^{\dagger} \mathfrak{D}_{J}$; the lower righthand " $\mathbb{F}_{l}^{*}$-cycle of $*$ 's" corresponds to ${ }^{\dagger} \mathcal{D}{ }^{\odot}$; the " $\Uparrow$ " corresponds to the associated $\mathcal{D}$ - $\Theta$-bridge; the " $\Rightarrow$ " corresponds to the associated $\mathcal{D}$-NF-bridge; the "/* "s" denote $\mathcal{D}$-prime-strips.

(ii) Note that the labels arising from ${ }^{\dagger} \mathfrak{D}_{>}$correspond, ultimately, to various irreducible components in the special fiber of a certain tempered covering of a ["geometric"!] Tate curve [a special fiber which consists of a chain of copies of the projective line - cf. [EtTh], Corollary 2.9]. In particular, these labels are obtained by counting - in an intuitive, archimedean, additive fashion - the number of irreducible components between a given irreducible component and the "origin". That is to say, the portion of the diagram of Fig. 4.4 corresponding to ${ }^{\dagger} \mathfrak{D}_{>}$may be described by the following terms:

geometric, additive, archimedean, hence Frobenius-like [cf. Corollary 3.8]. 
By contrast, the various "*'s" in the portion of the diagram of Fig. 4.4 corresponding to ${ }^{\dagger} \mathcal{D}^{\odot}$ arise, ultimately, from various primes of an ["arithmetic"!] number field. These primes are permuted by the multiplicative group $\mathbb{F}_{l}^{*}=\mathbb{F}_{l}^{\times} /\{ \pm 1\}$, in a cyclic - i.e., nonarchimedean - fashion. Thus, the portion of the diagram of Fig. 4.4 corresponding to ${ }^{\dagger} \mathcal{D}^{\odot}$ may be described by the following terms:

arithmetic, multiplicative, nonarchimedean, hence étale-like [cf. the discussion of Remark 4.3.2].

That is to say, the portions of the diagram of Fig. 4.4 corresponding to ${ }^{\dagger} \mathfrak{D}_{>},{ }^{\dagger} \mathcal{D}^{\odot}$ differ quite fundamentally in structure. In particular, it is not surprising that the only "common ground" of these two fundamentally structurally different portions consists of an underlying set of cardinality $l^{*}$ [i.e., the portion of the diagram of Fig. 4.4 corresponding to $\left.{ }^{\dagger} \mathfrak{D}_{J}\right]$.

(iii) The bijection ${ }^{\dagger} \zeta_{*}$ - or, perhaps more appropriately, its inverse

$$
\left({ }^{\dagger} \zeta_{*}\right)^{-1}: J \stackrel{\sim}{\rightarrow} \operatorname{LabCusp}\left({ }^{\dagger} \mathcal{D}^{\odot}\right)
$$

- may be thought of as relating arithmetic [i.e., if one thinks of the elements of the capsule index set $J$ as collections of primes of a number field] to geometry [i.e., if one thinks of the elements of LabCusp $\left({ }^{\dagger} \mathcal{D}^{\odot}\right)$ as corresponding to the [geometric!] cusps of the hyperbolic orbicurve]. From this point of view,

$\left({ }^{\dagger} \zeta_{*}\right)^{-1}$ may be thought of as a sort of "combinatorial Kodaira-Spencer morphism" [cf. the point of view of [HASurI], §1.4].

We refer to Remark 4.9.2, (iv), below, for another way to think about ${ }^{\dagger} \zeta_{*}$.

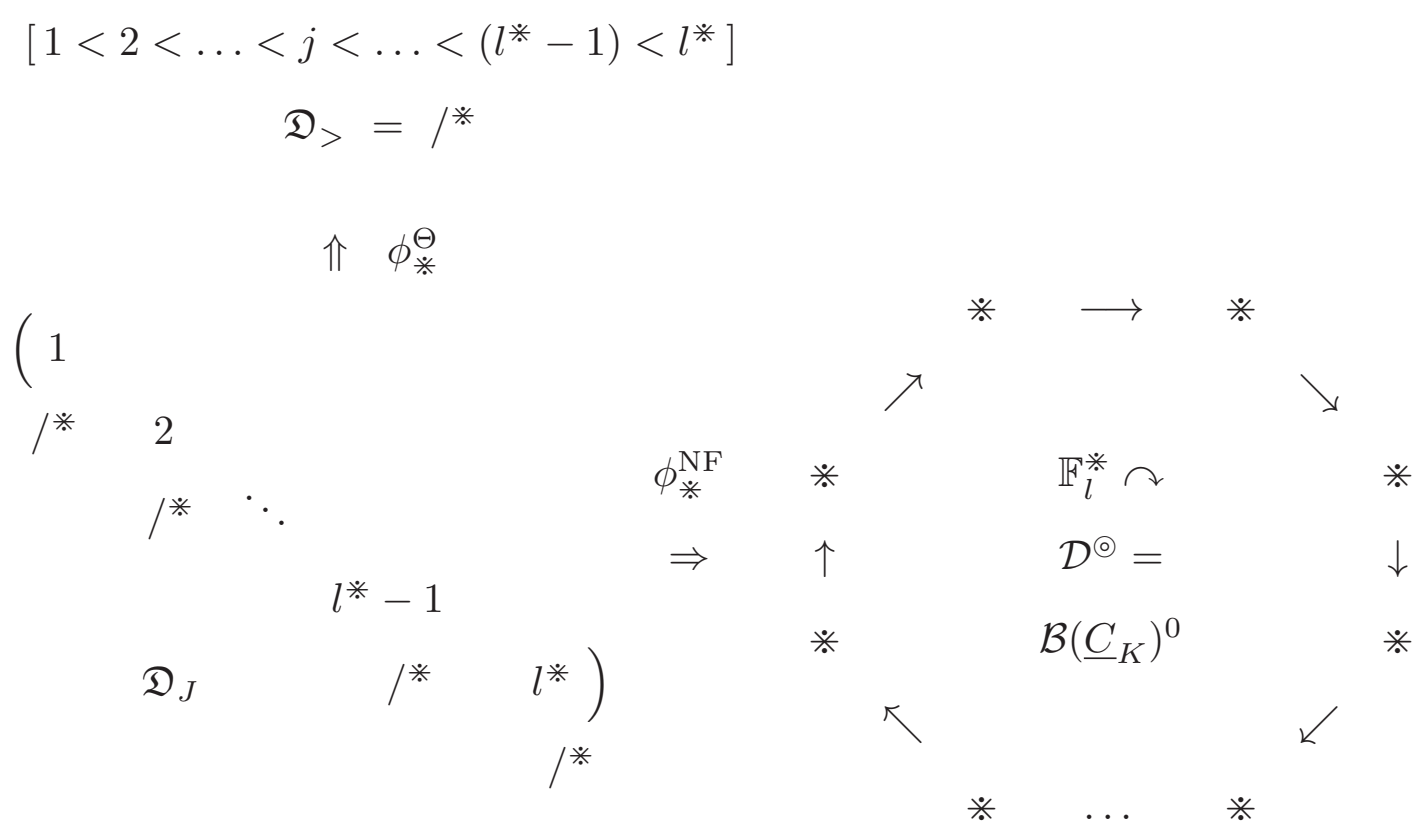

Fig. 4.4: The combinatorial structure of a $\mathcal{D}$ - $\Theta N F-H o d g e$ theater

The following result follows immediately from the definitions. 
Proposition 4.8. (First Properties of Base-NF-Bridges, Base- $\Theta-B r i d g e s$, and Base- $\Theta N F-H o d g e$ Theaters) Relative to a fixed collection of initial $\Theta-$ data:

(i) The set of isomorphisms between two $\mathcal{D}$-NF-bridges forms an $\mathbb{F}_{l}^{*}$ torsor.

(ii) The set of isomorphisms between two $\mathcal{D}$ - $\Theta$-bridges (respectively, two $\mathcal{D}-\Theta N F-H o d g e$ theaters) is of cardinality one.

(iii) Given a $\mathcal{D}$-NF-bridge and a $\mathcal{D}$ - $\Theta$-bridge, the set of capsule-full polyisomorphisms between the respective capsules of $\mathcal{D}$-prime-strips which allow one to

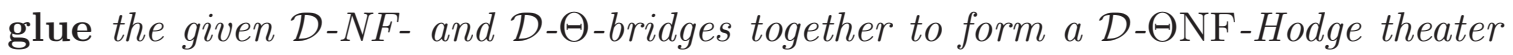
forms an $\mathbb{F}_{l}^{*}$-torsor.

(iv) Given a $\mathcal{D}$-NF-bridge, there exists a relatively simple - cf. the discussion of Examples 4.4, (i), (ii), (iii); 4.5, (i), (ii)] functorial algorithm for constructing, up to an $\mathbb{F}_{l}^{*}$-indeterminacy [cf. (i), (iii)], from the given $\mathcal{D}$-NF-bridge a $\mathcal{D}$ - $\Theta N F-H o d g e$ theater whose underlying $\mathcal{D}$-NF-bridge is the given $\mathcal{D}$-NF-bridge.

Proposition 4.9. (Symmetries arising from Forgetful Functors) Relative to a fixed collection of initial $\Theta$-data:

(i) (Base-NF-Bridges) The operation of associating to a $\mathcal{D}$ - $\Theta N F-H o d g e$ theater the underlying $\mathcal{D}$-NF-bridge of the $\mathcal{D}-\Theta N F-H o d g e$ theater determines a natural functor

$$
\begin{aligned}
& \begin{array}{|c|c|}
\hline \text { category of } \\
\mathcal{D} \text { - } \Theta \text { NF-Hodge theaters } \\
\text { and isomorphisms of } \\
\mathcal{D} \text { - } \Theta \text { NF-Hodge theaters }
\end{array} \rightarrow \quad \begin{array}{c}
\text { category of } \\
\mathcal{D} \text {-NF-bridges } \\
\text { and isomorphisms of } \\
\mathcal{D} \text {-NF-bridges } \\
\hline
\end{array} \\
& \dagger \mathcal{H} \mathcal{T}^{\mathcal{D}-\Theta \mathrm{NF}} \quad \mapsto \quad\left({ }^{\dagger} \mathcal{D}^{\odot} \stackrel{{ }^{\dagger} \phi_{*}^{\mathrm{NF}}}{\longleftarrow} \dagger \mathfrak{D}_{J}\right)
\end{aligned}
$$

whose output data admits an $\mathbb{F}_{l}^{*}$-symmetry which acts simply transitively on the index set [i.e., "J"] of the underlying capsule of $\mathcal{D}$-prime-strips [i.e., "计 $\mathfrak{D}_{J}$ "] of this output data.

(ii) (Holomorphic Capsules) The operation of associating to a $\mathcal{D}$ - $\Theta \mathrm{NF}$ Hodge theater the underlying capsule of $\mathcal{D}$-prime-strips of the $\mathcal{D}$ - $\Theta \mathrm{NF}$-Hodge theater determines a natural functor

\begin{tabular}{|c|c|c|}
\hline $\begin{array}{c}\text { category of } \\
\mathcal{D} \text { - } \Theta \text { NF-Hodge theaters } \\
\text { and isomorphisms of } \\
\mathcal{D} \text { - } \Theta \text { NF-Hodge theaters }\end{array}$ & $\rightarrow \quad \begin{array}{c}\text { category of } l * \text {-capsules } \\
\text { of } \mathcal{D} \text {-prime-strips } \\
\text { and capsule-full poly- } \\
\text { isomorphisms of } l * \text {-capsules }\end{array}$ \\
\hline$\dagger \mathcal{H} \mathcal{T}^{\mathcal{D} \text { - } \Theta \mathrm{NF}}$ & $\mapsto$ & $\dagger \mathfrak{D}_{J}$
\end{tabular}




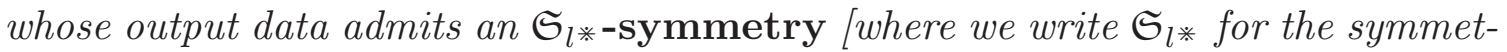
ric group on $l *$ letters] which acts transitively on the index set [i.e., "J"] of this output data. Thus, this functor may be thought of as an operation that consists of forgetting the labels $\in \mathbb{F}_{l}^{*}$ [i.e., forgetting the bijection $J \stackrel{\sim}{\rightarrow} \mathbb{F}_{l}^{*}$ of Proposition 4.7, (i)]. In particular, if one is only given this output data ${ }^{\dagger} \mathfrak{D}_{J}$ up to isomorphism, then there is a total of precisely $l^{*}$ possibilities for the element $\in \mathbb{F}_{l}^{*}$ to which a given index $j \in J$ corresponds [cf. Proposition 4.7, (i)], prior to the application of this functor.

(iii) (Mono-analytic Capsules) By composing the functor of (ii) with the mono-analyticization operation discussed in Definition 4.1, (iv), one obtains a natural functor

\begin{tabular}{|c|c|c|}
\hline $\begin{array}{c}\text { category of } \\
\mathcal{D} \text { - } \Theta \text { NF-Hodge theaters } \\
\text { and isomorphisms of } \\
\mathcal{D} \text { - } \Theta \text { NF-Hodge theaters }\end{array}$ & $\rightarrow$ & $\begin{array}{c}\text { category of } l^{*} \text {-capsules } \\
\text { of } \mathcal{D}^{\vdash} \text {-prime-strips } \\
\text { and capsule-full poly- } \\
\text { isomorphisms of } l^{*} \text {-capsules }\end{array}$ \\
\hline$\dagger \mathcal{H} \mathcal{T}^{\mathcal{D}-\Theta N F}$ & $\mapsto$ & $\dagger \mathfrak{D}_{J}^{\vdash}$ \\
\hline
\end{tabular}

whose output data satisfies the same symmetry properties with respect to labels as the output data of the functor of (ii).

Proof. Assertions (i), (ii), (iii) follow immediately from the definitions [cf. also Proposition 4.8, (i), in the case of assertion (i)].

\section{Remark 4.9.1.}

(i) Ultimately, in the theory of the present series of papers [cf., especially, [IUTchII], §2], we shall be interested in

evaluating the étale theta function of [EtTh] — i.e., in the spirit of the Hodge-Arakelov theory of [HASurI], [HASurII] — at the various $\mathcal{D}$-prime-strips of $\dagger \mathfrak{D}_{J}$, in the fashion stipulated by the labels discussed in Proposition 4.7, (i).

These values of the étale theta function will be used to construct various arithmetic line bundles. We shall be interested in computing the arithmetic degrees - in the form of various "log-volumes" - of these arithmetic line bundles. In order to compute these global log-volumes, it is necessary to be able to compare the logvolumes that arise at $\mathcal{D}$-prime-strips with different labels. It is for this reason that the non-labeled output data of the functors of Proposition 4.9, (i), (ii), (iii) [cf. also Proposition 4.11, (i), (ii), below], are of crucial importance in the theory of the present series of papers. That is to say,

the non-labeled output data of the functors of Proposition 4.9, (i), (ii), (iii) [cf. also Proposition 4.11, (i), (ii), below] — which allow one to consider isomorphisms between the $\mathcal{D}$-prime-strips that were originally 
assigned different labels - make possible the comparison of objects [e.g., log-volumes] constructed relative to different labels.

In Proposition 4.11, (i), (ii), below, we shall see that by considering "processions", one may perform such comparisons in a fashion that minimizes the label indeterminacy that arises.

(ii) Since the $\mathbb{F}_{l}^{*}$-symmetry that appears in Proposition 4.9, (i), is transitive, it follows that one may use this action to perform comparisons as discussed in (i). This prompts the question:

What is the difference between this $\mathbb{F}_{l}^{*}$-symmetry and the $\mathfrak{S}_{l *-\text { symmetry }}$ of the output data of the functors of Proposition 4.9, (ii), (iii)?

In a word, restricting to the $\mathbb{F}_{l}^{*}$-symmetry of Proposition 4.9, (i), amounts to the imposition of a "cyclic structure" on the index set $J$ [i.e., a structure of $\mathbb{F}_{i}^{*}$-torsor on $J$. Thus, relative to the issue of comparability raised in (i), this $\mathbb{F}_{l}^{*}$-symmetry allows comparison between - i.e., involves isomorphisms between the non-labeled $\mathcal{D}$-prime-strips corresponding to - distinct members of this index set $J$, without disturbing the cyclic structure on $J$. This cyclic structure may be thought of as a sort of combinatorial manifestation of the link to the global object ${ }^{\dagger} \mathcal{D}^{\odot}$ that appears in a $\mathcal{D}$-NF-bridge. On the other hand,

in order to compare these $\mathcal{D}$-prime-strips indexed by $J$ "in the absolute" to $\mathcal{D}$-prime-strips that have nothing to do with $J$, it is necessary to "forget the cyclic structure on $J$ ".

This is precisely what is achieved by considering the functors of Proposition 4.9, (ii), (iii), i.e., by working with the "full $\mathfrak{S}_{l *-\text { symmetry". }}$

\section{Remark 4.9.2.}

(i) The various elements of the index set of the capsule of $\mathcal{D}$-prime-strips of a $\mathcal{D}$-NF-bridge are synchronized in their correspondence with the labels " $1,2, \ldots, l *$ ", in the sense that this correspondence is completely determined up to composition with the action of an element of $\mathbb{F}_{l}^{*}$. In particular, this correspondence is always bijective.

One may regard this phenomenon of synchronization, or cohesion, as an important consequence of the fact that the number field in question is represented in the $\mathcal{D}$-NF-bridge via a single copy [i.e., as opposed to a capsule whose index set is of cardinality $\geq 2$ ] of $\mathcal{D}^{\odot}$.

Indeed, consider a situation in which each $\mathcal{D}$-prime-strip in the capsule $\dagger^{\mathfrak{D}} \mathfrak{D}_{J}$ is equipped with its own "independent globalization", i.e., copy of $\mathcal{D}^{\odot}$, to which it

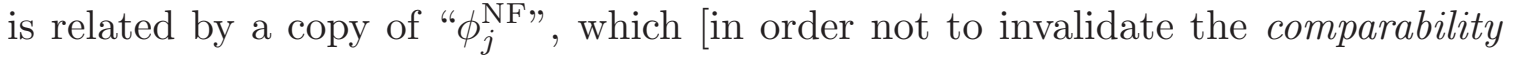
of distinct labels - cf. Remark 4.9.1, (i)] is regarded as being known only up to composition with the action of an element of $\mathbb{F}_{l}^{*}$. Then if one thinks of the [manifestly mutually disjoint — cf. Definition 3.1, (f); Example 4.3, (i)] $\mathbb{F}_{l}^{*}$-translates of $\underline{\mathbb{V}}^{ \pm \text {un }} \cap \mathbb{V}(K)^{\text {bad }}$ [whose union is equal to $\underline{\mathbb{V}}^{\text {Bor }} \cap \mathbb{V}(K)^{\text {bad }}$ ] as being labeled by the elements of $\mathbb{F}_{l}^{*}$, then each $\mathcal{D}$-prime-strip in the capsule ${ }^{\dagger} \mathfrak{D}_{J}$ - i.e., each "•" in Fig. 4.5 below - is subject, as depicted in Fig. 4.5, to an independent indeterminacy 
concerning the label $\in \mathbb{F}_{i}^{*}$ to which it is associated. In particular, the set of all possibilities for each association includes correspondences between the index set $J$ of the capsule ${ }^{\dagger} \mathfrak{D}_{J}$ and the set of labels $\mathbb{F}_{l}^{*}$ which fail to be bijective. Moreover, although $\mathbb{F}_{l}^{*}$ arises essentially as a subquotient of a Galois group of extensions of number fields [cf. the faithful poly-action of $\mathbb{F}_{l}^{*}$ on primes of $\mathbb{V}(K)$ ], the fact that it also acts faithfully on conjugates of the cusp $\underline{\epsilon}$ [cf. Example 4.3, (i)] implies that "working with elements of $\mathbb{V}(K)$ up to $\mathbb{F}_{l}^{*}$-indeterminacy" may only be done at the expense of "working with conjugates of the cusp $\underline{\epsilon}$ up to $\mathbb{F}_{l}^{*}$-indeterminacy". That is to say, "working with nonsynchronized labels" is inconsistent with the construction of the crucial bijection ${ }^{\dagger} \zeta_{*}$ in Proposition 4.7, (iii).

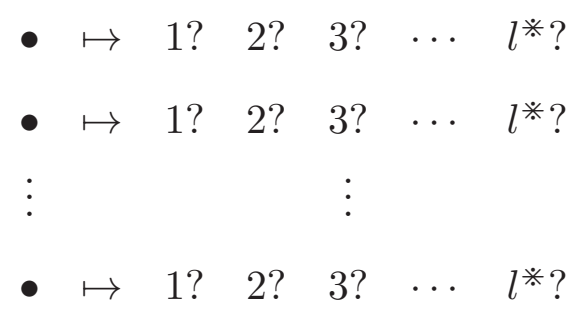

Fig. 4.5: Nonsynchronized labels

(ii) In the context of the discussion of (i), we observe that the "single copy" of $\mathcal{D}^{\odot}$ may also be thought of as a "single connected component", hence - from the point of view of Galois categories - as a "single basepoint".

(iii) In the context of the discussion of (i), it is interesting to note that since the natural action of $\mathbb{F}_{i}^{*}$ on $\mathbb{F}_{i}^{*}$ is transitive, one obtains the same "set of all possibilities for each association", regardless of whether one considers independent $\mathbb{F}_{l}^{*}$-indeterminacies at each index of $J$ or independent $\mathfrak{S}_{l *-\text { indeterminacies }}$ at each index of $J$ [cf. the discussion of Remark 4.9.1, (ii)].

(iv) The synchronized indeterminacy [cf. (i)] exhibited by a $\mathcal{D}$-NF-bridge - i.e., at a more concrete level, the crucial bijection $^{\dagger} \zeta_{*}$ of Proposition 4.7, (iii) may be thought of as a sort of combinatorial model of the notion of a "holomorphic structure". By contrast, the nonsynchronized indeterminacies discussed in (i) may be thought of as a sort of combinatorial model of the notion of a "real analytic structure". Moreover, we observe that the theme of the above discussion — in which one considers

"how a given combinatorial holomorphic structure is 'embedded' within its underlying combinatorial real analytic structure"

— is very much in line with the spirit of classical complex Teichmüller theory.

(v) From the point of view discussed in (iv), the main results of the "multiplicative combinatorial Teichmüller theory" developed in the present $\S 4$ may be summarized as follows:

(a) globalizability of labels, in a fashion that is independent of local structures [cf. Remark 4.3.2, (b); Proposition 4.7, (iii)];

(b) comparability of distinct labels [cf. Proposition 4.9; Remark 4.9.1, (i)];

(c) absolute comparability [cf. Proposition 4.9, (ii), (iii); Remark 4.9.1, (ii)]; 
(d) minimization of label indeterminacy - without sacrificing the symmetry necessary to perform comparisons! - via processions [cf. Proposition 4.11, (i), (ii), below].

\section{Remark 4.9.3.}

(i) Ultimately, in the theory of the present series of papers [cf. [IUTchIII]], we would like to apply the mono-anabelian theory of [AbsTopIII] to the various local and global arithmetic fundamental groups [i.e., isomorphs of $\Pi_{\underline{C}_{K}}, \Pi_{\underline{v}}$ for $\underline{v} \in \underline{\mathbb{V}}^{\text {non }}$ ] that appear in a $\mathcal{D}$ - $\Theta N F$-Hodge theater [cf. the discussion of Remark 4.3.2]. To do this, it is of essential importance to have available not only the absolute Galois groups of the various local and global base fields involved, but also the geometric fundamental groups that lie inside the isomorphs of $\Pi_{\underline{C}_{K}}, \Pi_{\underline{v}}$ involved. Indeed, in the theory of [AbsTopIII], it is precisely the outer Galois action of the absolute Galois group of the base field on the geometric fundamental group that allows one to reconstruct the ring structures group-theoretically in a fashion that is compatible with localization/globalization operations as shown in Fig. 4.3. Here, we pause to recall that in [AbsTopIII], Remark 5.10.3, (i), one may find a discussion of the analogy between this phenomenon of "entrusting of arithmetic moduli" [to the outer Galois action on the geometric fundamental group] and the Kodaira-Spencer isomorphism of an indigenous bundle - an analogy that is reminiscent of the discussion of Remark 4.7.2, (iii).

(ii) Next, let us observe that the state of affairs discussed in (i) has important implications concerning the circumstances that necessitate the use of " $\underline{X}_{\underline{v}}$ " [i.e., as opposed to " $\underline{C}_{\underline{v}}$ "] in the definition of " $\mathcal{D}_{\underline{v}}$ " in Examples 3.3, 3.4 [cf. Remärk 4.2.1]. Indeed, localization/globalization operations as shown in Fig. 4.3 give rise, when applied to the various geometric fundamental groups involved, to various bijections between local and global sets of label classes of cusps. Now suppose that one uses " $\underline{C}_{\underline{v}}$ " instead of " $\underline{X}_{v}$ " in the definition of " $\mathcal{D}_{\underline{v}}$ " in Examples 3.3, 3.4. Then the existence of $\underline{v} \in \underline{\mathbb{V}}$ of the sort discussed in Remark 4.2.1, together with the condition of compatibility with localization/globalization operations as shown in Fig. 4.3 where we take, for instance,

$$
\begin{array}{rll}
(v \text { of Fig. } 4.3) & \stackrel{\text { def }}{=} \quad(\underline{v} \text { of Remark 4.2.1) } \\
\left(v^{\prime} \text { of Fig. } 4.3\right) & \stackrel{\text { def }}{=} \quad(\underline{w} \text { of Remark 4.2.1) }
\end{array}
$$

- imply that, at a combinatorial level, one is led, in effect, to a situation of the sort discussed in Remark 4.9.2, (i), i.e., a situation involving nonsynchronized labels [cf. Fig. 4.5], which, as discussed in Remark 4.9.2, (i), is incompatible with the construction of the crucial bijection ${ }^{\dagger} \zeta$ of Proposition 4.7, (iii), an object which will play an important role in the theory of the present series of papers.

Definition 4.10. Let $\mathcal{C}$ be a category, $n$ a positive integer. Then we shall refer to as a procession of length $n$, or $n$-procession, of $\mathcal{C}$ any diagram of the form

$$
P_{1} \hookrightarrow P_{2} \hookrightarrow \ldots \hookrightarrow P_{n}
$$


— where each $P_{j}$ [for $\left.j=1, \ldots, n\right]$ is a $j$-capsule [cf. $\S 0$ ] of objects of $\mathcal{C}$; each arrow $P_{j} \hookrightarrow P_{j+1}$ [for $j=1, \ldots, n-1$ ] denotes the collection of all capsule-full poly-morphisms [cf. $\S 0$ ] from $P_{j}$ to $P_{j+1}$. A morphism from an $n$-procession of $\mathcal{C}$ to an $m$-procession of $\mathcal{C}$

$$
\left(P_{1} \hookrightarrow \ldots \hookrightarrow P_{n}\right) \quad \rightarrow \quad\left(Q_{1} \hookrightarrow \ldots \hookrightarrow Q_{m}\right)
$$

consists of an order-preserving injection $\iota:\{1, \ldots, n\} \hookrightarrow\{1, \ldots, m\}$ [so $n \leq m]$, together with a capsule-full poly-morphism $P_{j} \hookrightarrow Q_{\iota(j)}$ for each $j=1, \ldots, n$.

$$
\text { /* } \hookrightarrow / * / * \hookrightarrow / * / * / * \hookrightarrow \quad \ldots \hookrightarrow(/ * \ldots / *)
$$

Fig. 4.6: An $l^{*}$-procession of $\mathcal{D}$-prime-strips

Proposition 4.11. (Processions of Base-Prime-Strips) Relative to a fixed collection of initial $\Theta$-data:

(i) (Holomorphic Processions) Given a $\mathcal{D}-\Theta$-bridge ${ }^{\dagger} \phi_{*}^{\Theta}:{ }^{\dagger} \mathfrak{D}_{J} \rightarrow{ }^{\dagger} \mathfrak{D}_{>}$ [cf. Definition 4.6, (ii)], with underlying capsule of $\mathcal{D}$-prime-strips ${ }^{\dagger} \mathfrak{D}_{J}$, denote by $\operatorname{Prc}\left({ }^{\dagger} \mathfrak{D}_{J}\right)$ the $l *$-procession of $\mathcal{D}$-prime-strips [cf. Fig. 4.6, where each "/*" denotes a D-prime-strip] determined by considering the ["sub"]capsules of $\dagger \mathfrak{D}_{J}$ corresponding to the subsets $\mathbb{S}_{1}^{*} \subseteq \ldots \subseteq \mathbb{S}_{j}^{*} \stackrel{\text { def }}{=}\{1,2, \ldots, j\} \subseteq \ldots \subseteq \mathbb{S}_{l *}^{*} \stackrel{\text { def }}{=} \mathbb{F}_{l}^{*}$ [where, by abuse of notation, we use the notation for positive integers to denote the images of these positive integers in $\left.\mathbb{F}_{l}^{*}\right]$, relative to the bijection ${ }^{\dagger} \chi: J \stackrel{\sim}{\rightarrow} \mathbb{F}_{i}^{*}$ of Proposition 4.7, (i). Then the assignment ${ }^{\dagger} \phi_{*}^{\Theta} \mapsto \operatorname{Prc}\left({ }^{\dagger} \mathfrak{D}_{J}\right)$ determines a natural functor

\begin{tabular}{|c|c|c|}
\hline $\begin{array}{c}\text { category of } \\
\mathcal{D} \text { - } \Theta \text {-bridges } \\
\text { and isomorphisms of } \\
\mathcal{D} \text { - } \Theta \text {-bridges }\end{array}$ & $\rightarrow \quad \begin{array}{c}\text { category of processions } \\
\text { of } \mathcal{D} \text {-prime-strips } \\
\text { and morphisms of } \\
\text { processions }\end{array}$ \\
\hline${ }^{\dagger} \phi_{*}^{\Theta}$ & $\mapsto$ & $\operatorname{Prc}\left({ }^{\dagger} \mathfrak{D}_{J}\right)$ \\
\hline
\end{tabular}

whose output data satisfies the following property: for each $n \in\left\{1, \ldots, l^{*}\right\}$, there are precisely $\boldsymbol{n}$ possibilities for the element $\in \mathbb{F}_{i}^{*}$ to which a given index of the index set of the $n$-capsule that appears in the procession constituted by this output data corresponds, prior to the application of this functor. That is to say, by taking the product, over elements of $\mathbb{F}_{l}^{*}$, of cardinalities of "sets of possibilies", one concludes that

by considering processions - i.e., the functor discussed above, possibly pre-composed with the functor ${ }^{\dagger} \mathcal{H} \mathcal{T}^{\mathcal{D}-\Theta N F} \mapsto{ }^{\dagger} \phi_{*}^{\Theta}$ that associates to a D - $\Theta \mathrm{NF}$-Hodge theater its associated $\mathcal{D}$ - $\Theta$-bridge - the indeterminacy consisting of $\left(l^{*}\right)^{\left(l^{*}\right)}$ possibilities that arises in Proposition 4.9, (ii), is reduced to an indeterminacy consisting of a total of $l *$ ! possibilities.

(ii) (Mono-analytic Processions) By composing the functor of (i) with the mono-analyticization operation discussed in Definition 4.1, (iv), one obtains a 


\section{natural functor}

\begin{tabular}{|c|c|c|}
\hline $\begin{array}{c}\text { category of } \\
\mathcal{D} \text { - } \Theta \text {-bridges } \\
\text { and isomorphisms of } \\
\mathcal{D} \text { - } \Theta \text {-bridges }\end{array}$ & $\rightarrow$ & $\begin{array}{c}\text { category of processions } \\
\text { of } \mathcal{D}^{\vdash} \text {-prime-strips } \\
\text { and morphisms of } \\
\text { processions }\end{array}$ \\
\hline${ }^{\dagger} \phi_{*}^{\Theta}$ & $\mapsto$ & $\operatorname{Prc}\left({ }^{\dagger} \mathfrak{D}_{J}^{\vdash}\right)$ \\
\hline
\end{tabular}

whose output data satisfies the same indeterminacy properties with respect to labels as the output data of the functor of (i).

Proof. Assertions (i), (ii) follow immediately from the definitions.

The following result is an immediate consequence of our discussion.

Corollary 4.12. (Étale-pictures of Base- $\Theta N F-H o d g e$ Theaters) Relative to a fixed collection of initial $\Theta$-data:

(i) Consider the [composite] functor

$$
{ }^{\dagger} \mathcal{H} \mathcal{T}^{\mathcal{D}-\Theta N F} \quad \mapsto \quad{ }^{\dagger} \mathfrak{D}_{>} \quad \mapsto \quad{ }^{\dagger} \mathfrak{D}_{>}^{\vdash}
$$

- from the category of $\mathcal{D}-\Theta \mathrm{NF}-$ Hodge theaters and isomorphisms of $\mathcal{D}-\Theta \mathrm{NF}-$ Hodge theaters [cf. Definition 4.6, (iii)] to the category of $\mathcal{D}^{\vdash}$-prime-strips and isomorphisms of $\mathcal{D}^{\vdash}$-prime-strips - obtained by assigning to the $\mathcal{D}$ - $\Theta \mathrm{NF}$-Hodge theater ${ }^{\dagger} \mathcal{H} \mathcal{T}^{\mathcal{D}-\Theta N F}$ the mono-analyticization [cf. Definition 4.1, (iv)] ${ }^{\dagger} \mathfrak{D}_{>}^{\vdash}$ of the $\mathcal{D}$ prime-strip ${ }^{\dagger} \mathfrak{D}_{>}$that appears as the codomain of the underlying $\mathcal{D}$ - $\Theta$-bridge $/ c f$. Definition 4.6, (ii)] of ${ }^{\dagger} \mathcal{H} \mathcal{T}^{\mathcal{D}-\Theta N F}$. If ${ }^{\dagger} \mathcal{H} \mathcal{T}^{\mathcal{D}-\Theta N F},{ }^{\ddagger} \mathcal{H} \mathcal{T}^{\mathcal{D}-\Theta N F}$ are $\mathcal{D}$ - $\Theta$ NF-Hodge theaters, then we define the base- $\Theta \mathrm{NF}-$, or $\mathcal{D}-\Theta \mathrm{NF}-$, link

$$
{ }^{\dagger} \mathcal{H} \mathcal{T}^{\mathcal{D}-\Theta N F} \stackrel{\mathcal{D}}{\longrightarrow}{ }^{\ddagger} \mathcal{H} \mathcal{T}^{\mathcal{D}-\Theta N F}
$$

from ${ }^{\dagger} \mathcal{H} \mathcal{T}^{\mathcal{D}-\Theta \mathrm{NF}}$ to ${ }^{\ddagger} \mathcal{H} \mathcal{T}^{\mathcal{D}-\Theta \mathrm{NF}}$ to be the full poly-isomorphism

$$
{ }^{\dagger} \mathfrak{D}_{>}^{\vdash} \stackrel{\sim}{\rightarrow} \ddagger \mathfrak{D} \vdash
$$

between the $\mathcal{D}^{\vdash}$-prime-strips obtained by applying the functor discussed above to $\dagger \mathcal{H} \mathcal{T}^{\mathcal{D}-\Theta N F}, \ddagger \mathcal{H} \mathcal{T}^{\mathcal{D}-\Theta N F}$.

(ii) If

$$
\ldots \quad \stackrel{\mathcal{D}}{\longrightarrow} \quad{ }^{(n-1)} \mathcal{H} \mathcal{T}^{\mathcal{D}-\Theta \mathrm{NF}} \stackrel{\mathcal{D}}{\longrightarrow}{ }^{n} \mathcal{H} \mathcal{T}^{\mathcal{D}-\Theta \mathrm{NF}} \stackrel{\mathcal{D}}{\longrightarrow} \quad{ }^{(n+1)} \mathcal{H} \mathcal{T}^{\mathcal{D}-\Theta \mathrm{NF}} \stackrel{\mathcal{D}}{\longrightarrow} \quad \ldots
$$

[where $n \in \mathbb{Z}$ ] is an infinite chain of $\mathcal{D}$ - $\Theta N F$-linked $\mathcal{D}$ - $\Theta N F-H o d g e$ theaters [cf. the situation discussed in Corollary 3.8], then we obtain a resulting chain of full poly-isomorphisms

$$
\ldots \stackrel{\sim}{\rightarrow}{ }^{n} \mathfrak{D}_{>}^{\vdash} \stackrel{\sim}{\rightarrow}(n+1) \mathfrak{D}_{>}^{\vdash} \stackrel{\sim}{\rightarrow} \ldots
$$


[cf. the situation discussed in Remark 3.8.1, (ii)] between the $\mathcal{D}^{\vdash}$-prime-strips obtained by applying the functor of (i). That is to say, the output data of the functor of (i) forms a constant invariant [cf. the discussion of Remark 3.8.1, (ii)] i.e., a mono-analytic core [cf. the situation discussed in Remark 3.9.1] - of the above infinite chain.

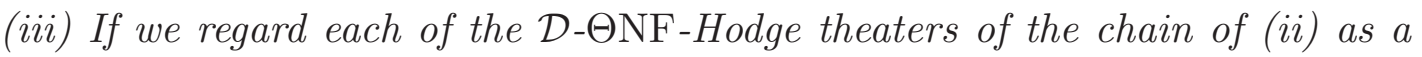
spoke emanating from the mono-analytic core discussed in (ii), then we obtain a

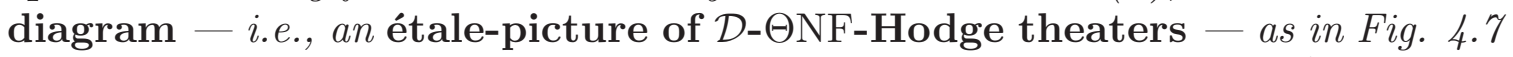
below [cf. the situation discussed in Corollary 3.9, (i)]. In Fig. 4.7, " $>$ " " denotes the mono-analytic core; "/* $\hookrightarrow / * / * \hookrightarrow \ldots$ " denotes the "holomorphic" processions of Proposition 4.11, (i), together with the remaining ["holomorphic"] data of the corresponding $\mathcal{D}-\Theta \mathrm{NF}-H o d g e$ theater. Finally, [cf. the situation discussed in Corollary 3.9, (i)] this diagram satisfies the important property of admitting arbitrary permutation symmetries among the spokes [i.e., among the labels $n \in \mathbb{Z}$ of the $\mathcal{D}-\Theta N F-H o d g e$ theaters].
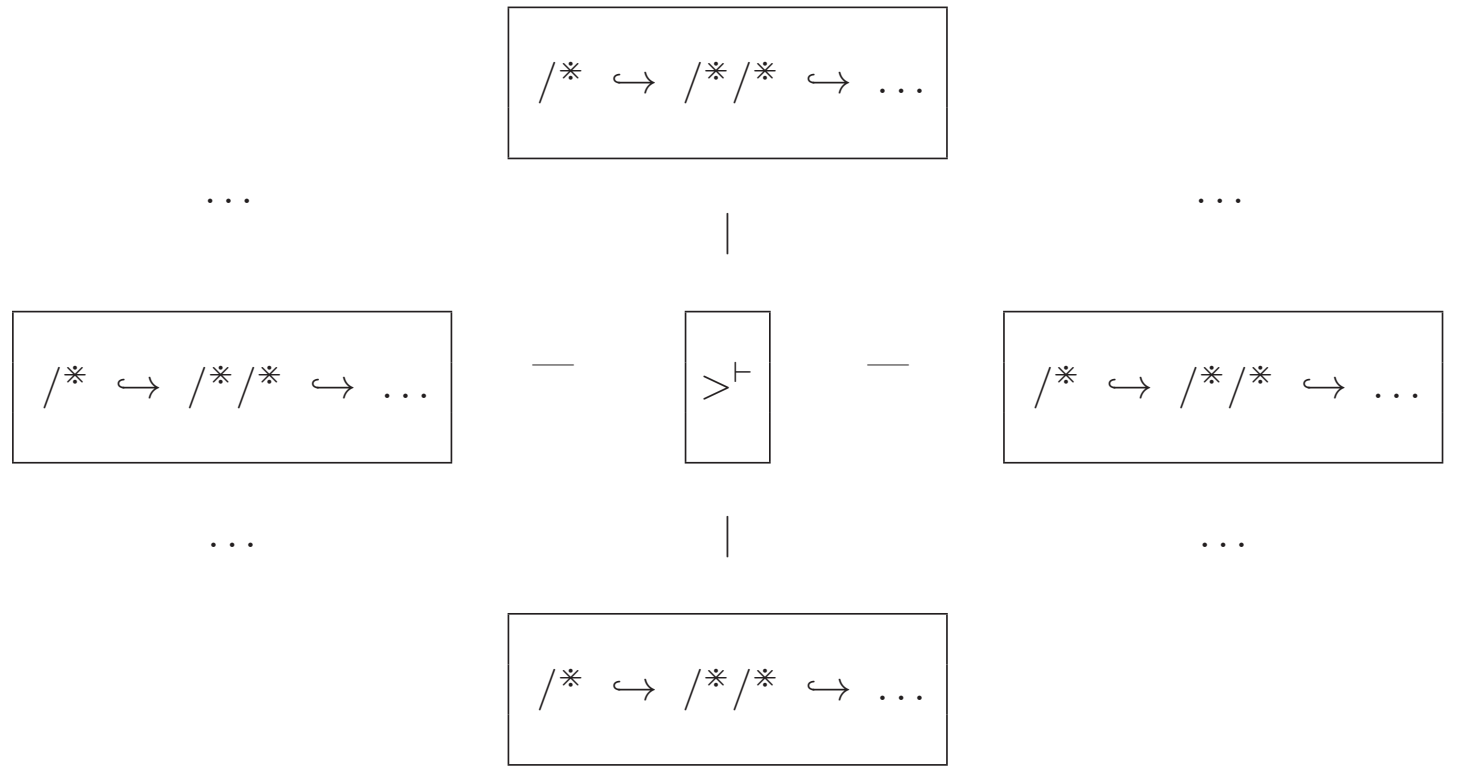

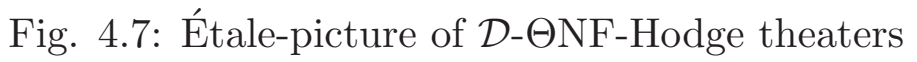




\section{Section 5: $\Theta N F-H o d g e$ Theaters}

In the present $\S 5$, we continue our discussion of various "enhancements" to the $\Theta$-Hodge theaters of $\S 3$. Namely, we define the notion of a $\Theta N F-H o d g e$ theater [cf. Definition 5.5, (iii)] and observe that these $\Theta N F$-Hodge theaters satisfy the same "functorial dynamics" [cf. Corollary 5.6; Remark 5.6.1] as the base- $\Theta N F-$ Hodge theaters discussed in $\S 4$.

Let

$$
{ }^{\dagger} \mathcal{H} \mathcal{T}^{\mathcal{D}-\Theta \mathrm{NF}}=\left({ }^{\dagger} \mathcal{D}^{\odot} \stackrel{{ }^{\dagger} \phi_{*}^{\mathrm{NF}}}{\longleftarrow} \quad \dagger \mathfrak{D}_{J} \stackrel{{ }^{\dagger} \phi_{*}^{\Theta}}{\longrightarrow} \quad{ }^{\dagger} \mathfrak{D}_{>}\right)
$$

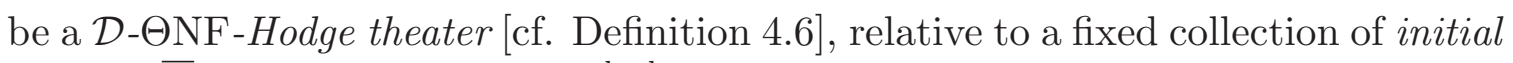
$\Theta$-data $\left(\bar{F} / F, X_{F}, l, \underline{C}_{K}, \underline{\mathbb{V}}, \mathbb{V}_{\bmod }^{\text {bad }}, \underline{\epsilon}\right)$ as in Definition 3.1.

\section{Example 5.1. Global Frobenioids.}

(i) By applying the anabelian result of [AbsTopIII], Theorem 1.9, via the " $\Theta$-approach" discussed in Remark 3.1.2, to $\pi_{1}\left({ }^{\dagger} \mathcal{D}^{\odot}\right)$, we may construct grouptheoretically from $\pi_{1}\left({ }^{\dagger} \mathcal{D}^{\odot}\right)$ an isomorph of " $\bar{F}^{\times}$" - which we shall denote

$$
\mathbb{M}^{\circledast}\left({ }^{\dagger} \mathcal{D}^{\odot}\right)
$$

- equipped with its natural $\pi_{1}\left({ }^{\dagger} \mathcal{D}^{\odot}\right)$-action. Here, we recall that this construction includes a reconstruction of the field structure on $\overline{\mathbb{M}}^{\circledast}\left({ }^{\dagger} \mathcal{D}^{\odot}\right) \stackrel{\text { def }}{=} \mathbb{M}^{\circledast}\left({ }^{\dagger} \mathcal{D}^{\odot}\right) \cup\{0\}$. Next, let us recall [cf. Remark 3.1.7, (i)] the unique model $C_{F_{\text {mod }}}$ of the $F$-core $C_{F}$ over $F_{\text {mod }}$. Observe that one may construct group-theoretically from $\pi_{1}\left({ }^{\dagger} \mathcal{D}^{\odot}\right)$, in a functorial fashion, a profinite group corresponding to " $C_{F_{\bmod }}$ " [cf. the algorithms of [AbsTopII], Corollary 3.3, (i), which are applicable in light of [AbsTopI], Example 4.8], which contains $\pi_{1}\left({ }^{\dagger} \mathcal{D}^{\odot}\right)$ as an open subgroup; write ${ }^{\dagger} \mathcal{D}^{\circledast}$ for $\mathcal{B}(-)^{0}$ of this profinite group, so we obtain a natural morphism

$$
{ }^{\dagger} \mathcal{D}^{\odot} \rightarrow{ }^{\dagger} \mathcal{D}^{\circledast}
$$

— i.e., a "category-theoretic version" of the natural morphism of hyperbolic orbicurves $\underline{C}_{K} \rightarrow C_{F_{\mathrm{mod}}}$ - together with a natural extension of the action of $\pi_{1}\left({ }^{\dagger} \mathcal{D}^{\odot}\right)$ on $\mathbb{M}^{\circledast}\left({ }^{\dagger} \mathcal{D}^{\odot}\right)$ to $\pi_{1}\left({ }^{\dagger} \mathcal{D}^{\circledast}\right)$. In particular, by taking $\pi_{1}\left({ }^{\dagger} \mathcal{D}^{\circledast}\right)$-invariants, we obtain a submonoid/subfield

$$
\mathbb{M}_{\bmod }^{\circledast}\left({ }^{\dagger} \mathcal{D}^{\odot}\right) \subseteq \mathbb{M}^{\circledast}\left({ }^{\dagger} \mathcal{D}^{\odot}\right), \quad \overline{\mathbb{M}}_{\bmod }^{\circledast}\left({ }^{\dagger} \mathcal{D}^{\odot}\right) \subseteq \overline{\mathbb{M}}^{\circledast}\left({ }^{\dagger} \mathcal{D}^{\odot}\right)
$$

corresponding to $F_{\text {mod }}^{\times} \subseteq \bar{F}^{\times}, F_{\text {mod }} \subseteq \bar{F}$. In a similar vein, by applying [AbsTopIII], Theorem 1.9 - cf., especially, the construction of the Belyi cuspidalizations of [AbsTopIII], Theorem 1.9, (a), and of the field " $K_{Z_{\mathrm{NF}}}^{\times} \cup\{0\}$ " of [AbsTopIII], Theorem 1.9 , (d), (e) — we conclude that we may construct group-theoretically from $\pi_{1}\left({ }^{\dagger} \mathcal{D}^{\odot}\right)$, in a functorial fashion, an isomorph

$$
\pi_{1}^{\mathrm{rat}}\left({ }^{\dagger} \mathcal{D}^{\circledast}\right) \quad\left(\rightarrow \pi_{1}\left({ }^{\dagger} \mathcal{D}^{\circledast}\right)\right)
$$


of the absolute Galois group of the function field of $C_{F_{\bmod }}$ [i.e., equipped with its natural surjection to $\pi_{1}\left({ }^{\dagger} \mathcal{D}^{\circledast}\right)$ and well-defined up to inner automorphisms determined by elements of the kernel of this natural surjection], as well as isomorphs of the pseudo-monoids of $\kappa$-, $\infty \kappa$-, and ${ }_{\infty} \kappa \times$-coric rational functions associated to $C_{F_{\text {mod }}}$ [cf. the discussion of Remark 3.1.7, (i), (ii); [AbsTopII], Corollary 3.3, (iii), which is applicable in light of [AbsTopI], Example 4.8] — which we shall denote

$$
\mathbb{M}_{\kappa}^{\circledast}\left({ }^{\dagger} \mathcal{D}^{\odot}\right), \quad \mathbb{M}_{\infty \kappa}^{\circledast}\left({ }^{\dagger} \mathcal{D}^{\odot}\right), \quad \mathbb{M}_{\infty \kappa \times}^{\circledast}\left({ }^{\dagger} \mathcal{D}^{\odot}\right)
$$

- equipped with their natural $\pi_{1}^{\mathrm{rat}}\left({ }^{\dagger} \mathcal{D}^{\circledast}\right)$-actions. Thus, $\mathbb{M}_{\kappa}^{\circledast}\left({ }^{\dagger} \mathcal{D}^{\odot}\right)$ may be identified with the subset of $\pi_{1}^{\text {rat }}\left({ }^{\dagger} \mathcal{D}^{\circledast}\right)$-invariants of $\mathbb{M}_{\infty}^{\circledast}{ }^{\circledast}\left({ }^{\dagger} \mathcal{D}^{\odot}\right)$, and $\mathbb{M}^{\circledast}\left({ }^{\dagger} \mathcal{D}^{\odot}\right)$ may be identified with a certain subset [i.e., indeed, a certain "sub-pseudo-monoid"!] of $\mathbb{M}_{\infty}^{\circledast} \kappa \times\left({ }^{\dagger} \mathcal{D}^{\odot}\right)$. Next, let us observe that it also follows from the group-theoretic constructions recalled above that one may reconstruct the quotients of $\pi_{1}\left({ }^{\dagger} \mathcal{D}^{\odot}\right)$, $\pi_{1}\left({ }^{\dagger} \mathcal{D}^{\circledast}\right)$ that correspond, respectively, to the absolute Galois groups of $K, F_{\text {mod }}$. Thus, by forming the quotient of $\pi_{1}^{\mathrm{rat}}\left({ }^{\dagger} \mathcal{D}^{\circledast}\right)$ by the intersection of the kernel of the action of $\pi_{1}^{\text {rat }}\left({ }^{\dagger} \mathcal{D}^{\circledast}\right)$ on $\mathbb{M}_{\infty \kappa}^{\circledast}\left({ }^{\dagger} \mathcal{D}^{\odot}\right)$ with the inverse image in $\pi_{1}^{\text {rat }}\left({ }^{\dagger} \mathcal{D}^{\circledast}\right)$ of the kernel of the maximal solvable quotient of [the quotient of $\pi_{1}^{\text {rat }}\left({ }^{\dagger} \mathcal{D}^{\circledast}\right)$ that corresponds to] the absolute Galois group of $F_{\bmod }$, we obtain a group-theoretic construction for a quotient

$$
\pi_{1}^{\mathrm{rat}}\left({ }^{\dagger} \mathcal{D}^{\circledast}\right) \quad \rightarrow \quad \pi_{1}^{\kappa-\text { sol }}\left({ }^{\dagger} \mathcal{D}^{\circledast}\right)
$$

— whose kernel we denote by $\pi_{1}^{\mathrm{rat} / \kappa \text {-sol }}\left({ }^{\dagger} \mathcal{D}^{\circledast}\right)$ - that corresponds to the quotient "Gal $\left(\bar{L}_{C} / L_{C}\right) \rightarrow \operatorname{Gal}\left(L_{C}(\kappa\right.$-sol $\left.) / L_{C}\right)$ " of Remark 3.1.7, (iv), as well as pseudomonoids equipped with natural $\pi_{1}^{\kappa \text {-sol }}\left({ }^{\dagger} \mathcal{D}^{\circledast}\right)$-actions

$$
\mathbb{M}_{\infty \kappa}^{\circledast}\left({ }^{\dagger} \mathcal{D}^{\odot}\right), \quad \mathbb{M}_{\kappa \text {-sol }}^{\circledast}\left({ }^{\dagger} \mathcal{D}^{\odot}\right), \quad \mathbb{M}_{\text {sol }}^{\circledast}\left({ }^{\dagger} \mathcal{D}^{\odot}\right)
$$

— where $\mathbb{M}_{\kappa \text {-sol }}^{\circledast}\left({ }^{\dagger} \mathcal{D}^{\odot}\right), \mathbb{M}_{\text {sol }}^{\circledast}\left({ }^{\dagger} \mathcal{D}^{\odot}\right)$ denote the respective subsets of $\pi_{1}^{\text {rat } / \kappa \text {-sol }}\left({ }^{\dagger} \mathcal{D}^{\circledast}\right)$ invariants of $\mathbb{M}_{\infty}^{\circledast} \kappa \times\left({ }^{\dagger} \mathcal{D}^{\odot}\right), \mathbb{M}^{\circledast}\left({ }^{\dagger} \mathcal{D}^{\odot}\right)$. Moreover, by applying the characterization of the subgroup "Gal $\left(K / F\left(\boldsymbol{\mu}_{l}\right)\right) \subseteq \operatorname{Gal}\left(K / F_{\text {mod }}\right)$ " given in Remark 3.1.7, (iii), we obtain a group-theoretic construction for subgroups

$$
\operatorname{Aut}_{\underline{\epsilon}}^{S L}\left({ }^{\dagger} \mathcal{D}^{\odot}\right) \subseteq \operatorname{Aut}^{S L}\left({ }^{\dagger} \mathcal{D}^{\odot}\right) \subseteq \operatorname{Aut}\left({ }^{\dagger} \mathcal{D}^{\odot}\right)
$$

that correspond to the subgroups "Aut ${ }_{\epsilon}^{S L}\left(\underline{C}_{K}\right) \subseteq \operatorname{Aut}^{S L}\left(\underline{C}_{K}\right) \subseteq \operatorname{Aut}\left(\underline{C}_{K}\right)$ " of Example 4.3, (i), hence induce natural isomorphisms

$$
\operatorname{Aut}^{S L}\left({ }^{\dagger} \mathcal{D}^{\odot}\right) / \operatorname{Aut}_{\underline{\epsilon}}^{S L}\left({ }^{\dagger} \mathcal{D}^{\odot}\right) \stackrel{\sim}{\rightarrow} \operatorname{Aut}\left({ }^{\dagger} \mathcal{D}^{\odot}\right) / \operatorname{Aut}_{\underline{\epsilon}}\left({ }^{\dagger} \mathcal{D}^{\odot}\right) \stackrel{\sim}{\rightarrow} \mathbb{F}_{l}^{*}
$$

- i.e., which, in the spirit of Example 4.3, (iv), may be thought of as a polyaction of $\mathbb{F}_{l}^{*}$ on ${ }^{\dagger} \mathcal{D}^{\odot}$. Finally, we observe that although this poly-action of $\mathbb{F}_{l}^{*}$ on $\pi_{1}^{\mathrm{rat}}\left({ }^{\dagger} \mathcal{D}^{\circledast}\right)$ is only well-defined up to conjugation by elements of the subgroup

$$
\pi_{1}^{\mathrm{rat}}\left({ }^{\dagger} \mathcal{D}^{\odot}\right) \stackrel{\text { def }}{=} \pi_{1}^{\mathrm{rat}}\left({ }^{\dagger} \mathcal{D}^{\circledast}\right) \times_{\pi_{1}\left({ }^{\dagger} \mathcal{D} \circledast\right)} \pi_{1}\left({ }^{\dagger} \mathcal{D}^{\odot}\right)
$$

of $\pi_{1}^{\text {rat }}\left({ }^{\dagger} \mathcal{D}^{\circledast}\right)$, it follows formally from the linear disjointness property discussed in Remark 3.1.7, (iii), that, by regarding this poly-action of $\mathbb{F}_{l}^{*}$ as arising from the $a c$ tion of elements of $\mathrm{Aut}^{S L}\left({ }^{\dagger} \mathcal{D}^{\odot}\right)$, one may conclude that, if we write $\pi_{1}^{\text {rat } / \kappa \text {-sol }}\left({ }^{\dagger} \mathcal{D}^{\odot}\right)$ $\stackrel{\text { def }}{=} \pi_{1}^{\mathrm{rat} / \kappa \text {-sol }}\left({ }^{\dagger} \mathcal{D}^{\circledast}\right) \bigcap \pi_{1}^{\mathrm{rat}}\left({ }^{\dagger} \mathcal{D}^{\odot}\right)$, then 
the resulting poly-action of $\mathbb{F}_{l}^{*}$ on $\pi_{1}^{\text {rat }}\left({ }^{\dagger} \mathcal{D}^{\circledast}\right)$ is, in fact, well-defined up to $\pi_{1}^{\mathrm{rat} / \kappa \text {-sol }}\left({ }^{\dagger} \mathcal{D}^{\odot}\right)$-conjugacy indeterminacies, hence, in particular, that the induced poly-action on [the domain, codomain, and arrow that constitute] the " $\kappa$-sol-outer representation"

$$
\pi_{1}^{\kappa-\text { sol }}\left({ }^{\dagger} \mathcal{D}^{\circledast}\right) \quad \rightarrow \quad \mathrm{Out}^{\kappa-\mathrm{sol}}\left(\pi_{1}^{\mathrm{rat} / \kappa \text {-sol }}\left({ }^{\dagger} \mathcal{D}^{\circledast}\right)\right)
$$

- i.e., which may be associated to and is, in fact, equivalent to the exact sequence $1 \rightarrow \pi_{1}^{\mathrm{rat} / \kappa \text {-sol }}\left({ }^{\dagger} \mathcal{D}^{\circledast}\right) \rightarrow \pi_{1}^{\mathrm{rat}}\left({ }^{\dagger} \mathcal{D}^{\circledast}\right) \rightarrow \pi_{1}^{\kappa \text {-sol }}\left({ }^{\dagger} \mathcal{D}^{\circledast}\right) \rightarrow 1$, regarded up to $\pi_{1}^{\mathrm{rat} / \kappa \text {-sol }}\left({ }^{\dagger} \mathcal{D}^{\circledast}\right)$-conjugacy indeterminacies [cf. the discussion of Remark 3.1.7, (iv)] — is, in fact, well-defined without any conjugacy indeterminacies, and, moreover, equal to the trivial action.

We shall refer to this phenomenon [cf. also Remark 5.1.5 below] as the phenomenon of $\kappa$-sol-conjugate synchronization.

(ii) Next, let us recall [cf. Definition 4.1, (v)] that the field structure on $\overline{\mathbb{M}}^{\circledast}\left({ }^{\dagger} \mathcal{D}^{\odot}\right)$ [i.e., " $\left.\bar{F} "\right]$ allows one to reconstruct group-theoretically from $\pi_{1}\left({ }^{\dagger} \mathcal{D}^{\odot}\right)$ the set of valuations $\overline{\mathbb{V}}\left({ }^{\dagger} \mathcal{D}^{\odot}\right)$ [i.e., " $\mathbb{V}(\bar{F})$ "] on $\overline{\mathbb{M}}^{\circledast}\left({ }^{\dagger} \mathcal{D}^{\odot}\right)$ equipped with its natural $\pi_{1}\left({ }^{\dagger} \mathcal{D}^{\circledast}\right)$-action, hence also the monoid on ${ }^{\dagger} \mathcal{D}^{\circledast}$ [i.e., in the sense of [FrdI], Definition $1.1,(\mathrm{ii})]$

$$
\Phi^{\circledast}\left({ }^{\dagger} \mathcal{D}^{\odot}\right)(-)
$$

that associates to an object $A \in \mathrm{Ob}\left({ }^{\dagger} \mathcal{D}^{\circledast}\right)$ the monoid $\Phi^{\circledast}\left({ }^{\dagger} \mathcal{D}^{\odot}\right)(A)$ of "stacktheoretic" [cf. Remark 3.1.5] arithmetic divisors on the corresponding subfield $\overline{\mathbb{M}}^{\circledast}\left({ }^{\dagger} \mathcal{D}^{\odot}\right)^{A} \subseteq \overline{\mathbb{M}}^{\circledast}\left({ }^{\dagger} \mathcal{D}^{\odot}\right)$ [i.e., the monoid denoted “ $\Phi(-)$ " in [FrdI], Example 6.3; cf. also Remark 3.1.5 of the present paper], together with the natural morphism of monoids $\mathbb{M}^{\circledast}\left({ }^{\dagger} \mathcal{D}^{\odot}\right)^{A} \rightarrow \Phi^{\circledast}\left({ }^{\dagger} \mathcal{D}^{\odot}\right)(A)^{\text {gp }}$ [cf. the discussion of [FrdI], Example 6.3 ; Remark 3.1.5 of the present paper]. As discussed in [FrdI], Example 6.3 [cf. also Remark 3.1 .5 of the present paper], this data determines, by applying [FrdI], Theorem 5.2, (ii), a model Frobenioid

$$
\mathcal{F}^{\circledast}\left({ }^{\dagger} \mathcal{D}^{\odot}\right)
$$

over the base category ${ }^{\dagger} \mathcal{D}^{\circledast}$.

(iii) Let ${ }^{\dagger} \mathcal{F}^{\circledast}$ be any category equivalent to $\mathcal{F}^{\circledast}\left({ }^{\dagger} \mathcal{D}^{\odot}\right)$. Thus, ${ }^{\dagger} \mathcal{F}^{\circledast}$ is equipped with a natural Frobenioid structure [cf. [FrdI], Corollary 4.11; [FrdI], Theorem 6.4, (i); Remark 3.1.5 of the present paper]; write Base $\left({ }^{\dagger} \mathcal{F}^{\circledast}\right)$ for the base category of this Frobenioid. Suppose further that we have been given a morphism

$$
{ }^{\dagger} \mathcal{D}^{\odot} \rightarrow \operatorname{Base}\left({ }^{\dagger} \mathcal{F}^{\circledast}\right)
$$

which is abstractly equivalent [cf. $\S 0$ ] to the natural morphism ${ }^{\dagger} \mathcal{D}^{\odot} \rightarrow{ }^{\dagger} \mathcal{D}^{\circledast}[$ cf. (i)]. In the following discussion, we shall use the resulting [uniquely determined, in light of the F-coricity of $C_{F}$, together with [AbsTopIII], Theorem 1.9!] isomorphism $\operatorname{Base}\left({ }^{\dagger} \mathcal{F}^{\circledast}\right) \stackrel{\sim}{\rightarrow}{ }^{\dagger} \mathcal{D}^{\circledast}$ to identify $\operatorname{Base}\left({ }^{\dagger} \mathcal{F}^{\circledast}\right)$ with ${ }^{\dagger} \mathcal{D}^{\circledast}$. Let us denote by

$$
\left.{ }^{\dagger} \mathcal{F}^{\odot} \stackrel{\text { def }}{=} \quad{ }^{\dagger} \mathcal{F}^{\circledast}\right|_{\dagger \mathcal{D} \odot} \quad\left(\rightarrow^{\dagger} \mathcal{F}^{\circledast}\right)
$$


the restriction of ${ }^{\dagger} \mathcal{F}^{\circledast}$ to ${ }^{\dagger} \mathcal{D}^{\odot}$ via the natural morphism ${ }^{\dagger} \mathcal{D}^{\odot} \rightarrow{ }^{\dagger} \mathcal{D}^{\circledast}$ and by

$$
\left.{ }^{\dagger} \mathcal{F}_{\text {mod }}^{\circledast} \stackrel{\text { def }}{=} \quad \dagger \mathcal{F}^{\circledast}\right|_{\text {terminal objects }} \quad\left(\underline{\subset}^{\dagger} \mathcal{F}^{\circledast}\right)
$$

the restriction of ${ }^{\dagger} \mathcal{F}^{\circledast}$ to the full subcategory of ${ }^{\dagger} \mathcal{D}^{\circledast}$ determined by the terminal objects [i.e., " $C_{F_{\text {mod }}}$ "] of ${ }^{\dagger} \mathcal{D}^{\circledast}$. Thus, when the data denoted here by the label " $\dagger$ " arises [in the evident sense] from data as discussed in Definition 3.1, the Frobenioid $\dagger \mathcal{F}_{\bmod }^{\circledast}$ may be thought of as the Frobenioid of arithmetic line bundles on the stack " $S_{\text {mod" }}$ of Remark 3.1.5.

(iv) We continue to use the notation of (iii). We shall denote by a superscript "birat" the birationalizations [which are category-theoretic - cf. [FrdI], Corollary 4.10; [FrdI], Theorem 6.4, (i); Remark 3.1.5 of the present paper] of the Frobenioids ${ }^{\dagger} \mathcal{F}^{\odot},{ }^{\dagger} \mathcal{F}^{\circledast},{ }^{\dagger} \mathcal{F}_{\bmod }^{\circledast}$; we shall also use this superscript to denote the images of objects and morphisms of these Frobenioids in their birationalizations. Thus, if $A \in \mathrm{Ob}\left({ }^{\dagger} \mathcal{F}^{\circledast}\right)$, then $\mathcal{O}^{\times}\left(A^{\text {birat }}\right)$ may be naturally identified with the multiplicative group of nonzero elements of the number field [i.e., finite extension of $F_{\text {mod }}$ ] corresponding to $A$. In particular, by allowing $A$ to vary among the Frobenius-trivial objects [a notion which is category-theoretic - cf. [FrdI], Definition 1.2, (iv); [FrdI], Corollary 4.11, (iv); [FrdI], Theorem 6.4, (i); Remark 3.1.5 of the present paper] of ${ }^{\dagger} \mathcal{F}^{\circledast}$ that lie over Galois objects of ${ }^{\dagger} \mathcal{D}^{\circledast}$, we obtain a pair [i.e., consisting of a topological group acting continuously on a discrete abelian group]

$$
\pi_{1}\left({ }^{\dagger} \mathcal{D}^{\circledast}\right) \curvearrowright \widetilde{\mathcal{O}}^{\circledast \times}
$$

- which we consider up to the action by the "inner automorphisms of the pair" arising from conjugation by $\pi_{1}\left({ }^{\dagger} \mathcal{D}^{\circledast}\right)$. Write $\Phi_{\dagger \mathcal{F} \circledast}$ for the divisor monoid of the Frobenioid ${ }^{\dagger} \mathcal{F}^{\circledast}$ [which is category-theoretic - cf. [FrdI], Corollary 4.11, (iii); [FrdI], Theorem 6.4, (i); Remark 3.1.5 of the present paper]. Thus, for each $\mathfrak{p} \in \operatorname{Prime}\left(\Phi_{\dagger \mathcal{F} \circledast}(A)\right)$ [where we use the notation "Prime $(-)$ " as in [FrdI], §0], the natural homomorphism $\mathcal{O}^{\times}\left(A^{\text {birat }}\right) \rightarrow \Phi_{\dagger \mathcal{F} \circledast}(A)^{\text {gp }} \quad[$ cf. [FrdI], Proposition 4.4, (iii)] determines - i.e., by taking the inverse image via this homomorphism of [the union with $\{0\}$ of] the subset of $\Phi_{\dagger \mathcal{F} \circledast}(A)$ constituted by $\mathfrak{p}$ - a submonoid $\mathcal{O}_{\mathfrak{p}}^{\triangleright} \subseteq \mathcal{O}^{\times}\left(A^{\text {birat }}\right)$. That is to say, in more intuitive terms, this submonoid is the submonoid of integral elements of $\mathcal{O}^{\times}\left(A^{\text {birat }}\right)$ with respect to the valuation determined by $\mathfrak{p}$ of the number field corresponding to $A$. Write $\mathcal{O}_{\mathfrak{p}}^{\times} \subseteq \mathcal{O}_{\mathfrak{p}}^{\triangleright}$ for the submonoid of invertible elements. Thus, by allowing $A$ to vary among the Frobeniustrivial objects of ${ }^{\dagger} \mathcal{F}^{\circledast}$ that lie over Galois objects of ${ }^{\dagger} \mathcal{D}^{\circledast}$ and considering the way in which the natural action of $\operatorname{Aut}_{\dagger} \mathcal{F}^{\circledast}(A)$ on $\mathcal{O}^{\times}\left(A^{\text {birat }}\right)$ permutes the various submonoids $\mathcal{O}_{\mathfrak{p}}^{\triangleright}$, it follows that for each $\mathfrak{p}_{0} \in \operatorname{Prime}\left(\Phi_{\dagger \mathcal{F} \circledast}\left(A_{0}\right)\right)$, where $A_{0} \in \mathrm{Ob}\left({ }^{\dagger} \mathcal{F}^{\circledast}\right)$ lies over a terminal object of ${ }^{\dagger} \mathcal{D}^{\circledast}$, we obtain a closed subgroup [well-defined up to conjugation]

$$
\Pi_{\mathfrak{p}_{0}} \subseteq \pi_{1}\left({ }^{\dagger} \mathcal{D}^{\circledast}\right)
$$

by considering the elements of $\operatorname{Aut}_{+\mathcal{F} \circledast}(A)$ that fix the submonoid $\mathcal{O}_{\mathfrak{p}}^{\triangleright}$, for some system of $\mathfrak{p}$ 's lying over $\mathfrak{p}_{0}$. That is to say, in more intuitive terms, the subgroup $\Pi_{\mathfrak{p}_{0}}$ is simply the decomposition group associated to some $v \in \mathbb{V}_{\text {mod }}$. In particular, it follows that $\mathfrak{p}_{0}$ is nonarchimedean if and only if the $p$-cohomological dimension of $\Pi_{\mathfrak{p}_{0}}$ is equal to $2+1=3$ for infinitely many prime numbers $p$ [cf., e.g., [NSW], Theorem 7.1.8, (i)]. 
(v) We continue to use the notation of (iv). Let us write

$$
\pi_{1}\left({ }^{\dagger} \mathcal{D}^{\circledast}\right) \curvearrowright \quad{ }^{\dagger} \mathbb{M}^{\circledast}, \quad \pi_{1}^{\kappa-\mathrm{sol}}\left({ }^{\dagger} \mathcal{D}^{\circledast}\right) \curvearrowright \quad{ }^{\dagger} \mathbb{M}_{\mathrm{sol}}^{\circledast}, \quad{ }^{\dagger} \mathbb{M}_{\bmod }^{\circledast}
$$

for the pair $\pi_{1}\left({ }^{\dagger} \mathcal{D}^{\circledast}\right) \curvearrowright \widetilde{\mathcal{O}}^{\circledast \times}$ discussed in (iv) and its respective subsets [i.e., $\left.{ }^{\dagger} \mathbb{M}_{\mathrm{sol}}^{\circledast},{ }^{\dagger} \mathbb{M}_{\text {mod }}^{\circledast}\right]$ of $\pi_{1}^{\mathrm{rat} / \kappa \text {-sol }}\left({ }^{\dagger} \mathcal{D}^{\circledast}\right)$ -,$\pi_{1}^{\mathrm{rat}}\left({ }^{\dagger} \mathcal{D}^{\circledast}\right)$-invariants. We shall refer to a pair [i.e., consisting of a pseudo-monoid equipped with a continuous action by $\pi_{1}^{\text {rat }}\left({ }^{\dagger} \mathcal{D}^{\circledast}\right)$ ]

$$
\pi_{1}^{\mathrm{rat}}\left({ }^{\dagger} \mathcal{D}^{\circledast}\right) \curvearrowright{ }^{\dagger} \mathbb{M}_{\infty \kappa}^{\circledast} \quad\left(\text { respectively, } \pi_{1}^{\mathrm{rat}}\left({ }^{\dagger} \mathcal{D}^{\circledast}\right) \curvearrowright{ }^{\dagger} \mathbb{M}_{\infty \kappa \times}^{\circledast}\right)
$$

as an $\infty \kappa$-coric (respectively, $\infty \kappa \times$-coric) structure on ${ }^{\dagger} \mathcal{F}^{\circledast}$ if it is isomorphic [i.e., as a pair consisting of a pseudo-monoid equipped with a continuous action by $\left.\pi_{1}^{\text {rat }}\left({ }^{\dagger} \mathcal{D}^{\circledast}\right)\right]$ to the pair

$$
\pi_{1}^{\mathrm{rat}}\left({ }^{\dagger} \mathcal{D}^{\circledast}\right) \curvearrowright \mathbb{M}_{\infty \kappa}^{\circledast}\left({ }^{\dagger} \mathcal{D}^{\odot}\right) \quad \text { (respectively, } \pi_{1}^{\mathrm{rat}}\left({ }^{\dagger} \mathcal{D}^{\circledast}\right) \curvearrowright \mathbb{M}_{\infty \kappa \times}^{\circledast}\left({ }^{\dagger} \mathcal{D}^{\odot}\right) \text { ) }
$$

of (i). Thus, the $\pi_{1}^{\text {rat }}\left({ }^{\dagger} \mathcal{D}^{\circledast}\right)$-action that appears in an $\infty \kappa$-coric (respectively, $\infty \kappa \times$ coric) structure necessarily factors (respectively, does not factor) through the natural surjection $\pi_{1}^{\mathrm{rat}}\left({ }^{\dagger} \mathcal{D}^{\circledast}\right) \rightarrow \pi_{1}^{\kappa-\text { sol }}\left({ }^{\dagger} \mathcal{D}^{\circledast}\right)$ of (i). Suppose that we have been given an $\infty \kappa$-coric (respectively, $\infty \kappa \times$-coric) structure on ${ }^{\dagger} \mathcal{F}^{\circledast}$. If “(-)" is a [commutative] monoid, then let us write

$$
\boldsymbol{\mu}_{\widehat{\mathbb{Z}}}((-)) \stackrel{\text { def }}{=} \operatorname{Hom}(\mathbb{Q} / \mathbb{Z},(-))
$$

[cf. [AbsTopIII], Definition 3.1, (v); [AbsTopIII], Definition 5.1, (v)]; note that this notational convention also makes sense if "(-)" is a cyclotomic pseudo-monoid [cf. $\S 0]$. Also, let us write $\boldsymbol{\mu}_{\widehat{\mathbb{Z}}}^{\Theta}\left(\pi_{1}\left({ }^{\dagger} \mathcal{D}^{\odot}\right)\right)$ for the cyclotome " $\boldsymbol{\mu}_{\widehat{\mathbb{Z}}}\left(\Pi_{(-)}\right)$" of [AbsTopIII], Theorem 1.9, which we think of as being applied "via the $\Theta$-approach" [cf. Remark 3.1.2] to $\pi_{1}\left({ }^{\dagger} \mathcal{D}^{\odot}\right)$. Then let us observe that $\mathbb{M}_{\infty}^{\circledast} \kappa\left({ }^{\dagger} \mathcal{D}^{\odot}\right)$ (respectively, $\mathbb{M}_{\infty}^{\circledast} \kappa \times\left({ }^{\dagger} \mathcal{D}^{\odot}\right)$ ) is, in effect, constructed [cf. [AbsTopIII], Theorem 1.9, (d)] as a subset of

$$
\underset{H}{\varliminf_{H}} H^{1}\left(H, \boldsymbol{\mu}_{\widehat{\mathbb{Z}}}^{\Theta}\left(\pi_{1}\left({ }^{\dagger} \mathcal{D}^{\odot}\right)\right)\right)
$$

— where $H$ ranges over the open subgroups of $\pi_{1}^{\kappa \text {-sol }}\left({ }^{\dagger} \mathcal{D}^{\circledast}\right)$ (respectively, $\left.\pi_{1}^{\text {rat }}\left({ }^{\dagger} \mathcal{D}^{\circledast}\right)\right)$. On the other hand, consideration of Kummer classes [i.e., of the action of $\pi_{1}^{\kappa-\operatorname{sol}}\left({ }^{\dagger} \mathcal{D}^{\circledast}\right)$ (respectively, $\pi_{1}^{\text {rat }}\left({ }^{\dagger} \mathcal{D}^{\circledast}\right)$ ) on $N$-th roots of elements, for positive integers $\left.N\right]$ yields a natural injection of ${ }^{\dagger} \mathbb{M}_{\infty}^{\circledast} \kappa\left(\right.$ respectively, $\left.{ }^{\dagger} \mathbb{M}_{\infty \kappa \times}^{\circledast}\right)$ into

$$
\left.\varliminf_{H}^{\varliminf_{H}} H^{1}\left(H, \boldsymbol{\mu}_{\widehat{\mathbb{Z}}}\left({ }^{\dagger} \mathbb{M}_{\infty \kappa}^{\circledast}\right)\right) \quad \text { (respectively, } \underset{H}{\varliminf_{H}} H^{1}\left(H, \boldsymbol{\mu}_{\widehat{\mathbb{Z}}}\left({ }^{\dagger} \mathbb{M}_{\infty \kappa \times}^{\circledast}\right)\right)\right)
$$

— where $H$ ranges over the open subgroups of $\pi_{1}^{\kappa \text {-sol }}\left({ }^{\dagger} \mathcal{D}^{\circledast}\right)$ (respectively, $\left.\pi_{1}^{\text {rat }}\left({ }^{\dagger} \mathcal{D}^{\circledast}\right)\right)$, and we observe that the asserted injectivity follows immediately from the corresponding injectivity in the case of $\mathbb{M}_{\infty}^{\circledast} \kappa\left({ }^{\dagger} \mathcal{D}^{\odot}\right)$ (respectively, $\mathbb{M}_{\infty}^{\circledast} \kappa \times\left({ }^{\dagger} \mathcal{D}^{\odot}\right)$ ). In particular, it follows immediately, by considering divisors of zeroes and poles [cf. the definition of a " $\kappa$-coric function" given in Remark 3.1.7, (i)] associated to Kummer classes of rational functions as in [AbsTopIII], Proposition 1.6, (iii), from the elementary observation that, relative to the natural inclusion $\mathbb{Q} \hookrightarrow \widehat{\mathbb{Z}} \otimes \mathbb{Q}$,

$$
\mathbb{Q}_{>0} \bigcap \widehat{\mathbb{Z}}^{\times}=\{1\}
$$


that there exists a unique isomorphism of cyclotomes

$$
\left.\boldsymbol{\mu}_{\widehat{\mathbb{Z}}}^{\Theta}\left(\pi_{1}\left({ }^{\dagger} \mathcal{D}^{\odot}\right)\right) \stackrel{\sim}{\rightarrow} \boldsymbol{\mu}_{\widehat{\mathbb{Z}}}\left({ }^{\dagger} \mathbb{M}_{\infty}^{\circledast}\right) \quad \text { (respectively, } \boldsymbol{\mu}_{\widehat{\mathbb{Z}}}^{\Theta}\left(\pi_{1}\left({ }^{\dagger} \mathcal{D}^{\odot}\right)\right) \stackrel{\sim}{\rightarrow} \boldsymbol{\mu}_{\widehat{\mathbb{Z}}}\left({ }^{\dagger} \mathbb{M}_{\infty}^{\circledast} \kappa \times\right)\right)
$$

such that the resulting isomorphism between direct limits of cohomology modules as considered above induces an isomorphism

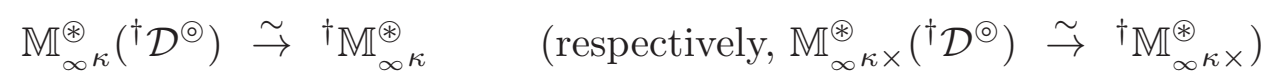

[i.e., of pseudo-monoids equipped with continuous actions by $\left.\pi_{1}^{\text {rat }}\left({ }^{\dagger} \mathcal{D}^{\circledast}\right)\right]$. In a similar vein, it follows immediately from the theory summarized in [AbsTopIII], Theorem 1.9, (d), that there exists a unique isomorphism of cyclotomes

$$
\boldsymbol{\mu}_{\widehat{\mathbb{Z}}}^{\Theta}\left(\pi_{1}\left({ }^{\dagger} \mathcal{D}^{\odot}\right)\right) \stackrel{\sim}{\rightarrow} \boldsymbol{\mu}_{\widehat{\mathbb{Z}}}\left({ }^{\dagger} \mathbb{M}^{\circledast}\right)
$$

such that the resulting isomorphism between direct limits of cohomology modules induces isomorphisms

$$
\mathbb{M}^{\circledast}\left({ }^{\dagger} \mathcal{D}^{\odot}\right) \stackrel{\sim}{\rightarrow}{ }^{\dagger} \mathbb{M}^{\circledast}, \quad \mathbb{M}_{\mathrm{sol}}^{\circledast}\left({ }^{\dagger} \mathcal{D}^{\odot}\right) \stackrel{\sim}{\rightarrow}{ }^{\dagger} \mathbb{M}_{\mathrm{sol}}^{\circledast}, \quad \mathbb{M}_{\mathrm{mod}}^{\circledast}\left({ }^{\dagger} \mathcal{D}^{\odot}\right) \stackrel{\sim}{\rightarrow}{ }^{\dagger} \mathbb{M}_{\bmod }^{\circledast}
$$

[i.e., of monoids equipped with continuous actions by $\left.\pi_{1}\left({ }^{\dagger} \mathcal{D}^{\circledast}\right)\right]$ in a fashion that is compatible with the integral submonoids " $\mathcal{O}_{\mathfrak{p}}^{\triangleright}$ " [cf. the discussion of (iv)], relative to the ring structure constructed in [AbsTopIII], Theorem 1.9, (e), on the domains of these isomorphisms. In particular, it follows immediately from the above discussion that

${ }^{\dagger} \mathcal{F}^{\circledast}$ always admits an $\infty \kappa$-coric (respectively, $\infty \kappa \times$-coric) structure, which is, moreover, unique up to a uniquely determined isomorphism [i.e., of pseudo-monoids equipped with continuous actions by $\left.\pi_{1}^{\mathrm{rat}}\left({ }^{\dagger} \mathcal{D}^{\circledast}\right)\right]$.

Thus, in the following, we shall regard, without further notice, this uniquely determined $\infty \kappa$-coric (respectively, $\infty \kappa \times$-coric) structure on ${ }^{\dagger} \mathcal{F}^{\circledast}$ as a collection of data that is naturally associated to ${ }^{\dagger} \mathcal{F}^{\circledast}$. Here, we observe that the various isomorphisms of the last few displays allow one to regard the pseudo-monoids ${ }^{\dagger} \mathbb{M}_{\infty}^{\circledast} \kappa$, $\dagger \mathbb{M}_{\infty}^{\circledast} \times \times$ as being related to the Frobenioid ${ }^{\dagger} \mathcal{F}^{\circledast}$ via ${ }^{\dagger} \mathbb{M}^{\circledast}$ [cf. the definition of ${ }^{\dagger} \mathbb{M}^{\circledast}$ at the beginning of the present $(\mathrm{v})$ ] and the morphisms

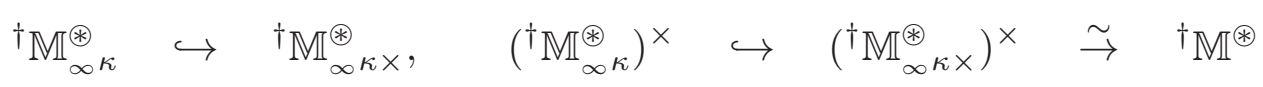

induced by the various isomorphisms of the last few displays, together with the corresponding inclusions/equalities

$$
\begin{gathered}
\mathbb{M}_{\infty \kappa}^{\circledast}\left({ }^{\dagger} \mathcal{D}^{\odot}\right) \subseteq \mathbb{M}_{\infty \kappa \times}^{\circledast}\left({ }^{\dagger} \mathcal{D}^{\odot}\right) \\
\left(\mathbb{M}_{\infty \kappa}^{\circledast}\left({ }^{\dagger} \mathcal{D}^{\odot}\right)\right)^{\times} \subseteq\left(\mathbb{M}_{\infty \kappa \times}^{\circledast}\left({ }^{\dagger} \mathcal{D}^{\odot}\right)\right)^{\times}=\mathbb{M}^{\circledast}\left({ }^{\dagger} \mathcal{D}^{\odot}\right)
\end{gathered}
$$

- where we use the superscript " $\times$ " to denote the subset of invertible elements of a pseudo-monoid [cf. the discussion of the initial portion of (i)]. Also, we shall write

$$
{ }^{\dagger} \mathbb{M}_{\kappa}^{\circledast} \subseteq{ }^{\dagger} \mathbb{M}_{\infty \kappa}^{\circledast}, \quad{ }^{\dagger} \mathbb{M}_{\kappa-\text { sol }}^{\circledast} \subseteq{ }^{\dagger} \mathbb{M}_{\infty \kappa \times}^{\circledast}
$$


for the respective "sub-pseudo-monoids" of $\pi_{1}^{\mathrm{rat}}\left({ }^{\dagger} \mathcal{D}^{\circledast}\right)$-, $\pi_{1}^{\mathrm{rat} / \kappa \text {-sol }}\left({ }^{\dagger} \mathcal{D}^{\circledast}\right)$-invariants. In this context, we observe further that it follows immediately from the discussion of Remark 3.1.7, (i), (ii), (iii) [cf. also [AbsTopII], Corollary 3.3, (iii), which is applicable in light of [AbsTopI], Example 4.8], and the theory summarized in [AbsTopIII], Theorem 1.9 [cf., especially, [AbsTopIII], Theorem 1.9, (a), (d), (e)], that

any $\infty_{\infty} \kappa \times$-coric structure $\pi_{1}^{\mathrm{rat}}\left({ }^{\dagger} \mathcal{D}^{\circledast}\right) \curvearrowright{ }^{\dagger} \mathbb{M}_{\infty}^{\circledast} \times$ on ${ }^{\dagger} \mathcal{F}^{\circledast}$ determines an associated $\infty \kappa$-coric structure

$$
\pi_{1}^{\mathrm{rat}}\left({ }^{\dagger} \mathcal{D}^{\circledast}\right) \curvearrowright \dagger \mathbb{M}_{\infty}^{\circledast} \subseteq{ }^{\dagger} \mathbb{M}_{\infty \kappa \times}^{\circledast}
$$

by considering the subset of elements for which the restriction of the associated Kummer class [as in the above discussion] to some [or, equivalently, every] subgroup of $\pi_{1}^{\text {rat }}\left({ }^{\dagger} \mathcal{D}^{\circledast}\right)$ that corresponds to an open subgroup of the decomposition group of some strictly critical point of $C_{F_{\bmod }}$ is a torsion element [i.e., corresponds to a root of unity],

and, moreover, that

the operation of restricting Kummer classes [as in the above discussion] arising from ${ }^{\dagger} \mathbb{M}_{\kappa}^{\circledast} \subseteq{ }^{\dagger} \mathbb{M}_{\infty \kappa}^{\circledast}$ to subgroups of $\pi_{1}^{\kappa \text {-sol }}\left({ }^{\dagger} \mathcal{D}^{\circledast}\right)$ that correspond to decomposition groups of non-critical $F_{\mathrm{mod}^{-}}, F_{\mathrm{sol}}$-valued points of $C_{F_{\text {mod }}}$ yields a functorial algorithm for reconstructing the monoids with $\pi_{1}^{\kappa-\mathrm{sol}}\left({ }^{\dagger} \mathcal{D}^{\circledast}\right)$-action ${ }^{\dagger} \mathbb{M}_{\text {mod }}^{\circledast},{ }^{\dagger} \mathbb{M}_{\text {sol }}^{\circledast}$, together with the field structure - and hence, in particular, the topologies determined by the valuations - on the union of ${ }^{\dagger} \mathbb{M}_{\text {mod }}^{\circledast},{ }^{\dagger} \mathbb{M}_{\text {sol }}^{\circledast}$ with $\{0\}$, from the $\infty \kappa$-coric structure associated to ${ }^{\dagger} \mathcal{F}^{\circledast}$.

A similar statement to the statement of the last display holds, if one makes the following substitutions:

$$
\begin{aligned}
& \text { " } \pi_{1}^{\kappa-\operatorname{sol}}\left({ }^{\dagger} \mathcal{D}^{\circledast}\right) " \rightsquigarrow “ \pi_{1}^{\mathrm{rat}}\left({ }^{\dagger} \mathcal{D}^{\circledast}\right) " ; \\
& \text { "F } \mathrm{mod}^{-}, F_{\mathrm{sol}^{-}} " \rightsquigarrow " \bar{F}-" ; \quad \text { "† } \mathbb{M}_{\mathrm{mod}}^{\circledast},{ }^{\dagger} \mathbb{M}_{\mathrm{sol}}^{\circledast} " \rightsquigarrow " \dagger \mathbb{M}^{\circledast} " .
\end{aligned}
$$

In particular, we obtain a purely category-theoretic construction, from the category $\dagger \mathcal{F}^{\circledast}$, of the natural bijection

$$
\operatorname{Prime}\left({ }^{\dagger} \mathcal{F}_{\bmod }^{\circledast}\right) \stackrel{\sim}{\rightarrow} \mathbb{V}_{\bmod }
$$

— where we write Prime $\left({ }^{\dagger} \mathcal{F}_{\bmod }^{\circledast}\right)$ for the set of primes [cf. [FrdI], §0] of the divisor monoid of ${ }^{\dagger} \mathcal{F}_{\text {mod }}^{\circledast}$; we think of $\mathbb{V}_{\text {mod }}$ as the set of $\pi_{1}\left({ }^{\dagger} \mathcal{D}^{\circledast}\right)$-orbits of $\overline{\mathbb{V}}\left({ }^{\dagger} \mathcal{D}^{\odot}\right)$. Now, in the notation of the discussion of (iv), suppose that $\mathfrak{p}$ is nonarchimedean [i.e., lies over a nonarchimedean $\left.\mathfrak{p}_{0}\right]$. Thus, $\mathfrak{p}$ determines a valuation, hence, in particular, a topology on the ring $\{0\} \cup \mathcal{O}^{\times}\left(A^{\text {birat }}\right)$. Write $\mathcal{O}_{\widehat{\mathfrak{p}}}^{\times}, \mathcal{O}_{\widehat{\mathfrak{p}}}^{\triangleright}$ for the respective completions, with respect to this topology, of the monoids $\mathcal{O}_{\mathfrak{p}}^{\times}, \mathcal{O}_{\mathfrak{p}}^{\triangleright}$. Then $\mathcal{O}_{\widehat{p}}^{\triangleright}$ may be identified with the multiplicative monoid of nonzero integral elements of the completion of the number field corresponding to $A$ at the prime of this number field determined by $\mathfrak{p}$. Thus, again by allowing $A$ to vary and considering the resulting system of ind-

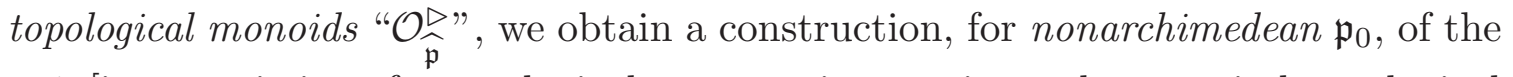
pair [i.e., consisting of a topological group acting continuously on an ind-topological monoid] 


$$
\Pi_{\mathfrak{p}_{0}} \curvearrowright \widetilde{\mathcal{O}}_{\widehat{p}_{0}}^{\triangleright}
$$

— which [since $\Pi_{\mathfrak{p}_{0}}$ is commensurably terminal in $\pi_{1}\left({ }^{\dagger} \mathcal{D}^{\circledast}\right)$ - cf., e.g., [AbsAnab], Theorem 1.1.1, (i)] we consider up to the action by the "inner automorphisms of the pair" arising from conjugation by $\Pi_{\mathfrak{p}_{0}}$. In the language of [AbsTopIII], §3, this pair is an "MLF-Galois TM-pair of strictly Belyi type" [cf. [AbsTopIII], Definition 3.1, (ii); [AbsTopIII], Remark 3.1.3].

(vi) Before proceeding, we observe that the discussion of (iv), (v) concerning ${ }^{\dagger} \mathcal{F}^{\circledast},{ }^{\dagger} \mathcal{D}^{\circledast}$ may also be carried out for ${ }^{\dagger} \mathcal{F}^{\odot},{ }^{\dagger} \mathcal{D}^{\odot}$. We leave the routine details to the reader.

(vii) Next, let us consider the index set $J$ of the capsule of $\mathcal{D}$-prime-strips ${ }^{\dagger} \mathfrak{D}_{J}$.

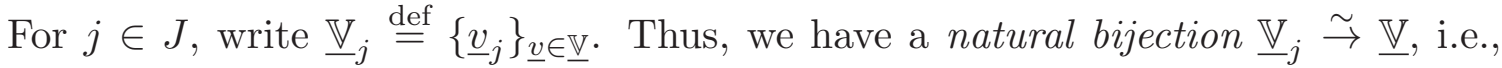
given by sending $\underline{v}_{j} \mapsto \underline{v}$. These bijections determine a "diagonal subset"

$$
\underline{\mathbb{V}}_{\langle J\rangle} \subseteq \underline{\mathbb{V}}_{J} \stackrel{\text { def }}{=} \prod_{j \in J} \underline{\mathbb{V}}_{j}
$$

— which also admits a natural bijection $\underline{\mathbb{V}}_{\langle J\rangle} \stackrel{\sim}{\rightarrow} \underline{\mathbb{V}}$. Thus, we obtain natural bijections

$$
\underline{\mathbb{V}}_{\langle J\rangle} \stackrel{\sim}{\rightarrow} \underline{\mathbb{V}}_{j} \stackrel{\sim}{\rightarrow} \operatorname{Prime}\left({ }^{\dagger} \mathcal{F}_{\mathrm{mod}}^{\circledast}\right) \stackrel{\sim}{\rightarrow} \mathbb{V}_{\mathrm{mod}}
$$

for $j \in J$. Write

$$
\begin{gathered}
\dagger \mathcal{F}_{\langle J\rangle}^{\circledast} \stackrel{\text { def }}{=}\left\{{ }^{\dagger} \mathcal{F}_{\text {mod }}^{\circledast}, \underline{\mathbb{V}}_{\langle J\rangle} \stackrel{\sim}{\rightarrow} \operatorname{Prime}\left({ }^{\dagger} \mathcal{F}_{\text {mod }}^{\circledast}\right)\right\} \\
\dagger \mathcal{F}_{j}^{\circledast} \stackrel{\text { def }}{=}\left\{\mathcal{F}_{\text {mod }}^{\circledast}, \underline{\mathbb{V}}_{j} \stackrel{\sim}{\rightarrow} \operatorname{Prime}\left({ }^{\dagger} \mathcal{F}_{\text {mod }}^{\circledast}\right)\right\}
\end{gathered}
$$

for $j \in J$. That is to say, we think of ${ }^{\dagger} \mathcal{F}_{j}^{\circledast}$ as a copy of $\dagger \mathcal{F}_{\bmod }^{\circledast}$ "situated on" the constituent labeled $j$ of the capsule $\dagger \mathfrak{D}_{J}$; we think of ${ }^{\dagger} \mathcal{F}_{\langle J\rangle}^{\circledast}$ as a copy of ${ }^{\dagger} \mathcal{F}_{\bmod }^{\circledast}$ "situated in a diagonal fashion on" all the constituents of the capsule ${ }^{\dagger} \mathfrak{D}_{J}$. Thus, we have a natural embedding of categories

$$
{ }^{\dagger} \mathcal{F}_{\langle J\rangle}^{\circledast} \hookrightarrow{ }^{\dagger} \mathcal{F}_{J}^{\circledast} \stackrel{\text { def }}{=} \prod_{j \in J}{ }^{\dagger} \mathcal{F}_{j}^{\circledast}
$$

- where, by abuse of notation, we write ${ }^{\dagger} \mathcal{F}_{\langle J\rangle}^{\circledast}$ for the underlying category of [i.e., the first member of the pair $]^{\dagger} \mathcal{F}_{\langle J\rangle}^{\circledast}$. Here, we remark that we do not regard the category ${ }^{\dagger} \mathcal{F}_{J}^{\circledast}$ as being equipped with a Frobenioid structure. Write

$$
{ }^{\dagger} \mathcal{F}_{j}^{\circledast \mathbb{R}} ; \quad \dagger \mathcal{F}_{\langle J\rangle}^{\circledast \mathbb{R}} ; \quad \dagger \mathcal{F}_{J}^{\circledast \mathbb{R}} \stackrel{\text { def }}{=} \prod_{j \in J}{ }^{\dagger} \mathcal{F}_{j}^{\circledast \mathbb{R}}
$$

for the respective realifications [or product of the underlying categories of the realifications] of the corresponding Frobenioids whose notation does not contain a superscript " $\mathbb{R}$ ". [Here, we recall that the theory of realifications of Frobenioids is discussed in [FrdI], Proposition 5.3.] 

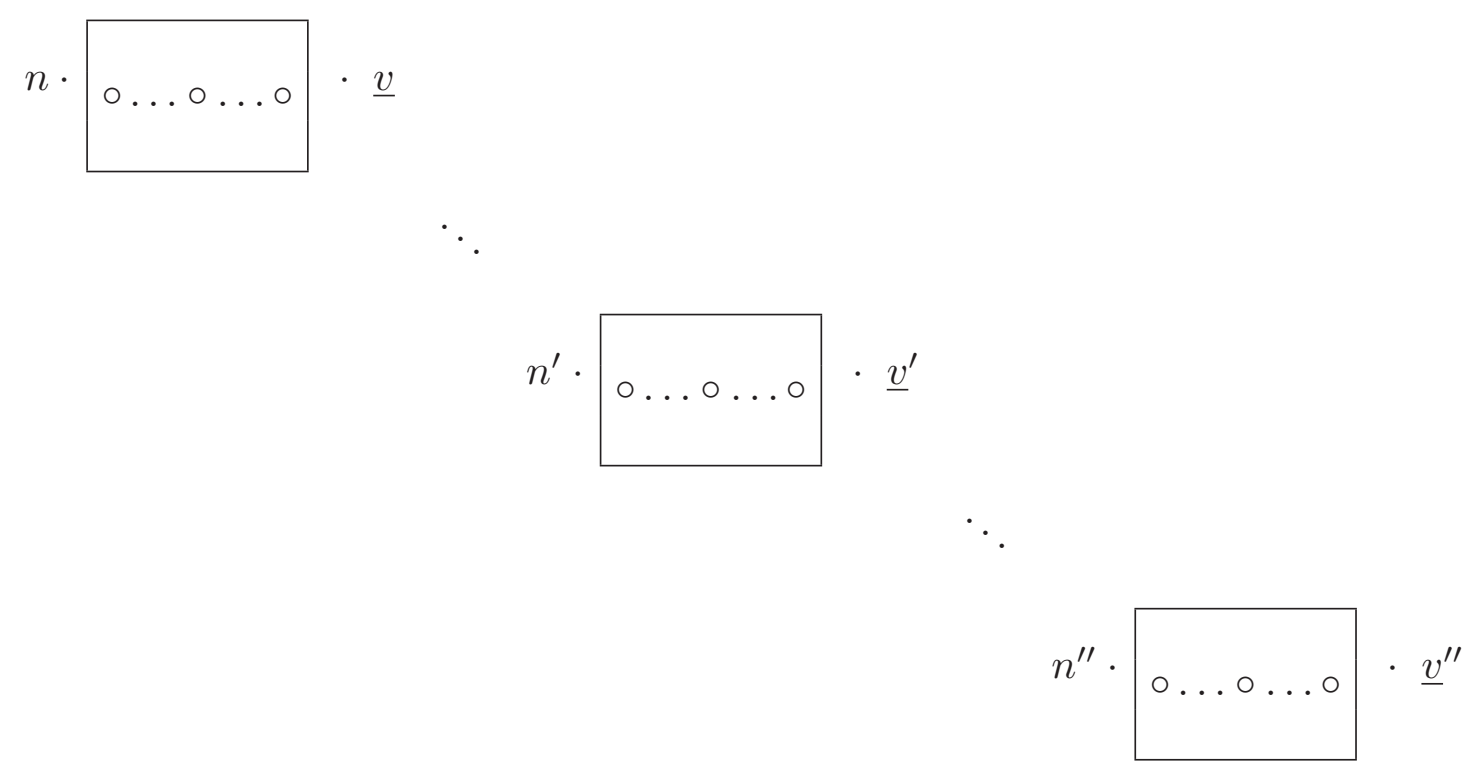

Fig. 5.1: Constant distribution

Remark 5.1.1. Thus, ${ }^{\dagger} \mathcal{F}_{\langle J\rangle}^{\circledast}$ may be thought of as the Frobenioid associated to divisors on $\underline{\mathbb{V}}_{J}$ [i.e., finite formal sums of elements of this set with coefficients in $\mathbb{Z}$ or $\mathbb{R}]$ whose dependence on $j \in J$ is constant - that is to say, divisors corresponding to "constant distributions" on $\underline{\mathbb{V}}_{J}$. Such constant distributions are depicted in Fig. 5.1 above. On the other hand, the product of [underlying categories of] Frobenioids $\dagger \mathcal{F}_{J}^{\circledast}$ may be thought of as a sort of category of "arbitrary distributions" on $\underline{\mathbb{V}}_{J}$, i.e., divisors on $\underline{\mathbb{V}}_{J}$ whose dependence on $j \in J$ is arbitrary.

Remark 5.1.2. The constructions of Example 5.1 manifestly only require the $\mathcal{D}$-NF-bridge portion ${ }^{\dagger} \phi_{*}^{\mathrm{NF}}$ of the $\mathcal{D}$ - $\Theta \mathrm{NF}$-Hodge theater ${ }^{\dagger} \mathcal{H} \mathcal{T}^{\mathcal{D}-\Theta N F}$.

Remark 5.1.3. In the context of the discussion of Example 5.1, (v), (vi), we note that unlike the case with ${ }^{\dagger} \mathcal{F}^{\odot},{ }^{\dagger} \mathcal{F}^{\circledast},{ }^{\dagger} \mathbb{M}^{\circledast},{ }^{\dagger} \mathbb{M}_{\text {sol }}^{\circledast},{ }^{\dagger} \mathbb{M}_{\infty \kappa}^{\circledast},{ }^{\dagger} \mathbb{M}_{\infty}^{\circledast} \kappa \times$, or ${ }^{\dagger} \mathbb{M}_{\kappa \text {-sol }}^{\circledast}$, one cannot perform Kummer theory [cf. [FrdII], Definition 2.1, (ii)] with ${ }^{\dagger} \mathcal{F}_{\text {mod }}^{\circledast},{ }^{\dagger} \mathbb{M}_{\text {mod }}^{\circledast}$, or ${ }^{\dagger} \mathbb{M}_{\kappa}^{\circledast}$. [That is to say, in more concrete terms, [unlike the case with $\bar{F}^{\times}, F_{\text {sol }}^{\times}$, or $\infty \kappa-/ \infty \kappa \times$-coric rational functions] it is not necessarily the case that elements of $F_{\text {mod }}^{\times}$or $\kappa$-coric rational functions admit $N$-th roots, for $N$ a nonnegative integer!] The fact that one can perform Kummer theory with ${ }^{\dagger} \mathcal{F}^{\odot},{ }^{\dagger} \mathcal{F}^{\circledast}$, or ${ }^{\dagger} \mathbb{M}^{\circledast}$ implies that $\dagger \mathbb{M}^{\circledast}$ equipped with its natural $\pi_{1}\left({ }^{\dagger} \mathcal{D}^{\odot}\right)$-action, as well as the "birational monoid portions" of ${ }^{\dagger} \mathcal{F}^{\odot}$ or $^{\dagger} \mathcal{F}^{\circledast}$, satisfy various strong rigidity properties [cf. Corollary 5.3, (i), below]. For instance, these rigidity properties allow one to recover the additive structure on [the union with $\{0\}$ of] ${ }^{\dagger} \mathbb{M}^{\circledast}$ equipped with its natural $\pi_{1}\left({ }^{\dagger} \mathcal{D}^{\odot}\right)$-action [cf. the discussion of Example 5.1, (v), (vi)]. That is to say,

the additive structure - or, equivalently, ring/field structure - on [the union with $\{0\}$ of] the "birational monoid portion" of $\dagger \mathcal{F}_{\bmod }^{\circledast}$ may only be recovered if one is given the additional datum consisting of the natural embedding ${ }^{\dagger} \mathcal{F}_{\bmod }^{\circledast} \hookrightarrow^{\dagger} \mathcal{F}^{\circledast}[$ cf. Example 5.1, (iii)]. 
Put another way, if one only considers the category ${ }^{\dagger} \mathcal{F}_{\text {mod }}^{\circledast}$ without the embedding ${ }^{\dagger} \mathcal{F}_{\text {mod }}^{\circledast} \hookrightarrow{ }^{\dagger} \mathcal{F}^{\circledast}$, then ${ }^{\dagger} \mathcal{F}_{\text {mod }}^{\circledast}$ is subject to a "Kummer black-out" — one consequence of which is that there is no way to recover the additive structure on the "birational monoid portion" of ${ }^{\dagger} \mathcal{F}_{\text {mod }}^{\circledast}$ [cf. also Remark 5.1.5 below]. In subsequent discussions, we shall refer to these observations concerning ${ }^{\dagger} \mathcal{F}^{\odot},{ }^{\dagger} \mathcal{F}^{\circledast},{ }^{\dagger} \mathbb{M}^{\circledast},{ }^{\dagger} \mathbb{M}_{\text {sol }}^{\circledast}$, $\dagger \mathbb{M}_{\infty}^{\circledast},{ }^{\circledast} \mathbb{M}_{\infty \kappa \times}^{\circledast},{ }^{\dagger} \mathbb{M}_{\kappa \text {-sol }}^{\circledast},{ }^{\dagger} \mathcal{F}_{\text {mod }}^{\circledast},{ }^{\dagger} \mathbb{M}_{\text {mod }}^{\circledast}$, and ${ }^{\dagger} \mathbb{M}_{\kappa}^{\circledast}$ by saying that ${ }^{\dagger} \mathcal{F}^{\odot},{ }^{\dagger} \mathcal{F}^{\circledast},{ }^{\dagger} \mathbb{M}^{\circledast}$, $\dagger \mathbb{M}_{\text {sol }}^{\circledast},{ }^{\dagger} \mathbb{M}_{\infty}^{\circledast},{ }^{\circledast} \mathbb{M}_{\infty \kappa \times}^{\circledast}$, and ${ }^{\dagger} \mathbb{M}_{\kappa \text {-sol }}^{\circledast}$ are Kummer-ready, whereas ${ }^{\dagger} \mathcal{F}_{\text {mod }}^{\circledast},{ }^{\dagger} \mathbb{M}_{\text {mod }}^{\circledast}$, and ${ }^{\dagger} \mathbb{M}{ }_{\kappa}^{\circledast}$ are Kummer-blind. In particular, the various copies of [and products of copies of $]{ }^{\dagger} \mathcal{F}_{\text {mod }}^{\circledast}$ - i.e., ${ }^{\dagger} \mathcal{F}_{j}^{\circledast},{ }^{\dagger} \mathcal{F}_{\langle J\rangle}^{\circledast},{ }^{\dagger} \mathcal{F}_{J}^{\circledast}$ - considered in Example 5.1, (vii), are also Kummer-blind.

Remark 5.1.4. The various functorial reconstruction algorithms for number fields discussed in Example 5.1 are based on the technique of Belyi cuspidalization, as applied in the theory of [AbsTopIII], §1. At a more concrete level, this theory of [AbsTopIII], §1, may be thought of revolving around the point of view that

elements of number fields may be expressed geometrically by means of Belyi maps.

Moreover, if one thinks of such elements of number fields as "analytic functions", then the remainder of the theory of [AbsTopIII] [cf., especially, [AbsTopIII], §5] may be thought of as a sort of theory of

"analytic continuation" of the "analytic functions" constituted by elements of number fields in the context of the various logarithm maps at the various localizations of these number fields.

This point of view is very much in line with the points of view discussed in Remarks 4.3.2, 4.3.3. Moreover, the geometric representation of elements of number fields via Belyi maps [i.e., whose existence may be regarded as a reflection of the hyperbolic nature of the projective line minus three points] is reminiscent of indeed, may perhaps be regarded as an arithmetic analogue of - the "categories of hyperbolic analytic continuations", i.e., of copies of the upper half-plane regarded as equipped with their natural hyperbolic metrics, discussed in the "Motivating Example" given in the Introduction to [GeoAnbd]. Here, it is perhaps of interest to note that the inequalities " $\leq 1$ " satisfied by the derivatives [i.e., with respect to the respective Poincaré metrics] of the holomorphic maps that appear in this "Motivating Example" in [GeoAnbd] are reminiscent of the monotonically decreasing nature of the various "degrees" - i.e., over $\mathbb{Q}$ of the ramification locus of the endomorphisms of the projective line over $\mathbb{Q}$ - that appear in the construction of Belyi maps [where we recall that this monotonic decreasing of degrees is the key observation that allows one to obtain Belyi maps which are unramified over the projective line minus three points].

Remark 5.1.5. Although the phenomenon of $\kappa$-sol-conjugate synchronization discussed in the final portion of Example 5.1, (i), will not play as central a role in the present series of papers as the conjugate synchronization of local Galois groups that will be discussed in [IUTchII], [IUTchIII], it has the following interesting consequence: 
The Kummer theory of

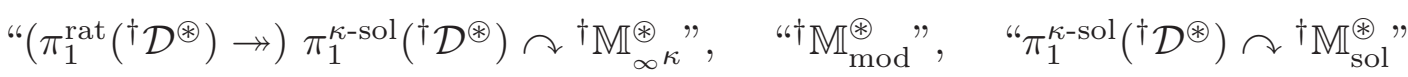

$$
\begin{aligned}
& \text { - i.e., of the abstract analogues of " } \infty \kappa \text {-coric functions", " } F_{\bmod }^{\times} \text {", and } \\
& \text { " } F_{\text {sol }}^{\times} \text {" as in Remark 3.1.7, (iii) - that was discussed in Example 5.1, } \\
& \text { (v), may be performed in a fashion that is compatible without any } \\
& \text { conjugacy indeterminacies with the poly-action of }\left(\mathrm{Aut}^{S L}\left({ }^{\dagger} \mathcal{D}^{\odot}\right) \rightarrow\right) \\
& \mathbb{F}_{l}^{*} \text {. }
\end{aligned}
$$

Here, we pause, however, to make the following observation: At first glance, it may appear as though the analogue obtained by Uchida of the Neukirch-Uchida theorem for maximal solvable quotients of absolute Galois groups of number fields [reviewed, for instance, in [GlSol], §3] — or, perhaps, some future mono-anabelian version of this result of Uchida - may be applied, in the context of the " $\kappa$-sol-Kummer theory" just discussed, to reconstruct the ring structure on the number fields involved [i.e., in the fashion of Example 5.1, (v)]. In fact, however,

such a "solvable-Uchida-type" reconstruction is, in effect, meaningless from the point of view of the theory of the present series of papers since it is fundamentally incompatible with the localization opera-

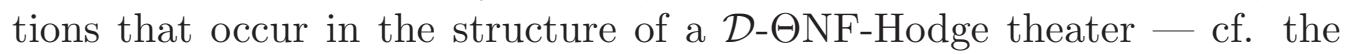
discussion of Remarks 4.3.1, 4.3.2.

Indeed, such a compatibility with localization would imply that the reconstruction of the ring structure may somehow be "restricted" to the absolute Galois groups of completions at nonarchimedean primes of a number field, i.e., in contradiction to the well-known fact that absolute Galois groups of such completions at nonarchimedean primes admit automorphisms that do not arise from field automorphisms [cf., e.g., [NSW], the Closing Remark preceding Theorem 12.2.7]. Finally, we note that this incompatibility of "solvable-Uchida-type" reconstructions of ring structures with the theory of the present series of papers is also interesting in the context of the point of view discussed in Remark 5.1.4: Suppose, for instance, that it was the case that the outer action of the absolute Galois group of a number field on the geometric fundamental group of a hyperbolic curve over the number field in fact factors through the maximal solvable quotient of the absolute Galois group. Then it is conceivable that some sort of version of the mono-anabelian theory of [AbsTopIII], $\S 1$, for extensions of such a maximal solvable quotient by the geometric fundamental group under consideration could be applied in the theory of the present series of papers to give a reconstruction of the ring structure of a number field that only requires the use of such extensions and is, moreover, compatible with the localization operations that occur in the various types of "Hodge theaters" that appear in the theory of the present series of papers - a state of affairs that would be fundamentally at odds with a quite essential portion of the "spirit" of the theory of the present series of papers, namely, the point of view of "dismantling the two underlying combinatorial dimensions of a ring". In fact, however,

the outer action referred to above does not admit such a "solvable factorization".

Indeed, the nonexistence of such a "solvable factorization" is a formal consequence of the the Galois injectivity result discussed in [NodNon], Theorem C - a result that depends, in an essential way, on the theory of Belyi maps. Put another way, 
Belyi maps not only play the role of allowing one to perform the sort of "arithmetic analytic continuation via Belyi cuspidalizations" [i.e., discussed in Remark 5.1.4] that is of central importance in the theory of the present series of papers, but also play the role of assuring one that such "arithmetic analytic continuations" cannot be extended to the case of extensions associated to "solvable factorizations" of outer actions of the sort just discussed.

\section{Definition 5.2.}

(i) We define a holomorphic Frobenioid-prime-strip, or $\mathcal{F}$-prime-strip, [relative to the given initial $\Theta$-data] to be a collection of data

$$
\ddagger \mathfrak{F}=\left\{{ }^{\ddagger} \mathcal{F}_{\underline{v}}\right\}_{\underline{v} \in \underline{\mathbb{V}}}
$$

that satisfies the following conditions: (a) if $\underline{v} \in \underline{\mathbb{V}}^{\text {non }}$, then ${ }^{\ddagger} \mathcal{F}_{\underline{v}}$ is a category ${ }^{\ddagger} \mathcal{C}_{\underline{v}}$ which admits an equivalence of categories ${ }^{\ddagger} \mathcal{C}_{\underline{v}} \stackrel{\sim}{\rightarrow} \mathcal{C}_{\underline{v}}$ [where $\mathcal{C}_{\underline{v}}$ is as in Examples 3.2, (iii); 3.3, (i)]; (b) if $\underline{v} \in \underline{\mathbb{V}}^{\text {arc }}$, then ${ }^{\ddagger} \mathcal{F}_{\underline{v}}=\left({ }^{\ddagger} \mathcal{C}_{\underline{v}},{ }^{\ddagger} \overline{\mathcal{D}}_{\underline{v}},{ }^{\ddagger} \kappa_{\underline{v}}\right)$ is a collection of data consisting of a category, an Aut-holomorphic orbispace, and a Kummer structure such that there exists an isomorphism of collections of data $\ddagger \mathcal{F}_{\underline{\underline{v}}} \underset{\sim}{\rightarrow} \underline{\underline{\mathcal{F}}} \underline{\underline{v}}=\left(\mathcal{C}_{\underline{v}}, \mathcal{D}_{\underline{v}}, \kappa_{\underline{v}}\right)$ [where $\underline{\underline{\mathcal{F}}}_{\underline{v}}$ is as in Example 3.4, (i)].

(ii) We define a mono-analytic Frobenioid-prime-strip, or $\mathcal{F}^{\vdash}$-prime-strip, [relative to the given initial $\Theta$-data] to be a collection of data

$$
{ }^{\ddagger} \mathfrak{F}^{\vdash}=\left\{{ }^{\ddagger} \mathcal{F}_{\underline{v}}^{\vdash}\right\}_{\underline{v}} \in \underline{\mathbb{V}}
$$

that satisfies the following conditions: (a) if $\underline{v} \in \underline{\mathbb{V}}^{\text {non }}$, then ${ }^{\ddagger} \mathcal{F}_{\underline{v}}^{\vdash}$ is a split Frobenioid, whose underlying Frobenioid we denote by ${ }^{\ddagger} \mathcal{C}_{\underline{v}}^{\vdash}$, which admits an isomorphism $\ddagger \mathcal{F}_{\underline{v}}^{\vdash} \stackrel{\sim}{\rightarrow} \mathcal{F}_{\underline{v}}^{\vdash}\left[\right.$ where $\mathcal{F}_{\underline{v}}^{\vdash}$ is as in Examples 3.2 , (v); 3.3, (i)]; (b) if $\underline{v} \in \underline{\mathbb{V}}^{\text {arc }}$, then $\ddagger \mathcal{F}_{\underline{v}}^{\leftarrow}$ is a triple of data, consisting of a Frobenioid ${ }^{\ddagger} \mathcal{C}_{\underline{v}}^{\vdash}$, an object of $\mathbb{T M}^{\vdash}$, and a splitting of the Frobenioid, such that there exists an isomorphism of collections of data $\mathcal{F}_{\underline{\underline{v}}}^{\vdash} \stackrel{\sim}{\rightarrow} \mathcal{F}_{\underline{v}}^{\vdash}\left[\right.$ where $\mathcal{F}_{\underline{v}}^{\vdash}$ is as in Example 3.4, (ii)].

(iii) A morphism of $\mathcal{F}$ - (respectively, $\mathcal{F}^{\vdash}$-) prime-strips is defined to be a collection of isomorphisms, indexed by $\underline{\mathbb{V}}$, between the various constituent objects of the prime-strips. Following the conventions of $\S 0$, one thus has notions of capsules of $\mathcal{F}$ - (respectively, $\mathcal{F}^{\vdash}$-) and morphisms of capsules of $\mathcal{F}$ - (respectively, $\mathcal{F}^{\vdash}$-) prime-strips.

(iv) We define a globally realified mono-analytic Frobenioid-prime-strip, or $\mathcal{F}^{\Vdash}$ prime-strip, [relative to the given initial $\Theta$-data] to be a collection of data

$$
{ }^{\ddagger} \mathfrak{F}^{\Vdash}=\left({ }^{\ddagger} \mathcal{C}^{\Vdash}, \operatorname{Prime}\left({ }^{\ddagger} \mathcal{C}^{\Vdash}\right) \stackrel{\sim}{\rightarrow} \underline{\mathbb{V}},{ }^{\ddagger} \mathfrak{F},\left\{{ }^{\ddagger} \rho_{\underline{v}}\right\}_{\underline{v} \in \underline{\mathbb{V}}}\right)
$$

that satisfies the following conditions: (a) $\mathcal{C}^{\Vdash}$ is a category [which is, in fact, equipped with a Frobenioid structure] that is isomorphic to the category $\mathcal{C}_{\text {mod }}^{\Vdash}$ of 
Example 3.5, (i); (b) "Prime(-)" is defined as in the discussion of Example 3.5, (i); (c) Prime $\left({ }^{\ddagger} \mathcal{C}^{\Vdash}\right) \stackrel{\sim}{\rightarrow} \underline{\mathbb{V}}$ is a bijection of sets; (d) ${ }^{\ddagger} \mathfrak{F}^{\vdash}=\left\{{ }^{\ddagger} \mathcal{F}_{\underline{v}}^{\vdash}\right\}_{\underline{v}} \in \underline{\mathbb{V}}$ is an $\mathcal{F}^{\vdash}$-prime-

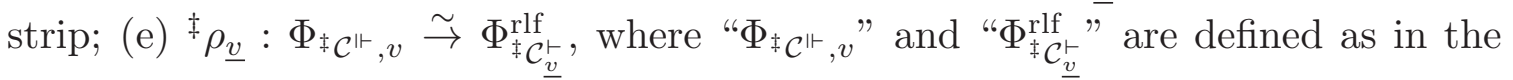
discussion of Example 3.5, (i), is an isomorphism of topological monoids [both of which are, in fact, isomorphic to $\mathbb{R}_{\geq 0}$ ]; (f) the collection of data in the above display is isomorphic to the collection of data $\mathfrak{F}_{\text {mod }}^{\Vdash}$ of Example 3.5, (ii). A morphism of $\mathcal{F}^{\Vdash}$-prime-strips is defined to be an isomorphism between collections of data as discussed above. Following the conventions of $\S 0$, one thus has notions of capsules of $\mathcal{F}^{\Vdash}$-prime-strips and morphisms of capsules of $\mathcal{F}^{\Vdash}$-prime-strips.

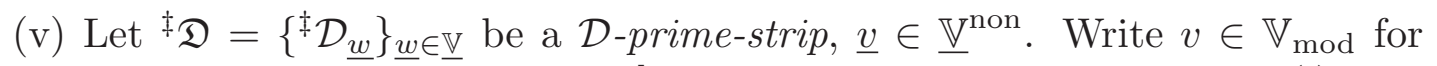
the valuation determined by $\underline{v}$. Then [cf. the discussion of Example 5.1, (i); Remark 3.1.7, (i)] one may construct group-theoretically from $\pi_{1}\left({ }^{\ddagger} \mathcal{D}_{\underline{v}}\right)$, in a functorial fashion, a profinite group corresponding to " $C_{v}$ " [cf. the algorithms of [AbsTopII], Corollary 3.3, (i), which are applicable in light of [AbsTopI], Example 4.8; [AbsTopIII], Theorem 1.9], which contains $\pi_{1}\left({ }^{\ddagger} \mathcal{D}_{\underline{v}}\right)$ as an open subgroup; we write ${ }^{\ddagger} \mathcal{D}_{v}$ for $\mathcal{B}(-)^{0}$ of this profinite group, so we obtain a natural morphism

$$
{ }^{\ddagger} \mathcal{D}_{\underline{v}} \rightarrow{ }^{\ddagger} \mathcal{D}_{v}
$$

- i.e., a "category-theoretic version" of the natural morphism of hyperbolic orbicurves $\underline{\underline{X}}_{\underline{v}}=\underline{X}_{K} \times_{K} K_{\underline{v}} \rightarrow C_{v}$ if $\underline{v} \in \underline{\mathbb{V}}^{\text {bad }}$, or $\underline{X}_{\underline{X}_{\underline{v}}}=\underline{X}_{K} \times_{K} K_{\underline{v}} \rightarrow C_{v}$ if $\underline{v} \in \underline{\mathbb{V}}^{\text {good }}$. Next, let us observe [cf. Remark 3.1.7, (i); the construction of the Belyi cuspidalizations of [AbsTopIII], Theorem 1.9, (a), and of the field " $K_{Z_{\mathrm{NF}}^{\times}}$" of [AbsTopIII], Theorem $1.9,(\mathrm{~d}),(\mathrm{e})]$ that one may construct group-theoretically from $\pi_{1}\left({ }^{\ddagger} \mathcal{D}_{\underline{v}}\right)$, in a functorial fashion, an isomorph

$$
\pi_{1}^{\mathrm{rat}}\left({ }^{\ddagger} \mathcal{D}_{v}\right) \quad\left(\rightarrow \pi_{1}\left({ }^{\ddagger} \mathcal{D}_{v}\right)\right)
$$

of the étale fundamental group [i.e., equipped with its natural surjection to $\pi_{1}\left({ }^{\ddagger} \mathcal{D}_{v}\right)$ and well-defined up to inner automorphisms determined by elements of the kernel of this natural surjection] of the scheme obtained by base-changing to $\left(F_{\bmod }\right)_{v}$ the generic point of $C_{F_{\mathrm{mod}}}$. Next, let us recall [cf. [AbsTopIII], Corollary 1.10, (b), (c), (d), $\left(\mathrm{d}^{\prime}\right)$ ] that one may construct group-theoretically from $\pi_{1}\left({ }^{\ddagger} \mathcal{D}_{\underline{v}}\right)$, in a functorial fashion, an ind-topological monoid [which is naturally isomorphic to $\mathcal{O}_{F_{\underline{v}}}^{\triangleright}$ ]

$$
\mathbb{M}_{v}\left({ }^{\ddagger} \mathcal{D}_{\underline{v}}\right)
$$

equipped with its natural $\pi_{1}\left({ }^{\ddagger} \mathcal{D}_{v}\right)$-action, as well as isomorphs of the pseudomonoids of $\kappa-, \infty^{\kappa-}$, and $\infty_{\infty} \kappa \times$-coric rational functions associated to $C_{v}$ [cf. the discussion of Remark 3.1.7, (i), (ii); [AbsTopII], Corollary 3.3, (iii), which is applicable in light of [AbsTopI], Example 4.8; [AbsTopIII], Theorem 1.9, (a), (d), (e); [AbsTopIII], Corollary 1.10, (d), (d')] — which we shall denote

$$
\mathbb{M}_{\kappa v}\left({ }^{\ddagger} \mathcal{D}_{\underline{v}}\right), \quad \mathbb{M}_{\infty \kappa v}\left({ }^{\ddagger} \mathcal{D}_{\underline{v}}\right), \quad \mathbb{M}_{\infty \kappa \times v}\left({ }^{\ddagger} \mathcal{D}_{\underline{v}}\right)
$$

— equipped with their natural $\pi_{1}^{\text {rat }}\left({ }^{\ddagger} \mathcal{D}_{v}\right)$-actions. Thus, $\mathbb{M}_{\kappa v}\left({ }^{\ddagger} \mathcal{D}_{\underline{v}}\right)$ may be identified with the subset of $\pi_{1}^{\text {rat }}\left({ }^{\ddagger} \mathcal{D}_{v}\right)$-invariants of $\mathbb{M}_{\infty} \kappa v\left({ }^{\ddagger} \mathcal{D}_{\underline{v}}\right)$, and if we use the 
superscript " $\times$ " to denote the subset of invertible elements of a pseudo-monoid, then] $\mathbb{M}_{v}\left({ }^{\ddagger} \mathcal{D}_{\underline{v}}\right)^{\times}$may be identified with $\mathbb{M}_{\infty \kappa \times v}\left({ }^{\ddagger} \mathcal{D}_{\underline{v}}\right)^{\times}$.

(vi) We continue to use the notation of $(\mathrm{v})$. Suppose further that $\ddagger \mathfrak{F}=$ $\left\{{ }^{\ddagger} \mathcal{F}_{\underline{w}}\right\}_{\underline{w} \in \mathbb{V}}$ is an $\mathcal{F}$-prime-strip whose associated $\mathcal{D}$-prime-strip [cf. Remark 5.2.1,

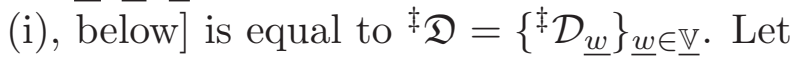

$$
\pi_{1}\left({ }^{\ddagger} \mathcal{D}_{v}\right) \curvearrowright{ }^{\ddagger} \mathbb{M}_{v}
$$

be an ind-topological monoid equipped with a continuous action by $\pi_{1}\left({ }^{\ddagger} \mathcal{D}_{v}\right)$ that is isomorphic [i.e., as an ind-topological monoid equipped with a continuous action by $\left.\pi_{1}\left({ }^{\ddagger} \mathcal{D}_{v}\right)\right]$ to the pair $\pi_{1}\left({ }^{\ddagger} \mathcal{D}_{v}\right) \curvearrowright \mathbb{M}_{v}\left({ }^{\ddagger} \mathcal{D}_{\underline{v}}\right)$ constructed in $(\mathrm{v})$. One may regard such a pair $\pi_{1}\left({ }^{\ddagger} \mathcal{D}_{v}\right) \curvearrowright \mathbb{M}_{v}$ as being related to the Frobenioid ${ }^{\ddagger} \mathcal{F}_{\underline{v}}$ [cf. (i), (a)] via the unique isomorphism corresponding to the identity automorphism of $\ddagger \mathfrak{D}=$ $\left\{{ }^{\ddagger} \mathcal{D}_{\underline{w}}\right\}_{\underline{w} \in \underline{\mathbb{V}}}$ [cf. Corollary 5.3, (ii), below] between ${ }^{\ddagger} \mathcal{F}_{\underline{v}}$ and the $p_{\underline{\underline{v}}}$-adic Frobenioid determined [cf. Remark 3.3.2] by the pair

$$
\pi_{1}\left({ }^{\ddagger} \mathcal{D}_{\underline{v}}\right) \curvearrowright{ }^{\ddagger} \mathbb{M}_{v}
$$

obtained by restricting the action of the pair $\pi_{1}\left({ }^{\ddagger} \mathcal{D}_{v}\right) \curvearrowright \mathbb{M}_{v}$ to the open subgroup $\pi_{1}\left({ }^{\ddagger} \mathcal{D}_{\underline{v}}\right) \subseteq \pi_{1}\left({ }^{\ddagger} \mathcal{D}_{v}\right)[\mathrm{cf} .(\mathrm{v})]$. We shall refer to a pair [i.e., consisting of a pseudomonoid equipped with a continuous action by $\left.\pi_{1}^{\text {rat }}\left({ }^{\ddagger} \mathcal{D}_{v}\right)\right]$

$$
\pi_{1}^{\mathrm{rat}}\left({ }^{\ddagger} \mathcal{D}_{v}\right) \curvearrowright \quad{ }^{\ddagger} \mathbb{M}_{\infty \kappa v} \quad\left(\text { respectively, } \pi_{1}^{\mathrm{rat}}\left({ }^{\ddagger} \mathcal{D}_{v}\right) \curvearrowright \quad{ }_{\mathbb{M}_{\infty} \kappa \times v}\right)
$$

as an $\infty \kappa$-coric (respectively, $\infty \kappa \times$-coric) structure on $\ddagger \mathcal{F}_{\underline{v}}$ if it is isomorphic [i.e., as a pair consisting of a pseudo-monoid equipped with a continuous action by $\left.\pi_{1}^{\text {rat }}\left({ }^{\ddagger} \mathcal{D}_{v}\right)\right]$ to the pair

$$
\pi_{1}^{\text {rat }}\left({ }^{\ddagger} \mathcal{D}_{v}\right) \curvearrowright \mathbb{M}_{\infty \kappa v}\left({ }^{\ddagger} \mathcal{D}_{\underline{v}}\right) \quad\left(\text { respectively, } \pi_{1}^{\text {rat }}\left({ }^{\ddagger} \mathcal{D}_{v}\right) \curvearrowright \mathbb{M}_{\infty \kappa \times v}\left({ }^{\ddagger} \mathcal{D}_{\underline{v}}\right)\right)
$$

of (v). Suppose that we have been given such an $\infty \kappa$-coric (respectively, $\infty \kappa \times$-coric) structure on ${ }^{\ddagger} \mathcal{F}_{\underline{v}}$. In the following, we shall use the notational convention " $\boldsymbol{\mu}_{\widehat{\mathbb{Z}}}((-))$ " introduced in Example 5.1, (v). Also, let us write $\boldsymbol{\mu}_{\widehat{\mathbb{Z}}}^{\Theta}\left(\pi_{1}\left({ }^{\ddagger} \mathcal{D}_{\underline{v}}\right)\right)$ for the cyclotome " $\mu_{\widehat{\mathbb{Z}}}\left(\Pi_{(-)}\right)$" of [AbsTopIII], Theorem 1.9, which we think of as being applied "via the $\Theta$-approach" [cf. Remark 3.1.2] to $\pi_{1}\left({ }^{\ddagger} \mathcal{D}_{\underline{v}}\right)$. Then let us observe that $\mathbb{M}_{\infty} \kappa v\left({ }^{\ddagger} \mathcal{D}_{\underline{v}}\right)$ (respectively, $\mathbb{M}_{\infty \kappa \times v}\left({ }^{\ddagger} \mathcal{D}_{\underline{v}}\right)$ ) is, in effect, constructed [cf. [AbsTopIII], Theorem 1.9, (d)] as a subset of

$$
\underset{H}{\varliminf_{H}} H^{1}\left(H, \mu_{\widehat{\mathbb{Z}}}^{\Theta}\left(\pi_{1}\left({ }^{\ddagger} \mathcal{D}_{\underline{v}}\right)\right)\right)
$$

- where $H$ ranges over the open subgroups of $\pi_{1}^{\text {rat }}\left({ }^{\ddagger} \mathcal{D}_{v}\right)$. On the other hand, consideration of Kummer classes [i.e., of the action of $\pi_{1}^{\text {rat }}\left({ }^{\ddagger} \mathcal{D}_{v}\right)$ on $N$-th roots of elements, for positive integers $N$ ] yields a natural injection of $\ddagger \mathbb{M}_{\infty} \kappa v$ (respectively, $\left.\ddagger \mathbb{M}_{\infty} \kappa \times v\right)$ into

$$
\left.\varliminf_{H} H^{1}\left(H, \boldsymbol{\mu}_{\widehat{\mathbb{Z}}}\left(\varliminf_{\infty} \kappa v\right)\right) \quad \text { (respectively, } \varliminf_{H} H^{1}\left(H, \boldsymbol{\mu}_{\widehat{\mathbb{Z}}}\left(\mathbb{M}_{\infty} \kappa \times v\right)\right)\right)
$$

- where $H$ ranges over the open subgroups of $\pi_{1}^{\text {rat }}\left({ }^{\ddagger} \mathcal{D}_{v}\right)$, and we observe that the asserted injectivity follows immediately from the corresponding injectivity in the 
case of $\mathbb{M}_{\infty \kappa v}\left({ }^{\ddagger} \mathcal{D}_{\underline{v}}\right)$ (respectively, $\mathbb{M}_{\infty \kappa \times v}\left({ }^{\ddagger} \mathcal{D}_{\underline{v}}\right)$ ). In particular, it follows immediately [cf. the discussion of Example 5.1, (v)], by considering divisors of zeroes and poles [cf. the definition of a " $\kappa$-coric function" given in Remark 3.1.7, (i)] associated to Kummer classes of rational functions as in [AbsTopIII], Proposition 1.6, (iii), from the elementary observation that, relative to the natural inclusion $\mathbb{Q} \hookrightarrow \widehat{\mathbb{Z}} \otimes \mathbb{Q}$,

$$
\mathbb{Q}_{>0} \bigcap \widehat{\mathbb{Z}}^{\times}=\{1\}
$$

that there exists a unique isomorphism of cyclotomes

$$
\boldsymbol{\mu}_{\widehat{\mathbb{Z}}}^{\Theta}\left(\pi_{1}\left({ }^{\ddagger} \mathcal{D}_{\underline{v}}\right)\right) \stackrel{\sim}{\rightarrow} \boldsymbol{\mu}_{\widehat{\mathbb{Z}}}\left({ }^{\ddagger} \mathbb{M}_{\infty} \kappa v\right) \quad\left(\text { respectively, } \boldsymbol{\mu}_{\widehat{\mathbb{Z}}}^{\Theta}\left(\pi_{1}\left({ }^{\ddagger} \mathcal{D}_{\underline{v}}\right)\right) \stackrel{\sim}{\rightarrow} \boldsymbol{\mu}_{\widehat{\mathbb{Z}}}\left({ }^{\ddagger} \mathbb{M}_{\infty} \kappa \times v\right)\right)
$$

such that the resulting isomorphism between direct limits of cohomology modules as considered above induces an isomorphism

$$
\left.\mathbb{M}_{\infty \kappa v}\left({ }^{\ddagger} \mathcal{D}_{\underline{v}}\right) \stackrel{\sim}{\rightarrow}{ }^{\ddagger} \mathbb{M}_{\infty} \kappa v \quad \text { (respectively, } \mathbb{M}_{\infty} \kappa \times v\left({ }^{\ddagger} \mathcal{D}_{\underline{v}}\right) \stackrel{\sim}{\rightarrow}{ }^{\ddagger} \mathbb{M}_{\infty} \kappa \times v\right)
$$

[i.e., of pseudo-monoids equipped with continuous actions by $\pi_{1}^{\text {rat }}\left({ }^{\ddagger} \mathcal{D}_{v}\right)$ ]. In a similar vein, it follows immediately from the theory summarized in [AbsTopIII], Corollary $1.10,(d),\left(d^{\prime}\right)$, that there exists a unique isomorphism of cyclotomes

$$
\boldsymbol{\mu}_{\widehat{\mathbb{Z}}}^{\Theta}\left(\pi_{1}\left({ }^{\ddagger} \mathcal{D}_{\underline{v}}\right)\right) \stackrel{\sim}{\rightarrow} \boldsymbol{\mu}_{\widehat{\mathbb{Z}}}\left({ }^{\ddagger} \mathbb{M}_{v}\right)
$$

such that the resulting isomorphism between direct limits of cohomology modules induces an isomorphism

$$
\mathbb{M}_{v}\left({ }^{\ddagger} \mathcal{D}_{\underline{v}}\right) \stackrel{\sim}{\rightarrow}{ }^{\ddagger} \mathbb{M}_{v}
$$

[i.e., of monoids equipped with continuous actions by $\left.\pi_{1}\left({ }^{\ddagger} \mathcal{D}_{v}\right)\right]$. In particular, it follows immediately from the above discussion that

${ }^{\ddagger} \mathcal{F}_{\underline{v}}$ always admits an $\infty \kappa$-coric (respectively, $\infty \kappa \times$-coric) structure, which is, moreover, unique up to a uniquely determined isomorphism [i.e., of pseudo-monoids equipped with continuous actions by $\left.\pi_{1}^{\text {rat }}\left({ }^{\ddagger} \mathcal{D}_{v}\right)\right]$.

Thus, in the following, we shall regard, without further notice, this uniquely determined $\infty \kappa$-coric (respectively, $\infty \kappa \times$-coric) structure on ${ }^{\ddagger} \mathcal{F}_{\underline{v}}$ as a collection of data that is naturally associated to ${ }^{\ddagger} \mathcal{F}_{\underline{v}}$. Here, we observe that the various isomorphisms of the last few displays allow one to regard the pseudo-monoids ${ }^{\ddagger} \mathbb{M}_{\infty} \kappa v,{ }^{\ddagger} \mathbb{M}_{\infty} \kappa \times v$ as being related to the Frobenioid ${ }^{\ddagger} \mathcal{F}_{\underline{v}}$ via ${ }^{\ddagger} \mathbb{M}_{v}$ [cf. the discussion at the beginning of the present (vi) concerning the relationship between ${ }^{\ddagger} \mathbb{M}_{v}$ and $\left.{ }^{\ddagger} \mathcal{F}_{\underline{v}}\right]$ and the morphisms

$$
\ddagger_{\infty} \kappa v \quad \hookrightarrow \quad \mathbb{M}_{\infty \kappa \times v}, \quad{ }_{\mathbb{M}_{\infty} \kappa v}^{\times} \quad \hookrightarrow \quad \ddagger \mathbb{M}_{\infty \kappa \times v}^{\times} \stackrel{\sim}{\rightarrow} \quad \ddagger \mathbb{M}_{v}^{\times}
$$

induced by the various isomorphisms of the last few displays, together with the corresponding inclusions/equalities

$$
\begin{gathered}
\mathbb{M}_{\infty \kappa v}\left({ }^{\ddagger} \mathcal{D}_{\underline{v}}\right) \subseteq \mathbb{M}_{\infty \kappa \times v}\left({ }^{\ddagger} \mathcal{D}_{\underline{v}}\right), \\
\mathbb{M}_{\infty \kappa v}\left({ }^{\ddagger} \mathcal{D}_{\underline{\underline{v}}}\right)^{\times} \subseteq \mathbb{M}_{\infty \kappa \times v}\left({ }^{\ddagger} \mathcal{D}_{\underline{v}}\right)^{\times}=\mathbb{M}_{v}\left({ }^{\ddagger} \mathcal{D}_{\underline{v}}\right)^{\times}
\end{gathered}
$$


[cf. the discussion at the end of (v)]. Also, we shall write

$$
{ }^{\ddagger} \mathbb{M}_{\kappa v} \subseteq{ }^{\ddagger} \mathbb{M}_{\infty} \kappa v
$$

for the "sub-pseudo-monoid" of $\pi_{1}^{\text {rat }}\left({ }^{\ddagger} \mathcal{D}_{v}\right)$-invariants. In this context, we observe further that it follows immediately from the discussion of Remark 3.1.7, (i), (ii) [cf. also [AbsTopII], Corollary 3.3, (iii), which is applicable in light of [AbsTopI], Example 4.8], and the theory summarized in [AbsTopIII], Theorem 1.9 [cf., especially, [AbsTopIII], Theorem 1.9, (a), (d), (e)], and [AbsTopIII], Corollary 1.10, (h), that

any $\infty_{\infty} \kappa \times$-coric structure $\pi_{1}^{\text {rat }}\left({ }^{\ddagger} \mathcal{D}_{v}\right) \curvearrowright \mathbb{M}_{\infty} \kappa \times v$ on ${ }^{\ddagger} \mathcal{F}_{\underline{v}}$ determines an associated $\infty \kappa$-coric structure

$$
\pi_{1}^{\mathrm{rat}}\left({ }^{\ddagger} \mathcal{D}_{v}\right) \curvearrowright \ddagger \mathbb{M}_{\infty \kappa v} \subseteq \mathbb{M}_{\infty \kappa \times v}
$$

by considering the subset of elements for which the restriction of the associated Kummer class [as in the above discussion] to some [or, equivalently, every — cf. Remark 5.2.3 below] subgroup of $\pi_{1}^{\text {rat }}\left({ }^{\ddagger} \mathcal{D}_{v}\right)$ that corresponds to an open subgroup of the decomposition group of some strictly critical point of $C_{v}$ determines a torsion element $\in{ }^{\ddagger} \mathbb{M}_{v}^{\times} \stackrel{\sim}{\rightarrow}$ $\ddagger \mathbb{M}_{\infty \kappa \times v}^{\times}$[i.e., corresponds to a root of unity],

and, moreover, that

the operation of restricting Kummer classes [as in the above discussion] arising from ${ }^{\ddagger} \mathbb{M}_{\kappa v} \subseteq{ }^{\ddagger} \mathbb{M}_{\infty} \kappa v$ to subgroups of $\pi_{1}^{\text {rat }}\left({ }^{\ddagger} \mathcal{D}_{v}\right)$ that correspond to decomposition groups of non-critical $\left(F_{\bmod }\right)_{v}$-valued points of $C_{v}$ yields a functorial algorithm for reconstructing the submonoid of $\pi_{1}\left({ }^{\ddagger} \mathcal{D}_{v}\right)$-invariants of $\ddagger \mathbb{M}_{v}^{g p}$ [where the superscript "gp" denotes the groupification], together with the ind-topological field structure on the union of this monoid with $\{0\}$, from the $\infty_{\infty} \kappa$-coric structure ${ }^{\ddagger} \mathbb{M}_{\infty} \kappa v$ associated to ${ }^{\ddagger} \mathcal{F}_{\underline{v}}$.

A similar statement to the statement of the last display holds, if one replaces the phrase " $\left(F_{\bmod }\right)_{v}$-valued points" by the phrase " $\bar{F}_{v}$-valued points" and the phrase "submonoid of $\pi_{1}\left({ }^{\ddagger} \mathcal{D}_{v}\right)$-invariants of $\ddagger \mathbb{M}_{v}^{\text {gp }}$ " by the phrase "pair $\pi_{1}\left({ }^{\ddagger} \mathcal{D}_{v}\right) \curvearrowright \mathbb{M}_{v}^{g p} "$.

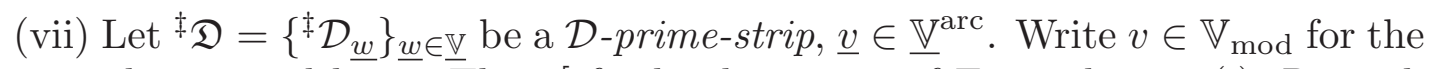
valuation determined by $\underline{v}$. Then [cf. the discussion of Example 5.1, (i); Remark 3.1.7, (i)] one may construct algorithmically from the Aut-holomorphic space ${ }^{\ddagger} \mathcal{D}_{\underline{v}}$, in a functorial fashion, an Aut-holomorphic orbispace ${ }^{\ddagger} \mathcal{D}_{v}$ corresponding to " $C_{v}$ " $[\mathrm{cf}$. the algorithms of [AbsTopIII], Corollary 2.7, (a)], together with a natural morphism

$$
{ }^{\ddagger} \mathcal{D}_{\underline{v}} \rightarrow{ }^{\ddagger} \mathcal{D}_{v}
$$

— i.e., an "Aut-holomorphic orbispace version" of the natural morphism of hyperbolic orbicurves $\underline{X}_{\underline{v}} \stackrel{\text { def }}{=} \underline{X}_{K} \times_{K} K_{\underline{v}} \rightarrow C_{v} \times_{\left(F_{\text {mod }}\right)_{v}} K_{\underline{v}}$. Here, we observe [cf. the fact that $C_{K}$ is a $\bar{K}$-core; [AbsTopIII], Corollary 2.3] that one has a natural isomorphism

$$
\operatorname{Aut}\left({ }^{\ddagger} \mathcal{D}_{v}\right) \stackrel{\sim}{\rightarrow} \operatorname{Gal}\left(K_{\underline{v}} /\left(F_{\bmod }\right)_{v}\right)(\hookrightarrow \mathbb{Z} / 2 \mathbb{Z})
$$


— i.e., obtained by considering whether an automorphism of $\ddagger \mathcal{D}_{v}$ is holomorphic or anti-holomorphic - from the group of automorphisms of the Aut-holomorphic orbispace ${ }^{\ddagger} \mathcal{D}_{v}$ onto the Galois group $\operatorname{Gal}\left(K_{\underline{v}} /\left(F_{\text {mod }}\right)_{v}\right)$. Write

$$
{ }^{\ddagger} \mathcal{D}_{v}^{\text {rat }} \rightarrow{ }^{\ddagger} \mathcal{D}_{v}
$$

for the projective system of Aut-holomorphic orbispaces that arise as universal covering spaces of "co-finite" open sub-orbispaces of $\ddagger \mathcal{D}_{v}$ [i.e., open sub-orbispaces determined by forming complements of finite sets of points of the underlying topological orbispace of ${ }^{\ddagger} \mathcal{D}_{v}$ ] that contain every strictly critical point [cf. Remark 3.1.7, (i)], as well as every point that is not an NF-point [cf. Remark 3.4.3, (ii)], of ${ }^{\ddagger} \mathcal{D}_{v}$. Thus, ${ }^{\ddagger} \mathcal{D}_{v}^{\text {rat }}$ is well-defined up to the action of deck transformations over ${ }^{\ddagger} \mathcal{D}_{v}$ [cf. the countability of the set of NF-points of ${ }^{\ddagger} \mathcal{D}_{v}$; the discussion of compatible systems of basepoints at the end of Remark 2.5.3, (i)]. Next, let us recall the complex archimedean topological field $\overline{\mathcal{A}}_{\ddagger \mathcal{D}_{v}}$ [cf. the discussion of Example 3.4, (i), as well as Definition 3.6, (b); the discussion of (i) of the present Definition 5.2]. Write $\operatorname{Aut}\left(\overline{\mathcal{A}}_{\ddagger \mathcal{D}_{\underline{v}}}\right)$ for the group of automorphisms $(\cong \mathbb{Z} / 2 \mathbb{Z})$ of the topological field $\overline{\mathcal{A}}_{\ddagger \mathcal{D}_{\underline{v}}}$. Observe that it follows immediately from the construction of $\overline{\mathcal{A}}_{\ddagger \mathcal{D}_{\underline{v}}}$ in $[\mathrm{Ab}-$ sTopIII], Corollary 2.7, (e), that $\overline{\mathcal{A}}_{\ddagger \mathcal{D}_{\underline{v}}}$ is equipped with a natural Aut-holomorphic structure [cf. [AbsTopIII], Definition 4.1, (i)], as well as with a tautological coholomorphicization [cf. [AbsTopIII], Definition 2.1, (iv); [AbsTopIII], Proposition 2.6, (a)] with ${ }^{\ddagger} \mathcal{D}_{\underline{v}}$. Write

$$
\mathbb{M}_{v}\left({ }^{\ddagger} \mathcal{D}_{\underline{v}}\right) \subseteq \overline{\mathcal{A}}_{\ddagger \mathcal{D}_{\underline{v}}}
$$

for the topological submonoid consisting of nonzero elements of norm $\leq 1$ [i.e.,

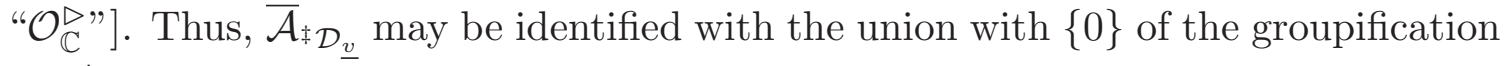
$\mathbb{M}_{v}\left({ }^{\ddagger} \mathcal{D}_{\underline{v}}\right)^{\mathrm{gp}}$. Moreover, the pseudo-monoids of $\kappa$-, $\infty^{\kappa-}$, and $\infty^{\kappa \times- \text { coric rational }}$ functions associated to $C_{v}$ [cf. the discussion of Remark 3.1.7, (i), (ii)] may be represented, via algorithmic constructions [cf. [AbsTopIII], Corollary 2.7, (b)], as pseudo-monoids of "meromorphic functions"

$$
\mathbb{M}_{\kappa v}\left({ }^{\ddagger} \mathcal{D}_{\underline{v}}\right), \quad \mathbb{M}_{\infty \kappa v}\left({ }^{\ddagger} \mathcal{D}_{\underline{v}}\right), \quad \mathbb{M}_{\infty \kappa \times v}\left({ }^{\ddagger} \mathcal{D}_{\underline{v}}\right)
$$

- i.e., as sets of morphisms of Aut-holomorphic orbispaces from [some constituent of the projective system] ${ }^{\ddagger} \mathcal{D}_{v}^{\text {rat }}$ to $\mathbb{M}_{v}\left({ }^{\ddagger} \mathcal{D}_{\underline{v}}\right)^{\text {gp }}$ that are compatible with the tautological co-holomorphicization just discussed and, moreover, satisfy conditions corresponding to the conditions of the final display of Remark 3.1.7, (i). Here, $\mathbb{M}_{\kappa v}\left({ }^{\ddagger} \mathcal{D}_{\underline{v}}\right)$ may be identified with the subset of elements of $\mathbb{M}_{\infty \kappa v}\left({ }^{\ddagger} \mathcal{D}_{\underline{v}}\right)$ that descend to some co-finite open sub-orbispace of ${ }^{\ddagger} \mathcal{D}_{v}$ and, moreover, are equivariant with respect to the unique embedding $\operatorname{Aut}\left({ }^{\ddagger} \mathcal{D}_{v}\right) \hookrightarrow \operatorname{Aut}\left(\overline{\mathcal{A}}_{\ddagger \mathcal{D}_{\underline{v}}}\right)$; [if we use the superscript “ $\times$ " to denote the subset of invertible elements of a pseudo-monoid, then] $\mathbb{M}_{v}\left({ }^{\ddagger} \mathcal{D}_{\underline{v}}\right)^{\times}$may be identified with $\mathbb{M}_{\infty \kappa \times v}\left({ }^{\ddagger} \mathcal{D}_{\underline{v}}\right)^{\times}$; we observe that both $\mathbb{M}_{v}\left({ }^{\ddagger} \mathcal{D}_{\underline{v}}\right)^{\times}$and $\mathbb{M}_{\infty \kappa \times v}\left({ }^{\ddagger} \mathcal{D}_{\underline{v}}\right)^{\times}$ are isomorphic, as abstract topological monoids, to $\mathbb{S}^{1}$ [i.e., " $\mathcal{O}_{\mathbb{C}}^{\times}$].

(viii) We continue to use the notation of (vii). Suppose further that ${ }^{\ddagger} \mathfrak{F}=$

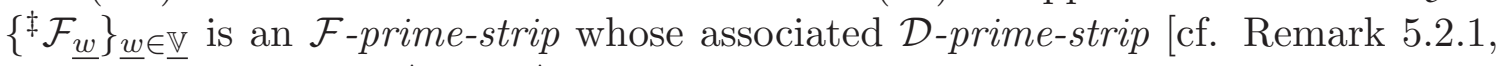

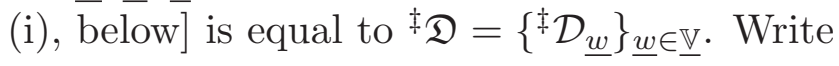


for the topological monoid [i.e., " $\mathcal{O}^{\triangleright}\left({ }^{\ddagger} \mathcal{C}_{v}\right)$ " — cf. the discussion of Example 3.4, (i); Definition 3.6, (b)] that appears as the domain of the Kummer structure portion of the data that constitutes $\ddagger \mathcal{F}_{\underline{v}}$ [cf. (i) of the present Definition 5.2]. Thus, the Kummer structure portion of $\ddagger \mathcal{F}_{\underline{v}}$ may be regarded as an isomorphism of topological monoids

$$
\mathbb{M}_{v}\left({ }^{\ddagger} \mathcal{D}_{\underline{v}}\right) \stackrel{\sim}{\rightarrow} \ddagger \mathbb{M}_{v}
$$

[both of which are abstractly isomorphic to $\left.\mathcal{O}_{\mathbb{C}}^{\triangleright}\right]$. In particular, the Kummer structure determines an isomorphism of topological groups $\mathbb{M}_{v}\left({ }^{\ddagger} \mathcal{D}_{\underline{v}}\right)^{\text {gp }} \stackrel{\sim}{\rightarrow}{ }^{\ddagger} \mathbb{M}_{v}^{g p}[$ both of which are abstractly isomorphic to $\left.\mathbb{C}^{\times}\right]$, hence also a natural action of $\operatorname{Aut}\left(\overline{\mathcal{A}}_{\ddagger \mathcal{D}_{\underline{v}}}\right)$ on ${ }^{\ddagger} \mathbb{M}_{v}^{g p}$. Next, let us observe that the pseudo-monoid of $\infty \kappa$ - (respectively, $\infty \kappa \times-$ ) coric rational functions associated to $C_{v}$ [cf. the discussion of Remark 3.1.7, (i), (ii)] may be represented, via algorithmic constructions [cf. [AbsTopIII], Corollary $2.7,(\mathrm{~b})]$, as the pseudo-monoid of "meromorphic functions"

$$
\left.{ }^{\ddagger} \mathbb{M}_{\infty} \kappa v \quad \text { (respectively, }{ }^{\ddagger} \mathbb{M}_{\infty} \kappa \times v\right)
$$

by considering the set of maps from [some constituent of the projective system] $\ddagger \mathcal{D}_{v}^{\text {rat }}$ to

$$
{ }^{\ddagger} \mathbb{M}_{v}^{g p}
$$

that satisfy the following condition: the map from [some constituent of the projective system $]{ }^{\ddagger} \mathcal{D}_{v}^{\text {rat }}$ to $\mathbb{M}_{v}\left({ }^{\ddagger} \mathcal{D}_{\underline{v}}\right)$ gp obtained by composing the given map with the inverse of [the result of applying "gp" to] the Kummer structure isomorphism $\mathbb{M}_{v}\left({ }^{\ddagger} \mathcal{D}_{\underline{v}}\right) \stackrel{\sim}{\rightarrow}{ }^{\ddagger} \mathbb{M}_{v}$ determines an element of the pseudo-monoid $\mathbb{M}_{\infty} \kappa v\left({ }^{\ddagger} \mathcal{D}_{\underline{v}}\right.$ ) (respectively, $\mathbb{M}_{\infty \kappa \times v}\left({ }^{\ddagger} \mathcal{D}_{\underline{v}}\right)$ ) discussed in (vii) above. We shall refer to

$$
\left.{ }^{\ddagger} \mathbb{M}_{\infty \kappa v} \quad \text { (respectively, }{ }^{\ddagger} \mathbb{M}_{\infty \kappa \times v}\right)
$$

as the [uniquely determined] $\infty_{\infty} \kappa$-coric (respectively, $\infty \kappa \times$-coric) structure on $\ddagger^{\mathcal{F}_{\underline{v}}}$ and write

$$
{ }^{\ddagger} \mathbb{M}_{\kappa v} \subseteq{ }^{\ddagger} \mathbb{M}_{\infty} \kappa v
$$

for the subset of elements that descend to some co-finite open sub-orbispace of $\ddagger \mathcal{D}_{v}$ and, moreover, are equivariant with respect to the unique embedding $\operatorname{Aut}\left({ }^{\ddagger} \mathcal{D}_{v}\right) \hookrightarrow$ $\operatorname{Aut}\left(\overline{\mathcal{A}}_{\ddagger \mathcal{D}_{\underline{v}}}\right)$. In the following, we shall use the notational convention " $\boldsymbol{\mu}_{\widehat{\mathbb{Z}}}((-))$ " introduced in Example 5.1, (v). Also, let us write

$$
\begin{aligned}
\mu_{\widehat{\mathbb{Z}}}^{\Theta}\left({ }^{\ddagger} \mathcal{D}_{\underline{v}}\right) & \stackrel{\text { def }}{=} \operatorname{Hom}\left(\mathbb{Q} / \mathbb{Z}, \mathbb{M}_{v}\left({ }^{\ddagger} \mathcal{D}_{\underline{\underline{v}}}\right)^{\mathrm{gp}}\right) \\
& =\operatorname{Hom}\left(\mathbb{Q} / \mathbb{Z}, \mathbb{M}_{v}\left({ }^{\ddagger} \mathcal{D}_{\underline{\underline{v}}}\right)^{\boldsymbol{\mu}}\right)=\operatorname{Hom}\left(\mathbb{Q} / \mathbb{Z}, \mathbb{M}_{v}\left({ }^{\ddagger} \mathcal{D}_{\underline{v}}\right)^{\times}\right)
\end{aligned}
$$

— where $\mathbb{M}_{v}\left({ }^{\ddagger} \mathcal{D}_{\underline{v}}\right)^{\times} \subseteq \mathbb{M}_{v}\left({ }^{\ddagger} \mathcal{D}_{\underline{v}}\right)$ denotes the topological group of units of $\mathbb{M}_{v}\left({ }^{\ddagger} \mathcal{D}_{\underline{v}}\right)$; $\mathbb{M}_{v}\left({ }^{\ddagger} \mathcal{D}_{\underline{v}}\right)^{\boldsymbol{\mu}} \subseteq \mathbb{M}_{v}\left({ }^{\ddagger} \mathcal{D}_{\underline{v}}\right)^{\times}$denotes the subgroup of torsion elements; we observe that the Kummer structure isomorphism discussed above induces a natural "Kummer structure cyclotomic isomorphism" $\left.\boldsymbol{\mu}_{\widehat{\mathbb{Z}}}^{\Theta}\left({ }^{\ddagger} \mathcal{D}_{\underline{v}}\right) \stackrel{\sim}{\rightarrow} \boldsymbol{\mu}_{\widehat{\mathbb{Z}}}{ }^{\ddagger} \mathbb{M}_{v}\right)$ [of profinite groups abstractly isomorphic to $\widehat{\mathbb{Z}}$; the superscript " $\Theta$ " may be thought of as expressing the fact that we wish to apply to " $\boldsymbol{\mu}_{\widehat{\mathbb{Z}}}^{\Theta}(-)$ " the interpretation via the archimedean version of the $\Theta$-approach, i.e., the interpretation in terms of cuspidal inertia 
groups, discussed in Remark 3.4.3, (i). In this context, we observe that these cuspidal inertia groups may be interpreted as profinite completions of subgroups of the group of deck transformations

$$
\pi_{1}^{\mathrm{rat}}\left({ }^{\ddagger} \mathcal{D}_{v}\right)
$$

determined, up to inner automorphism, by the projective system of covering spaces $\ddagger \mathcal{D}_{v}^{\text {rat }} \rightarrow{ }^{\ddagger} \mathcal{D}_{v}$. Here, we observe that the pseudo-monoids $\mathbb{M}_{\infty} \kappa v\left({ }^{\ddagger} \mathcal{D}_{\underline{v}}\right),{ }^{\ddagger} \mathbb{M}_{\infty} \kappa v$ (respectively, $\left.\mathbb{M}_{\infty \kappa \times v}\left({ }^{\ddagger} \mathcal{D}_{\underline{v}}\right),{ }^{\ddagger} \mathbb{M}_{\infty \kappa \times v}\right)$ admit natural $\pi_{1}^{\text {rat }}\left({ }^{\ddagger} \mathcal{D}_{v}\right)$-actions in such a way that each of the pairs

$$
\begin{gathered}
\pi_{1}^{\mathrm{rat}}\left({ }^{\ddagger} \mathcal{D}_{v}\right) \curvearrowright \mathbb{M}_{\infty} \kappa v\left({ }^{\ddagger} \mathcal{D}_{\underline{v}}\right), \quad \pi_{1}^{\mathrm{rat}}\left({ }^{\ddagger} \mathcal{D}_{v}\right) \curvearrowright \mathbb{M}_{\infty} \kappa v \\
\left(\text { respectively, } \pi_{1}^{\mathrm{rat}}\left({ }^{\ddagger} \mathcal{D}_{v}\right) \curvearrowright \mathbb{M}_{\infty} \kappa \times v\left({ }^{\ddagger} \mathcal{D}_{\underline{v}}\right), \quad \pi_{1}^{\mathrm{rat}}\left({ }^{\ddagger} \mathcal{D}_{v}\right) \curvearrowright \mathbb{M}_{\infty} \kappa \times v\right)
\end{gathered}
$$

is well-defined up to $\pi_{1}^{\text {rat }}\left({ }^{\ddagger} \mathcal{D}_{v}\right)$-conjugacy. Next, let us observe that by considering the action of the various cuspidal inertia groups just discussed on elements of the pseudo-monoid ${ }^{\ddagger} \mathbb{M}_{\infty} \kappa v$ (respectively, ${ }^{\ddagger} \mathbb{M}_{\infty} \kappa \times v$ ) - i.e., in effect, by considering, in the fashion of [AbsTopIII], Proposition 1.6, (iii), "local Kummer classes" at the points that give rise to these cuspidal inertia groups — we obtain various $\mathbb{Q}$-multiples - i.e., corresponding to the order of zeroes or poles at the point that gives rise to the cuspidal inertia group under consideration - of the Kummer structure cyclotomic isomorphism $\boldsymbol{\mu}_{\widehat{\mathbb{Z}}}^{\Theta}\left({ }^{\ddagger} \mathcal{D}_{\underline{v}}\right) \stackrel{\sim}{\rightarrow} \boldsymbol{\mu}_{\widehat{\mathbb{Z}}}\left({ }^{\ddagger} \mathbb{M}_{v}\right)$ discussed above. In particular, relative to the natural identification [cf. the various definitions involved!] of $\boldsymbol{\mu}_{\widehat{\mathbb{Z}}}\left({ }^{\ddagger} \mathbb{M}_{v}\right)$ with $\left.\boldsymbol{\mu}_{\widehat{\mathbb{Z}}}{ }^{\ddagger} \mathbb{M}_{\infty} \kappa v\right)$ (respectively, $\boldsymbol{\mu}_{\widehat{\mathbb{Z}}}\left({ }^{\ddagger} \mathbb{M}_{\infty} \kappa \times v\right)$ ), it follows immediately [cf. the discussion of Example 5.1, (v)], by considering [i.e., in the fashion just discussed] divisors of zeroes and poles [cf. the definition of a " $\kappa$-coric function" given in Remark 3.1.7, (i)] of meromorphic functions, from the elementary observation that, relative to the natural inclusion $\mathbb{Q} \hookrightarrow \widehat{\mathbb{Z}} \otimes \mathbb{Q}$,

$$
\mathbb{Q}_{>0} \bigcap \widehat{\mathbb{Z}}^{\times}=\{1\}
$$

that one may algorithmically reconstruct the Kummer structure cyclotomic isomorphism

$$
\boldsymbol{\mu}_{\widehat{\mathbb{Z}}}^{\Theta}\left({ }^{\ddagger} \mathcal{D}_{\underline{v}}\right) \stackrel{\sim}{\rightarrow} \boldsymbol{\mu}_{\widehat{\mathbb{Z}}}\left({ }^{\ddagger} \mathbb{M}_{\infty} \kappa v\right) \quad\left(\text { respectively, } \boldsymbol{\mu}_{\widehat{\mathbb{Z}}}^{\Theta}\left({ }^{\ddagger} \mathcal{D}_{\underline{v}}\right) \stackrel{\sim}{\rightarrow} \boldsymbol{\mu}_{\widehat{\mathbb{Z}}}\left({ }^{\ddagger} \mathbb{M}_{\infty \kappa \times v}\right)\right)
$$

- hence also the Kummer structure isomorphism $\mathbb{M}_{v}\left({ }^{\ddagger} \mathcal{D}_{\underline{v}}\right)^{\mu} \stackrel{\sim}{\rightarrow}{ }^{\ddagger} \mathbb{M}_{\infty}^{\mu}{ }_{\infty} v$ (respectively, $\mathbb{M}_{v}\left({ }^{\ddagger} \mathcal{D}_{\underline{v}}\right)^{\mu} \stackrel{\sim}{\rightarrow}{ }^{\ddagger} \mathbb{M}_{\infty \kappa \times v}^{\mu}$ ) [where the superscript " $\boldsymbol{\mu}$ 's" denote the subgroups of torsion elements] — from

the projective system of coverings of Aut-holomorphic orbispaces ${ }^{\ddagger} \mathcal{D}_{v}^{\text {rat }} \rightarrow{ }^{\ddagger} \mathcal{D}_{v}$, together with the abstract pseudo-monoid with $\pi_{1}^{\text {rat }}\left({ }^{\ddagger} \mathcal{D}_{v}\right)$ action $\pi_{1}^{\text {rat }}\left({ }^{\ddagger} \mathcal{D}_{v}\right) \curvearrowright \mathbb{M}_{\infty} \kappa v\left(\right.$ respectively, $\left.\pi_{1}^{\text {rat }}\left({ }^{\ddagger} \mathcal{D}_{v}\right) \curvearrowright \mathbb{M}_{\infty \kappa \times v}\right)$.

Since, moreover, a rational algebraic function is completely determined by its divisor of zeroes and poles together with its value at a single point, we thus conclude that one may algorithmically reconstruct the isomorphism(s) of pseudo-monoids 
determined by the Kummer structure on ${ }^{\ddagger} \mathcal{F}_{\underline{v}}$ [i.e., by the Kummer structure isomorphism $\mathbb{M}_{v}\left({ }^{\ddagger} \mathcal{D}_{\underline{v}}\right) \stackrel{\sim}{\rightarrow} \ddagger \mathbb{M}_{v}$ discussed above]

$$
\begin{aligned}
& \mathbb{M}_{\kappa v}\left({ }^{\ddagger} \mathcal{D}_{\underline{v}}\right) \stackrel{\sim}{\rightarrow} \ddagger \mathbb{M}_{\kappa v}, \quad \mathbb{M}_{\infty \kappa v}\left({ }^{\ddagger} \mathcal{D}_{\underline{v}}\right) \stackrel{\sim}{\rightarrow} \ddagger \mathbb{M}_{\infty \kappa v} \\
& \text { (respectively, } \mathbb{M}_{\infty \kappa \times v}\left({ }^{\ddagger} \mathcal{D}_{\underline{v}}\right) \stackrel{\sim}{\rightarrow} \ddagger \mathbb{M}_{\infty \kappa \times v} \text { ) }
\end{aligned}
$$

from

the projective system of coverings of Aut-holomorphic orbispaces ${ }^{\ddagger} \mathcal{D}_{v}^{\text {rat }} \rightarrow{ }^{\ddagger} \mathcal{D}_{v}$, together with the abstract pseudo-monoid with $\pi_{1}^{\text {rat }}\left({ }^{\ddagger} \mathcal{D}_{v}\right)$ action $\pi_{1}^{\text {rat }}\left({ }^{\ddagger} \mathcal{D}_{v}\right) \curvearrowright \ddagger \mathbb{M}_{\infty \kappa v}$ (respectively, $\left.\pi_{1}^{\text {rat }}\left({ }^{\ddagger} \mathcal{D}_{v}\right) \curvearrowright \ddagger \mathbb{M}_{\infty} \kappa \times v\right)$ and the collection of splittings

$$
{ }^{\ddagger} \mathbb{M}_{\infty \kappa v} \rightarrow{ }^{\ddagger} \mathbb{M}_{\infty \kappa v}^{\mu} \text { (respectively, }{ }^{\ddagger} \mathbb{M}_{\infty \kappa \times v} \rightarrow{ }^{\ddagger} \mathbb{M}_{\infty \kappa \times v}^{\times} \text {) }
$$

— where the superscript " $\boldsymbol{\mu}$ " (respectively, " $\times$ ") denotes the subgroup of torsion elements (respectively, the topological group of units, which contains the subgroup of torsion elements as a dense subgroup) of $¥ \mathbb{M}_{\infty} \kappa v$ (respectively, ${ }^{\ddagger} \mathbb{M}_{\infty \kappa \times v}$ ) - determined [and parametrized], via the operation of restriction, by the collection of systems of strictly critical points of $\ddagger \mathcal{D}_{v}^{\text {rat }} \rightarrow{ }^{\ddagger} \mathcal{D}_{v}$ [i.e., systems of points lying over some strictly critical point of $\ddagger \mathcal{D}_{v}$ ].

In this context, we observe further that it follows immediately from the discussion of Remark 3.1.7, (ii) [cf. also [AbsTopIII], Corollary 2.7, (b)], that

the $\infty \kappa$-coric structure

$$
\ddagger \mathbb{M}_{\infty} \kappa v \subseteq \ddagger \mathbb{M}_{\infty \kappa \times v}
$$

on ${ }^{\ddagger} \mathcal{F}_{\underline{v}}$ may be constructed from the ${ }_{\infty} \kappa \times$-coric structure ${ }^{\ddagger} \mathbb{M}_{\infty} \kappa \times v$ on $\ddagger \mathcal{F}_{\underline{v}}$ by considering the subset of elements for which the restriction to some [or, equivalently, every] system of strictly critical points of ${ }^{\ddagger} \mathcal{D}_{v}^{\text {rat }} \rightarrow{ }^{\ddagger} \mathcal{D}_{v}$ is a torsion element $\in \mathbb{M}_{v}^{\times} \stackrel{\sim}{\rightarrow} \ddagger \mathbb{M}_{\infty \kappa \times v}^{\times}$[i.e., corresponds to a root of unity],

and, moreover, that

the operation of restricting elements of $\ddagger \mathbb{M}_{\kappa v} \subseteq{ }^{\ddagger} \mathbb{M}_{\infty} \kappa v$ to systems of points of $\ddagger^{\text {rat }}$ that lie over Aut $\left({ }^{\ddagger} \mathcal{D}_{v}\right)$-invariant non-critical points of $\ddagger \mathcal{D}_{v}$ yields a functorial algorithm for reconstructing the submonoid of Aut $\left({ }^{\ddagger} \mathcal{D}_{v}\right)$-invariants of ${ }^{\ddagger} \mathbb{M}_{v}^{g p}$ [where the superscript "gp" denotes the groupification], together with the topological field structure on the union of this monoid with $\{0\}$, from the $\infty_{\infty} \kappa$-coric structure $\ddagger_{\infty} \mathbb{M}_{\infty v}$ associated to $\ddagger \mathcal{F}_{\underline{v}}$.

A similar statement to the statement of the last display holds if one replaces the phrase "Aut $\left({ }^{\ddagger} \mathcal{D}_{v}\right)$-invariant" by the phrase "arbitrary" and the phrase "submonoid of $\operatorname{Aut}\left({ }^{\ddagger} \mathcal{D}_{v}\right)$-invariants of $\ddagger \mathbb{M}_{v}^{g p} "$ by the phrase "monoid $\ddagger \mathbb{M}_{v}^{g p}$ ". 


\section{Remark 5.2.1.}

(i) Note that it follows immediately from Definitions 4.1, (i), (iii); 5.2, (i), (ii); Examples 3.2, (vi), (c), (d); 3.3, (iii), (b), (c), that there exists a functorial algorithm for constructing $\mathcal{D}$ - (respectively, $\mathcal{D}^{{ }^{-}}$) prime-strips from $\mathcal{F}$ - (respectively, $\mathcal{F}^{\vdash}$-) prime-strips.

(ii) In a similar vein, it follows immediately from Definition 5.2, (i), (ii); Examples 3.2, (vi), (f); 3.3, (iii), (e); 3.4, (i), (ii), that there exists a functorial algorithm for constructing from an $\mathcal{F}$-prime-strip ${ }^{\ddagger} \mathfrak{F}=\left\{{ }^{\ddagger} \mathcal{F}_{\underline{v}}\right\}_{\underline{v} \in \mathbb{V}}$ an $\mathcal{F}^{\vdash}$-prime-strip ${ }^{\ddagger} \mathfrak{F}$

$$
\ddagger \mathfrak{F} \mapsto{ }^{\ddagger} \mathfrak{F}=\left\{{ }^{\ddagger} \mathcal{F}_{\underline{v}}^{\vdash}\right\}_{\underline{v} \in \underline{\mathbb{V}}}
$$

— which we shall refer to as the mono-analyticization of ${ }^{\ddagger} \mathfrak{F}$. Next, let us recall from the discussion of Example 3.5, (i), the relatively simple structure of the category "C $\mathcal{C}_{\text {mod }}^{\Vdash}$ ", i.e., which may be summarized, roughly speaking, as a collection, indexed by $\underset{\mathbb{V}}{\stackrel{\sim}{\rightarrow}} \mathbb{V}_{\text {mod }}$, of copies of the topological monoid $\mathbb{R}_{\geq 0}$, which are related to one another by a "product formula". In particular, it follows immediately [cf. Definition 5.2, (i)] from the rigidity of the divisor monoids associated to the Frobenioids that appear at each of the components at $\underline{v} \in \underline{\mathbb{V}}$ of an $\mathcal{F}$-prime-strip [cf., especially, the topological field structure of the field " $\overline{\mathcal{A}}_{\mathcal{D}_{v}}$ " of Example 3.4, (i)!] that one may also construct from the $\mathcal{F}$-prime-strip ${ }^{\ddagger} \mathfrak{F}$, via a functorial algorithm $[\mathrm{cf}$. the constructions of Example 3.5, (i), (ii)], a collection of data

$$
\ddagger \mathfrak{F} \mapsto{ }^{\ddagger} \mathfrak{F} \stackrel{\text { def }}{=}\left({ }^{\ddagger} \mathcal{C}^{\Vdash}, \operatorname{Prime}\left({ }^{\ddagger} \mathcal{C}^{\Vdash}\right) \stackrel{\sim}{\rightarrow} \underline{\mathbb{V}}, \ddagger \mathfrak{F}^{\vdash},\left\{{ }^{\ddagger} \rho_{\underline{v}}\right\}_{\underline{v} \in \underline{\mathbb{V}}}\right)
$$

- i.e., consisting of a category [which is, in fact, equipped with a Frobenioid structure], a bijection, the $\mathcal{F}^{\vdash}$-prime-strip ${ }^{\ddagger} \mathfrak{F}^{\vdash}$, and an isomorphism of topological monoids associated to ${ }^{\ddagger} \mathcal{C}^{\Vdash}$ and ${ }^{\ddagger} \mathfrak{F}^{\vdash}$, respectively, at each $\underline{v} \in \mathbb{V}$ - which is isomorphic to the collection of data $\mathfrak{F}_{\text {mod }}^{\Vdash}$ of Example 3.5, (ii), i.e., which forms an $\mathcal{F}^{\Vdash}$-prime-strip [cf. Definition 5.2, (iv)].

Remark 5.2.2. Thus, from the point of view of the discussion of Remark 5.1.3, $\mathcal{F}$-prime-strips are Kummer-ready [i.e., at $\underline{v} \in \underline{\mathbb{V}}^{\text {non }}$ - cf. the theory of [FrdII], §2], whereas $\mathcal{F}^{\vdash}$-prime-strips are Kummer-blind.

Remark 5.2.3. In the context of the construction of $\infty \kappa$-coric structures from $\infty \kappa \times$-coric structures in Definition 5.2, (vi), we make the following observation. When $\underline{v} \in \underline{\mathbb{V}}^{\text {bad }}$, it is natural to take the decomposition groups corresponding to strictly critical points [i.e., to which one restricts the Kummer classes under consideration] to be decomposition groups that correspond to the point of $C_{v}$ that arises as the image of the zero-labeled evaluation points [i.e., evaluation points corresponding to the label $0 \in\left|\mathbb{F}_{l}\right|$ - cf. the discussion of Example 4.4, (i)]. In the notation of Example 4.4, (i), this point of $C_{v}$ may also be described simply as the point that arises as the image of the point " $\mu_{-}$". 
(i) For $i=1,2$, let ${ }^{i} \mathcal{F}^{\circledast}$ (respectively, ${ }^{i} \mathcal{F}^{\odot}$ ) be a category which is equivalent to the category ${ }^{\dagger} \mathcal{F}^{\circledast}$ (respectively, ${ }^{\dagger} \mathcal{F}^{\odot}$ ) of Example 5.1, (iii). Thus, ${ }^{i} \mathcal{F}^{\circledast}$ (respectively, ${ }^{i} \mathcal{F}^{\odot}$ ) is equipped with a natural Frobenioid structure [cf. [FrdI], Corollary 4.11; [FrdI], Theorem 6.4, (i); Remark 3.1 .5 of the present paper]. Write $\operatorname{Base}\left({ }^{i} \mathcal{F}^{\circledast}\right)$ (respectively, Base $\left({ }^{i} \mathcal{F}^{\odot}\right)$ ) for the base category of this Frobenioid. Then the natural map

$$
\begin{gathered}
\operatorname{Isom}\left({ }^{1} \mathcal{F}^{\circledast},{ }^{2} \mathcal{F}^{\circledast}\right) \rightarrow \operatorname{Isom}\left(\operatorname{Base}\left({ }^{1} \mathcal{F}^{\circledast}\right), \operatorname{Base}\left({ }^{2} \mathcal{F}^{\circledast}\right)\right) \\
\text { (respectively, Isom } \left.\left({ }^{1} \mathcal{F}^{\odot},{ }^{2} \mathcal{F}^{\odot}\right) \rightarrow \operatorname{Isom}\left(\operatorname{Base}\left({ }^{1} \mathcal{F}^{\odot}\right), \operatorname{Base}\left({ }^{2} \mathcal{F}^{\odot}\right)\right)\right)
\end{gathered}
$$

[cf. [FrdI], Corollary 4.11; [FrdI], Theorem 6.4, (i); Remark 3.1 .5 of the present paper] is bijective.

(ii) For $i=1,2$, let ${ }^{i} \mathfrak{F}$ be an $\mathcal{F}$-prime-strip; ${ }^{i} \mathfrak{D}$ the $\mathcal{D}$-prime-strip associated to ${ }^{i} \mathfrak{F}$ [cf. Remark 5.2.1, (i)]. Then the natural map

$$
\operatorname{Isom}\left({ }^{1} \mathfrak{F},{ }^{2} \mathfrak{F}\right) \rightarrow \operatorname{Isom}\left({ }^{1} \mathfrak{D},{ }^{2} \mathfrak{D}\right)
$$

[cf. Remark 5.2.1, (i)] is bijective.

(iii) For $i=1,2$, let ${ }^{i} \mathfrak{F}^{\vdash}$ be an $\mathcal{F}^{\vdash}$-prime-strip; ${ }^{i} \mathfrak{D}^{\vdash}$ the $\mathcal{D}^{\vdash}$-prime-strip associated to ${ }^{i} \mathfrak{F}^{\vdash}$ [cf. Remark 5.2.1, (i)]. Then the natural map

$$
\operatorname{Isom}\left({ }^{1} \mathfrak{F}^{\vdash},{ }^{2} \mathfrak{F}\right) \rightarrow \operatorname{Isom}\left({ }^{1} \mathfrak{D}^{\vdash},{ }^{2} \mathfrak{D}^{\vdash}\right)
$$

[cf. Remark 5.2.1, (i)] is surjective.

(iv) Let $\underline{v} \in \underline{\mathbb{V}}^{\text {bad }}$. Recall the category $\underline{\underline{\mathcal{F}}}_{\underline{v}}$ of Example 3.2, (i). Thus, $\underline{\underline{\mathcal{F}}}_{\underline{v}}$ is equipped with a natural Frobenioid structure [cf. [FrdI], Corollary 4.11; [EtTh], Proposition 5.1], with base category $\mathcal{D}_{\underline{v}}$. Then the natural homomorphism $\operatorname{Aut}\left(\underline{\underline{\mathcal{F}}}_{\underline{v}}\right) \rightarrow \operatorname{Aut}\left(\mathcal{D}_{\underline{v}}\right)[c f$. Example 3.2, (vi), ( $\left.\bar{d})\right]$ is bijective.

Proof. Assertion (i) follows immediately from the category-theoreticity of the "isomorphism $\mathbb{M}^{\circledast}\left({ }^{\dagger} \mathcal{D}^{\odot}\right) \stackrel{\sim}{\rightarrow} \dagger \mathbb{M}^{\circledast}$ ” of Example 5.1, (v) [cf. also the surrounding discussion; Example 5.1, (vi)]. [Here, we note in passing that this argument is entirely similar to the technique applied to the proof of the equivalence "Th $\mathfrak{T}_{\mathbb{T}}^{\odot} \stackrel{\sim}{\rightarrow} \mathbb{E}^{\odot}$ " of [AbsTopIII], Corollary 5.2, (iv).] Assertion (ii) (respectively, (iii)) follows immediately from [AbsTopIII], Proposition 3.2, (iv); [AbsTopIII], Proposition 4.2, (i) [cf. also [AbsTopIII], Remarks 3.1.1, 4.1.1; the discussion of Definition 5.2, (vi), (viii), of the present paper] (respectively, [AbsTopIII], Proposition 5.8, (ii), (v)).

Finally, we consider assertion (iv). First, we recall that since automorphisms of $\mathcal{D}_{\underline{v}}=\mathcal{B}^{\text {temp }}\left(\underline{\underline{X}}_{\underline{v}}\right)^{0}$ necessarily arise from automorphisms of the scheme $\underline{\underline{X}}_{\underline{v}}[\mathrm{cf}$. [AbsTopIII], Theorem 1.9; [AbsTopIII], Remark 1.9.1], surjectivity follows immediately from the construction of $\underline{\underline{\mathcal{F}}}_{\underline{v}}$. Thus, it remains to verify injectivity. To this end, let $\alpha \in \operatorname{Ker}\left(\operatorname{Aut}\left(\underline{\underline{\mathcal{F}}}_{\underline{v}}\right) \rightarrow \operatorname{Aut}\left(\mathcal{D}_{\underline{v}}\right)\right)$. For simplicity, we suppose [without loss of generality] that $\alpha$ lies over the identity self-equivalence of $\mathcal{D}_{\underline{v}}$. Then I claim that to show that $\alpha$ is [isomorphic to - cf. $\S 0$ ] the identity self-equivalence of $\underline{\underline{\mathcal{F}}}_{\underline{v}}$, it suffices to verify that

$\alpha$ induces [cf. [FrdI], Corollary 4.11; [EtTh], Proposition 5.1] the identity on the rational function and divisor monoids of $\underline{\underline{\mathcal{F}}}_{\underline{v}}$. 
Indeed, recall that since $\underline{\underline{\mathcal{F}}}_{\underline{v}}$ is a Frobenioid of model type [cf. [EtTh], Definition 3.6, (ii)], it follows from [FrdI], Corollary 5.7, (i), (iv), that $\alpha$ preserves base-Frobenius pairs. Thus, once one shows that $\alpha$ induces the identity on the rational function and divisor monoids of $\underline{\underline{\mathcal{F}}}_{\underline{v}}$, it follows, by arguing as in the construction of the equivalence of categories given in the proof of [FrdI], Theorem 5.2, (iv), that the various units obtained in [FrdI], Proposition 5.6, determine [cf. Remark 5.3.3 below; the argument of the first paragraph of the proof of [FrdI], Proposition 5.6] an isomorphism between $\alpha$ and the identity self-equivalence of $\underline{\underline{\mathcal{F}}}_{\underline{v}}$, as desired.

Thus, we proceed to show that $\alpha$ induces the identity on the rational function and divisor monoids of $\underline{\underline{\mathcal{F}}}_{\underline{v}}$, as follows. In light of the category-theoreticity [cf. [EtTh], Theorem 5.6] of the cyclotomic rigidity isomorphism of [EtTh], Proposition 5.5 , the fact that $\alpha$ induces the identity on the rational function monoid follows immediately from the naturality of the Kummer map [cf. the discussion of Remark 3.2.4; [FrdII], Definition 2.1, (ii)], which is injective by [EtTh], Proposition 3.2, (iii) — cf. the argument of [EtTh], Theorem 5.7, applied to verify the categorytheoreticity of the Frobenioid-theoretic theta function. Next, we consider the effect of $\alpha$ on the divisor monoid of $\underline{\underline{\mathcal{F}}} \underline{\underline{v}}$. To this end, let us first recall that $\alpha$ preserves cuspidal and non-cuspidal elements of the monoids that appear in this divisor monoid [cf. Remark 3.2.4, (vi); [EtTh], Proposition 5.3, (i)]. In particular, by considering the non-cuspidal portion of the divisor of the Frobenioid-theoretic theta function and its conjugates [each of which is preserved by $\alpha$, since $\alpha$ has already been shown to induce the identity on the rational function monoid of $\left.\underline{\underline{\mathcal{F}}}_{v}\right]$, we conclude that $\alpha$ induces the identity on the non-cuspidal elements of the monoids that appear in the divisor monoid of $\underline{\underline{\mathcal{F}}}_{\underline{v}}$ [cf. [EtTh], Proposition 5.3, (v), (vi), for a discussion of closely related facts]. In a similar vein, since any divisor of degree zero on an elliptic curve that is supported on the torsion points of the elliptic curve admits a positive multiple which is principal, it follows by considering the cuspidal portions of divisors of appropriate rational functions [each of which is preserved by $\alpha$, since $\alpha$ has already been shown to induce the identity on the rational function monoid of $\left.\underline{\underline{\mathcal{F}}}_{\underline{v}}\right]$ that $\alpha$ also induces the identity on the cuspidal elements of the monoids that appear in the divisor monoid of $\underline{\underline{\mathcal{F}}}_{\underline{v}}$. This completes the proof of assertion (iv).

\section{Remark 5.3.1.}

(i) In the situation of Corollary 5.3, (ii), let

$$
\phi:{ }^{1} \mathfrak{D} \rightarrow{ }^{2} \mathfrak{D}
$$

be a morphism of $\mathcal{D}$-prime-strips [i.e., which is not necessarily an isomorphism!] that induces an isomorphism between the respective collections of data indexed by $\underline{v} \in \underline{\mathbb{V}}^{\text {good }}$, as well as an isomorphism $\phi^{\vdash}:{ }^{1} \mathfrak{D}^{\vdash} \stackrel{\sim}{\rightarrow} 2 \mathfrak{D}^{\vdash}$ between the associated $\mathcal{D}^{\vdash}$-prime-strips [cf. Definition 4.1, (iv)]. Then let us observe that by applying Corollary 5.3, (ii), it follows that $\phi$ lifts to a uniquely determined "arrow"

$$
\psi:{ }^{1} \mathfrak{F} \rightarrow{ }^{2} \mathfrak{F}
$$

— which we think of as "lying over" $\phi$ - defined as follows: First, let us recall that, in light of our assumptions on $\phi$, it follows immediately from the construction 
[cf. Examples 3.2, (iii); 3.3, (i); 3.4, (i)] of the various p-adic and archimedean Frobenioids [cf. [FrdII], Example 1.1, (ii); [FrdII], Example 3.3, (ii)] that appear in an $\mathcal{F}$-prime-strip that it makes sense to speak of the "pull-back" - i.e., by forming the "categorical fiber product" [cf. [FrdI], §0; [FrdI], Proposition 1.6] — of the Frobenioids that appear in the $\mathcal{F}$-prime-strip ${ }^{2} \mathfrak{F}$ via the various morphisms at $\underline{v} \in \underline{\mathbb{V}}$ that constitute $\phi$. That is to say, it follows from our assumptions on $\phi$ [cf. also [AbsTopIII], Proposition 3.2, (iv)] that $\phi$ determines a pulled-back $\mathcal{F}$ prime-strip " $\phi^{*}(2 \mathfrak{F})$ ", whose associated $\mathcal{D}$-prime-strip [cf. Remark 5.2.1, (i)] is tautologically equal to ${ }^{1} \mathfrak{D}$. On the other hand, by Corollary 5.3, (ii), it follows that this tautological equality of associated $\mathcal{D}$-prime-strips uniquely determines an isomorphism ${ }^{1} \mathfrak{F} \stackrel{\sim}{\rightarrow} \phi^{*}\left({ }^{2} \mathfrak{F}\right)$. Then we define the arrow $\psi:{ }^{1} \mathfrak{F} \rightarrow{ }^{2} \mathfrak{F}$ to be the collection of data consisting of $\phi$ and this isomorphism ${ }^{1} \mathfrak{F} \stackrel{\sim}{\rightarrow} \phi^{*}\left({ }^{2} \mathfrak{F}\right)$; we refer to $\psi$ as the "morphism uniquely determined by $\phi$ " or the "uniquely determined morphism that lies over $\phi "$ ". Also, we shall apply various terms used to describe a morphism $\phi$ of $\mathcal{D}$-prime-strips to the "arrow" of $\mathcal{F}$-prime-strips determined by $\phi$.

(ii) The conventions discussed in (i) concerning liftings of morphisms of $\mathcal{D}$ prime-strips may also be applied to poly-morphisms. We leave the routine details to the reader.

Remark 5.3.2. Just as in the case of Corollary 5.3, (i), (ii), the rigidity property of Corollary 5.3, (iv), may be regarded as being essentially a consequence of the "Kummer-readiness" [cf. Remarks 5.1.3, 5.2.2] of the tempered Frobenioid $\underline{\underline{\mathcal{F}}}_{\underline{v}}$ cf. also the arguments applied in the proofs of [AbsTopIII], Proposition 3.2, (iv); [AbsTopIII], Corollary 5.2, (iv).

Remark 5.3.3. We take this opportunity to rectify a minor oversight in [FrdI]. The hypothesis that the Frobenioids under consideration be of "unit-profinite type" in [FrdI], Proposition 5.6 - hence also in [FrdI], Corollary 5.7, (iii) - may be removed. Indeed, if, in the notation of the proof of [FrdI], Proposition 5.6, one writes $\phi_{p}^{\prime}=c_{p} \cdot \phi_{p}$, where $c_{p} \in \mathcal{O}^{\times}(A)$, for $p \in \mathfrak{P r i m e s}$, then one has

$$
\begin{aligned}
c_{2} \cdot c_{p}^{2} \cdot \phi_{2} \cdot \phi_{p} & =c_{2} \cdot \phi_{2} \cdot c_{p} \cdot \phi_{p}=\phi_{2}^{\prime} \cdot \phi_{p}^{\prime}=\phi_{p}^{\prime} \cdot \phi_{2}^{\prime} \\
& =c_{p} \cdot \phi_{p} \cdot c_{2} \cdot \phi_{2}=c_{p} \cdot c_{2}^{p} \cdot \phi_{p} \cdot \phi_{2}=c_{p} \cdot c_{2}^{p} \cdot \phi_{2} \cdot \phi_{p}
\end{aligned}
$$

- so $c_{2} \cdot c_{p}^{2}=c_{p} \cdot c_{2}^{p}$, i.e., $c_{p}=c_{2}^{p-1}$, for $p \in \mathfrak{P r i m e s}$. Thus, $\phi_{p}^{\prime}=c_{2}^{-1} \cdot \phi_{p} \cdot c_{2}$, so by taking $u \stackrel{\text { def }}{=} c_{2}^{-1}$, one may eliminate the final two paragraphs of the proof of [FrdI], Proposition 5.6.

Let

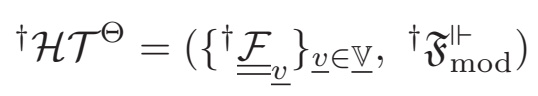

be a $\Theta$-Hodge theater [relative to the given initial $\Theta$-data — cf. Definition 3.6] such

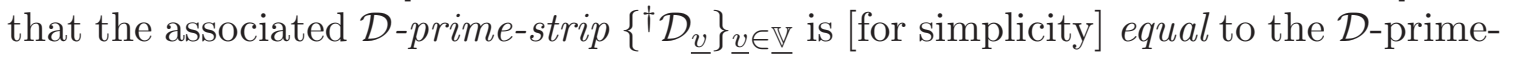
strip ${ }^{\dagger} \mathfrak{D}_{>}$of the $\mathcal{D}$ - $\Theta N F-H o d g e$ theater in the discussion preceding Example 5.1. Write 
for the $\mathcal{F}$-prime-strip tautologically associated to this $\Theta$-Hodge theater [cf. the data " $\left\{\underline{\underline{\mathcal{F}}}_{\underline{\underline{\mathcal{F}}}}\right\}_{\underline{v}} \in \underline{\mathbb{V}}$ " of Definition 3.6; Definition 5.2, (i); Example 3.2, (iii); Example 3.3, (i)]. Thus, ${ }^{\dagger} \mathfrak{D}_{>}$may be identified with the $\mathcal{D}$-prime-strip associated [cf. Remark $5.2 .1,(\mathrm{i})]$ to ${ }^{\dagger} \mathfrak{F}_{>}$.

\section{Example 5.4. Model $\Theta$ - and NF-Bridges.}

(i) For $j \in J$, let

$$
{ }^{\dagger} \mathfrak{F}_{j}=\left\{{ }^{\dagger} \mathcal{F}_{\underline{v}_{j}}\right\}_{\underline{v}_{j} \in \underline{V}_{j}}
$$

be an $\mathcal{F}$-prime-strip whose associated $\mathcal{D}$-prime-strip [cf. Remark 5.2.1, (i)] is equal to $\mathfrak{D}_{j}$,

$$
{ }^{\dagger} \mathfrak{F}_{\langle J\rangle}=\left\{{ }^{\dagger} \mathcal{F}_{\underline{v}_{\langle J\rangle}}\right\}_{\underline{v}_{\langle J\rangle} \in \underline{\mathbb{V}}_{\langle J\rangle}}
$$

an $\mathcal{F}$-prime-strip whose associated $\mathcal{D}$-prime-strip we denote by ${ }^{\dagger} \mathfrak{D}_{\langle J\rangle}$ [cf. Example 5.1, (vii)]. Write

$$
{ }^{\dagger} \mathfrak{F}_{J} \stackrel{\text { def }}{=} \prod_{j \in J}{ }^{\dagger} \mathfrak{F}_{j}
$$

- where the "formal product $\prod$ " is to be understood as denoting the capsule with index set $J$ for which the datum indexed by $j$ is given by ${ }^{\dagger} \mathfrak{F}_{j}$. Thus, ${ }^{\dagger} \mathfrak{F}_{\langle J\rangle}$ may be related to ${ }^{\dagger} \mathfrak{F}_{>}$, in a natural fashion, via the full poly-isomorphism

$$
{ }^{\dagger} \mathfrak{F}_{\langle J\rangle} \stackrel{\sim}{\rightarrow}{ }^{\dagger} \mathfrak{F}_{\rangle}
$$

and to ${ }^{\dagger} \mathfrak{F}_{J}$ via the "diagonal arrow"

$$
{ }^{\dagger} \mathfrak{F}_{\langle J\rangle} \rightarrow{ }^{\dagger} \mathfrak{F}_{J}=\prod_{j \in J}{ }^{\dagger} \mathfrak{F}_{j}
$$

- i.e., the arrow defined as the collection of data indexed by $J$ for which the datum indexed by $j$ is given by the full poly-isomorphism ${ }^{\dagger} \mathfrak{F}_{\langle J\rangle} \stackrel{\sim}{\rightarrow}{ }^{\dagger} \mathfrak{F}_{j}$. Thus, we think of ${ }^{\dagger} \mathfrak{F}_{j}$ as a copy of ${ }^{\dagger} \mathfrak{F}_{>}$"situated on" the constituent labeled $j$ of the capsule $\dagger \mathfrak{D}_{J}$; we think of $\mathfrak{f}^{\dagger} \mathfrak{F}_{\langle J\rangle}$ as a copy of ${ }^{\dagger} \mathfrak{F}_{>}$"situated in a diagonal fashion on" all the constituents of the capsule ${ }^{\dagger} \mathfrak{D}_{J}$.

(ii) Note that in addition to thinking of ${ }^{\dagger} \mathfrak{F}_{>}$as being related to ${ }^{\dagger} \mathfrak{F}_{j}$ [for $\left.j \in J\right]$ via the full poly-isomorphism ${ }^{\dagger} \mathfrak{F}_{>} \stackrel{\sim}{\rightarrow}{ }^{\dagger} \mathfrak{F}_{j}$, we may also regard ${ }^{\dagger} \mathfrak{F}_{>}$as being related to ${ }^{\dagger} \mathfrak{F}_{j}[$ for $j \in J]$ via the poly-morphism

$$
{ }^{\dagger} \psi_{j}^{\Theta}:{ }^{\dagger} \mathfrak{F}_{j} \rightarrow{ }^{\dagger} \mathfrak{F}_{>}
$$

uniquely determined by ${ }^{\dagger} \phi_{j}^{\Theta}$ [i.e., as discussed in Remark 5.3.1]. Write

$$
{ }^{\dagger} \psi_{*}^{\Theta}:{ }^{\dagger} \mathfrak{F}_{J} \rightarrow{ }^{\dagger} \mathfrak{F}_{>}
$$

for the collection of arrows $\left\{{ }^{\dagger} \psi_{j}^{\Theta}\right\}_{j \in J}$ — which we think of as "lying over" the collection of arrows ${ }^{\dagger} \phi_{*}^{\Theta}=\left\{{ }^{\dagger} \phi_{j}^{\Theta}\right\}_{j \in J}$.

(iii) Next, let ${ }^{\dagger} \mathcal{F}^{\circledast},{ }^{\dagger} \mathcal{F}^{\odot}$ be as in Example 5.1, (iii); $\delta \in \operatorname{LabCusp}\left({ }^{\dagger} \mathcal{D}^{\odot}\right)$. Thus, [cf. the discussion of Example 4.3, (i)] there exists a unique Aut $_{\underline{\epsilon}}\left({ }^{\dagger} \mathcal{D}^{\odot}\right)$-orbit of 
isomorphisms ${ }^{\dagger} \mathcal{D}^{\odot} \stackrel{\sim}{\rightarrow} \mathcal{D}^{\odot}$ that maps $\delta \mapsto[\underline{\epsilon}] \in \operatorname{LabCusp}\left(\mathcal{D}^{\odot}\right)$. We shall refer to as a $\delta$-valuation $\in \mathbb{V}\left({ }^{\dagger} \mathcal{D}^{\odot}\right)$ [cf. Definition 4.1, (v)] any element that maps to an element of $\underline{\mathbb{V}}^{ \pm \text {un }}\left[\mathrm{cf}\right.$. Example 4.3, (i)] via this $\operatorname{Aut}_{\underline{\epsilon}}\left({ }^{\dagger} \mathcal{D}^{\odot}\right)$-orbit of isomorphisms. Note that the notion of a $\delta$-valuation may also be defined intrinsically by means of the structure of $\mathcal{D}$-NF-bridge ${ }^{\dagger} \phi_{*}^{\mathrm{NF}}$. Indeed, [one verifies immediately that] a $\delta$-valuation may be defined as a valuation $\in \mathbb{V}\left({ }^{\dagger} \mathcal{D}^{\odot}\right)$ that lies in the "image" [in the evident sense] via ${ }^{\dagger} \phi_{*}^{\text {NF }}$ of the unique $\mathcal{D}$-prime-strip ${ }^{\dagger} \mathfrak{D}_{j}$ of the capsule ${ }^{\dagger} \mathfrak{D}_{J}$ such that the bijection $\operatorname{LabCusp}\left({ }^{\dagger} \mathcal{D}^{\odot}\right) \stackrel{\sim}{\rightarrow} \operatorname{LabCusp}\left({ }^{\dagger} \mathfrak{D}_{j}\right)$ induced by ${ }^{\dagger} \phi_{*}^{\mathrm{NF}}[\mathrm{cf}$. the discussion of Example 4.5, (i)] maps $\delta$ to the element of LabCusp $\left({ }^{\dagger} \mathfrak{D}_{j}\right)$ that is "labeled 1", relative to the bijection of the second display of Proposition 4.2.

(iv) We continue to use the notation of (iii). Then let us observe that by localizing at each of the $\delta$-valuations $\in \mathbb{V}\left({ }^{\dagger} \mathcal{D}^{\odot}\right)$, one may construct, in a natural way, an $\mathcal{F}$-prime-strip

$$
\left.{ }^{\dagger} \mathcal{F}^{\odot}\right|_{\delta}
$$

— which is well-defined up to isomorphism - from ${ }^{\dagger} \mathcal{F}^{\odot}$ [i.e., in the notation of Example 5.1, (iv), from $\widetilde{\mathcal{O}}^{\circledast \times}$, equipped with its natural $\pi_{1}\left({ }^{\dagger} \mathcal{D}^{\odot}\right)$-action]. Indeed, at a nonarchimedean $\delta$-valuation $\underline{v}$, this follows by considering the $p_{\underline{v}}$-adic Frobenioids [cf. Remark 3.3.2] associated to the restrictions to suitable open subgroups of $\Pi_{\mathfrak{p}_{0}} \bigcap \pi_{1}\left({ }^{\dagger} \mathcal{D}^{\odot}\right)\left(\subseteq \pi_{1}\left({ }^{\dagger} \mathcal{D}^{\odot}\right) \subseteq \pi_{1}\left({ }^{\dagger} \mathcal{D}^{\circledast}\right)\right)$ determined by $\delta \in \operatorname{LabCusp}\left({ }^{\dagger} \mathcal{D}^{\odot}\right)$ [i.e., open subgroups corresponding to the coverings " $X$ ", " $X$ " discussed in Definition 3.1 , (e), (f); cf. also Remark 3.1.2, (i)], where $\Pi_{\mathfrak{p}_{0}}$ is chosen [among its $\pi_{1}\left({ }^{\dagger} \mathcal{D}^{\circledast}\right)$ conjugates] so as to correspond to $\underline{v}$, of the pairs

$$
" \Pi_{\mathfrak{p}_{0}} \curvearrowright \widetilde{\mathcal{O}}{\widetilde{\mathfrak{p}_{0}}}^{\triangleright} "
$$

of Example 5.1, (v) [cf. also Example 5.1, (vi)]. [Here, we note that, when $\underline{v}$ lies over an element of $\mathbb{V}_{\bmod }^{\text {bad }}$, one must replace these "suitable open subgroups" by their tempered analogues, i.e., by applying the mono-anabelian algorithm implicit in the proof of [SemiAnbd], Theorem 6.6.] On the other hand, at an archimedean $\delta$ valuation $\underline{v}$, this follows by applying the functorial algorithm for reconstructing the corresponding Aut-holomorphic orbispace at $\underline{v}$ given in [AbsTopIII], Corollaries 2.8, 2.9 , together with the discussion concerning the "isomorphism $\mathbb{M}^{\circledast}\left({ }^{\dagger} \mathcal{D}^{\odot}\right) \stackrel{\sim}{\rightarrow} \dagger \mathbb{M}^{\circledast}$ " in Example 5.1, (v) [cf. also Example 5.1, (vi)]. Here, we observe that since the natural projection map $\underline{\mathbb{V}}^{ \pm \text {un }} \rightarrow \mathbb{V}_{\text {mod }}$ fails to be injective, in order to relate the restrictions obtained at different elements in a fiber of this map in a welldefined fashion, it is necessary to regard $\left.{ }^{\dagger} \mathcal{F}^{\odot}\right|_{\delta}$ as being well-defined only up to isomorphism. Nevertheless, despite this indeterminacy inherent in the definition of $\left.{ }^{\dagger} \mathcal{F}^{\odot}\right|_{\delta}$, it still makes sense to define, for an $\mathcal{F}$-prime-strip ${ }^{\ddagger} \mathfrak{F}$ with underlying $\mathcal{D}$-prime-strip ${ }^{\ddagger} \mathfrak{D}$ [cf. Remark 5.2.1, (i)], a poly-morphism

$$
\ddagger \mathfrak{F} \rightarrow{ }^{\dagger} \mathcal{F}^{\odot}
$$

to be a full poly-isomorphism $\left.{ }^{\ddagger} \mathfrak{F} \stackrel{\sim}{\rightarrow}{ }^{\dagger} \mathcal{F}^{\odot}\right|_{\delta}$ for some $\delta \in \operatorname{LabCusp}\left({ }^{\dagger} \mathcal{D}^{\odot}\right)$ [cf. Definition 4.1, (vi)]. Moreover, it makes sense to pre-compose such poly-morphisms with isomorphisms of $\mathcal{F}$-prime-strips and to post-compose such poly-morphisms with isomorphisms between isomorphs of ${ }^{\dagger} \mathcal{F}^{\odot}$. Here, we note that such a polymorphism ${ }^{\ddagger} \mathfrak{F} \rightarrow{ }^{\dagger} \mathcal{F}^{\odot}$ may be thought of as "lying over" an induced poly-morphism $\ddagger \mathfrak{D} \rightarrow{ }^{\dagger} \mathcal{D}^{\odot}$ [cf. Definition 4.1, (vi)], and that any poly-morphism ${ }^{\ddagger} \mathfrak{F} \rightarrow{ }^{\dagger} \mathcal{F}^{\odot}$ is 
fixed by pre-composition with automorphisms of $\ddagger \mathfrak{F}$, as well as by post-composition with automorphisms $\in \operatorname{Aut}_{\underline{\epsilon}}\left({ }^{\dagger} \mathcal{F}^{\odot}\right)$. Also, we observe that such a poly-morphism ${ }^{\ddagger} \mathfrak{F} \rightarrow{ }^{\dagger} \mathcal{F}^{\odot}$ is compatible with the local and global $\infty_{\infty} \kappa$-coric structures [cf. Example 5.1, (v); Definition 5.2, (vi), (viii)] that appear in the domain and codomain of this poly-morphism in the following sense: the operation of restriction of associated Kummer classes [cf. the discussion of Example 5.1, (v); Definition 5.2, (vi), (viii); the constructions discussed in the present item (iv)] determines a collection, indexed by $\underline{v} \in \underline{\mathbb{V}}$, of poly-morphisms of pseudo-monoids

$$
\left\{\pi_{1}^{\mathrm{rat}}\left({ }^{\dagger} \mathcal{D}^{\circledast}\right) \curvearrowright{ }^{\dagger} \mathbb{M}_{\infty \kappa}^{\circledast} \rightarrow{ }^{\ddagger} \mathbb{M}_{\infty \kappa v} \subseteq{ }^{\ddagger} \mathbb{M}_{\infty \kappa \times v}\right\}_{\underline{v} \in \underline{\mathbb{V}}}
$$

- where the global data in the domain of the arrow that appears in the display is regarded as only being defined up to automorphisms induced by inner automorphisms of $\pi_{1}^{\text {rat }}\left({ }^{\dagger} \mathcal{D}^{\circledast}\right)$ [cf. the discussion of Example 5.1, (i)]; the local data in the codomain of the arrow that appears in the display is regarded as only being defined up to automorphisms induced by automorphisms of the $\mathcal{F}$-prime-strip $\ddagger \mathfrak{F}$ [cf. Definition 5.2 , (vi), (viii); Corollary 5.3, (ii)]; the arrow of the display is equivariant with respect to the various homomorphisms $\pi_{1}^{\mathrm{rat}}\left({ }^{\ddagger} \mathcal{D}_{v}\right) \rightarrow \pi_{1}^{\mathrm{rat}}\left({ }^{\dagger} \mathcal{D}^{\circledast}\right)$ [i.e., relative to the respective actions of these groups on the pseudo-monoids in the domain and codomain of the arrow] induced [cf. the constructions discussed in the present item (iv), as well as the theory summarized in [AbsTopIII], Theorem 1.9, and [AbsTopIII], Corollaries $1.10,2.8]$ by the given poly-morphism ${ }^{\ddagger} \mathfrak{F} \rightarrow{ }^{\dagger} \mathcal{F}^{\odot}$; when $\underline{v} \in \underline{\mathbb{V}}^{\text {arc }}$, we regard the pseudo-monoids $¥ \mathbb{M}_{\infty \kappa v} \subseteq \mathbb{M}_{\infty} \kappa \times v$ as being equipped with the various splittings discussed in Definition 5.2, (viii). Finally, if $\left\{{ }^{e} \mathfrak{F}\right\}_{e \in E}$ is a capsule of $\mathcal{F}$-prime-strips whose associated capsule of $\mathcal{D}$-prime-strips [cf. Remark 5.2.1, (i)] we denote by $\left\{{ }^{e} \mathfrak{D}\right\}_{e \in E}$, then we define a poly-morphism

$$
\left\{{ }^{e} \mathfrak{F}\right\}_{e \in E} \rightarrow{ }^{\dagger} \mathcal{F}^{\odot} \text { (respectively, }\left\{{ }^{e} \mathfrak{F}\right\}_{e \in E} \rightarrow{ }^{\dagger} \mathfrak{F} \text { ) }
$$

to be a collection of poly-morphisms $\left\{{ }^{e} \mathfrak{F} \rightarrow{ }^{\dagger} \mathcal{F}^{\odot}\right\}_{e \in E}$ (respectively, $\left\{{ }^{e} \mathfrak{F} \rightarrow{ }^{\dagger} \mathfrak{F}\right\}_{e \in E}$ ) [cf. Definition 4.1, (vi)]. Thus, a poly-morphism $\left\{{ }^{e} \mathfrak{F}\right\}_{e \in E} \rightarrow{ }^{\dagger} \mathcal{F}^{\odot}$ (respectively, $\left\{{ }^{e} \mathfrak{F}\right\}_{e \in E} \rightarrow \dagger \mathfrak{F}$ ) may be thought of as "lying over" an induced poly-morphism $\left\{{ }^{e} \mathfrak{D}\right\}_{e \in E} \rightarrow^{\dagger} \mathcal{D}^{\odot}$ (respectively, $\left\{{ }^{e} \mathfrak{D}\right\}_{e \in E} \rightarrow^{\dagger} \mathfrak{D}$ ) [cf. Definition 4.1, (vi)].

(v) We continue to use the notation of (iv). Now observe that by Corollary 5.3 , (ii), there exists a unique poly-morphism

$$
{ }^{\dagger} \psi_{*}^{\mathrm{NF}}:{ }^{\dagger} \mathfrak{F}_{J} \rightarrow{ }^{\dagger} \mathcal{F}^{\odot}
$$

that lies over ${ }^{\dagger} \phi_{*}^{\mathrm{NF}}$.

(vi) We continue to use the notation of (v). Now observe that it follows from the definition of ${ }^{\dagger} \mathcal{F}_{\bmod }^{\circledast}$ in terms of terminal objects of ${ }^{\dagger} \mathcal{D}^{\circledast}$ [cf. Example 5.1, (iii)] that any poly-morphism ${ }^{\dagger} \mathfrak{F}_{\langle J\rangle} \rightarrow{ }^{\dagger} \mathcal{F}^{\odot}$ [cf. the notation of (i)] induces, via "restriction" [in the evident sense], an isomorphism class of functors [cf. Definition 5.2, (i); the notation of Example 5.1, (vii)]

$$
\left({ }^{\dagger} \mathcal{F}^{\odot} \rightarrow{ }^{\dagger} \mathcal{F}^{\circledast} \supseteq\right) \quad{ }^{\dagger} \mathcal{F}_{\bmod }^{\circledast} \stackrel{\sim}{\rightarrow}{ }^{\dagger} \mathcal{F}_{\langle J\rangle}^{\circledast} \rightarrow{ }^{\dagger} \mathcal{F}_{\underline{v}_{\langle J\rangle}}
$$


for each $\underline{v}_{\langle J\rangle} \in \underline{\mathbb{V}}_{\langle J\rangle}$ - where, by abuse of notation when $\underline{v}_{\langle J\rangle} \in \underline{\mathbb{V}}_{\langle J\rangle}^{\text {arc }}$, we write " $\mathcal{F}_{\underline{v}_{\langle J}}$ " for the category portion of the "collection of data" that appears in Definition 5.2, (i), (b) - which is independent of the choice of the poly-morphism ${ }^{\dagger} \mathfrak{F}_{\langle J\rangle} \rightarrow{ }^{\dagger} \mathcal{F}^{\odot}$ [i.e., among its $\mathbb{F}_{l}^{*}$-conjugates]. That is to say, the fact that ${ }^{\dagger} \mathcal{F}_{\text {mod }}^{\circledast}$ is defined in terms of terminal objects of ${ }^{\dagger} \mathcal{D}^{\circledast}$ [cf. also the definition of $F_{\text {mod }}$ given in Definition 3.1, (b)!] implies that this particular isomorphism class of functors is immune to [i.e., fixed by] the various indeterminacies that appear in the choice of ${ }^{\dagger} \mathfrak{F}_{\langle J\rangle} \rightarrow{ }^{\dagger} \mathcal{F}^{\ominus}$. Let us write

$$
\left({ }^{\dagger} \mathcal{F}^{\odot} \rightarrow{ }^{\dagger} \mathcal{F}^{\circledast} \supseteq\right) \quad{ }^{\dagger} \mathcal{F}_{\text {mod }}^{\circledast} \stackrel{\sim}{\rightarrow}{ }^{\dagger} \mathcal{F}_{\langle J\rangle}^{\circledast} \rightarrow{ }^{\dagger} \mathfrak{F}_{\langle J\rangle}
$$

for the collection of isomorphism classes of restriction functors just defined, as $\underline{v}\langle J\rangle$ ranges over the elements of $\underline{\mathbb{V}}_{\langle J\rangle}$. In a similar vein, we also obtain collections of natural isomorphism classes of restriction functors

$$
{ }^{\dagger} \mathcal{F}_{J}^{\circledast} \rightarrow{ }^{\dagger} \mathfrak{F}_{J} ; \quad{ }^{\dagger} \mathcal{F}_{j}^{\circledast} \rightarrow{ }^{\dagger} \mathfrak{F}_{j}
$$

for $j \in J$. Finally, just as in Example 5.1, (vii), we obtain natural realifications

$$
{ }^{\dagger} \mathcal{F}_{\langle J\rangle}^{\circledast \mathbb{R}} \rightarrow{ }^{\dagger} \mathfrak{F}_{\langle J\rangle}^{\mathbb{R}} ; \quad{ }^{\dagger} \mathcal{F}_{J}^{\circledast \mathbb{R}} \rightarrow{ }^{\dagger} \mathfrak{F}_{J}^{\mathbb{R}} ; \quad{ }^{\dagger} \mathcal{F}_{j}^{\circledast \mathbb{R}} \rightarrow{ }^{\dagger} \mathfrak{F}_{j}^{\mathbb{R}}
$$

of the various $\mathcal{F}$-prime-strips - i.e., realifications [cf. [FrdI], Corollary 5.4; [FrdII], Theorem 1.2, (i); [FrdII], Theorem 3.6, (i)] of each of the Frobenioid [that is to say, category] portions of the data of Definition 5.2, (i), (a), (b) - and isomorphism classes of restriction functors discussed so far.

(vii) We shall refer to as "pivotal distributions" the objects constructed in (vi)

$$
\dagger \mathcal{F}_{\mathrm{pvt}}^{\circledast} \rightarrow{ }^{\dagger} \mathfrak{F}_{\mathrm{pvt}} ; \quad{ }^{\dagger} \mathcal{F}_{\mathrm{pvt}}^{\circledast \mathbb{R}} \rightarrow{ }^{\dagger} \mathfrak{F}_{\mathrm{pvt}}^{\mathbb{R}}
$$

in the case $j=1-$ cf. Fig. 5.2 below.

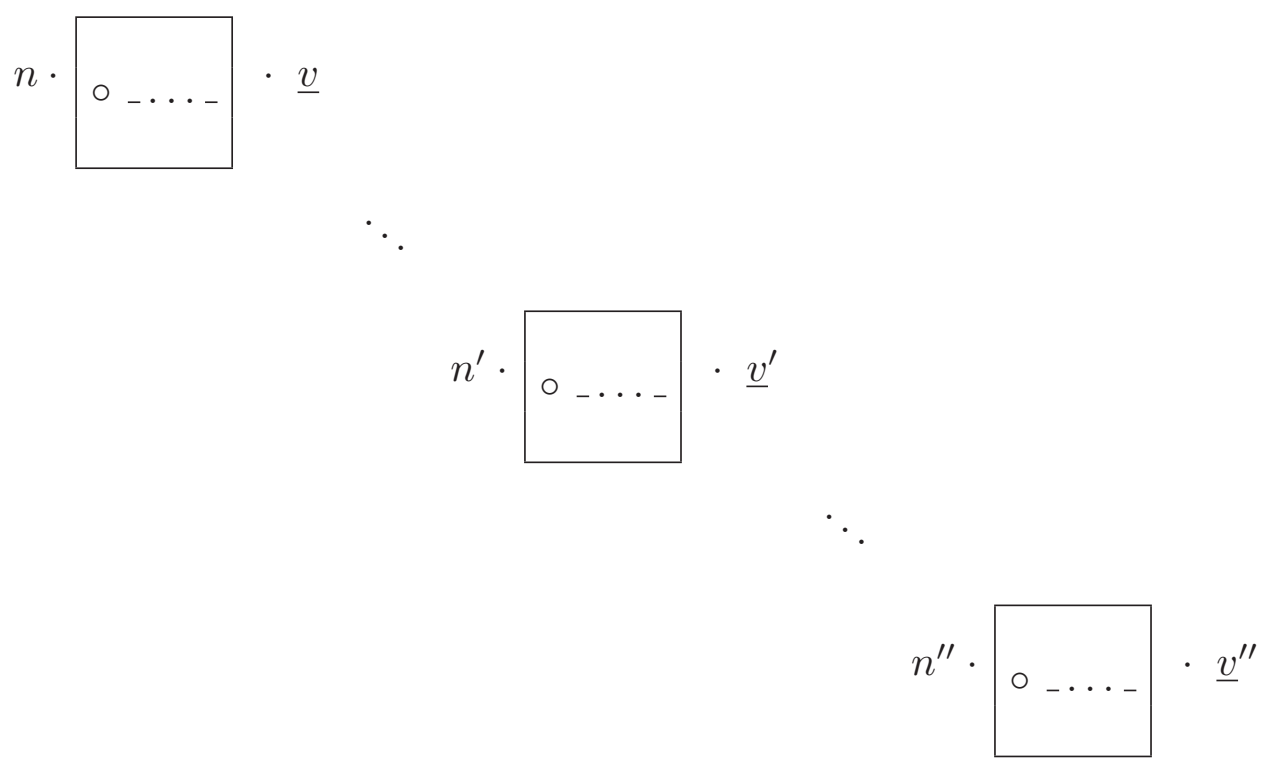

Fig. 5.2: Pivotal distribution 
Remark 5.4.1. The constructions of Example 5.4, (i), (ii) (respectively, Example 5.4, (iii), (iv), (v), (vi), (vii)) manifestly only require the $\mathcal{D}$ - $\Theta$-bridge portion ${ }^{\dagger} \phi_{*}^{\Theta}$ (respectively, $\mathcal{D}$-NF-bridge portion ${ }^{\dagger} \phi_{*}^{\mathrm{NF}}$ ) of the $\mathcal{D}$ - $\Theta \mathrm{NF}$-Hodge theater ${ }^{\dagger} \mathcal{H} \mathcal{T}^{\mathcal{D} \text { - } \Theta \mathrm{NF}}$ [cf. Remark 5.1.2].

\section{Remark 5.4.2.}

(i) At this point, it is useful to consider the various copies of $\dagger \mathcal{F}_{\bmod }^{\circledast}$ and its realifications introduced so far from the point of view of "log-volumes", i.e., arithmetic degrees [cf., e.g., the discussion of [FrdI], Example 6.3; [FrdI], Theorem 6.4; Remark 3.1.5 of the present paper]. That is to say, since ${ }^{\dagger} \mathcal{F}_{j}^{\circledast}$ may be thought of as a sort of "section of ${ }^{\dagger} \mathcal{F}_{J}^{\circledast}$ over ${ }^{\dagger} \mathcal{F}_{\bmod }^{\circledast}$ " - i.e., a sort of "section of $K$ over $F_{\bmod }$ " [cf. the discussion of prime-strips in Remark 4.3.1] — one way to think of log-volumes of ${ }^{\dagger} \mathcal{F}_{\langle J\rangle}^{\circledast}$ is as quantities that differ by a factor of $l^{*}$ — i.e., corresponding, to the cardinality of $J \stackrel{\sim}{\rightarrow} \mathbb{F}_{l}^{*}$ - from log-volumes of ${ }^{\dagger} \mathcal{F}_{j}^{\circledast}$. Put another way, this amounts to thinking of arithmetic degrees that appear in the various factors of ${ }^{\dagger} \mathcal{F}_{J}^{\circledast}$ as being

averaged over the elements of $J$ and hence of arithmetic degrees that appear in ${ }^{\dagger} \mathcal{F}_{\langle J\rangle}^{\circledast}$ as the "resulting averages".

This sort of averaging may be thought of as a sort of abstract, Frobenioid-theoretic analogue of the normalization of arithmetic degrees that is often used in the theory of heights [cf., e.g., [GenEll], Definition 1.2, (i)] that allows one to work with heights in such a way that the height of a point remains invariant with respect to change of the base field.

(ii) On the other hand, to work with the various isomorphisms of Frobenioids — such as ${ }^{\dagger} \mathcal{F}_{j}^{\circledast} \stackrel{\sim}{\rightarrow} \dagger \mathcal{F}_{\langle J\rangle}^{\circledast}$ — involved amounts [since the arithmetic degree is an intrinsic invariant of the Frobenioids involved - cf. [FrdI], Theorem 6.4, (iv); Remark 3.1.5 of the present paper] to thinking of arithmetic degrees that appear in the various factors of ${ }^{\dagger} \mathcal{F}_{J}^{\circledast}$ as being

summed [i.e., without dividing by a factor of $l^{*}$ ] over the elements of $J$ and hence of arithmetic degrees that appear in ${ }^{\dagger} \mathcal{F}_{\langle J\rangle}^{\circledast}$ as the "resulting sums".

This point of view may be thought of as a sort of abstract, Frobenioid-theoretic analogue of the normalization of arithmetic degrees or heights in which the height of a point is multiplied by the degree of the field extension when one executes a change of the base field.

The notions defined in the following "Frobenioid-theoretic lifting" of Definition 4.6 will play a central role in the theory of the present series of papers.

\section{Definition 5.5.}

(i) We define an NF-bridge [relative to the given initial $\Theta$-data] to be a collection of data

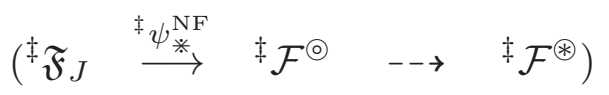


as follows:

(a) ${ }^{\ddagger} \mathfrak{F}_{J}=\left\{{ }^{\ddagger} \mathfrak{F}_{j}\right\}_{j \in J}$ is a capsule of $\mathcal{F}$-prime-strips, indexed by a finite index set $J$. Write ${ }^{\ddagger} \mathfrak{D}_{J}=\left\{{ }^{\ddagger} \mathfrak{D}_{j}\right\}_{j \in J}$ for the associated capsule of $\mathcal{D}$-prime-strips [cf. Remark 5.2.1, (i)].

(b) ${ }^{\ddagger} \mathcal{F}^{\odot},{ }^{\ddagger} \mathcal{F}^{\circledast}$ are categories equivalent to the categories ${ }^{\dagger} \mathcal{F}^{\odot},{ }^{\dagger} \mathcal{F}^{\circledast}$, respectively, of Example 5.1, (iii). Thus, each of $\ddagger \mathcal{F}^{\odot},{ }^{\ddagger} \mathcal{F}^{\circledast}$ is equipped with a natural Frobenioid structure [cf. [FrdI], Corollary 4.11; [FrdI], Theorem 6.4 , (i); Remark 3.1.5 of the present paper]; write ${ }^{\ddagger} \mathcal{D}^{\odot},{ }^{\ddagger} \mathcal{D}^{\circledast}$ for the respective base categories of these Frobenioids.

(c) The arrow “- $\rightarrow$ " consists of the datum of a morphism ${ }^{\ddagger} \mathcal{D}^{\odot} \rightarrow{ }^{\ddagger} \mathcal{D}^{\circledast}$ which is abstractly equivalent [cf. §0] to the natural morphism ${ }^{\dagger} \mathcal{D}^{\odot} \rightarrow$ ${ }^{\dagger} \mathcal{D}^{\circledast}$ of Example 5.1, (i), together with the datum of an isomorphism $\left.\ddagger \mathcal{F}^{\odot} \stackrel{\sim}{\rightarrow} \ddagger \mathcal{F}^{\circledast}\right|_{\ddagger \mathcal{D}} \odot[$ cf. the discussion of Example 5.1, (iii)].

(d) ${ }^{\ddagger} \psi_{*}^{\mathrm{NF}}$ is a poly-morphism that lifts [uniquely! — cf. Corollary 5.3, (ii)] a poly-morphism ${ }^{\ddagger} \phi_{*}^{\mathrm{NF}}:{ }^{\ddagger} \mathfrak{D}_{J} \rightarrow{ }^{\ddagger} \mathcal{D}^{\odot}$ such that ${ }^{\ddagger} \phi_{*}^{\mathrm{NF}}$ forms a $\mathcal{D}$-NF-bridge [cf. Example 5.4, (v); Remark 5.4.1].

Thus, one verifies immediately that any NF-bridge as above determines an associated $\mathcal{D}$-NF-bridge $\left({ }^{\ddagger} \phi_{*}^{\mathrm{NF}}:{ }^{\ddagger} \mathfrak{D}_{J} \rightarrow{ }^{\ddagger} \mathcal{D}^{\odot}\right)$. We define a(n) [iso]morphism of NF-bridges

$$
\left({ }^{1} \mathfrak{F}_{J_{1}} \stackrel{{ }^{1} \stackrel{\psi_{*}^{\mathrm{NF}}}{\longrightarrow}}{\longrightarrow} 1 \mathcal{F}^{\odot} \quad \rightarrow \quad \rightarrow \mathcal{F}^{\circledast}\right) \quad \rightarrow \quad\left({ }^{2} \mathfrak{F}_{J_{2}} \stackrel{{ }^{2} \psi_{*}^{\mathrm{NF}}}{\longrightarrow}{ }^{2} \mathcal{F}^{\odot} \quad \rightarrow \quad \rightarrow \mathcal{F}^{\circledast}\right)
$$

to be a collection of arrows

$$
{ }^{1} \mathfrak{F}_{J_{1}} \stackrel{\sim}{\rightarrow}{ }^{2} \mathfrak{F}_{J_{2}} ; \quad{ }^{1} \mathcal{F}^{\odot} \stackrel{\sim}{\rightarrow}{ }^{2} \mathcal{F}^{\odot} ; \quad{ }^{1} \mathcal{F}^{\circledast} \stackrel{\sim}{\rightarrow}{ }^{2} \mathcal{F}^{\circledast}
$$

— where ${ }^{1} \mathfrak{F}_{J_{1}} \stackrel{\sim}{\rightarrow} \mathfrak{F}_{J_{2}}$ is a capsule-full poly-isomorphism [cf. $\S 0$ ], hence induces a poly-isomorphism ${ }^{1} \mathfrak{D}_{J_{1}} \stackrel{\sim}{\rightarrow}{ }^{2} \mathfrak{D}_{J_{2}} ;{ }^{1} \mathcal{F}^{\odot} \stackrel{\sim}{\rightarrow}{ }^{2} \mathcal{F}^{\odot}$ is a poly-isomorphism which lifts a poly-isomorphism ${ }^{1} \mathcal{D}^{\odot} \stackrel{\sim}{\rightarrow}{ }^{2} \mathcal{D}^{\odot}$ such that the pair of arrows ${ }^{1} \mathfrak{D}_{J_{1}} \stackrel{\sim}{\rightarrow}{ }^{2} \mathfrak{D}_{J_{2}}$, ${ }^{1} \mathcal{D}^{\odot} \stackrel{\sim}{\rightarrow}{ }^{2} \mathcal{D}^{\odot}$ forms a morphism between the associated $\mathcal{D}$-NF-bridges; ${ }^{1} \mathcal{F}^{\circledast} \stackrel{\sim}{\rightarrow}{ }^{2} \mathcal{F}^{\circledast}$ is an isomorphism — which are compatible [in the evident sense] with the ${ }^{i} \psi_{*}^{\mathrm{NF}}$ [for $i=1,2]$, as well as with the respective " $-\rightarrow$ ' $\mathrm{s}$ ". It is immediate that any morphism of NF-bridges induces a morphism between the associated $\mathcal{D}$-NF-bridges. There is an evident notion of composition of morphisms of NF-bridges.

(ii) We define a $\Theta$-bridge [relative to the given initial $\Theta$-data] to be a collection of data

$$
\left(\mathfrak{F}_{J} \stackrel{{ }^{\ddagger} \psi_{\text {* }}^{\ominus}}{\longrightarrow}{ }^{\ddagger} \mathfrak{F}_{>} \quad \rightarrow \quad \rightarrow{ }^{\ddagger} \mathcal{H} \mathcal{T}^{\Theta}\right)
$$

as follows:

(a) ${ }^{\ddagger} \mathfrak{F}_{J}=\left\{{ }^{\ddagger} \mathfrak{F}_{j}\right\}_{j \in J}$ is a capsule of $\mathcal{F}$-prime-strips, indexed by a finite index set $J$. Write $\ddagger_{J}=\left\{\mathfrak{D}_{j}\right\}_{j \in J}$ for the associated capsule of $\mathcal{D}$-prime-strips [cf. Remark 5.2.1, (i)].

(b) $\ddagger \mathcal{H} \mathcal{T}^{\Theta}$ is a $\Theta$-Hodge theater. 
(c) ${ }^{\ddagger} \mathfrak{F}_{>}$is the $\mathcal{F}$-prime-strip tautologically associated to $\ddagger \mathcal{H} \mathcal{T}^{\Theta}$ [cf. the discussion preceding Example 5.4]; we use the notation " $-\rightarrow$ " to denote this relationship between ${ }^{\ddagger} \mathfrak{F}_{>}$and ${ }^{\ddagger} \mathcal{H} \mathcal{T}^{\Theta}$. Write ${ }^{\ddagger} \mathfrak{D}_{>}$for the $\mathcal{D}$-primestrip associated to ${ }^{\ddagger} \mathfrak{F}_{>}$[cf. Remark 5.2.1, (i)].

(d) ${ }^{\ddagger} \psi_{*}^{\Theta}=\left\{{ }^{\ddagger} \psi_{j}^{\Theta}\right\}_{j \in J}$ is the collection of poly-morphisms ${ }^{\ddagger} \psi_{j}^{\Theta}:{ }^{\ddagger} \mathfrak{F}_{j} \rightarrow{ }^{\ddagger} \mathfrak{F}_{>}$ determined [i.e., as discussed in Remark 5.3.1] by a $\mathcal{D}$ - $\Theta$-bridge ${ }^{\ddagger} \phi_{*}^{\Theta}=$ $\left\{{ }^{\ddagger} \phi_{j}^{\Theta}:{ }^{\ddagger} \mathfrak{D}_{j} \rightarrow{ }^{\ddagger} \mathfrak{D}_{>}\right\}_{j \in J}$.

Thus, one verifies immediately that any $\Theta$-bridge as above determines an associated $\mathcal{D}$ - $\Theta$-bridge $\left({ }^{\ddagger} \phi_{*}^{\Theta}:{ }^{\ddagger} \mathfrak{D}_{J} \rightarrow{ }^{\ddagger} \mathfrak{D}_{>}\right)$. We define a(n) [iso]morphism of $\Theta$-bridges

$$
\left({ }^{1} \mathfrak{F}_{J_{1}} \stackrel{{ }^{1} \psi_{\text {*ै }}^{\Theta}}{\longrightarrow}{ }^{1} \mathfrak{F}_{>} \quad \rightarrow \quad \rightarrow{ }^{1} \mathcal{H} \mathcal{T}^{\Theta}\right) \quad \rightarrow \quad\left({ }^{2} \mathfrak{F}_{J_{2}} \stackrel{{ }^{2} \psi_{\text {*ै }}^{\Theta}}{\longrightarrow}{ }^{2} \mathfrak{F}_{>} \quad-\rightarrow{ }^{2} \mathcal{H} \mathcal{T}^{\Theta}\right)
$$

to be a collection of arrows

$$
{ }^{1} \mathfrak{F}_{J_{1}} \stackrel{\sim}{\rightarrow}{ }^{2} \mathfrak{F}_{J_{2}} ; \quad{ }^{1} \mathfrak{F}_{>} \stackrel{\sim}{\rightarrow}{ }^{2} \mathfrak{F}_{>} ; \quad{ }^{1} \mathcal{H} \mathcal{T}^{\Theta} \stackrel{\sim}{\rightarrow}{ }^{2} \mathcal{H} \mathcal{T}^{\Theta}
$$

- where ${ }^{1} \mathfrak{F}_{J_{1}} \stackrel{\sim}{\rightarrow}{ }^{2} \mathfrak{F}_{J_{2}}$ is a capsule-full poly-isomorphism [cf. $\left.\oint 0\right] ;{ }^{1} \mathfrak{F}_{>} \stackrel{\sim}{\rightarrow}{ }^{2} \mathfrak{F}_{>}$is the full poly-isomorphism; ${ }^{1} \mathcal{H} \mathcal{T}^{\Theta} \stackrel{\sim}{\rightarrow}{ }^{2} \mathcal{H} \mathcal{T}^{\Theta}$ is an isomorphism of $\Theta$-Hodge theaters [cf. Remark 3.6.2] — which are compatible [in the evident sense] with the ${ }^{i} \psi_{*}^{\Theta}$ [for $i=1,2$ ], as well as with the respective "- $\rightarrow$ 's" [cf. Corollary 5.6, (i), below]. It is immediate that any morphism of $\Theta$-bridges induces a morphism between the associated $\mathcal{D}$ - $\Theta$-bridges. There is an evident notion of composition of morphisms of $\Theta$-bridges.

(iii) We define a $\Theta \mathrm{NF}$-Hodge theater [relative to the given initial $\Theta$-data] to be a collection of data

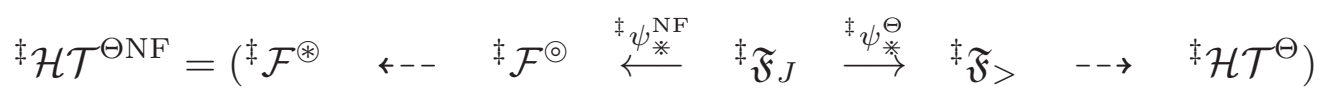

— where the data $\left({ }^{\ddagger} \mathcal{F}^{\circledast} \quad \leftarrow--\quad \ddagger \mathcal{F}^{\odot} \longleftarrow \mathfrak{F}_{J}\right)$ forms an $N F$-bridge; the data $\left({ }^{\ddagger} \mathfrak{F}_{J} \longrightarrow{ }^{\ddagger} \mathfrak{F}_{>} \quad-\rightarrow \mathcal{H}^{\Theta}\right)$ forms a $\Theta$-bridge — such that the associated data $\left\{{ }^{\ddagger} \phi_{*}^{\mathrm{NF}},{ }^{\ddagger} \phi_{*}^{\Theta}\right\}$ [cf. (i), (ii)] forms a $\mathcal{D}$ - $\Theta$ NF-Hodge theater. $\mathrm{A}(\mathrm{n})$ [iso]morphism of $\Theta N F-H o d g e$ theaters is defined to be a pair of morphisms between the respective associated NF- and $\Theta$-bridges that are compatible with one another in the sense that they induce the same bijection between the index sets of the respective capsules of $\mathcal{F}$-prime-strips. There is an evident notion of composition of morphisms of $\Theta N F-$ Hodge theaters.

Corollary 5.6. (Isomorphisms of $\Theta$-Hodge Theaters, NF-Bridges, $\Theta-$ Bridges, and $\Theta N F-H o d g e$ Theaters) Relative to a fixed collection of initial $\Theta$-data:

(i) The natural functorially induced map from the set of isomorphisms between two $\Theta$-Hodge theaters to the set of isomorphisms between the respective associated $\mathcal{D}$-prime-strips [cf. the discussion preceding Example 5.4; Remark 5.2.1, (i)] is bijective.

(ii) The natural functorially induced map from the set of isomorphisms be-

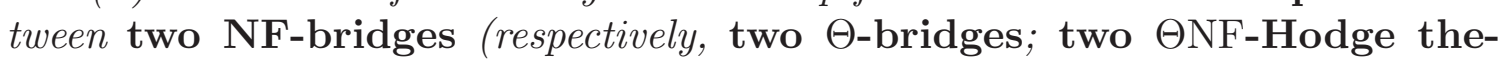
aters) to the set of isomorphisms between the respective associated $\mathcal{D}$-NF-bridges 
(respectively, associated $\mathcal{D}-\Theta$-bridges; associated $\mathcal{D}$ - $\Theta N F-H o d g e$ theaters) is bijective.

(iii) Given an NF-bridge and a $\Theta$-bridge, the set of capsule-full poly-isomorphisms between the respective capsules of $\mathcal{F}$-prime-strips which allow one to glue the given $N F$ - and $\Theta$-bridges together to form a $\Theta \mathrm{NF}$-Hodge theater forms an $\mathbb{F}_{l}^{*}$-torsor.

Proof. First, we consider assertion (i). Sorting through the data listed in the definition of a $\Theta$-Hodge theater ${ }^{\dagger} \mathcal{H} \mathcal{T}^{\Theta}$ [cf. Definition 3.6], one verifies immediately that the only data that is not contained in the associated $\mathcal{F}$-prime-strip ${ }^{\dagger} \mathfrak{F}_{>}[\mathrm{cf}$. the discussion preceding Example 5.4] is the global data of Definition 3.6, (c), and the tempered Frobenioids isomorphic to " $\underline{\underline{\mathcal{F}}} \underline{\underline{v}}$ " [cf. Example 3.2, (i)] at the $\underline{v} \in \underline{\mathbb{V}}^{\text {bad }}$. That is to say, for $\underline{v} \in \underline{\mathbb{V}}^{\text {good }}$, one verifies immediately that

$$
{ }^{\dagger} \mathcal{F}_{>, \underline{v}}={ }^{\dagger} \underline{\underline{\mathcal{F}}}_{\underline{v}}
$$

[cf. Example 3.3, (i); Example 3.4, (i); Definition 3.6; Definition 5.2, (i)]. On the other hand, one verifies immediately that this global data may be obtained by applying the functorial algorithm “ $\mathfrak{F} \mapsto \ddagger \mathfrak{F}$ " summarized in the second display of Remark 5.2.1, (ii), to the associated $\mathcal{F}$-prime-strips that appear. Thus, assertion (i) follows by applying Corollary 5.3, (ii), to the associated $\mathcal{F}$-primestrips and Corollary 5.3, (iv), to the various tempered Frobenioids at $\underline{v} \in \underline{\mathbb{V}}^{\text {bad }}$. This completes the proof of assertion (i). In light of assertion (i), assertions (ii), (iii) follow immediately from the definitions and Corollary 5.3, (i), (ii) [cf. also Proposition 4.8, (iii), in the case of assertion (iii)].

Remark 5.6.1. Observe that the various "functorial dynamics" studied in $\S 4$ - i.e., more precisely, analogues of Propositions 4.8, (i), (ii); 4.9; 4.11 - apply to the NF-bridges, $\Theta$-bridges, and $\Theta \mathrm{NF}$-Hodge theaters studied in the present $\S 5$. Indeed, such analogues follow immediately from Corollaries 5.3, (ii), (iii); 5.6, (ii). 


\section{Section 6: Additive Combinatorial Teichmüller Theory}

In the present $\S 6$, we discuss the additive analogue - i.e., which revolves around the "functorial dynamics" that arise from labels

$$
\in \mathbb{F}_{l}
$$

- of the "multiplicative combinatorial Teichmüller theory" developed in $\S 4$ for labels $\in \mathbb{F}_{l}^{*}$. These considerations lead naturally to certain enhancements of the various Hodge theaters considered in $\S 5$. On the other hand, despite the resemblance of the theory of the present $\S 6$ to the theory of $\S 4, \S 5$, the theory of the present $\S 6$ is, in certain respects - especially those respects that form the analogue of the theory of $\S 5$ - substantially technically simpler.

In the following, we fix a collection of initial $\Theta$-data

$$
\left(\bar{F} / F, X_{F}, l, \underline{C}_{K}, \underline{\mathbb{V}}, \mathbb{V}_{\bmod }^{\mathrm{bad}}, \underline{\epsilon}\right)
$$

as in Definition 3.1; also, we shall use the various notations introduced in Definition 3.1 for various objects associated to this initial $\Theta$-data.

\section{Definition 6.1.}

(i) We shall write

$$
\mathbb{F}_{l}^{\rtimes \pm} \stackrel{\text { def }}{=} \mathbb{F}_{l} \rtimes\{ \pm 1\}
$$

for the group determined by forming the semi-direct product with respect to the natural inclusion $\{ \pm 1\} \hookrightarrow \mathbb{F}_{l}^{\times}$and refer to an element of $\mathbb{F}_{l}^{\rtimes \pm}$ that maps to +1 (respectively, -1 ) via the natural surjection $\mathbb{F}_{l}^{\rtimes \pm} \rightarrow\{ \pm 1\}$ as positive (respectively, negative). We shall refer to as an $\mathbb{F}_{l}^{ \pm}$-group any set $E$ equipped with a $\{ \pm 1\}$-orbit of bijections $E \stackrel{\sim}{\rightarrow} \mathbb{F}_{l}$. Thus, any $\mathbb{F}_{l}^{ \pm}$-group $E$ is equipped with a natural $\mathbb{F}_{l}$-module structure. We shall refer to as an $\mathbb{F}_{l}^{ \pm}$-torsor any set $T$ equipped with an $\mathbb{F}_{l}^{\rtimes \pm}$-orbit of bijections $T \stackrel{\sim}{\rightarrow} \mathbb{F}_{l}$ [relative to the action of $\mathbb{F}_{l}^{\rtimes \pm}$ on $\mathbb{F}_{l}$ by automorphisms of the form $\mathbb{F}_{l} \ni z \mapsto \pm z+\lambda \in \mathbb{F}_{l}$, for $\left.\lambda \in \mathbb{F}_{l}\right]$. Thus, if $T$ is an $\mathbb{F}_{l}^{ \pm}$-torsor, then the abelian group of automorphisms of the underlying set of $\mathbb{F}_{l}$ given by the translations $\mathbb{F}_{l} \ni z \mapsto z+\lambda \in \mathbb{F}_{l}$, for $\lambda \in \mathbb{F}_{l}$, determines an abelian group

$$
\operatorname{Aut}_{+}(T)
$$

of "positive automorphisms" of the underlying set of $T$. Moreover, $\operatorname{Aut}_{+}(T)$ is equipped with a natural structure of $\mathbb{F}_{l}^{ \pm}$-group [such that the abelian group structure of Aut $_{+}(T)$ coincides with the $\mathbb{F}_{l}$-module structure of Aut $+(T)$ induced by this $\mathbb{F}_{l}^{ \pm}$-group structure]. Finally, if $T$ is an $\mathbb{F}_{l}^{ \pm}$-torsor, then we shall write

$$
\operatorname{Aut}_{ \pm}(T)
$$

for the group of automorphisms of the underlying set of $T$ determined [relative to the $\mathbb{F}_{l}^{ \pm}$-torsor structure on $\left.T\right]$ by the group of automorphisms of the underlying set of $\mathbb{F}_{l}$ given by $\mathbb{F}_{l}^{\rtimes \pm}\left[\right.$ so $\operatorname{Aut}_{+}(T) \subseteq \operatorname{Aut}_{ \pm}(T)$ is the unique subgroup of index 2]. 
(ii) Let

$$
\dagger \mathfrak{D}=\left\{{ }^{\dagger} \mathcal{D}_{\underline{v}}\right\}_{\underline{v} \in \underline{\mathbb{V}}}
$$

be a $\mathcal{D}$-prime-strip [relative to the given initial $\Theta$-data]. Observe [cf. the discussion of Definition 4.1, (i)] that if $\underline{v} \in \underline{\mathbb{V}}^{\text {non }}$, then $\pi_{1}\left({ }^{\dagger} \mathcal{D}_{\underline{v}}\right)$ determines, in a functorial fashion, a topological [in fact, profinite if $\underline{v} \in \underline{\mathbb{V}}^{\text {good }}$ ] group corresponding to " $\underline{X}_{\underline{v}}$ " [cf. Corollary 1.2 if $\underline{v} \in \underline{\mathbb{V}}^{\text {good }}$; [EtTh], Proposition 2.4, if $\underline{v} \in \underline{\mathbb{V}}^{\text {bad }}$ ], which contains $\pi_{1}\left({ }^{\dagger} \mathcal{D}_{\underline{v}}\right)$ as an open subgroup; thus, if we write ${ }^{\dagger} \underline{\mathcal{D}}_{v}^{ \pm}$for $\mathcal{B}(-)^{0}$ of this topological group, then we obtain a natural morphism ${ }^{\dagger} \mathcal{D}_{\underline{v}} \rightarrow{ }^{\dagger} \underline{\mathcal{D}}_{\underline{v}}^{ \pm}[\mathrm{cf}$. §0]. In a similar vein, if $\underline{v} \in \underline{\mathbb{V}}^{\text {arc }}$, then since $\underline{X}_{\underline{v}}$ admits a $K_{\underline{v}^{-}}$core, a routine translation into the "language of Aut-holomorphic orbispaces" of the argument given in the proof of Corollary 1.2 [cf. also [AbsTopIII], Corollary 2.4] reveals that ${ }^{\dagger} \mathcal{D}_{\underline{v}}$ determines, in a functorial fashion, an Aut-holomorphic orbispace ${ }^{\dagger} \underline{\mathcal{D}}_{\underline{v}}^{ \pm}$corresponding to " $\underline{X}_{\underline{v}}$ ", together with a natural morphism ${ }^{\dagger} \mathcal{D}_{\underline{v}} \rightarrow{ }^{\dagger} \underline{\mathcal{D}}_{\underline{v}}^{ \pm}$of Aut-holomorphic orbispaces. Thus, in summary, one obtains a collection of datā

$$
{ }^{\dagger} \underline{\mathfrak{D}}^{ \pm}=\left\{{ }^{\dagger} \underline{\mathcal{D}}_{\underline{v}}^{ \pm}\right\}_{\underline{v} \in \underline{\mathbb{V}}}
$$

completely determined by ${ }^{\dagger} \mathfrak{D}$.

(iii) Suppose that we are in the situation of (ii). Then observe [cf. the discussion of Definition 4.1, (ii)] that by applying the group-theoretic algorithm of [AbsTopI], Lemma 4.5 [cf. also Remark 1.2.2, (ii), of the present paper], to the topological group $\pi_{1}\left({ }^{\dagger} \mathcal{D}_{\underline{v}}\right)$ when $\underline{v} \in \underline{\mathbb{V}}^{\text {non }}$, or by considering $\pi_{0}(-)$ of a cofinal collection of "neighborhoods of infinity" [i.e., complements of compact subsets] of the underlying topological space of ${ }^{\dagger} \mathcal{D}_{\underline{v}}$ when $\underline{v} \in \underline{\mathbb{V}}^{\text {arc }}$, it makes sense to speak of the set of cusps of ${ }^{\dagger} \mathcal{D}_{\underline{v}}$; a similar observation applies to ${ }^{\dagger} \underline{\mathcal{D}}_{\underline{v}}^{ \pm}$, for $\underline{v} \in \underline{\mathbb{V}}$. If $\underline{v} \in \underline{\mathbb{V}}$, then we define a \pm -label class of cusps of ${ }^{\dagger} \mathcal{D}_{\underline{\underline{v}}}$ to be the set of cusps of ${ }^{\dagger} \mathcal{D}_{\underline{\underline{v}}}$ that lie over a single cusp [i.e., corresponding to an arbitrary element of the quotient " $Q$ " that appears in the definition of a "hyperbolic orbicurve of type $(1, l$-tors)" given in [EtTh], Definition 2.1] of ${ }^{\dagger} \underline{\mathcal{D}}_{\underline{v}}^{ \pm}$; write

$$
\operatorname{LabCusp}^{ \pm}\left({ }^{\dagger} \mathcal{D}_{\underline{v}}\right)
$$

for the set of \pm -label classes of cusps of ${ }^{\dagger} \mathcal{D}_{\underline{v}}$. Thus, [for any $\left.\underline{v} \in \underline{\mathbb{V}} !\right] \operatorname{LabCusp}^{ \pm}\left({ }^{\dagger} \mathcal{D}_{\underline{v}}\right)$ admits a natural action by $\mathbb{F}_{l}^{\times}[\mathrm{cf}$. [EtTh], Definition 2.1], as well as a zero element

$$
{ }^{\dagger} \underline{\eta}_{\underline{v}}^{0} \in \operatorname{LabCusp}^{ \pm}\left({ }^{\dagger} \mathcal{D}_{\underline{v}}\right)
$$

and a \pm -canonical element

$$
{ }^{\dagger} \underline{\eta}_{\underline{v}}^{ \pm} \in \operatorname{LabCusp}^{ \pm}\left({ }^{\dagger} \mathcal{D}_{\underline{v}}\right)
$$

- which is well-defined up to multiplication by \pm 1 , and which may be constructed solely from ${ }^{\dagger} \mathcal{D}_{\underline{v}}$ [cf. Definition 4.1, (ii)] — such that, relative to the natural bijection

$$
\left\{\operatorname{LabCusp}^{ \pm}\left({ }^{\dagger} \mathcal{D}_{\underline{v}}\right) \backslash\left\{{ }^{\dagger} \underline{\eta}_{\underline{v}}^{0}\right\}\right\} /\{ \pm 1\} \stackrel{\sim}{\rightarrow} \operatorname{LabCusp}\left({ }^{\dagger} \mathcal{D}_{\underline{v}}\right)
$$

[cf. the notation of Definition 4.1, (ii)], we have ${ }^{\dagger} \underline{\eta}_{\underline{v}}^{ \pm} \mapsto^{\dagger} \underline{\eta}_{\underline{v}}$. In particular, we obtain a natural bijection

$$
\operatorname{LabCusp}^{ \pm}\left({ }^{\dagger} \mathcal{D}_{\underline{v}}\right) \stackrel{\sim}{\rightarrow} \mathbb{F}_{l}
$$


- which is well-defined up to multiplication by \pm 1 and compatible, relative to the natural bijection to "LabCusp(-)" of the preceding display, with the natural bijection of the second display of Proposition 4.2. That is to say, in the terminology of (i), LabCusp ${ }^{ \pm}\left({ }^{\dagger} \mathcal{D}_{\underline{v}}\right)$ is equipped with a natural $\mathbb{F}_{l}^{ \pm}$-group structure. This $\mathbb{F}_{l}^{ \pm}$-group structure determines a natural surjection

$$
\operatorname{Aut}\left({ }^{\dagger} \mathcal{D}_{\underline{v}}\right) \rightarrow\{ \pm 1\}
$$

— i.e., by considering the induced automorphism of $\operatorname{LabCusp}^{ \pm}\left({ }^{\dagger} \mathcal{D}_{\underline{v}}\right)$. Write

$$
\operatorname{Aut}_{+}\left({ }^{\dagger} \mathcal{D}_{\underline{v}}\right) \subseteq \operatorname{Aut}\left({ }^{\dagger} \mathcal{D}_{\underline{v}}\right)
$$

for the index two subgroup of "positive automorphisms" [i.e., the kernel of the above surjection] and Aut $-\left({ }^{\dagger} \mathcal{D}_{\underline{v}}\right) \stackrel{\text { def }}{=} \operatorname{Aut}\left({ }^{\dagger} \mathcal{D}_{\underline{v}}\right) \backslash \operatorname{Aut}_{+}\left({ }^{\dagger} \mathcal{D}_{\underline{v}}\right)$ [i.e., where "\" denotes the set-theoretic complement] for the subset of "negative automorphisms". In a similar vein, we shall write

$$
\operatorname{Aut}_{+}\left({ }^{\dagger} \mathfrak{D}\right) \subseteq \operatorname{Aut}\left({ }^{\dagger} \mathfrak{D}\right)
$$

for the subgroup of "positive automorphisms" [i.e., automorphisms each of whose components, for $\underline{v} \in \underline{\mathbb{V}}$, is positive], and, if $\alpha \in\{ \pm 1\} \underline{\mathbb{V}}$ [i.e., where we write $\{ \pm 1\} \underline{\mathbb{V}}$ for the set of set-theoretic maps from $\underline{\mathbb{V}}$ to $\{ \pm 1\}]$,

$$
\operatorname{Aut}_{\alpha}\left({ }^{\dagger} \mathfrak{D}\right) \subseteq \operatorname{Aut}\left({ }^{\dagger} \mathfrak{D}\right)
$$

for the subset of " $\alpha$-signed automorphisms" [i.e., automorphisms each of whose components, for $\underline{v} \in \underline{\mathbb{V}}$, is positive if $\alpha(\underline{v})=+1$ and negative if $\alpha(\underline{v})=-1]$.

(iv) Suppose that we are in the situation of (ii). Let

$$
\ddagger \mathfrak{D}=\left\{{ }^{\ddagger} \mathcal{D}_{\underline{v}}\right\}_{\underline{v} \in \mathbb{V}}
$$

be another $\mathcal{D}$-prime-strip [relative to the given initial $\Theta$-data]. Then for any $\underline{v} \in \underline{\mathbb{V}}$, we shall refer to as a + -full poly-isomorphism ${ }^{\dagger} \mathcal{D}_{\underline{v}} \stackrel{\sim}{\rightarrow}{ }^{\ddagger} \mathcal{D}_{\underline{v}}$ any poly-isomorphism obtained as the Aut $+\left({ }^{\dagger} \mathcal{D}_{\underline{v}}\right)$ - [or, equivalently, Aut $\left.+\left({ }^{\ddagger} \mathcal{D}_{\underline{v}}\right)-\right]$ orbit of an isomorphism $\dagger \mathcal{D}_{\underline{v}} \stackrel{\sim}{\rightarrow} \ddagger \mathcal{D}_{\underline{v}}$. In particular, if $\dagger \mathfrak{D}=\ddagger \mathfrak{D}$, then there are precisely two + -full polyisomorphisms ${ }^{\dagger} \mathcal{D}_{\underline{v}} \stackrel{\sim}{\rightarrow} \ddagger \mathcal{D}_{\underline{v}}$, namely, the + -full poly-isomorphism determined by the identity isomorphism, which we shall refer to as positive, and the unique nonpositive +-full poly-isomorphism, which we shall refer to as negative. In a similar vein, we shall refer to as a + -full poly-isomorphism $\dagger \mathfrak{D} \stackrel{\sim}{\rightarrow} \ddagger \mathfrak{D}$ any poly-isomorphism obtained as the Aut $+\left({ }^{\dagger} \mathfrak{D}\right)-\left[\right.$ or, equivalently, Aut $\left.+\left({ }^{\ddagger} \mathfrak{D}\right)-\right]$ orbit of an isomorphism $\dagger \mathfrak{D} \stackrel{\sim}{\rightarrow} \ddagger \mathfrak{D}$. In particular, if $\dagger \mathfrak{D}=\ddagger \mathfrak{D}$, then the set of + -full poly-isomorphisms $\dagger \mathfrak{D} \stackrel{\sim}{\rightarrow} \ddagger \mathfrak{D}$ is in natural bijective correspondence [cf. the discussion of (iii) above] with the set $\{ \pm 1\} \underline{\mathbb{V}}$; we shall refer to the + -full poly-isomorphism ${ }^{\dagger} \mathfrak{D} \stackrel{\sim}{\rightarrow} \ddagger \mathfrak{D}$ that corresponds to $\alpha \in\{ \pm 1\} \underline{\mathbb{V}}$ as the $\alpha$-signed + -full poly-isomorphism. Finally, a capsule-+-full poly-morphism between capsules of $\mathcal{D}$-prime-strips

$$
\left\{{ }^{\dagger} \mathfrak{D}_{t}\right\}_{t \in T} \stackrel{\sim}{\rightarrow}\left\{{ }^{\ddagger} \mathfrak{D}_{t^{\prime}}\right\}_{t^{\prime} \in T^{\prime}}
$$

is defined to be a poly-morphism between two capsules of $\mathcal{D}$-prime-strips determined by +-full poly-isomorphisms ${ }^{\dagger} \mathfrak{D}_{t} \stackrel{\sim}{\rightarrow} \ddagger \mathfrak{D}_{\iota(t)}$ [where $\left.t \in T\right]$ between the constituent objects indexed by corresponding indices, relative to some injection $\iota: T \hookrightarrow T^{\prime}$. 
(v) Write

$$
\mathcal{D}^{\odot \pm} \stackrel{\text { def }}{=} \mathcal{B}\left(\underline{X}_{K}\right)^{0}
$$

[cf. $§ 0$; the situation discussed in Definition 4.1, (v)]. Thus, we have a finite étale double covering $\mathcal{D}^{\odot \pm} \rightarrow \mathcal{D}^{\odot}=\mathcal{B}\left(\underline{C}_{K}\right)^{0}$. Just as in the case of $\mathcal{D}^{\odot}$ [cf. Example 4.3, (i)], one may construct, in a category-theoretic fashion from $\mathcal{D}^{\odot \pm}$, the outer homomorphism

$$
\operatorname{Aut}\left(\mathcal{D}^{\odot \pm}\right) \rightarrow G L_{2}\left(\mathbb{F}_{l}\right) /\{ \pm 1\}
$$

arising from the l-torsion points of the elliptic curve $E_{\bar{F}}$ [i.e., from the Galois action on $\left.\Delta_{X}^{\mathrm{ab}} \otimes \mathbb{F}_{l}\right]$. Moreover, it follows from the construction of $\underline{X}_{K}$ that, relative to the natural isomorphism $\operatorname{Aut}\left(\mathcal{D}^{\odot \pm}\right) \stackrel{\sim}{\rightarrow} \operatorname{Aut}\left(\underline{X}_{K}\right)$ [cf., e.g., [AbsTopIII], Theorem 1.9], the image of the above outer homomorphism is equal to a subgroup of $G L_{2}\left(\mathbb{F}_{l}\right) /\{ \pm 1\}$ that contains a Borel subgroup of $S L_{2}\left(\mathbb{F}_{l}\right) /\{ \pm 1\}$ [cf. the discussion of Example 4.3, (i)] — i.e., the Borel subgroup corresponding to the rank one quotient of $\Delta_{X}^{\mathrm{ab}} \otimes \mathbb{F}_{l}$ that gives rise to the covering $\underline{X}_{K} \rightarrow X_{K}$. In particular, this rank one quotient determines a natural surjective homomorphism

$$
\operatorname{Aut}\left(\mathcal{D}^{\odot \pm}\right) \rightarrow \mathbb{F}_{l}^{*}
$$

[which may be reconstructed category-theoretically from $\mathcal{D}^{\odot \pm !] ~ — ~ w h o s e ~ k e r n e l ~}$ we denote by $\operatorname{Aut}_{ \pm}\left(\mathcal{D}^{\odot \pm}\right) \subseteq \operatorname{Aut}\left(\mathcal{D}^{\odot \pm}\right)$. One verifies immediately that the subgroup $\operatorname{Aut}_{ \pm}\left(\mathcal{D}^{\odot \pm}\right) \subseteq \operatorname{Aut}\left(\mathcal{D}^{\odot \pm}\right) \stackrel{\sim}{\rightarrow} \operatorname{Aut}\left(\underline{X}_{K}\right)$ contains the subgroup $\operatorname{Aut}_{K}\left(\underline{X}_{K}\right) \subseteq$ $\operatorname{Aut}\left(\underline{X}_{K}\right)$ of $K$-linear automorphisms and acts transitively on the cusps of $\underline{X}_{K}$. Next, let us write $\operatorname{Aut}_{\mathrm{csp}}\left(\mathcal{D}^{\odot \pm}\right) \subseteq \operatorname{Aut}_{ \pm}\left(\mathcal{D}^{\odot \pm}\right)$ for the subgroup [which may be reconstructed category-theoretically from $\mathcal{D}^{\odot \pm}$ ! — cf. [AbsTopI], Lemma 4.5, as well as Remark 1.2.2, (ii), of the present paper] of automorphisms that fix the cusps of $\underline{X}_{K}$. Then one obtains natural outer isomorphisms

$$
\operatorname{Aut}_{K}\left(\underline{X}_{K}\right) \stackrel{\sim}{\rightarrow} \operatorname{Aut}_{ \pm}\left(\mathcal{D}^{\odot \pm}\right) / \operatorname{Aut}_{c s p}\left(\mathcal{D}^{\odot \pm}\right) \stackrel{\sim}{\rightarrow} \mathbb{F}_{l}^{\rtimes \pm}
$$

[cf. the discussion preceding [EtTh], Definition 2.1] — where the second outer isomorphism depends, in an essential way, on the choice of the cusp $\underline{\epsilon}$ of $\underline{C}_{K}$ [cf. Definition 3.1, (f)]. Put another way, if we write $\operatorname{Aut}_{+}\left(\mathcal{D}^{\odot \pm}\right) \subseteq \operatorname{Aut}_{ \pm}\left(\mathcal{D}^{\odot \pm}\right)$ for the unique index two subgroup containing $\operatorname{Aut}_{\mathrm{csp}}\left(\mathcal{D}^{\odot \pm}\right)$, then the cusp $\underline{\epsilon}$ determines a natural $\mathbb{F}_{l}^{ \pm}$-group structure on the subgroup

$$
\operatorname{Aut}_{+}\left(\mathcal{D}^{\odot \pm}\right) / \operatorname{Aut}_{\mathrm{csp}}\left(\mathcal{D}^{\odot \pm}\right) \subseteq \operatorname{Aut}_{ \pm}\left(\mathcal{D}^{\odot \pm}\right) / \operatorname{Aut}_{\mathrm{csp}}\left(\mathcal{D}^{\odot \pm}\right)
$$

[which corresponds to the subgroup $\operatorname{Gal}\left(\underline{X}_{K} / X_{K}\right) \subseteq \operatorname{Aut}_{K}\left(\underline{X}_{K}\right)$ via the natural outer isomorphisms of the preceding display] and, in the notation of (vi) below, a natural $\mathbb{F}_{l}^{ \pm}$-torsor structure on the set LabCusp ${ }^{ \pm}\left(\mathcal{D}^{\odot \pm}\right)$. Write

$$
\underline{\mathbb{V}}^{ \pm} \stackrel{\text { def }}{=} \operatorname{Aut}_{ \pm}\left(\mathcal{D}^{\odot \pm}\right) \cdot \underline{\mathbb{V}}=\operatorname{Aut}_{\mathrm{csp}}\left(\mathcal{D}^{\odot \pm}\right) \cdot \underline{\mathbb{V}} \subseteq \mathbb{V}(K)
$$

[cf. the discussion of Example 4.3, (i); Remark 6.1 .1 below] — where the "=" follows immediately from the natural outer isomorphisms discussed above. Then [by considering what happens at the elements of $\left.\underline{\mathbb{V}}^{ \pm} \cap \underline{\mathbb{V}}^{\text {bad }}\right]$ one verifies immediately that the subgroup $\operatorname{Aut}_{ \pm}\left(\mathcal{D}^{\odot \pm}\right) \subseteq \operatorname{Aut}\left(\mathcal{D}^{\odot \pm}\right) \cong \operatorname{Aut}\left(\underline{X}_{K}\right)$ may be identified with the subgroup of $\operatorname{Aut}\left(\underline{X}_{K}\right)$ that stabilizes $\underline{\mathbb{V}}^{ \pm}$. 
(vi) Let

$$
{ }^{\dagger} \mathcal{D}^{\odot \pm}
$$

be any category isomorphic to $\mathcal{D}^{\odot \pm}$. Then just as in the discussion of (iii) in the case of " $\underline{v} \in \underline{\mathbb{V}}^{\text {non" }}$, it makes sense [cf. [AbsTopI], Lemma 4.5, as well as Remark 1.2 .2 , (ii), of the present paper] to speak of the set of cusps of ${ }^{\dagger} \mathcal{D}^{\odot \pm}$, as well as the set of \pm -label classes of cusps

$$
\operatorname{LabCusp}^{ \pm}\left({ }^{\dagger} \mathcal{D}^{\odot \pm}\right)
$$

— which, in this case, may be identified with the set of cusps of ${ }^{\dagger} \mathcal{D}^{\odot \pm}$.

(vii) Recall from [AbsTopIII], Theorem 1.9 [applied via the " $\Theta$-approach" discussed in Remark 3.1.2], that [just as in the case of $\mathcal{D}^{\odot}$ - cf. the discussion of Definition 4.1, (v)] there exists a group-theoretic algorithm for reconstructing, from $\pi_{1}\left(\mathcal{D}^{\odot \pm}\right)$ [cf. $\left.\S 0\right]$, the algebraic closure " $\bar{F}$ " of the base field " $K$ ", hence also the set of valuations " $\mathbb{V}(\bar{F})$ " from $\mathcal{D}^{\odot \pm}$ [e.g., as a collection of topologies on $\bar{F}$ - cf., e.g., [AbsTopIII], Corollary 2.8]. Moreover, for $\underline{w} \in \mathbb{V}(K)^{\text {arc }}$, let us recall [cf. Remark 3.1.2; [AbsTopIII], Corollaries 2.8, 2.9] that one may reconstruct group-theoretically, from $\pi_{1}\left(\mathcal{D}^{\odot \pm}\right)$, the Aut-holomorphic orbispace $\underline{\mathbb{X}}_{\underline{w}}$ associated to $\underline{X}_{\underline{w}}$. Let ${ }^{\dagger} \mathcal{D}^{\odot \pm}$ be as in (vi). Then let us write

$$
\overline{\mathbb{V}}\left({ }^{\dagger} \mathcal{D}^{\odot \pm}\right)
$$

for the set of valuations [i.e., " $\mathbb{V}(\bar{F})$ "] , equipped with its natural $\pi_{1}\left({ }^{\dagger} \mathcal{D}^{\odot \pm}\right)$-action,

$$
\mathbb{V}\left({ }^{\dagger} \mathcal{D}^{\odot \pm}\right) \stackrel{\text { def }}{=} \overline{\mathbb{V}}\left({ }^{\dagger} \mathcal{D}^{\odot \pm}\right) / \pi_{1}\left({ }^{\dagger} \mathcal{D}^{\odot \pm}\right)
$$

for the quotient of $\overline{\mathbb{V}}\left({ }^{\dagger} \mathcal{D}^{\odot \pm}\right)$ by $\pi_{1}\left({ }^{\dagger} \mathcal{D}^{\odot \pm}\right)$ [i.e., " $\mathbb{V}(K)$ "] , and, for $\underline{w} \in \mathbb{V}\left({ }^{\dagger} \mathcal{D}^{\odot \pm}\right)^{\text {arc }}$,

$$
\underline{\mathbb{X}}\left({ }^{\dagger} \mathcal{D}^{\odot \pm}, \underline{w}\right)
$$

[i.e., " $\underline{\mathbb{X}}_{\underline{w}}$ " - cf. the discussion of [AbsTopIII], Definition 5.1, (ii)] for the Autholomorphic orbispace obtained by applying these group-theoretic reconstruction algorithms to $\pi_{1}\left({ }^{\dagger} \mathcal{D}^{\odot \pm}\right)$. Now if $\mathbb{U}$ is an arbitrary Aut-holomorphic orbispace, then let us define a morphism

$$
\mathbb{U} \rightarrow{ }^{\dagger} \mathcal{D}^{\odot \pm}
$$

to be a morphism of Aut-holomorphic orbispaces [cf. [AbsTopIII], Definition 2.1, (ii)] $\mathbb{U} \rightarrow \underline{\mathbb{X}}\left({ }^{\dagger} \mathcal{D}^{\odot \pm}, \underline{w}\right)$ for some $\underline{w} \in \mathbb{V}\left({ }^{\dagger} \mathcal{D}^{\odot \pm}\right)^{\text {arc }}$. Thus, it makes sense to speak of the pre-composite (respectively, post-composite) of such a morphism $\mathbb{U} \rightarrow{ }^{\dagger} \mathcal{D}^{\odot \pm}$ with a morphism of Aut-holomorphic orbispaces (respectively, with an isomorphism [cf. $\S 0]{ }^{\dagger} \mathcal{D}^{\odot \pm} \stackrel{\sim}{\rightarrow}{ }^{\ddagger} \mathcal{D}^{\odot \pm}$ [i.e., where ${ }^{\ddagger} \mathcal{D}^{\odot \pm}$ is a category equivalent to $\mathcal{D}^{\odot \pm}$ ]).

Remark 6.1.1. In fact, in the notation of Example 4.3, (i); Definition 6.1, (v), it is not difficult to verify $\left[\mathrm{cf}\right.$. Remark 3.1.2, (i)] that $\underline{\mathbb{V}}^{ \pm}=\underline{\mathbb{V}}^{ \pm \text {un }}(\subseteq \mathbb{V}(K))$.

\section{Example 6.2. Model Base- $\Theta^{ \pm}$-Bridges.}

(i) In the following, let us think of $\mathbb{F}_{l}$ as an $\mathbb{F}_{l}^{ \pm}$-group [relative to the tautological $\mathbb{F}_{l}^{ \pm}$-group structure]. Let

$$
\mathfrak{D}_{\succ}=\left\{\mathcal{D}_{\succ, \underline{v}}\right\}_{\underline{v} \in \underline{\mathbb{V}}} ; \quad \mathfrak{D}_{t}=\left\{\mathcal{D}_{\underline{v}_{t}}\right\}_{\underline{v} \in \underline{\mathbb{V}}}
$$


- where $t \in \mathbb{F}_{l}$, and we use the notation $\underline{v}_{t}$ to denote the pair $(t, \underline{v})$ [cf. Example

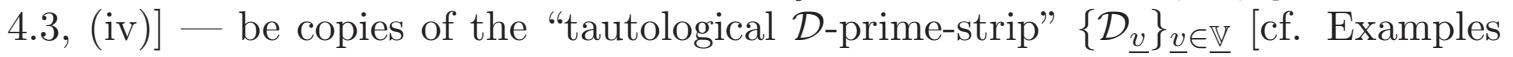
4.3 , (iv); 4.4, (ii)]. For each $t \in \mathbb{F}_{l}$, write

$$
\phi_{\underline{v}_{t}}^{\Theta^{ \pm}}: \mathcal{D}_{\underline{v}_{t}} \rightarrow \mathcal{D}_{\succ, \underline{v}} ; \quad \phi_{t}^{\Theta^{ \pm}}: \mathfrak{D}_{t} \rightarrow \mathfrak{D}_{\succ}
$$

for the respective positive +-full poly-isomorphisms, i.e., relative to the respective

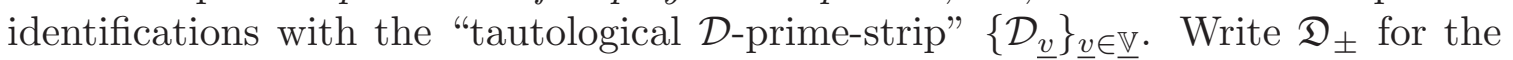
capsule $\left\{\mathfrak{D}_{t}\right\}_{t \in \mathbb{F}_{l}}$ [cf. the constructions of Example 4.4, (iv)] and

$$
\phi_{ \pm}^{\Theta^{ \pm}}: \mathfrak{D}_{ \pm} \rightarrow \mathfrak{D}_{\succ}
$$

for the collection of poly-morphisms $\left\{\phi_{t}^{\Theta^{ \pm}}\right\}_{t \in \mathbb{F}_{l}}$.

(ii) The collection of data

$$
\left(\mathfrak{D}_{ \pm}, \mathfrak{D}_{\succ}, \phi_{ \pm}^{\Theta^{ \pm}}\right)
$$

admits a natural poly-automorphism of order two $-1_{\mathbb{F}_{l}}$ defined as follows: the poly-automorphism $-1_{\mathbb{F}_{l}}$ acts on $\mathbb{F}_{l}$ as multiplication by -1 and induces the polyisomorphisms $\mathfrak{D}_{t} \stackrel{\sim}{\rightarrow} \mathfrak{D}_{-t}$ [for $\left.t \in \mathbb{F}_{l}\right]$ and $\mathfrak{D}_{\succ} \stackrel{\sim}{\rightarrow} \mathfrak{D}_{\succ}$ determined [i.e., relative to the respective identifications with the "tautological $\mathcal{D}$-prime-strip" $\left.\left\{\mathcal{D}_{\underline{v}}\right\}_{\underline{v} \in \mathbb{V}}\right]$ by the +-full poly-automorphism whose sign at every $\underline{v} \in \mathbb{V}$ is negative. One verifies immediately that $-1_{\mathbb{F}_{l}}$, defined in this way, is compatible [in the evident sense] with $\phi_{ \pm}^{\Theta}$

(iii) Let $\alpha \in\{ \pm 1\} \underline{\mathbb{V}}$. Then $\alpha$ determines a natural poly-automorphism $\alpha^{\Theta^{ \pm}}$of order $\in\{1,2\}$ of the collection of data

$$
\left(\mathfrak{D}_{ \pm}, \mathfrak{D}_{\succ}, \phi_{ \pm}^{\Theta^{ \pm}}\right)
$$

as follows: the poly-automorphism $\alpha^{\Theta^{ \pm}}$acts on $\mathbb{F}_{l}$ as the identity and on $\mathfrak{D}_{t}$, for $t \in \mathbb{F}_{l}$, and $\mathfrak{D}_{\succ}$ as the $\alpha$-signed + -full poly-automorphism. One verifies immediately that $\alpha^{\Theta^{ \pm}}$, defined in this way, is compatible [in the evident sense] with $\phi_{ \pm}^{\Theta^{ \pm}}$.

\section{Example 6.3. Model Base- $\Theta^{\text {ell }}$-Bridges.}

(i) In the following, let us think of $\mathbb{F}_{l}$ as an $\mathbb{F}_{l}^{ \pm}$-torsor [relative to the tautological $\mathbb{F}_{l}^{ \pm}$-torsor structure]. Let

$$
\mathfrak{D}_{t}=\left\{\mathcal{D}_{\underline{v}_{t}}\right\}_{\underline{v} \in \underline{\mathbb{V}}}
$$

[for $t \in \mathbb{F}_{l}$ ] and $\mathfrak{D}_{ \pm}$be as in Example 6.2, (i); $\mathcal{D}^{\odot \pm}$ as in Definition 6.1, (v). In the following, let us fix an isomorphism of $\mathbb{F}_{l}^{ \pm}$-torsors

$$
\operatorname{LabCusp}^{ \pm}\left(\mathcal{D}^{\odot \pm}\right) \stackrel{\sim}{\rightarrow} \mathbb{F}_{l}
$$

[cf. the discussion of Definition 6.1, (v)], which we shall use to identify LabCusp ${ }^{ \pm}\left(\mathcal{D}^{\odot \pm}\right)$ with $\mathbb{F}_{l}$. Note that this identification induces an isomorphism of groups

$$
\operatorname{Aut}_{ \pm}\left(\mathcal{D}^{\odot \pm}\right) / \operatorname{Aut}_{\mathrm{csp}}\left(\mathcal{D}^{\odot \pm}\right) \stackrel{\sim}{\rightarrow} \mathbb{F}_{l}^{\rtimes \pm}
$$


[cf. the discussion of Definition 6.1, (v)], which we shall use to identify the group $\operatorname{Aut}_{ \pm}\left(\mathcal{D}^{\odot \pm}\right) / \operatorname{Aut}_{\mathrm{csp}}\left(\mathcal{D}^{\odot \pm}\right)$ with the group $\mathbb{F}_{l}^{\rtimes \pm}$. If $\underline{v} \in \underline{\mathbb{V}}^{\text {good }} \cap \underline{\mathbb{V}}^{\text {non }}$ (respectively, $\left.\underline{v} \in \underline{\mathbb{V}}^{\text {bad }}\right)$, then the natural restriction functor on finite étale coverings arising from the natural composite morphism $\underline{X}_{\underline{v}_{\underline{v}}} \rightarrow \underline{X}_{\underline{v}} \rightarrow \underline{X}_{K}$ (respectively, $\underline{\underline{X}}_{\underline{v}} \rightarrow \underline{X}_{\underline{v}} \rightarrow$ $\underline{X}_{K}$ ) determines [cf. Examples 3.2, (i); 3.3, (i)] a natural morphism $\phi_{\bullet, v}^{\Theta^{\text {ell }}}: \mathcal{D}_{\underline{v}} \rightarrow$ $\mathcal{D}^{\odot \pm}\left[\mathrm{cf}\right.$. the discussion of Example 4.3, (ii)]. If $\underline{v} \in \underline{\mathbb{V}}^{\text {arc }}$, then [cf. Example 3.4, (i)] we have a tautological morphism $\mathcal{D}_{\underline{v}}=\underline{\mathbb{X}}_{\underline{v}} \rightarrow \underline{\mathbb{X}}_{\underline{v}} \stackrel{\sim}{\rightarrow} \underline{\mathbb{X}}\left(\mathcal{D}^{\odot \pm}, \underline{v}\right)$, hence a morphism $\phi_{\bullet, \underline{v}}^{\Theta^{\mathrm{ell}}}: \mathcal{D}_{\underline{v}} \rightarrow \mathcal{D}^{\odot \pm}[$ cf. the discussion of Example 4.3 , (iii)]. For arbitrary $\underline{v} \in \underline{\mathbb{V}}$, write

$$
\phi_{\underline{v}_{0}}^{\Theta^{\mathrm{ell}}}: \mathcal{D}_{\underline{v}_{0}} \rightarrow \mathcal{D}^{\odot \pm}
$$

for the poly-morphism given by the collection of morphisms $\mathcal{D}_{\underline{v}_{0}} \rightarrow \mathcal{D}^{\odot \pm}$ of the form

$$
\beta \circ \phi_{\bullet, \underline{v}}^{\Theta^{\mathrm{ell}}} \circ \alpha
$$

— where $\alpha \in \operatorname{Aut}_{+}\left(\mathcal{D}_{\underline{v}_{0}}\right) ; \beta \in \operatorname{Aut}_{\mathrm{csp}}\left(\mathcal{D}^{\odot \pm}\right)$; we apply the tautological identification of $\mathcal{D}_{\underline{v}}$ with $\mathcal{D}_{\underline{v}_{0}}$ [cf. the discussion of Example 4.3, (ii), (iii), (iv)]. Write

$$
\phi_{0}^{\Theta^{\mathrm{ell}}}: \mathfrak{D}_{0} \rightarrow \mathcal{D}^{\odot \pm}
$$

for the poly-morphism determined by the collection $\left\{\phi_{\underline{v}_{0}}^{\Theta^{\text {ell }}}: \mathcal{D}_{\underline{v}_{0}} \rightarrow \mathcal{D}^{\odot \pm}\right\}_{\underline{v} \in \underline{\mathbb{V}}}[\mathrm{cf}$. the discussion of Example 4.3, (iv)]. Note that the presence of " $\beta$ " in the definition of $\phi_{\underline{v}_{0}}^{\Theta^{\text {ell }}}$ implies that it makes sense to post-compose $\phi_{0}^{\Theta^{\text {ell }}}$ with an element of $\operatorname{Aut}_{ \pm}\left(\mathcal{D}^{\odot \pm}\right) / \operatorname{Aut}_{\mathrm{csp}}\left(\mathcal{D}^{\odot \pm}\right) \stackrel{\sim}{\rightarrow} \mathbb{F}_{l}^{\rtimes \pm}$. Thus, for any $t \in \mathbb{F}_{l} \subseteq \mathbb{F}_{l}^{\rtimes \pm}$, let us write

$$
\phi_{t}^{\Theta^{\mathrm{ell}}}: \mathfrak{D}_{t} \rightarrow \mathcal{D}^{\odot \pm}
$$

for the result of post-composing $\phi_{0}^{\Theta^{\mathrm{ell}}}$ with the "poly-action" [i.e., action via polyautomorphisms] of $t$ on $\mathcal{D}^{\odot \pm}$ [and pre-composing with the tautological identification of $\mathfrak{D}_{0}$ with $\left.\mathfrak{D}_{t}\right]$ and

$$
\phi_{ \pm}^{\Theta^{\mathrm{ell}}}: \mathfrak{D}_{ \pm} \rightarrow \mathcal{D}^{\odot \pm}
$$

for the collection of arrows $\left\{\phi_{t}^{\Theta^{\mathrm{ell}}}\right\}_{t \in \mathbb{F}_{l}}$.

(ii) Let $\gamma \in \mathbb{F}_{l}^{\rtimes \pm}$. Then $\gamma$ determines a natural poly-automorphism $\gamma_{ \pm}$of $\mathfrak{D}_{ \pm}$ as follows: the automorphism $\gamma_{ \pm}$acts on $\mathbb{F}_{l}$ via the usual action of $\mathbb{F}_{l}^{\rtimes \pm}$ on $\mathbb{F}_{l}$ and, for $t \in \mathbb{F}_{l}$, induces the + -full poly-isomorphism $\mathfrak{D}_{t} \stackrel{\sim}{\rightarrow} \mathfrak{D}_{\gamma(t)}$ whose sign at every $\underline{v} \in \underline{\mathbb{V}}$ is equal to the sign of $\gamma$ [cf. the construction of Example 6.2, (ii)]. Thus, we obtain a natural poly-action of $\mathbb{F}_{l}^{\rtimes \pm}$ on $\mathfrak{D}_{ \pm}$. On the other hand, the isomorphism $\operatorname{Aut}_{ \pm}\left(\mathcal{D}^{\odot \pm}\right) / \operatorname{Aut}_{\mathrm{csp}}\left(\mathcal{D}^{\odot \pm}\right) \stackrel{\sim}{\rightarrow} \mathbb{F}_{l}^{\rtimes \pm}$ of (i) determines a natural poly-action of $\mathbb{F}_{l}^{\rtimes \pm}$ on $\mathcal{D}^{\odot \pm}$. Moreover, one verifies immediately that $\phi_{ \pm}^{\Theta^{\text {ell }}}$ is equivariant with respect to these poly-actions of $\mathbb{F}_{l}^{\rtimes \pm}$ on $\mathfrak{D}_{ \pm}$and $\mathcal{D}^{\odot \pm}$; in particular, we obtain a natural poly-action

$$
\mathbb{F}_{l}^{\rtimes \pm} \curvearrowright\left(\mathfrak{D}_{ \pm}, \mathcal{D}^{\odot \pm}, \phi_{ \pm}^{\Theta^{\mathrm{ell}}}\right)
$$

of $\mathbb{F}_{l}^{\rtimes \pm}$ on the collection of data $\left(\mathfrak{D}_{ \pm}, \mathcal{D}^{\odot \pm}, \phi_{ \pm}^{\Theta^{\text {ell }}}\right)$ [cf. the discussion of Example $4.3,(\mathrm{iv})]$. 
Definition 6.4. In the following, we shall write $l^{ \pm} \stackrel{\text { def }}{=} l^{*}+1=(l+1) / 2$. [Here, we recall that the notation " $l *$ " was introduced at the beginning of $\S 4$.]

(i) We define a base- $\Theta^{ \pm}$-bridge, or $\mathcal{D}-\Theta^{ \pm}$-bridge, [relative to the given initial $\Theta$-data] to be a poly-morphism

$$
\dagger \mathfrak{D}_{T} \stackrel{{ }^{\dagger} \phi_{ \pm}^{\Theta^{ \pm}}}{\longrightarrow} \dagger \mathfrak{D}_{\succ}
$$

— where ${ }^{\dagger} \mathfrak{D}_{\succ}$ is a $\mathcal{D}$-prime-strip; $T$ is an $\mathbb{F}_{l}^{ \pm}$-group; ${ }^{\dagger} \mathfrak{D}_{T}=\left\{{ }^{\dagger} \mathfrak{D}_{t}\right\}_{t \in T}$ is a capsule of $\mathcal{D}$-prime-strips, indexed by [the underlying set of] $T$ - such that there exist isomorphisms

$$
\mathfrak{D}_{\succ} \stackrel{\sim}{\rightarrow} \mathfrak{D}_{\succ}, \quad \mathfrak{D}_{ \pm} \stackrel{\sim}{\rightarrow} \dagger \mathfrak{D}_{T}
$$

— where we require that the bijection of index sets $\mathbb{F}_{l} \stackrel{\sim}{\rightarrow} T$ induced by the second isomorphism determine an isomorphism of $\mathbb{F}_{l}^{ \pm}$-groups - conjugation by which maps $\phi_{ \pm}^{\Theta^{ \pm}} \mapsto^{\dagger} \phi_{ \pm}^{\Theta^{ \pm}}$. In this situation, we shall write

$$
{ }^{\dagger} \mathfrak{D}_{|T|}
$$

for the $l^{ \pm}$-capsule obtained from the $l$-capsule ${ }^{\dagger} \mathfrak{D}_{T}$ by forming the quotient $|T|$ of the index set $T$ of this underlying capsule by the action of $\{ \pm 1\}$ and identifying the components of the capsule ${ }^{\dagger} \mathfrak{D}_{T}$ indexed by the elements in the fibers of the quotient $T \rightarrow|T|$ via the constituent poly-morphisms of ${ }^{\dagger} \phi_{ \pm}^{\Theta^{ \pm}}=\left\{{ }^{\dagger} \phi_{t}^{\Theta^{ \pm}}\right\}_{t \in T}$ [so each constituent $\mathcal{D}$-prime-strip of $\dagger \mathfrak{D}_{|T|}$ is only well-defined up to a positive automorphism, but this indeterminacy will not affect applications of this construction — cf. Propositions 6.7; 6.8, (ii); 6.9, (i), below]. Also, we shall write

$$
\dagger \mathfrak{D}_{T *}
$$

for the $l^{*}$-capsule determined by the subset $T^{*} \stackrel{\text { def }}{=}|T| \backslash\{0\}$ of nonzero elements of $|T|$. We define a $(\mathrm{n})\left[\right.$ iso]morphism of $\mathcal{D}-\Theta^{ \pm}$-bridges

$$
\left({ }^{\dagger} \mathfrak{D}_{T} \stackrel{{ }^{\dagger} \phi_{ \pm}^{\Theta \pm}}{\longrightarrow} \dagger \mathfrak{D}_{\succ}\right) \rightarrow\left({ }^{\ddagger} \mathfrak{D}_{T^{\prime}} \stackrel{\ddagger}{\stackrel{\phi_{ \pm}^{\Theta}}{\longrightarrow}}{ }^{\Theta^{ \pm}} \mathfrak{D}_{\succ}\right)
$$

to be a pair of poly-morphisms

$$
\dagger \mathfrak{D}_{T} \stackrel{\sim}{\rightarrow} \ddagger \mathfrak{D}_{T^{\prime}} ; \quad \dagger \mathfrak{D}_{\succ} \stackrel{\sim}{\rightarrow} \ddagger \mathfrak{D}_{\succ}
$$

— where ${ }^{\dagger} \mathfrak{D}_{T} \stackrel{\sim}{\rightarrow} \ddagger \mathfrak{D}_{T^{\prime}}$ is a capsule-+-full poly-isomorphism whose induced morphism on index sets $T \stackrel{\sim}{\rightarrow} T^{\prime}$ is an isomorphism of $\mathbb{F}_{l}^{ \pm}$-groups; ${ }^{\dagger} \mathfrak{D}_{\succ} \stackrel{\sim}{\rightarrow} \ddagger \mathfrak{D}_{\succ}$ is a + -full poly-isomorphism — which are compatible with ${ }^{\dagger} \phi_{ \pm}^{\Theta^{ \pm}},{ }^{\ddagger} \phi_{ \pm}^{\Theta^{ \pm}}$. There is an evident notion of composition of morphisms of $\mathcal{D}-\Theta^{ \pm}$-bridges.

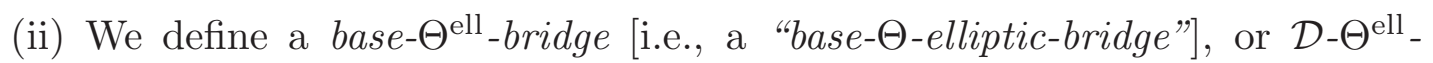
bridge, [relative to the given initial $\Theta$-data] to be a poly-morphism

$$
\dagger \mathfrak{D}_{T} \stackrel{{ }^{\dagger} \stackrel{\phi_{ \pm}}{\longrightarrow}}{\longrightarrow} \dagger \mathcal{D}^{\odot \pm}
$$

— where ${ }^{\dagger} \mathcal{D}^{\odot \pm}$ is a category equivalent to $\mathcal{D}^{\odot \pm} ; T$ is an $\mathbb{F}_{l}^{ \pm}$-torsor; ${ }^{\dagger} \mathfrak{D}_{T}=\left\{{ }^{\dagger} \mathfrak{D}_{t}\right\}_{t \in T}$ is a capsule of $\mathcal{D}$-prime-strips, indexed by [the underlying set of] $T$ - such that there exist isomorphisms

$$
\mathcal{D}^{\odot \pm} \stackrel{\sim}{\rightarrow}{ }^{\dagger} \mathcal{D}^{\odot \pm}, \quad \mathfrak{D}_{ \pm} \stackrel{\sim}{\rightarrow}{ }^{\dagger} \mathfrak{D}_{T}
$$


- where we require that the bijection of index sets $\mathbb{F}_{l} \stackrel{\sim}{\rightarrow} T$ induced by the second isomorphism determine an isomorphism of $\mathbb{F}_{l}^{ \pm}$-torsors - conjugation by which maps $\phi_{ \pm}^{\Theta^{\mathrm{ell}}} \mapsto^{\dagger} \phi_{ \pm}^{\Theta^{\mathrm{ell}}}$. We define a(n) [iso]morphism of $\mathcal{D}$ - $\Theta^{\mathrm{ell}}$-bridges

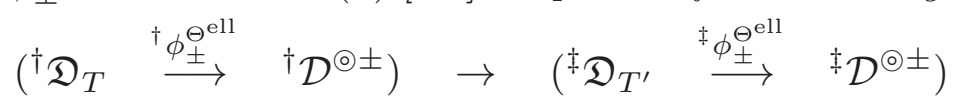

to be a pair of poly-morphisms

$$
\dagger \mathfrak{D}_{T} \stackrel{\sim}{\rightarrow} \ddagger \mathfrak{D}_{T^{\prime}} ; \quad{ }^{\dagger} \mathcal{D}^{\odot \pm} \stackrel{\sim}{\rightarrow} \ddagger \mathcal{D}^{\odot \pm}
$$

— where ${ }^{\dagger} \mathfrak{D}_{T} \stackrel{\sim}{\rightarrow} \ddagger \mathfrak{D}_{T^{\prime}}$ is a capsule-+-full poly-isomorphism whose induced morphism on index sets $T \stackrel{\sim}{\rightarrow} T^{\prime}$ is an isomorphism of $\mathbb{F}_{l}^{ \pm}$-torsors; ${ }^{\dagger} \mathcal{D}^{\odot \pm} \rightarrow{ }^{\ddagger} \mathcal{D}^{\odot \pm}$ is a poly-morphism which is an $\operatorname{Aut}_{\mathrm{csp}}\left({ }^{\dagger} \mathcal{D}^{\odot \pm}\right)$ - [or, equivalently, $\operatorname{Aut}_{\mathrm{csp}}\left({ }^{\ddagger} \mathcal{D}^{\odot \pm}\right)$-] orbit of isomorphisms — which are compatible with ${ }^{\dagger} \phi_{ \pm}^{\Theta^{\mathrm{ell}}},{ }^{\ddagger} \phi_{ \pm}^{\Theta^{\mathrm{ell}}}$. There is an evident notion of composition of morphisms of $\mathcal{D}$ - $\Theta^{\text {ell }}$-bridges.

(iii) We define a base- $\Theta^{ \pm \text {ell }}-$ Hodge theater, or $\mathcal{D}-\Theta^{ \pm \text {ell }}-$ Hodge theater, [relative to the given initial $\Theta$-data] to be a collection of data

$$
{ }^{\dagger} \mathcal{H} \mathcal{T}^{\mathcal{D}-\Theta^{ \pm \mathrm{ell}}}=\left({ }^{\dagger} \mathfrak{D}_{\succ} \stackrel{{ }^{\dagger} \phi_{ \pm}^{\Theta^{ \pm}}}{\longleftarrow} \mathfrak{D}_{T} \stackrel{{ }^{\dagger} \phi_{ \pm}^{\Theta^{\mathrm{ell}}}}{\longrightarrow}{ }^{\dagger} \mathcal{D}^{\odot \pm}\right)
$$

— where $T$ is an $\mathbb{F}_{l}^{ \pm}$-group $;{ }^{\dagger} \phi_{ \pm}^{\Theta^{ \pm}}$is a $\mathcal{D}$ - $\Theta^{ \pm}$-bridge $;{ }^{\dagger} \phi_{ \pm}^{\Theta^{\text {ell }}}$ is a $\mathcal{D}$ - $\Theta^{\text {ell }}$-bridge [relative to the $\mathbb{F}_{l}^{ \pm}$-torsor structure determined by the $\mathbb{F}_{l}^{ \pm}$-group structure on $\left.T\right]$ - such that there exist isomorphisms

$$
\mathfrak{D}_{\succ} \stackrel{\sim}{\rightarrow}{ }^{\dagger} \mathfrak{D}_{\succ} ; \quad \mathfrak{D}_{ \pm} \stackrel{\sim}{\rightarrow}{ }^{\dagger} \mathfrak{D}_{T} ; \quad \mathcal{D}^{\odot \pm} \stackrel{\sim}{\rightarrow}{ }^{\dagger} \mathcal{D}^{\odot \pm}
$$

conjugation by which maps $\phi_{ \pm}^{\Theta^{ \pm}} \mapsto^{\dagger} \phi_{ \pm}^{\Theta^{ \pm}}, \phi_{ \pm}^{\Theta^{\text {ell }}} \mapsto^{\dagger} \phi_{ \pm}^{\Theta^{\text {ell }}}$. $\mathrm{A}(\mathrm{n})$ [iso]morphism of $\mathcal{D}-\Theta^{ \pm e l l}$-Hodge theaters is defined to be a pair of morphisms between the respective associated $\mathcal{D}-\Theta^{ \pm}$- and $\mathcal{D}$ - $\Theta^{\text {ell }}$-bridges that are compatible with one another in the sense that they induce the same poly-isomorphism between the respective capsules of $\mathcal{D}$-prime-strips. There is an evident notion of composition of morphisms of $\mathcal{D}$ $\Theta^{ \pm \text {ell }}$-Hodge theaters.

The following additive analogue of Proposition 4.7 follows immediately from the various definitions involved. Put another way, the content of Proposition 6.5 below may be thought of as a sort of "intrinsic version" of the constructions carried out in Examples 6.2, 6.3.

\section{Proposition 6.5. (Transport of \pm -Label Classes of Cusps via Base- Bridges) Let}

$$
{ }^{\dagger} \mathcal{H} \mathcal{T}^{\mathcal{D}-\Theta^{ \pm \mathrm{ell}}}=\left({ }^{\dagger} \mathfrak{D}_{\succ} \stackrel{{ }^{\dagger} \phi_{ \pm}^{\Theta^{ \pm}}}{\longleftarrow}{ }^{\dagger} \mathfrak{D}_{T} \stackrel{{ }^{\dagger} \phi_{ \pm}^{\Theta^{\mathrm{ell}}}}{\longrightarrow}{ }^{\dagger} \mathcal{D}^{\odot \pm}\right)
$$

be a $\mathcal{D}-\Theta^{ \pm e l l}-$ Hodge theater [relative to the given initial $\Theta$-data]. Then:

(i) For each $\underline{v} \in \underline{\mathbb{V}}, t \in T$, the $\mathcal{D}$ - $\Theta^{\text {ell }}$-bridge ${ }^{\dagger} \phi_{ \pm}^{\Theta^{\text {ell }}}$ induces a [single, welldefined!] bijection of sets of \pm -label classes of cusps

$$
{ }^{\dagger} \zeta_{\underline{v}_{t}}^{\Theta^{\text {ell }}}: \operatorname{LabCusp}^{ \pm}\left({ }^{\dagger} \mathcal{D}_{\underline{v}_{t}}\right) \stackrel{\sim}{\rightarrow} \operatorname{LabCusp}^{ \pm}\left({ }^{\dagger} \mathcal{D}^{\odot \pm}\right)
$$


that is compatible with the respective $\mathbb{F}_{l}^{ \pm}$-torsor structures. Moreover, for $\underline{w} \in \underline{\mathbb{V}}$, the bijection

$$
\dagger \xi_{\underline{v}_{t}, \underline{w}_{t}}^{\Theta^{\text {ell }}} \stackrel{\text { def }}{=}\left({ }^{\dagger} \zeta_{\underline{w}_{t}}^{\Theta^{\text {ell }}}\right)^{-1} \circ\left({ }^{\dagger} \zeta_{\underline{v}_{t}}^{\Theta \text { ell }}\right): \operatorname{LabCusp}^{ \pm}\left({ }^{\dagger} \mathcal{D}_{\underline{v}_{t}}\right) \stackrel{\sim}{\rightarrow} \operatorname{LabCusp}^{ \pm}\left({ }^{\dagger} \mathcal{D}_{\underline{w}_{t}}\right)
$$

is compatible with the respective $\mathbb{F}_{l}^{ \pm}$-group structures. Write

$$
\operatorname{LabCusp}^{ \pm}\left({ }^{\dagger} \mathfrak{D}_{t}\right)
$$

for the $\mathbb{F}_{l}^{ \pm}$-group obtained by identifying the various $\mathbb{F}_{l}^{ \pm}$-groups LabCusp ${ }^{ \pm}\left({ }^{\dagger} \mathcal{D}_{\underline{v}_{t}}\right)$, as $\underline{v}$ ranges over the elements of $\underline{\mathbb{V}}$, via the various $\dagger^{\dagger} \xi_{\underline{v}_{t}, \underline{w}_{t}}^{\Theta^{e l l}}$. Finally, the various $\dagger \zeta_{\underline{v}_{t}}^{\Theta^{\mathrm{ell}}}$ determine a [single, well-defined!] bijection

$$
{ }^{\dagger} \zeta_{t}^{\Theta^{\mathrm{ell}}}: \operatorname{LabCusp}^{ \pm}\left({ }^{\dagger} \mathfrak{D}_{t}\right) \stackrel{\sim}{\rightarrow} \operatorname{LabCusp}^{ \pm}\left({ }^{\dagger} \mathcal{D}^{\odot \pm}\right)
$$

- which is compatible with the respective $\mathbb{F}_{l}^{ \pm}$-torsor structures.

(ii) For each $\underline{v} \in \underline{\mathbb{V}}, t \in T$, the $\mathcal{D}-\Theta^{ \pm}$-bridge ${ }^{\dagger} \phi_{ \pm}^{\Theta^{ \pm}}$induces a [single, welldefined!] bijection of sets of \pm -label classes of cusps

$$
\dagger \zeta_{\underline{v}_{t}}^{\Theta^{ \pm}}: \operatorname{LabCusp}^{ \pm}\left({ }^{\dagger} \mathcal{D}_{\underline{v}_{t}}\right) \stackrel{\sim}{\rightarrow} \operatorname{LabCusp}^{ \pm}\left({ }^{\dagger} \mathcal{D}_{\succ, \underline{v}}\right)
$$

that is compatible with the respective $\mathbb{F}_{l}^{ \pm}$-group structures. Moreover, for $\underline{w} \in \underline{\mathbb{V}}$, the bijections

$$
\begin{gathered}
{ }^{\dagger} \xi_{\succ, \underline{v}, \underline{w}}^{\Theta^{ \pm}} \stackrel{\text { def }}{=}\left({ }^{\dagger} \zeta_{\underline{w}_{0}}^{\Theta^{ \pm}}\right) \circ{ }^{\dagger} \xi_{\underline{v}_{0}, \underline{w}_{0}}^{\Theta^{\text {ell }}} \circ\left({ }^{\dagger} \zeta_{\underline{\underline{v}}_{0}}^{\Theta^{ \pm}}\right)^{-1}: \operatorname{LabCusp}^{ \pm}\left({ }^{\dagger} \mathcal{D}_{\succ, \underline{v}}\right) \stackrel{\sim}{\rightarrow} \operatorname{LabCusp}^{ \pm}\left({ }^{\dagger} \mathcal{D}_{\succ, \underline{w}}\right) ; \\
{ }^{\dagger} \xi_{\underline{v}_{t}, \underline{w}_{t}} \stackrel{\text { def }}{=}\left({ }^{\dagger} \zeta_{\underline{w}_{t}}^{\Theta^{ \pm}}\right)^{-1} \circ{ }^{\dagger} \xi_{\succ, \underline{v}, \underline{w}^{ \pm}} \circ\left({ }^{\dagger} \zeta_{\underline{v}_{t}}^{\Theta^{ \pm}}\right): \operatorname{LabCusp}^{ \pm}\left({ }^{\dagger} \mathcal{D}_{\underline{v}_{t}}\right) \stackrel{\sim}{\rightarrow} \operatorname{LabCusp}^{ \pm}\left({ }^{\dagger} \mathcal{L}_{\underline{w}_{t}}\right)
\end{gathered}
$$

- where, by abuse of notation, we write " 0 " for the zero element of the $\mathbb{F}_{l}^{ \pm}$-group $T$

- are compatible with the respective $\mathbb{F}_{l}^{ \pm}$-group structures, and we have ${ }^{\dagger} \xi_{\underline{v}_{t}, \underline{w}_{t}}^{\Theta^{ \pm}}=$ $\dagger \xi_{\underline{v}_{t}, \underline{w}_{t}}^{\Theta^{\text {ell }}}$. Write

$$
\operatorname{LabCusp}^{ \pm}\left({ }^{\dagger} \mathfrak{D}_{\succ}\right)
$$

for the $\mathbb{F}_{l}^{ \pm}$-group obtained by identifying the various $\mathbb{F}_{l}^{ \pm}$-groups LabCusp ${ }^{ \pm}\left({ }^{\dagger} \mathcal{D}_{\succ, \underline{v}}\right)$, as $\underline{v}$ ranges over the elements of $\underline{\mathbb{V}}$, via the various ${ }^{\dagger} \xi_{\succ, \underline{v}, \underline{w}}^{\Theta^{ \pm}}$. Finally, for any $t \in T$, the various ${ }^{\dagger} \zeta_{\underline{v}_{t}}^{\Theta^{ \pm}},{ }^{\dagger} \zeta_{\underline{v}_{t}}^{\Theta^{\text {ell }}}$ determine, respectively, a [single, well-defined!] bijection

$$
{ }^{\dagger} \zeta_{t}^{\Theta^{ \pm}}: \operatorname{LabCusp}^{ \pm}\left({ }^{\dagger} \mathfrak{D}_{t}\right) \stackrel{\sim}{\rightarrow} \operatorname{LabCusp}^{ \pm}\left({ }^{\dagger} \mathfrak{D}_{\succ}\right)
$$

- which is compatible with the respective $\mathbb{F}_{l}^{ \pm}$-group structures.

(iii) The assignment

$$
T \ni t \mapsto^{\dagger} \zeta_{t}^{\Theta^{\mathrm{ell}}}(0) \in \operatorname{LabCusp}^{ \pm}\left({ }^{\dagger} \mathcal{D}^{\odot \pm}\right)
$$

- where, by abuse of notation, we write " 0 " for the zero element of the $\mathbb{F}_{l}^{ \pm}$-group $\operatorname{LabCusp}^{ \pm}\left({ }^{\dagger} \mathfrak{D}_{t}\right)$ - determines a [single, well-defined!] bijection

$$
\left({ }^{\dagger} \zeta_{ \pm}\right)^{-1}: T \stackrel{\sim}{\rightarrow} \operatorname{LabCusp}^{ \pm}\left({ }^{\dagger} \mathcal{D}^{\odot \pm}\right)
$$


[i.e., whose inverse we denote by ${ }^{\dagger} \zeta_{ \pm}$] — which is compatible with the respective $\mathbb{F}_{l}^{ \pm}$-torsor structures. Moreover, for any $t \in T$, the composite bijection

$$
\left({ }^{\dagger} \zeta_{0}^{\Theta^{\mathrm{ell}}}\right)^{-1} \circ\left({ }^{\dagger} \zeta_{t}^{\Theta^{\mathrm{ell}}}\right) \circ\left({ }^{\dagger} \zeta_{t}^{\Theta^{ \pm}}\right)^{-1} \circ\left({ }^{\dagger} \zeta_{0}^{\Theta^{ \pm}}\right): \operatorname{LabCusp}^{ \pm}\left({ }^{\dagger} \mathfrak{D}_{0}\right) \stackrel{\sim}{\rightarrow} \operatorname{LabCusp}^{ \pm}\left({ }^{\dagger} \mathfrak{D}_{0}\right)
$$

coincides with the automorphism of the set LabCusp ${ }^{ \pm}\left({ }^{\dagger} \mathfrak{D}_{0}\right)$ determined, relative to the $\mathbb{F}_{l}^{ \pm}$-group structure on this set, by the action of $\left({ }^{\dagger} \zeta_{0}^{\Theta^{\mathrm{ell}}}\right)^{-1}\left(\left({ }^{\dagger} \zeta_{ \pm}\right)^{-1}(t)\right)$.

(iv) Let $\alpha \in$ Aut $_{ \pm}\left({ }^{\dagger} \mathcal{D}^{\odot \pm}\right) / \operatorname{Aut}_{\mathrm{csp}}\left({ }^{\dagger} \mathcal{D}^{\odot \pm}\right)$. Then if one replaces ${ }^{\dagger} \phi_{ \pm}^{\Theta^{\text {ell }}}$ by $\alpha \circ$ ${ }^{\dagger} \phi_{ \pm}^{\Theta^{\mathrm{ell}}}$ [cf. Proposition 6.6, (iv), below], then the resulting " $\zeta_{t}^{\Theta^{\mathrm{ell}}}$ " is related to the "† $\zeta_{t}^{\Theta^{\text {ell }}}$ " determined by the original ${ }^{\dagger} \phi_{ \pm}^{\Theta^{\mathrm{ell}}}$ by post-composition with the image of $\alpha$ via the natural bijection [cf. the discussion of Definition 6.1, (v)]

$$
\operatorname{Aut}_{ \pm}\left({ }^{\dagger} \mathcal{D}^{\odot \pm}\right) / \operatorname{Aut}_{\operatorname{csp}}\left({ }^{\dagger} \mathcal{D}^{\odot \pm}\right) \stackrel{\sim}{\rightarrow} \operatorname{Aut}_{ \pm}\left(\operatorname{LabCusp}^{ \pm}\left({ }^{\dagger} \mathcal{D}^{\odot \pm}\right)\right) \quad\left(\cong \mathbb{F}_{l}^{\rtimes \pm}\right)
$$

determined by the tautological action of $\mathrm{Aut}_{ \pm}\left({ }^{\dagger} \mathcal{D}^{\odot \pm}\right) / \mathrm{Aut}_{\mathrm{csp}}\left({ }^{\dagger} \mathcal{D}^{\odot \pm}\right)$ on the set of \pm -label classes of cusps LabCusp ${ }^{ \pm}\left({ }^{\dagger} \mathcal{D}^{\odot \pm}\right)$.

Next, let us observe that it follows immediately from the various definitions involved [cf. the discussion of Definition 6.1; Examples 6.2, 6.3], together with the explicit description of the various poly-automorphisms discussed in Examples 6.2, (ii), (iii); 6.3, (ii) [cf. also the various properties discussed in Proposition 6.5], that we have the following additive analogue of Proposition 4.8.

Proposition 6.6. (First Properties of Base- $\Theta^{ \pm}$-Bridges, Base- $\Theta^{\text {ell }}$-Bridges, and Base- $\Theta^{ \pm e l l}-$ Hodge Theaters) Relative to a fixed collection of initial $\Theta-$ data:

(i) The set of isomorphisms between two $\mathcal{D}-\Theta^{ \pm}$-bridges forms a torsor over the group

$$
\{ \pm 1\} \times\left(\{ \pm 1\}^{\mathbb{V}}\right)
$$

- where the first (respectively, second) factor corresponds to poly-automorphisms of the sort described in Example 6.2, (ii) (respectively, Example 6.2, (iii)). Moreover, the first factor may be thought of as corresponding to the induced isomorphisms of $\mathbb{F}_{l}^{ \pm}$-groups between the index sets of the capsules involved.

(ii) The set of isomorphisms between two $\mathcal{D}$ - $\Theta^{\text {ell }}$-bridges forms an $\mathbb{F}_{l}^{\rtimes \pm}$ torsor - i.e., more precisely, a torsor over a finite group that is equipped with a natural outer isomorphism to $\mathbb{F}_{l}^{\rtimes \pm}$. Moreover, this set of isomorphisms maps bijectively, by considering the induced bijections, to the set of isomorphisms of $\mathbb{F}_{l}^{ \pm}$-torsors between the index sets of the capsules involved.

(iii) The set of isomorphisms between two $\mathcal{D}-\Theta^{ \pm \text {ell }}$-Hodge theaters forms $a\{ \pm 1\}$-torsor. Moreover, this set of isomorphisms maps bijectively, by considering the induced bijections, to the set of isomorphisms of $\mathbb{F}_{l}^{ \pm}$-groups between the index sets of the capsules involved. 
(iv) Given a $\mathcal{D}-\Theta^{ \pm}$-bridge and a $\mathcal{D}-\Theta^{\mathrm{ell}}$-bridge, the set of capsule-+-full polyisomorphisms between the respective capsules of $\mathcal{D}$-prime-strips which allow one to glue the given $\mathcal{D}-\Theta^{ \pm}$- and $\mathcal{D}-\Theta^{\mathrm{ell}}$-bridges together to form a $\mathcal{D}-\Theta^{ \pm \text {ell }}$-Hodge theater forms a torsor over the group

$$
\mathbb{F}_{l}^{\rtimes \pm} \times\left(\{ \pm 1\}^{\mathbb{V}}\right)
$$

- where the first factor corresponds to the $\mathbb{F}_{l}^{\rtimes \pm}$ of (ii); the subgroup $\{ \pm 1\} \times$ $\left(\{ \pm 1\}^{\mathbb{V}}\right)$ corresponds to the group of $(i)$. Moreover, the first factor may be thought of as corresponding to the induced isomorphisms of $\mathbb{F}_{l}^{ \pm}$-torsors between the index sets of the capsules involved.

(v) Given a $\mathcal{D}-\Theta^{\mathrm{ell}}$-bridge, there exists a [relatively simple - cf. the discussion of Example 6.2, (i)] functorial algorithm for constructing, up to an $\mathbb{F}_{l}^{\rtimes \pm}$ indeterminacy $[c f . \quad(i i),(i v)]$, from the given $\mathcal{D}-\Theta^{\text {ell }}$-bridge a $\mathcal{D}$ - $\Theta^{ \pm \text {tell }}$-Hodge theater whose underlying $\mathcal{D}-\Theta^{\text {ell }}$-bridge is the given $\mathcal{D}-\Theta^{\text {ell }}$-bridge.

$$
\begin{aligned}
& {\left[-l^{*}<\ldots<-2<-1<0<1<2<\ldots<l^{*}\right]} \\
& \mathfrak{D}_{\succ}=/^{ \pm} \\
& \Uparrow \phi_{ \pm}^{\Theta^{ \pm}} \\
& \{ \pm 1\} \curvearrowright\left(-l^{*}<\ldots<-2<-1<0<1<2<\ldots<l^{*}\right) \\
& \left(\begin{array}{lllllll}
/^{ \pm} & /^{ \pm} & /^{ \pm} & /^{ \pm} & /^{ \pm} & /^{ \pm} & /^{ \pm}
\end{array}\right) \\
& \mathfrak{D}_{T} \\
& \Downarrow \phi_{ \pm}^{\Theta^{\mathrm{ell}}} \\
& \pm \quad \longrightarrow \quad \pm \\
& \nearrow \searrow \\
& \pm \quad \mathbb{F}_{l}^{\rtimes \pm} \curvearrowright \quad \pm \\
& \uparrow \quad \mathcal{D}^{\odot \pm}=\quad \downarrow \\
& \pm \underset{\nwarrow}{ } \mathcal{B}\left(\underline{X}_{K}\right)^{0} \quad{ }^{ \pm} \\
& \pm \quad \ldots \quad \pm
\end{aligned}
$$

Fig. 6.1: The combinatorial structure of a $\mathcal{D}-\Theta^{ \pm \text {ell }}$-Hodge theater

Remark 6.6.1. The underlying combinatorial structure of a $\mathcal{D}$ - $\Theta^{ \pm \text {ell }}$-Hodge theater - or, essentially equivalently [cf. Definition 6.11, Corollary 6.12 below], of 
a $\Theta^{ \pm \text {ell }}$-Hodge theater - is illustrated in Fig. 6.1 above. Thus, Fig. 6.1 may be thought of as a sort of additive analogue of the multiplicative situation illustrated in Fig. 4.4. In Fig. 6.1, the "介" corresponds to the associated $[\mathcal{D}$ - $] \Theta^{ \pm}$-bridge, while the " $\downarrow$ " corresponds to the associated $[\mathcal{D}$ - $] \Theta^{\text {ell }}$-bridge; the " $/{ }^{ \pm}$'s" denote $\mathcal{D}$-primestrips.

Proposition 6.7. (Base- $\Theta-B r i d g e s$ Associated to Base- $\Theta^{ \pm}$-Bridges) Relative to a fixed collection of initial $\Theta$-data, let

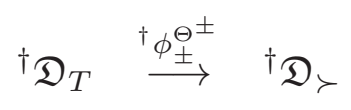

be a $\mathcal{D}-\Theta^{ \pm}$-bridge, as in Definition 6.4, (i). Then by replacing ${ }^{\dagger} \mathfrak{D}_{T}$ by ${ }^{\dagger} \mathfrak{D}_{T *}$ [cf. Definition 6.4, (i)], identifying the $\mathcal{D}$-prime-strip ${ }^{\dagger} \mathfrak{D}_{\succ}$ with the $\mathcal{D}$-prime-strip ${ }^{\dagger} \mathfrak{D}_{0}$ via ${ }^{\dagger} \phi_{0}^{\Theta^{ \pm}}$[cf. the discussion of Definition 6.4, (i)] to form a D-prime-strip ${ }^{\dagger} \mathfrak{D}_{>}$, replacing the various + -full poly-morphisms that occur in ${ }^{\dagger} \phi_{ \pm}^{\Theta^{ \pm}}$at the $\underline{v} \in \underline{\mathbb{V}}^{\text {good }}$ by the corresponding full poly-morphisms, and replacing the various +-full polymorphisms that occur in ${ }^{\dagger} \phi_{ \pm}^{\Theta^{ \pm}}$at the $\underline{v} \in \underline{\mathbb{V}}^{\text {bad }}$ by the poly-morphisms described [via group-theoretic algorithms!] in Example 4.4, (i), (ii), we obtain a functorial algorithm for constructing a [well-defined, up to a unique isomorphism!] $\mathcal{D}-\Theta-$ bridge

$$
\dagger \mathfrak{D}_{T *} \stackrel{{ }^{\dagger} \phi_{*}^{\ominus}}{\longrightarrow} \dagger \mathfrak{D}_{>}
$$

as in Definition 4.6, (ii). Thus, the newly constructed $\mathcal{D}$ - $\Theta$-bridge is related to the given $\mathcal{D}-\Theta^{ \pm}$-bridge via the following correspondences:

$$
\left.{ }^{\dagger} \mathfrak{D}_{T}\right|_{(T \backslash\{0\})} \mapsto{ }^{\dagger} \mathfrak{D}_{T *} ; \quad \dagger \mathfrak{D}_{0},{ }^{\dagger} \mathfrak{D}_{\succ} \mapsto{ }^{\dagger} \mathfrak{D}_{>}
$$

- each of which maps precisely two $\mathcal{D}$-prime-strips to a single $\mathcal{D}$-prime-strip.

Proof. The various assertions of Proposition 6.7 follow immediately from the various definitions involved.

Next, we consider additive analogues of Propositions 4.9, 4.11; Corollary 4.12.

Proposition 6.8. (Symmetries arising from Forgetful Functors) Relative to a fixed collection of initial $\Theta$-data:

(i) (Base- $\Theta^{\text {ell }}$-Bridges) The operation of associating to a $\mathcal{D}-\Theta^{ \pm \mathrm{ell}}-$ Hodge theater the underlying $\mathcal{D}-\Theta^{\text {ell }}$-bridge of the $\mathcal{D}-\Theta^{ \pm \text {ell }}$-Hodge theater determines a natural functor

\begin{tabular}{|c|c|}
\hline category of \\
$\mathcal{D}-\Theta^{ \pm \text {ell }}$-Hodge theaters \\
and isomorphisms of \\
$\mathcal{D}$ - $\Theta^{ \pm \text {ell }}$-Hodge theaters
\end{tabular}$\rightarrow$\begin{tabular}{c} 
category of \\
$\mathcal{D}$ - $\Theta^{\text {ell }}$-bridges \\
and isomorphisms of \\
$\mathcal{D}$ - $\Theta^{\text {ell }}$-bridges \\
\hline
\end{tabular}

$$
{ }^{\dagger} \mathcal{H} \mathcal{T}^{\mathcal{D}-\Theta^{ \pm \mathrm{ell}}} \quad \mapsto \quad\left({ }^{\dagger} \mathfrak{D}_{T} \stackrel{{ }^{\dagger}}{\stackrel{\phi_{ \pm}^{\Theta^{\mathrm{ell}}}}{\longrightarrow}}{ }^{\dagger} \mathcal{D}^{\odot \pm}\right)
$$


whose output data admits an $\mathbb{F}_{l}^{\rtimes \pm}$-symmetry - i.e., more precisely, a symmetry given by the action of a finite group that is equipped with a natural outer isomorphism to $\mathbb{F}_{l}^{\rtimes \pm}$ - which acts doubly transitively [i.e., transitively with stabilizers of order two] on the index set [i.e., "T"] of the underlying capsule of $\mathcal{D}$-prime-strips [i.e., "† $\mathfrak{D}_{T}$ "] of this output data.

(ii) (Holomorphic Capsules) The operation of associating to a $\mathcal{D}-\Theta^{ \pm \text {ell }}$ Hodge theater ${ }^{\dagger} \mathcal{H} \mathcal{T}^{\mathcal{D}-\Theta^{ \pm \mathrm{ell}}}$ the $l^{ \pm}$-capsule

$$
{ }^{\dagger} \mathfrak{D}_{|T|}
$$

associated to the underlying $\mathcal{D}-\Theta^{ \pm}$-bridge of $\dagger \mathcal{H} \mathcal{T}^{\mathcal{D}-\Theta^{ \pm e l l}}$ [cf. Definition 6.4, (i)] determines a natural functor

$$
\begin{aligned}
& \begin{array}{|c|c|}
\hline \begin{array}{c}
\text { category of } \\
\mathcal{D}-\Theta^{ \pm \text {ell }} \text {-Hodge theaters } \\
\text { and isomorphisms of } \\
\mathcal{D} \text { - } \Theta^{ \pm \text {ell }} \text {-Hodge theaters }
\end{array} \rightarrow \begin{array}{c}
\text { category of } l^{ \pm} \text {-capsules } \\
\text { of } \mathcal{D} \text {-prime-strips } \\
\text { and capsule-full poly- } \\
\text { isomorphisms of } l^{ \pm} \text {-capsules }
\end{array} \\
\hline
\end{array} \\
& \dagger \mathcal{H} \mathcal{T}^{\mathcal{D}-\Theta^{ \pm \mathrm{ell}}} \quad \mapsto \quad \dagger \mathfrak{D}_{|T|}
\end{aligned}
$$

whose output data admits an $\mathfrak{S}_{l^{ \pm}}$-symmetry [where we write $\mathfrak{S}_{l^{ \pm}}$for the symmetric group on $l^{ \pm}$letters] which acts transitively on the index set [i.e., "TT"] of this output data. Thus, this functor may be thought of as an operation that consists of forgetting the labels $\in\left|\mathbb{F}_{l}\right|=\mathbb{F}_{l} /\{ \pm 1\}$ [i.e., forgetting the bijection $|T| \stackrel{\sim}{\rightarrow}\left|\mathbb{F}_{l}\right|$ determined by the $\mathbb{F}_{l}^{ \pm}$-group structure of $T$ - cf. Definition 6.4, (i)]. In particular, if one is only given this output data ${ }^{\dagger} \mathfrak{D}_{|T|}$ up to isomorphism, then there is a total of precisely $l^{ \pm}$possibilities for the element $\in\left|\mathbb{F}_{l}\right|$ to which a given index $|t| \in|T|$ corresponds, prior to the application of this functor.

(iii) (Mono-analytic Capsules) By composing the functor of (ii) with the mono-analyticization operation discussed in Definition 4.1, (iv), one obtains a natural functor

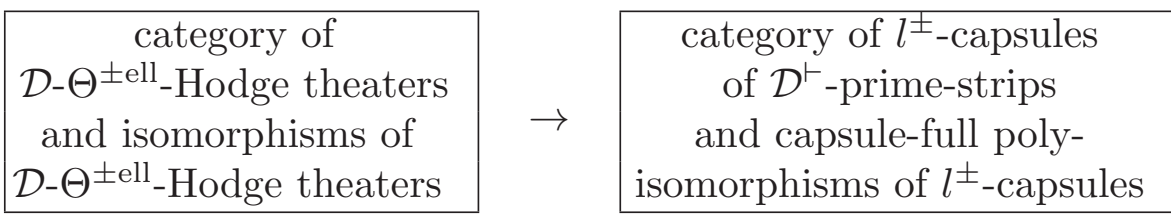

$$
\begin{aligned}
& \dagger \mathcal{H} \mathcal{T}^{\mathcal{D}-\Theta^{ \pm \text {ell }}} \quad \mapsto \quad \dagger \mathfrak{D}_{|T|}^{\vdash}
\end{aligned}
$$

whose output data satisfies the same symmetry properties with respect to labels as the output data of the functor of (ii).

Proof. Assertions (i), (ii), (iii) follow immediately from the definitions [cf. also Proposition 6.6, (ii), in the case of assertion (i)]. 


$$
/^{ \pm} \hookrightarrow /^{ \pm} / \pm \hookrightarrow /^{ \pm} / \pm / \pm \hookrightarrow \ldots \hookrightarrow /^{ \pm} /^{ \pm} / \pm \ldots /^{ \pm}
$$

Fig. 6.2: An $l^{ \pm}$-procession of $\mathcal{D}$-prime-strips

Proposition 6.9. (Processions of Base-Prime-Strips) Relative to a fixed collection of initial $\Theta$-data:

(i) (Holomorphic Processions) Given a $\mathcal{D}-\Theta^{ \pm}-$bridge ${ }^{\dagger} \phi_{ \pm}^{\Theta^{ \pm}}:{ }^{\dagger} \mathfrak{D}_{T} \rightarrow{ }^{\dagger} \mathfrak{D}_{\succ}$, with underlying capsule of $\mathcal{D}$-prime-strips ${ }^{\dagger} \mathfrak{D}_{T}$ [cf. Definition 6.4, (i)], denote by $\operatorname{Prc}\left({ }^{\dagger} \mathfrak{D}_{T}\right)$ the $l^{ \pm}$-procession of $\mathcal{D}$-prime-strips [cf. Fig. 6.2, where each "I士" denotes a D-prime-strip] determined by considering the ["sub"]capsules of the capsule ${ }^{\dagger} \mathfrak{D}_{|T|}$ of Definition 6.4, (i), corresponding to the subsets $\mathbb{S}_{1}^{ \pm} \subseteq \ldots \subseteq$ $\mathbb{S}_{t}^{ \pm} \stackrel{\text { def }}{=}\{0,1,2, \ldots, t-1\} \subseteq \ldots \subseteq \mathbb{S}_{l^{ \pm}}^{ \pm}=\left|\mathbb{F}_{l}\right|$ [where, by abuse of notation, we use the notation for nonnegative integers to denote the images of these nonnegative integers in $\left.\left|\mathbb{F}_{l}\right|\right]$, relative to the bijection $|T| \stackrel{\sim}{\rightarrow}\left|\mathbb{F}_{l}\right|$ determined by the $\mathbb{F}_{l}^{ \pm}$-group structure of $T$ [cf. Definition 6.4, (i)]. Then the assignment ${ }^{\dagger} \phi_{ \pm}^{\Theta^{ \pm}} \mapsto \operatorname{Prc}\left({ }^{\dagger} \mathfrak{D}_{T}\right)$ determines a natural functor

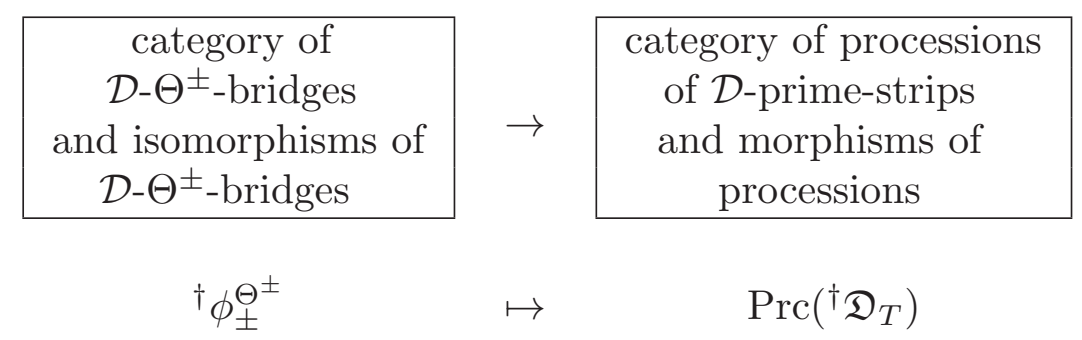

whose output data satisfies the following property: for each $n \in\left\{1, \ldots, l^{ \pm}\right\}$, there are precisely $\boldsymbol{n}$ possibilities for the element $\in\left|\mathbb{F}_{l}\right|$ to which a given index of the index set of the $n$-capsule that appears in the procession constituted by this output data corresponds, prior to the application of this functor. That is to say, by taking the product, over elements of $\left|\mathbb{F}_{l}\right|$, of cardinalities of "sets of possibilies", one concludes that

by considering processions - i.e., the functor discussed above, possibly pre-composed with the functor ${ }^{\dagger} \mathcal{H} \mathcal{T}^{\mathcal{D}-\Theta^{ \pm \text {ell }}} \mapsto^{\dagger} \phi_{ \pm}^{\Theta^{ \pm}}$that associates to a $\mathcal{D}-\Theta^{ \pm \text {ell }}$-Hodge theater its associated $\mathcal{D}-\Theta^{ \pm}$-bridge - the indeterminacy consisting of $\left(l^{ \pm}\right)^{\left(l^{ \pm}\right)}$possibilities that arises in Proposition 6.8, (ii), is reduced to an indeterminacy consisting of a total of $l^{ \pm}$! possibilities.

(ii) (Mono-analytic Processions) By composing the functor of (i) with the mono-analyticization operation discussed in Definition 4.1, (iv), one obtains a natural functor

$$
\begin{aligned}
& \begin{array}{|c|}
\hline \text { category of } \\
\mathcal{D} \text { - } \Theta^{ \pm} \text {-bridges } \\
\text { and isomorphisms of } \\
\mathcal{D} \text { - } \Theta^{ \pm} \text {-bridges }
\end{array} \rightarrow \quad \begin{array}{c}
\begin{array}{c}
\text { category of processions } \\
\text { of } \mathcal{D}^{\vdash} \text {-prime-strips } \\
\text { and morphisms of } \\
\text { processions }
\end{array} \\
\hline
\end{array} \\
& { }^{\dagger} \phi_{ \pm}^{\Theta^{ \pm}} \quad \mapsto \quad \operatorname{Prc}\left({ }^{\dagger} \mathfrak{D}_{T}^{\vdash}\right)
\end{aligned}
$$


whose output data satisfies the same indeterminacy properties with respect to labels as the output data of the functor of (i).

(iii) The functors of (i), (ii) are compatible, respectively, with the functors of Proposition 4.11, (i), (ii), relative to the functor [i.e., determined by the functorial algorithm] of Proposition 6.7, in the sense that the natural inclusions

$$
\mathbb{S}_{j}^{*}=\{1, \ldots, j\} \hookrightarrow \mathbb{S}_{t}^{ \pm}=\{0,1, \ldots, t-1\}
$$

[cf. the notation of Proposition 4.11] - where $j \in\left\{1, \ldots, l^{*}\right\}$ and $t \stackrel{\text { def }}{=} j+1$ determine natural transformations

$$
\begin{aligned}
& { }^{\dagger} \phi_{ \pm}^{\Theta^{ \pm}} \mapsto \quad\left(\operatorname{Prc}\left({ }^{\dagger} \mathfrak{D}_{T^{*}}\right) \hookrightarrow \operatorname{Prc}\left({ }^{\dagger} \mathfrak{D}_{T}\right)\right) \\
& \left.{ }^{\dagger} \phi_{ \pm}^{\Theta^{ \pm}} \mapsto \operatorname{Prc}\left({ }^{\dagger} \mathfrak{D}_{T^{*}}^{\vdash}\right) \hookrightarrow \operatorname{Prc}\left({ }^{\dagger} \mathfrak{D}_{T}^{\vdash}\right)\right)
\end{aligned}
$$

from the respective composites of the functors of Proposition 4.11, (i), (ii), with the functor [determined by the functorial algorithm] of Proposition 6.7 to the functors of (i), (ii).

Proof. Assertions (i), (ii), (iii) follow immediately from the definitions.

The following result is an immediate consequence of our discussion.

Corollary 6.10. (Étale-pictures of Base- $\Theta^{ \pm e l l}-$ Hodge Theaters) Relative to a fixed collection of initial $\Theta$-data:

(i) Consider the [composite] functor

$$
{ }^{\dagger} \mathcal{H} \mathcal{T}^{\mathcal{D}-\Theta^{ \pm \mathrm{ell}}} \mapsto{ }^{\dagger} \mathfrak{D}_{>} \quad \mapsto \quad{ }^{\dagger} \mathfrak{D}_{>}^{\vdash}
$$

- from the category of $\mathcal{D}-\Theta^{ \pm \mathrm{ell}}-$ Hodge theaters and isomorphisms of $\mathcal{D}-\Theta^{ \pm \mathrm{ell}}-$ Hodge theaters [cf. Definition 6.4, (iii)] to the category of $\mathcal{D}^{\vdash}$-prime-strips and isomorphisms of $\mathcal{D}^{\vdash}$-prime-strips — obtained by assigning to the $\mathcal{D}-\Theta^{ \pm \text {ell }}$-Hodge theater ${ }^{\dagger} \mathcal{H} \mathcal{T}^{\mathcal{D}-\Theta^{ \pm e l l}}$ the mono-analyticization [cf. Definition 4.1, (iv)] ${ }^{\dagger} \mathfrak{D}_{>}^{\vdash}$ of the $\mathcal{D}$ prime-strip ${ }^{\dagger} \mathfrak{D}_{>}$associated, via the functorial algorithm of Proposition 6. 7 , to the underlying $\mathcal{D}-\Theta^{ \pm}$-bridge of ${ }^{\dagger} \mathcal{H} \mathcal{T}^{\mathcal{D}-\Theta^{ \pm \text {ell }}}$. If ${ }^{\dagger} \mathcal{H} \mathcal{T}^{\mathcal{D}-\Theta^{ \pm \mathrm{ell}}},{ }^{\ddagger} \mathcal{H} \mathcal{T}^{\mathcal{D}-\Theta^{ \pm \text {ell }}}$ are $\mathcal{D}$ - $\Theta^{ \pm \text {ell }}$ Hodge theaters, then we define the base- $\Theta^{ \pm \text {ell }}$-, or $\mathcal{D}-\Theta^{ \pm e l l}-$, link

$$
{ }^{\dagger} \mathcal{H} \mathcal{T}^{\mathcal{D}-\Theta^{ \pm \mathrm{ell}}} \stackrel{\mathcal{D}}{\longrightarrow} \ddagger \mathcal{H} \mathcal{T}^{\mathcal{D}-\Theta^{ \pm \mathrm{ell}}}
$$

from ${ }^{\dagger} \mathcal{H} \mathcal{T}^{\mathcal{D} \text { - } \Theta^{ \pm \text {ell }}}$ to ${ }^{\ddagger} \mathcal{H} \mathcal{T}^{\mathcal{D} \text { - } \Theta^{ \pm \text {ell }}}$ to be the full poly-isomorphism

$$
\dagger \mathfrak{D}_{>}^{\vdash} \stackrel{\sim}{\rightarrow} \ddagger \mathfrak{D}_{>}^{\vdash}
$$

between the $\mathcal{D}^{\vdash}$-prime-strips obtained by applying the functor discussed above to $\dagger \mathcal{H} \mathcal{T}^{\mathcal{D}-\Theta^{ \pm \mathrm{ell}}}, \ddagger \mathcal{H} \mathcal{T}^{\mathcal{D}-\Theta^{ \pm \mathrm{ell}}}$. 
(ii) If

$\quad \stackrel{\mathcal{D}}{\longrightarrow}{ }^{(n-1)} \mathcal{H} \mathcal{T}^{\mathcal{D}-\Theta^{ \pm \mathrm{ell}}} \stackrel{\mathcal{D}}{\longrightarrow} n^{n} \mathcal{H} \mathcal{T}^{\mathcal{D}-\Theta^{ \pm \text {ell }}} \stackrel{\mathcal{D}}{\longrightarrow} \quad{ }^{(n+1)} \mathcal{H} \mathcal{T}^{\mathcal{D}-\Theta^{ \pm \text {ell }}} \stackrel{\mathcal{D}}{\longrightarrow} \quad \ldots$

[where $n \in \mathbb{Z}$ ] is an infinite chain of $\mathcal{D}$ - $\Theta^{ \pm \text {ell }}$-linked $\mathcal{D}$ - $\Theta^{ \pm \text {ell }}$-Hodge theaters [cf. the situation discussed in Corollary 3.8], then we obtain a resulting chain of full poly-isomorphisms

$$
\ldots \stackrel{\sim}{\rightarrow} n^{\vdash} \mathfrak{D}_{>}^{\vdash} \stackrel{\sim}{\rightarrow}(n+1) \mathfrak{D}_{>}^{\vdash} \stackrel{\sim}{\rightarrow} \ldots
$$

[cf. the situation discussed in Remark 3.8.1, (ii)] between the $\mathcal{D}^{\vdash}$-prime-strips obtained by applying the functor of (i). That is to say, the output data of the functor of (i) forms a constant invariant [cf. the discussion of Remark 3.8.1, (ii)] i.e., a mono-analytic core [cf. the situation discussed in Remark 3.9.1] - of the above infinite chain.

$$
{ }^{ \pm} \hookrightarrow / \pm / \pm \hookrightarrow \ldots
$$

.

${ }^{ \pm} \hookrightarrow /^{ \pm} / \pm \hookrightarrow \ldots$

.

Fig. 6.3: Étale-picture of $\mathcal{D}-\Theta^{ \pm \text {ell }}-$ Hodge theaters

(iii) If we regard each of the $\mathcal{D}-\Theta^{ \pm e l l}-$ Hodge theaters of the chain of (ii) as a spoke emanating from the mono-analytic core discussed in (ii), then we obtain a diagram - i.e., an étale-picture of $\mathcal{D}-\Theta^{ \pm \text {ell }}$-Hodge theaters - as in Fig. 6.3 [cf. the situation discussed in Corollary 3.9, (i)]. In Fig. 6.3, "> " denotes the mono-analytic core, obtained [cf. (i); Proposition 6.7] by identifying the mono-analyticized $\mathcal{D}$-prime-strips of the $\mathcal{D}-\Theta^{ \pm e l l}-H o d g e$ theater labeled " 0 " and " $\succ$ "; "/ $\hookrightarrow /^{ \pm} / \pm \hookrightarrow \ldots$ " denotes the "holomorphic" processions of Proposition 6.9, (i), together with the remaining ["holomorphic"] data of the corresponding $\mathcal{D}$ $\Theta^{ \pm \text {ell }}$-Hodge theater. In particular, the mono-analyticizations of the zero-labeled $\mathcal{D}$-prime-strips - i.e., the $\mathcal{D}$-prime-strips corresponding to the first " \pm " in the processions just discussed - in the various spokes are identified with one another. Put another way, the coric $\mathcal{D}^{\vdash}$-prime-strip " $>\vdash$ " may be thought of as being equipped with various distinct "holomorphic structures" - i.e., D-prime-strip structures that give rise to the $\mathcal{D}^{\vdash}$-prime-strip structure - corresponding to the various 
spokes. Finally, [cf. the situation discussed in Corollary 3.9, (i)] this diagram satisfies the important property of admitting arbitrary permutation symmetries among the spokes [i.e., among the labels $n \in \mathbb{Z}$ of the $\mathcal{D}-\Theta^{ \pm \text {ell }}$-Hodge theaters].

(iv) The constructions of (i), (ii), (iii) are compatible, respectively, with the constructions of Corollary 4.12, (i), (ii), (iii), relative to the functor [i.e., determined by the functorial algorithm] of Proposition 6.7, in the evident sense [cf. the compatibility discussed in Proposition 6.9, (iii)].

Finally, we conclude with additive analogues of Definition 5.5, Corollary 5.6.

\section{Definition 6.11.}

(i) We define a $\Theta^{ \pm}$-bridge [relative to the given initial $\Theta$-data] to be a polymorphism

$$
\dagger \mathfrak{F}_{T} \stackrel{{ }^{\dagger} \psi_{ \pm}^{\Theta \pm}}{\longrightarrow} \dagger \mathfrak{F}_{\succ}
$$

- where ${ }^{\dagger} \mathfrak{F}_{\succ}$ is an $\mathcal{F}$-prime-strip; $T$ is an $\mathbb{F}_{l}^{ \pm}$-group; ${ }^{\dagger} \mathfrak{F}_{T}=\left\{{ }^{\dagger} \mathfrak{F}_{t}\right\}_{t \in T}$ is a capsule of $\mathcal{F}$-prime-strips, indexed by [the underlying set of] $T$ - that lifts a $\mathcal{D}$ - $\Theta^{ \pm}$-bridge ${ }^{\dagger} \phi_{ \pm}^{\Theta}{ }^{ \pm}{ }^{\dagger} \mathfrak{D}_{T} \rightarrow{ }^{\dagger} \mathfrak{D}_{\succ}[$ cf. Corollary 5.3, (ii)]. In this situation, we shall write

$$
{ }^{\dagger} \mathfrak{F}_{|T|}
$$

for the $l^{ \pm}$-capsule obtained from the $l$-capsule ${ }^{\dagger} \mathfrak{F}_{T}$ by forming the quotient $|T|$ of the index set $T$ of this underlying capsule by the action of $\{ \pm 1\}$ and identifying the components of the capsule ${ }^{\dagger} \mathfrak{F}_{T}$ indexed by the elements in the fibers of the quotient $T \rightarrow|T|$ via the constituent poly-morphisms of ${ }^{\dagger} \psi_{ \pm}^{\Theta^{ \pm}}=\left\{{ }^{\dagger} \psi_{t}^{\Theta^{ \pm}}\right\}_{t \in T}$ [so each constituent $\mathcal{F}$-prime-strip of ${ }^{\dagger} \mathfrak{F}_{|T|}$ is only well-defined up to a positive automorphism [i.e., up to an automorphism such that the induced automorphism of the associated $\mathcal{D}$-prime-strip is positive], but this indeterminacy will not affect applications of this construction - cf. the discussion of Definition 6.4, (i)]. Also, we shall write

$$
{ }^{\dagger} \mathfrak{F}_{T *}
$$

for the $l^{*}$-capsule determined by the subset $T \stackrel{*}{\text { def }}|T| \backslash\{0\}$ of nonzero elements of $|T|$. We define a(n) [iso]morphism of $\Theta^{ \pm}$-bridges

$$
\left({ }^{\dagger} \mathfrak{F}_{T} \stackrel{{ }^{\dagger} \psi_{ \pm}^{\Theta^{ \pm}}}{\longrightarrow} \dagger \mathfrak{F}_{\succ}\right) \rightarrow\left({ }^{\ddagger} \mathfrak{F}_{T^{\prime}} \stackrel{{ }^{\ddagger} \psi_{ \pm}^{\Theta^{ \pm}}}{\longrightarrow} \quad \ddagger \mathfrak{F}_{\succ}\right)
$$

to be a pair of poly-isomorphisms

$$
{ }^{\dagger} \mathfrak{F}_{T} \stackrel{\sim}{\rightarrow} \ddagger \mathfrak{F}_{T^{\prime}} ; \quad{ }^{\dagger} \mathfrak{F}_{\succ} \stackrel{\sim}{\rightarrow} \ddagger \mathfrak{F}_{\succ}
$$

that lifts a morphism between the associated $\mathcal{D}$ - $\Theta^{ \pm}$-bridges ${ }^{\dagger} \phi_{ \pm}^{\Theta^{ \pm}},{ }^{\ddagger} \phi_{ \pm}^{\Theta^{ \pm}}$. There is an evident notion of composition of morphisms of $\Theta^{ \pm}$-bridges.

(ii) We define a $\Theta^{\text {ell }}$-bridge [relative to the given initial $\Theta$-data]

$$
{ }^{\dagger} \mathfrak{F}_{T} \stackrel{{ }^{\dagger}}{\stackrel{\psi_{ \pm}}{\longrightarrow}}{ }^{\mathrm{ell}} \mathcal{D}^{\odot \pm}
$$


— where ${ }^{\dagger} \mathcal{D}^{\odot \pm}$ is a category equivalent to $\mathcal{D}^{\odot \pm} ; T$ is an $\mathbb{F}_{l}^{ \pm}$-torsor; ${ }^{\dagger} \mathfrak{F}_{T}=\left\{{ }^{\dagger} \mathfrak{F}_{t}\right\}_{t \in T}$ is a capsule of $\mathcal{F}$-prime-strips, indexed by [the underlying set of] $T$ - to be a $\mathcal{D}$ - $\Theta^{\text {ell }}$-bridge ${ }^{\dagger} \phi_{ \pm}^{\Theta^{\text {ell }}}:{ }^{\dagger} \mathfrak{D}_{T} \rightarrow{ }^{\dagger} \mathcal{D}^{\odot \pm}$ — where we write ${ }^{\dagger} \mathfrak{D}_{T}$ for the capsule of $\mathcal{D}$ prime-strips associated to ${ }^{\dagger} \mathfrak{F}_{T}[\mathrm{cf}$. Remark 5.2.1, (i)]. We define a(n) [iso]morphism of $\Theta^{\mathrm{ell}}$-bridges

$$
\left({ }^{\dagger} \mathfrak{F}_{T} \stackrel{{ }^{\dagger} \psi_{ \pm}^{\Theta^{\text {ell }}}}{\longrightarrow}{ }^{\dagger} \mathcal{D}^{\odot \pm}\right) \quad \rightarrow \quad\left({ }^{\ddagger} \mathfrak{F}_{T^{\prime}} \stackrel{{ }^{\ddagger}}{\psi_{ \pm}^{\Theta^{\text {ell }}}} \stackrel{\ddagger}{\ddagger} \mathcal{D}^{\odot \pm}\right)
$$

to be a pair of poly-isomorphisms

$$
{ }^{\dagger} \mathfrak{F}_{T} \stackrel{\sim}{\rightarrow} \ddagger \mathfrak{F}_{T^{\prime}} ; \quad{ }^{\dagger} \mathcal{D}^{\odot \pm} \stackrel{\sim}{\rightarrow} \ddagger \mathcal{D}^{\odot \pm}
$$

that determines a morphism between the associated $\mathcal{D}$ - $\Theta^{\text {ell }}$-bridges ${ }^{\dagger} \phi_{ \pm}^{\Theta^{\text {ell }}},{ }^{\ddagger} \phi_{ \pm}^{\Theta^{\text {ell }}}$. There is an evident notion of composition of morphisms of $\Theta^{\text {ell }}$-bridges.

(iii) We define a $\Theta^{ \pm e l l}$-Hodge theater [relative to the given initial $\Theta$-data] to be a collection of data

$$
{ }^{\dagger} \mathcal{H} \mathcal{T}^{\Theta^{ \pm \mathrm{ell}}}=\left({ }^{\dagger} \mathfrak{F}_{\succ} \stackrel{{ }^{\dagger} \psi_{ \pm}^{\Psi^{ \pm}}}{\longleftarrow}{ }^{\dagger} \mathfrak{F}_{T} \stackrel{{ }^{\dagger} \psi_{ \pm \pm}^{\Theta^{\mathrm{ell}}}}{\longrightarrow}{ }^{\dagger} \mathcal{D}^{\odot \pm}\right)
$$

- where ${ }^{\dagger} \psi_{ \pm}^{\Theta^{ \pm}}$is a $\Theta^{ \pm}$-bridge $;{ }^{\dagger} \psi_{ \pm}^{\Theta^{\text {ell }}}$ is a $\Theta^{\text {ell }}$-bridge - such that the associated data $\left\{{ }^{\dagger} \phi_{ \pm}^{\Theta^{ \pm}},{ }^{\dagger} \phi_{ \pm}^{\Theta^{\mathrm{ell}}}\right\}\left[\right.$ cf. (i), (ii)] forms a $\mathcal{D}-\Theta^{ \pm \text {ell }}$-Hodge theater. A(n) [iso]morphism of $\Theta^{ \pm \text {ell }}$-Hodge theaters is defined to be a pair of morphisms between the respective associated $\Theta^{ \pm}$- and $\Theta^{\text {ell }}$-bridges that are compatible with one another in the sense that they induce the same poly-isomorphism between the respective capsules of $\mathcal{F}$-prime-strips. There is an evident notion of composition of morphisms of $\Theta^{ \pm \text {ell }}$ Hodge theaters.

Corollary 6.12. (Isomorphisms of $\Theta^{ \pm}$-Bridges, $\Theta^{\text {ell }}$-Bridges, and $\Theta^{ \pm \text {ell }}$ Hodge Theaters) Relative to a fixed collection of initial $\Theta$-data:

(i) The natural functorially induced map from the set of isomorphisms between two $\Theta^{ \pm}$-bridges (respectively, two $\Theta^{\text {ell }}$-bridges; two $\Theta^{ \pm \text {ell }}$-Hodge theaters) to the set of isomorphisms between the respective associated $\mathcal{D}$ - $\Theta^{ \pm}$-bridges (respectively, associated $\mathcal{D}-\Theta^{\text {ell }}$-bridges; associated $\mathcal{D}-\Theta^{ \pm \text {ell }}$-Hodge theaters) is bijective.

(ii) Given a $\Theta^{ \pm}$-bridge and $a \Theta^{\mathrm{ell}}$-bridge, the set of capsule-+-full poly-isomorphisms between the respective capsules of $\mathcal{F}$-prime-strips which allow one to glue the given $\Theta^{ \pm}$- and $\Theta^{\mathrm{ell}}$-bridges together to form a $\Theta^{ \pm \mathrm{ell}}$-Hodge theater forms a torsor over the group

$$
\mathbb{F}_{l}^{\rtimes \pm} \times\left(\{ \pm 1\}^{\mathbb{V}}\right)
$$

[cf. Proposition 6.6, (iv)]. Moreover, the first factor may be thought of as corresponding to the induced isomorphisms of $\mathbb{F}_{l}^{ \pm}$-torsors between the index sets of the capsules involved.

Proof. Assertions (i), (ii) follow immediately from Definition 6.11; Corollary 5.3, (ii) [cf. also Proposition 6.6, (iv), in the case of assertion (ii)]. 
Remark 6.12.1. By applying Corollary 6.12, a similar remark to Remark 5.6.1 may be made concerning the $\Theta^{ \pm}$-bridges, $\Theta^{\text {ell }}$-bridges, and $\Theta^{ \pm \text {ell }}$-Hodge theaters studied in the present $\S 6$. We leave the routine details to the reader.

Remark 6.12.2. Relative to a fixed collection of initial $\Theta$-data:

(i) Suppose that $\left({ }^{\dagger} \mathfrak{F}_{T} \rightarrow{ }^{\dagger} \mathfrak{F}_{\succ}\right)$ is a $\Theta^{ \pm}$-bridge; write $\left({ }^{\dagger} \mathfrak{D}_{T} \rightarrow{ }^{\dagger} \mathfrak{D}_{\succ}\right)$ for the associated $\mathcal{D}-\Theta^{ \pm}$-bridge [cf. Definition 6.11, (i)]. Then Proposition 6.7 gives a functorial algorithm for constructing a $\mathcal{D}$ - $\Theta$-bridge $\left({ }^{\dagger} \mathfrak{D}_{T *} \rightarrow{ }^{\dagger} \mathfrak{D}_{>}\right)$from this $\mathcal{D}$ - $\Theta^{ \pm}$-bridge $\left({ }^{\dagger} \mathfrak{D}_{T} \rightarrow{ }^{\dagger} \mathfrak{D}_{\succ}\right)$. Suppose that this $\mathcal{D}$ - $\Theta$-bridge $\left({ }^{\dagger} \mathfrak{D}_{T^{*}} \rightarrow{ }^{\dagger} \mathfrak{D}_{>}\right)$ arises as the $\mathcal{D}$ - $\Theta$-bridge associated to a $\Theta$-bridge $\left({ }^{\ddagger} \mathfrak{F}_{J} \rightarrow{ }^{\ddagger} \mathfrak{F}_{>} \rightarrow{ }^{\ddagger} \mathcal{H T}^{\Theta}\right)$ [so $J=T^{*}$ — cf. Definition 5.5, (ii)]. Then since the portion " $\ddagger \mathfrak{F}_{J} \rightarrow{ }^{\ddagger} \mathfrak{F}_{>}$" of this $\Theta$-bridge is completely determined [cf. Definition 5.5, (ii), (d)] by the associated $\mathcal{D}$ - $\Theta$-bridge, one verifies immediately that

one may regard this portion " $\ddagger \mathfrak{F}_{J} \rightarrow \ddagger \mathfrak{F}_{>}$" of the $\Theta$-bridge as having been constructed via a functorial algorithm similar to the functorial algorithm of Proposition 6.7 [cf. also Definition 5.5, (ii), (d); the discussion of Remark 5.3.1] from the $\Theta^{ \pm}$-bridge $\left({ }^{\dagger} \mathfrak{F}_{T} \rightarrow{ }^{\dagger} \mathfrak{F}_{\succ}\right)$.

Since, moreover, isomorphisms between $\Theta$-bridges are in natural bijective correspondence with isomorphisms between the associated $\mathcal{D}$ - $\Theta$-bridges [cf. Corollary 5.6, (ii)], it thus follows immediately [cf. Corollary 5.3, (ii)] that isomorphisms between $\Theta$-bridges are in natural bijective correspondence with isomorphisms between the portions of $\Theta$-bridges [i.e., " $\mathfrak{F}_{J} \rightarrow{ }^{\ddagger} \mathfrak{F}_{>}$"] considered above. Thus, in summary, if $\left({ }^{\ddagger} \mathfrak{F}_{J} \rightarrow{ }^{\ddagger} \mathfrak{F}_{>} \rightarrow{ }^{\ddagger} \mathcal{H} \mathcal{T}^{\Theta}\right)$ is a $\Theta$-bridge for which the portion "¥ $\mathfrak{F}_{J} \rightarrow \ddagger \mathfrak{F}_{>} "$ is obtained via the functorial algorithm discussed above from the $\Theta^{ \pm}$-bridge $\left({ }^{\dagger} \mathfrak{F}_{T} \rightarrow{ }^{\dagger} \mathfrak{F}_{\succ}\right)$, then, for simplicity, we shall describe this state of affairs by saying that

the $\Theta$-bridge $\left({ }^{\ddagger} \mathfrak{F}_{J} \rightarrow{ }^{\ddagger} \mathfrak{F}_{>} \rightarrow{ }^{\ddagger} \mathcal{H} \mathcal{T}^{\Theta}\right)$ is glued to the $\Theta^{ \pm}$-bridge $\left({ }^{\dagger} \mathfrak{F}_{T} \rightarrow{ }^{\dagger} \mathfrak{F}_{\succ}\right)$ via the functorial algorithm of Proposition 6.7.

We leave the routine details of giving a more explicit description [say, in the style of the statement of Proposition 6.7] of such functorial algorithms to the reader. A similar [but easier!] construction may be given for $\mathcal{D}-\Theta$-bridges and $\mathcal{D}$ - $\Theta^{ \pm}$-bridges.

(ii) Now observe that

by gluing a $\Theta^{ \pm \text {ell }}$-Hodge theater [cf. Definition 6.11, (iii)] to a $\Theta N F-$ Hodge theater [cf. Definition 5.5, (iii)] along the respective associated $\Theta^{ \pm}$- and $\Theta$-bridges via the functorial algorithm of Proposition 6.7 [cf. (i)], one obtains the notion of a

\section{" $\Theta^{ \pm \text {ell }}$ NF-Hodge theater"}

- cf. Definition 6.13, (i), below. Here, we note that by Proposition 4.8, (ii); Corollary 5.6, (ii), the gluing isomorphism that occurs in such a gluing operation is unique. Then by applying Propositions 4.8, 6.6, and Corollaries 5.6, 6.12, one 
may verify analogues of these results for such $\Theta^{ \pm \text {ell }}$ NF-Hodge theaters. In a similar vein, one may glue a $\mathcal{D}-\Theta^{ \pm \text {ell }}-$ Hodge theater to a $\mathcal{D}-\Theta N F-H o d g e$ theater to obtain a "D- $\Theta^{ \pm \text {ell }} N F-H o d g e$ theater" [cf. Definition 6.13, (ii), below]. We leave the routine details to the reader.

\section{Remark 6.12.3.}

(i) One way to think of the notion of a $\Theta \mathrm{NF}$-Hodge theater studied in $\S 4$ is as a sort of

$$
\text { total space of a local system of } \mathbb{F}_{l}^{*} \text {-torsors }
$$

over a "base space" that represents a sort of "homotopy" between a number field and a Tate curve [i.e., the elliptic curve under consideration at the $\left.\underline{v} \in \underline{\mathbb{V}}^{\text {bad }}\right]$. From this point of view, the notion of a $\Theta^{ \pm \text {ell }}$-Hodge theater studied in the present $\S 6$ may be thought of as a sort of

$$
\text { total space of a local system of } \mathbb{F}_{l}^{\rtimes \pm} \text {-torsors }
$$

over a similar "base space". Here, it is interesting to note that these $\mathbb{F}_{l}^{*}$ - and $\mathbb{F}_{l}^{\rtimes \pm}$ torsors arise, on the one hand, from the l-torsion points of the elliptic curve under consideration, hence may be thought of as

\section{discrete approximations of [the geometric portion of] this elliptic curve over a number field}

[cf. the point of view of scheme-theoretic Hodge-Arakelov theory discussed in [HASurI], §1.3.4]. On the other hand, if one thinks in terms of the tempered fundamental groups of the Tate curves that occur at $\underline{v} \in \underline{\mathbb{V}}^{\text {bad }}$, then these $\mathbb{F}_{l}^{*}$ - and $\mathbb{F}_{l}^{\rtimes \pm}$-torsors may be thought of as

\section{finite approximations of the copy of " $\mathbb{Z}$ "}

that occurs as the Galois group of a well-known tempered covering of the Tate curve [cf. the discussion of [EtTh], Remark 2.16.2]. Note, moreover, that if one works with $\Theta^{ \pm \text {ell }} N F$-Hodge theaters [cf. Remark 6.12.2, (ii)], then one is, in effect, working with both the additive and the multiplicative structures of this copy of $\mathbb{Z}$ - although, unlike the situation that occurs when one works with rings, i.e., in which the additive and multiplicative structures are "entangled" with one another in some sort of complicated fashion [cf. the discussion of [AbsTopIII], Remark 5.6.1], if one works with $\Theta^{ \pm \text {ell } N F-H o d g e ~ t h e a t e r s, ~ t h e n ~ e a c h ~ o f ~ t h e ~ a d d i t i v e ~}$ and multiplicative structures occurs in an independent fashion [i.e., in the form of $\Theta^{ \pm \text {ell }}$ - and $\Theta N F-H o d g e$ theaters], i.e., "extracted" from this entanglement.

(ii) At this point, it is useful to recall that the idea of a distinct [i.e., from the copy of $\mathbb{Z}$ implicit in the "base space"] "local system-theoretic" copy of $\mathbb{Z}$ occurring over a "base space" that represents a number field is reminiscent not only of the discussion of [EtTh], Remark 2.16.2, but also of the Teichmüller-theoretic point of view discussed in [AbsTopIII], §I5. That is to say, relative to the analogy with $p$-adic Teichmüller theory, the "base space" that represents a number field corresponds to a hyperbolic curve in positive characteristic, while the "local system-theoretic" 
copy of $\mathbb{Z}$ - which, as discussed in (i), also serves as a discrete approximation of the [geometric portion of the] elliptic curve under consideration - corresponds to a nilpotent ordinary indigenous bundle over the positive characteristic hyperbolic curve.

(iii) Relative to the analogy discussed in (ii) between the "local system-theoretic" copy of $\mathbb{Z}$ of (i) and the indigenous bundles that occur in $p$-adic Teichmüller theory, it is interesting to note that the two combinatorial dimensions [cf. [AbsTopIII], Remark 5.6.1] corresponding to the additive and multiplicative [i.e., " $\mathbb{F}_{l}^{\rtimes \pm}{ }_{-}$" and "F $F_{l}^{*}$-"] symmetries of $\Theta^{ \pm \text {ell }}$,, $\Theta N F-H_{0}$ ge theaters may be thought of as corresponding, respectively, to the two real dimensions

$$
\begin{array}{ll}
z \mapsto z+a, & z \mapsto-\bar{z}+a ; \\
\cdot z \mapsto \frac{z \cdot \cos (t)-\sin (t)}{z \cdot \sin (t)+\cos (t)}, & z \mapsto \frac{\bar{z} \cdot \cos (t)+\sin (t)}{\bar{z} \cdot \sin (t)-\cos (t)}
\end{array}
$$

— where $a, t \in \mathbb{R} ; z$ denotes the standard coordinate on $\mathfrak{H}$ - of transformations of the upper half-plane $\mathfrak{H}$, i.e., an object that is very closely related to the canonical indigenous bundles that occur in the classical complex uniformization theory of hyperbolic Riemann surfaces [cf. the discussions of Remarks 4.3.3, 5.1.4]. Here, it is also of interest to observe that the above additive symmetry of the upper half-plane is closely related to the coordinate on the upper half-plane determined by the "classical $q$-parameter"

$$
q \stackrel{\text { def }}{=} e^{2 \pi i z}
$$

- a situation that is reminiscent of the close relationship, in the theory of the present series of papers, between the $\mathbb{F}_{l}^{\rtimes \pm}$-symmetry and the Kummer theory surrounding the Hodge-Arakelov-theoretic evaluation of the theta function on the $l$-torsion points at bad primes [cf. Remark 6.12.6, (ii), below; the theory of [IUTchII]]. Moreover, the fixed basepoint " $\mathbb{V}^{ \pm} "[$ cf. Definition $6.1,(\mathrm{v})]$ with respect to which one considers $l$-torsion points in the context of the $\mathbb{F}_{l}^{\rtimes \pm}$-symmetry is reminiscent of the fact that the above additive symmetries of the upper half-plane fix the cusp at infinity. Indeed, taken as a whole, the geometry and coordinate naturally associated to this additive symmetry of the upper half-plane may be thought of, at the level of "combinatorial prototypes", as the geometric apparatus associated to a cusp [i.e., as opposed to a node - cf. the discussion of [NodNon], Introduction]. By contrast, the "toral" multiplicative symmetry of the upper half-plane recalled above is closely related to the coordinate on the upper half-plane that determines a biholomorphic isomorphism with the unit disc

$$
w \stackrel{\text { def }}{=} \frac{z-i}{z+i}
$$

- a situation that is reminiscent of the close relationship, in the theory of the present series of papers, between the $\mathbb{F}_{l}^{*}$-symmetry and the Kummer theory surrounding the number field $F_{\bmod }$ [cf. Remark 6.12.6, (iii), below; the theory of $\S 5$ of the present paper]. Moreover, the action of $\mathbb{F}_{l}^{*}$ on the "collection of basepoints for the $l$-torsion points" $\underline{\mathbb{V}}^{\text {Bor }}=\mathbb{F}_{l}^{*} \cdot \underline{\mathbb{V}}^{ \pm \text {un }}[$ cf. Example 4.3 , (i)] in the context of 
the $\mathbb{F}_{l}^{*}$-symmetry is reminiscent of the fact that the multiplicative symmetries of the upper half-plane recalled above act transitively on the entire boundary of the upper half-plane. That is to say, taken as a whole, the geometry and coordinate naturally associated to this multiplicative symmetry of the upper half-plane may be thought of, at the level of "combinatorial prototypes", as the geometric apparatus associated to a node, i.e., of the sort that occurs in the reduction modulo $p$ of a Hecke correspondence [cf. the discussion of [IUTchII], Remark 4.11.4, (iii), (c); [NodNon], Introduction]. Finally, we note that, just as in the case of the $\mathbb{F}_{l}^{\rtimes \pm}{ }_{-}, \mathbb{F}_{l}^{*}$-symmetries discussed in the present paper, the only "coric" symmetries, i.e., symmetries common to both the additive and multiplicative symmetries of the upper half-plane recalled above, are the symmetries " $\{ \pm 1\}$ " [i.e., the symmetries $z \mapsto z,-\bar{z}$ in the case of the upper half-plane]. The observations of the above discussion are summarized in Fig. 6.4 below.

\section{Remark 6.12.4.}

(i) Just as in the case of the $\mathbb{F}_{l}^{*}$-symmetry of Proposition 4.9, (i), the $\mathbb{F}_{l}^{\rtimes \pm}$ symmetry of Proposition 6.8, (i), will eventually be applied, in the theory of the present series of papers [cf. theory of [IUTchII], [IUTchIII]], to establish an

\section{explicit network of comparison isomorphisms}

relating various objects — such as log-volumes — associated to the non-labeled prime-strips that are permuted by this symmetry [cf. the discussion of Remark 4.9.1, (i)]. Moreover, just as in the case of the $\mathbb{F}_{l}^{*}$-symmetry studied in $\S 4$ [cf. the discussion of Remark 4.9.2], one important property of this "network of comparison isomorphisms" is that it operates without "label crushing" [cf. Remark 4.9.2, (i)] - i.e., without disturbing the bijective relationship between the set of indices of the symmetrized collection of prime-strips and the set of labels $\in T \stackrel{\sim}{\rightarrow} \mathbb{F}_{l}$ under consideration. Finally, just as in the situation studied in $\S 4$,

\section{this crucial synchronization of labels is essentially a consequence of the single connected component}

— or, at a more abstract level, the single basepoint — of the global object [i.e., "† $\mathcal{D}^{\odot \pm " ~ i n ~ t h e ~ p r e s e n t ~} \S 6$; "† $\mathcal{D} \odot "$ in $\left.\S 4\right]$ that appears in the $\left[\mathcal{D}-\Theta^{ \pm \text {ell }}\right.$ - or $\mathcal{D}$ - $\left.\Theta N F-\right]$ Hodge theater under consideration [cf. Remark 4.9.2, (ii)].

(ii) At a more concrete level, the "synchronization of labels" discussed in (i) is realized by means of the crucial bijections

$$
{ }^{\dagger} \zeta_{*}: \operatorname{LabCusp}\left({ }^{\dagger} \mathcal{D}^{\odot}\right) \stackrel{\sim}{\rightarrow} J ; \quad{ }^{\dagger} \zeta_{ \pm}: \operatorname{LabCusp}^{ \pm}\left({ }^{\dagger} \mathcal{D}^{\odot \pm}\right) \stackrel{\sim}{\rightarrow} T
$$

of Propositions 4.7, (iii); 6.5, (iii). Here, we pause to observe that it is precisely the existence of these

bijections relating index sets of capsules of $\mathcal{D}$-prime-strips to sets of global [ \pm-$]$ label classes of cusps 


\begin{tabular}{|c|c|c|}
\hline & $\frac{\text { Classical }}{\underline{\text { upper half-plane }}}$ & 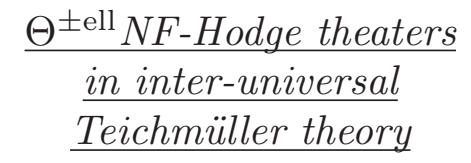 \\
\hline $\begin{array}{l}\text { Additive } \\
\text { symmetry }\end{array}$ & $\begin{array}{l}z \mapsto \quad z+a, \\
z \mapsto-\bar{z}+a \quad(a \in \mathbb{R})\end{array}$ & $\begin{array}{c}\mathbb{F}_{l}^{\rtimes \pm}- \\
\text { symmetry }\end{array}$ \\
\hline $\begin{array}{l}\text { "Functions" assoc'd } \\
\text { to add. symm. }\end{array}$ & $q \stackrel{\text { def }}{=} e^{2 \pi i z}$ & $\begin{array}{l}\text { theta fn. evaluated at } \\
\boldsymbol{l} \text {-tors. [cf. I, 6.12.6, (ii)] }\end{array}$ \\
\hline $\begin{array}{l}\text { Basepoint assoc'd } \\
\text { to add. symm. }\end{array}$ & $\begin{array}{l}\text { single cusp } \\
\text { at infinity }\end{array}$ & {$\left[\right.$ cf. I, $\left.\frac{\mathbb{V}^{ \pm}}{6.1,(\mathrm{v})}\right]$} \\
\hline $\begin{array}{l}\text { Combinatorial } \\
\text { prototype assoc'd } \\
\text { to add. symm. }\end{array}$ & cusp & cusp \\
\hline $\begin{array}{l}\text { Multiplicative } \\
\text { symmetry }\end{array}$ & $\begin{array}{l}z \mapsto \frac{z \cdot \cos (t)-\sin (t)}{z \cdot \sin (t)+\cos (t)}, \\
z \mapsto \frac{\bar{z} \cdot \cos (t)+\sin (t)}{\bar{z} \cdot \sin (t)-\cos (t)} \quad(t \in \mathbb{R})\end{array}$ & $\begin{array}{c}\mathbb{F}_{l}^{*}- \\
\text { symmetry }\end{array}$ \\
\hline $\begin{array}{l}\text { "Functions" } \\
\text { assoc'd to } \\
\text { mult. symm. }\end{array}$ & $w \stackrel{\text { def }}{=} \frac{z-i}{z+i}$ & $\begin{array}{l}\text { elements of the } \\
\text { number field } F_{\bmod } \\
\text { [cf. I, } 6.12 .6,(\text { iii }]\end{array}$ \\
\hline $\begin{array}{l}\text { Basepoints assoc'd } \\
\text { to mult. symm. }\end{array}$ & $\begin{array}{c}\left(\begin{array}{cc}\cos (t) & -\sin (t) \\
\sin (t) & \cos (t)\end{array}\right),\left(\begin{array}{cc}\cos (t) & \sin (t) \\
\sin (t) & -\cos (t)\end{array}\right) \\
\curvearrowright\{\text { entire boundary of } \mathfrak{H}\}\end{array}$ & $\begin{array}{c}\mathbb{F}_{i}^{*} \curvearrowright \underline{\mathbb{V}}^{\text {Bor }}=\mathbb{F}_{i}^{*} \cdot \underline{\mathbb{V}}^{ \pm \text {un }} \\
{[\text { cf. I, 4.3, (i)] }}\end{array}$ \\
\hline $\begin{array}{l}\text { Combinatorial } \\
\text { prototype assoc'd } \\
\text { to mult. symm. }\end{array}$ & $\begin{array}{l}\text { nodes of } \bmod p \\
\text { Hecke correspondence } \\
\text { [cf. II, } 4.11 .4,(\mathrm{iii}),(\mathrm{c})]\end{array}$ & $\begin{array}{l}\quad \text { nodes of mod } p \\
\text { Hecke correspondence } \\
{[\text { cf. II, } 4.11 .4,(\text { iii }),(\mathrm{c})]}\end{array}$ \\
\hline Coric symmetries & $z \mapsto z,-\bar{z}$ & $\{ \pm 1\}$ \\
\hline
\end{tabular}

Fig. 6.4: Comparison of $\mathbb{F}_{l}^{\rtimes \pm}-, \mathbb{F}_{l}^{*}$-symmetries with the geometry of the upper half-plane

that distinguishes the finer "combinatorially holomorphic" [cf. Remarks 4.9.1, 
(ii); 4.9.2, (iv)] $\mathbb{F}_{l}^{*}$ - and $\mathbb{F}_{l}^{\rtimes \pm}$-symmetries of Propositions 4.9, (i); 6.8, (i), from the coarser "combinatorially real analytic" [cf. Remarks 4.9.1, (ii); 4.9.2, (iv)]

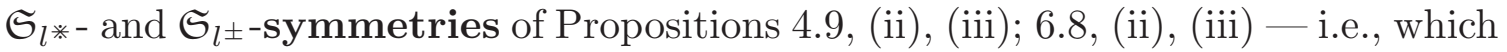
do not admit a compatible bijection between the index sets of the capsules involved and some sort of set of [ \pm -]label classes of cusps [cf. the discussion of Remark 4.9.2, (i)]. This relationship with a set of [ \pm -]label classes of cusps will play a crucial role in the theory of the Hodge-Arakelov-theoretic evaluation of the étale theta function that will be developed in [IUTchII].

(iii) On the other hand, one significant feature of the additive theory of the present $\S 6$ which does not appear in the multiplicative theory of $\S 4$ is the phenomenon of "global \pm -synchronization" — i.e., at a more concrete level, the various isomorphisms " $\dagger$ " that appear in Proposition 6.5, (i), (ii) - between the \pm -indeterminacies that occur at the various $\underline{v} \in \mathbb{V}$. Note that this global \pm -synchronization is a necessary "pre-condition" [i.e., since the natural additive action of $\mathbb{F}_{l}$ on $\mathbb{F}_{l}$ is not compatible with the natural surjection $\left.\mathbb{F}_{l} \rightarrow\left|\mathbb{F}_{l}\right|\right]$ for the additive portion [i.e., corresponding to $\mathbb{F}_{l} \subseteq \mathbb{F}_{l}^{\rtimes \pm}$ ] of the $\mathbb{F}_{l}^{\rtimes \pm}$-symmetry of Proposition 6.8, (i). This "additive portion" of the $\mathbb{F}_{l}^{\rtimes \pm}$-symmetry plays the crucial role of allowing one to relate the zero and nonzero elements of $\mathbb{F}_{l}[\mathrm{cf}$. the discussion of Remark 6.12 .5 below].

(iv) One important property of both the "† $\zeta$ 's" discussed in (ii) and the "† $\xi$ 's" discussed in (iii) is that they are constructed by means of functorial algorithms from the intrinsic structure of a $\mathcal{D}-\Theta^{ \pm \text {ell }}$ - or $\mathcal{D}$ - $\Theta N F-H o d g e$ theater [cf. Propositions 4.7, (iii); 6.5, (i), (ii), (iii)] — i.e., not by means of comparison with some fixed reference model [cf. the discussion of [AbsTopIII], §I4], such as the objects constructed in Examples 4.3, 4.4, 4.5, 6.2, 6.3. This property will be of crucial importance when, in the theory of [IUTchIII], we combine the theory developed in the present series of papers with the theory of log-shells developed in [AbsTopIII].

\section{Remark 6.12.5.}

(i) One fundamental difference between the $\mathbb{F}_{l}^{*}$-symmetry of $\S 4$ and the $\mathbb{F}_{l}^{\rtimes \pm}$ symmetry of the present $\S 6$ lies in the inclusion of the zero element $\in \mathbb{F}_{l}$ in the symmetry under consideration. This inclusion of the zero element $\in \mathbb{F}_{l}$ means, in particular, that the resulting network of comparison isomorphisms [cf. Remark $6.12 .4,(\mathrm{i})]$

allows one to relate the "zero-labeled" prime-strip to the various "nonzerolabeled" prime-strips, i.e., the prime-strips labeled by nonzero elements $\in \mathbb{F}_{l}\left[\right.$ or, essentially equivalently, $\left.\in \mathbb{F}_{l}^{*}\right]$.

Moreover, as reviewed in Remark 6.12.4, (ii), the $\mathbb{F}_{l}^{\rtimes \pm}$-symmetry allows one to relate the zero-labeled and non-zero-labeled prime-strips to one another in a "combinatorially holomorphic" fashion, i.e., in a fashion that is compatible with the various natural bijections [i.e., " $\zeta$ "] with various sets of global \pm -label classes of cusps. Here, it is useful to recall that evaluation at [torsion points closely related to] the zero-labeled cusps [cf. the discussion of "evaluation points" in Example 4.4, (i)] plays an important role in the theory of normalization of the étale theta function 
— cf. the theory of étale theta functions "of standard type", as discussed in [EtTh], Theorem 1.10; the theory to be developed in [IUTchII].

(ii) Whereas the $\mathbb{F}_{l}^{\rtimes \pm}$-symmetry of the theory of the present $\S 6$ has the $a d$ vantage that it allows one to relate zero-labeled and non-zero-labeled prime-strips, it has the [tautological!] disadvantage that it does not allow one to "insulate" the non-zero-labeled prime-strips from confusion with the zero-labeled prime-strip. This issue will be of substantial importance in the theory of Gaussian Frobenioids [to be developed in [IUTchII]], i.e., Frobenioids that, roughly speaking, arise from the theta values

$$
\left\{\underline{\underline{q}} \underline{\underline{\underline{v}}} \stackrel{\underline{j}}{=}^{2}\right\}_{\underline{\underline{j}}}
$$

[cf. the discussion of Example 4.4, (i)] at the non-zero-labeled evaluation points. Moreover, ultimately, in [IUTchII], [IUTchIII], we shall relate these Gaussian Frobenioids to various global arithmetic line bundles on the number field $F$. This will require the use of both the additive and the multiplicative structures on the number field; in particular, it will require the use of the theory developed in $\S 5$.

(iii) By contrast, since, in the theory of the present series of papers, we shall not be interested in analogues of the Gaussian Frobenioids that involve the zerolabeled evaluation points, we shall not require an "additive analogue" of the portion [cf. Example 5.1] of the theory developed in $\S 5$ concerning global Frobenioids.

\section{Remark 6.12.6.}

(i) Another fundamental difference between the $\mathbb{F}_{l}^{*}$-symmetry of $\S 4$ and the $\mathbb{F}_{l}^{\rtimes \pm}$-symmetry of the present $\S 6$ lies in the geometric nature of the "single basepoint" [cf. the discussion of Remark 6.12.4] that underlies the $\mathbb{F}_{l}^{\rtimes \pm}$-symmetry. That is to say, the various labels $\in T \stackrel{\sim}{\rightarrow} \mathbb{F}_{l}$ that appear in a $[\mathcal{D}$ - $] \Theta^{ \pm \text {ell }}$-Hodge theater correspond - throughout the various portions [e.g., bridges] of the $[\mathcal{D}$ - $] \Theta^{ \pm \text {ell }}$-Hodge theater - to collections of cusps in a single copy [i.e., connected component] of "D믈 of the $[\mathcal{D}$ - $] \Theta^{\text {ell }}$-bridge [cf. Proposition 6.8, (i)] without permuting the collection of valuations $\mathbb{V}^{ \pm}(\subseteq \mathbb{V}(K))$ [cf. the discussion of Definition 6.1, (v)]. This contrasts sharply with the arithmetic nature of the "single basepoint" [cf. the discussion of Remark 6.12.4] that underlies the $\mathbb{F}_{i}^{*}$-symmetry of $\S 4$, i.e., in the sense that the $\mathbb{F}_{l}^{*}$-symmetry [cf. Proposition 4.9 , (i)] permutes the various $\mathbb{F}_{l}^{*}$-translates of $\underline{\mathbb{V}}^{ \pm}=\underline{\mathbb{V}}^{ \pm \text {un }} \subseteq \underline{\mathbb{V}}^{\text {Bor }}(\subseteq \mathbb{V}(K))$ [cf. Example 4.3, (i); Remark 6.1.1].

(ii) The geometric nature of the "single basepoint" of the $\mathbb{F}_{l}^{\rtimes \pm}$-symmetry of a $[\mathcal{D}-] \Theta^{ \pm e l l}$-Hodge theater $[\mathrm{cf}$. (i)] is more suited to the theory of the

\section{Hodge-Arakelov-theoretic evaluation of the étale theta function}

to be developed in [IUTchII], in which the existence of a "single basepoint" corresponding to a single connected component of " $\mathcal{D}_{\underline{v}}$ " for $\underline{v} \in \underline{\mathbb{V}}^{\text {bad }}$ plays a central role.

(iii) By contrast, the arithmetic nature of the "single basepoint" of the $\mathbb{F}_{l}^{*}$ symmetry of a $[\mathcal{D}$-] $\Theta$ NF-Hodge theater $[$ cf. (i) $]$ is more suited to the 
explicit construction of the number field $F_{\bmod }$ [cf. Example 5.1]

- i.e., to the construction of an object which is invariant with respect to the $\operatorname{Aut}\left(\underline{C}_{K}\right) / \operatorname{Aut}_{\underline{\epsilon}}\left(\underline{C}_{K}\right) \stackrel{\sim}{\rightarrow} \mathbb{F}_{l}^{*}$-symmetries that appear in the discussion of Example 4.3 , (iv). That is to say, if one attempts to carry out a similar construction to the construction of Example 5.1 with respect to the copy of $\mathcal{D}^{\odot \pm}$ that appears in a $[\mathcal{D}$ - $] \Theta^{\text {ell }}$-bridge, then one must sacrifice the crucial ridigity with respect to $\operatorname{Aut}\left(\mathcal{D}^{\odot \pm}\right) / \operatorname{Aut}_{ \pm}\left(\mathcal{D}^{\odot \pm}\right) \stackrel{\sim}{\rightarrow} \mathbb{F}_{l}^{*}[$ cf. Definition 6.1, (v)] that arises from the structure [i.e., definition] of a $[\mathcal{D}$ - $] \Theta^{\text {ell }}$-bridge [cf. Example 6.3; Definition 6.4, (ii)]. Moreover, if one sacrifices this $\mathbb{F}_{l}^{*}$-rigidity, then one no longer has a situation in which the symmetry under consideration is defined relative to a single copy of " $\mathcal{D}_{\underline{v}}$ " at each $\underline{v} \in \underline{\mathbb{V}}$, i.e., defined with respect to a "single geometric basepoint". In particular, once one sacrifices this $\mathbb{F}_{l}^{*}$-rigidity, the resulting symmetries are no longer compatible with the theory of the Hodge-Arakelov-theoretic evaluation of the étale theta function to be developed in [IUTchII] [cf. (ii)].

(iv) One way to understand the difference discussed in (iii) between the global portions [i.e., the portions involving copies of $\mathcal{D}^{\odot}, \mathcal{D}^{\odot \pm}$ ] of a $[\mathcal{D}$-] $\Theta$ NF-Hodge theater and a $[\mathcal{D}-] \Theta^{ \pm \text {ell }}$-Hodge theater is as a reflection of the fact that whereas the Borel subgroup

$$
\left\{\left(\begin{array}{cc}
* & * \\
0 & *
\end{array}\right)\right\} \subseteq S L_{2}\left(\mathbb{F}_{l}\right)
$$

is normally terminal in $S L_{2}\left(\mathbb{F}_{l}\right)$ [cf. the discussion of Example 4.3], the "semiunipotent" subgroup

$$
\left\{\left(\begin{array}{cc} 
\pm 1 & * \\
0 & \pm 1
\end{array}\right)\right\} \subseteq S L_{2}\left(\mathbb{F}_{l}\right)
$$

[which corresponds to the subgroup $\operatorname{Aut}_{ \pm}\left(\mathcal{D}^{\odot \pm}\right) \subseteq \operatorname{Aut}\left(\mathcal{D}^{\odot \pm}\right)-$ cf. the discussion of Definition 6.1, (v)] fails to be normally terminal in $S L_{2}\left(\mathbb{F}_{l}\right)$.

(v) In summary, taken as a whole, a $[\mathcal{D}$ - $] \Theta^{ \pm \text {ell }}$ NF-Hodge theater $[$ cf. Remark 6.12 .2 , (ii)] may be thought of as a sort of

\section{"intricate relay between geometric and arithmetic basepoints"}

that allows one to carry out, in a consistent fashion, both

(a) the theory of the Hodge-Arakelov-theoretic evaluation of the étale theta function to be developed in [IUTchII] [cf. (ii)] and

(b) the explicit construction of the number field $F_{\bmod }$ in Example 5.1 [cf. (iii)].

Moreover, if one thinks of $\mathbb{F}_{l}$ as a finite approximation of $\mathbb{Z}$ [cf. Remark 6.12.3], then this intricate relay between geometric and arithmetic - or, alternatively, $\mathbb{F}_{l}^{\rtimes \pm}$ [i.e., additive!]- and $\mathbb{F}_{l}^{*}$ [i.e., multiplicative!]- basepoints - may be thought of as a sort of

global combinatorial resolution of the two combinatorial dimensions - i.e., additive and multiplicative [cf. [AbsTopIII], Remark 5.6.1] — of the $\operatorname{ring} \mathbb{Z}$. 
Finally, we observe in passing that - from a computational point of view [cf. the theory of [IUTchIV]!] — it is especially natural to regard $\mathbb{F}_{l}$ as a "good approximation" of $\mathbb{Z}$ when $l$ is "sufficiently large", as is indeed the case in the situations discussed in [GenEll], $§ 4$ [cf. also Remark 3.1.2, (iv)].

$$
\begin{aligned}
& {\left[\begin{array}{rl}
-l^{*} & <\ldots<-1<0 \\
& <1<\ldots<l^{*}
\end{array}\right]} \\
& \left\{\underline{\underline{\mathcal{F}}}_{\underline{v}}\right\}_{\underline{v} \in \underline{\mathbb{V}}^{\text {bad }}} \quad\left[\begin{array}{r}
1 \\
\ddots
\end{array} \quad\left[\begin{array}{l}
< \\
<l^{*}
\end{array}\right]\right. \\
& \mathfrak{D}_{\succ}=/^{ \pm} \\
& \begin{array}{c}
\Uparrow \phi_{ \pm}^{\Theta^{ \pm}} \\
\stackrel{ \pm 1\}}{\curvearrowright}\left(\begin{array}{r}
-l^{*} \\
<\ldots<-1<0 \\
<1<\ldots<l^{*}
\end{array}\right)
\end{array} \\
& \stackrel{\text { glue }}{\Rightarrow}\{0, \succ\}=>\quad \stackrel{\text { glue }}{\Leftarrow} \\
& \mathfrak{D}_{>}=/ * \\
& /^{ \pm} \ldots /^{ \pm} /^{ \pm} /^{ \pm} \ldots /^{ \pm} \\
& \mathfrak{D}_{T} \\
& \Downarrow \phi_{ \pm}^{\Theta^{\mathrm{ell}}} \\
& \begin{array}{ccc} 
\pm & \rightarrow & \pm \\
\uparrow & \stackrel{\mathbb{F}_{l}^{\rtimes \pm}}{\curvearrowright} & \downarrow
\end{array} \\
& \pm \leftarrow \pm \\
& \mathcal{D}^{\odot \pm}=\mathcal{B}\left(\underline{X}_{K}\right)^{0} \\
& \begin{array}{ccccc}
\mathcal{F}_{\text {mod }}^{\circledast} & & & & \\
\cap & & * & \rightarrow & * \\
\mathcal{F}^{\circledast} \quad \leftarrow- & \mathcal{F}^{\odot} & \uparrow & \stackrel{\mathbb{F}_{l}^{*}}{\curvearrowright} & \downarrow \\
& & * & \leftarrow & * \\
& & \mathcal{D}^{\odot} & =\mathcal{B}\left(\underline{C}_{K}\right)^{0}
\end{array} \\
& / * \ldots / * \\
& \mathfrak{D}_{J} \\
& \Downarrow \phi_{*}^{\mathrm{NF}}
\end{aligned}
$$

Fig. 6.5: The combinatorial structure of a $\Theta^{ \pm \text {ell }} \mathrm{NF-Hodge} \mathrm{theater}$ [cf. also Figs. 4.4, 4.7, 6.1, 6.3, 6.6]

\section{Definition 6.13.}

(i) We define a $\Theta^{ \pm e l l} N F$-Hodge theater [relative to the given initial $\Theta$-data]

$$
\dagger \mathcal{H} \mathcal{T}^{\Theta^{ \pm \mathrm{ell}} \mathrm{NF}}
$$

to be a triple, consisting of the following data: (a) a $\Theta^{ \pm \text {ell }}$-Hodge theater ${ }^{\dagger} \mathcal{H} \mathcal{T}^{\Theta^{ \pm e l l}}$ [cf. Definition 6.11, (iii)]; (b) a $\Theta N F-H o d g e$ theater ${ }^{\dagger} \mathcal{H} \mathcal{T}^{\Theta N F}$ [cf. Definition 5.5, (iii)]; (c) the [necessarily unique!] gluing isomorphism between ${ }^{\dagger} \mathcal{H} \mathcal{T}^{\Theta^{ \pm e l l}}$ and $\dagger \mathcal{H} \mathcal{T}^{\Theta \mathrm{NF}}$ [cf. the discussion of Remark 6.12.2, (i), (ii)]. An illustration of the combinatorial structure of a $\Theta^{ \pm e l l} \mathrm{NF}$-Hodge theater is given in Fig. 6.5 above [cf. also Fig. 6.6 below]. 
(ii) We define a $\mathcal{D}$ - $\Theta^{ \pm \text {ell }} N F$-Hodge theater [relative to the given initial $\Theta$-data]

$$
{ }^{\dagger} \mathcal{H} \mathcal{T}^{\mathcal{D}-\Theta^{ \pm \mathrm{ell}} \mathrm{NF}}
$$

to be a triple, consisting of the following data: (a) a $\mathcal{D}-\Theta^{ \pm \text {ell }}-$ Hodge theater ${ }^{\dagger} \mathcal{H} \mathcal{T}^{\mathcal{D}-\Theta^{ \pm e l l}}$ [cf. Definition 6.4, (iii)]; (b) a $\mathcal{D}-\Theta N F-H o d g e$ theater ${ }^{\dagger} \mathcal{H} \mathcal{T}^{\mathcal{D}-\Theta N F}$ [cf. Definition 4.6, (iii)]; (c) the [necessarily unique!] gluing isomorphism between ${ }^{\dagger} \mathcal{H} \mathcal{T}^{\mathcal{D}-\Theta^{ \pm e l l}}$ and $\dagger \mathcal{H} \mathcal{T}^{\mathcal{D}-\Theta N F}$ [cf. the discussion of Remark 6.12.2, (i), (ii)].

\begin{tabular}{|c|c|c|}
\hline $\begin{array}{c}\text { Frobenioid } \\
\text { that appears in a } \\
\Theta^{ \pm \text {ell } N F-H o d g e ~ t h e a t e r ~}\end{array}$ & Brief description & $\underline{\text { Reference }}$ \\
\hline $\begin{array}{c}\text { Data at } \underline{v} \in \underline{\mathbb{V}} \text { of } \\
\mathcal{F} \text {-prime-strip } \\
\text { corresponding to each } \\
/^{ \pm}, \quad /^{*}\end{array}$ & $\begin{array}{c}\text { When } \underline{v} \in \underline{\mathbb{V}}^{\text {non }}, \\
\text { corresponds } \\
\text { to } \\
\Pi_{\underline{v}} \curvearrowright \mathcal{O} \bar{F}_{\underline{v}}^{\triangleright}\end{array}$ & $\mathrm{I}, 5.2,(\mathrm{i})$ \\
\hline$\underline{\underline{\mathcal{F}}}_{\underline{v}}$ at $\underline{v} \in \underline{\mathbb{V}}^{\text {bad }}$ & $\begin{array}{l}\text { tempered Frobenioid } \\
\text { over the portion of } \\
\qquad \mathfrak{D}_{>} \text {at } \underline{v}\end{array}$ & $\begin{array}{c}\mathrm{I}, 5.5,(\mathrm{ii}),(\mathrm{iii}) ; \\
\mathrm{I}, 3.6,(\mathrm{a}) ; \text { discussion } \\
\text { preceding I, } 5.4\end{array}$ \\
\hline $\mathcal{F}_{\bmod }^{\circledast}$ & $\begin{array}{c}\text { [non-realified] } \\
\text { global Frobenioid } \\
\text { corresponding to } \\
F_{\bmod }\end{array}$ & $\begin{array}{l}\mathrm{I}, 5.5,(\mathrm{i}),(\mathrm{iii}) \\
\quad \text { I, 5.1, (iii) }\end{array}$ \\
\hline $\mathcal{F}^{\circledast}$ & $\begin{array}{l}\text { [non-realified] } \\
\text { global Frobenioid } \\
\text { corresponding to } \\
\pi_{1}\left(\mathcal{D}^{\circledast}\right) \curvearrowright \bar{F}\end{array}$ & $\begin{array}{l}\text { I, 5.5, (i), (iii); } \\
\text { I, 5.1, (ii), (iii) }\end{array}$ \\
\hline $\mathcal{F}^{\odot}$ & $\begin{array}{l}\text { [non-realified] } \\
\text { global Frobenioid } \\
\text { corresponding to } \\
\pi_{1}\left(\mathcal{D}^{\odot}\right) \curvearrowright \bar{F}\end{array}$ & $\begin{array}{l}\mathrm{I}, 5.5,(\mathrm{i}),(\mathrm{iii}) \\
\quad \text { I, 5.1, (iii) }\end{array}$ \\
\hline
\end{tabular}

Fig. 6.6: The Frobenioids that appear in a $\Theta^{ \pm \text {ell } N F-H o d g e ~ t h e a t e r ~}$ 


\section{Bibliography}

[André] Y. André, On a Geometric Description of $\operatorname{Gal}\left(\overline{\mathbb{Q}}_{p} / \mathbb{Q}_{p}\right)$ and a $p$-adic Avatar of $\widehat{G T}$, Duke Math. J. 119 (2003), pp. 1-39.

[Asada] M. Asada, The faithfulness of the monodromy representations associated with certain families of algebraic curves, J. Pure Appl. Algebra 159 (2001), pp. 123-147.

[Falt] G. Faltings, Endlichkeitssätze für Abelschen Varietäten über Zahlkörpern, Invent. Math. 73 (1983), pp. 349-366.

[FRS] B. Fine, G. Rosenberger, and M. Stille, Conjugacy pinched and cyclically pinched one-relator groups, Rev. Mat. Univ. Complut. Madrid 10 (1997), pp. 207-227.

[Groth] A. Grothendieck, letter to G. Faltings (June 1983) in Lochak, L. Schneps, Geometric Galois Actions; 1. Around Grothendieck's Esquisse d'un Programme, London Math. Soc. Lect. Note Ser. 242, Cambridge Univ. Press (1997).

[NodNon] Y. Hoshi, S. Mochizuki, On the Combinatorial Anabelian Geometry of Nodally Nondegenerate Outer Representations, Hiroshima Math. J. 41 (2011), pp. 275342 .

[CbTpII] Y. Hoshi, S. Mochizuki, Topics Surrounding the Combinatorial Anabelian Geometry of Hyperbolic Curves II: Tripods and Combinatorial Cuspidalization, RIMS Preprint 1762 (November 2012).

[JP] K. Joshi and C. Pauly, Hitchin-Mochizuki morphism, Opers and Frobeniusdestabilized vector bundles over curves, Adv. Math. 274 (2015), pp. 39-75.

[Kim] M. Kim, The motivic fundamental group of $\mathbb{P}^{1} \backslash\{0,1, \infty\}$ and the theorem of Siegel, Invent. Math. 161 (2005), pp. 629-656.

[Lang] S. Lang, Algebraic number theory, Addison-Wesley Publishing Co. (1970).

[Lehto] O. Lehto, Univalent Functions and Teichmüller Spaces, Graduate Texts in Mathematics 109, Springer-Verlag (1987).

[PrfGC] S. Mochizuki, The Profinite Grothendieck Conjecture for Closed Hyperbolic Curves over Number Fields, J. Math. Sci. Univ. Tokyo 3 (1996), pp. 571-627.

[pOrd] S. Mochizuki, A Theory of Ordinary p-adic Curves, Publ. Res. Inst. Math. Sci. 32 (1996), pp. 957-1151.

[pTeich] S. Mochizuki, Foundations of p-adic Teichmüller Theory, AMS/IP Studies in Advanced Mathematics 11, American Mathematical Society/International Press (1999).

$[p \mathrm{GC}]$ S. Mochizuki, The Local Pro- $p$ Anabelian Geometry of Curves, Invent. Math. 138 (1999), pp. 319-423.

[HASurI] S. Mochizuki, A Survey of the Hodge-Arakelov Theory of Elliptic Curves I, Arithmetic Fundamental Groups and Noncommutative Algebra, Proceedings of 
Symposia in Pure Mathematics 70, American Mathematical Society (2002), pp. 533-569.

[HASurII] S. Mochizuki, A Survey of the Hodge-Arakelov Theory of Elliptic Curves II, Algebraic Geometry 2000, Azumino, Adv. Stud. Pure Math. 36, Math. Soc. Japan (2002), pp. 81-114.

[AbsAnab] S. Mochizuki, The Absolute Anabelian Geometry of Hyperbolic Curves, Galois Theory and Modular Forms, Kluwer Academic Publishers (2004), pp. 77-122.

[CanLift] S. Mochizuki, The Absolute Anabelian Geometry of Canonical Curves, Kazuya Kato's fiftieth birthday, Doc. Math. 2003, Extra Vol., pp. 609-640.

[GeoAnbd] S. Mochizuki, The Geometry of Anabelioids, Publ. Res. Inst. Math. Sci. 40 (2004), pp. 819-881.

[SemiAnbd] S. Mochizuki, Semi-graphs of Anabelioids, Publ. Res. Inst. Math. Sci. 42 (2006), pp. 221-322.

[QuCnf] S. Mochizuki, Conformal and quasiconformal categorical representation of hyperbolic Riemann surfaces, Hiroshima Math. J. 36 (2006), pp. 405-441.

[GlSol] S. Mochizuki, Global Solvably Closed Anabelian Geometry, Math. J. Okayama Univ. 48 (2006), pp. 57-71.

[CombGC] S. Mochizuki, A combinatorial version of the Grothendieck conjecture, Tohoku Math. J. 59 (2007), pp. 455-479.

[Cusp] S. Mochizuki, Absolute anabelian cuspidalizations of proper hyperbolic curves, J. Math. Kyoto Univ. 47 (2007), pp. 451-539.

[FrdI] S. Mochizuki, The Geometry of Frobenioids I: The General Theory, Kyushu J. Math. 62 (2008), pp. 293-400.

[FrdII] S. Mochizuki, The Geometry of Frobenioids II: Poly-Frobenioids, Kyushu J. Math. 62 (2008), pp. 401-460.

[EtTh] S. Mochizuki, The Étale Theta Function and its Frobenioid-theoretic Manifestations, Publ. Res. Inst. Math. Sci. 45 (2009), pp. 227-349.

[AbsTopI] S. Mochizuki, Topics in Absolute Anabelian Geometry I: Generalities, J. Math. Sci. Univ. Tokyo 19 (2012), pp. 139-242.

[AbsTopII] S. Mochizuki, Topics in Absolute Anabelian Geometry II: Decomposition Groups and Endomorphisms, J. Math. Sci. Univ. Tokyo 20 (2013), pp. 171-269.

[AbsTopIII] S. Mochizuki, Topics in Absolute Anabelian Geometry III: Global Reconstruction Algorithms, J. Math. Sci. Univ. Tokyo 22 (2015), pp. 939-1156.

[GenEll] S. Mochizuki, Arithmetic Elliptic Curves in General Position, Math. J. Okayama Univ. 52 (2010), pp. 1-28.

[CombCusp] S. Mochizuki, On the Combinatorial Cuspidalization of Hyperbolic Curves, Osaka J. Math. 47 (2010), pp. 651-715.

[IUTchII] S. Mochizuki, Inter-universal Teichmüller Theory II: Hodge-Arakelov-theoretic Evaluation, RIMS Preprint 1757 (August 2012), to appear in Publ. Res. Inst. Math. Sci. 
[IUTchIII] S. Mochizuki, Inter-universal Teichmüller Theory III: Canonical Splittings of the Log-theta-lattice, RIMS Preprint 1758 (August 2012), to appear in Publ. Res. Inst. Math. Sci.

[IUTchIV] S. Mochizuki, Inter-universal Teichmüller Theory IV: Log-volume Computations and Set-theoretic Foundations, RIMS Preprint 1759 (August 2012), to appear in Publ. Res. Inst. Math. Sci.

[MNT] S. Mochizuki, H. Nakamura, A. Tamagawa, The Grothendieck conjecture on the fundamental groups of algebraic curves, Sugaku Expositions 14 (2001), pp. $31-53$.

[Config] S. Mochizuki, A. Tamagawa, The algebraic and anabelian geometry of configuration spaces, Hokkaido Math. J. 37 (2008), pp. 75-131.

[NSW] J. Neukirch, A. Schmidt, K. Wingberg, Cohomology of number fields, Grundlehren der Mathematischen Wissenschaften 323, Springer-Verlag (2000).

[NS] N. Nikolov and D. Segal, Finite index subgroups in profinite groups, C. R. Math. Acad. Sci. Paris 337 (2003), pp. 303-308.

[RZ] Ribes and Zaleskii, Profinite Groups, Ergebnisse der Mathematik und ihrer Grenzgebiete 3, Springer-Verlag (2000).

[Stb1] P. F. Stebe, A residual property of certain groups, Proc. Amer. Math. Soc. 26 (1970), pp. 37-42.

[Stb2] P. F. Stebe, Conjugacy separability of certain Fuchsian groups, Trans. Amer. Math. Soc. 163 (1972), pp. 173-188.

[Stl] J. Stillwell, Classical topology and combinatorial group theory. Second edition, Graduate Texts in Mathematics 72, Springer-Verlag (1993).

[Tama1] A. Tamagawa, The Grothendieck Conjecture for Affine Curves, Compositio Math. 109 (1997), pp. 135-194.

[Tama2] A. Tamagawa, Resolution of nonsingularities of families of curves, Publ. Res. Inst. Math. Sci. 40 (2004), pp. 1291-1336.

[Wiles] A. Wiles, Modular elliptic curves and Fermat's last theorem, Ann. of Math. 141 (1995), pp. 443-551.

Updated versions of preprints are available at the following webpage: http://www.kurims.kyoto-u.ac.jp/〜motizuki/papers-english.html 